Endbericht

Research Report

\title{
Review des Auf- und Ausbaus des Fachhochschulsektors
}

Lorenz Lassnigg

Martin Unger Hans Pechar Ada Pellert Eva Schmutzer-Hollensteiner Don F. Westerheijden

Institut für Höhere Studien (IHS), Wien Institute for Advanced Studies, Vienna 

Endbericht

Research Report

\title{
Review des Auf- und Ausbaus des Fachhochschulsektors
}

\author{
Lorenz Lassnigg \\ Martin Unger \\ Hans Pechar \\ Ada Pellert
}

Eva Schmutzer-Hollensteiner

Don F. Westerheijden

Mitarbeit:

Monika Krenn

Barbara Neubauer

Stephanie Kiessling

Endbericht

Studie im Auftrag des Bundesministeriums für Bildung, Wissenschaft und Kultur (bm:bwk)

März 2003

Institut für Höhere Studien (IHS), Wien

Institute for Advanced Studies, Vienna

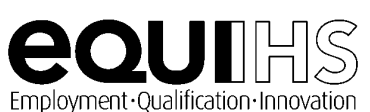

iff bochschulforschung

Iclnhe|p|s| 


\section{Contact:}

Lorenz Lassnigg

III: : +43/1/599 91-214

email: lassnigg@ihs.ac.at

Martin Unger

요: $+43 / 1 / 599$ 91-133

email: unger@ihs.ac.at 


\section{Inhaltsverzeichnis}

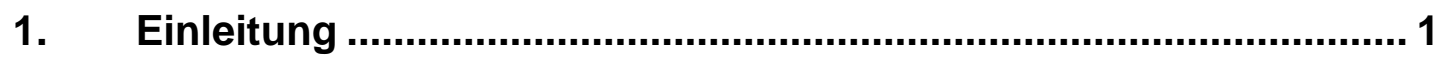

2. Der österreichische FH-Sektor - Grundmerkmale, quantitative Entwicklung, internationaler Vergleich........................................ 5

2.1 Grundmerkmale und Besonderheiten des FH-Sektors....................................... 5

2.1.1 Aufbau neuer Studiengänge in Form eines „bottom-up“-Prozesses ......................... 7

2.1.2 Ein neues Ausbildungsangebot im Hochschulsektor: kurze und berufsorientierte

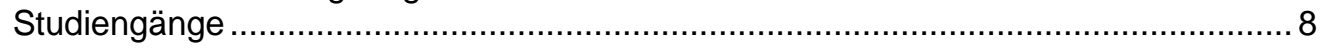

2.1.3 Akkreditierung und Evaluierung, Qualitätsentwicklung an den Standorten ................ 9

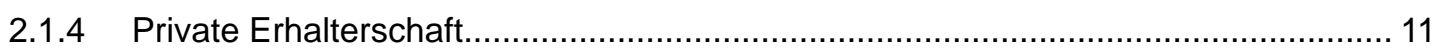

2.1.5 Entwicklungsplanung im Zusammenwirken zwischen Bund, Ländern und FHR

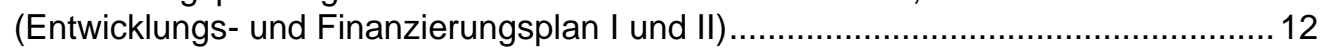

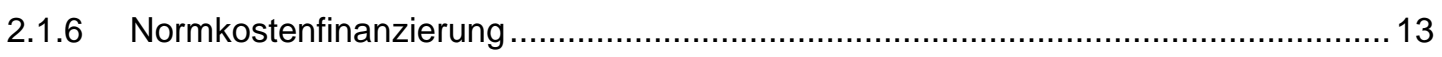

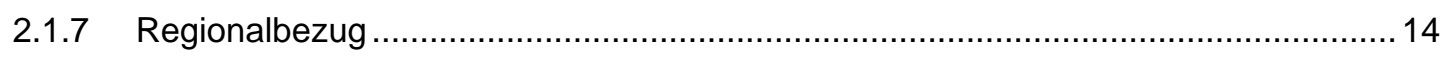

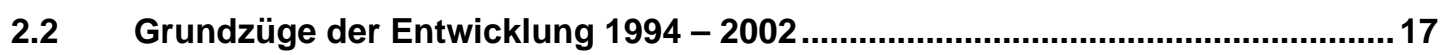

2.2.1 Die wichtigsten Daten im Überblick .................................................................. 17

2.2.2 Entwicklung der Studierenden nach Studiengängen, Erhaltern und Studienorten .... 18

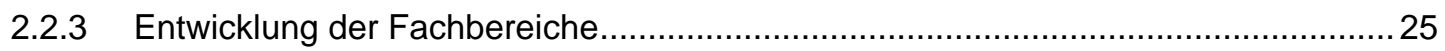

2.2.4 Das BewerberInnenpotential und seine Ausschöpfung ...................................... 27

2.2.5 Vorbildung der FH-Studierenden und nicht-traditioneller Zugang zu FH-Studien ..... 34

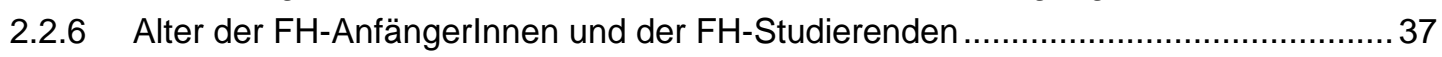

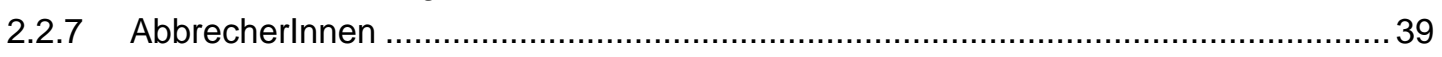

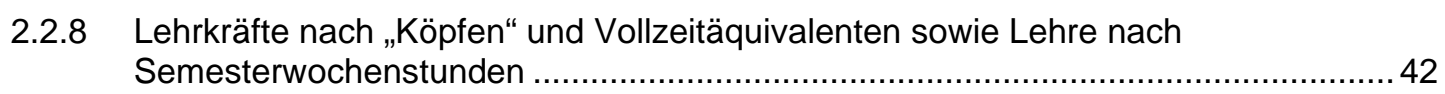

2.3 Der österreichische FH-Sektor im internationalen Vergleich............................. 48

2.3.1 Das FH-System in vier Vergleichsländern (Deutschland, Niederlande, Finnland,

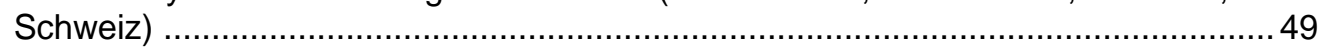

2.3.2 Österreichs FH-Sektor im internationalen Vergleich............................................. 58

3. Stellenwert des FH-Sektors in der Bildungs- und Hochschullandschaft ................................................................65

3.1 Retrospektive Analyse und Einschätzung der Zielerreichung...........................67

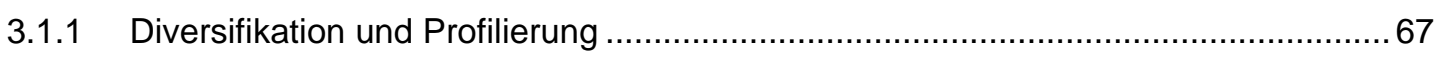

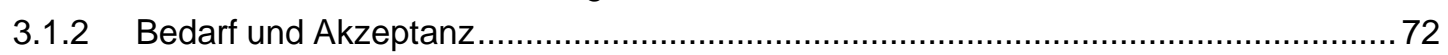

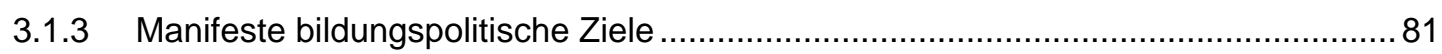

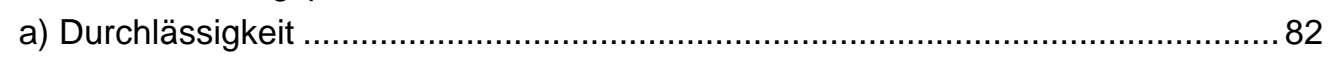

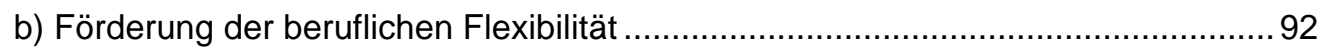

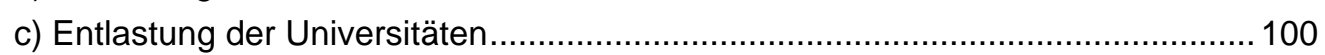

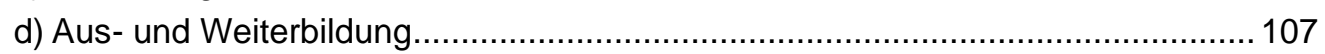

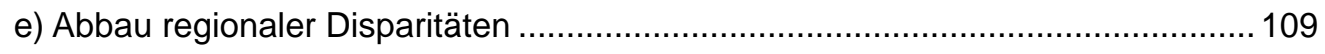

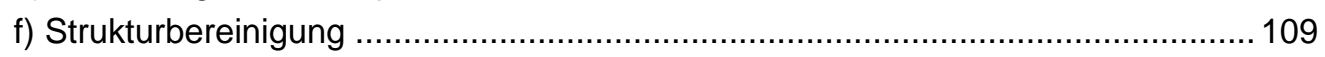

3.1.4 Latente bildungspolitische Ziele .............................................................. 113 
3.2 Veränderungen im Umfeld des FH-Sektors ......................................................116

3.2.1 Endogene Entwicklung und Dynamik im internationalen Vergleich ........................116

3.2.2 Wesentliche Veränderungen im Umfeld des FH-Sektors und zentrale

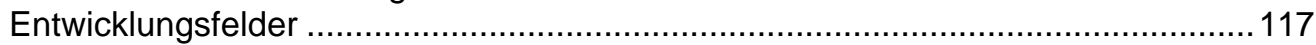

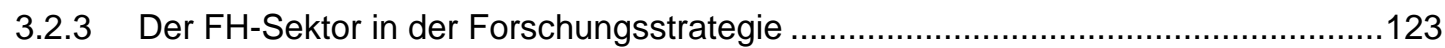

3.3 Entwicklungspfade für den Stellenwert des FH-Sektors ................................131

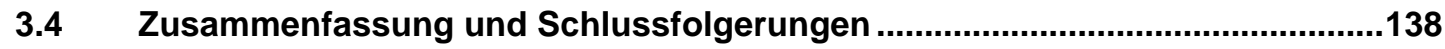

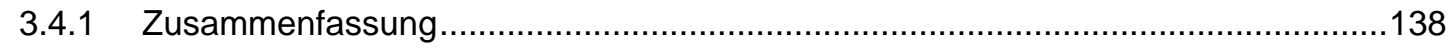

Die Zielsetzungen für den FH-Sektor und ihre Realisierung ...............................138

Auswirkungen von Veränderungen im Umfeld des FH-Sektors.............................141

Rolle des FH-Sektors in der österreichischen Innovationspolitik ............................142

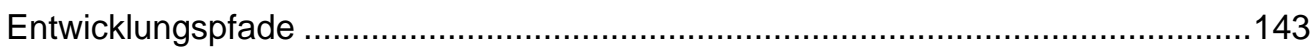

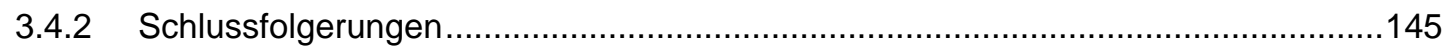

Einbindung der Akteure in eine vertiefende Diskussion der Dimensionierung des

FH-Sektors im Hochschulsystem .........................................................................

Geschlechtsspezifischer Aspekt der Durchlässigkeit: Förderung und

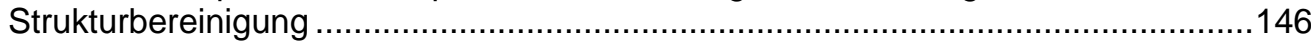

Durchlässigkeit für nicht-traditionelle Studierende ...........................................147

Konzentration und Schwerpunktbildung im Bereich F\&E ....................................147

Fragen zur Diskussion und weiteren Analyse ....................................................147

4. Umsetzung der FH-Entwicklungs- und

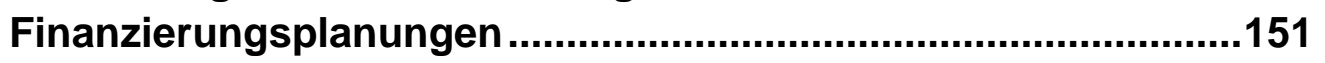

4.1 Retrospektive Betrachtung: Entwicklungsplanung und -steuerung im

Fachhochschulsektor ...............................................................................151

4.1.1 Das Grundmodell der Entwicklungsplanung und -steuerung ..............................151

4.2 Die Steuerung durch die Entwicklungs- und Finanzierungsplanung ................153

4.2.1 Steuerung des FH-Sektors durch die Ausbaugeschwindigkeit .............................155

4.2.2 Steuerung des FH-Sektors durch die Finanzierung .............................................158

4.2.3 Steuerung des FH-Sektors durch inhaltliche Kriterien ........................................167

4.3 Zusammenfassung und Schlussfolgerungen .....................................................174

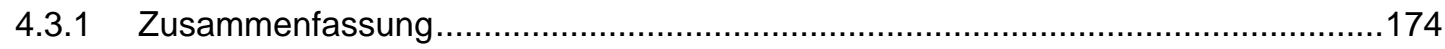

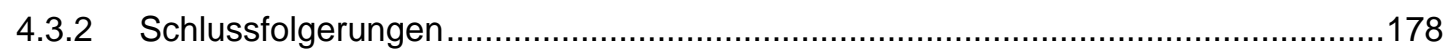

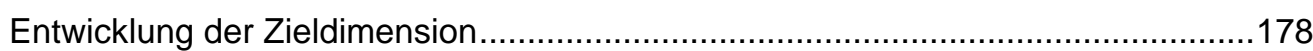

Adäquate Organisationsformen zur Zielbildung .................................................180

Gesamtbetrachtung des tertiären Sektors ..........................................................180

5. Konzentration, Regionalisierung und Standorte im FH-Sektor..183

5.1 Der FH-Sektor im Kontext regionaler Strukturen ..............................................183

5.1.1 Entwicklung in der Spannung zwischen Regionalisierung und Konzentration ........183

5.1.2 Regionale Strukturen und deren Dynamik .......................................................186

5.1.3 Regionale Wirkungen von Hochschuleinrichtungen in der Spannung von Regionalisierung und Internationalisierung .....................................................190

5.2 Retrospektive Analyse der Regionalisierung im FH-Sektor ..............................193 


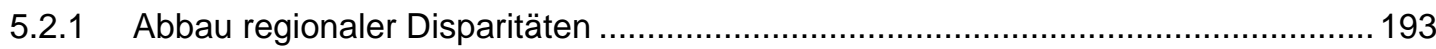

5.2.2 Entwicklung der Potentiale nach Studiengängen, Erhaltern, Standorten ................ 199

5.2.3 Regionale Wirkungen von Hochschuleinrichtungen ..........................................207

5.2.4 Regionale Planungen und Strategien .......................................................... 212

5.3 Entwicklungsfragen im Bereich der regionalen Standortpolitik........................223

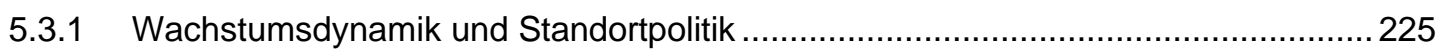

5.3.2 Leistungen von Hochschulen und regionale Innovationssysteme............................228

5.3.3 Szenarien der Erhöhung der Forschungsquote.................................................229

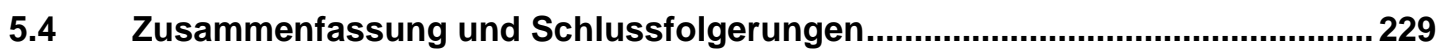

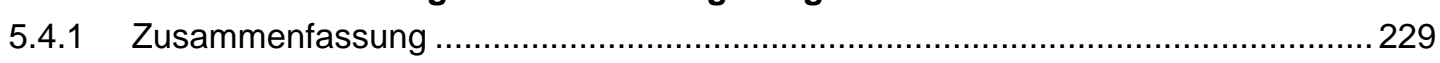

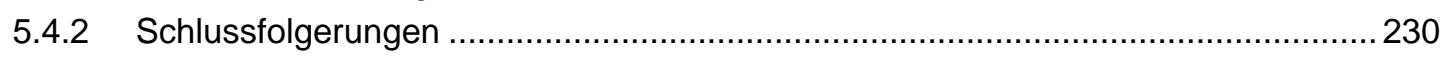

6. Die Internationalisierung der Hochschulen und das Entstehen eines „Europäischen Hochschulraums“ .................................. 233

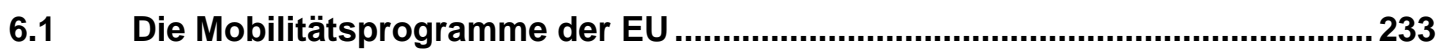

6.1.1 Daten zur akademischen Mobilität: Vergleich zwischen Fachhochschul- und

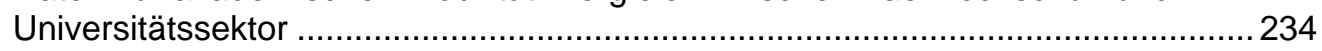

6.1.2 Perspektiven der weiteren Entwicklung ..........................................................241

6.2 Der Bologna-Prozess: eine neue Studienarchitektur für den

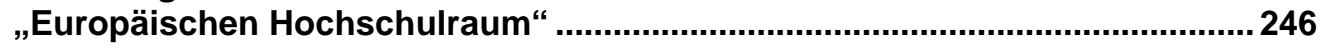

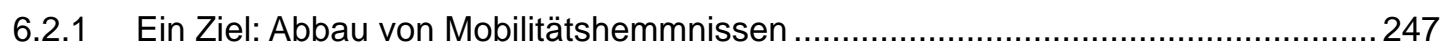

6.2.2 Umstellung auf ein „two-tier“-System (angelsächsisches Modell) ............................248

6.2.3 Gründe für die „Harmonisierung der Studienarchitektur“......................................2.250

6.2.4 Zum „Europäischen Leistungspunktesystem“ .................................................251

6.2.5 Zum Stand der Implementierung des Bologna-Prozesses in Europa......................2252

6.3 Die Implementierung des Bologna-Prozesses in Österreich ...........................257

6.3.1 Die Rezeption des Bologna-Prozesses in der österreichischen Hochschulpolitik .. 257

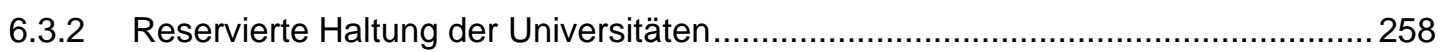

6.4 Konsequenzen der neuen Studienarchitektur für den

6.4.1 Implementierung der neuen Studienarchitektur im Fachhochschulbereich..............260

6.4.2 Wie verändern sich die Profile der beiden Sektoren? ...........................................261

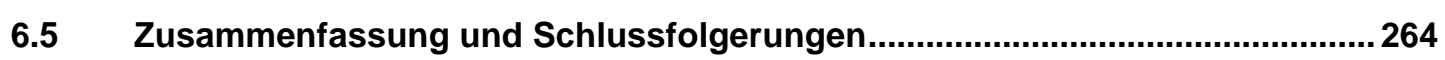

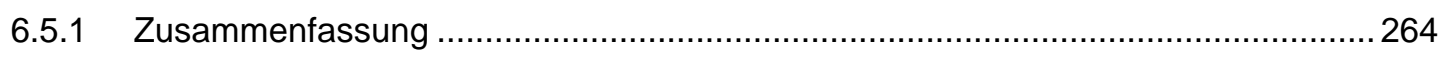

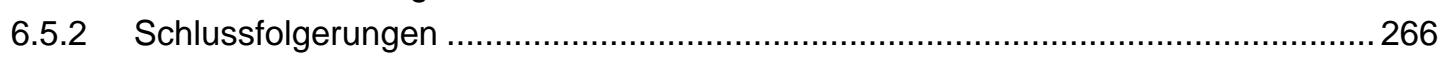

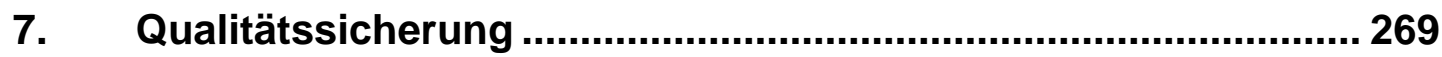

7.1 Das Qualitätssicherungsmodell des FH-Sektors ….........................................269

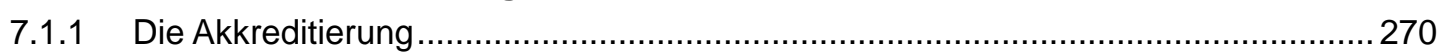

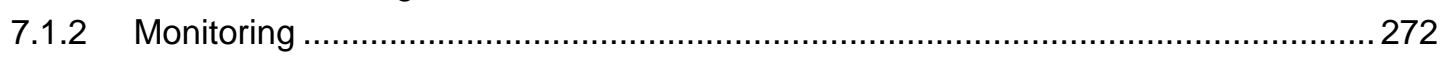

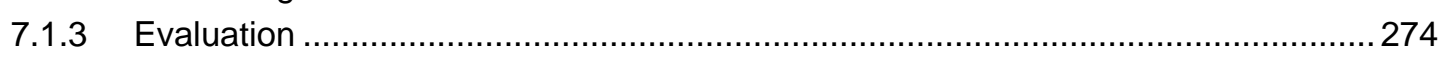

7.1.4 Interne Qualitätssicherung der Studiengänge und Fachhochschulen .....................277

7.2 Internationale Einbettung des Qualitätssicherungsmodells............................283

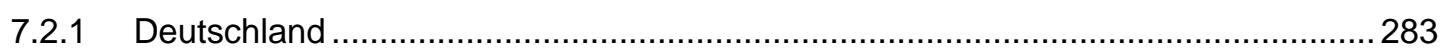




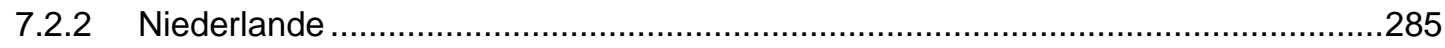

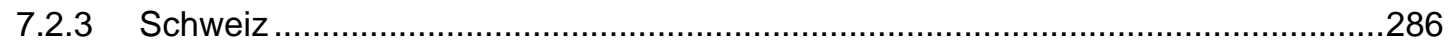

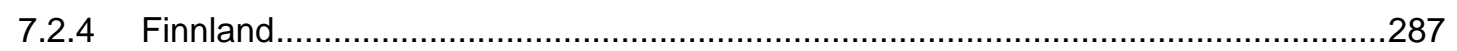

7.2.5 Einbettung des Qualitätssicherungsmodells des österreichischen

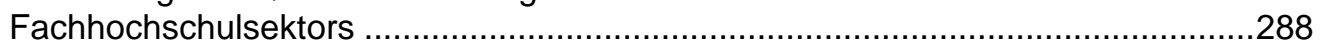

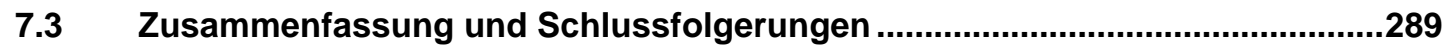

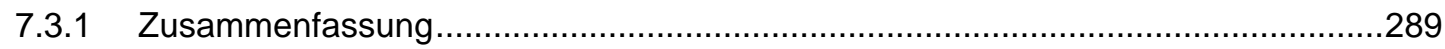

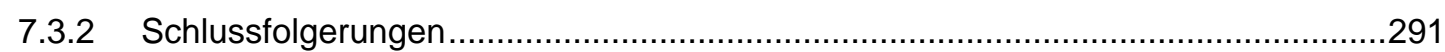

Funktionsdifferenzierung der Institutionen ....................................................291

Differenzierung der Qualitätssicherungsdimensionen ...........................................295

8. Zusammenfassung und übergreifende Schlussfolgerungen .....305

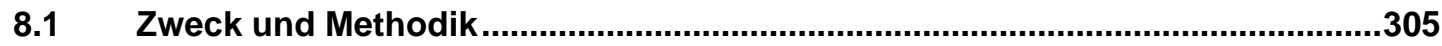

8.2 Einzelergebnisse der spezifischen Untersuchungsaspekte ............................305

8.2.1 Stellenwert des FH-Sektors in der Bildungs- und Hochschullandschaft ..................305

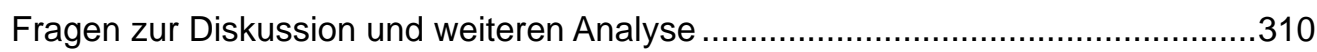

8.2.2 Umsetzung der Entwicklungs- und Finanzierungsplanungen ..................................312

8.2.3 Konzentration, Regionalisierung und Standorte im FH-Sektor .................................313

8.2.4 Die Internationalisierung der Hochschulen und das Entstehen eines

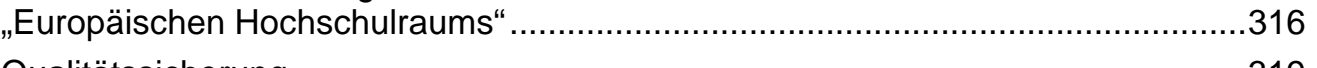

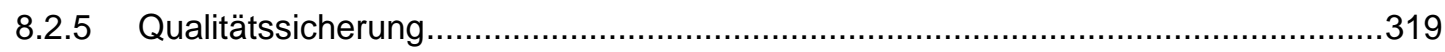

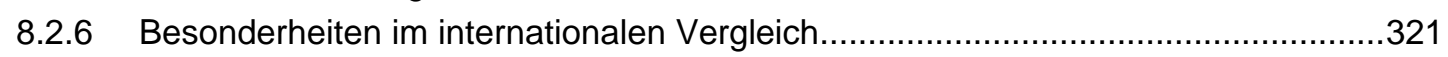

8.3 Übergreifende Schlussfolgerungen .............................................................322

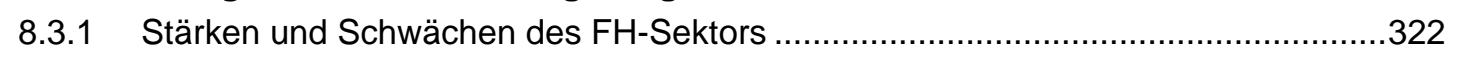

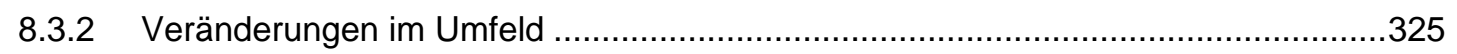

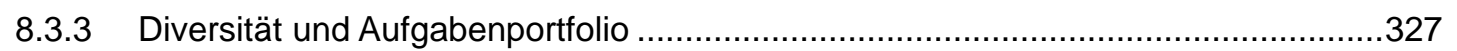

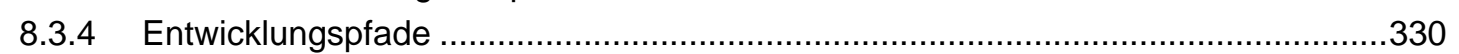

8.3.5 Steuerungsmodell: Kooperation und Funktionendifferenzierung ............................335

8.4 Zusammenfassung der Empfehlungen.........................................................339

8.4.1 Bereich Entwicklungs- und Finanzierungsplanung: Entwicklung der

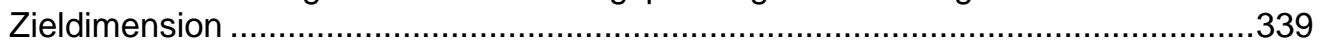

8.4.2 Wahl der Entwicklungspfade für die weitere quantitative Dimensionierung und

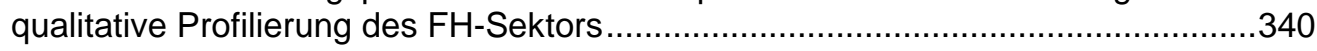

8.4.3 Die Gestaltung des Aufgabenportfolios und nähere Spezifikation der Aufgaben

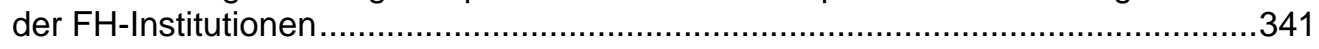

8.4.4 Adäquate Organisationsformen zur Zielbildung und Koordination ..........................341

8.4.5 Überprüfung der Funktionen und Aufgaben des FHR..........................................342

8.4.6 Gesamtbetrachtung des tertiären Sektors: .......................................................34

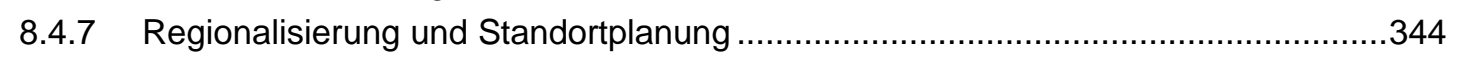

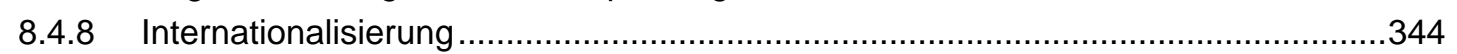

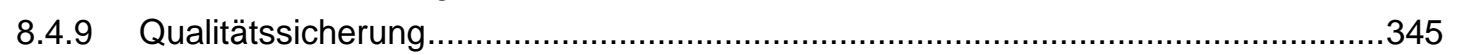

9. Zusammenfassung des nationalen und internationalen

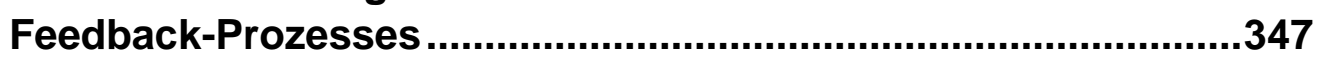

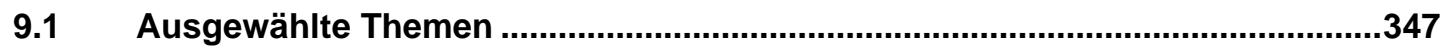


9.1.1 Strategische Planung und Steuerung, politisches Gesamtkonzept für den tertiären Sektor .....

9.1.2 Verhältnis von politischer Steuerung und Qualitätssicherung, organisatorische Steuerungs- und Behördenstruktur, Funktion und Konstruktion des FHR ..............349

9.1.3 Entwicklungspfade, „Bild“ und Aufgabenstruktur der Fachhochschule ....................350

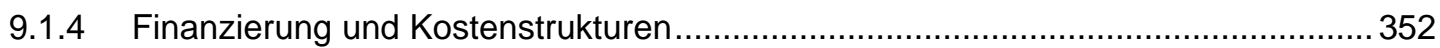

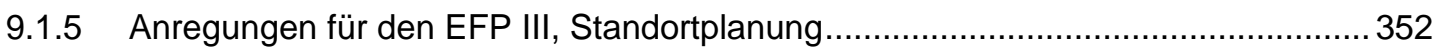

9.2 Wertung des Berichtes und seiner Rolle für die weitere Entwicklung des

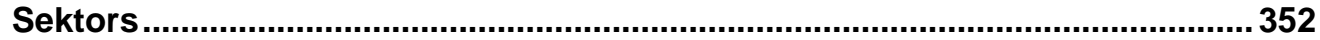

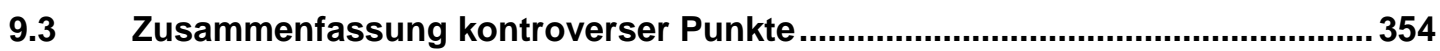

9.4 Offene Punkte, die dringend näher behandelt werden sollten ...........................354

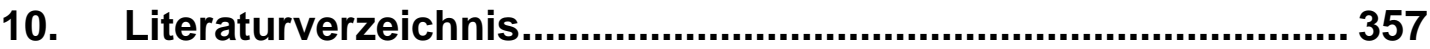

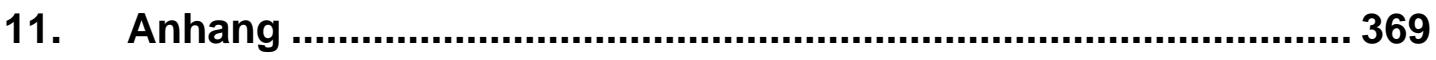

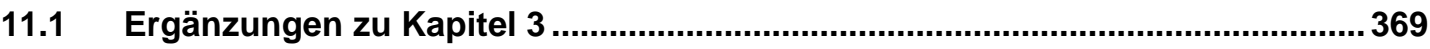

11.1.1 Zusammenfassung von Forschungsergebnissen zur Positionierung der FH-

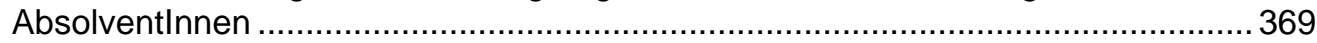

11.1.2 Zur Begrifflichkeit der Berufsfelder bzw. Berufsprofile ............................................376

11.1.3 Synopse der Veränderungen im Umfeld der Bildungspolitik ..................................381

Lissabon-Schlussfolgerungen und Innovationspolitik ........................................... 381

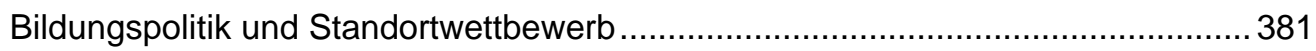

Europäische Koordinierung über Ziele, neue Bereitstellungsstrukturen und

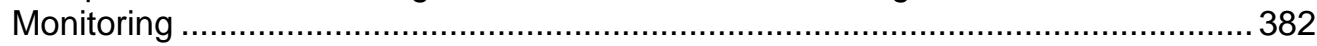

Konkretisierung der bildungspolitischen Ziele für die Bildungssysteme..................382

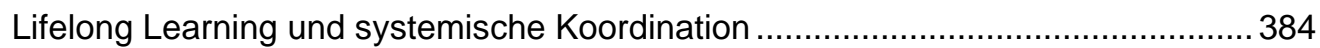

Neue Kompetenzen als Zentralbegriff inhaltlicher und methodischer Innovation ... 385

Forschungsstrategie als Umsetzung von Innovationspolitik................................. 385

Unternehmen und Regionen im Innovations- und

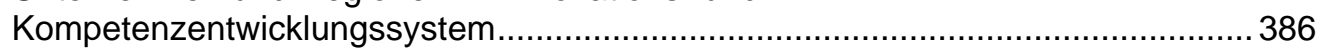

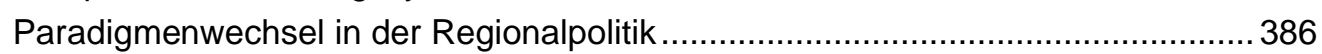

11.1.4 Ergänzende Grafiken und Tabellen zu Kapitel 3 .............................................. 388

11.2 Ergänzende Grafiken und Tabellen zu Kapitel 5 ............................................393

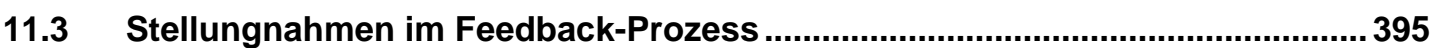

11.3.1 Points raised at the feedback meeting with international reviewers ........................395

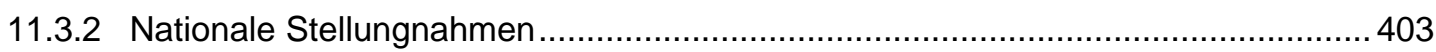

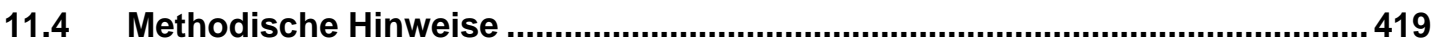

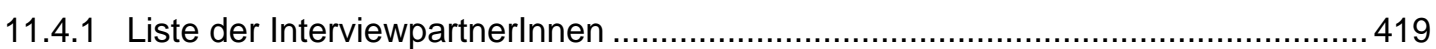

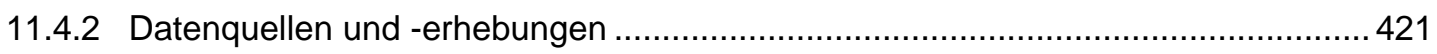

11.5 Zeittafel zur Entwicklung des FH-Sektors ........................................................423

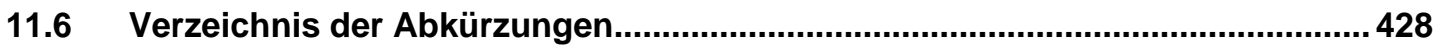





\section{Einleitung}

Das vorliegende Projekt hat gemäß der Ausschreibung den Auftrag, die bisherige Entwicklung des FH-Sektors einer Evaluierung zu unterziehen, wobei die folgenden Aspekte schwerpunktmäßig zu behandeln sind:

- der Stellenwert im Hochschulsystem

- die Funktionsfähigkeit und Wirksamkeit der Finanzierungs- und Entwicklungsplanung

- die Entwicklung und Allokation der Standorte im Raum

- die Funktionsfähigkeit und Wirksamkeit der Qualitätssicherung

- die internationale Positionierung angesichts der Entwicklung des Europäischen Hochschulraumes.

Die Analysen sollten einen Beitrag für die Konzeption des dritten Entwicklungs- und Finanzierungsplanes abgeben.

Da für die Evaluierung ein enger zeitlicher Rahmen vorgegeben war, haben wir methodisch ein Verfahren gewählt, das an die häufig praktizierten Review-Verfahren angelehnt ist. Diese Verfahren bestehen daraus, dass der evaluierte Bereich einen Bericht verfasst, der von einem Review-Team in Kombination mit eigenen Erhebungen (meistens in Form von VorOrt-Besuchen und Auswertungen von verfügbaren Daten) als Basis für einen Evaluierungsbericht herangezogen wird. Wir haben unsere Vorgangsweise als "QuasiReview" bezeichnet, da unser Review-Team in einem bedeutend größeren Maß als sonst in Review-Verfahren üblich, eigene Recherchen und Analysen durchgeführt hat. Um die Beteiligung des Sektors sicherzustellen, wurde ein zweistufiges Verfahren gewählt, indem nach Abschluss eines Rohberichtes durch ein Feedbackverfahren Rückmeldungen von den Beteiligten eingebracht werden. Darüber hinaus werden auch internationale und nationale ExpertInnen in das Feedback eingebunden.

Die Informationsgrundlage für die Evaluierung wurde durch ein breites und vielfältiges Set an Erhebungen aufgebaut:

- Eine systematische Literaturrecherche von nationalen Materialien entsprechend den angeführten Themenstellungen. Ein besonderer Schwerpunkt liegt auf der Auswertung der jährlichen Berichte des Fachhochschulrates (FHR). 
- Zur Erfassung der internationalen Positionierung: Dokumentanalysen, Internetrecherchen und Auswertungen von einschlägigen Datenbanken (insbesondere jene des CHEPS).

- Aufwändige Umorganisierung der Datenbank des FHR und Re-Analyse der Daten, bezogen auf den bisherigen Zeitraum und die verfügbaren statistischen Einheiten (Lehrpersonen, Studierende, BewerberInnen, Zugänge, AbsolventInnen, Abbrüche), auf Basis folgender Kategorisierungen: Studiengänge, Standorte, grobe Fachrichtungen, Bundesländer.

- Die Informationen über finanzielle Aspekte konnten aufgrund der unzureichenden Informationslage nicht im Zeitverlauf analysiert werden. Es wurden Sekundäranalysen von verfügbaren Studien und eine Auswertung der nur teilweise verfügbaren Voranschläge für das laufende Jahr durchgeführt.

- Eine zentrale Informationsquelle für die Evaluierung sind ExpertInnen-Interviews, die direkt für das vorliegende Projekt teilweise persönlich und teilweise telefonisch durchgeführt wurden und Sekundärauswertungen von bereits verfügbaren ExpertInnenInterviews zu zentralen Fragestellungen. Eine Aufstellung der InterviewpartnerInnen, die einerseits die beteiligten Akteure repräsentieren und andererseits im Hinblick auf ihre Expertise zu den Schwerpunktthemen ausgewählt wurden, befindet sich im Anhang des Berichtes.

- Um die Ergebnisse der ExpertInnen-Interviews in das Gesamtbild einbetten zu können und um zusätzliche spezifische Informationen zu den verschiedenen Themenbereichen (insbesondere zu finanziellen Aspekten, zu Fragen der Personalpolitik und zu Fragen der regionalen Wirksamkeit) zu erheben, wurden zwei internetbasierte Erhebungen durchgeführt (bei den Erhaltern und bei den StudiengangsleiterInnen; die näheren Informationen befinden sich im Anhang des Berichtes).

- Um bestimmte Aspekte insbesondere des Stellenwertes des Fachhochschulbereichs im Bildungswesen $\mathrm{zu}$ analysieren, wurden darüber hinaus Sekundärauswertungen verschiedener Datenbasen der offiziellen Statistik durchgeführt. Diese Auswertungen der Hochschulstatistik und der Zensusdaten (Volkszählung, Mikrozensus) haben einige wichtige neue Originalinformationen zu Tage gefördert.

In der Analyse und Präsentation der Informationen wurde die Grundentscheidung getroffen, die strukturellen und systemischen Aspekte herauszuarbeiten und die Diskussion auf diese Aspekte zu lenken. Daher werden die Informationen (teilweise mit Ausnahme der Bundesländer) in anonymisierter Form präsentiert. Dies schwächt zwar einerseits die Transparenz der Informationen, vermindert aber andererseits das Risiko, dass sich die Diskussion auf unproduktive Nebengeleise begibt. 
Es muss an dieser Stelle mit besonderem Nachdruck hervorgehoben werden, dass die Erhebungen und Analysen nur mit Hilfe der teilweise sehr umfangreichen Mitarbeit vieler KollegInnen aus dem $\mathrm{FH}$-Sektor und aus seinem Umfeld in der umfassenden Form erfolgreich durchgeführt werden konnten. Daher möchten wir uns als Projektteam bei allen Beteiligten für ihre Mitarbeit, ihr Engagement und ihren Einsatz und insbesondere auch für die geopferten Zeitressourcen ganz herzlich bedanken, umso mehr als die Erhebungen teilweise in die Sommermonate gefallen sind. Ausdrücklich bedanken wollen wir uns

- bei den vielen StudiengangsleiterInnen und Erhaltervertreterlnnen, die sich an den Erhebungen beteiligt haben,

- bei den MitarbeiterInnen der Fachhochschul-Konferenz (FHK), insbesondere bei Christina Freyberger und Werner Jungwirth, die dem Projekt in verschiedenster Weise wertvollste Unterstützung zukommen haben lassen, durch die Gelegenheit zur Präsentation in der Vollversammlung, durch die Kommentierung der Erhebungsinstrumente (zu der auch Kurt Koleznik beigetragen hat) und die organisatorische und moralische Unterstützung der beiden Erhebungen,

- bei den MitarbeiterInnen der Geschäftstelle des FHR, insbesondere bei Wilfried Grätz, Andreas Neuhold, Herwig Patscheider und Kurt Sohm für die wertvollen Hinweise im Rahmen der ExpertInnen-Interviews sowie bei Herwig Patscheider für die äußerst hilfreiche und zum Teil aufwändige Hilfe bei der Aufschließung der Daten und Materialien,

- bei den MitarbeiterInnen des auftraggebenden Bundesministeriums für Bildung, Wissenschaft und Kultur (BMBWK) für eine ganze Reihe von sehr wichtigen Hilfestellungen bei der Projektdurchführung (Mark Német, Günther Simonitsch und EvaMaria Götz) sowie für die sachkundige, kritische und ermutigende Betreuung der Projektabwicklung bei Maria Keplinger und Friedrich Faulhammer,

- bei den Mitgliedern des Projektbeirates (Gerfried Brandstetter, Christina Freyberger, Wilfried Grätz, Werner Jungwirth, Kurt Koleznik, Christian Mann, Monika Petermandl, Susanne Schöberl und Monika Vyslouzil) für die engagierte Beteiligung am Projektverlauf, in Form wichtiger Anregungen und kritischer Anmerkungen, die teilweise leidenschaftliche Diskussionen ausgelöst haben,

- bei Christian Mann und der Niederösterreichischen Bildungsgesellschaft für die Erlaubnis, die Informationen aus dem Szenarienprojekt für Sekundärauswertungen zu nutzen,

- bei Günther Schelling für das ausführliche Gespräch und die zur Verfügung gestellten Unterlagen, die für uns sehr hilfreich bei der Auswertung der quantitativen Informationen waren, 
- bei den internationalen Experten Andri Gieré (CH), John Pratt (UK) und Ulrich Teichler (D) für ihr Engagement und die wertvollen Rückmeldungen und Anregungen im ersten Feedback-Prozess, bei Elsa Hackl für ihre Beteiligung und Unterstützung in diesem Feedback sowie bei Elke Gruber und Hans-Joachim Bodenhöfer für ihr Feedback als nationale ExpertInnen,

- bei Günter Strauch für die unzähligen Verbesserungsvorschläge zur Rohfassung des Berichtes sowie für seine - teilweise sehr unterhaltsamen und humorvollen Kommentare,

- und schließlich vor allem auch bei den Expertinnen und Experten für die Bereitschaft, ihr Wissen und ihre Einschätzungen mit uns zu teilen und ihre wertvolle Zeit dafür zur Verfügung zu stellen.

Wir hoffen, dass wir mit dem Ergebnis diesen Einsatz und Aufwand rechtfertigen können. 


\section{Der österreichische FH-Sektor - Grundmerkmale, quantitative Entwicklung, internationaler Vergleich}

In diesem Kapitel werden erstens die Grundmerkmale und Besonderheiten des österreichischen FH-Sektors in sehr pointierter Form zusammengefasst. Wenn auch viele Aspekte vor allem für einen Blick von außen näherer Diskussion und Erörterung bedürfen mögen, gehen wir davon aus, dass die wesentlichen Punkte dieser Darstellung der Grundmerkmale in der österreichischen Diskussion weitgehend geteilt werden. Diese Darstellung hat insofern auch den Zweck, Wiederholungen dieser Aspekte in den folgenden Kapiteln unnötig zu machen.

Zweitens enthält dieser Abschnitt eine deskriptive Aufarbeitung der quantitativen Daten in Form einer Re-Analyse der Datenbasis des FHR, teilweise kombiniert mit Informationen aus unserer Erhebung bei den Studiengängen und Erhaltern. Diese Aufarbeitung ist in großem Ausmaß abhängig von den verfügbaren Informationen und dient als Basis für tiefergehende und spezielle Betrachtungen in den weiteren Kapiteln, insbesondere in Kapitel 3 (Stellenwert) und Kapitel 5 (Standortfragen).

Drittens wird eine vergleichende Betrachtung des österreichischen FH-Sektors mit vier ausgewählten Ländern präsentiert, die Grundmerkmale und Besonderheiten im Ländervergleich herausarbeiten soll. Wir gehen im Prinzip davon aus, dass man mit Vergleichen sorgfältig umgehen soll und dass diese v.a. als Referenz für das Verständnis des eigenen Systems dienen können, aber auch dazu, das Spektrum der Möglichkeiten zu veranschaulichen. Auch hier werden in den weiteren Kapiteln zu bestimmten besonders interessierenden Aspekten nähere Vergleiche mit den anderen Ländern angestellt.

\subsection{Grundmerkmale und Besonderheiten des FH-Sektors}

In den meisten OECD-Ländern wurden im Laufe der Hochschulexpansion postsekundäre Einrichtungen eines neuen Typs gegründet, die sich von den traditionellen Universitäten vor allem in zweifacher Hinsicht unterscheiden:

- durch eine stärkere Berücksichtigung gesellschaftlicher und wirtschaftlicher Bedarfslagen, v.a. stärker berufsbezogene Curricula;

- im Angebot kürzerer Studiengänge, die stärker strukturiert sind und zumeist innerhalb der vorgesehenen Studiendauer zu einem Abschluss führen.

Österreich hat diesen Weg einer organisatorischen Differenzierung des Hochschulsystems zunächst nicht beschritten (vgl. Lassnigg/Pechar 1988). Ein erster Versuch zur Etablierung 
einer nicht-universitären Alternative im Postsekundarbereich scheiterte Anfang der 1970er Jahre. Erst Ende der 1980er Jahre, als die Krisensymptome eines nicht-differenzierten Massenhochschulsystems bereits deutlich spürbar waren, wurde dieser Reformstrang neuerlich aufgegriffen. Im Koalitionsabkommen von 1990 beschloss die Bundesregierung die Einrichtung eines nicht-universitären Sektors. 1993 wurde das Fachhochschul-Studiengesetz (FHStG) verabschiedet.

Der Fachhochschulsektor wurde - aus international vergleichender Perspektive - sehr spät eingerichtet; zu dieser Zeit gab es bereits massive Kritik seitens der Öffentlichkeit, der Wirtschaft und zum Teil auch der Politik an den Universitäten. Die Fachhochschuleinrichtungen hatten die Chance, sich von den Universitäten abzuheben und sie haben diese Chance genutzt. Man darf vermuten, dass diese Polarisierung zwischen einem kritisierten Modell und der Hoffnung auf Innovation ein Motivationsfaktor für den neuen Sektor war, der zu dem häufig beschriebenen "Pioniergeist" beigetragen hat. Ohne die Leistungen der fachhochschulischen Bildungsinstitutionen abzuwerten, kann man sagen, dass sich ihr Erfolg zum Teil dem Kontrasteffekt verdankt.

Damit wurde ein neuer Sektor des österreichischen Bildungswesens eröffnet, der sich durch eine andersartige „schlanke“ Gesetzgebung auszeichnet und die Möglichkeit für „unternehmerische“ Initiative bietet. Es wurde nicht an bestehende Institutionen angeknüpft, sondern das Fachhochschulsystem wurde von Grund auf neu aufgebaut. Das Ziel der Politik war es, Rahmenbedingungen zu schaffen, die ein rasches Reagieren des neuen Hochschultyps auf die Anforderungen des Beschäftigungssystems ermöglichen. Im Vergleich zum bestehenden Bildungs- und Hochschulwesen zeichnet sich der FH-Sektor durch einige Besonderheiten aus, die inn in hohem Maße zu einem Reformsektor machen:

- es wurden keine bestehenden Institutionen umgewidmet oder weiterentwickelt, sondern es wurden durchwegs neue Studiengänge geschaffen und diese Entwicklung erfolgte in Form eines „bottom-up“-Prozesses;

- es wurde ein neues Ausbildungsangebot im Hochschulsektor eingerichtet: berufsorientierte Studiengänge, die auf spezialisierte neue Berufsfelder ausgerichtet sind;

- ein umfassendes System der Qualitätssicherung mit dem Fachhochschulrat im Zentrum wurde eingerichtet: FHR, Akkreditierung, Evaluierung und verbindliche Etablierung von Systemen der Qualitätssicherung an den Standorten;

- es gab die Möglichkeit privater Erhalterschaft, was im hochgradig staatlich organisierten österreichischen Bildungswesen eine ausgeprägte Neuerung darstellte; 
- die öffentliche Bereitstellung der neuen Bildungsangebote wurde in Form der Entwicklungsplanung etabliert, die im Zusammenwirken zwischen Bund, Ländern und FHR erfolgt;

- der Finanzierungsbeitrag des Bundes erfolgt in Form der Normkostenfinanzierung;

- das Studienangebot zeichnet sich durch einen starken Regionalbezug aus, der durch ein starkes Engagement der meisten Länder und vieler Gemeinden (Trägerschaft, CoFinanzierung) gefördert wird.

Dieses einleitende Kapitel verfolgt die Absicht, die oben identifizierten Merkmale in relativ einfacher, deskriptiver Form darzustellen und damit einen Bezugsrahmen für die Diskussion in den folgenden Kapiteln zu schaffen. Wo dies möglich ist, wird auf die entsprechenden Stellen im Gesetz bzw. in den Entwicklungs- und Finanzierungsplänen verwiesen. In einem zweiten Schritt wird die quantitative Entwicklung des Sektors nachgezeichnet, in einem dritten der österreichische FH-Sektor in einen international vergleichenden Rahmen gestellt.

\subsubsection{Aufbau neuer Studiengänge in Form eines ,bottom-up“-Prozesses ${ }^{1}$}

Zwei Vorentscheidungen werden diesen Sektor noch auf lange Zeit hin prägen:

- Erstens der Umstand, dass es (im Gegensatz zu den meisten anderen OECD-Ländern) kein „upgrading“ ${ }^{2}$ bestehender Einrichtungen (z.B. BHS, Akademien) zu Fachhochschuleinrichtungen gegeben hat $^{3}$. Das hat zur Konsequenz, dass der Fachhochschulsektor auch mittelfristig viel kleiner sein wird als der universitäre Sektor. Die geringe Größe ist auch eine Folge der späten Gründung des Sektors: die historisch einmalige Expansionsphase mit den höchsten Wachstumsraten fand in den 1970er und frühen 1980er Jahren statt und hat an den Universitäten einen Kapazitätsausbau nach sich gezogen, der kurz- und mittelfristig nur schwer rückgängig zu machen wäre. Daher hat der Fachhochschulsektor im Vergleich zu den Universitäten eine sehr geringe $\mathrm{Größe}^{4}$. Das hat auch die Bewältigung der Schwierigkeiten der Anfangsphase erleichtert.

Vgl. FHStG §13, 15; Entwicklungs- und Finanzierungsplan I.

Unter „upgrading“ wird in der Literatur eine Funktions- und Statusaufwertung bereits bestehender Einrichtungen verstanden, die in der Regel auch mit einer rechtlichen Neukonstitution verbunden ist.

3 Ab dem Jahr 2000 stand mit den Sozialakademien erstmals das "upgrading“ bestehender Institutionen zur Diskussion.

4 In den meisten OECD-Ländern ist der nicht-universitäre Hochschulsektor größer als der universitäre (oder etwa gleich groß). Mit der "Größe" des Sektors ist primär die Zahl der Studierenden und des akademischen Personals gemeint, nicht die der AbsolventInnen. Die AbsolventInnenzahl hängt auch von der "Durchlaufgeschwindigkeit" ab, die im nicht-universitären Sektor generell höher ist als im universitären. 
- Zweitens die Präferenz für ein „bottom-up“-Modell: die Entscheidung über Standorte, Profile und Größe der Studiengänge wird nicht auf der Basis einer langfristigen zentralen Planung getroffen. Vielmehr werden die dezentralen Initiativen potentieller Erhalter von Studiengängen im Nachhinein koordiniert. Alle Studienpläne müssen erst vom Fachhochschulrat unter dem Gesichtspunkt akademischer Qualität akkreditiert werden. Dieser hat entscheidend zur Vitalität des Fachhochschulsektors beigetragen. Es war ein Kontrast zur langen zentralistischen Tradition, bei der die Initiative und die Richtungsentscheidungen "von oben" kamen und "an der Basis" umgesetzt wurden. Dass im Fachhochschulbereich die Initiative "von unten" kam, wirkte sich auf die Größe der Erhalterorganisationen aus: nicht nur der Sektor insgesamt, sondern auch die einzelnen Fachhochschuleinrichtungen sind sehr klein.

\subsubsection{Ein neues Ausbildungsangebot im Hochschulsektor: kurze und berufsorientierte Studiengänge ${ }^{5}$}

Das wichtigste und weitgehend unumstrittene Ziel beim Aufbau des $\mathrm{FH}$-Sektors war es, eine curriculare Alternative zu den wissenschaftlichen Langzeitstudien an den Universitäten zu schaffen. Fachhochschul-Studiengänge müssen Studienprogramme anbieten, die in kürzerer Zeit als die der Universitäten abgeschlossen werden können; und ihre Angebote müssen einen expliziten Berufsbezug aufweisen, sie müssen eine Berufsausbildung anstreben. In beiden Punkten unterscheiden sie sich - zumindest auf der programmatischen Ebene fundamental von den Universitätsstudien, die keine „Aus“-, sondern eine „Vor“bildung für Berufe anstreben und die einerseits längere Mindeststudienzeiten haben und zugleich wegen der an den Universitäten dominierenden Lernkultur die Einhaltung dieser Mindestzeiten nicht garantieren können. Beides - die Abwehr sowohl eines expliziten Berufsbezugs wie einer "Verschulung" des Studiums - wird von einem großen Teil des akademischen Personals an Universitäten als unantastbarer Kern ihrer akademischen Identität betrachtet (vgl. z.B. Brinek/Schirlbauer 1996).

Die Gründung des FH-Sektors war insofern ein fundamentaler Einschnitt im österreichischen Hochschulsystem, als damit kurze und berufsorientierte Angebote als Teil des Hochschulsystems etabliert wurden. Postsekundäre Angebote dieser Art hat es schon früher gegeben (Pädagogische Akademien, Sozialakademien, Akademien für medizinischtechnische Dienste), aber sie waren nicht Teil des Hochschulsystems, sie waren von den Universitäten durch eine klare Statusdifferenz und durch ein völliges Fehlen von Anrechnungs- und Übergangsmöglichkeiten getrennt. Fachhochschul-Studiengänge hingegen sollten den Universitäten statusmäßig ebenbürtig sein oder zumindest angenähert

5 Vgl. FHStG §3 (Ziele und Gestaltung), §4 (Studierende), §5 (Akademische Grade). 
werden. Daher wurden gleichlautende Abschlussbezeichnungen gewählt ${ }^{6}$ und es wurde gegen den massiven Widerstand der Universitäten - der Übertritt zu einem Doktoratsstudium an einer Universität ermöglicht ${ }^{7}$.

Schließlich zeichnet sich der Fachhochschulsektor durch eine radikale Begrenzung des Fächerspektrums aus. Bis vor kurzem wurden überwiegend betriebswirtschaftliche und technische Studiengänge angeboten. Auch diese "Komplexitätsreduktion" war in der Startphase ein wichtiger Erfolgsfaktor. In diesen Fachbereichen konnten die FachhochschulStudiengänge am leichtesten die Unterstützung der Wirtschaft gewinnen (was sich auch in öffentlicher Anerkennung niedergeschlagen hat). Hier sind die Arbeitsmarktchancen für AbsolventInnen am besten (was das hohe Interesse der Studiennachfrage begünstigt hat).

\subsubsection{Akkreditierung und Evaluierung, Qualitätsentwicklung an den Standorten8}

Der Fachhochschulrat ist als zentrale Instanz der Qualitätssicherung konzipiert. Er ist verantwortlich für die Akkreditierung neuer Studiengänge sowie für die vorgeschriebene ReAkkreditierung der Studiengänge nach einer Laufzeit von fünf Jahren (Verlängerungsanträge). An den Standorten wird darüber hinaus die Etablierung eines Systems der Qualitätssicherung als Teil der laufenden Arbeit verlangt. Die Vorgangsweise bei der Qualitätssicherung wurde im Zusammenspiel zwischen FHR und den Vertreterlnnen der Erhalter und Studiengänge, die sich in der Fachhochschul-Konferenz (FHK $)^{9}$ organisierten, entwickelt; auch die Richtlinien für die Evaluierung wurden in einer ersten Phase gemeinsam von FHR und FHK entwickelt.

Dieses Konzept von Qualitätssicherung unterscheidet sich radikal von den bis dahin im österreichischen Bildungswesen praktizierten Varianten. Bis in die späten 1980er Jahre hinein wurde über Maßnahmen zur Qualitätssicherung im Grunde nicht diskutiert. Sowohl im Schul- wie im Hochschulbereich gab es eine starke staatliche Regulierung des Curriculums in Form von Gesetzen und Verordnungen. Diese Form der „ex-ante-Kontrolle“ wurde für ausreichend gehalten, um hohe Qualität sicherzustellen. Sie hatte zugleich den Vorteil, dass - dem politischen Kräfteverhältnis entsprechend - alle Parteien und Interessensgruppen in den Prozess der Qualitätssicherung eingebunden waren, womit der öffentliche Charakter des Bildungsangebots unterstrichen wurde. Erst gegen Ende der 1980er Jahre begann man, dem internationalen Trend folgend, über Evaluierung, d.h. über eine Form der „ex-postKontrolle“ von Qualität zu diskutieren. Evaluierung wurde vor allem als Aufgabe von

Allerdings mit sektoraler Indizierung: Mag. (FH).

7 Allerdings war die Regierungspolitik nicht völlig konsistent, denn es wurde ursprünglich keine „A-Wertigkeit“ eines Fachhochschulabschlusses durchgesetzt, d.h. dieser Abschluss war nicht zum Eintritt in den höheren Bundesdienst geeignet. Durch das Vertragsbedienstetengesetz ist diese Regelung gegenstandslos geworden.

8 Vgl. FHStG §6-11 (generell zum FHR), §12 (Akkreditierung), §13-14 (Evaluierung).

Verein, gegründet November 1995. 
ExpertInnen-Teams, von „peers“ betrachtet; sie war aber zunächst, soweit sie überhaupt praktiziert wurde, nur eine Ergänzung zur weiterhin sehr starken ex-ante-Kontrolle durch den Staat.

\section{Akkreditierung}

Bei der Entwicklung von FH-Studiengängen sollte es eine neue Form der Qualitätskontrolle geben. Die dominanten bildungspolitischen Entscheidungsträger waren zur Auffassung gelangt, dass das etablierte Modell zu starr sei und eine rasche Anpassung des Studienangebots an neue wissenschaftliche Entwicklungen und veränderte Anforderungen des Arbeitsmarktes nicht zulasse. Im Sinne der „bottom-up“-Philosophie wurde die Verantwortung für die Curriculumentwicklung radikal dezentralisiert und in die Verantwortung der Studiengänge übergeben. Diese sehr weitgehende Liberalisierung musste durch eine neue Form der ex-ante-Kontrolle ausbalanciert werden: durch eine Sicherung von Mindeststandards. Damit stellte sich das für die österreichische Bildungspolitik völlig neue Problem der „Akkreditierung“. Die bis dato von Gesetzgeber und Behörden praktizierte exante-Kontrolle war keine „Mindestkontrolle“, sie verstand sich als Sicherung optimaler Qualität. $^{10}$

\section{ExpertInnengremium}

Die Aufgabe der Akkreditierung wurde einem ExpertInnengremium übertragen. Die Hälfte der 16 Mitglieder des Fachhochschulrates mussten eine Habilitation oder eine vergleichbare akademische Qualifikation besitzen; die andere Hälfte musste über berufliche Praxis in einem der für den Sektor relevanten Berufsfelder verfügen. Die Mitglieder des FHR werden von dem/der für Hochschulen zuständigen Bundesministerln für eine Funktionsdauer von drei Jahren ernannt, ${ }^{11}$ unterliegen aber keiner Weisungspflicht.

Während der Diskussion um das FHStG hat die Konstruktion des Fachhochschulrates für die stärksten Kontroversen gesorgt. ${ }^{12}$ Das Konzept eines ExpertInnengremiums, das Akkreditierungsentscheidungen trifft, wurde zunächst von vielen als Fremdkörper in der österreichischen Bildungslandschaft empfunden. Die Gegner des neuen Modells haben argumentiert, dass der Staat damit die öffentliche Kontrolle über das Studienangebot im FHSektor aufgibt und sie einem "quasi-privaten", demokratisch nicht ausreichend legitimierten Gremium überträgt.

10 Die bis in die frühen 1990er Jahre dominante Hintergrundüberzeugung der bildungspolitischen Entscheidungsträger lautete: es gibt eine Optimallösung („the one best system“) und die Aufgabe der Bildungspolitik besteht darin, diese zu realisieren.

11 Mit der Möglichkeit einer einmaligen Wiederbestellung.

12 Vgl. z.B. die Beiträge in Höllinger/Hackl/Brünner 1994. 


\section{Evaluierung und laufendes Qualitätsmanagement}

Neben der Akkreditierung obliegt dem FHR auch die Re-Akkreditierung der laufenden Studiengänge. Auch hinsichtlich des Stellenwerts nachträglicher Bewertung beschreitet der FH-Sektor neue Wege. In anderen Teilen des Bildungswesens war - bzw. ist - es sehr schwierig, ein neues Bildungsangebot zu etablieren (wegen der Langwierigkeit staatlicher ex-ante- Kontrolle); aber ebenso schwierig ist es, einmal eingerichtete Angebote zu beenden. Fachhochschul-Studiengänge hingegen sind mit einer fünfjährigen Laufdauer begrenzt; ihre Weiterführung ist von der Re-Akkreditierung durch den FHR abhängig, die auf einer umfassenden Evaluierung beruht.

Um die Qualität der laufenden Arbeit zu garantieren, hat der FHR von den Erhaltern die Entwicklung und Anwendung von Prozeduren des Qualitätsmanagements verlangt. Es wurde den Akteuren freigestellt, welche Systeme oder Prozeduren sie anwenden wollten. Zur Unterstützung wurde eine Arbeitsgruppe der Fachhochschul-Konferenz aufgebaut, die sich auch mit der Entwicklung der Evaluierungsverfahren befasste.

\subsubsection{Private Erhalterschaft ${ }^{13}$}

Fachhochschul-Studiengänge wurden als gemeinnützige Unternehmen mit einem handlungsfähigen Management gegründet. Dafür war kein eigenes Organisations- und Dienstrecht erforderlich. Das FHStG lässt neben Personen öffentlichen Rechts auch privatrechtliche Erhalter zu. Es entwickelten sich private Erhalter, die die Rechtsform von Vereinen oder Kapitalgesellschaften haben.

Bei der Gestaltung der internen Abläufe besitzt dieser Rechtsträger weitgehenden Handlungsspielraum, wobei allerdings die vom FHStG vorgeschriebenen Organisationsvorschriften (z.B. Autonomie des Lehrkörpers, Mitbestimmung der StudentInnen) erfüllt werden müssen. Der Rechtsträger eines Fachhochschul-Studiengangs ist zugleich Dienstgeber des wissenschaftlichen und nicht-wissenschaftlichen Personals. In fast allen Angelegenheiten haben die Fachhochschul-Studiengänge größere unternehmerische Handlungsspielräume als die Universitäten. Sie können eigenständig über die Aufnahme von StudentInnen entscheiden und sind selbst für die Ausgestaltung ihres Studienangebots verantwortlich.

Allerdings handelt es sich beim FH-Sektor um keinen privaten Sektor im engeren Sinn. Zum einen stehen die Erhalter trotz ihrer privaten Rechtsform überwiegend im Einflussbereich öffentlich-rechtlicher Instanzen (hauptsächlich der Länder, in einem geringeren Ausmaß von

13 Vgl. FHStG §2. 
Gemeinden und Kammern). Der politische Einfluss, der somit durchaus gegeben ist, wird allerdings auf deutlich andere Art wahrgenommen, als dies im öffentlichen Schulwesen und bis zuletzt ${ }^{14}$ an den Universitäten der Fall war. Zum anderen erfolgt auch die Finanzierung des Studienbetriebs fast ausschließlich aus öffentlichen Quellen.

\subsubsection{Entwicklungsplanung im Zusammenwirken zwischen Bund, Ländern und FHR (Entwicklungs- und Finanzierungsplan I und II)}

Laut B-VG fällt das postsekundäre Bildungswesen in den Kompetenzbereich des Bundes. Dieser Umstand wurde während der Diskussion um das FHStG vielfach (vor allem von VertreterInnen der Länder) so gedeutet, dass dem Bund daraus die Verpflichtung zur Gesamtfinanzierung des FH-Sektors erwachse. Diese Interpretation war mit der Erwartung verbunden, der Bund würde - nach dem Muster der Universitäten - die Trägerschaft der Fachhochschul-Studiengänge übernehmen und alle anfallenden Personal-, Sach- und Investitionskosten decken.

Die Bundespolitik hat sich dieser Auffassung nicht angeschlossen; mit einer Ausnahme ${ }^{15}$ findet kein FH-Studiengang in bundesstaatlicher Trägerschaft statt. Zugleich war allen Akteuren klar, dass der FH-Sektor ohne substanzielles finanzielles Engagement des Bundes nicht lebensfähig ist. Das Gesamtvolumen der Fördermittel durch den Bund sowie die Modalitäten der Finanzierung sind in Form von Entwicklungsplänen für eine bestimmte Periode festgelegt. Der Entwicklungs- und Finanzierungsplan I wurde 1994 beschlossen und bezieht sich auf die Zeit bis 2000; der Entwicklungs- und Finanzierungsplan aus 1999 gilt formell bis 2005, ist aber auf Grund des beschleunigten Ausbaus von Studienplätzen bereits zum gegenwärtigen Zeitpunkt überholt.

Im Rahmen des Entwicklungs- und Finanzierungsplans werden aus der Perspektive des Bundes folgende Punkte geklärt:

- Die Zahl der vom Bund zu finanzierenden Studienplätze bzw. das Tempo des Fachhochschul-Ausbaus. Der für die Zuteilung der Bundesmittel wichtigste Parameter ist die Zahl der Studienplätze. Einerseits muss es eine verbindliche Planung über die Gesamtzahl der Studienplätze innerhalb eines bestimmten Zeitabschnitts geben. Das erfolgt im Rahmen des Entwicklungs- und Finanzierungsplans und stell eine Selbstbindung der Regierung bezüglich der Ressourcen dar, die insgesamt dem Fachhochschulsektor zugeführt werden. Der erste Entwicklungs- und Finanzierungsplan

14 Durch das UG 2002 nähert sich das „Governance“-Modell der Universitäten dem des Fachhochschulsektors an.

15 Es handelt sich um den Fachhochschul-Studiengang für Militärische Führung an der Theresianischen Militärakademie, wobei der öffentliche Erhalter hier durch den hoheitlichen Charakter des Ausbildungsgangs nahe liegt. 
kündigte 10.000 Studienplätze bis zum Jahr 2000 an. Laut dem zweiten Entwicklungsund Finanzierungsplan wird sich diese Zahl bis zum Jahr 2005 auf 21.000 erhöhen. Zweitens ist für jeden einzelnen konkreten Studiengang ein Vertrag zwischen dem zuständigen Ministerium ${ }^{16}$ und dem Erhalter erforderlich, in dem die Höhe des Bundeszuschusses für dieses spezielle Vorhaben festgelegt wird.

- Die Modalitäten der Normkostenfinanzierung sowie die Höhe der Normkosten (vgl. Abschnitt 2.1.6).

- Die Gesamtkoordination des Fachhochschulsektors, die unter weitgehend dezentralisierten und deregulierten Bedingungen schwieriger ist als in einem zentralistischen System. Um in den Genuss einer Bundesfinanzierung zu gelangen, muss ein Studiengang neben einer positiven Begutachtung durch den Fachhochschulrat auch eine Reihe hochschulpolitischer Kriterien erfüllen, die im Entwicklungs- und Finanzierungsplan angeführt sind (z.B. Abbau regionaler Disparitäten, Förderung von Berufstätigen oder Nicht-Maturanten, Internationalität u.a.).

\subsubsection{Normkostenfinanzierung ${ }^{17}$}

Das für die Finanzierung der Fachhochschul-Studiengänge maßgebliche Dokument ist der erste Fachhochschulentwicklungs- und Finanzierungsplan.

Der wichtigste Bruch mit den etablierten Formen der Bildungsfinanzierung bestand im Übergang von der Kameralistik zu einem formelgebundenen Globalbudget. Der Bund übernimmt bei Fachhochschul-Studiengängen nicht (wie bei Universitäten) alle anfallenden Kosten, sondern er weist den Institutionen Pauschalbeträge („Normkosten“) zu, mit denen sie eigenverantwortlich wirtschaften müssen (bzw. dürfen). Fachhochschul-Studiengänge haben daher alle Handlungsspielräume, die die Universitäten seit Jahren fordern, aber sie haben zugleich einen erhöhten Handlungsdruck (denn die Konsequenzen einer Fehlentscheidung müssen nun von ihnen selbst getragen werden).

Darüber hinaus hat der Bund eine gemischte Finanzierung durchgesetzt. Seine Subventionen (d.h. die vom Bund bezahlten Normkosten) bewegen sich nämlich in einer Höhe, die in der Regel eine Co-Finanzierung von anderer Seite notwendig macht.

16 Da dieser Vertrag eine mehrjährige finanzielle Belastung des Bundes zur Folge hat, ist die Zustimmung des BMFin erforderlich.

17 Vgl. Entwicklungs- und Finanzierungsplan I, Kap. 3. 
Studiengebühren waren dabei zunächst ausgeschlossen ${ }^{18}$. In Beiträge der privaten Wirtschaft wurden kurzfristig große, aber unrealistische Hoffnungen gesetzt. Die vergleichsweise geringen Beträge, die als „Beiträge der Wirtschaft“ etikettiert werden, kommen überwiegend von den Kammern, also von Institutionen öffentlichen Rechts. Die einzigen Co-Finanziers, die tatsächlich nennenswerte Beiträge leisten, sind andere Gebietskörperschaften.

Im ersten Entwicklungs- und Finanzierungsplan wurde die Höhe der Normkosten (der laufenden Kosten pro Studienplatz; Investitionen sind in diesem Parameter definitionsgemäß nicht enthalten) festgesetzt. Bei der Ermittlung dieses Parameters waren einerseits die fachspezifischen Kostendifferenzen und andererseits die Fixkostendegression zu berücksichtigen (vgl. Keber 1992). Es wurden nur zwei Fachgruppen unterschieden: technische und kaufmännische Studien (d.h. laborintensive Fächer versus „paper and pencil"-Studien). Für das heikle und im strengen Sinn unlösbare ${ }^{19}$ Problem der Fixkostendegression wurde folgende Hilfskonstruktion gewählt: Die Normkosten wurden unter der Voraussetzung einer Mindestgröße von 1.000 Studienplätzen pro Erhalter ermittelt (obwohl klar war, dass in der Anfangsphase diese Mindestgröße nicht erreicht wird). Als einheitlicher Pauschalbetrag pro Studienplatz wurden 88.000 öS $(6.395,35 €)$ für den kaufmännischen und 105.000 öS $(7.630,81 €)$ für den technischen Bereich festgelegt (jeweils in Preisen von 1991). Der Bund erklärte sich bereit, $90 \%$ dieser Normkosten zu übernehmen.

\subsubsection{Regionalbezug}

Während die Universitäten Einrichtungen des Bundes sind und ihre Beziehungen zu den Bundesländern eher peripheren Charakter haben, hatten die Fachhochschul-Studiengänge von Anfang an einen starken Regionalbezug ${ }^{20}$. Es waren die Regierungen einiger Länder, die Ende der 1980er Jahre Druck auf den Bund ausgeübt haben, einen neuen Hochschultyp einzurichten. Das Qualifikationsprofil der Fachhochschul-Studiengänge ist stärker als jenes der Universitäten auf regionalen Bedarf zugeschnitten. Darüber hinaus hat auch der Umstand, dass der Bund nur $90 \%$ der von inm festgesetzten Normkosten trägt und auf diese Weise auch Länder und Gemeinden in die Finanzierung einbindet, zu einer stärkeren Bindung der Fachhochschuleinrichtungen an regionale und lokale "stakeholders" geführt.

18 Bei der parlamentarischen Behandlung des FHStG traf der Ausschuss für Wissenschaft und Forschung folgende Feststellung: „Hinsichtlich Studiengebühren sind der Universitätssektor und der Fachhochschulsektor gleichzustellen“.

19 Man will einen für alle Erhalter einheitlichen Pauschalbetrag (der nur nach Fächern differiert), obwohl die faktischen Aufwendungen je nach der Größe des Erhalters unterschiedlich sind.

20 Als Region wird in diesem Kontext ein durch ökonomische, politische, z.T. auch kulturelle Strukturen verbundenes Gebiet unterhalb der nationalen Ebene verstanden. 
Die meisten Erhalter stehen in einem mehr oder minder starken Naheverhältnis zur jeweiligen Landesregierung. Ausnahmen sind vor allem jene Erhalter, die einen Rückhalt bei Kammern bzw. Wirtschaftsverbänden gefunden haben. Das Land ist in fast allen Fällen am Erhalter beteiligt, sei es als Mitglied im Erhalterverein oder als Gesellschafter bei einem Erhalter mit der Rechtsform einer Kapitalgesellschaft. In der Regel ist auch sichergestellt, dass das Land gegenüber anderen Mitgliedern bzw. Gesellschaftern wie Gemeinden, Kammern oder Unternehmen einen dominanten Einfluss ausübt. Im konkreten Verhältnis zwischen Land und Erhalter gibt es deutliche Unterschiede:

- Die Funktion des Erhalters kann auf "quasi-kameralistische" Weise durch einen von der Landespolitik dominierten Verein ausgeübt werden. Die Vertreterlnnen des Landes sind in diesem Fall relativ stark in die operativen Belange involviert.

- Das Land kann dem Erhalter weitgehende unternehmerische Freiheiten gewähren, aber bei der finanziellen Unterstützung eher restriktiv vorgehen und die Zuschüsse strikt nach oben begrenzen. Hier ergeben sich für den Erhalter die größten unternehmerischen Herausforderungen, denn sowohl die Bundes-, wie die Landeszuschüsse sind limitiert. Nur für den Notfall gibt es in der Regel eine Ausfallshaftung des Landes.

- Das Land kann dem Erhalter weitgehende unternehmerische Freiheiten gewähren und zugleich eine eher großzügige Subventionspolitik betreiben (d.h. in den finanziellen Zusagen weitgehend den Wünschen der Erhalter folgen). Bei diesem Muster besteht die Gefahr explodierender Kosten mit einer entsprechenden Überforderung des Landesbudgets.

- Das Land kann sich dem Fachhochschulsektor gegenüber weitgehend gleichgültig verhalten.

Spürbare Unterschiede gibt es in bezug auf die Zahl der Erhalter pro Land. Sie variiert zwischen einem und vier Erhaltern. Überall, wo das Land von Anfang an eine sehr aktive Fachhochschulpolitik gemacht hat, hat sich auf Grund dieser landespolitischen Initiativen eine Art Erhaltermonopol gebildet. In diesen Ländern müssen alle Fachhochschulinitiativen, die auf eine Landesförderung Wert legen, ihr Vorhaben im Rahmen dieses Erhalters realisieren. Der Vorteil eines einzigen Erhalters liegt in einer Erleichterung der landesweiten Koordination des Fachhochschulangebots; weiters gibt es die Hoffnung, dass ein großer Erhalter mit geringeren Fixkosten belastet ist als mehrere kleine (was aber bei mehreren Standorten nicht automatisch der Fall ist, sondern von speziellen Voraussetzungen abhängt). In allen anderen Ländern gibt es mehrere Erhalter. In einigen Fällen gibt bzw. gab es aber Druck seitens des Landes und/oder des Bundes in Richtung einer besseren Verzahnung, entweder in Form eines Zusammenschlusses unterschiedlicher Erhalter zu einer Organisation oder durch Gründung einer Dachorganisation. 
Es ist bemerkenswert, dass die Landespolitik in fast allen Fällen jene Freiräume besetzt hat, die durch den legistischen und administrativen Rückzug des Bundes geschaffen wurden. Insofern hat das FHStG weniger eine Deregulierung als vielmehr eine Dezentralisierung politischer Regelungsprozesse bewirkt. Es ist damit eine deutliche "Verländerung"21 des Fachhochschulsektors entstanden, auch in dem Sinn, dass die Entwicklung sehr stark vom politischen Klima und den Prioritäten der jeweiligen Landesregierung abhängt.

21 Der politische Einfluss, den die Länder auf diesem Weg erhalten haben, dürfte ihren Anteil an der Finanzierung der Fachhochschul-Studiengänge in einigen Fällen übersteigen. 


\subsection{Grundzüge der Entwicklung 1994 - 2002}

Im folgenden Abschnitt werden wichtige quantitative Kenndaten in ihrer Entwicklung dargestellt. Dafür wurde die Datenbasis des FHR einer Sonderauswertung unterzogen. Ziel dabei ist die Darstellung grundlegender Entwicklungszüge des Sektors, die die laufende statistische Berichterstattung ergänzen soll und eine Grundlage für die qualitativen Analysen bietet.

\subsubsection{Die wichtigsten Daten im Überblick}

Tabelle 1: Überblick über die Expansion des FH-Sektors, 1994/95 - 2002/03

\begin{tabular}{|c|c|c|c|c|c|c|c|c|c|}
\hline & $\begin{array}{l}\stackrel{2}{\circ} \\
\text { \& } \\
\stackrel{\circ}{\circ}\end{array}$ & 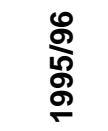 & $\begin{array}{l}\text { 응 } \\
\stackrel{\circ}{\circ} \\
\circ\end{array}$ & $\begin{array}{l}\stackrel{\infty}{\circ} \\
\stackrel{5}{\circ} \\
\stackrel{2}{\circ}\end{array}$ & $\begin{array}{l}\stackrel{\circ}{\circ} \\
\stackrel{0}{\circ} \\
\stackrel{\circ}{\circ}\end{array}$ & $\begin{array}{l}\text { 옹 } \\
\text { ㅇ } \\
\text { ᄋ }\end{array}$ & 옹 & $\frac{\text { ণ }}{\text { ণ }}$ & 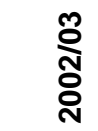 \\
\hline Studiengänge & 10 & 20 & 33 & 39 & 46 & 55 & 67 & 94 & 125 \\
\hline Erhalter & 7 & 11 & 16 & 17 & 18 & 18 & 18 & 19 & 19 \\
\hline $\begin{array}{l}\text { (geografische) } \\
\text { Studienorte }\end{array}$ & 9 & 15 & 17 & 19 & 19 & 20 & 20 & 22 & 23 \\
\hline Bewerberlnnen & 1.076 & 2.095 & 4.206 & 5.702 & 7.450 & 9.679 & 11.224 & 16.443 & n.v. \\
\hline Aufgenommene & 695 & 1.211 & 2.206 & 2.537 & 3.086 & 3.498 & 4.105 & 5.320 & $6.143^{*}$ \\
\hline Studierende & 695 & 1.756 & 3.753 & 5.771 & 7.880 & 9.977 & 11.766 & 14.4381 & $6.838^{\star}$ \\
\hline - männlich & 523 & 1.371 & 2.818 & 4.203 & 5.682 & 7.080 & 8.156 & 9.6511 & $0.651^{\star}$ \\
\hline - weiblich & 172 & 385 & 935 & 1.568 & 2.198 & 2.897 & 3.610 & 4.787 & $6.187^{*}$ \\
\hline Absolventlnnen & --- & --- & 114 & 425 & 897 & 1.584 & 2.018 & n.v. & n.v. \\
\hline - männlich & --- & --- & 90 & 326 & 699 & 1.152 & 1.443 & n.v. & n.v. \\
\hline - weiblich & --- & --- & 24 & 99 & 198 & 432 & 575 & n.v. & n.v. \\
\hline Abbrecherlnnen & n.v. & n.v. & 138 & 277 & 646 & 720 & 797 & n.v. & n.v. \\
\hline - männlich & n.v. & n.v. & 115 & 228 & 471 & 552 & 608 & n.v. & n.v. \\
\hline - weiblich & n.v. & n.v. & 23 & 49 & 175 & 168 & 189 & n.v. & n.v. \\
\hline Lehrende & n.v. & n.v. & 758 & 1.445 & 2.178 & 2.839 & 3.515 & n.v. & n.v. \\
\hline - männlich & n.v. & n.v. & 617 & 1.190 & 1.773 & 2.345 & 2.867 & n.v. & n.v. \\
\hline - weiblich & n.v. & n.v. & 141 & 255 & 405 & 494 & 648 & n.v. & n.v. \\
\hline Hauptberuflich Lehrende & n.v. & n.v. & 102 & 182 & 323 & 430 & 585 & n.v. & n.v. \\
\hline - männlich & n.v. & n.v. & 83 & 142 & 251 & 334 & 455 & n.v. & n.v. \\
\hline - weiblich & n.v. & n.v. & 19 & 40 & 72 & 96 & 130 & n.v. & n.v. \\
\hline
\end{tabular}

n.v.: nicht verfügbar.

Zahlen für 2002/03: vorläufige Daten

*) Nur InländerInnen.

Quelle: FHR, Statistik Austria 


\subsubsection{Entwicklung der Studierenden nach Studiengängen, Erhaltern und Studienorten}

Die Zahl der Erhalter und der Studienorte ${ }^{22}$ ist vor allem bis 1998/99 gestiegen, dann haben sich die Studienorte nur noch geringfügig differenziert. Die Zahl der Studiengänge zeigt bis 2000/01 einen ziemlich linearen Zuwachs in der Größenordnung von 6 bis 13 pro Jahr (insbesondere 1996/97 war ein starkes Jahr), in den Jahren 2001/02 und 2002/03 Jahr gab es dann eine sprunghafte Steigerung auf etwa das doppelte Ausmaß.

\section{Abbildung 1: Ausbau der FH-Institutionen 1994/95 bis 2002/03: Studiengänge, Erhalter, Studienorte}

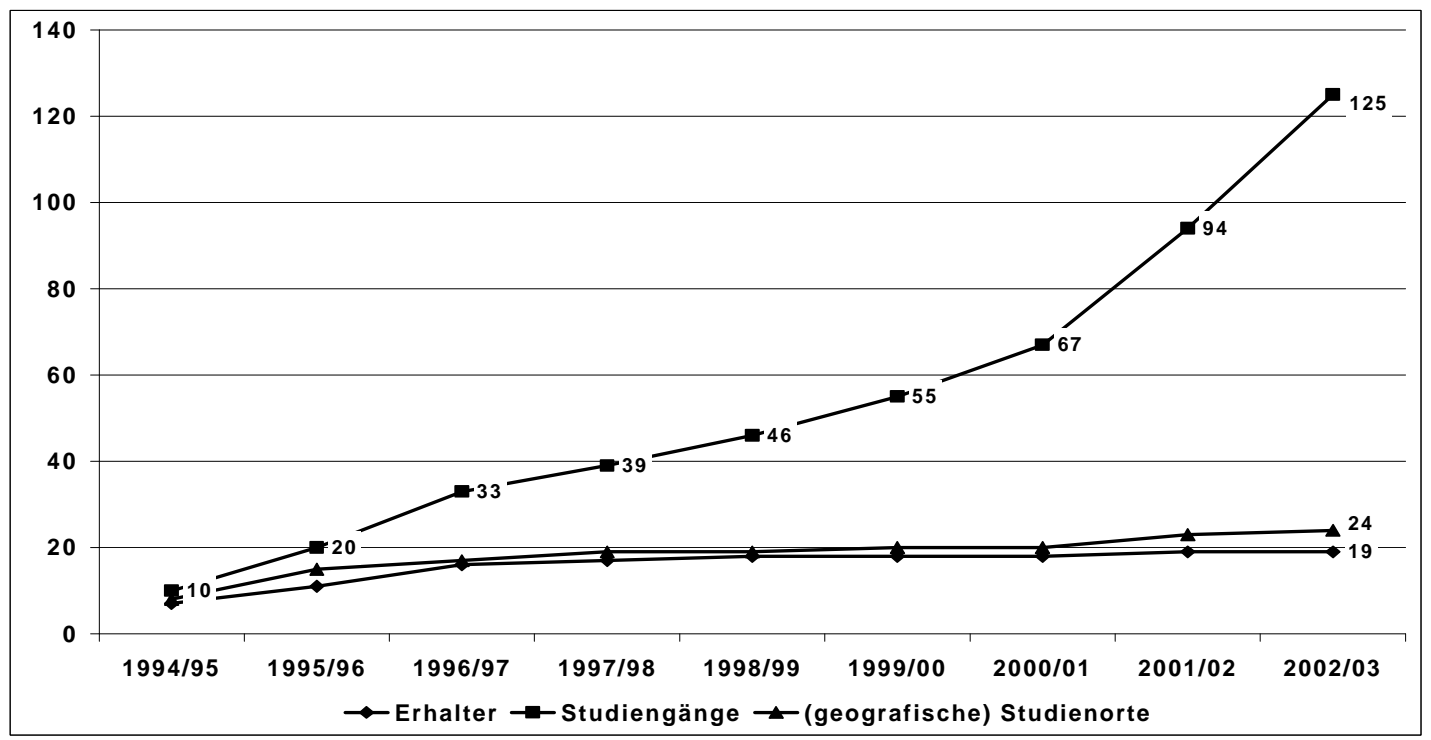

Quelle: FHR

Die Zahl der Studiengänge pro Erhalter (vgl. Abbildung 2) hat sich in der Aufbauphase des Sektors fast verfünffacht, von durchschnittlich 1,4 Studiengängen auf im Schnitt 6,6 (2002/03) pro Erhalter. Inzwischen gibt es - gemessen an der Zahl der Studiengänge - zwei große Erhalter (der Erhalterverein zur Vorbereitung der Errichtung und Erhaltung von Fachhochschulen in Oberösterreich mit 22 und das Technikum Kärnten mit 16 Studiengängen), elf Erhalter betreiben zwischen 5 und 10 Studiengänge und sechs betreiben weniger als fünf. Mit Ausnahme eines Erhalters (Holztechnikum Kuchl) haben inzwischen alle Erhalter, die in den ersten beiden Jahren des Sektors begonnen haben, mindestens fünf Studiengänge.

22 Um die regionale Dimension abzudecken, wurden Auswertungen nach den geografischen Studienorten durchgeführt (d.h. alle Standorte von verschiedenen Erhaltern an einem Ort wurden zusammengefasst). 


\section{Abbildung 2: Zahl der Studiengänge pro Erhalter 1994/95 bis 2002/03}

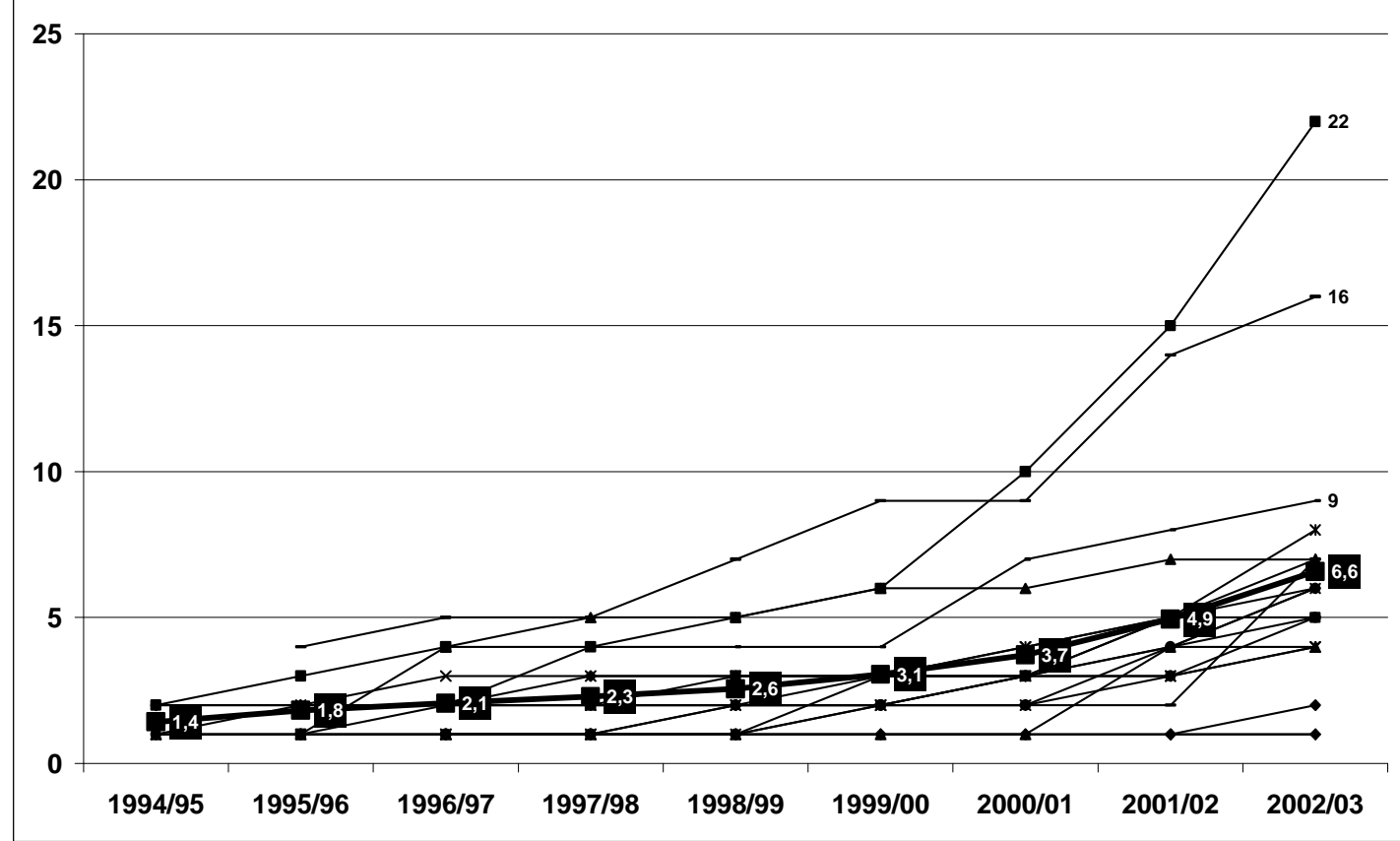

Zahlen auf schwarzem Untergrund: Durchschnitte.

Erhalter mit den meisten Studiengängen sind: Oberösterreich (22), Joanneum (16) und Technikum Kärnten (9). Quelle: FHR

Die Zahl der Studierenden pro Studiengang (vgl. Abbildung 3) ist zunächst gestiegen (bis 1999/00), hat dann aber stagniert (bei einer Zahl von 181) und ist im letzten Jahr leicht rückläufig. In dieser Relation kommt der Ausbauprozess zum Tragen, da sich voll ausgebaute und noch nicht voll ausgebaute Studiengänge mischen. Betrachtet man die Zahl der Studierenden pro Studiengang nur für voll ausgebaute Studiengänge (als solche wurden jene gezählt, die bereits mindestens 4 Jahre bestehen, vgl. Abbildung 4), so zeigt sich eine ziemlich lineare jährliche Steigerung dieser Durchschnittszahl (von 70 auf 247, jährlich um etwas über 20 Studierende/Studiengang mehr). Die Spannweite der Studierendenzahl pro Studiengang ist ziemlich groß, ein Studiengang ist auf etwa 1.000 angewachsen, die nächstgrößeren liegen bei etwa 450, die übrige Verteilung ist ziemlich dicht über die gesamte Spannweite von unter 100 bis zu diesem Wert gestreut. Die Jahrgangsgrößen liegen bei den vierjährigen Studiengängen im Durchschnitt bei 60 (bei den großen Studiengängen erreicht dieser Wert über 100 bis 250, es gibt aber auch viele kleinere Jahrgangsgrößen unter dem Durchschnitt bis unter 25). 
Abbildung 3: Studierende pro FH-Studiengang, 1994/95 - 2001/02

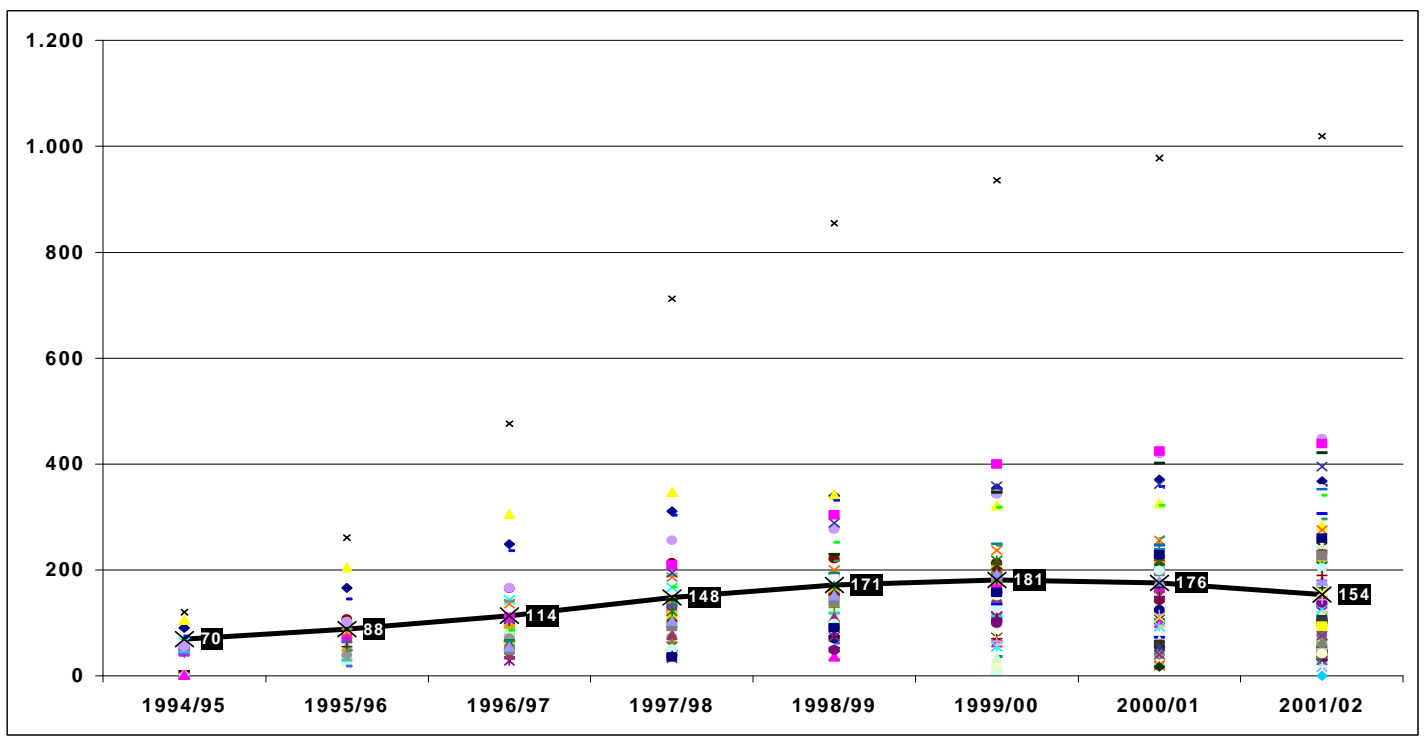

Die Darstellung verdeutlicht die Streuung des Gesamtsektors. Jeder Punkt entspricht einem Studiengang. Quelle: FHR, IHS-Berechnungen

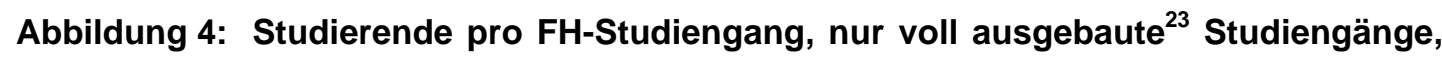
1994/95 - 2001/02

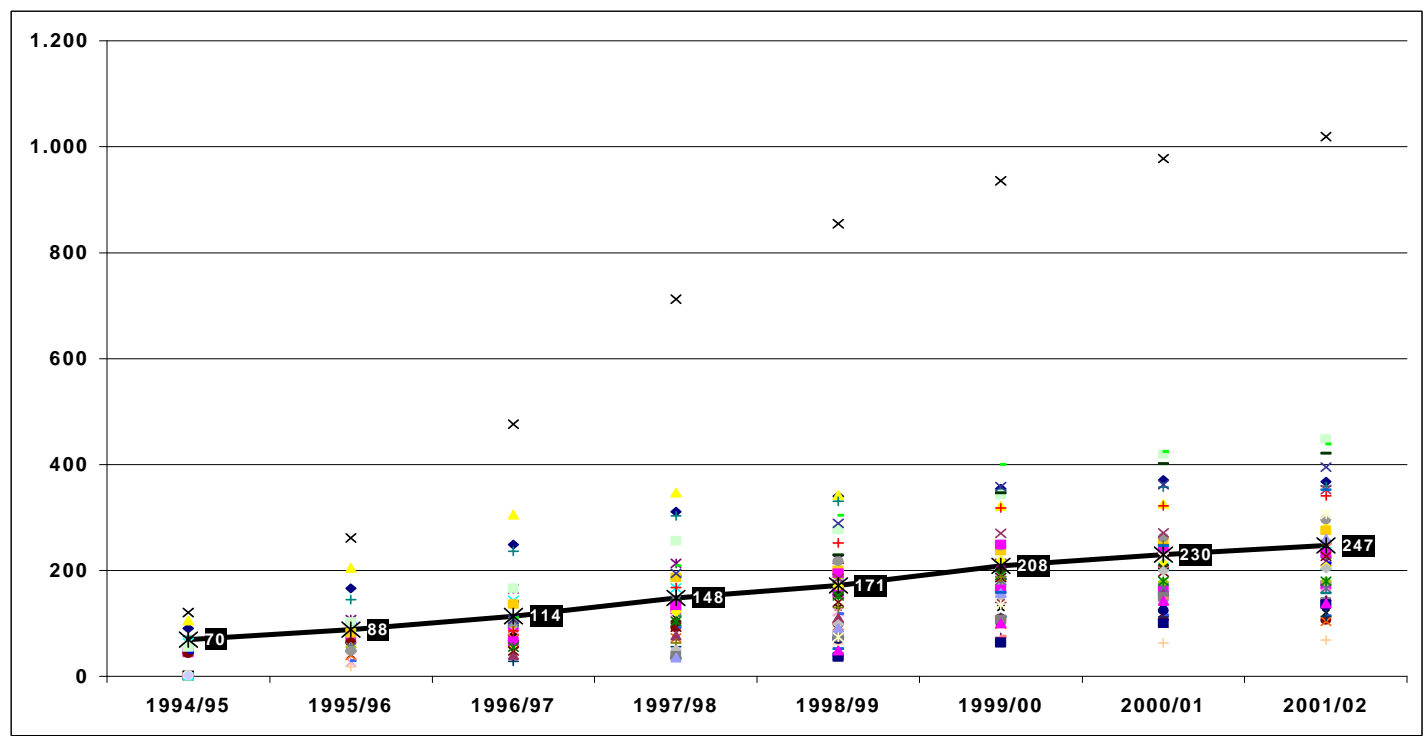

Die Darstellung verdeutlicht die Streuung des Gesamtsektors. Jeder Punkt entspricht einem Studiengang. Quelle: FHR, IHS-Berechnungen

23 Studiengänge, die seit mindestens 4 Jahren bestehen, werden als voll ausgebaute Studiengänge gezählt. 
Die Zahl der Studierenden pro Erhalter (vgl. Abbildung 5) ist von $99 \mathrm{im}$ ersten Jahr auf 759 im Jahr 2001/02 gestiegen. ${ }^{24}$ Auch hier ist die Spannweite außerordentlich groß. Zwei Erhalter liegen von Anfang an über dem Durchschnitt, bis zum dritten Jahr (1997/98) sind zwei weitere über den Durchschnitt gewachsen, diese vier Erhalter liegen jetzt in der Größenordnung von 1.400 bis 1.800 Studierenden. In den Jahren bis 2001/02 sind weitere zwei Erhalter über den Durchschnitt gewachsen, diese liegen bei etwas über 800 . Abgesehen von zwei bisher sehr kleinen Erhaltern mit bis zu 100 Studierenden bewegen sich die meisten Erhalter in der Größenordnung zwischen 300 und 900 Studierenden, die meisten davon liegen unter dem Durchschnittswert. Die große Mehrheit (15 der 19 Erhalter oder fast $80 \%$ ) liegt also noch unter dem Schwellenwert von 1.000 .

\section{Abbildung 5: FH-Studierende pro Erhalter, 1994/95 - 2001/02}

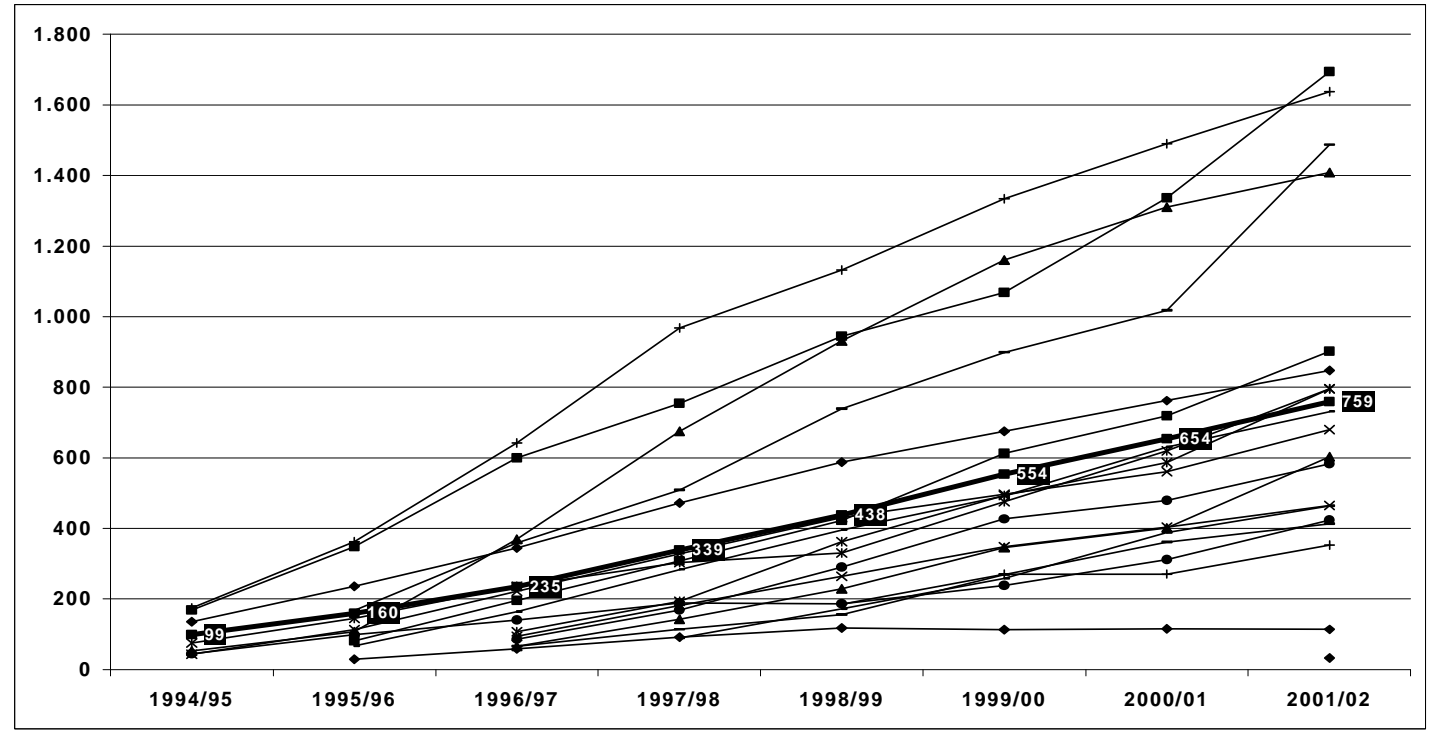

Die Darstellung verdeutlicht die Streuung des Gesamtsektors. Jede Linie entspricht einer Erhalterorganisation. Quelle: FHR, IHS-Berechnungen

Wenn man die 22 (geografischen) Studienorte 2001/02 betrachtet, liegt der Durchschnitt aufgrund der stärkeren regionalen Verteilung mit etwa 656 Studierenden um etwa 15\% unter dem der Erhalter. ${ }^{25}$ Vier Orte (Wien, Wiener Neustadt, Graz und Salzburg) liegen mehr oder weniger deutlich über dem Durchschnitt, die übrigen 18 streuen über den gesamten Bereich bis etwa 700 Studierende. Die deutliche Mehrzahl der Studienorte (19 von 22 oder über 85\%) liegt mehr oder weniger deutlich unter dem Schwellenwert von 1.000 Studierenden, die großen FH-Studienorte sind mit Ausnahme von Wiener Neustadt auch Universitätsorte.

24 Nach den vorläufigen Daten für das WS 2002/03 beträgt diese Relation nunmehr rund 880.

25 Die Betrachtung der geografischen Studienorte ist im Zusammenhang mit den Diskussionen um die Regionalisierung sinnvoll, da sich daran bestimmte Muster der Konzentration und Streuung anschaulicher zeigen als bei der Betrachtung der Standorte allein. 
Wenn man Standorte ${ }^{26}$ im engeren Sinn betrachtet, so müssen vor allem die Studienorte Wien und Graz weiter differenziert werden: die vier Wiener Standorte liegen im Durchschnitt bei 800 und streuen zwischen 400 und 1.400; in Graz gibt es einen größeren (bei 900) und einen kleineren (bei 500) Standort. Im Jahr 2001/02 zeigt sich einerseits eine Konzentration von etwas weniger als 50\% der Studierenden in den drei größten Studienorten bzw. von drei Viertel der Studierenden in den größten neun Standorten. Acht der neun Bundesländer sind unter den ersten neun Standorten vertreten, vor allem die Kärntner Standorte sind vergleichsweise klein (15., 17., 18. Stelle), die Tiroler liegen etwas darüber (an 9. und 12. Stelle), Niederösterreich liegt mit zwei Studienorten unter den ersten neun.

\section{Abbildung 6: FH-Studierende nach (geografischen) Studienorten, 2001/02}

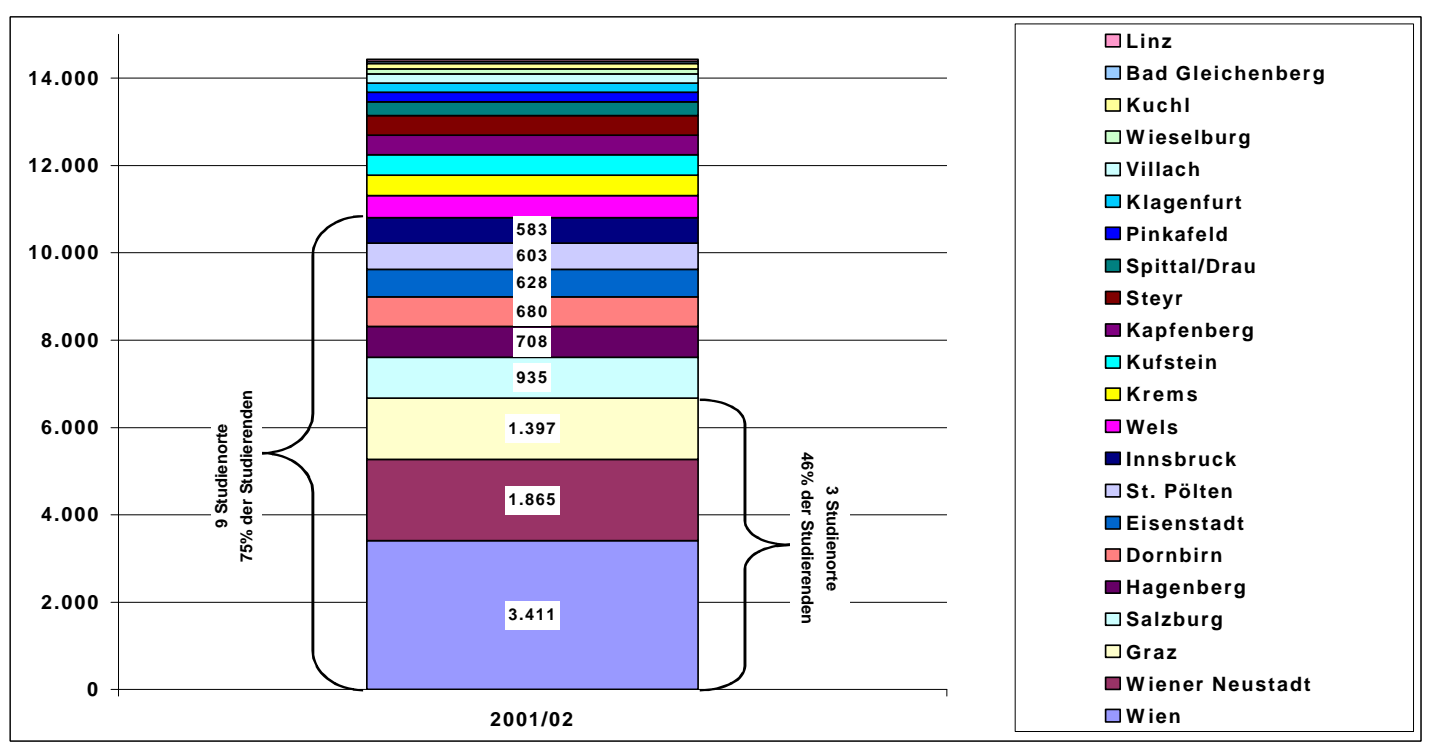

Quelle: FHR, IHS-Berechnungen

Nach Bundesländern gebündelt lassen sich sehr unterschiedliche Dynamiken der Studierendenzahl erkennen (vgl. Abbildung 7). Niederösterreich hatte stark begonnen und eine starke Dynamik entwickelt, hat aber wegen seines unterdurchschnittlichen Wachstums anteilsmäßig seit 1996 etwas verloren (von 23\% auf 21\% der FH-Studierenden in Österreich). Wien und Tirol zeigen - wenn auch auf unterschiedlichem Größenniveau - ein stärkeres Wachstum als die übrigen Bundesländer (Anteilsgewinne von 3 bzw. 5 Prozentpunkten), das gilt etwas abgeschwächt auch für die Steiermark, Salzburg und Kärnten (leichte Anteilsgewinne von bis zu einem Prozentpunkt); Oberösterreich, Burgenland und Vorarlberg zeigen ein vergleichsweise schwächeres Wachstum (Anteilsverluste von bis

${ }^{26}$ Als Standorte werden die Einrichtungen der verschiedenen Erhalter an einem Studienort gezählt. 
zu drei Prozentpunkten), wobei vor allem Oberösterreich und das Burgenland stark begonnen hatten.

Abbildung 7: Verteilung der FH-Studierenden nach Bundesländern, 1995/96 - 2001/02

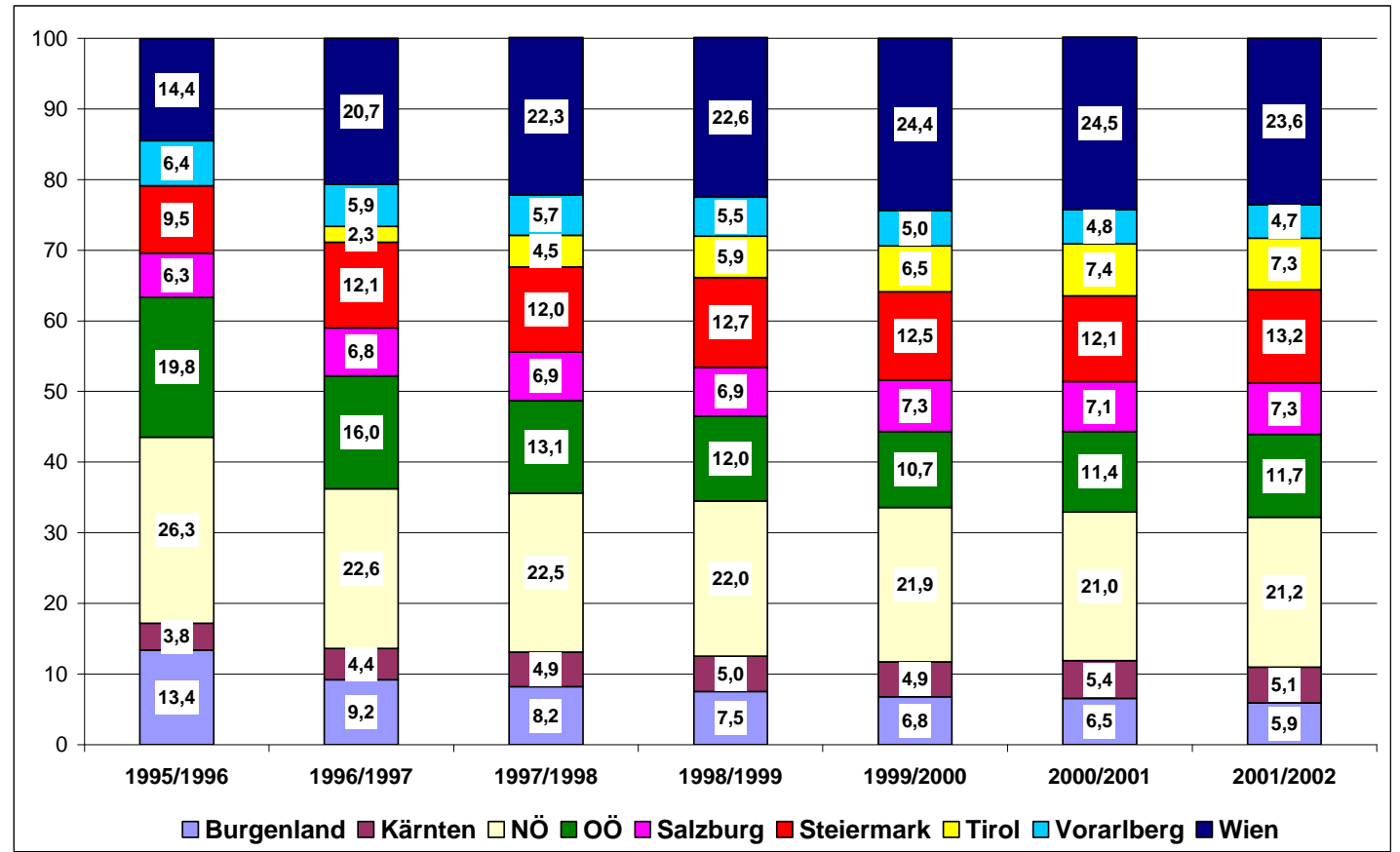

Quelle: FHR, IHS-Berechnungen

Der Frauenanteil unter den Fachhochschulstudierenden (vgl. Abbildung 8) ist im Durchschnitt etwas gestiegen (von 25\% auf 33\%), aber immer noch gering (entsprechend ist auch die Wachstumsrate der weiblichen Studierenden etwas höher als die der männlichen, der Unterschied der Wachstumsraten war 1996/97 besonders groß, hat sich dann 1998/99 abgeschwächt und steigt in den letzten Jahren wieder). Bemerkenswert ist in dieser Hinsicht die Streuung nach den verschiedenen Analysekriterien. Mit der Steigerung des Anteils hat sich die Streuungsbreite nach Studiengängen, die anfänglich bereits zwischen $0 \%$ und $70 \%$ lag, vor allem im letzten Jahr mit der Einrichtung der Studiengänge im Sozial- und Gesundheitsbereich noch bis 95\% erweitert. Darin spiegelt sich die bekannte geschlechtsspezifische Segregation nach Fachbereichen: in einer beträchtlichen Zahl von Studiengängen liegt der Frauenanteil höher als 50\% und die Zahl dieser Studiengänge ist im Laufe der Jahre stark gestiegen. Andererseits gibt es eine beträchtliche Zahl von Studiengängen, wo der Frauenanteil unter 20\% (38 von 94 Studiengängen) bzw. sogar unter 10\% (21 von 94 Studiengängen) liegt. 
Nach Fachbereichen ${ }^{27}$ betrachtet (vgl. Abbildung 9) liegt der Frauenanteil in den technischen und naturwissenschaftlichen Bereichen bei 10\%, in den anderen Bereichen zwischen $40 \%$ bis gegen $50 \%$. Die große Spanne zeigt sich aber auch nach Erhaltern, Studienorten und Bundesländern. Indirekt weist dies auf die wenig diversifizierte Fachbereichsstruktur vieler Erhalter hin (bei vier Erhaltern liegt der Frauenanteil bei bis zu 10\%, bei drei Erhaltern liegt er über 50\% und reicht bis zu 70\%). Ebenso gibt es vier Studienorte mit einem Frauenanteil zwischen $60 \%$ und $95 \%$ sowie fünf Studienorte mit einem Frauenanteil von unter $20 \%$. Nach Bundesländern hat sich die Streubreite deutlich verringert (von einer Spanne zwischen 0\% und fast $40 \%$ im ersten Jahr auf eine Spanne zwischen etwa $25 \%$ und 55\%). Vor allem das Burgenland hat den von Anfang an hohen Frauenanteil auf etwas über 50\% gesteigert. Auch in jenen Bundesländern mit ursprünglich sehr niedrigem Anteil hat sich dieser mehr oder weniger deutlich erhöht. Berufsbegleitende Studiengänge haben einen um fast 10 Prozentpunkte niedrigeren Frauenanteil als die Normalform, in den zielgruppenspezifischen Studiengängen liegt der Frauenanteil fast bei $0 \%$.

\section{Abbildung 8: Frauenanteil unter den FH-Studierenden, 1994/95 - 2001/02}

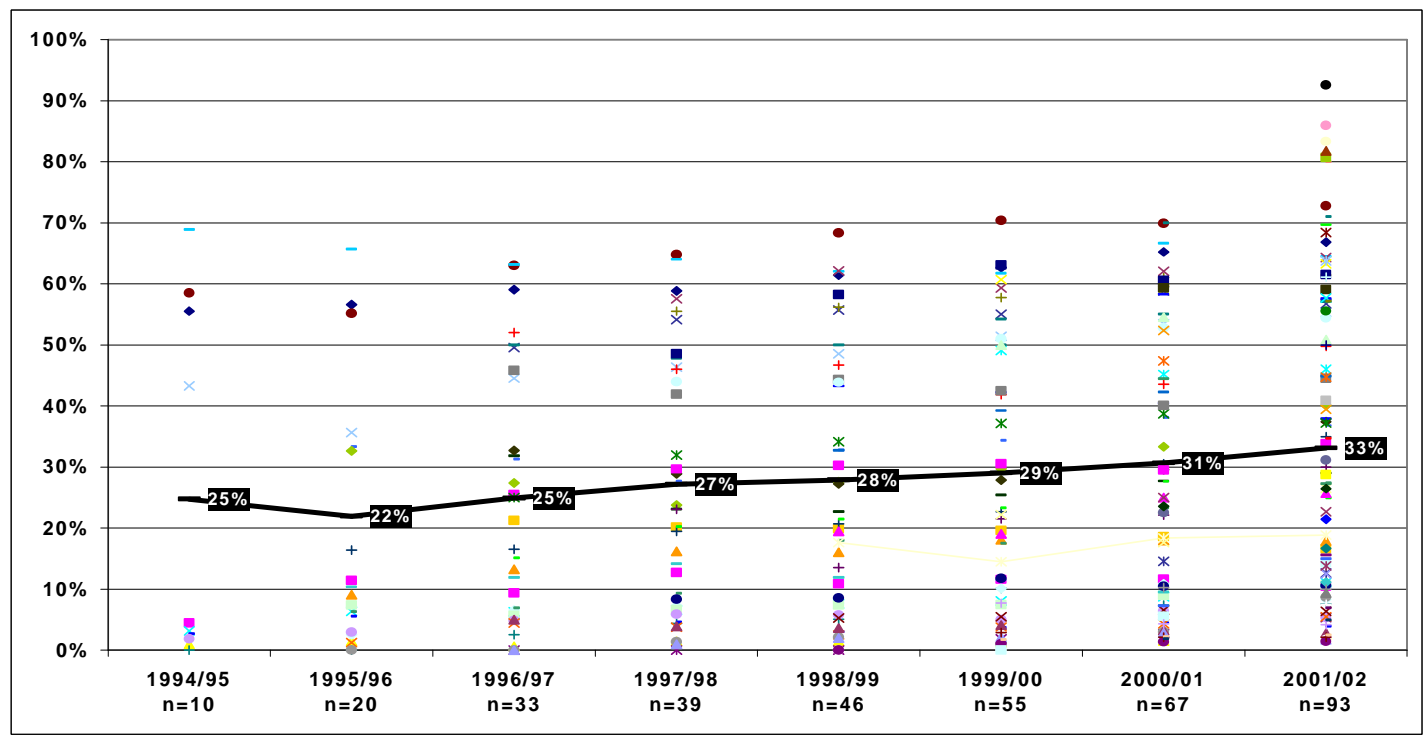

Die Darstellung verdeutlicht die Streuung des Gesamtsektors. Jeder Punkt entspricht einem Studiengang. Quelle: FHR, IHS-Berechnungen ${ }^{27}$ Zusammengefasst nach ISCED-Fachbereich-Kategorien (ISCED-FBR), nicht zu verwechseln mit den ISCED-
Levels. 


\section{Abbildung 9: Frauenanteil unter FH-Studierenden nach Fachbereichen (ISCED-FBR), 1994/95 - 2001/02}

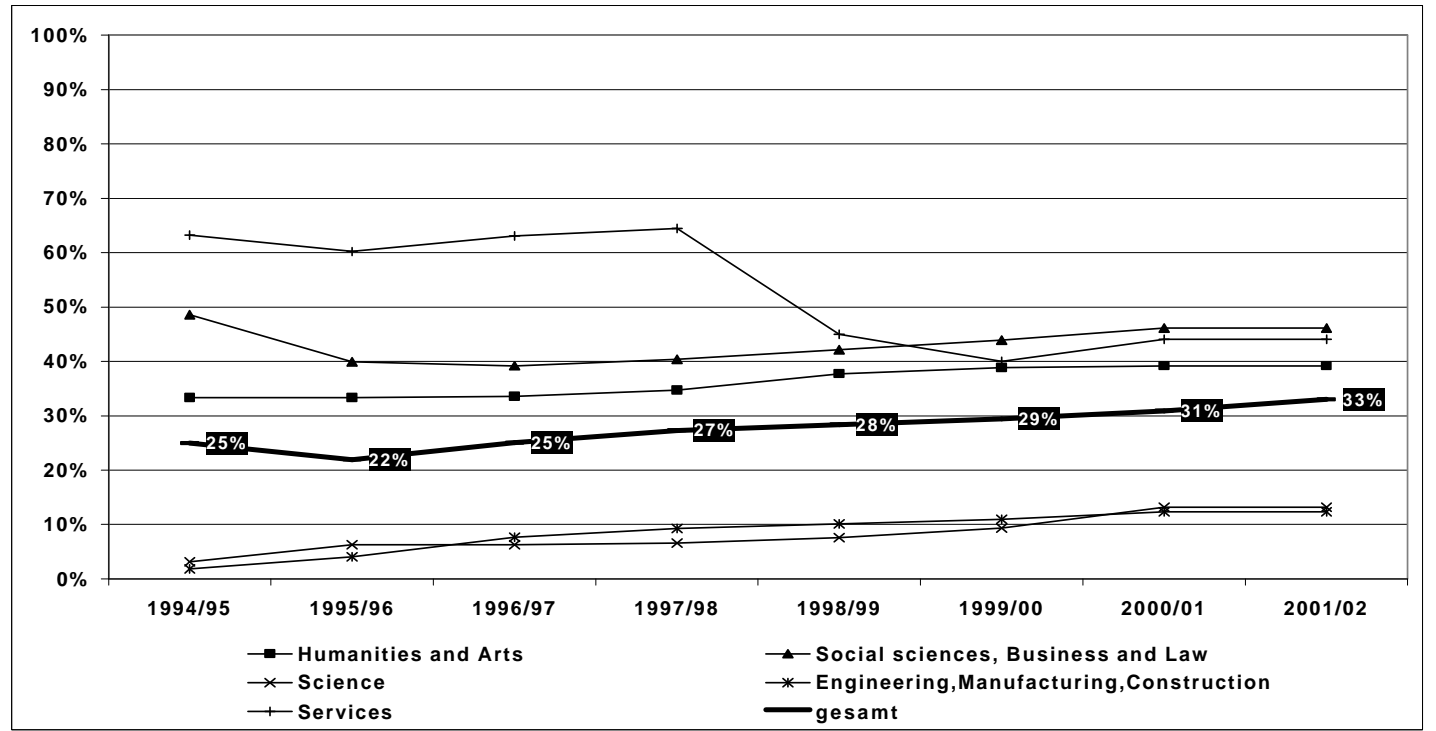

Quelle: FHR, IHS-Berechnungen

Unter den AbsolventInnen ist der Frauenanteil um etwa vier Prozentpunkte geringer als unter den Studierenden (21\% im ersten Jahr bzw. 27\% im letzten Jahr der Betrachtung), auch die Entwicklung zeigt nicht die sukzessive Steigerung wie unter den Studierenden, sondern lediglich einen stufenartigen Anstieg 1999, der auf einige Studiengänge zurückgeführt werden kann, die ab diesem Jahr bzw. den folgenden neue AbsolventInnen hervorgebracht haben. Es sind aber bei vielen Studiengängen von Jahr zu Jahr größere Schwankungen der Frauenanteile unter ihren AbsolventInnen erkennbar.

\subsubsection{Entwicklung der Fachbereiche}

Jeweils etwa $40 \%$ der Studierenden belegen technische (ISCED: Engineering, manufacturing, construction) bzw. wirtschaftliche Studiengänge (ISCED: Social sciences, business and law), wobei die Zahl der Studierenden in wirtschaftswissenschaftlichen Fächern im Studienjahr 2001/02 erstmals die in technischen leicht übertraf (vgl. Abbildung 10). Seit 1994/95 ist insbesondere der Anteil der Studierenden im Bereich Dienstleistungen, also v.a. Tourismus (ISCED: Services) deutlich von 14\% auf nunmehr $6 \%$ zurückgegangen. Im Studienjahr 2001/02 expandierte durch die Einführung neuer Studiengänge hauptsächlich der Bereich Naturwissenschaften/IKT (ISCED: Science), den heute 8\% der Studierenden 
besuchen. Als neue Bereiche kamen Gesundheit (ISCED: Health and Welfare) sowie allgemeine ${ }^{28}$ bzw. nicht näher zugeordnete ${ }^{29}$ Studiengänge hinzu.

\section{Abbildung 10: FH-Studierende nach ISCED-FBR, 1994/95 - 2001/02}

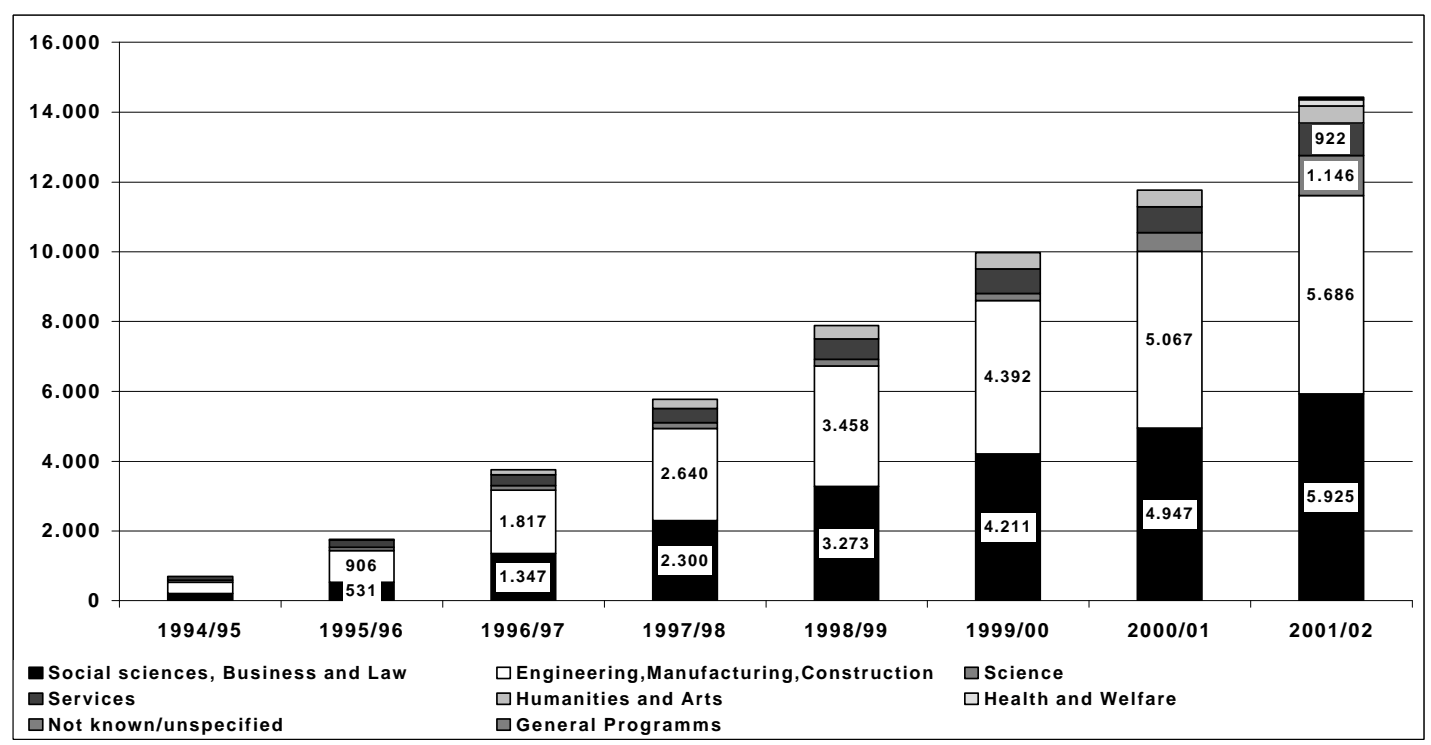

Quelle: FHR, IHS-Berechnungen

Zum gegenwärtigen Stand ist der Grad der Diversifizierung nach ISCED-Fachbereichen an den einzelnen Standorten sehr gemischt (vgl. Abbildung 11). Es gibt alteingesessene Standorte, die nicht bzw. kaum diversifiziert sind, wenn diese auch eher eine Ausnahme darstellen. Am häufigsten sind Standorte mit zwei Fachbereichen, wobei die Kombination von Wirtschaft und Technik überwiegt. Die Zahl der Standorte mit drei oder mehr Fachbereichen ist nur wenig geringer. Es zeigt sich tendenziell ein Muster nach Bundesländern, indem die Standorte in Kärnten, Burgenland und Oberösterreich, überwiegend auch in Wien und Niederösterreich weniger diversifiziert sind als die Standorte in Salzburg, Tirol, Vorarlberg und der Steiermark.

28 „Humanmanagement“ und „Digitale Dienste“.

29 „Sport-, Kultur- und Veranstaltungsmanagement". 


\section{Abbildung 11: FH-Studierende nach Fachbereichen (ISCED-FBR) pro Standort und Erhalter, 2001/02}

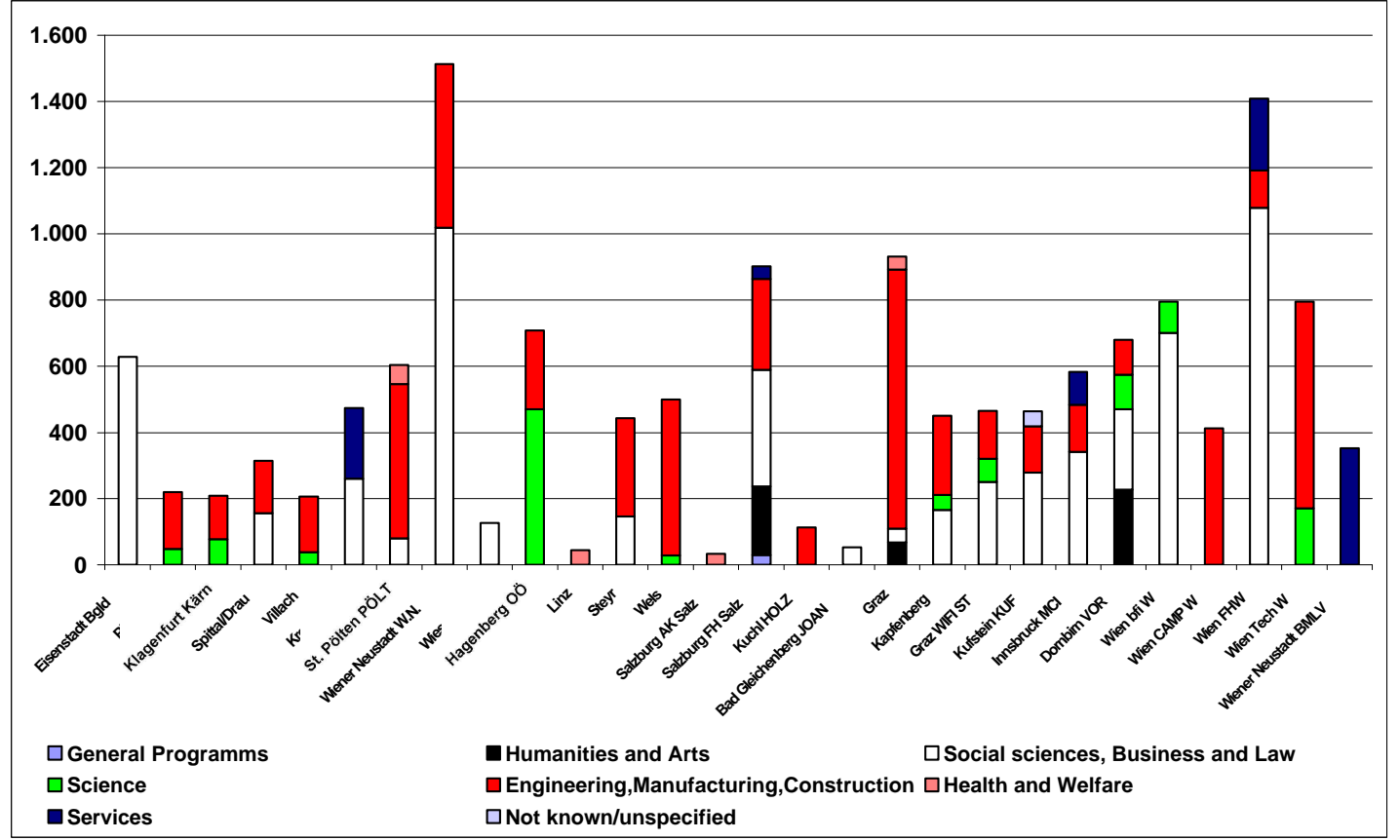

Quelle: FHR, IHS-Berechnungen

Es besteht kein direkter Zusammenhang zwischen dem Grad der Diversifizierung und der Zahl der Studierenden. Kleinere Standorte sind zwar mit hoher Wahrscheinlichkeit nicht diversifiziert, das Umgekehrte gilt jedoch nicht. Die nicht diversifizierten Standorte haben gemeinsam, dass sie wenig hauptberufliches Personal beschäftigen und eine hohe Zahl nebenberuflicher Lehrpersonen im Vergleich zu den hauptberuflich Lehrenden haben.

\subsubsection{Das BewerberInnenpotential und seine Ausschöpfung}

Die Zahl der BewerberInnen ist mit Ausnahme eines Jahres (2000/01) stärker gestiegen als die Zahl der aufgenommenen Studierenden, das hat im gesamten Zeitraum zu einer Verdoppelung des Verhältnisses BewerberInnen/Aufgenommene von 1,5:1 auf 3,1:1 geführt. Der Zuwachs der Bewerberlnnen betrug im Jahr etwa 1.700, ist im letzten Jahr aber sprunghaft auf das Dreifache angestiegen. Den Anteil der aufgenommenen Bewerberlnnen bezeichnen wir als Ausschöpfungsgrad. Dieser Indikator gibt in quantitativer Hinsicht Aufschluss über das Ergebnis der Aufnahmeprozeduren. Dabei ist zu berücksichtigen, dass die Zahl der Studienplätze in den Studiengängen, mit einem gewissen Flexibilitätskorridor, vorgegeben ist. Eine höhere Zahl an Bewerbungen im Vergleich zu den Studienplätzen hat daher keinen unmittelbaren Einfluss auf die Ausschöpfung. Die Zahl der Bewerberlnnen schließt Mehrfachzählungen nicht aus, da sich Interessierte bei mehreren Studiengängen bewerben können. 
Es wurden 2001/02 ziemlich genau ein Drittel der Bewerberlnnen aufgenommen, gegenüber zwei Dritteln im ersten Jahr. Auch bei diesem Merkmal ist der Streubereich exorbitant groß; er reicht in der Betrachtung nach Studiengängen derzeit von etwa 5\% bis 100\% und hat sich tendenziell im Laufe der Entwicklung erweitert. Einerseits gab es vor allem in den Anfangsjahren einige Studiengänge mit sehr hoher Ausschöpfung der Bewerbungen. Dies hat sich seit $1997 \mathrm{im}$ Bereich zwischen 85\% und 100\% Ausschöpfungsquote auf Einzelfälle (6 Studiengänge) verringert, andererseits hat sich die Zahl der hochselektiven Studiengänge mit Ausschöpfungsquoten von unter 20\% deutlich erhöht (von 5 Studiengängen im Jahr 1997 auf $13 \mathrm{im}$ Jahr 2001) (vgl. Abbildung 12).

Auch in der Betrachtung nach Erhaltern, Studienorten und Bundesländern gibt es große Streubereiche der Ausschöpfungsquoten. Die Selektivität zeigt teilweise konstante Muster nach diesen Kriterien, teilweise gibt es auch beträchtliche Schwankungen.

\section{Abbildung 12: Anteil der aufgenommenen FH-BewerberInnen nach FH-Studiengang,} 1994/95 - 2001/02

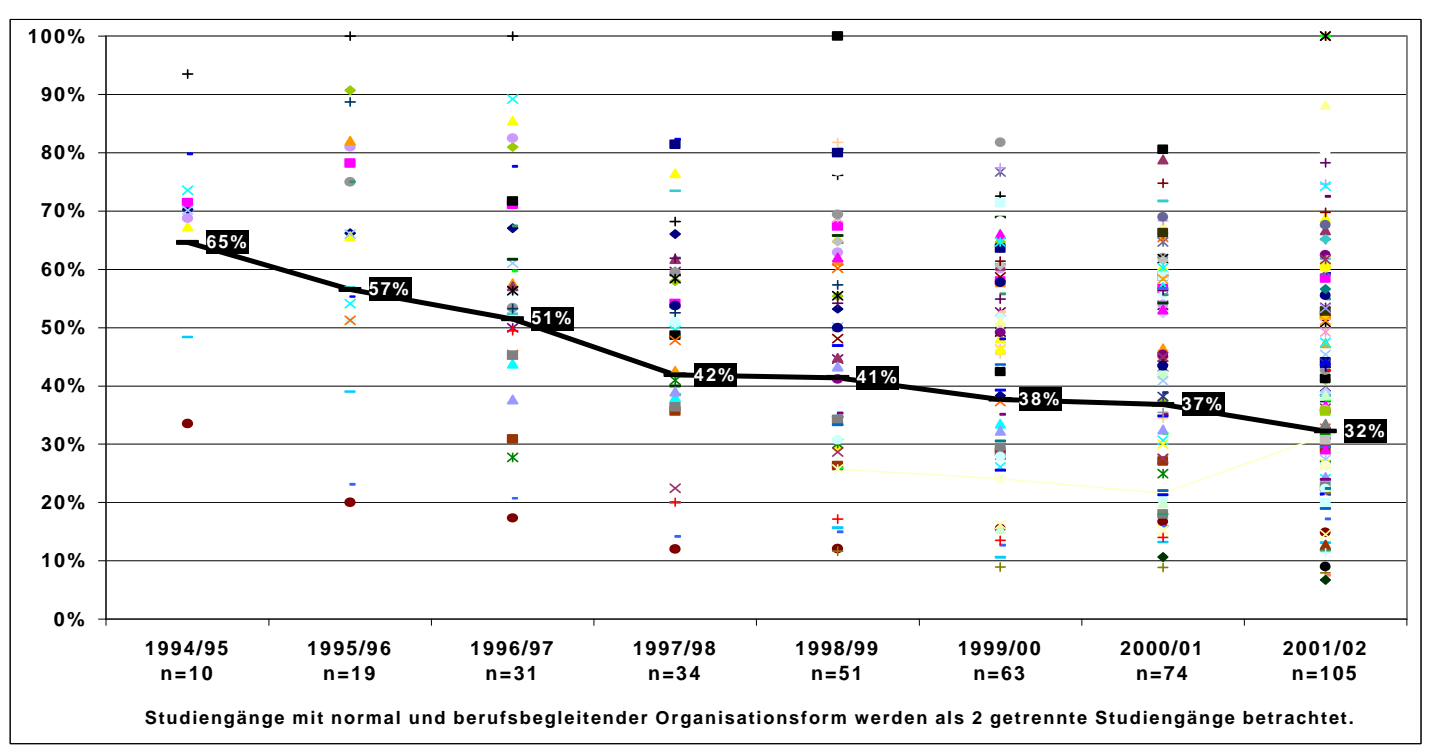

Die Darstellung verdeutlicht die Streuung des Gesamtsektors. Jeder Punkt entspricht einem Studiengang. Quelle: FHR, IHS-Berechnungen

Systematische Unterschiede bestehen nach Fachbereichen (vgl. Abbildung 13). Der technische Bereich (ISCED: Engineering, manufacturing, construction) hat eine durchgängig etwas überdurchschnittliche (um 10 Prozentpunkte) Ausschöpfung, mit parallel zum Durchschnitt sinkender Tendenz. Die Ausschöpfungsquote der wirtschaftlichen Studiengänge (ISCED: Social sciences, business and law) lag in den Anfangsjahren etwas über dem Durchschnitt und geht in den letzten Jahren mit der Entwicklung des Durchschnitts ziemlich konform. Die Studiengänge im Bereich Dienstleistungen und Medien/Design haben eine niedrige Ausschöpfung etwa konstant in der Größenordnung von 20-30\%, etwas darunter 
liegt der Bereich Gesundheits- und Sozialberufe; die Studiengänge im Bereich Naturwissenschaften/IKT (ISCED: Science) haben ihre Selektivität verstärkt und liegen seit 1998 etwas unter dem Durchschnitt in der Größenordnung von 30\%. Berufsbegleitende Studiengänge haben eine konstant überdurchschnittliche Ausschöpfung der Bewerberlnnenzahl in der Größenordnung von 45\%, der Rückgang zeigt sich in der Normalform (die zielgruppenspezifischen Studiengänge haben eine deutlich erhöhte Ausschöpfung von mehr als $60 \%$ ).

\section{Abbildung 13: Anteil der aufgenommenen FH-Bewerberlnnen nach Fachbereichen (ISCED-FBR), 1994/95 - 2001/02}

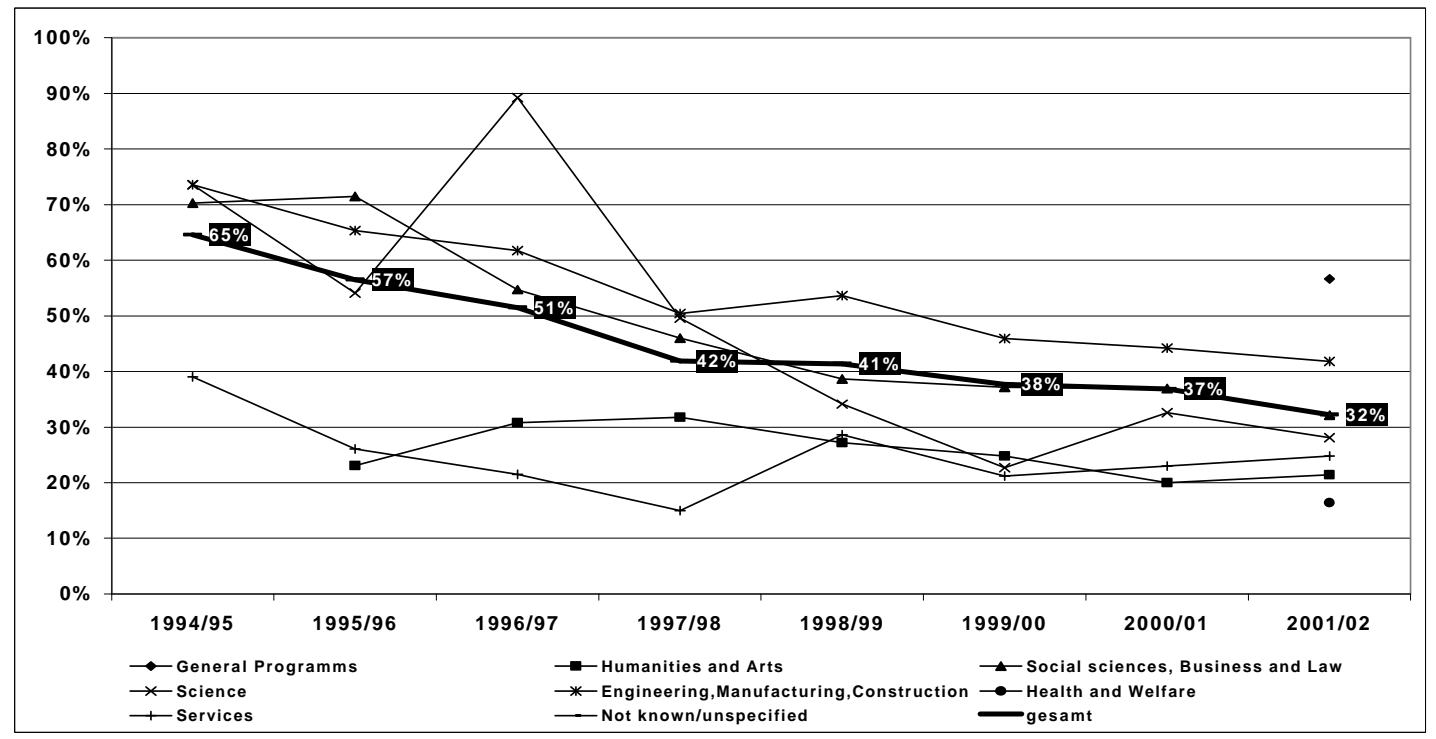

Quelle: FHR, IHS-Berechnungen

Die durchschnittliche Zahl der BewerberInnen pro Studiengang ist von etwas über $100 \mathrm{im}$ ersten Jahr auf etwas über 150 gestiegen, der Großteil der Studiengänge liegt in einer Spannbreite zwischen 20 und 300, nur acht Studiengänge haben höhere BewerberInnenzahlen zwischen 350 und 600. Nach Erhaltern liegt das durchschnittliche BewerberInnenpotential für einen Jahrgang bei 870, fünf Erhalter liegen mehr oder weniger deutlich darüber (bis über 3.000), drei deutlich darunter (unter 250), die übrigen in der Spanne zwischen 500 und 1.000. Nach Studienorten ist dieses Potential etwas geringer, der Durchschnitt liegt bei 750 Bewerberlnnen, fünf Orte liegen darüber (zwischen 1.000 und über 3.000), fünf Orte liegen unter 250 BewerberInnen (vgl. Abbildung 14). Nach Bundesländern bestehen zwei Gruppen, eine erste mit Wien, Niederösterreich, Oberösterreich und Steiermark mit stark überdurchschnittlichen BewerberInnenzahlen in der Größenordnung zwischen unter 3.000 und 3.500 (wobei die Steiermark im letzten Jahr eine exorbitante Steigerung von 1.500 auf 3.500 aufweist, womit sie von der vierten an die erste 
Stelle gerückt ist) und eine zweite Gruppe der übrigen kleineren Bundesländer mit deutlich unterdurchschnittlichen Bewerberlnnenzahlen in der Größenordnung von 500 bis 1.000 .

\section{Abbildung 14: Zahl der FH- BewerberInnen pro Studienort, 1994/95 - 2001/02}

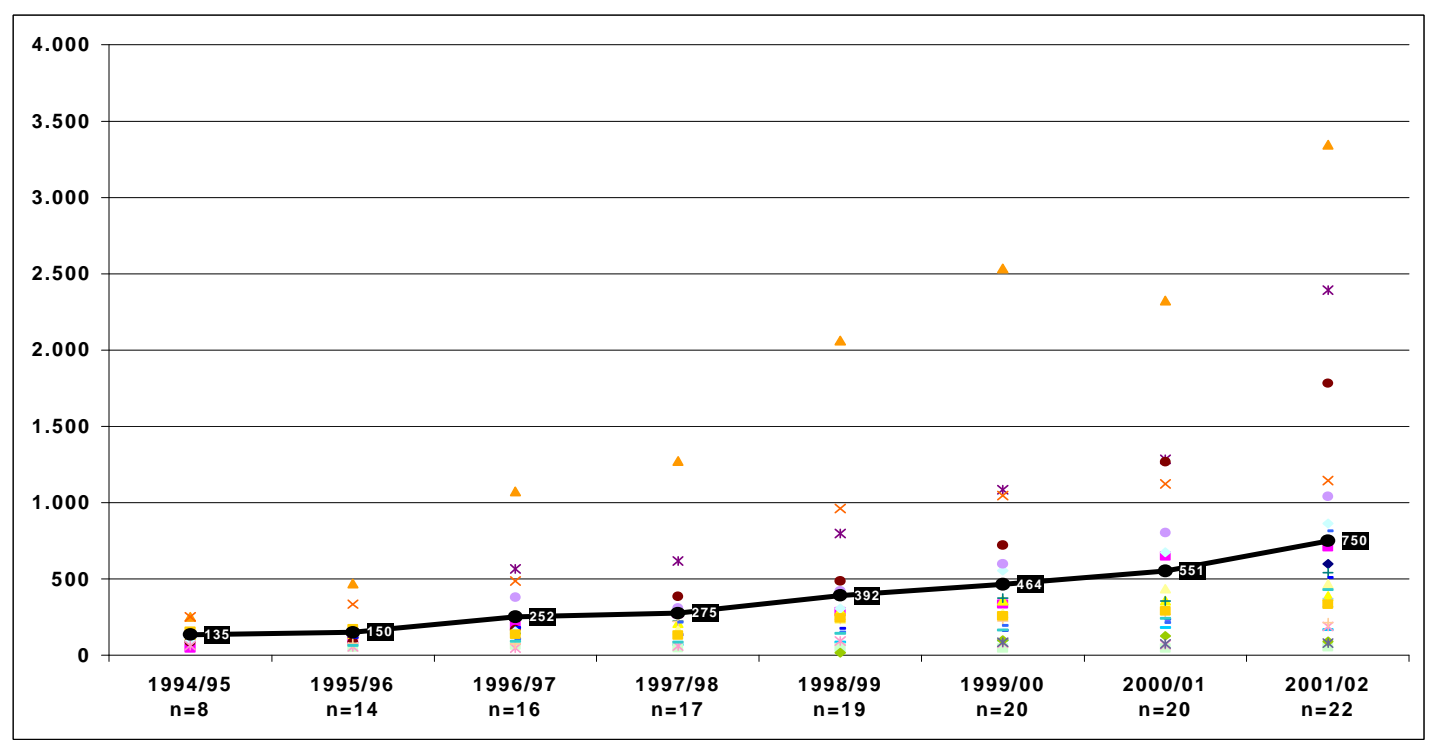

Die Darstellung verdeutlicht die Streuung des Gesamtsektors. Jeder Punkt entspricht einem Studienort. Quelle: FHR, IHS-Berechnungen

Nähere Betrachtung verdienen auch die Fachbereiche (vgl. Abbildung 15). Hier zeigen die Bewerberlnnenzahlen bei den wirtschaftlichen (ISCED: Social sciences, business and law) und den technischen (ISCED: Engineering, manufacturing, construction) Studiengängen durchgängig stark steigende Tendenz (auf zuletzt 6.500 bzw. 4.500), die übrigen stagnieren eher bei einer Größenordnung zwischen 500 und 1.000, mit Ausnahme der naturwissenschaftlichen überwiegend IKT-orientierten Fachrichtungen (ISCED: Science), die in den letzten beiden Jahren stark steigende Zahlen aufweisen (in ihrem absoluten Umfang jedoch mit etwa 2.500 noch deutlich unter den großen Richtungen liegen). 
Abbildung 15: FH-BewerberInnen nach Fachbereichen (ISCED-FBR), 1994/95 - 2001/02

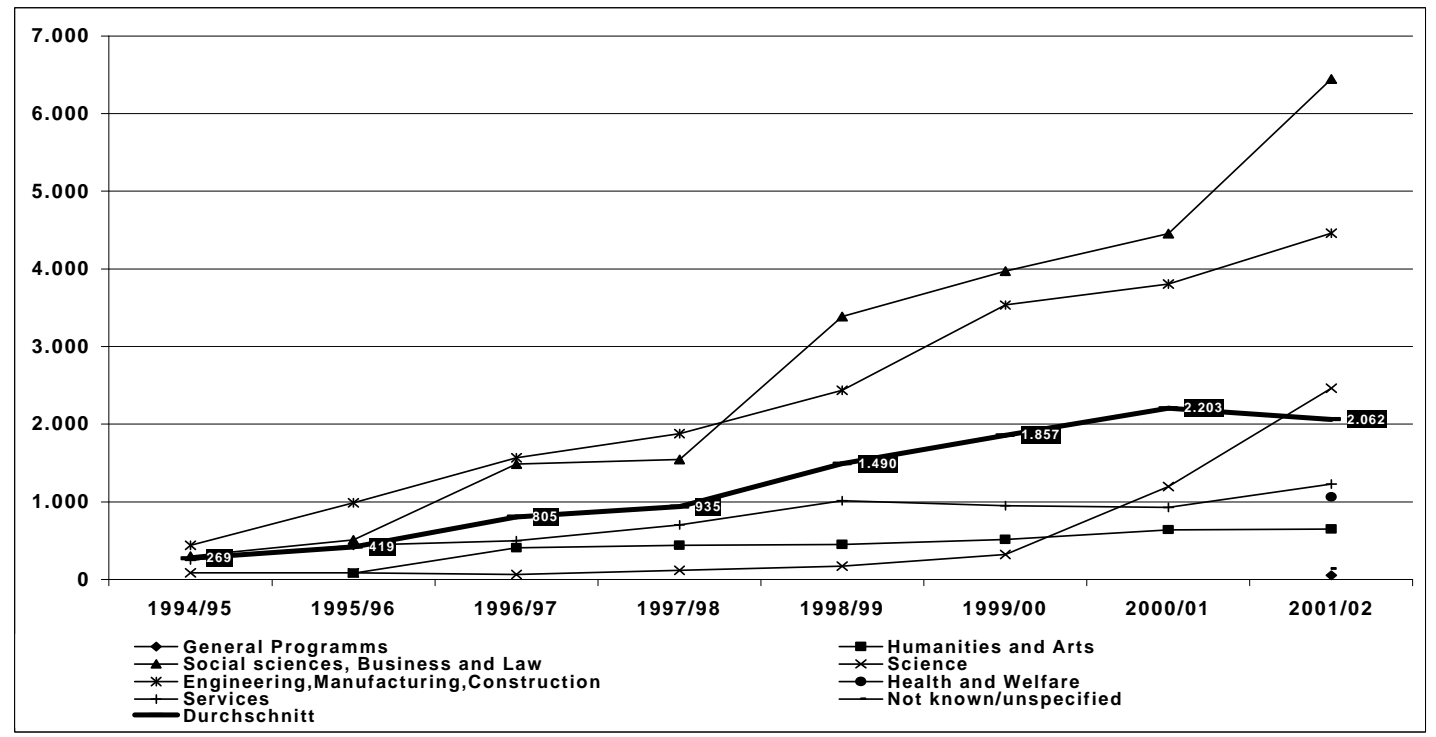

Quelle: FHR, IHS-Berechnungen

Betrachtet man die technischen (ISCED: Engineering, manufacturing, construction) und naturwissenschaftlichen IKT-Fachbereiche (ISCED: Science) differenziert nach Studiengängen (vgl. Abbildung 16, Abbildung 17), so sieht man die starke Ausweitung der Zahl kleinerer Studiengänge seit 1998. Es gibt in diesen Bereichen einige starke, bereits länger bestehende Studiengänge mit deutlich wachsenden BewerberInnenzahlen (in beiden Bereichen zusammen gibt es vier Studiengänge mit einem Potential von mehr als 300 BewerberInnen und weitere drei zwischen 200 und 300, wobei einer davon im letzten Jahr stagniert hat). Die übrigen Studiengänge sind zum Teil ziemlich jung, es gibt aber auch eine große Zahl von Studiengängen in diesen Bereichen, die bereits längere Zeit stagnieren oder auch rückläufige BewerberInnenzahlen aufweisen. So stagniert in beiden Bereichen die durchschnittliche Zahl der Bewerberlnnen pro Studiengang (in der Größenordnung von 120 bei den technischen bzw. von 150 bei den naturwissenschaftlichen IKT-Studiengängen).

Auch in den anderen Bereichen gibt es vergleichsweise große Studiengänge mit stagnierenden oder rückläufigen BewerberInnenzahlen nach einer Periode anfänglicher starker Expansion. 
Abbildung 16: FH-BewerberInnen nach technischen Studiengängen (ISCED-FBR 5: Engineering, manufacturing, construction), 1994/95 - 2001/02

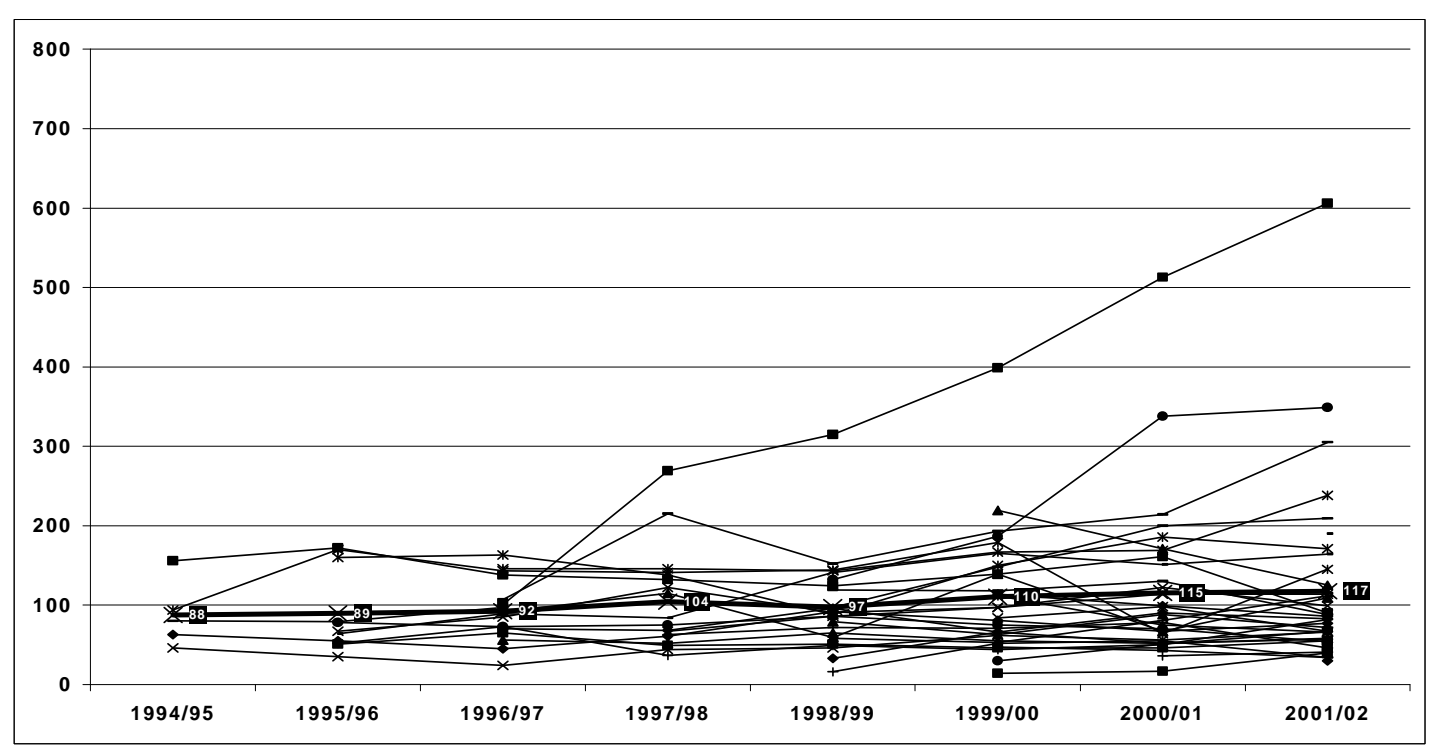

Quelle: FHR, IHS-Berechnungen

Abbildung 17: FH-BewerberInnen nach naturwissenschaftlichen IKT-Studiengängen (ISCED-FBR 4: Science), 1994/95 - 2001/02

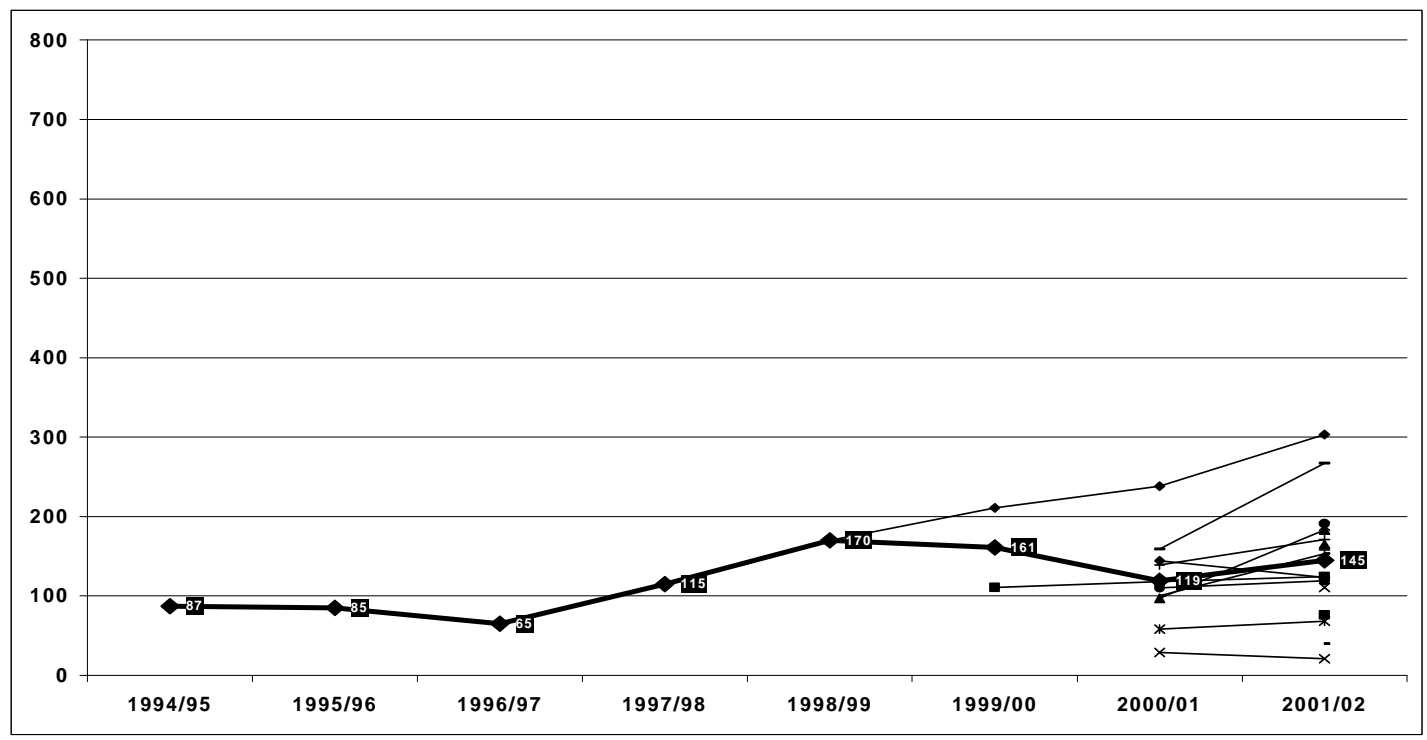

Quelle: FHR, IHS-Berechnungen 
Abbildung 18: FH-BewerberInnen nach wirtschaftlichen Studiengängen (ISCED-FBR 3: Social sciences, business and law), 1994/95 - 2001/02

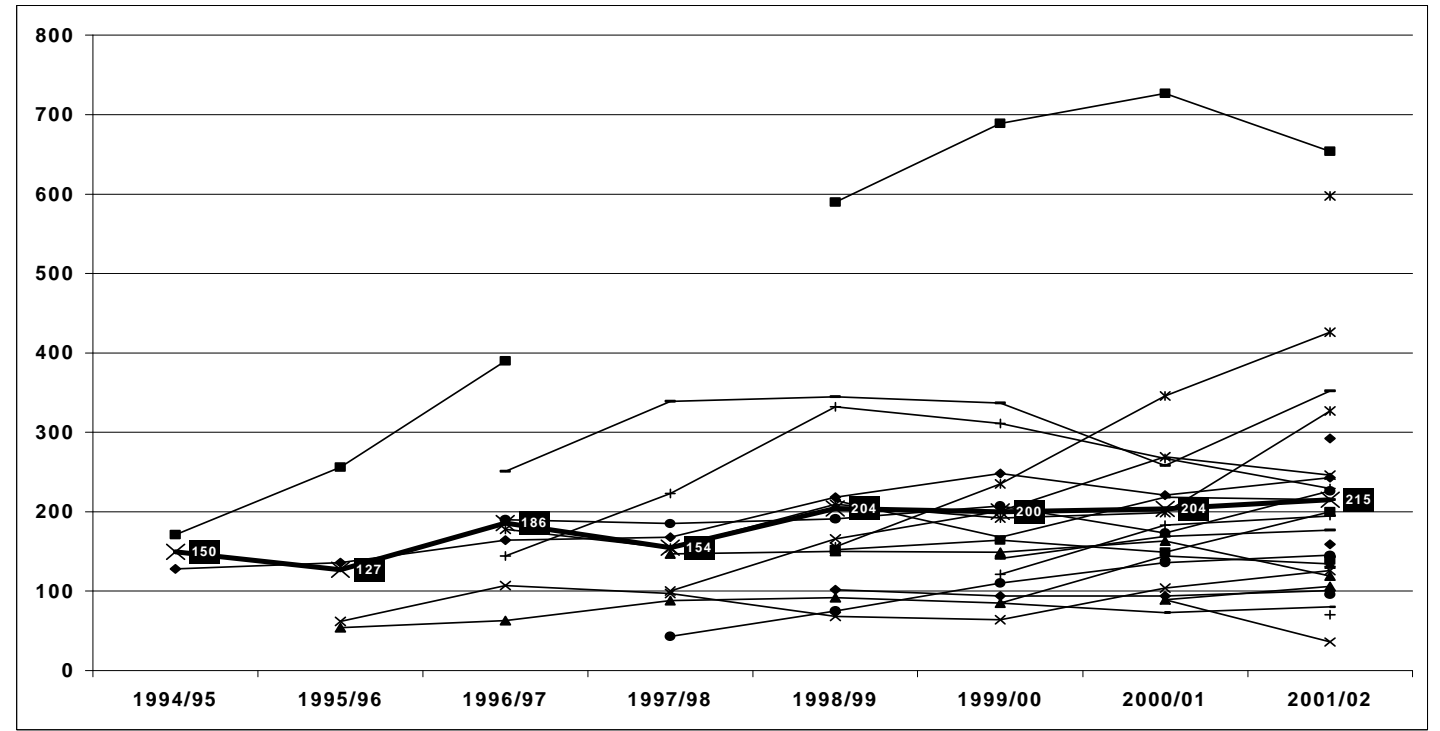

Quelle: FHR, IHS-Berechnungen

Abbildung 19: FH-BewerberInnen nach sonstigen Studiengängen (ISCED-FBR 0, 2, 7, 8, 9), 1994/95 - 2001/02

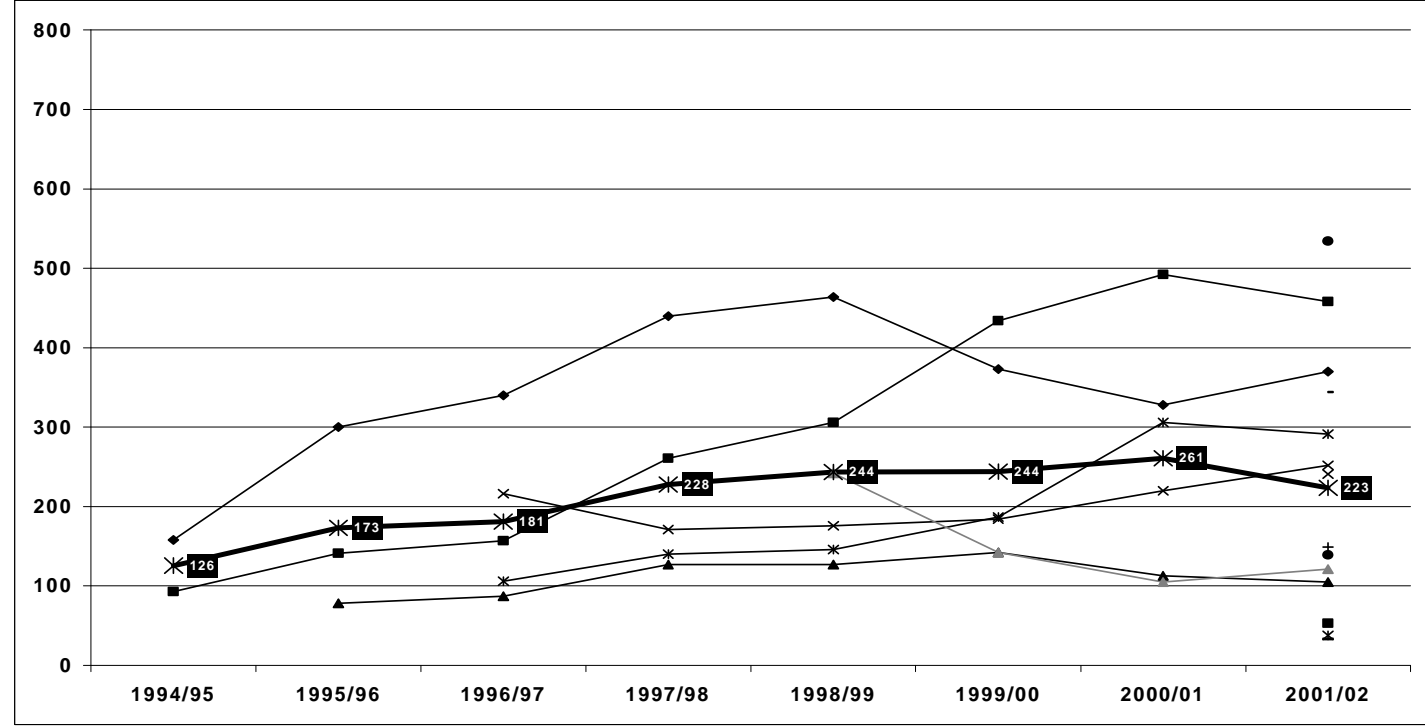

Isced 0: General Programmes, 2: Humanities and Arts, 7: Health and Welfare, 8: Services, 9: Not known/unspecified.

Quelle: FHR, IHS-Berechnungen 


\subsubsection{Vorbildung der FH-Studierenden und nicht-traditioneller Zugang zu FH-Studien}

Die Struktur der Studierenden nach ihrer Vorbildung (vgl. Abbildung 20) hat sich im gesamten Zeitraum sukzessive verändert. Der Anteil der AHS-AbsolventInnen ist gestiegen (von $30 \%$ auf $40 \%$ ), der von BHS-AbsolventInnen hat sich verringert (von $60 \%$ auf $50 \%$ ). Gesunken ist dabei vor allem der Anteil der AbsolventInnen von facheinschlägigen BHS (von $40 \%$ auf $30 \%$ ), er ist aber nach wie vor größer als der Anteil der nicht-einschlägigen BHSAbsolventInnen. Auch die Studierenden der zielgruppenspezifischen Lehrgänge, die in den letzten Jahren dazugekommen sind, konnten diesen Rückgang nicht wettmachen. AbsolventInnen der BHS machen den bei weitem größten Anteil der FH-Studierenden aus. Die Studierenden mit nicht-traditionellem Zugang (Nicht-Maturanten) haben ihren Anteil an allen Aufgenommenen verringert (um über ein Viertel von 11\% auf $8 \%$ ).

Die Struktur der Vorbildung von männlichen und weiblichen FH-Studierenden hat sich angenähert, indem die Veränderungen (Rückgang der BHS-AbsolventInnen und der nichttraditionellen Zugänge) vor allem bei den Männern wirksam geworden sind (vgl. Abbildung 21).

Abbildung 20: Struktur der FH-Studierenden nach Vorbildung, 1995/96 - 2001/02

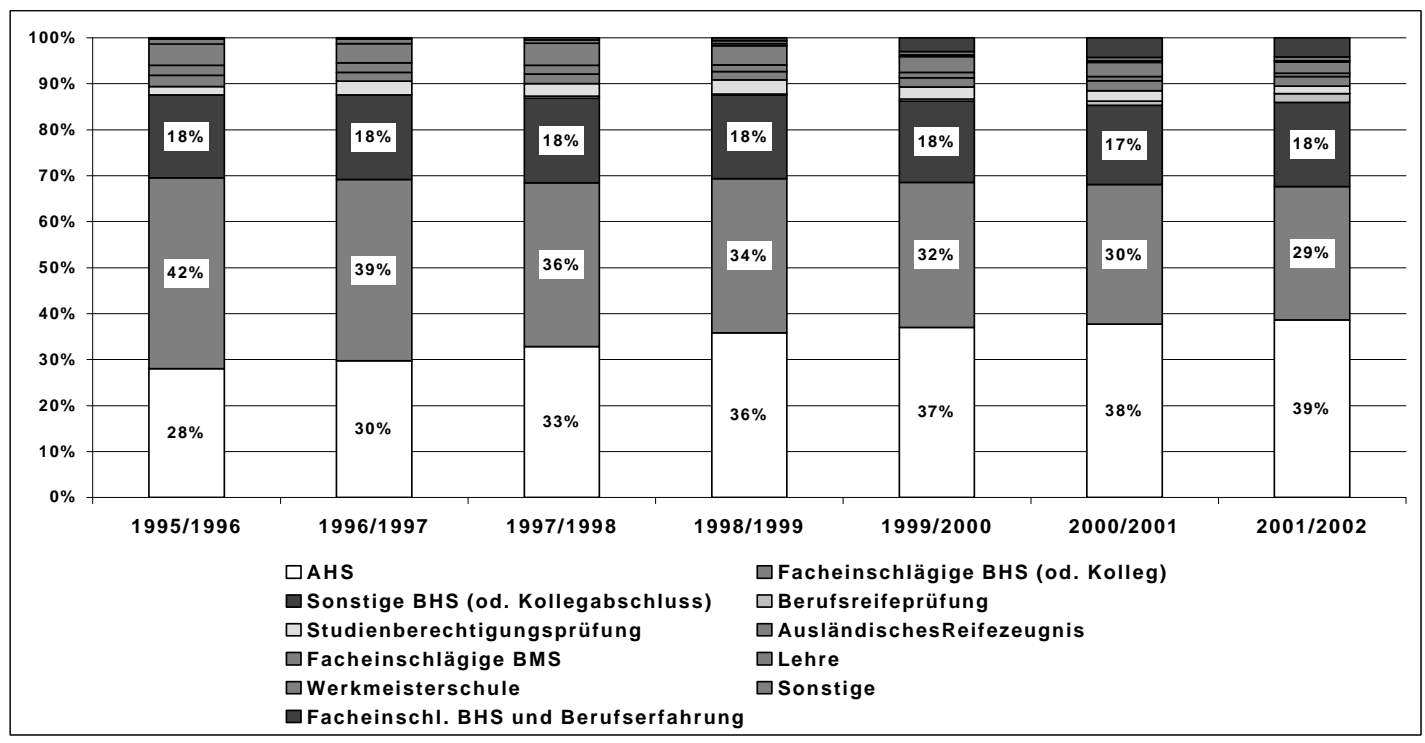

Quelle: FHR 


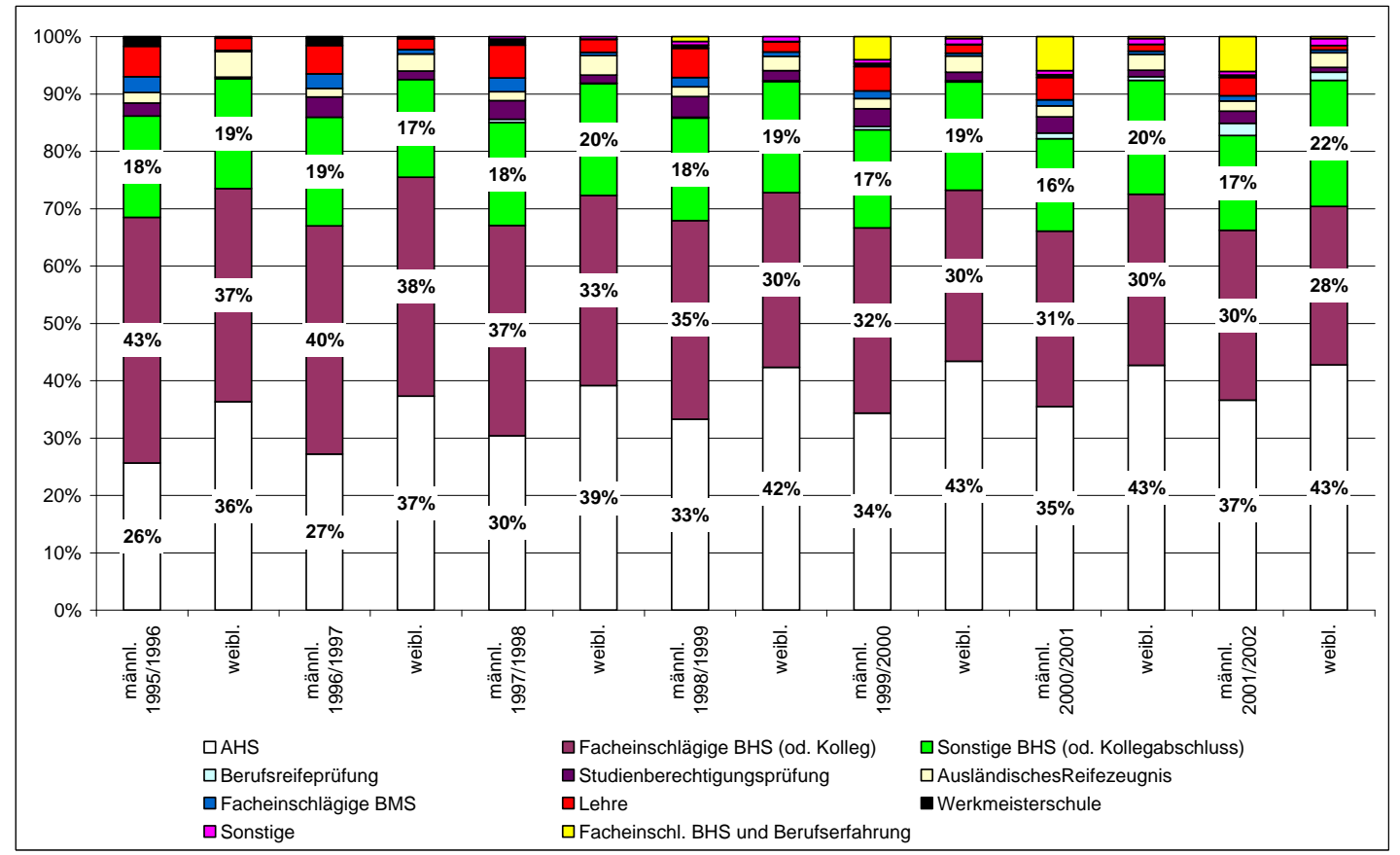

Quelle: FHR

Die Gruppe der Studierenden mit nicht-traditionellem Hochschulzugang setzt sich aus unterschiedlichen Kategorien zusammen, die auch in den Zielsetzungen des FHStG eine wichtige Rolle spielen (vgl. Abbildung 22). ${ }^{30}$ In die fünf Kategorien fallen einerseits zwei formalisierte Formen, die Studienberechtigungsprüfung und (seit 1997 mit steigender Bedeutung) die Berufsreifeprüfung, andererseits die nicht-formalisierten Zugänge aus anderen Ausbildungskategorien der Sekundarstufe (Lehrlingsausbildung, BMS und Werkmeisterschulen), die im Wege von individualisierten Anrechnungsmöglichkeiten erfolgen. Abgesehen vom geringen und seit 1998 sinkenden Anteil dieser nicht-traditionellen Zugänge zeigt sich eine deutliche Verschiebung von den nicht-formalisierten Zugängen zu den formalisierten Zugängen. Während erstere sich von fast $8 \%$ aller Zugänge auf 3,5\% halbiert haben, ist bei letzteren von 1,9\% auf 3,5\% fast eine Verdoppelung zu verzeichnen. Unter den AbsolventInnen liegt der Anteil mit nicht-traditionellem Zugang in den ersten Jahren niedriger, ab 1998/99 jedoch tendenziell höher als unter den Studierenden.

30 Siehe zu diesen Zielsetzungen näher das Kapitel 3.1.3. 


\section{Abbildung 22: Struktur der FH-Studierenden mit nicht-traditionellem Hochschulzugang nach Art der Vorbildung (Anteile an allen Studierenden), 1995/96 - 2001/02}

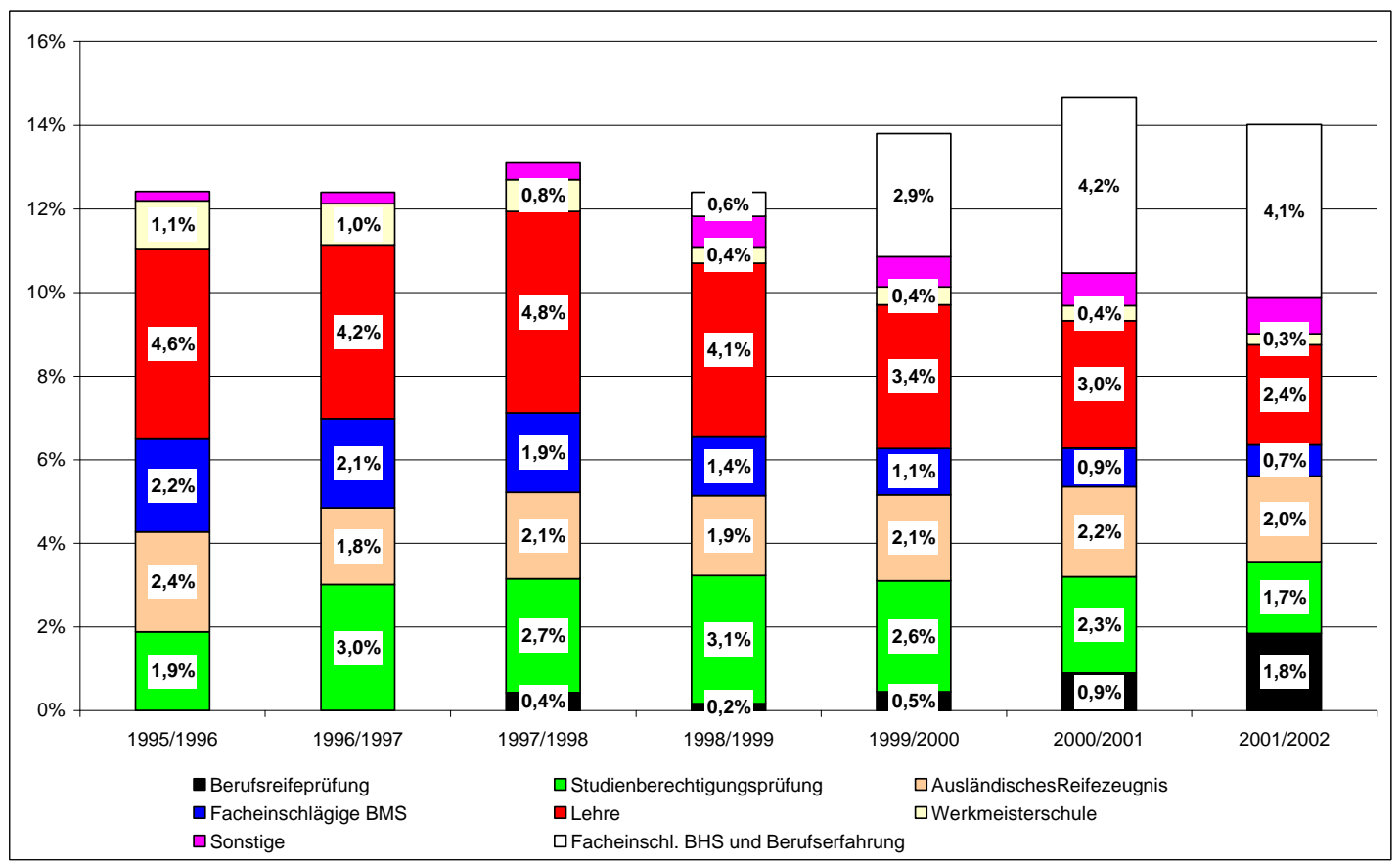

Quelle: FHR

Nach Studiengängen betrachtet (vgl. Abbildung 23) ist die Streubreite des Anteils von Studierenden mit nicht-traditionellem Hochschulzugang im Laufe der Entwicklung gestiegen und liegt mit wenigen Ausnahmen zwischen $0 \%$ und $20 \%$. In vier Studiengängen ist dieser Anteil deutlich höher (im letzten Jahr zwischen 30\% und 45\%). Nach Erhaltern und auch nach Studienorten und Bundesländern ist die Streubreite insgesamt geringer (Oberösterreich und Tirol bzw. bestimmte Standorte oder Erhalter in diesen beiden Bundesländern haben einen deutlich erhöhten Anteil an Studierenden mit nicht-traditionellem Hochschulzugang, dieser ist jedoch ebenfalls seit etwa 1997 sinkend). In berufsbegleitenden Studiengängen ist dieser Anteil gegenüber dem Durchschnitt erhöht, nicht-traditioneller Zugang gilt aber auch in diesen Studiengängen nur für eine Minderheit von etwas über $10 \%$, mit ebenfalls sinkender Tendenz. Der Frauenanteil liegt bei nicht-traditionellen Studierenden deutlich unter dem Gesamtdurchschnittsanteil, ist aber im Zeitverlauf gestiegen: im ersten Jahr lag dieser Anteil bei einem Viertel des durchschnittlichen Frauenanteils ( $8 \%$ vs. $25 \%$ ), ist aber bis 2001/02 auf die Hälfte gestiegen (17\% vs. 33\%). 


\section{Abbildung 23: Anteil der FH-Studierenden mit nicht-traditionellem Hochschulzugang nach Studiengängen, 1994/95 - 2001/02}

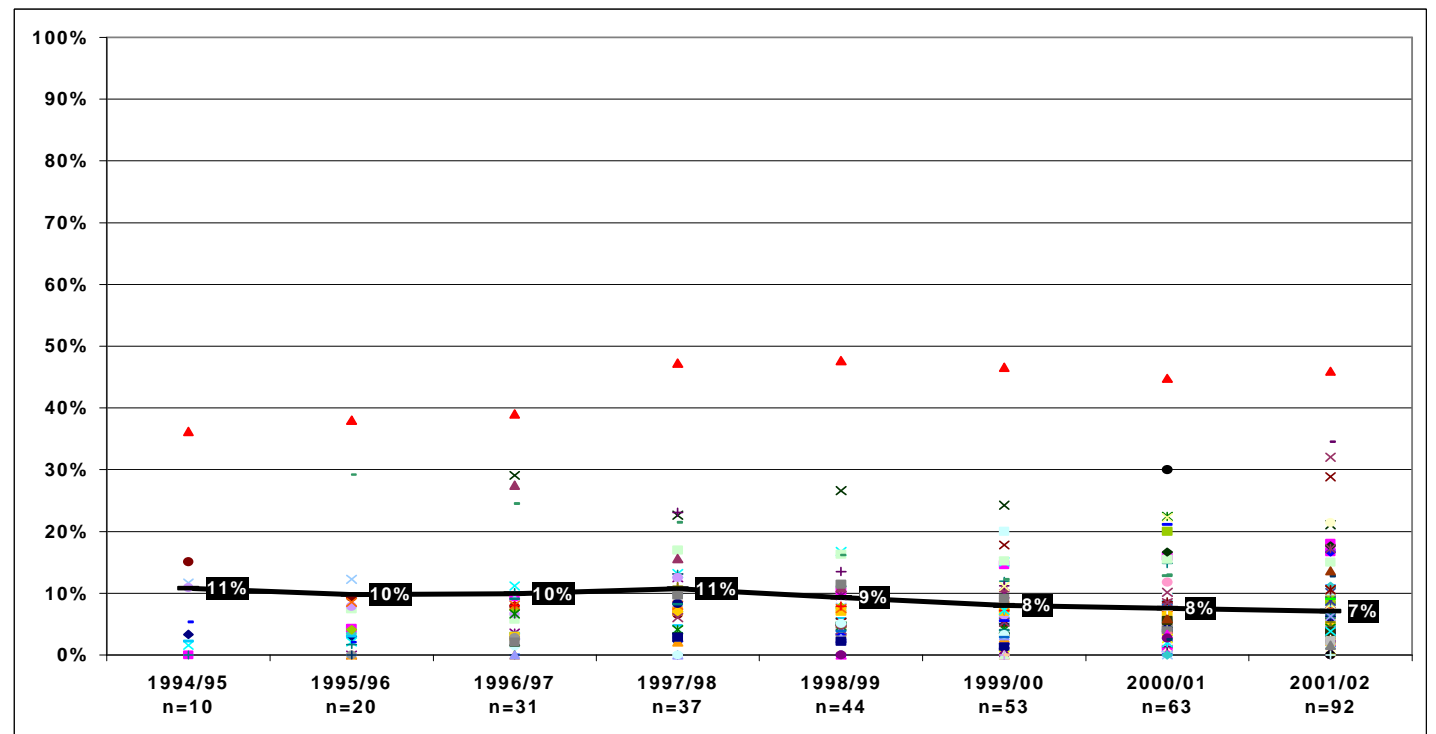

Die Darstellung verdeutlicht die Streuung des Gesamtsektors. Jeder Punkt entspricht einem Studiengang Quelle: FHR, IHS-Berechnungen

\subsubsection{Alter der FH-AnfängerInnen und der FH-Studierenden}

Die AnfängerInnen in Fachhochschul-Studiengängen sind im Durchschnitt um etwa 2 Jahre älter als die AnfängerInnen an den Universitäten, die männlichen FH-Anfänger sind etwas älter als die weiblichen (vgl. Abbildung 24). In den letzten Jahren, bei den Frauen etwas früher, verjüngen sich die durchschnittlichen FH-Anfängerlnnen tendenziell. Die Verteilung der Altersklassen zeigt auch die deutlich größere Altersspannweite der Neuzugänge (während an den Universitäten konstant etwa drei Viertel der Studienanfängerlnnen unter 20 Jahre alt sind, liegt dieser Anteil in den FH-Studiengängen unter der Hälfte, etwa 2/5 sind zwischen 20 und 25 Jahre alt und etwa $1 / 5$ älter).

Bei der Altersstruktur der Studierenden macht sich im Vergleich zum Universitätsbereich die kürzere Studiendauer der FH-Studiengänge (im Sinne einer kürzeren Verweildauer) bemerkbar. Die jüngeren Altersgruppen sind an FH-Studiengängen stärker vertreten, mit einem deutlichen Schwerpunkt bei den 21-25-Jährigen. 
Abbildung 24: Alterstruktur der inländischen Anfängerlnnen an wiss. Universitäten und FH-Studiengängen, WS 1990/91 (1994/95) - 2001/02

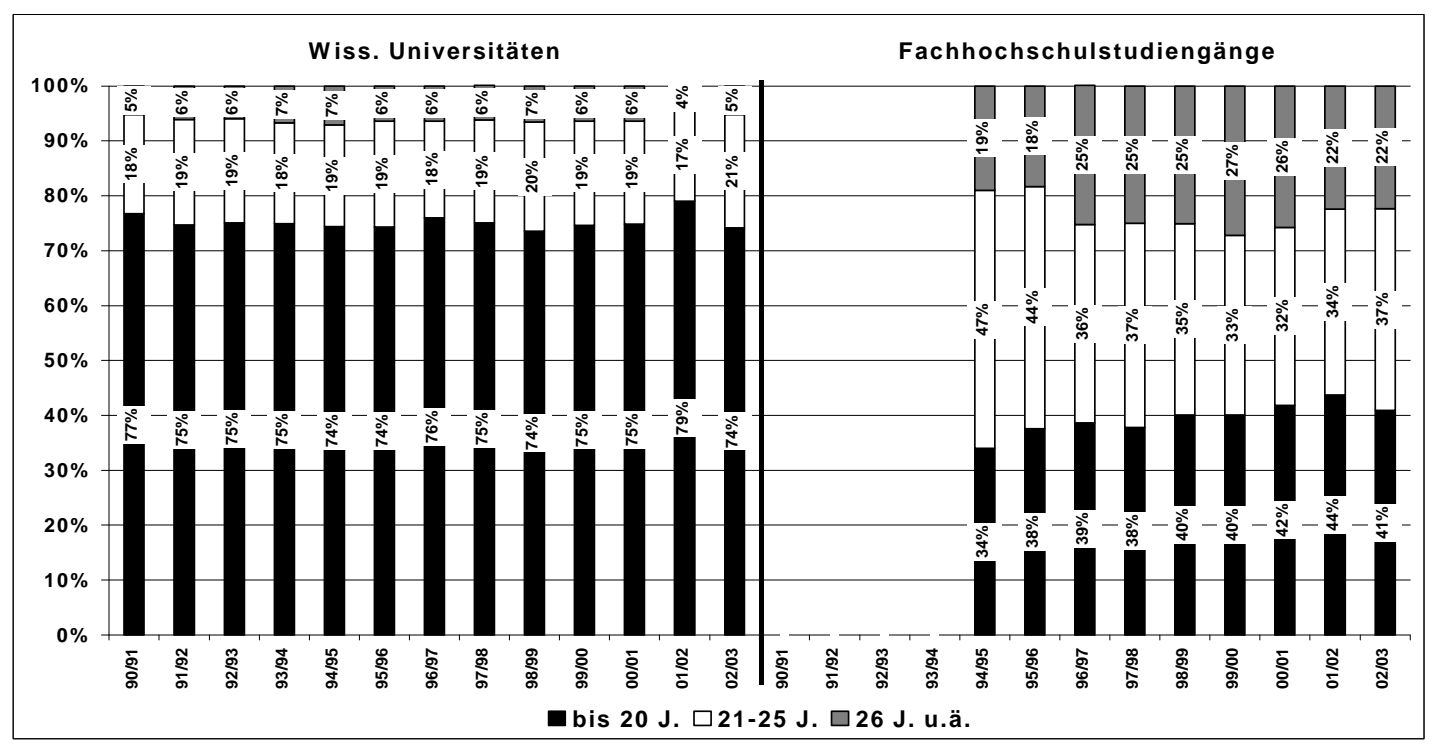

Quelle: Statistik Austria, IHS-Berechnungen

Abbildung 25: Entwicklung des durchschnittlichen Eintrittsalters inländischer StudienanfängerInnen an wiss. Universitäten und FH-Studiengängen nach Geschlecht, 1990/91 (1994/95) - 2001/02

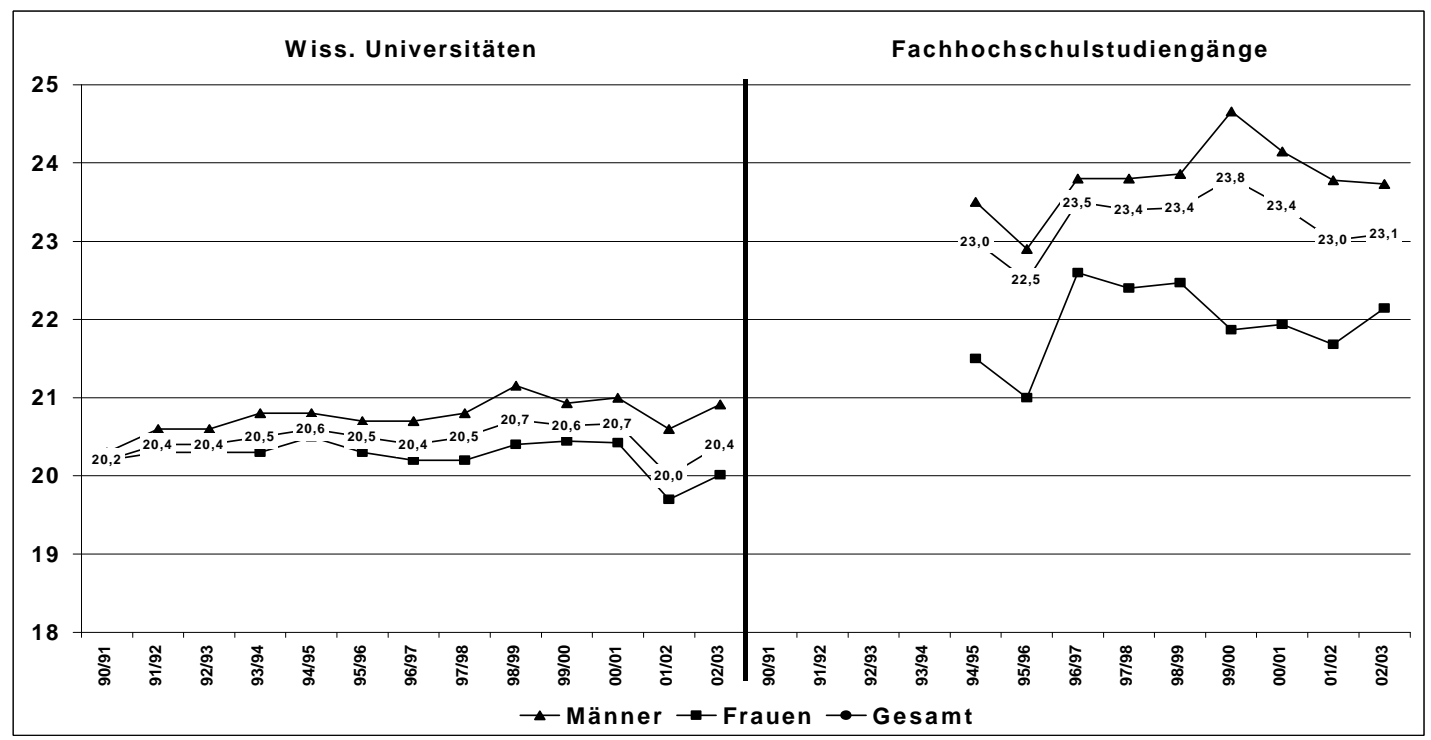

Quelle: Statistik Austria, IHS-Berechnungen 


\subsubsection{AbbrecherInnen}

Bezogen auf die Studierendenzahl beträgt die Quote der AbbrecherInnen in den letzten Jahren laut Datenbasis des FHR etwa 6-8\% pro Jahr, d.h. 6-8\% aller Studierenden brachen im Laufe des betreffenden Jahres ihr Studium ab. Länger laufende Studiengänge haben tendenziell eine niedrigere AbbrecherInnenquote als jüngere Studiengänge. Zudem besteht bei den älteren Studiengängen auch eine gewisse Konstanz der AbbrecherInnenquote, wenn man die letzten 2 Jahre miteinander vergleicht, bei den jüngeren ist das weniger der Fall.

Um grob die Erfolgsquote insgesamt abschätzen zu können, wird einem AbsolventInnenjahrgang die entsprechende Anfängerlnnenkohorte gegenübergestellt. Um die unterschiedlichen Studiendauern $\mathrm{zu}$ berücksichtigen, wird hilfsweise eine AnfängerInnenkohorte analog der Verteilung der Studiendauer des AbsolventInnenjahrgangs 2000/01 gebildet (siehe Tabelle 2). Aus dieser Berechnung ergibt sich, dass die Erfolgsquote bei rund $73 \%$ liegt oder mit anderen Worten $27 \%$ der entsprechenden AnfängerInnenkohorte haben ihr Studium (noch) nicht beendet.

Tabelle 2: Studienerfolgsquote an FH-Studiengängen STJ 2000/01; berechnet nach der Querschnittsmethode

\begin{tabular}{|c|c|c|c|c|c|c|c|}
\hline $\begin{array}{l}\text { Studiendauer } \\
\text { in Semestern }\end{array}$ & 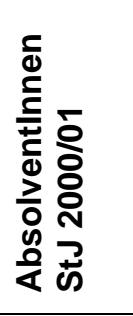 & 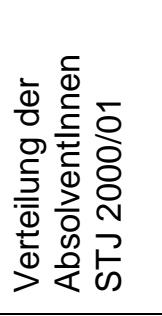 & 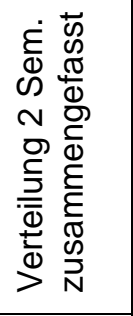 & 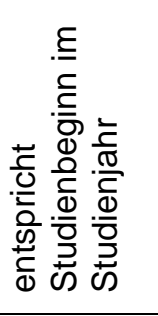 & 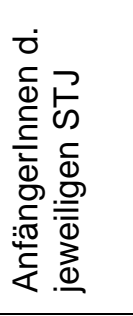 & 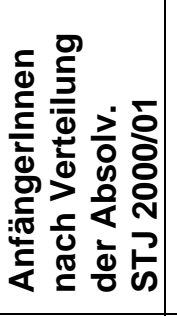 & $\begin{array}{l}0 \\
\frac{1}{0} \\
\frac{0}{0} \\
\frac{0}{0} \\
\frac{1}{4}\end{array}$ \\
\hline $\begin{array}{l}\text { AbsolventInnen } \\
\text { gesamt }\end{array}$ & 1.932 & $100 \%$ & $100 \%$ & & & 2.652 & $72,9 \%$ \\
\hline bis 5 Sem. & 11 & $1 \%$ & $7 \%$ & $1000 / 00$ & 20000 & 201 & \\
\hline 6 & 133 & $7 \%$ & $\% \%$ & $1998 / 99$ & 2.999 & $2<4$ & \\
\hline 7 & 185 & $10 \%$ & $88 \%$ & $1997 / 98$ & 2651 & 2.322 & \\
\hline 8 & 1.507 & $78 \%$ & $88 \%$ & $199 / / 98$ & 2.651 & $2.3<4$ & \\
\hline 9 & 52 & $3 \%$ & $5 \%$ & $1906 / 07$ & 2142 & 106 & \\
\hline 10 u. mehr Sem. & 44 & $2 \%$ & & & & & \\
\hline
\end{tabular}

Berechnung: Entsprechend der Verteilung der AbsolventInnen des STJ 2000/01 nach Studiendauer wird der Anteil der AnfängerInnen der jeweiligen AnfängerInnenkohorte berechnet. Diese aufsummiert ergeben die Zahl der Anfängerlnnen, von denen 1.932 oder rund 73\% im STJ 2000/01 ihr Studium abschlossen.

Quelle: Statistik Austria, BMBWK

Vergleicht man die AbbrecherInnenquoten der FHR-Datenbasis mit der Selektivität der Studiengänge bei der Aufnahme (Aufgenommene/BewerberInnen), so zeigt sich kein Zusammenhang. Die Auswahl erscheint also nicht treffsicher, um Abbrüche zu vermeiden (vgl. Abbildung 26); auch die angewendeten Auswahlverfahren stehen nicht im Zusammenhang mit der AbbrecherInnenquote. 
Abbildung 26: Anteil der Ausgeschiedenen an den FH-Studierenden sowie Anteil der aufgenommenen BewerberInnen nach Studiengängen, STJ 2001/02

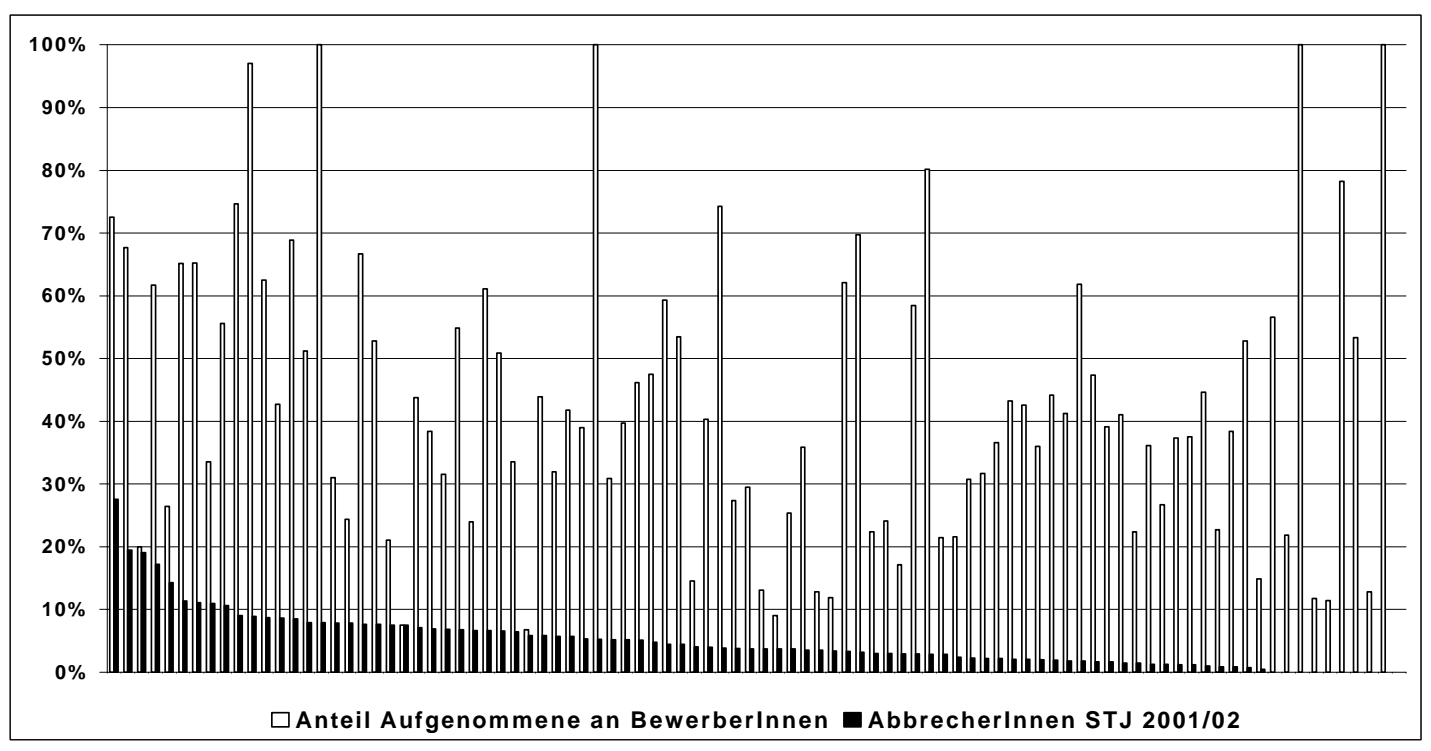

Quelle: FHR, IHS-Berechnungen

Die Abbrecherlnnenquote in berufsbegleitenden und zielgruppenspezifischen Studiengängen ist deutlich höher als in der Normalform (vgl. Abbildung 27). Auch differenziert nach den Fachbereichen zeigen sich tendenziell Unterschiede, mit erhöhten AbbrecherInnenquoten in den IKT-bezogenen und den ingenieurwissenschaftlichen Studiengängen (vgl. Abbildung 28). Frauen haben im Durchschnitt eine niedrigere Abbruchquote, dies gilt jedoch nicht für alle Kategorien von Studiengängen (in berufsbegleitenden und v.a. in den IKT-bezogenen Studiengängen ist die Abbruchquote der Frauen höher als die der Männer).

Die Abbrüche konzentrieren sich auf die frühen Phasen des Studiums (vgl. Abbildung 29). Über 60\% der AbbrecherInnen befanden sich im ersten Jahr, 85\% der AbbrecherInnen waren im ersten oder zweiten Jahr ihres FH-Studiums. 
Abbildung 27: Anteil der Ausgeschiedenen an den FH-Studierenden des Studienjahres 2000/01 nach Organisationsform der Studiengänge und Geschlecht

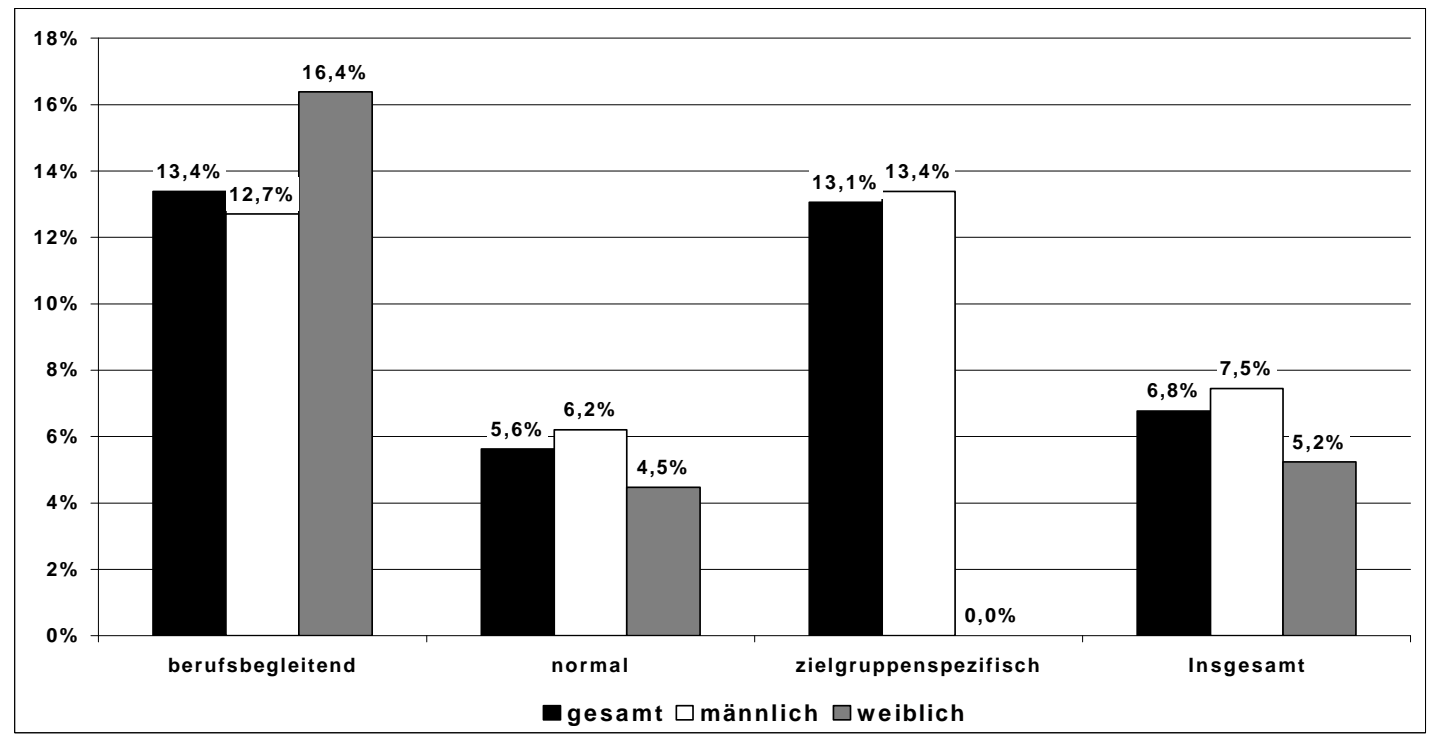

Quelle: FHR, IHS-Berechnungen

Abbildung 28: Anteil der Ausgeschiedenen an den FH-Studierenden des Studienjahres 2000/01 nach dem Fachbereich (ISCED-FBR) der Studiengänge und Geschlecht

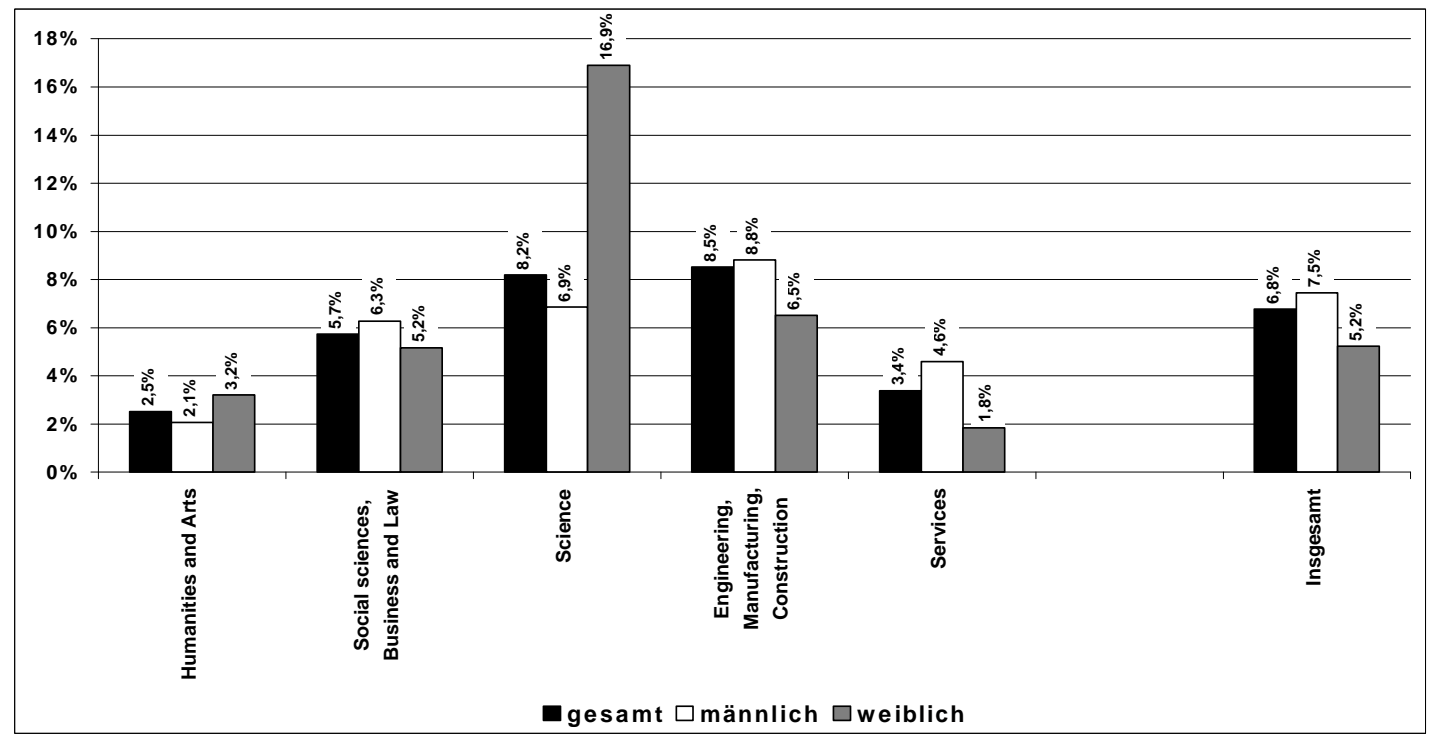

Quelle: FHR, IHS-Berechnungen 


\section{Abbildung 29: Verteilung der ausgeschiedenen FH-Studierenden nach Semestern und Geschlecht, STJ 2000/01}

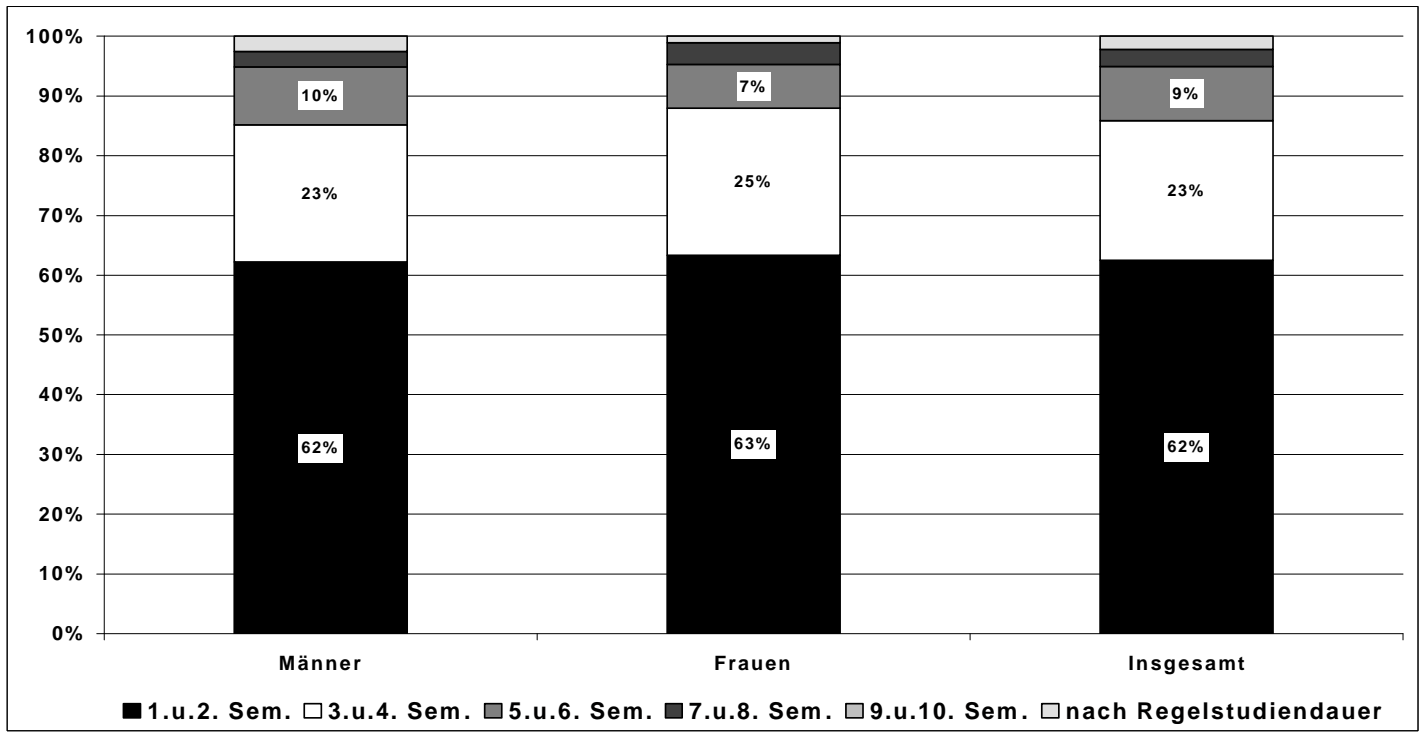

Quelle: FHR, IHS-Berechnungen

\subsubsection{Lehrkräfte nach „Köpfen“ und Vollzeitäquivalenten sowie Lehre nach Semesterwochenstunden}

Für die Weiterentwicklung des $\mathrm{FH}$-Sektors ist das Potential an Lehrkräften einer der zentralen Aspekte. Bei der Erfassung der Lehrkräfte im FH-Sektor ${ }^{31}$ gibt es jedoch aufgrund der Vielzahl an (flexiblen) Beschäftigungsformen eine Reihe von Unschärfen, die durch die vorliegende Untersuchung etwas vermindert werden konnten. Die in der Diskussion und Berichterstattung häufig verwendete Unterscheidung zwischen haupt- und nebenberuflichen Lehrkräften gibt nur einen sehr groben Aufschluss über das vorhandene personelle Potential auf den verschiedenen Betrachtungsebenen des FH-Sektors, vom Gesamtsektor über die Erhalter und Studienorte bis zu den Studiengängen. Daher haben wir in unseren Erhebungen versucht, mit vertretbarem Aufwand auch Vollzeitäquivalente und damit grobe Anhaltspunkte für das Beschäftigungsausmaß der Lehrkräfte zu erfassen ${ }^{32}$ Eine weitere Unschärfe betrifft die Verteilung der Tätigkeiten auf Lehre bzw. andere Tätigkeitsbereiche (F\&E, Dienstleistungen etc..). Dies ist wegen der Überschneidungen der Tätigkeiten wie auch der schwer abgrenzbaren Vor- und Nachbereitungszeiten der Lehrtätigkeit nicht ohne weiteres zu erfassen.

31 Wie in den anderen Bereichen des österreichischen Bildungswesens auch.

32 Ein Befund der Berechnungen besteht darin, dass die statistische Erfassung der hauptberuflichen Lehrkräfte aufgrund der Zählungsweise die Zahl der vollbeschäftigten Lehrkräfte etwas überschätzt, da teilweise Doppelzählungen vorkommen. Die Zahl der Vollzeitäquivalente an hauptberuflichen Lehrkräften ist also geringer als die Zahl der hauptberuflichen Lehrkräfte, die in der Statistik ausgewiesen ist. 
Die Gesamtzahl von Lehrkräften unabhängig von Form und Ausmaß ihrer Beschäftigung (also in der Definition nach „Köpfen") liegt $2000 / 01^{33}$ bei 3.500 (vgl. Tabelle 3). Pro Studiengang variiert diese Zahl in der Größenordnung zwischen 10 und 240, durchschnittlich sind etwa 50 Lehrpersonen in einem Studiengang tätig. Die Zahl der hauptberuflichen Lehrkräfte liegt in den einzelnen Studiengängen zwischen 0 und etwa 30, im Durchschnitt sind etwa 10 hauptberufliche Lehrkräfte in einem Studiengang tätig. Das Verhältnis von haupt- und nebenberuflichen Lehrkräften variiert außerordentlich stark und ist nicht unbedingt substitutiv (es gibt Studiengänge mit einer hohen Zahl an nebenberuflichen Lehrkräften über die ganze Verteilung der hauptberuflichen). Grob gesehen besteht ein Verhältnis in der personellen Kapazität von 1:4 bis 1:5 zwischen haupt- und nebenberuflichen Lehrpersonen. In neuen, im Aufbau befindlichen Studiengängen ist der Anteil nebenberuflicher Lehrkräfte deutlich geringer, es gibt einen größeren Sockel an hauptberuflichen Kräften (das Verhältnis liegt in den bis zu 2-jährigen Studiengängen bei 1:2 bei durchschnittlich 6 hauptberuflichen Lehrkräften). Eine weitere Aufstockung erfolgt dann in der Regel bei den nebenberuflichen Lehrkräften (in den 3-jährigen Studiengängen liegt das Verhältnis bei 1:4,5 bei der gleichen absoluten Durchschnittszahl von 6 hauptberuflichen Lehrkräften). Bei den vollausgebauten Studiengängen erhöht sich die Zahl der nebenberuflichen Kräfte im Durchschnitt stärker als die der hauptberuflichen (das Verhältnis beträgt 1:5,5 bei absolut 10 hauptberuflichen Lehrkräften).

In der Online-Befragung bei den Studiengängen wurden die Personalkapazitäten für das laufende Jahr (2001/02) nach Vollzeitäquivalenten (VZÄ), getrennt nach festangestellten und externen Lehrkräften, erhoben. ${ }^{34}$ Es ergeben sich im Durchschnitt pro Studiengang 5 - 6 hauptberufliche Vollzeitäquivalente und 10-11 nebenberufliche VZÄ (dies ergibt bei Berücksichtigung des Beschäftigungsausmaßes ein durchschnittliches Verhältnis von 1:2 zwischen haupt- und nebenberuflicher Personalkapazität). Aus dieser Relation kann man im Vergleich zu den Kopfzahlen der FHR-Zählung schließen, dass die hauptberuflichen Lehrkräfte nicht ihre ganze Arbeitszeit in einem Studiengang verausgaben, sondern eher nur die Hälfte; ein nebenberufliches Vollzeitäquivalent wird nach diesen Angaben im Durchschnitt etwa von vier Personen erfüllt (auf eine 40-Stundenwoche umgerechnet ist eine nebenberufliche Person im Durchschnitt 10 Stunden in einem FH-Studiengang tätig). Bei diesen Durchschnittswerten muss jedoch die große Variation im Auge behalten werden.

Hochgerechnet auf die Gesamtzahl der Studiengänge ergeben sich für 2000/01 aus der Durchschnittszahl von 16 VZÄ pro Studiengang etwa $1.000-1.100$ Vollzeitäquivalente an

33 Das ist das letzte Jahr, für das vollständige Informationen vorliegen, die Basis sind 67 Studiengänge; aufgrund der Steigerung der Zahl der Studiengänge dürfte diese Zahl beträchtlich gestiegen sein (eine Hochrechnung aus der Zahl der zusätzlichen Studiengänge für 2001/02 mit dem Durchschnittswert der 1 - 2 Jahre laufenden Studiengänge von 24 ergibt eine Zahl von etwa 650 zusätzlichen Lehrpersonen, also einen Zuwachs von fast $20 \%)$.

34 Aufgrund der Online-Erhebung liegen diese Informationen von 69 Studiengängen vor, diese repräsentieren $75 \%$ der Studierenden. 
Lehrkräften im Sektor und für 2001/02 zwischen 1.400-1.600 VZÄ, darunter 1/3 hauptberuflich, $2 / 3$ nebenberuflich beschäftigt (vgl. Tabelle 3 ). Die Fluktuation wird im Durchschnitt mit $5 \%$ bei den hauptberuflichen und mit etwa $10 \%$ bei den nebenberuflichen Kräften angegeben (deutlich erhöht ist diese nur in kleinen neuen Studiengängen, die sich in der Anfangsphase befinden). Im Durchschnitt würden aufgrund dieser Angaben im Jahr 35 hauptberufliche und 280 nebenberufliche Positionen nachbesetzt.

Pro Erhalter ${ }^{35}$ sind im Durchschnitt 200 Lehrpersonen tätig, darunter 33 hauptberuflich und 167 nebenberuflich (die Streubreite liegt zwischen 35 und 400 insgesamt bzw. zwischen 0 und 117 bei den hauptberuflichen Lehrpersonen). Nach den Angaben in unserer ErhalterBefragung hat ein Erhalter im Durchschnitt dauerhaft beschäftigtes Personal im Ausmaß von etwa 60 Vollzeitäquivalenten, das je zur Hälfte für administrative und zur Hälfte für wissenschaftliche und Lehrtätigkeiten eingesetzt wird. Für die Zahl an externen wissenschaftlichen MitarbeiterInnen kann aus der Hochrechung von den Angaben der Studiengänge darüber hinaus eine Zahl von 14-24 Vollzeitäquivalenten pro Erhalter geschätzt werden. Nach diesen Angaben beschäftigt im Durchschnitt ein Erhalter ein Stammpersonal von etwa 30 VZÄ für wissenschaftliche Tätigkeiten (das sich durch Teilzeitbeschäftigung auf eine etwas größere Zahl an Personen verteilen kann) und ein flexibles Personal in der Größenordnung von 20 VZÄ. Wenn man die 33 hauptberuflich in der Lehre tätigen Personen aus der Statistik nach „Köpfen“ analog zu den Studiengängen als halbe Vollzeitäquivalente rechnet, so wären im Durchschnitt etwa die Hälfte der von den Erhaltern angegebenen wissenschaftlichen Kräfte in der Lehre tätig, die andere Hälfte in anderen wissenschaftlichen Funktionen (F\&E etc.). Die Profile der Erhalter sind jedoch sehr unterschiedlich: Es kommen zwischen 0,4 und 2,0 wissenschaftliche Kräfte auf eine administrative Kraft. Auch das Ausmaß, in dem wissenschaftliche Kräfte mit Leitungsaufgaben betraut sind, variiert zwischen weniger als $10 \%$ und $50 \%$ (bei einem Durchschnitt von 28\%). Differenziert man nach dem (geografischen) Studienort, ${ }^{36}$ so reduziert sich das durchschnittliche Potential etwas (auf insgesamt 180 Lehrkräfte, darunter 29 hauptberufliche pro Studienort).

\footnotetext{
35 Es liegen in der statistischen Datenbasis des FHR Informationen über 18 Erhalter mit 67 Studiengängen vor.

36 Es liegen Informationen über 20 Studienorte mit 67 Studiengängen vor.
} 
Tabelle 3: Zusammenfassung der geschätzten (Durchschnitts-)Werte zum Lehrpersonal im FH-Sektor nach Erhaltern und Studiengängen, 2000/01 und 2001/02

\begin{tabular}{|c|c|c|c|}
\hline & $\begin{array}{l}\text { Köpfe gerundet } \\
(2000 / 01)\end{array}$ & $\begin{array}{c}\text { VZÄ 2000/01 } \\
\text { geschätzt } \\
\text { (67 Studiengänge, } \\
18 \text { Erhalter) }\end{array}$ & $\begin{array}{c}\text { VZÄ 2001/02 } \\
\text { geschätzt } \\
\text { (94 Studiengänge, } \\
19 \text { Erhalter) }\end{array}$ \\
\hline Sektor insgesamt & 3.500 & $1.000-1.100$ & $1.400-1.600$ \\
\hline Pro Erhalter & 200 & $55-61$ & $74-84$ \\
\hline Darunter hauptberuflich & 33 & $19-22$ & $25-30$ \\
\hline Darunter nebenberuflich & 167 & $37-41$ & $49-54$ \\
\hline Pro StG & 50 & $15-17$ & $15-17$ \\
\hline Darunter hauptberuflich & 10 & $5-6$ & $5-6$ \\
\hline Darunter nebenberuflich & 40 & $10-11$ & $10-11$ \\
\hline 1-2 Jahre laufende StG & 17 & $10-11$ & $10-11$ \\
\hline Darunter hauptberuflich & 6 & 3-4 & $3-4$ \\
\hline Darunter nebenberuflich & 11 & $6-7$ & $6-7$ \\
\hline 3 Jahre laufende StG & 34 & $9-11$ & $9-11$ \\
\hline Darunter hauptberuflich & 6 & $2-3$ & $2-3$ \\
\hline Darunter nebenberuflich & 28 & $7-8$ & $7-8$ \\
\hline 4 u.m. Jahre laufende StG & 65 & $22-24$ & $22-24$ \\
\hline Darunter hauptberuflich & 10 & $8-9$ & $8-9$ \\
\hline Darunter nebenberuflich & 55 & $14-15$ & $14-15$ \\
\hline
\end{tabular}

Quelle: FHR, Studiengangs-Befragung, Befragung der Erhalter, eigene Berechnungen.

Betrachtet man die Zusammensetzung der nebenberuflich Lehrenden nach ihrer hauptsächlichen Beschäftigung, so ist 2001/02 die Struktur nach den Erhaltern nicht sehr unterschiedlich. Im Durchschnitt liegt die Haupttätigkeit bei $46 \%$ in der Wirtschaft und bei $18 \%$ an den Universitäten. Abgesehen von einem großen Anteil „sonstiger Tätigkeiten“ (22\%) sind noch $9 \%$ der nebenberuflichen Lehrkräfte an Sekundarschulen beschäftigt. Vier große Erhalter und zwei kleinere Erhalter entsprechen in der Struktur ihrer nebenberuflichen Lehrkräfte dem Durchschnitt. Die übrigen können danach klassifiziert werden, ob sie verstärkt Lehrkräfte aus der Wirtschaft oder Lehrkräfte von den Universitäten rekrutieren bzw. ob sie andere Besonderheiten aufweisen. Dabei zeigen vier Erhalter einen deutlich erhöhten Anteil an Lehrkräften aus der Wirtschaft (darunter einer außerdem für LehrerInnen von Sekundarschulen), zwei Erhalter rekrutieren in erhöhtem Ausmaß Personal von den Universitäten, zwei haben ausgesprochen wenige Lehrkräfte von den Universitäten. Schließlich beschäftigen 2001/02 zwei Erhalter in ausgeprägtem Ausmaß Sekundarschullehrerlnnen und zwei weitere (darunter der oben erwähnte) in etwas weniger ausgeprägtem Ausmaß und ein Erhalter greift auf Lehrkräfte von anderen FachhochschulStudiengängen zurück.

Der Lehraufwand (gemessen in Semesterwochenstunden SWS) wird 2000/01 im Schnitt zu einem Drittel von hauptberuflichen Lehrkräften getragen. Gegenüber der Anfangsphase des FH-Sektors (1996/97 ist das erste Jahr, zu dem Daten vorliegen) ist dieser Anteil von einem Niveau von 20\% aus angestiegen, seit 1998/99 ist das Niveau stabil geblieben 
(möglicherweise wird die rasche Expansion 2001/02 zu einer Verringerung dieses Anteils führen). Nach Studiengängen variiert der Anteil hauptberuflicher Lehre zwischen 5\% und $90 \%$, wobei die Streubreite bis $75 \%$ ziemlich dicht besetzt ist. Die Zahl der Studiengänge mit hohen Anteilen hauptberuflicher Lehre (mehr als 60\%) ist im Laufe der letzten Jahre deutlich gestiegen, aber es gibt immer auch etliche Studiengänge mit sehr niedrigen Anteilen unter 10\% (10 von 67 Studiengängen des Jahres 2000/01), was jedenfalls nicht durch die Neugründungen erklärt werden kann.

In der Online-Befragung der Studiengänge wurde auch die Verteilung Semesterwochenstunden nach den verschiedenen Kategorien von Lehrkräften erhoben. Demnach werden im Durchschnitt 36\% der Semesterwochenstunden von hauptberuflichen Lehrkräften der Studiengänge bestritten, 34\% von nebenberuflichen Lehrkräften aus der Wirtschaft, $12 \%$ von Lehrkräften von Universitäten und $9 \%$ von Lehrkräften aus Sekundarschulen. Die Hälfte der Semesterwochenstunden wird von wissenschaftlich qualifizierten Lehrkräften (40\% Promotion, $9 \%$ Habilitation) bestritten, die andere Hälfte im Wesentlichen von Personen mit Hochschulabschluss. Betrachtet man die einzelnen Erhalter, so ist auch hier die Spannweite groß und es gibt einige, die über die Jahre sehr niedrige Anteile hauptberuflicher Lehre aufweisen.

In der folgenden Tabelle werden die personellen Potentiale der Fachhochschuleinrichtungen in den Bundesländern nach dem Profil von haupt- und nebenberuflicher Lehre nach Lehrpersonen und Semesterwochenstunden zusammengefasst (vgl. Tabelle 4). In vier Ländern ist der hauptberufliche Lehraufwand sehr hoch (V, K; 60 - 70\%) oder hoch (St, O; $40-50 \%)$, weitere drei Bundesländer liegen im Durchschnitt (B, N, S) und in zwei Ländern ist der hauptberufliche Anteil niedrig und sinkend (T, W; 10 - 20\%); die letzteren sind jene mit starkem Wachstum. 
Tabelle 4: Verteilung der Lehrkräfte und des Lehraufwandes (in SWS) nach hauptbzw. nebenberuflich Lehrenden, STJ 2000/01

\begin{tabular}{|l|c|c|c|c|}
\hline & $\begin{array}{c}\text { Hauptberufliche } \\
\text { Lehre (SWS) } \\
\text { sehr hoch } \\
\text { (60 bis 70\%) }\end{array}$ & $\begin{array}{c}\text { Hauptberufliche } \\
\text { Lehre (SWS) } \\
\text { hoch } \\
\text { (40 bis 50\%) }\end{array}$ & $\begin{array}{c}\text { Hauptberufliche } \\
\text { Lehre (SWS) } \\
\text { mittel } \\
(30 \text { bis }>35 \%)\end{array}$ & $\begin{array}{c}\text { Hauptberufliche } \\
\text { Lehre (SWS) } \\
\text { niedrig } \\
\text { (bis }>20 \%)\end{array}$ \\
\hline $\begin{array}{l}\text { Potential haupt- } \\
\text { beruflicher Lehr- } \\
\text { personen hoch } \\
(40 \% \text { bis }>50 \%)\end{array}$ & $\mathrm{K}, \mathrm{V}$ & & & \\
\hline $\begin{array}{l}\text { Potential haupt- } \\
\text { beruflicher Lehr- } \\
\text { personen mittel } \\
(15 \% \text { bis 25\%) }\end{array}$ & St, O, & S, N & \\
\hline $\begin{array}{l}\text { Potential haupt- } \\
\text { beruflicher Lehr- } \\
\text { personen niedrig } \\
\text { (bis 10\%) }\end{array}$ & & & $\mathrm{B}$ & $\mathrm{T}, \mathrm{W}$ \\
\hline
\end{tabular}

SWS: Semesterwochenstunden

Quelle: IHS

Der Frauenanteil unter den Lehrenden liegt mit 18\% deutlich unter dem Anteil weiblicher Studierender und dieser Durchschnitt hat sich im Laufe der Jahre nicht verändert. Die Streubreite liegt nach Studiengängen 2000/01 zwischen 0\% und 40\%, nach Erhaltern zwischen 5\% und 30\%, nach Studienorten zwischen 5\% und unter 40\% und nach Bundesländern zwischen 10\% und unter 30\% Frauenanteil. Die Mehrzahl der Länder bewegt sich 2000/01 zwischen 15\% und 20\% Frauenanteil, Vorarlberg und Oberösterreich liegen am unteren Ende und das Burgenland liegt herausragend höher. Im Zeitverlauf sind (mit Ausnahme von Oberösterreich) starke Schwankungen der Anteile ersichtlich, die nicht auf das Vorliegen von konsistenten Strategien hinweisen. 


\subsection{Der österreichische FH-Sektor im internationalen Vergleich}

Seit etwa drei Jahrzehnten werden die nationalen Bildungssysteme verstärkt in einen internationalen Kontext gestellt. Das politische Interesse an internationalen Vergleichen resultiert primär daraus, dass Bildung zunehmend als "Standortfaktor", als wesentliche Komponente des wirtschaftlichen Wettbewerbs betrachtet wird. Diese Sichtweise hat an Einfluss gewonnen, seit von Seite der Ökonomen auf den Zusammenhang zwischen Bildung und Wirtschaftswachstum hingewiesen wurde (vgl. auch den OECD-Bericht des BMU 1967). Es ist kein Zufall, dass sich seit den 1960iger Jahren internationale Wirtschaftsorganisationen wie die OECD in der Bildungspolitik engagierten; somit wurde die nationale Bildungspolitik aber auch verstärkt einem von außen kommenden Einfluss und innerhalb gewisser Grenzen ${ }^{37}$ - einer internationalen Evaluation ausgesetzt.

Die österreichische Fachhochschulpolitik ist ein sehr gutes Beispiel für Bedeutung und Einfluss des internationalen Umfelds auf die nationale Bildungspolitik. Die Entscheidung für die Gründung eines nicht-universitären Sektors Ende der 1980er Jahre war in mehrfacher Hinsicht mit dem bevorstehenden EU-Beitritt Österreichs verbunden, insbesondere mit der Frage, inwieweit die Strukturen des heimischen Postsekundarbereichs mit denen der EULänder kompatibel seien (Pechar 1990). Anfang der 1990er Jahre hat dann der OECDReview des österreichischen Postsekundarbereichs (BMWF/BMUK 1993) starken Einfluss auf die Kontroverse über die Architektur des neuen Sektors ausgeübt.

Für den vorliegenden Bericht wurden vier europäische Länder als Referenzsysteme gewählt. Zwei von innen - Deutschland und die Niederlande - haben seit langem ein Hochschulsystem mit einem Fachhochschulsektor, während in den beiden anderen Finnland und Schweiz - ein solcher Sektor erst im letzten Jahrzehnt etabliert worden ist. Im Folgenden werden die FH-Sektoren dieser vier Länder kurz charakterisiert und die wichtigsten, mit der hiesigen Situation kontrastierenden Elemente hervorgehoben.

37 Natürlich drücken z.B. die OECD-Länderprüfungen nur Empfehlungen aus; zumindest langfristig entfalten sie jedoch eine beträchtliche Wirkung und setzen die nationale Bildungspolitik einem erhöhten Legitimationsdruck aus. 


\subsubsection{Das FH-System in vier Vergleichsländern (Deutschland, Niederlande, Finnland, Schweiz)}

\section{Deutschland}

Der deutsche Fachhochschulsektor gilt als Beispiel eines schon lange bestehenden nichtuniversitären Hochschulsektors. Bei seiner Etablierung um 1970 beabsichtigte der Gesetzgeber, eine Alternative zum damaligen Universitätsstudium zu schaffen, insbesondere eine kürzere und mehr praxisbezogene Ausbildung. Die Aufgaben der deutschen Fachhochschule waren hauptsächlich auf die Lehre beschränkt; (angewandte) Forschung war eine Nebenaufgabe. In einem weiteren Reformschritt wurde in den 1970er Jahren in einigen der damaligen westdeutschen Bundesländer die Gesamthochschule eingeführt; an einer Gesamthochschule sollten wissenschaftliche und eher praxisbezogene Studiengänge gemeinsam in einer Institution vereinigt sein. Inzwischen wurden fast alle Gesamthochschulen zu Universitäten umbenannt bzw. ist eine solche Umbenennung noch im Gange. Fachhochschulen hingegen sind im ganzen Bundesgebiet eingeführt (mehr oder weniger ähnliche Hochschulen gab es auch in der damaligen DDR) und sie bestehen nach wie vor als eigenständiger Sektor. Im Hinblick auf Verbreitung und Lebensdauer ist die Fachhochschule also als erfolgreichere Innovation qualifizieren.

Deutsche Fachhochschulen unterscheiden sich stark hinsichtlich der Studien, die sie anbieten. Typisch sind aber breit gefächerte Angebote. Die Skala der Berufsfelder umfasst: Ingenieurwissenschaften, Rechts-, Sozial- und Wirtschaftswissenschaft, Verwaltung ${ }^{38}$, Sozialwesen, Religion, Gesundheit, Mathematik, Naturwissenschaft, Informatik, Kommunikation, Agrar-, Forst- und Ernährungswissenschaft, Kunst und Design.

1999/2000 gab es 182 Fachhochschulen (darunter 30 Verwaltungsfachhochschulen und 48 nichtstaatliche Fachhochschulen) von sehr unterschiedlicher Größe bezüglich ihrer Anzahl von Studierenden und ihrer Anzahl an Studienfächern. Insgesamt waren im Wintersemester 1999/2000 an deutschen Fachhochschulen 443.200 Studierende eingeschrieben, an Universitäten und Kunsthochschulen im gleichen Jahr 1,3 Millionen Studierende ${ }^{39}$, sodass ca. $75 \%$ der Studierenden im Universitätssektor, 25\% im Fachhochschulsektor zu finden waren. Von allen StudienanfängerInnen wählten etwa $32 \%$ ein FH-Studium. Fachhochschulstudierende konzentrieren sich fast ausschließlich (zu 92\%) in zwei Bereichen: in "Technik und Naturwissenschaften“ (46\%) und in „Wirtschaft und Recht“ (46\%). Das starke Gewicht des technischen Bereichs erklärt wohl auch den niedrigen Frauenanteil (37\%) an Fachhochschulen, der in den technischen und naturwissenschaftlichen Fächern

38 An besonderen, von den Ländern getragenen Verwaltungsfachhochschulen.

39 Fachhochschulen inklusive Verwaltungsfachhochschulen. In der Kategorie der Universitäten werden auch pädagogische und theologische Hochschulen mitgezählt (BMBF 2001). 
nur $19 \%$ beträgt, jedoch in den Wirtschaftswissenschaften 50\%, im Bereich Kunst $62 \%$ und in den Sprach- und Kulturwissenschaften $71 \%$ erreicht.

Aus der Zahl der Fachhochschulen und StudentInnen des WS 1999/2000 errechnet, lag die Durchschnittsgröße einer deutschen Fachhochschule bei ca. 2.440 Studierenden. Laut deutschem Wissenschaftsrat (WR 2002, 13) hatten im Jahr 1999 17\% der deutschen Fachhochschulen ${ }^{40}$ weniger als 1.000 Studierende und ebenfalls $17 \%$ zwischen 5.000 und 10.000 Studierende; 3 Fachhochschulen haben sogar über 10.000 StudentInnen. Der Großteil der Fachhochschulen (51\%) liegt im Größenbereich von 1.000 bis 5.000 Studierenden.

Zulassungsvoraussetzung für ein deutsches Fachhochschulstudium ist die Fachhochschulreife oder die allgemeine (bzw. fachgebundene) Hochschulreife. Die Bedeutung der Fachhochschulreife ist zurückgegangen, 1999 hatten bereits mehr als die Hälfte (53\%) der StudienanfängerInnen Abitur gegenüber 43\% mit Fachhochschulreife (WR 2002, 23). Darüber hinaus gibt es auch für Studieninteressierte mit beruflichen Qualifikationen die Möglichkeit, bei Erbringung der entsprechenden Aufnahmeerfordernisse zu einem Fachhochschulstudium zugelassen zu werden.

Die durchschnittliche Studiendauer (Fachstudiendauer) deutscher FachhochschulabsolventInnen lag 1999 bei 4,8 Jahren. (WR 2002, 219, Tabelle A19). Die deutsche Hochschulpolitik hat am Ziel einer Kürzung der Studiendauer festgehalten; die Einführung von Bachelor- und Masterstudiengängen im Rahmen des Bologna-Prozesses ist u.a. als erneuter Versuch zu sehen, eine kürzere Studiendauer zu erreichen. Bachelorstudiengänge sollen eine Dauer von mindestens 3, maximal 4 Jahren aufweisen, konsekutiv aufeinanderfolgende Bachelor- und Masterstudiengänge sollen zusammen eine Dauer von 5 Jahren nicht überschreiten (vgl. WR 2002, 102).

Zum Ausmaß des Studienabbruchs an Fachhochschulen gibt es neuere Daten aus einer

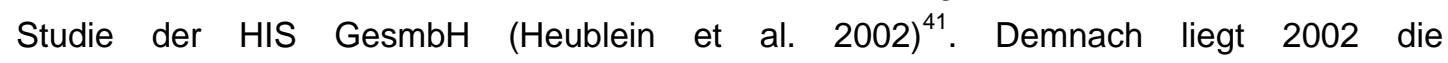
StudienabbrecherInnenquote an deutschen Fachhochschulen bei $22 \%$, d.h. von 100 StudienanfängerInnen (Erststudium) eines Jahrgangs brachen 22 das Studium ohne Studienabschluss ab. Gegenüber den neunziger Jahren hat sich diese Quote erhöht, sie lag 1992 bei 18-20\%. Bei Frauen ist die Studienabbruchquote deutlich geringer (16\%) als bei männlichen FH-Anfängern (25\%). Trotz der Erhöhung ist die AbbrecherInnenquote an Fachhochschulen nach wie vor niedriger als an Universitäten, wo sie 2002 bei $30 \%$ lag (Heublein et al. 2002, 22).

\footnotetext{
40 Nur HBFG-Hochschulen (d.s. die 115 Fachhochschulen, die im Anhang zum Hochschulbauförderungsgesetz enthalten sind); ohne Verwaltungsfachhochschulen.

${ }^{41} \mathrm{Vgl.} \mathrm{http://www.his.de/Abt2/Foerderung/pdf/Kia/kia200205.pdf.}$
} 
Angewandte Forschung gilt mittlerweile als eigenständige Aufgabe der Fachhochschulen und ist als solche in allen Ländergesetzen verankert. Der deutsche Wissenschaftsrat erachtet in seinen jüngsten Empfehlungen allerdings die weitere Stärkung von anwendungsorientierter Forschung und Entwicklung an Fachhochschulen als wesentlich (vgl. WR 2002, 129f.).

\section{Die Niederlande}

Das zweite Beispiel eines etablierten Fachhochschulsektors bieten die Niederlande. Im Zuge der Demokratisierungswelle wurde in der zweiten Hälfte der 1960er Jahre auch ein neuer Bereich des Hochschulsystems eingerichtet. Der größte Teil der neuen Fachhochschuleinrichtungen entstand aus berufsorientierten Schulen, die bis dahin Teil der Sekundarstufe waren.

Ein weiterer Entwicklungsschritt fand in den 1980er Jahren statt, als die zumeist kleinen und hochspezialisierten Schulen zu großen, viele Fachbereiche umfassenden regionalen Konglomeraten fusionierten. Der Prozess wurde 1983 mit drei Vorgaben gestartet (vgl. Goedegebuure/Meek 1992): eine wesentliche Vergrößerung der Institutionen (Minimum: 600 Studierende mit Ausnahme der Institutionen, die nur Volksschullehrerausbildung anbieten) mit vernünftiger Entfernung zwischen ihren Einheiten, eine größere Autonomie der Institutionen (aber mit einer gemeinsamen Leitung) und eine Konzentration der Ressourcen. Die Restrukturierung war auf einen Zeitraum von etwa $2^{1 / 1} / 2$ Jahren angelegt und mehr als $60 \%$ der bestehenden Institutionen waren davon betroffen. Bis 1987 hatten sich 314 von ursprünglich 348 Institutionen zu 51 neuen Hochschulen zusammengeschlossen, während 34 in ihrer ursprünglichen Form verblieben. Die Durchschnittsgröße lag dann bei fast 4.000 Studierenden (mit einer Spannweite von 300 bis fast 16.000, wobei der Großteil zwischen 600 und 8.000 Studierenden lag). Den hogescholen ${ }^{42}$ wurden auch Aufgaben wie Weiterbildung, Entwicklung der Profession und der Lehre, aber auch - und vor allem angewandte Forschung (insbesondere im technischen Bereich) zugeteilt. Dennoch blieb die praxisorientierte Lehre die Hauptaufgabe.

Nach den mehr oder weniger aufgezwungenen Fusionen der 1980er Jahre sind seit etwa 1990 auf freiwilliger Basis weitere Fusionen gefolgt, die teilweise zu sehr großen, regional oder gar überregional organisierten hogescholen mit mehreren Niederlassungen geführt haben. Die erforderliche Mindestgröße der Institutionen liegt derzeit bei 700 Studierenden. ${ }^{43}$ In der HBO-Statistik des niederländischen Bildungsministeriums (Ministerie van Onderwijs, Cultuur en Wetenschappen) werden für das Jahr 200056 Einrichtungen ausgewiesen, die

42 Diese Bezeichnung wurde anlässlich der Fusionen eingeführt. Was bis dahin wie in Deutschland technische hogescholen hieß, wurden zu technische universiteiten umbenannt.

43 Vgl. http://www.eurydice.org/Eurybase/frameset_eurybase.html. 
rund 200 Studienprogramme anbieten ${ }^{44}$. Im Durchschnitt hatten diese $56 \mathrm{FH}$-Einrichtungen über 5.400 StudentInnen.

In den 1990er Jahren waren die Fachhochschulen die Triebkraft der ,massification“ der niederländischen Hochschulen und im Jahr 2000 studierten bereits über 300.000 Studierende (inklusive Teilzeitstudierende), das sind 65\% aller StudentInnen, in diesem Sektor. Dieser umfasst fast alle Fachbereiche. Ein Drittel der FH-StudentInnen befindet sich im Jahr 2000 in wirtschaftlichen Programmen (1996 betrug deren Anteil noch 29\%). Auch die Lehrerlnnen (mit Ausnahme jener für die Sekundarstufe II) werden im Fachhochschulsektor (HBO-Sektor) ausgebildet; dieser Fachbereich $^{45}$ liegt größenmäßig nach den Wirtschaftsfächern an zweiter Stelle und ist annähernd gleich groß wie der Fachbereich Technik .

Die niederländischen Fachhochschulen bemühen sich in erster Linie darum, den AbsolventInnen des fünfjährigen Typs der Sekundarschule („HAVO“; typischer Abschluss mit 17 Jahren) berufsorientierte Hochschulstudien anzubieten. Der Zugang zu FH-Studien steht auch jenen offen, die den sechsjährigen Typ Sekundarschule („VWO“, primär Vorbereitung auf Universitätsstudien, typischer Abschluss mit 18 Jahren) absolviert oder ein Berufsbildungsdiplom erworben haben. Bei den Studierenden findet keine Eingangsselektion statt, außer in besonderen Fachbereichen wie den künstlerischen Studien.

Die vorgesehene Studiendauer der Fachhochschulprogramme beträgt 4 Jahre im Vollzeitstudium (168 Studienpunkte oder 240 ECTS). Die durchschnittliche Studienzeit betrug 1999/2000, mit geringen Schwankungen nach Fachbereichen, an die 4 Jahre (zwischen 3,9 Jahre im Fachbereich „Gesundheit“ und 4,3 Jahre im Fachbereich „Sprachen und Kultur"). Die Erfolgsquote, d.i. der Anteil der AnfängerInnen, der das Studium erfolgreich abschließt, ist in den letzten Jahren gesunken und liegt derzeit bei $65 \%$; dies bedeutet, dass 35\% der AnfängerInnen ohne Studienabschluss bleiben. ${ }^{46}$

Die niederländischen Fachhochschulinstitutionen finanzieren sich aus verschiedenen Quellen. Zusätzlich zur staatlichen Finanzierung haben sie vor allem Einnahmen aus Studiengebühren und Dienstleistungen für Externe. Die staatlichen Zuwendungen werden jährlich angepasst (hauptsächlich in Hinblick auf die aktuellen Studierendenzahlen)und den einzelnen Institutionen fast zur Gänze (92\%) als Globalsumme zugewiesen; die Institutionen entscheiden selbständig über die Allokation dieser Mittel. Infolge der überproportionalen Zunahme der anderen Einnahmequellen reduzierte sich der staatliche Finanzierungsbeitrag

\footnotetext{
44 Vgl. http://www.minocw.nl/english/education/higher.html.

45 In Tabelle 5 in der Kategorie „andere“.

$46 \mathrm{Vgl}$. http://www.minocw.nl/english oud/general/figures/pdf/2.6\%20Higher\%20Professional\%202002.pdf.
} 
pro Studierendem in den letzten Jahren, von $€$ 4.900.- im Jahr 1996 auf $€$ 4.500.- im Jahr $2000^{47}$.

\section{Finnland}

Die Entwicklung von Fachhochschuleinrichtungen („ammattikorkeakoulut") in den neunziger Jahren war in Finnland die späte Folge einer Beratung durch die OECD im Jahre 1981 (Opetusministeriö 2002). Gründe für die Schaffung eines Fachhochschulsektors waren vor allem die gestiegene Nachfrage nach Hochschulbildung und die Zersplitterung der Berufsbildung in mehrere hundert kleine, getrennte Einrichtungen; der Aspekt der Internationalisierung kam später noch hinzu. Der Prozess begann 1991; von zunächst 22 FH-Einrichtungen im Probebetrieb wurde der Sektor ab 1995 auf $31^{48}$ dauerhaft institutionalisierte und meist multidisziplinäre $\mathrm{FH}$-Einrichtungen erweitert. Daneben gibt es den Universitätssektor mit 20 Universitäten.

Im Jahr 2000 umfasste der finnische Fachhochschulsektor über 90.000 Studierende und zusätzlich 20.000 ,adult students“ (vgl. Opetusministeriö 2002). Die Durchschnittsgröße liegt bei 3.000 Studierenden (ohne „adult students") 49 pro Institution, mit Größen zwischen 600 und 6.500 Studierenden. 2000 gab es rund 24.000 Studienplätze für neue Studierende und 4.000 AnfängerInnenstudienplätze für „adult students“. Damit studieren rund $60 \%$ der AnfängerInnen im Fachhochschulsektor.

Der FH-Sektor muss in den kommenden Jahren den ganzen Zuwachs in der Studiennachfrage aufnehmen, im Universitätssektor soll keine Expansion stattfinden (vgl. Opetusministeriö 2002, 54). Die Zahl der Studienplätze für FH-StudienanfängerInnen soll bis 2004 auf 25.000 (jährlich + 250) erhöht werden, sodass zu diesem Zeitpunkt $70 \%$ des durchschnittlichen Altersjahrgangs der 19- bis 21-Jährigen ein Hochschulstudium an einer Universität oder Fachhochschule anfangen können (2001 waren es $66 \%$, wobei $37 \%$ der 19 bis 21-Jährigen ein Fachhochschulstudium anfangen). Die technischen (36\%) und wirtschaftlichen Studienprogramme $(27 \%)$ sowie die Studienprogramme im Fachbereich "Gesundheits- und Sozialdienste" (21\%) ziehen den größten Teil der FHStudienanfängerInnen an. Verglichen mit 1998 fingen 2001 um 21\% mehr Studierende ein FH-Studium an. Am meisten gewachsen sind die Fachbereiche „culture“ (+71\% Zuwachs an StudienanfängerInnen zwischen 1998 und 2001), „tourism and institutional management“ $(+52 \%)$ und „technology and communication“ (+43\%), wobei im letzteren der absolute Zuwachs an AnfängerInnen-Studienplätzen am größten war (+2.345).

47 Vgl. http://www.minocw.nl/english oud/general/figures/pdf/2.6\%20Higher\%20Professional\%202002.pdf.

48 Darunter eine Polizei-Fachhochschule und eine besondere Fachhochschule der Åland-Inseln.

49 Bzw. bei 3.700 Studierenden inkl. „adult students“. 
Im Jahr 2001 wurden von den Fachhochschulen insgesamt 149 verschiedene Studienprogramme angeboten. ${ }^{50}$ Die Studienprogramme sind auf Bachelor-Niveau eingestuft und umfassen in den meisten Fachbereichen 140 credit units (ca. 3,5 Jahre Vollzeit), in einigen Fachbereichen wie Technologie, Kultur und Bodenschätze 160 credit units (ca. 4 Jahre) oder gar 180 credit units wie in Musik (ca. 4,5 Jahre). Davon werden 20 credits für das Praktikum vergeben (im Fachbereich Gesundheitswesen 50 credits). Die Fachhochschulen müssen ihre Studien so gestalten, dass Studierende das entsprechende Studium in dieser Zeit abschließen können; die Studierenden müssen andererseits ihr Studium in dieser Zeitspanne plus maximal ein Jahr abschließen. Im Jahr 2000 lag die durchschnittliche Studiendauer der finnischen FH-AbsolventInnen bei 3,9 Jahren ${ }^{51}$ (vgl. Opetusministeriö 2002, 82f.).

Zugang zu FH-Studienprogrammen haben BewerberInnen mit Maturitätsexamen oder mit mindestens drei Jahren Berufsausbildung. Eine kürzere Berufsausbildung qualifiziert nur für einschlägige FH-Studien. Derzeit haben ca. $70 \%$ der FH-Studierenden ein Maturitätsexamen und ca. $30 \%$ eine berufliche Qualifikation (Opetusministeriö 2002, 74). Die Fachhochschuleinrichtungen können die Selektion der Bewerberlnnen selbst vornehmen.

Im Jahr 2000 brachen 6\% der FH-Studierenden ihr Studium ab, 1,2\% wechselten die Fachhochschule und 0,5\% wechselten innerhalb ihrer Fachhochschule in ein anderes Studienprogramm. Von den FH-Abbrecherlnnen gingen 13\% zum Studium an eine Universität und $9 \%$ an eine andere Bildungseinrichtung; $20 \%$ brachen ab, um eine Berufstätigkeit aufzunehmen. Die Drop-out-Raten variieren nach Fachbereichen ${ }^{52}$ und sind somit - dem Fächerspektrum entsprechend - an den einzelnen Fachhochschulen unterschiedlich hoch; sie werden auch als Kriterium bei der leistungsbezogenen Finanzierung der Institutionen berücksichtigt (Opetusministeriö 2002, 76f.).

Dem finnischen FH-Sektor wird ausdrücklich auch eine Funktion in der Regionalpolitik beigemessen. Ein Ziel bei der Errichtung von FH-Netzwerken war es, regionale Expertise zu fördern. Regionale und lokale Behörden gehören zu den „stakeholders“ beim „upgrading“ der Berufsbildungsschulen zu fachhochschulischen Einrichtungen. Die bevölkerungsstarken Regionen haben mehr Fachhochschuleinrichtungen, doch alle 19 Regionen des Landes haben mindestens eine FH-Einrichtung. In manchen Einrichtungen sind die früheren Schulen weitgehend fusioniert, in anderen Fällen ist die Verteilung auf mehrere Standorte ein

50 Ab dem Studienjahr 2002/03 hat das finnische Parlament außerdem Postgraduate-Studienprogramme im Umfang von 40 - 60 credits genehmigt (im Vollzeit- und Teilzeitbetrieb).

51 An den Universitäten, wo der größte Teil der Abschlüsse Master's-Degree-Abschlüsse sind, liegt die durchschnittliche Studiendauer bei 6,5 Jahren.

52 Unter dem Durchschnitt liegen die Drop-out-Quoten in den Studienprogrammen der Geisteswissenschaften (2,4\%), der Gesundheits- und Sozialdienste (4,5\%) und im Fachbereich „Kultur“ (4,7\%), über dem Durchschnitt in den Fachbereichen Technologie (6,9\%) und Wirtschaft $(6,8 \%)$. 
wichtiges Organisationsprinzip (in diesen Fällen muss vor Genehmigung der FH-Einrichtung eine gute Zusammenarbeit der Teilschulen nachgewiesen werden).

\section{Die Schweiz}

Fachhochschulen sind in der Schweiz ein neuer Sektor des Hochschulsystems, der seit 1997 die inzwischen 10 kantonalen Universitäten und die beiden vom Bund getragenen Technischen Hochschulen (ETH Zürich und ETH Lausanne) ergänzt. Der Bund hatte großes Gewicht darauf gelegt, dass die vielen, zum Teil sehr kleinen Studiengänge in sieben regionalen Institutionen zusammengelegt werden. Gleichzeitig bemühten sich auch die Kantone um eine Umgestaltung der berufsbildenden Schulen in ihren Gebieten zu Fachhochschulen (v.a. in den Bereichen Gesundheit, Sozialarbeit und Kunst). ${ }^{53}$ Die Schweizer Fachhochschulen wurden in Form eines „upgradings“ früher bestehender höherer Fachschulen (nach österreichischer Terminologie postsekundäre Bildungseinrichtungen) errichtet: 1997/88 erfolgte die Einrichtung von sieben regionalen Fachhochschulen, in denen über 70 Schulen zusammenarbeiten. Die sieben Institutionen sind über das ganze Land verteilt. Bei der ursprünglichen Genehmigung wurden häufig Auflagen gemacht hinsichtlich der Fusion von Partnerschulen, der Vertiefung von einzelnen Studiengängen und stellenweise auch Auflagen zum Abbau des Überangebots an Studienplätzen zugunsten neuer, zukunftsorientierter Programme.

Mit der Schaffung der Fachhochschulen wurde der Weg zu einem breiteren Angebot an tertiärer Hochschulbildung eröffnet. Darüber hinaus war die Errichtung des FH-Sektors mit drei wesentlichen politischen Steuerungsaufgaben verbunden (EFHK 2000, 3 -19): erstens einer Verstärkung der Bundeskompetenz und der interkantonalen Koordination der Hochschulpolitik, zweitens der Errichtung von entsprechenden Führungsstrukturen auf der institutionellen Ebene und drittens der Aufbau der F\&E-Funktion für den Sektor.

Die Eintrittsquote in den Fachhochschulsektor ist derzeit nicht ganz die Hälfte der universitären Eintrittsquote. 2001 traten 20,5\% der entsprechenden Altersgruppe in eine universitäre Hochschule ein. 9,7\% nahmen ein Fachhochschulstudium auf; aber in den nächsten Jahren wird ein weiterer Anstieg erwartet.

Im Wintersemester 1999/2000 befanden sich von den insgesamt über 112.000 Studierenden ca. $95.700(85 \%)$ an den 12 Universitäten und ca. 16.700 (15\%) an den sieben Fachhochschulen. Die Situation im Fachhochschulsektor änderte sich in dieser Aufbauphase noch sehr schnell. Im Wintersemester 2000 lag diese Zahl bereits bei 25.000, davon ein

53 Da es nur sieben regionale Fachhochschulen gibt, aber 26 Kantone, bedeutet dies, dass meistens mehrere Kantone zu einer Fachhochschule beitragen, was sich sowohl auf Zugang wie auch auf Finanzierung bezieht. 
Drittel Studienanfängerlnnen ${ }^{54}$. Ein weiterer Grund für die schnelle Änderung der Zahlen und Kategorien ist die Integration von kantonalen Hochschulen ins FH-System. Für das Jahr 2001 weist das Statistische Bundesamt bereits 29.400 Studierende (inkl. Studierende in Nachdiplomstudien) aus, von denen sich 1.200 an noch nicht integrierten Schulen finden. Die durchschnittliche Größe der Fachhochschulen liegt bei 4.000 Studierenden, wobei die Spannweite von 1.100 bis 7.100 Studierenden reicht.

Die normale Zugangsvoraussetzung für die Fachhochschule ist die Berufsmaturität, die während oder nach der Berufslehre erworben werden kann. Von StudienanfängerInnen mit allgemeinbildender Hochschulberechtigung wird mindestens ein Jahr einschlägige Berufspraxis verlangt. 2001 hatten 44\% der FH-Studierenden im 1. Semester eine Berufsmaturität, $18 \%$ eine gymnasiale Maturität. $38 \%$ hatten einen anderen Zulassungsausweis - dies wird aber bald nur noch die Ausnahme sein, da die Hauptzugangsberechtigung in Zukunft die Berufsmaturität sein wird. „Das Durchschnittsalter der Studierenden im ersten Jahr ist je nach Studienbereich unterschiedlich (unter 25 Jahre bei den Musikern und Schauspielern, rund 25 Jahre bei den Ingenieuren und Ökonomen und über 30 Jahre bei den Sozialarbeitern, Psychologen und beim Pflegepersonal)" (SKBF 2001). Im Durchschnitt sind die StudienanfängerInnen laut Statistischem Bundesamt 24 Jahre alt .

Fachhochschulen bieten betont praxisbezogene Studiengänge (ca. 220 Studiengänge) und Weiterbildungskurse auf Hochschulstufe (ca. 140 Nachdiplomstudien) an. Die FHStudienprogramme sind dreijährig (Vollzeitstudium) oder vierjährig (berufsbegleitende Ausbildung). Allfällige Praktika sind in dieser Zeit nicht eingeschlossen. Im Vergleich dazu beträgt die durchschnittliche Studiendauer an den universitären Hochschulen 6 Jahre bis zum Lizenziat bzw. Diplomabschluss. Die FH-AbsolventInnen waren $2001 \mathrm{im}$ Durchschnitt 26,6 Jahre alt, wobei es Schwankungen zwischen den Fachbereichen gibt (z.B. lag das Durchschnittsalter der AbsolventInnen im Fachbereich "Soziale Arbeit“ bei 35 Jahren, in „Technik“ bei 25 Jahren und in „Wirtschaft“ bei 27 Jahren). Das Durchschnittsalter beim Erwerb eines Nachdiploms liegt naturgemäß höher, 2001 waren es 35,4 Jahre..$^{55}$ „Auf dem Arbeitsmarkt beurteilt die Wirtschaft Abschlüsse einer Ingenieurschule oder einer Höheren Wirtschafts- und Verwaltungsschule schon seit geraumer Zeit als den universitären Hochschulen gleichwertig." (SKBF 2001)

Ursprünglich boten die Fachhochschulen Studienprogramme in Bereichen an, für die der Bund zuständig ist, d.h. Technologie, Ingenieurwesen, Architektur, Umweltwissenschaften, Wirtschaft und Verwaltung, Design, Gastgewerbe und Sport. Bis 2003 wird das Angebot auf die Bereiche in kantonaler Zuständigkeit erweitert sein, als Teil einer „Flurbereinigung“ im

\footnotetext{
54 Vgl. den OECD-Länderbericht der Schweiz (Confédération et les cantons de la Suisse 2002, 41).

55 Quelle: Statistisches Bundesamt.
} 
Hochschulsystem. Diese Bereiche sind Gesundheit, Sozialwesen, Kunst, Musik, Theater, Tanz, Lehrerausbildung, angewandte Psychologie und angewandte Linguistik. Trotz verschiedener Änderungen bleiben Technik (38\% der Studierenden) und Wirtschaft (37\% der Studierenden) die bei weitem größten Fachbereiche der schweizerischen Fachhochschulen. Der hohe Anteil des Fachbereichs Technik, wo der Frauenanteil sehr gering ist, ist für den niedrigen Frauenanteil im Gesamtsektor (29\%) hauptverantwortlich.

1995 wurde die Gesetzesgrundlage für den FH-Sektor mit folgender Intention geschaffen: „Fachhochschulen sollten mit einer gegenüber den bisherigen Höheren Fachschulen vermehrt wissenschafts- und praxisbezogenen Ausbildung den Kadernachwuchs sicherstellen und damit die Attraktivität des Wirtschaftsstandortes Schweiz erhöhen. Mit dem erweiterten Leistungsauftrag - Weiterbildung, anwendungsorientierte Forschung und Entwicklung, Zusammenarbeit sowie Dienstleistungen zugunsten Dritter - sollte zudem der Transfer von Ergebnissen der Grundlagenforschung in marktfähige Produkte beschleunigt und somit das Innovationspotential unserer Wirtschaft gestärkt werden." (SKBF 2001). Neben dem bildungspolitischen Ziel, die Berufsbildung in der Schweiz auf ein dem übrigen Europa angepasstes Niveau zu reorganisieren, sind die Fachhochschulen also insbesondere als wirtschaftspolitischer Impuls zu sehen, der nach der Ablehnung des EWR-Beitritts nötig wurde.

Für die Reformen des Hochschulsystems, die die Schweiz bis 2007 anstrebt, sollen trotz der schwierigen Lage des Staatsbudgets die öffentlichen Hochschulausgaben um jährlich 6\% gesteigert werden. Auf welche Weise dies erfolgen soll, ist noch nicht klar. Über die Finanzierung der Fachhochschulen in den Bereichen Technik, Wirtschaft und Design geben die jährlich abgegebenen harmonisierten Kosten- und Leistungsrechnungen umfassend Auskunft.

Die Kosten des Fachhochschulsektors belaufen sich im Jahr 2002 laut Bundesamt für Berufsbildung und Technologie auf ca. 740 Millionen SFR, davon zahlt der Bund 200 Millionen SFR. Laut Gesetz entfallen auf den Bund ein Drittel der Investitions- und Betriebskosten, den Rest zahlen die Kantone. Diese Regelung gilt aber nur für die Fachbereiche, die zur Bundeszuständigkeit gehören; die kantonalen Fachbereiche werden nur von den Kantonen finanziert. Fast $70 \%$ der Studierenden studieren in Bereichen, die in Bundesverantwortung sind; auch der noch verbleibende kantonale Teil wird allmählich unter Bundeszuständigkeit kommen. „Zudem wird den Universitätskantonen für alle Studierenden, die ihre ... Fachhochschule besuchen und deren Eltern in einem anderen Kanton steuerpflichtig sind, ein bestimmter Betrag vergütet." (SKBF 2001) 
Der Bundesbeitrag zu den Studienprogrammen wie auch für die Transfers zwischen den Fachhochschulregionen wird auf Basis einer Pauschale pro Studierenden errechnet. ${ }^{56}$ Diese Pauschale wurde anhand der Durchschnittskosten (festgestellt in einer Untersuchung der faktischen Ausgaben an den Fachhochschulen) vergleichbarer Studiengänge berechnet. Der Bund zahlt insgesamt 207 Millionen SFR als Pauschalbeträge direkt an die Fachhochschulen (2000 und 2001; SBKF 2001). Die Mehrzahl der Studierenden studiert in der eigenen Region, weshalb die Transferzahlungen insgesamt nicht so hoch sind (ca. 100 Mio. SFR).

Jede Fachhochschule entscheidet selbst über Studiengebühren. Diese belaufen sich im technischen und wirtschaftlichen Bereich in der Regel auf rund 500 SFR pro Semester (auch für Ausländer). In den anderen Bereichen können sie bis zu 1.500 - 2.000 SFR pro Semester betragen, abhängig vom Status der Schule.

\subsection{2 Österreichs FH-Sektor im internationalen Vergleich}

Betrachtet man Hochschulsysteme im Hinblick auf ihren Systemcharakter, so lassen sich internationale Vergleiche hinsichtlich Struktur und Prozesse dieser Systeme anstellen. Bei der Struktur geht es z.B. um das Fächerspektrum eines Hochschulsektors, seinen Stellenwert im Unterrichts- und Forschungssystem oder die Gesetzeslage. Prozessdaten beziehen sich u.a. auf AnfängerInnenzahlen (Input), StudentInnenzahlen, Drop-out-Quoten (Prozess in engerem Sinn) oder AbsolventInnen (Output).

Die Datenlage über die Fachhochschulsektoren der 4 betrachteten Länder ist allerdings sehr unterschiedlich. Daher ist es zwar möglich, eine Tabelle mit Kennzahlen zu den jeweiligen FH-Sektoren zu präsentieren (Tabelle 5), aber für Vergleiche sind die Zahlen mit großer Vorsicht zu interpretieren, denn Definitionen und Rahmenbedingungen sind in den Ländern nicht gleich. So ist es nicht völlig klar, was als Fachhochschule zu zählen ist: zählt man jede einzelne Niederlassung (diese ist im Zusammenhang mit Reisedistanzen, regionaler Einbindung usw. von Bedeutung) oder zählt man nur die Konglomerate/Erhalter (was für politische Fragestellungen relevanter ist)? Auch bei Studierendenzahlen definieren sich Inhalte und Zählweisen unterschiedlich, sie bedeuten in manchen Quellen Studienplätze, in anderen eingeschriebene Studierende, beinhalten teilweise "adult students“ oder Nachdiplomstudien, teilweise nicht. Der Begriff des Nachdiploms besteht z.B. nur in der Schweiz, obwohl in anderen Ländern die Fachhochschulen auch Aufgaben in der Weiterbildung wahrnehmen können. Wo ein Teilzeitstudium möglich ist, werden in den

56 Diese Pauschalbeträge pro Studierendem machen etwa $80 \%$ des Bundesbeitrages zum FH-Sektor aus. Weitere 10\% werden für die erweiterten Zielsetzungen zugeteilt (Wirtschaftsbindung, Forschung). Die letzten 10\% gehen an besondere Programme, z.B. die gemeinsamen Forschungsprogramme mit Universitäten/ETHs (OECD 2002). 
Quellen teilweise Kopfzahlen, teilweise Vollzeitäquivalente angegeben. Darüber hinaus gibt es noch die Problematik fehlender Daten und unterschiedlicher methodischer Zugänge z.B. für Eintrittsquoten, Drop-out-Quoten u.ä.

Alle betrachteten Länder haben gemeinsam, dass es bei der Schaffung des Sektors darum ging, eine neue Art Hochschulstudium einzuführen, das mehrere Aspekte berücksichtigen sollte. Die neuen Ausbildungsgänge sollten die wachsende Studiennachfrage befriedigen und den Erfordernissen der sich rasch wandelnden Volkswirtschaften („knowledge economies") entsprechen; sie sollten stärker praxisbezogen sein als herkömmliche universitäre Studiengänge ${ }^{57}$ und von kürzerer Dauer, weil die hohen Studierendenzahlen im Zusammenhang mit der langen Studiendauer an Universitäten entsprechend hohe Kosten für die Staatsbudgets bedeuten. Der österreichische Fachhochschulsektor unterscheidet sich in diesen Zielsetzungen nicht von dem der anderen vier Ländern; der Weg, der in Österreich beschritten wurde, ist teilweise ähnlich, teilweise auch unterschiedlich zu den Lösungen in den anderen Ländern.

57 Die Praxisbezogenheit der Universitäten wurden in allen betrachteten Ländern mehr oder weniger in Frage gestellt. 
Tabelle 5: $\quad$ Vergleichende Kennzahlen zu den Fachhochschulsektoren der Länder Deutschland, Schweiz, Finnland, Niederlande und Österreich

\begin{tabular}{|c|c|c|c|c|c|}
\hline & D & NL & CH & FIN & $\mathbf{A}$ \\
\hline Einwohnerlnnen in Mio. & 82 & 16 & 7,2 & 5,2 & 8 \\
\hline Fläche in 1.000 km$^{2}$ & 357 & 41,5 & 41,3 & 338 & 83,9 \\
\hline Einwohnerlnnen/ km² & 230 & 385,5 & 174 & 15 & 95 \\
\hline Gründung FH-Sektor & $\begin{array}{r}1970 \\
\text { ursprünglich } \\
\text { Upgrading, später } \\
\text { Neugründungen }\end{array}$ & $\begin{array}{r}\text { ca. } 1968 \\
\text { Upgrading } \\
\text { und Fusionen } \\
\text { (Mitte 1980er) }\end{array}$ & $\begin{array}{r}1997 \\
\begin{array}{r}\text { Upgrading und } \\
\text { Fusionen }\end{array}\end{array}$ & $\begin{array}{r}1992 \\
\text { Upgrading }\end{array}$ & $\begin{array}{r}1993 \\
\text { Neugründungen }\end{array}$ \\
\hline $\begin{array}{l}\text { Zahl der Fachhochschul- } \\
\text { Einrichtungen (FH) }\end{array}$ & 182 & 56 & 7 & 31 & 19 Erhalter \\
\hline $\begin{array}{l}\text { FH pro } 1 \text { Mio. } \\
\text { Einwohnerlnnen }\end{array}$ & 2,2 & 3,9 & 1,0 & 6,0 & 2,4 \\
\hline $\begin{array}{l}\text { Anzahl der Studiengänge/ } \\
\text { Programme }\end{array}$ & & 200 & 220 & 149 & 125 \\
\hline (Regel-)Studiendauer in J. & n.v. & $4 \mathrm{~J}$. & $\begin{array}{r}\text { Vollzeit } 3 \mathrm{~J} . \\
\text { berufsbegl. } 4 \mathrm{~J} .\end{array}$ & 3,5 bis $4,5 \mathrm{~J}$. & $4 \mathrm{~J}$. \\
\hline FH-Studierende in 1.000 & 443,2 & 307,0 & 29,4 & 114,1 & 16,8 \\
\hline Univ.-Studierende in $\mathbf{1 . 0 0 0}$ & $1.300,7$ & 164,7 & 99,6 & 157,2 & 157,5 \\
\hline$\%$ Studierende an FH & $25 \%$ & $65 \%$ & $23 \%$ & $42 \%$ & $10 \%$ \\
\hline \% Anfängerlnnen an FH & $32 \%$ & $71 \%$ & $32 \%$ & $60 \%$ & $24 \%$ \\
\hline $\begin{array}{l}\text { Durchschn. Zahl der } \\
\text { Studierenden an einer FH }\end{array}$ & 2.440 & 5.480 & 4.000 & 3.680 & 880 \\
\hline Frauenanteil an FH & $37 \%$ & um $50 \%$ & n.v. & $54 \%$ & $37 \%$ \\
\hline Abbrecherlnnen & $\begin{array}{r}\text { Abbruchquote } \\
22 \%\end{array}$ & $\begin{array}{l}65 \% \text { Erfolgsquote } \\
35 \% \text { Erfolglose }\end{array}$ & n.v. & $\begin{array}{l}\text { 6\% der Stud. } \\
\text { p.a. }\end{array}$ & $\begin{array}{r}6,7 \% \text { der Stud. } \\
\text { p.a. }\end{array}$ \\
\hline \multicolumn{6}{|l|}{ Fachbereiche (Stud.): } \\
\hline \% Technik, Naturwiss. & $46 \%$ & $18 \%$ & $38 \%$ & $35 \%$ & $47 \%$ \\
\hline$\%$ Wirtschaft, Recht & $46 \%$ & $33 \%$ & $37 \%$ & $30 \%$ & $41 \%$ \\
\hline$\%$ Gesundheit, Soziales & $0 \%$ & $23 \%$ & $8 \%$ & $22 \%$ & $1 \%$ \\
\hline$\%$ Kunst, Design & $3 \%$ & $5 \%$ & $14 \%$ & $0 \%$ & $0 \%$ \\
\hline$\%$ andere & $5 \%$ & $21 \%$ & $3 \%$ & $13 \%$ & $10 \%$ \\
\hline Weiterbildung an FH & ja & ja & ja & ja & tw. \\
\hline $\begin{array}{l}\text { Vorgabe einer „kritischen } \\
\text { Größe“? }\end{array}$ & n.v. & $\begin{array}{r}700 \text { für } \\
\text { Finanzierung }\end{array}$ & n.v. & n.v. & 1.000 \\
\hline Hauptfunktionen einer FH & $\begin{array}{l}\text { Lehre, } \\
\text { wenig F\&E }\end{array}$ & $\begin{array}{l}\text { Lehre, v.a. } \\
\text { Bachelor, } \\
\text { wenig F\&E }\end{array}$ & $\begin{array}{l}\text { Lehre, Weiter- } \\
\text { bild., F\&E, } \\
\text { Dienstleistung }\end{array}$ & $\begin{array}{l}\text { Lehre, F\&E, } \\
\text { regionale } \\
\text { Innovation }\end{array}$ & $\begin{array}{l}\text { Lehre, ange- } \\
\text { wandte F\&E, } \\
\text { Dienstleistung }\end{array}$ \\
\hline
\end{tabular}

n.v.: nicht verfügbar

Quellen:

Deutschland: Daten für WS 1999/2000, inkl. 30 Verwaltungsfachhochschulen, BMBF 2001, Heublein et al. 2002.

Niederlande: Daten für das Jahr 2000/01, Studierendenzahlen inkl. Gasthörerlnnen und externe Studierende, inkl.

Teilzeitstudierende, Ministerie van Onderwijs, Cultuur en Wetenschappen 2002.

Finnland: Daten für das Jahr 2000, Studierende inkl. 20.000 „adult students“, Opetusministeriö 2002.

Schweiz: Daten für das Jahr 2001, Studierende inkl. Nachdiplomstudien, Bundesamt für Statistik.

Österreich: Vorläufige Daten für WS 2002/03 (Abbrecherlnnen 2000/01, Fachbereiche 2001/02), BMBWK, FHR,

Statistik Austria. 
Die deutlichste Ähnlichkeit der fünf Länder ist, dass sich der neue Hochschulsektor auf praxisbezogene Lehre konzentrierte und die Verbindung zur regionalen Wirtschaft stärkte, einerseits in Hinblick auf den Arbeitsmarkt für AbsolventInnen, andererseits in Hinblick auf seine Aktivitäten in den Bereichen Forschung und Entwicklung, Innovation und Dienstleistungen.

Mit Finnland und der Schweiz gehört der österreichische Fachhochschulsektor mit zu den jüngeren Fachhochschulsystemen. Alle drei sind in den neunziger Jahren des 20. Jahrhunderts etabliert worden, im Gegensatz zu Deutschland und den Niederlanden. Einzigartig ist in Österreich, dass die FH-Einrichtungen nicht direkt auf bereits bestehenden berufsbildenden Schulen aufbauten, sondern Neugründungen waren. Dies hatte den Vorteil, dass der neue Sektor nicht schon bestehenden Einrichtungen Rechnung tragen musste, die institutionell und kulturell dem Sekundarunterricht verbunden waren. Daher war z.B. auch eine Fusionswelle von kleinen, spezialisierten Schulen wie in den Niederlanden nicht nötig. Zugleich war es möglicherweise auch ein gewisser Nachteil, nicht an etablierte Erfolge bestehender Bildungseinrichtungen anknüpfen zu können, wie es in der Schweiz anscheinend sehr gut gelang (z.B. in Hinblick auf die bereits etablierte Akzeptanz der AbsolventInnen auf dem Arbeitsmarkt, vgl. das Zitat aus SKBF 2001). Die Fachhochschuleinrichtungen in Österreich haben ihre eigene „Nische“ im Hochschulsystem entwickeln müssen und diese Entwicklung ist auch in quantitativer Hinsicht noch nicht abgeschlossen. Zum einen ist das geringe Alter des FH-Sektors ein Grund dafür, dass derzeit nicht mehr als 10\% der österreichischen Studierenden im Fachhochschulsektor studieren; die Neugründung an Stelle eines „upgradings“ bestehender Einrichtungen ist ein weiterer Grund. Die finnische Entwicklung (Zuwachs auf über 40\% Studierendenanteil in 10 Jahren) als auch die schweizerische (23\% Studierendenanteil in nur 5 Jahren) zeigen, dass unter den entsprechenden Umständen ein rasches Wachstum möglich ist. Ein Vergleich mit Deutschland und den Niederlanden zeigt andererseits, dass es keinen „natürlichen Anteil“ von FH-Studierenden im Hochschulbereich gibt. In Deutschland wählt ein Drittel der Neuzugänge FH-Studiengänge, in den Niederlanden sind es fast zwei Drittel. In Österreich hat man also einen großen Gestaltungsspielraum für die weitere Entwicklung des Sektors.

Die Größe des FH-Sektors im Hochschulsystem ist eng verknüpft mit dem Fächerspektrum ${ }^{58}$, innerhalb dessen die Studierenden an Fachhochschulen wählen können (so auch Huisman/Kaiser 2001). Das Fächerspektrum ist in Österreich wie in Deutschland relativ eng, die Studienprogramme sind überwiegend in technisch-naturwissenschaftlichen und wirtschaftlichen Gebieten angesiedelt. In der Schweiz ist neben diesen beiden Gebieten auch Kunst und Design stark vertreten; in Finnland Gesundheit und Sozialwesen sowie ein

58 Die Zahl der Studienprogramme ist ein weniger geeigneter Indikator, da sie zu sehr von nationalen Regelungen abhängig ist. 
hoher Prozentsatz bei „andere“59. Auch in den Niederlanden findet man einen hohen Prozentsatz „andere“ (21\%), neben einer relativ ausgeglichenen Verteilung der übrigen Fachgebiete. Dies ist darauf zurückzuführen, dass der Großteil der Lehrerlnnenausbildung im niederländischen Fachhochschulsektor angesiedelt ist. Gerade die Niederlande haben ja auch den höchsten Anteil von Studierenden im FH-Sektor. Der Zusammenhang scheint deutlich: um so breiter das Fächerspektrum, das im FH-Sektor angesiedelt ist, desto höher ist der Studierendenanteil, der im FH-Sektor zu realisieren ist.

Darüber hinaus korreliert das Fächerspektrum auch mit dem Frauenanteil im Fachhochschulsektor: Technik und Naturwissenschaften ziehen bekanntlich weniger Frauen an als z.B. Gesundheit, Sozialwesen, Kunst und Design. Länder, in denen technische und Naturwissenschaften den größten Anteil unter den Studienprogrammen haben, weisen daher die niedrigsten Frauenquoten auf (Deutschland, Österreich und insbesondere die Schweiz).

Auch geografische Aspekte können bei Entscheidungen über Studierendenquoten an Fachhochschulen, über die Zahl der Standorte oder der Erhalter eine Rolle spielen. Im Vergleich mit drei der anderen vier Länder hat Österreich eine geringe Bevölkerungsdichte (um 95 Einwohnerlnnen pro $\mathrm{km}^{2}$; nur Finnland hat mit 15 Einwohnerlnnen pro $\mathrm{km}^{2}$ eine geringere Dichte). Um zumutbare Reisedistanzen für Studierende zu gewährleisten, können in diesen beiden Ländern mehr Fachhochschuleinrichtungen pro (Million) EinwohnerInnen erwartet werden. Wie aus Tabelle 5 ersichtlich, hat zwar Finnland mit 6,0 die meisten Fachhochschuleinrichtungen pro Million Einwohnerlnnen, aber die dichtbevölkerten Niederlande kommen mit etwa 3,9 an zweiter Stelle. Österreich und Deutschland haben etwa die gleiche FH-Dichte (2,4 und 2,2 pro Million EinwohnerInnen), aber eine sehr unterschiedliche Bevölkerungsdichte. Damit liegt nahe, dass institutionelle und politische Erwägungen für diesen Zusammenhang wichtiger sind als die Geografie, das Alter des FHSektors u.ä. Dies zeigt sich auch an den unterschiedlichen Durchschnittsgrößen der Fachhochschuleinrichtungen, wobei Österreich den bei weitem kleinsten Wert hat. Hier liegt die durchschnittliche Größe bei rund 880 Studierenden, in den vier anderen Ländern ist sie um das Drei- bis Sechsfache höher.

Der Regionalbezug einer Fachhochschuleinrichtung war in allen hier betrachteten Ländern Teil der politischen Ziele bei der Etablierung des Sektors. Nur in den Niederlanden hat es losgelöst von regionalen Erwägungen - bei der Fusionspolitik in den Jahren 1983 bis 1987 eine deutliche Vorgabe hinsichtlich der kritischen Größe der einzelnen Fachhochschuleinrichtungen gegeben.

59 Unter denen vor allem den Studienprogrammen im Bereich „culture“ große Bedeutung zukommt - die Zahl der Studienplätze für Neueintretende hatte in diesem Fachbereich zwischen 1998 und 2001 mit 71 \% den größten relativen Zuwachs (vgl. Opetusministeriö 2002, 55). 
Eine weitere Besonderheit in Österreich gibt es hinsichtlich der institutionellen Ausgestaltung, da die Fachhochschul-Studiengänge zwar privatrechtlich organisiert sind, bei den Erhaltern jedoch die öffentliche Hand stark präsent ist. Die privatrechtliche Form hätte wie in Finnland - eine direktere Anbindung an die regionale private Wirtschaft ermöglicht.

In Deutschland und in den Niederlanden gibt es einen Unterschied zwischen einer „allgemeinen Hochschulreife" und der Fachhochschulreife (in den Niederlanden bezogen auf die Type des Sekundarunterrichts, s.o.). In der Schweiz repräsentiert die Berufsmaturität die normale Zugangsvoraussetzung $\mathrm{zu}$ den Fachhochschulen, Studienwerbern mit allgemeinbildender Maturität brauchen mindestens ein Jahr einschlägige Berufspraxis. Auch Finnland hat für den FH-Sektor andere Zulassungsvoraussetzungen als für die Universitäten (obwohl hier eher eine Gleichwertigkeit gesucht wurde, mit Berufsbildung als Alternative für die allgemeine, auch für ein Universitätsstudium qualifizierende Matura). Das österreichische Modell weist Besonderheiten auf, liegt aber nicht außerhalb des europäischen Spektrums.

Die Regelstudiendauer ist in den verschiedenen Ländern unterschiedlich hoch. In der Schweiz sind die Programme 3- oder 4jährig, je nachdem, ob es sich um ein Vollzeitstudium oder ein berufsbegleitendes Studienprogramm handelt. In den Niederlanden haben die Fachhochschulstudien im Prinzip alle die gleiche Studiendauer von 4 Jahren Vollzeitstudium. Die Variation der Studiendauer zwischen den unterschiedlichen Fachbereichen ist in anderen Ländern erheblich größer (vgl. Huisman/ Kaiser 2001), wie z.B. in Finnland, wo FHProgramme im allgemeinen zwischen 140 und 180 credits umfassen und damit zwischen 3,5 und 4,5 Jahre dauern. Immerhin sollen die Abschlüsse in den jeweiligen Ländern alle zu Diplomen auf dem Niveau eines Bachelors führen. In Österreich haben 92\% der Studiengänge eine Regelstudienzeit von 8 Semestern, unabhängig davon, ob es sich um ein berufsbegleitenden Studiengang oder um die Normalform handelt. ${ }^{60}$

Daten über Prozesse und Effekte von Lehren und Lernen sind nur schwer verfügbar und ihre Vergleichbarkeit ist problematisch. Gerade bei Aussagen zum Ausmaß von Studienerfolg oder Studienabbruch sind die Probleme der Vergleichbarkeit am größten, da es große Unterschiede in den Erhebungsmethoden relevanter Daten und den Berechnungsmethoden für die betreffenden Quoten gibt. Laut Meldungen der Fachhochschul-Studiengänge brechen im österreichischen $\mathrm{FH}$-Sektor jährlich $6,7 \%$ der Studierenden ihr $\mathrm{FH}$-Studium ab. Im finnischen Fachhochschulsektor liegt der Anteil der jährlichen Studienabbrecherlnnen unter den $\mathrm{FH}$-Studierenden in einer vergleichbaren Größenordnung (6\%). An niederländischen Fachhochschulen ist liegt die Erfolgsquote der FH-Anfängerlnnen bei $65 \%$, was bedeutet, dass zum Beobachtungszeitpunkt 35\% keinen Studienabschluss erreicht haben (aber das Studium nicht definitiv „abgebrochen“ haben müssen, sondern auch weiter oder anderswo

60 Bei den restlichen 7 Studiengängen beträgt die Regelstudienzeit in 3 Studiengängen 7 Semester und in 4 zielgruppenspezifischen Studiengängen 6 Semester . 
studieren können). Für den deutschen Fachhochschulsektor gibt es Ergebnisse einer HISStudie, wonach für 2002 von 100 StudienanfängerInnen an Fachhochschulen 22 das Studium ohne Studienabschluss beenden, also die Studienabbruchquote im Fachhochschulsektor $22 \%$ beträgt.

Ebenso schlecht ist es um die Vergleichbarkeit der Daten bestellt, wenn es um die Qualität des „Outputs“ der Fachhochschulen geht. Deutlich ist bei den betrachteten Ländern, dass HochschulabsolventInnen bessere Chancen auf dem Arbeitsmarkt haben als Personen ohne Hochschulbildung. Schwieriger gestaltet sich der Versuch, die Arbeitsmarktchancen von Universitäts- und FH-AbsolventInnen $\mathrm{zu}$ vergleichen. $\mathrm{Zu}$ den methodisch bedingten Problemen bei der Vergleichbarkeit kommen noch Differenzen in den Fachbereichen (Huisman/Kaiser 2001). In Deutschland und in den Niederlanden scheinen die Unterschiede zwischen Universitäts- und FH-AbsolventInnen auf dem Arbeitsmarkt nicht sehr groß zu sein. Von Finnland, der Schweiz und Österreich ist bekannt, dass die FH-AbsolventInnen auf dem Arbeitsmarkt gut aufgenommen werden.

Zusammenfassend kann man aus diesem Überblick den Schluss ziehen, dass es im Ausland kein "Standard-Modell“ für Fachhochschulen gibt, sondern dass in den einzelnen Ländern ein Spektrum an verschiedenen Möglichkeiten realisiert wurde. Elemente dieses Spektrums können für die weitere Entwicklung des noch immer im Aufbau befindlichen österreichischen FH-Sektors interessant sein. Entscheidend für die zukünftige Positionierung des FH-Sektors im österreichischen Hochschulsystem werden die politischen Präferenzen und Weichenstellungen sein. 


\section{Stellenwert des FH-Sektors in der Bildungs- und Hochschullandschaft}

Die Untersuchung des Stellenwertes des FH-Sektors konzentriert sich schwerpunktmäßig auf den Bereich des Hochschulsystems. ${ }^{61}$ Die Einbindung in das Gesamtsystem wird vor allem unter den Gesichtspunkten der Durchlässigkeit und der Veränderungen im allgemeinen bildungspolitischen Umfeld betrachtet. In diesem Zusammenhang sind vor allem die Auswirkungen der europäischen Integration (Stichworte: Hochschulraum, Forschungsraum, „offene Koordinierung“ der Bildungspolitik) und die neue Positionierung der Bildungs- und Hochschulpolitik (Stichworte: Innovation und Wissensgesellschaft, lebensbegleitendes Lernen) zu berücksichtigen.

Die Einschätzung des Stellenwertes des FH-Sektors umfasst die folgenden Aspekte:

a. Diversifikation und Profilierung. Damit ist die Positionierung als praxisbezogenes Ausbildungsangebot auf Hochschulniveau zur Diversifikation der tertiären Ebene und zur Entlastung und Ergänzung des Hochschulbereiches (das spezifische Profil im Vergleich zu den Universitäten) angesprochen. Das spezifische Profil ist vor allem durch die Begriffe Berufsausbildung in einer Kombination von Wissenschaft und Praxis gegeben (in Unterscheidung zum Begriff der Berufsvorbildung, in Kombination mit wissenschaftlichdisziplinärer Orientierung). Die wissenschaftliche Orientierung schließt anwendungsbezogene Forschung und Entwicklung ein, in Unterscheidung zur grundlagenorientierten Forschung an den Universitäten. Das Profil der Fachhochschulausbildung umfasste von Anfang an die Anpassung an den europäischen Standard bzw. die EU-Konformität der Diplome. Das Profil sollte innovativen Charakter haben und umfasste auch eine angestrebte kürzere Studiendauer und transparentere Mechanismen der Leistungsbereitstellung (Qualitätssicherung) im Vergleich zu den Universitäten.

b. Bedarf und Akzeptanz. Die Akkreditierung der neuen Ausbildungsangebote ist an einen Bedarfsnachweis für die erworbenen Qualifikationen in einem Berufsfeld und an einen entsprechenden Akzeptanznachweis seitens der Bildungsnachfragenden gebunden. Die Bedarfslage und das Berufsfeld werden auch weiterhin beobachtet und im (normalerweise fünfjährigen) Rhythmus der Re-Akkreditierung überprüft. Die Entwicklung des FH-Sektors ist daher in ihrer Struktur und bis zu einem gewissen Grad auch in ihrem Umfang an den wahrgenommenen Qualifikationsbedarf gebunden. Die Angebote sollten längerfristig konzipiert und in ein Entwicklungskonzept eingebunden sein.

61 Die Analyse der Beziehung zur Ebene der Sekundarstufe des Bildungswesens wurde aus dem Auftrag der vorliegenden Untersuchung aufgrund der Komplexität der Fragestellung ausgeklammert. 
c. Manifeste bildungspolitische Ziele, die mit der Errichtung des Sektors verbunden waren: Errichtung innovativer Stätten der Aus- und Weiterbildung, mit Durchlässigkeit vor allem für nicht-traditionelle Studierende, Förderung der beruflichen Flexibilität, Abbau regionaler Disparitäten sowie Strukturbereinigung im Bereich der postsekundären beruflichen Ausbildung. Diese Zielfestlegungen sind in verschiedenen Dokumenten in verschiedener Form ausgedrückt und repräsentieren eine stärkere Orientierung an Ergebniszielen, als dies in der österreichischen Bildungspolitik ansonsten üblich war (wo Ziele eher in Form von Inputs, sei es finanziell oder als reale Angebote bzw. als Merkmale der Ausstattung, formuliert worden sind). ${ }^{62}$

d. Latente bildungspolitischer Ziele, die mit der Errichtung des Sektors verbunden waren: Aufbau und Umsetzung von neuartigen dezentralisierten Regulierungsformen im österreichischen Bildungswesen, die sich insbesondere durch geringere Regelungsdichte, Autonomie, privatrechtliche Anbieterstrukturen, ein neues Finanzierungsmodell, Qualitätssicherung und Output-Kontrolle bestimmen lassen. Das Modell sollte vor allem schneller auf neue Anforderungen aus der Wirtschaft und Gesellschaft („,bottom-up“-Modell) reagieren können und durch die Einbeziehung autonomer ExpertInnen gesteuert werden. Das staatliche Engagement, das grundsätzlich nicht in Frage gestellt wurde, sollte in neuer Form wirksam werden.

Die Analyse und Bewertung dieser Aspekte erfolgt einerseits retrospektiv, im Hinblick auf ihre Ausgestaltung und Konkretisierung im Zuge der Implementation des FH-Sektors. Andererseits erfolgt auch eine prospektive Einschätzung des Stellenwertes des FH-Sektors vor dem Hintergrund wichtiger Entwicklungen und Veränderungen in seinem Umfeld.

Die Analyse muss sich aus Gründen der Arbeitsökonomie auf bestimmte zentrale Fragen konzentrieren. Die Hauptfrage besteht in der Formulierung von Entwicklungsoptionen und der Identifikation von Faktoren, die für die weitere Entwicklung als maßgeblich anzusehen sind. Die Frage nach dem Stellenwert des FH-Sektors im Hochschul- und Bildungswesen wird als Frage nach Optionen der politischen Gestaltung dieses Stellenwertes gestellt. Die politische Gestaltung kann durch sachliche Analysen und Begründungen unterstützt werden, der Raum für politische Entscheidungen jenseits wissenschaftlicher oder analytischer Befunde bleibt jedoch sehr weit. Dies zeigen beispielsweise die Vergleiche mit Entwicklungen in anderen Ländern, die von der österreichischen Lösung abweichende Gestaltungsformen ihrer Alternativen zur Universität im Hochschulsystem gewählt haben. Dies betrifft insbesondere das Größenwachstum und den Umfang des FH-Sektors insgesamt wie auch die Größenordnung und Gestaltung der angestrebten Institutionen. In den zwei

62 Vgl. das FHStG sowie die Erläuterungen zur Regierungsvorlage, den Entwicklungs- und Finanzierungsplan I (1994) und den Entwicklungs- und Finanzierungsplan II (1999) sowie die einschlägigen Berichte des Bundesministeriums sowie des FHR; vgl. auch zusammenfassend Hauser 1999. 
Ländern, die ebenfalls in den 1980er Jahren mit dem Aufbau eines FH-Sektors begonnen haben, Schweiz und Finnland, ist das Wachstum viel rascher vor sich gegangen und die Institutionen sind meist viel größer (konzipiert) als in Österreich (vgl. auch Abschnitt 2.3) ${ }^{63}$

Die Analyse beschreitet mehrere Lösungswege für diese Problematik der Indeterminiertheit des Entscheidungsraumes: Erstens werden bestimmte Faktoren identifiziert, die zumindest Aussagen über Spielräume zulassen (z.B. Demografie); zweitens werden bestimmte Entwicklungsoptionen formuliert, die sich aus unterschiedlichen politischen Strategien ergeben (z.B. Innovationspolitik, lebensbegleitendes Lernen); drittens werden bestimmte Bedingungen und Defizite identifiziert, die die Auswahl zwischen alternativen Entwicklungspfaden erleichtern könnten (z.B. verbesserte Antizipation von Qualifikationsbedarf). Diese Lösungswege werden grob skizziert, eine nähere und rigorose Analyse ist aufgrund des gewählten methodischen Ansatzes (Quasi-Review) und der damit verbundenen Kapazitäten weder möglich noch angestrebt. Die Ergebnisse erbringen ein „Screening“ der für die weitere Entwicklung wesentlichen Aspekte und Empfehlungen darüber, welchen Aspekten in Abhängigkeit von bestimmten Zielsetzungen und Erwartungen näher nachgegangen werden sollte.

\subsection{Retrospektive Analyse und Einschätzung der Zielerreichung}

\subsubsection{Diversifikation und Profilierung}

Die Profilierung des FH-Sektors knüpfte einerseits in vielerlei Hinsicht an behaupteten oder zutreffenden Schwächen des bestehenden Hochschulsystems (v.a. der Universitäten) an, ${ }^{64}$ andererseits an der deklarierten Betonung der Wichtigkeit und Unverzichtbarkeit des berufsbildenden höheren Schulwesens ${ }^{65}$. Aus dieser Kombination ergibt sich für den Stellenwert des FH-Sektors ein spezifisches Gesamtprofil, das die folgenden Aspekte betont:

63 Beide Länder haben, wie auch schon die meisten anderen Länder in den 60er Jahren, bestehende Institutionen mittels verschiedener Mechanismen in Fachhochschulen übergeführt. In der Schweiz ist die Errichtung der ersten sieben FHs für 1997/98 erfolgt (vgl. EFHK 2000, SKBF 2001); in Finnland begann der Prozess der Errichtung der Fachhochschulen 1991(vgl. MoE Finnland o.J. und http://www.arene.fi/english/ylakuva.cfm?pagelD=125\&top=otsikko ammattikorkeakoulut.cfm).

64 Vgl. z.B. Lassnigg/Pollan 1996.

65 Vgl. z.B. aus der Stellungnahme der Handelskammer-Organisation Kärnten: "Im Rahmen unseres Bildungssystems nehmen für die gewerbliche Wirtschaft die berufsbildenden höheren Schulen (...) einen besonders wichtigen Platz ein. Es ist für die gewerbliche Wirtschaft völlig ausgeschlossen, auf die Berufsqualifikationen, die diese Schulen vermitteln, zu verzichten. Auch die ,österreichische Lösung' der Vermittlung der Hochschulberechtigung (...) ist für die Wirtschaft wichtig und auch künftig unverzichtbar" (Pongratz 1993, Anhang, 62); vgl. auch Clement 1991. 
- Die starke Hervorhebung der Praxisnähe der Fachhochschul-Studiengänge drückt indirekt eine bereits seit langem vorgebrachte und immer wieder wiederholte Kritik an der „Abgehobenheit“ der Universitäten aus. Vor allem während der vorbereitenden Diskussionen um die Errichtung des Sektors war die Forderung nach unbedingter Vermeidung von Vorgängen des „Academic Drif“ (also einer befürchteten Tendenz einer zukünftigen Annäherung von Fachhochschulstudien an die Universitäten) ein sehr wichtiges Thema. ${ }^{66}$ Diese Abgrenzung gegenüber den Universitäten war und ist teilweise immer noch mit Diskussionen um die Rolle von Forschung und Entwicklung im FH-Sektor - und auch insgesamt in der österreichischen Wirtschaft - verbunden. Bis heute gibt es prononcierte Stellungnahmen, die der Forschungsverpflichtung im FH-Sektor skeptisch bis ablehnend gegenüberstehen und die Konkretisierung des Wissenschaftsbezuges als der zweiten wesentlichen Zieldimension der FH-Studien wird durch viele Befunde und Stellungnahmen als nach wie vor bestehende Herausforderung unterstrichen. „Den Universitäten wird vorgeworfen, sie seien theorielastig; ihre AbsolventInnen deshalb unbrauchbar. Die Fachhochschulen werden für ihre Praxisnähe gelobt. Das wären umschreibbare Profilierungen. Aber genau können Kritiker und Lobredner nicht umschreiben, was sie damit wirklich meinen." (Prisching 2002, 26) ${ }^{67}$ Diese Unbestimmtheiten auf der Ebene des Bildungswesens spiegeln sich auch in Unbestimmtheiten der Bewertung der Bildungsstruktur der Bevölkerung bzw. der Berufstätigen. Seit langem zeigen alle internationalen Vergleiche die niedrige österreichische Akademisierungsquote. ${ }^{68}$ Seit den 1970er Jahren ist jedoch die Bewertung dieses Befundes umstritten (vgl. Lassnigg 1998) und in jüngerer Zeit wird auch an prominenter Stelle der Befund selbst in seiner Stichhaltigkeit in Frage gestellt und als statistisches Zählungs- und Definitionsproblem dargestellt: Die österreichische Qualifikationsstruktur - und insbesondere die österreichische Akademikerschaft - sei in den internationalen statistischen Zählungen aufgrund der "Strukturspezifik“ des Ausbildungswesens unvergleichlich. In den meisten Ländern würden dieser Argumentation zufolge hohe Anteile an Qualifikationen als tertiäre Abschlüsse gezählt, die faktisch (aber eben nicht definitorisch) dem Qualifikationsniveau der österreichischen BHS gleichzustellen wären. ${ }^{69}$ Diese Argumentation beruht jedoch nicht auf wissenschaftlichen Vergleichen des tatsächlichen Qualifikationsniveaus (in Form von

66 Vgl. die verschiedenen Referate und auch die dokumentierten Diskussionsbeiträge in BMWF 1991.

67 Vgl. sehr prononciert Prisching 2002, in seiner Diskussion um die "Unklarheit der Ziele"; Wilhelmer 2001; weiters eine Reihe von Befunden aus der AbsolventInnenbefragung (Hoyer/Ziegler 2002), aus Unternehmensbefragungen (Lang/Finder 2000) und auch aus der NÖ-Szenario-Entwicklung (Steiner et al. 2001a,b).

68 Vgl. als rasch verfügbare Quelle z.B. die Europäischen Innovationsindikatoren zu den Humanressourcen: "Population with tertiary education" an drittletzter Stelle, "S\&E graduates" unterdurchschnittlich und an 8. Stelle (siehe http://trendchart.cordis.lu/Scoreboard/scoreboard.htm).

69 "Es ist ein Faktum, dass die Strukturspezifik des österreichischen Bildungssystems in der europäischen und globalen komparativen Statistik zu einer Unterschätzung der Abschlüsse österreichischer Absolventlnnen, aber auch der Humanressourcen unserer Volkswirtschaft führt." (Schneeberger 2001, 30; vgl. auch Schneeberger 2000). 
Kompetenzen oder anderen Verfahren der Messung von Ergebnissen), sondern auf verschiedenen Plausibilitätsannahmen und erfahrungsgestützten institutionellen Vergleichen in Kombination mit allgemeinem Hintergrundwissen über verschiedene Bildungssysteme. Darüber hinaus wird in dieser Argumentation auch die Validität des qualitativen Unterschiedes zwischen den sekundären Abschlüssen und tertiären Abschlüssen in den internationalen Statistiken in Frage gestellt. Hier ist nicht der Ort, um die Tragfähigkeit dieser Argumentationen zu beurteilen ${ }^{70}$, aber es ist festzuhalten, dass diese Unbestimmtheit darüber, was als tertiärer Abschluss zu werten ist, die Identitätsbildung der verschiedenen Institutionen über ihr Profil natürlich nicht erleichtert.

- Während die Praxisnähe ein wesentliches Merkmal ist, das den FH-Sektor von den Universitäten unterscheidet, markiert die ausdrückliche Positionierung auf Hochschulniveau sowie die Vermittlung wissenschaftlicher Qualifikationen in der Lehre und die Verpflichtung zu anwendungsbezogener F\&E die Abgrenzung vom Bereich der BHS. $^{71}$ Das Hochschulniveau ist auch durch die Festlegung der Wissenschaftsfreiheit und durch die starke Involvierung von Universitätspersonal bzw. Personen mit wissenschaftlichen Qualifikationen auf allen Ebenen und in den verschiedenen Prozessen der Leistungserstellung (Entwicklung, Akkreditierung, Lehre, F\&E, Evaluierung) gewährleistet. Im Vergleich zu den meisten anderen FH-Systemen, deren Entwicklung durch „upgrading“ von postsekundären Schulen zustande gekommen ist, besitzt der österreichische FH-Sektor in dieser Hinsicht sicherlich Ausgangsvorteile, da neue Institutionen - im Vergleich zur Veränderung von bestehenden Institutionen leichter neue Praktiken aufbauen können. ${ }^{72}$ Gleichzeitig ist jedoch der quantitative Stellenwert der wissenschaftlichen Qualifikationen auf Hochschulniveau im gesamten Qualifikationsangebot durch die Stärke und Aufrechterhaltung der BHS insgesamt deutlich begrenzt. Durch das „upgrading“ bestehender postsekundärer Institutionen in anderen Ländern wurde ein bedeutender Reformdruck auf diese ausgeübt und erforderte auch eine entsprechende Weiterentwicklung des Lehrkörpers dieser Institutionen, um die bleibende Akkreditierung zu erlangen. ${ }^{73}$ Bisher ist der Stellenwert des FH-Sektors im

70 Dies würde wohl tiefergehende Analysen erfordern, für die die Beteiligung Österreichs an den internationalen Vergleichsstudien über das Bildungsniveau der Bevölkerung sehr hilfreich wäre.

71 Vgl. näher die ausführliche Diskussion der Zielstruktur und der Abgrenzung von den anderen Bereichen in Hauser 2000.

72 Vgl. die entsprechenden Analysen zur Reform in Hochschulsystemen (z. B. Cerych/Sabatier 1986) sowie die bereits klassische Literatur aus der Organisationsforschung (z. B. Hannan/Freeman 1984).

73 Der Bericht der OECD-Prüfer zum Reformprozess der Fachhochschulen in Finnland gibt zu diesem Aspekt ein ambivalentes Bild. Das „upgrading“ der Lehrenden wurde v.a. mit der Anforderung des Erwerbs von akademischen Graden an der Universität verbunden, was den praktischen Aspekt unterminieren könnte (OECD 2002, 50-52). Darüber hinaus wird über eine "heated debate" betreffend einen Mangel an Berufsqualifikationen auf der Sekundarstufe infolge der Reform berichtet (OECD 2002, 21). Die Schweizer Evaluationen weisen auf ein starkes Beharrungsvermögen der (ursprünglichen) Teilschulen hin (EFHK 2002, 10). 
Hinblick auf die Wissensproduktion noch nicht sehr ausgeprägt und dieser Aspekt stellt eine wichtige Herausforderung für die Weiterentwicklung dar. ${ }^{74}$

- Im Hinblick auf Qualitätsstandards im Bereich des Studiums ist der FH-Sektor insbesondere durch die Gewährleistung eines zeitgerechten Studienabschlusses im Vergleich zu den Universitäten profiliert, die seit langem wegen der hohen Abbrecherlnnenquote, der langen Studiendauer und der unüberschaubaren Organisations- und Leitungsstrukturen kritisiert - und seit der ersten Hälfte der 1990er Jahre - auch reformiert werden. ${ }^{75}$ Trotz der erzielten effektiv kurzen Studiendauer der Fachhochschul-Studiengänge liegt das Durchschnittsalter der FH-AbsolventInnen jedoch bisher nicht unter dem der Universitäten (Hoyer/Ziegler 2002, 263, 265). Das Ausmaß von Studienabbrüchen ist zwar im Vergleich zu den exorbitant hohen Drop-out-Raten der Universitäten gering (vgl. Abschnitt 2.2.7); für sich genommen und unter Berücksichtigung der Selektivität des Zuganges ist das Ausmaß jedoch nicht so gering. Im Hinblick auf das Studium kommt dem FH-Sektor aber jedenfalls ein Stellenwert als Vorreiter der Bewegung der Qualitätssicherung im Bildungswesen zu, die aktuell auch als eine wichtige Priorität in der Europäischen Bildungs- und Berufsbildungspolitik vorangetrieben wird. ${ }^{76}$ Der FH-Sektor ist im Hinblick auf die interne Qualitätssicherung und -entwicklung sicherlich ein Beispiel von "good practice" nicht nur im österreichischen, sondern auch im europäischen Kontext.

74 Dies zeigt sich an einigen Befunden der AbsolventInnenstudie (z.B. dem Umgang mit den Diplomarbeiten; vgl. Hoyer/Ziegler 2002) und der Befragung österreichischer Unternehmen und KMUs über das Qualifikationsprofil der AbsolventInnen (vgl. Lang/Finder 2000, Wimmer 2000).

75 Vor allem im Zusammenhang mit den Auswahlmöglichkeit unter den Bewerberlnnen für die gegebene Zahl an Studienplätzen wird dem FH-Sektor teilweise ein Stellenwert als „Elitesektor" im österreichischen Hochschulsystem zugeschrieben. Die Fachhochschul-Studiengänge würden sich „die Besten“ aussuchen, der Rest würde dann den Universitäten bleiben. Abgesehen von den restriktiven (und unbewiesenen) unterliegenden Annahmen dieser Argumentation, die von einem identischen BewerberInnenpool, von einer klaren eindimensionalen Skala der Qualität der BewerberInnen ausgeht und die unterschiedliche Profilierung der Studien leugnet, hat die AbsolventInnenstudie des FHR gezeigt, dass immerhin $20 \%$ der erfolgreichen AbsolventInnen vor ihrem FH-Zugang ein Universitätsstudium abgebrochen haben - auf diesen Teil der "Elite“ haben die Universitäten also jedenfalls verzichtet. Die AbsolventInnenstudie (Hoyer/Ziegler 2002) verweist außerdem auch darauf, dass FH-AbsolventInnen in der Größenordnung von etwa 10\% verschiedene Arten von Schwierigkeiten bei der beruflichen Eingliederung haben, etwa 5\% ernste Schwierigkeiten - wenn man diese Schwierigkeiten nicht der Nachfragesituation zuschreibt, so verweist dieser Befund doch auf ein nicht unbeträchtliches Ausmaß von Spannungen in der Relation von Auswahl und Ausbildung. Andererseits haben auch Uni-StudentInnen in einen FH-Studiengang gewechselt und diesen absolviert, die nach ihrer Einschätzung andernfalls das Universitätsstudium absolviert hätten bzw. dazu entschlossen waren (für diese war das $\mathrm{FH}$ Studium offensichtlich die erste Wahl).

76 Vgl. zum europäischen Kontext die Aktivitäten im Rahmen des European Network for Quality Assurance in Higher Education (enqa; http://www.enqa.net/index.html), wie auch die Dokumente im Zusammenhang mit der Entwicklung der konkreten Ziele im Rahmen der offenen Koordinierung der Europäischen Bildungspolitik im Lissabon-Follow-up, das Memorandum und die Mitteilung der Kommission zum lebenslangen Lernen und die Arbeiten des Europäischen Forums für Qualität in der Berufsbildung. In Österreich wurden in den 1990er Jahren im Schulwesen wesentliche Schritte in Richtung Qualitätssicherung und Qualitätsentwicklung gesetzt (vgl. Posch/Altrichter 1997, Eder et al 2002; http://www.qis.at/) und auch im Universitätsbereich wurden Schritte in diese Richtung gesetzt (Evaluierungsverordnung 1997, Altrichter/Schratz 1992, Titscher et al. 2000). 
Mehrere Studien (Wimmer 2000, Lang/Finder 2000, Hoyer/Ziegler 2002) geben erste Aufschlüsse $^{77}$ darüber, wie die Profilierung des Fachhochschulbereichs im Unternehmenssektor aufgenommen wurde bzw. welche Erwartungen an die AbsolventInnen bestehen (eine detaillierte Zusammenstellung der Ergebnisse befindet sich im Anhang, Kapitel 11.1.1). Die Ergebnisse beziehen sich auf die ersten Abschlussjahrgänge der Fachhochschul-Studiengänge und die Merkmale sind jeweils unterschiedlich definiert. Dennoch ergibt sich ein teilweise bemerkenswertes Bild. Insgesamt deuten die Ergebnisse darauf hin, dass FH-AbsolventInnen in ihrer Profilierung einen sehr hohen Überschneidungsbereich mit den UniversitätsabsolventInnen haben. Die direkte Kompetenz im F\&E-Bereich wird innen jedoch (noch) nicht so klar zugetraut. Hinsichtlich Führungskompetenzen bestehen ebenfalls nicht so günstige Einschätzungen, dies kann jedoch auch mit dem jungen Alter der bisherigen FH-AbsolventInnen zusammenhängen. Vom pädagogischen Profil her könnte der starke Verschulungsgrad und das geringe Maß an Wahl-, Entscheidungs- und Mitsprachemöglichkeiten in dieser Hinsicht einen beeinträchtigenden Faktor darstellen.

Die Gestaltung und weitere Entwicklung des speziellen Ausbildungsprofils der FH-Studien ist ein wichtiges und komplexes Aktivitätsfeld für den Sektor, das in engem Zusammenhang mit der Personalentwicklung und der Professionalisierung der methodischen und didaktischen Ansätze steht. Die verstärkte Betonung der F\&E-Funktion im Aufgabenportfolio der Fachhochschul-Studiengänge und die zunehmenden Berührungspunkte zu den Universitäten im Zuge des Bologna-Prozesses sind dafür verstärkende Faktoren. In pädagogischer und inhaltlich-methodischer Hinsicht ergeben sich wichtige Ansatzpunkte und Anregungen für die Weiterentwicklung beispielsweise aus den neueren Arbeiten und Diskussionen über die Definition und Formulierung von Kompetenzen sowie aus den vielfältigen und - im Vergleich zum traditionellen Unterricht - teilweise radikalen methodischen Weiterentwicklungen im Paradigma der konstruktivistischen Ansätze des Lernens und Lehrens. ${ }^{78}$ Die Profilierung der FH-Studiengänge bietet durch die explizite Formulierung des Ausbildungsprofils und den Praxisbezug wichtige Anknüpfungspunkte zu diesen didaktisch-methodischen Entwicklungen, die nicht selbstverständlich sind und durch entsprechende Rahmenbedingungen gefördert werden können. Innovative inhaltlichmethodische und professionelle Konzepte haben in der Entwicklung der FachhochschulStudiengänge von Anfang an eine Rolle gespielt:

77 Die empirische Basis der Aussagen beruht auf Erfahrungen mit den ersten Jahrgängen im Beschäftigungssystem und den damit zusammenhängenden Erwartungen und Einschätzungen; die Ergebnisse sind insbesondere bei den Unternehmen aufgrund niedriger Beantwortungsquoten (etwas über 10\%) nicht gut gesichert.

78 Vgl. zu den neuen Ansätzen zur Definition von Kompetenzen Rychen/Sagalnik 2001 sowie die Materialien im Rahmen des Projektes DeSeCo (http://www.statistik.admin.ch/stat ch/ber15/deseco/), zur österreichischen Entwicklung Lassnigg/Mayer 2001; zu den Weiterentwicklungen im Rahmen der konstruktivistischen Pädagogik vgl. z. B. Lave/Wenger 1991, Berryman/Bailey 1992, Dubs 1995, Voss 1997, Reinmann-Rothmeier/Mandl 1998; vgl. auch die Arbeiten von Kersten Reich: http://www.uni-koeln.de/ew-fak/konstrukt/literatur/index.html. 
- In der ersten Entwicklungsphase wurde das „reflective practitioner“-Konzept professioneller Praxis (vgl. Altrichter/Posch im österreichischen Länderbericht an die OECD; BMWF 1992a) eingebracht;

- Die Ausbildungsprofile der Studiengänge legen großen Wert auf die verschiedenen Formen von überfachlichen Kompetenzen, jedoch zumindest in der Anfangsphase in wenig systematischer Weise (Lassnigg/Stöger 1999).

- Teilweise wird im Zusammenhang mit der verstärkten Umsetzung der F\&E-Funktion ein deutlicher Entwicklungsbedarf im Verständnis des Ausbildungsprofils der FachhochschulStudiengänge festgestellt und insbesondere auch ein breiteres Verständnis der akademischen Dimension der FH-Studien gefordert (Prisching 2002). Die Ergebnisse aus der AbsolventInnenstudie (Hoyer/Ziegler 2002) beispielsweise zu Schwächen in der Betreuung von Diplomarbeiten unterstützen diese Argumentation.

In einem Entwicklungsschwerpunkt wurde seitens des FHR ein Forschungsprojekt zur Profilierung im Bereich des „praktischen akademischen Wissens“ in Auftrag gegeben. In diesem Projekt wurde in einer Weiterentwicklung des Konzeptes der "communities of practice" das Modell der Hochschulpraxisgemeinschaft als möglicher Professionalisierungspfad für die FH-Studien eingebracht (Markowitsch 2001), das zwischenzeitlich in verschiedenen Initiativen auf sein Umsetzungspotential ausgelotet bzw. bereits umgesetzt wird. Damit wurde ein sehr anspruchsvoller und innovativer Weg eingeschlagen, um die methodische Profilierung zu untermauern und die Professionalisierung voranzutreiben und die inhaltliche Profilierung der Studien mit dem aktuellen methodisch-didaktischen Schwerpunkt in der Personalentwicklung zu verbinden (diese Fragen werden im Abschnitt zur beruflichen Spezialisierung wieder aufgenommen; vgl. 3.1.3 b).

\subsubsection{Bedarf und Akzeptanz}

Die Bindung der Akkreditierung der FH-Studiengänge an den Bedarfs- und Akzeptanznachweis steht in einem engen Zusammenhang mit dem quantitativen Ausbaustand des FH-Sektors, indem dadurch das vorsichtige Ausbautempo und vermutlich auch die starke Diversifizierung und Nischenpolitik verstärkt wurde: Nachweise für kleine und spezifische Qualifikationsprofile sind kurzfristig und im Einzelnen weniger riskant als Nachweise für größere und breitere Profile.

\section{Bedarf}

Während Bedarfsanalysen zum Zeitpunkt der Einrichtung des FH-Sektors (und mehr oder weniger bis heute) ansonsten keinen wesentlichen Stellenwert in der österreichischen Bildungspolitik haben (Lassnigg 2002), wurde der Bedarfsnachweis zu einer der 
entscheidenden Voraussetzungen der Akkreditierung von FH-Studiengängen. Viele Stellungnahmen in der Diskussion um die Errichtung des FH-Sektors weisen auf die damals große Skepsis hinsichtlich des Bedarfs nach den neuen Institutionen hin. Als Beispiel sei die Stellungnahme der Handelskammer Kärnten zitiert: „Fachhochschulen können nur ein fakultatives, ergänzendes Angebot am Bildungssektor sein, wobei der Bedarf in jedem Einzelfall sehr genau zu prüfen ist und nicht von Vorneherein generell gegeben erscheint" (Pongratz 1993, Anhang, 65). Der Bericht der Prüfer im OECD-Review (BMWF/BMUK 1993) hat zurecht darauf hingewiesen, dass im österreichischen Länderbericht eine Analyse zum Qualifikationsbedarf fehlt und dass auch unter den Akteuren kein klares Bild dazu vorliegt. ${ }^{79}$

Es hat in dieser Hinsicht also von Anfang an eine Diskrepanz zwischen der Makro-Ebene und der studiengangsbezogenen Mikro- oder Meso-Ebene gegeben, wobei die Frage nach dem Bedarfsnachweis auf die letztere „bottom-up“-Ebene verschoben wurde. Während auf der allgemeinen Ebene keine systematische Forschung betrieben wird, sind die Anforderungen an den Bedarfsnachweis für die Anträge vergleichsweise hoch. Damit fehlte - und fehlt bis heute - ein Rahmen, in dem die einzelnen Bedarfserhebungen eingeschätzt werden können. Dies wurde und wird von den verschiedensten Akteuren zu Recht kritisiert, ${ }^{80}$ ohne dass bisher nachhaltige Schritte in Richtung Veränderung gesetzt wurden.

Das Verfahren verläuft im Prinzip marktähnlich, indem die potentiellen Anbieter eines Produktes quasi die Marktgängigkeit dieses Produktes abtesten müssen. Das Ergebnis dieses Tests in Verbindung mit der positiven Qualitätsprüfung und der Finanzierungszusage führt in der Regel zu einem positiven Akkreditierungsbescheid. Im Gegensatz zur starken Rhetorik der bedarfsgesteuerten Entwicklung ist der gesamte Prozess eigentlich überwiegend angebotsgesteuert, da die potentiellen Anbieter - unter Berücksichtigung ihrer Wahrnehmung von Entwicklungen und Signalen von der Bedarfsseite - entscheiden, welcher Bedarf getestet wird. Man weiß jedoch nichts Näheres über den Bedarf in jenen Bereichen, wo nicht getestet wird. Dies verbindet sich mit mehreren weiteren Mechanismen, die paradoxerweise diesen angebotsseitigen Aspekt verstärken: erstens die Nischenstrategie - je spezifischer die Profile sind, je kleiner wird der Bereich des Gesamtmarktes, der abgetestet wird; zweitens die Finanzierungsentscheidung - das

79 "Wie in vielen Ländern spricht man auch in Österreich von einem ,Mangel an qualifizierten Arbeitskräften', aber es ist ziemlich schwierig, ein klares Bild von der Natur, dem Ausmaß und den Gründen dieses Mangels zu gewinnen, vor allem was die Arbeitskräfte mit Hochschulbildung betrifft." (BMWF/BMUK 1993, 44); die im Bericht enthaltene Analyse des Instituts für Wirtschaftsforschung hat lediglich eine angebotsseitige Fortschreibung der Qualifikationsstruktur vorgenommen; in der kurz zuvor veröffentlichten Studie des Beirates für Wirtschafts- und Sozialfragen zur "Qualifikation 2000" war eine äußerst reservierte Position zur Tragfähigkeit von Bedarfsforschung formuliert, mit dem dynamischen Wandel der Anforderungen als Hauptbegründung.

80 Im Rahmen des österreichischen Konsultationsprozesses zum EU-Memorandum für lebenslanges Lernen wurde in Rahmen eines Koordinationsworkshops eine Bestandsaufnahme vorgenommen (vgl. BMBWK 2001a,b,c; download: http://www.lebenslangeslernen.at/ > Forum \& Workshops > Workshops > Ergebnisprotokoll: „Erkennung und Erhebung des Qualifikationsbedarfs am Arbeitsmarkt“; vgl. auch Lassnigg 2002). 
Ministerium als Anbieter rationiert aufgrund finanzieller und anderer bildungspolitischer Erwägungen teilweise Angebote mit positiver Bedarfsprüfung; drittens die Studierenden diese wählen Angebote bis zu einem gewissen Grad aufgrund der Bedarfsrhetorik und werden möglicherweise von anderen ungetesteten Wahlmöglichkeiten abgehalten. Die „Überzeichnung“ der Studienplätze durch die BewerberInnenzahlen kann insofern zu einem gewissen Teil auf derartige Selbstverstärkungsmechanismen zurückgeführt werden. Viertens die Wettbewerbseinschränkung: Da im FH-Sektor nur Angebote zulässig sind, die es sonst nicht gibt, ist der durch Bedarfsprüfungen abzudeckende Bereich des Gesamtmarktes von vorneherein einerseits quantitativ stark eingeschränkt, andererseits auf Wachstums- oder Erweiterungsbereiche fokussiert.

Eine zweite Paradoxie besteht insofern, als hier ein Feld durch marktähnliche Mechanismen abgedeckt werden soll, in dem erstens die Existenz von Marktversagen ${ }^{81}$ vielfach begründet ist (wobei man meistens Unterversorgung vermutet, das Versagen also in die gleiche Richtung geht wie die skizzierten Mechanismen vermuten lassen) und in dem zweitens wesentliche Entscheidungen von Akteuren und nach Vorgaben getroffen werden, die nicht marktwirtschaftlichen Kriterien genügen (öffentliche Haushalte, bildungspolitische Kriterien).

Berücksichtigt man den weiteren Kontext der Innovationspolitik, so ist der Stellenwert des FH-Sektors aufgrund der starken Bindung der Entwicklung an die Bedarfserhebung in hohem Maße als reaktiv einzuschätzen. Unter der Annahme einer innovativen Wirtschaft und entsprechend innovativer Orientierung der Anbietenden wäre dies ein befriedigendes System. Wenn man jedoch die Stellung Österreichs im Europäischen Innovationsobservatorium als bei den meisten Indikatoren unterdurchschnittlich und überdies die jüngste Verschlechterung der wichtigsten Benchmarks (Wirtschaftswachstum, Beschäftigungswachstum, Produktivitätswachstum) gegenüber der europäischen Entwicklung bzw. der Vorperiode im European Competitiveness Report (vgl. European Commission - Enterprise 2002) berücksichtigt, dann scheint dieser Mechanismus nur bedingt befriedigend zu sein.

Tichy/Darlap (1996) haben schon vor einiger Zeit vom „unbewussten Nachholbedarf“ in der Akademisierung gesprochen und damit ausgedrückt, was verschiedene Akteure auch in unseren Erhebungen immer wieder betont haben. Diese Befunde sind auch verträglich mit dem Grundtheorem der Innovationsökonomie und -forschung, demzufolge die

81 Booth/Snower 1996 berichten zumindest 14 empirisch nachweisbare Formen von Marktversagen im Bereich der Bereitstellung von Qualifikationen, in alphabetischer Reihenfolge: complementarities labour - capital; credit constraints; imbalance education - vocational training; external production economies; failing risk management; high-wage/low-skill trap; imperfect competition concerning a) tax , b) regulatory distortions; information bias ; innovation - skills interaction; uncompensated costs/benefits: a) wage, b) risk; low-skill/bad job trap; lowskill/low-tech trap; poaching; training risk; biased incentives by unemployment benefit/welfare payment; vgl. dazu näher Lassnigg 2000; vgl. auch Lynch 1994; OECD 1996. Als konkretes Beispiel aus dem FH-Sektor für die Analyse von Marktunvollkommenheiten vgl. Hartmann et al. (2002) zum steirischen Autocluster. 
wirtschaftlichen Akteure mit unterschiedlichen Strategien auf den gestiegenen Wettbewerbsdruck reagieren können. Innovation ist nach diesen Konzepten vermutlich eine für nachhaltige Entwicklung wirksame Strategie, aber nicht die wahrscheinlichste. Daraus wird die Notwendigkeit der Entwicklung von Innovationspolitik abgeleitet, die die verschiedenen Politikbereiche in gezielter Weise bündelt und eine ausgewogene wirtschaftliche Innovationsdynamik fördert. ${ }^{82}$ Der Einsatz von gesteigerten finanziellen Mitteln für F\&E ist dafür eine notwendige, aber keinesfalls eine hinreichende Maßnahme.

Lang/Finder (2000) haben in ihrer Studie über die Beschäftigung der ersten Jahrgänge von $\mathrm{FH}-$ AbsolventInnen in kleineren und mittleren Betrieben einen klaren Zusammenhang zwischen der Innovationstätigkeit der Betriebe und der Beschäftigungsentwicklung wie auch der Höherqualifizierung herausgearbeitet. Die empirischen Ergebnisse entsprechen den Erwartungen aus der Innovationsforschung: Betriebe, die sowohl Produkt- als auch Prozessinnovation betreiben, haben einen erhöhten zusätzlichen Personalbedarf an höher Qualifizierten (80\% dieser Betriebe haben in der Vergangenheit Beschäftigungszuwächse und fast $60 \%$ erwarten auch weitere Zuwächse in den nächsten Jahren). Expansion in neue Bereiche wird als wichtigster Grund für einen Bedarf an FH-AbsolventInnen wie auch für Höherqualifizierung und Beschäftigungsexpansion angegeben. Gleichzeitig ist auch in den Branchen, wo die FH-AbsolventInnen konzentriert beschäftigt sind, ein hoher Anteil an Betrieben (etwa 30\%) tätig, die überhaupt keine MitarbeiterInnen mit höheren formalen Qualifikationen (BHS, FH-Studium, Universitätsstudium) beschäftigen. Zusätzlicher Bedarf an höheren Qualifikationen wird aber eher von Betrieben geäußert, die bereits einen höher qualifizierten Personaleinsatz haben. Der Personalbedarf ist im Hinblick auf formale Qualifikationen in vielen Fällen nicht sehr spezifisch ausgeprägt (in drei Viertel der befragten Betriebe gibt es Stellen, die von BHS-, Fachhochschul-, UniversitätsabsolventInnen oder Fachkräften mit langjähriger Berufserfahrung besetzt werden können, darunter in mehr als der Hälfte dieser Betriebe häufiger als vereinzelt). Qualifikationsanpassungen an die realen Anforderungen werden als normaler und erforderlicher Vorgang gesehen, nur etwa 10 - 15\% der befragten Betriebe erwarten die unmittelbare Einsetzbarkeit der AbsolventInnen (bei FHAbsolventInnen liegt dieser Anteil mit 15\% geringfügig höher als bei UniversitätsabsolventInnen mit 10\%).

Diese Ergebnisse deuten auf einen expansiven Bedarf hin, gleichzeitig bestehen jedoch Unterschiede nach betrieblichen Strategien und Positionierungen im Hinblick auf die Innovationstätigkeit. Der Bedarf ist verbunden mit der Innovationstätigkeit der Betriebe, also wenn der Anteil dieser Betriebe steigt, steigt auch der Bedarf nach FH-AbsolventInnen. Eine ganz wesentliche Frage, die in der Forschung unterschiedlich beantwortet wird und in der Politik umstritten ist, besteht letztlich darin, inwieweit der Anteil dieser Betriebe durch

82 Vgl. v.a. Lundvall/Borras 1999; als Beispiel auch die Darstellung der Strategie der National Technology Agency in Finnland, Heurlin 2001. 
Innovationspolitik gesteigert werden kann und inwieweit ein entsprechendes zusätzliches Qualifikationsangebot dabei eine Rolle spielt. Berücksichtigt man die Stellung Österreichs im Europäischen Benchmarking, so wäre jedenfalls in dieser Hinsicht beträchtlicher Aufholbedarf gegeben.

Als Schlussfolgerung ergibt sich, dass die Bedarfserhebungen für Studiengänge nur einen bestimmten Aspekt im Rahmen der Feststellung des Bedarfes nach Qualifikationen abdecken und daher auch nur ein Element im Rahmen der Erfassung des Bedarfes nach FH-Qualifikationen darstellen sollten. Die Entwicklung der Qualifikationsstruktur ist in hohem Maße eine Frage der Gestaltung, sowohl auf betrieblicher Ebene als auch auf aggregierter Ebene. Auf betrieblicher Ebene erfolgt diese Gestaltung meistens kurzfristig und es besteht hohe Flexibilität, wobei sich die Betriebe offensichtlich auch auf das vorhandene Qualifikationsangebot einstellen müssen. In Österreich besteht der bekannte Schwerpunkt auf der mittleren Qualifikationsebene (Lehre und BHS), die meisten anderen Länder haben viel stärker auf Akademisierung gesetzt. Gleichzeitig bestehen ziemlich offensichtliche Schwächen im österreichischen Innovationssystem (am hervorstechendsten die niedrige F\&E-Quote im Unternehmenssektor) und die Frage, inwieweit diese Schwächen mit der Qualifikationsstruktur und der Struktur des Ausbildungssystems zusammenhängen, wird seit langem diskutiert. Im vorliegenden Projekt kann diese Frage nicht zwingend beantwortet werden. Viele Argumente sind bereits vorgebracht worden, die diesen Zusammenhang unterstreichen und eine tiefergehende Analyse dieser Zusammenhänge ist für eine zureichende Klärung der Bedarfsfrage unbedingt erforderlich.

In einer Strategie für die Wissensgesellschaft muss der FH-Sektor zweifellos eine wesentliche Rolle spielen, aber diese Rolle kann nicht geklärt werden, ohne die Beziehungen zu den anderen Qualifikationen zu klären. Die angestrebte Erhöhung der F\&EQuote erfordert beispielsweise nicht nur finanzielle Ressourcen, sondern auch personelle Ressourcen. Woher sollen diese bezogen werden? Sollen diese auf bestimmte thematische oder wirtschaftliche Bereiche konzentriert werden? Welches Gewicht soll der Innovationspolitik im Rahmen des komplexen Regulierungssystems des FH-Sektors im Vergleich zu anderen Erwägungen zukommen? Wie können die verschiedenen Akteure, die sehr unterschiedliche Gesichtspunkte verfolgen (von Bürgermeisterlnnen und LandespolitikerInnen über die Sozialpartner und die Lehrenden und Forschenden bis zu den Verantwortlichen auf Bundesebene) zu einer gemeinsamen Position kommen?

Spezielle Fragen betreffen die klein- und mittelbetrieblichen Unternehmen (KMUs) und die angestrebten regionalen Verflechtungen und Entwicklungen. Der FH-Sektor sollte in besonderer Weise dem Bedarf von KMUs nachkommen. Dieses Ziel scheint bis zu einem gewissen Grad erreicht worden zu sein. Das Leistungsprofil der FachhochschulStudiengänge hat einen deutlichen Schwerpunkt in ihrem regionalen Umfeld ( $\mathrm{vgl}$. die entsprechenden Befunde in Kapitel 3.1.3). Laut AbsolventInnenstudie (Hoyer/Ziegler 2002) sind jedoch andererseits zwei Drittel der AbsolventInnen in Großbetrieben (über 100 
MitarbeiterInnen) beschäftigt, 9,5\% in mittleren Betrieben (50-100 MitarbeiterInnen) und $29,3 \%$ in Kleinbetrieben (unter 50 MitarbeiterInnen).

Die Frage nach dem Bedarf lässt sich letztlich nur zureichend klären, wenn in Ergänzung zu den bisherigen Bedarfs- und Akzeptanzerhebungen ein übergreifendes Antizipationssystem entwickelt wird, das Lösungen für diese Fragen enthält. "While the concept of supply is fairly clear, that of demand is to a large extent arbitrary and subjective" (OECD 1993, 7). Die NÖ Bildungsgesellschaft m.b.H. beschäftigt sich intensiv mit den Fragen der Bedarfsentwicklung (vgl. Steiner et al. 2001 a, b). Diese Frage wird auch in den Empfehlungen des deutschen Wissenschaftsrates für die Entwicklung der deutschen Fachhochschulen in genau der Richtung gelöst, die in der Szenarienanalyse für Niederösterreich eingeschlagen wurde: Die verschiedenen Akteure in Politik, Wirtschaft und Fachhochschulsektor sollen in einen interaktiven Zusammenhang gebracht werden, der es erlaubt, vor dem Hintergrund entsprechender prospektiver Verfahren kollektive Prozesse des Lernens und der Wissensproduktion hervorzubringen (WR 2002, 79, 88, 96). Die OECD-Studie nennt regionale Agenturen („lead agencies to articulate the skill needs of the region“; OECD 1999, 70) als Schlüssel für die Formulierung des Bedarfes. Die Entwicklung derartiger kooperativer Mechanismen wird heute, auch im Zusammenhang mit dem Konzept der "lernenden Region“ (vgl. die entsprechenden Abschnitte in Kapitel 5), als wesentliches Feld für die Nutzung und Umsetzung von formalisierten Bedarfserhebungen gesehen. In praktischer Hinsicht befinden sich diese Konzepte, insbesondere soweit die Schnittstelle zum Bildungswesen betroffen ist, aktuell noch gewissermaßen in einem Pilotstadium. In der einschlägigen OECD-Studie (OECD 1999) wird aufgrund der Analysen in ausgewählten Ländern herausgearbeitet, dass den Aktivitäten seitens der Hochschulen in Richtung ihrer Einbettung in das regionale Umfeld eine Schlüsselrolle in diesen Prozessen zukommt. ${ }^{83}$

\section{Akzeptanz}

Die Seite der Akzeptanz betrifft die Frage, ob für die Angebote im FH-Sektor eine ausreichende Bildungsnachfrage zu erwarten ist. In welchem Ausmaß die Nachfrage nach Bildungsplätzen einen leitenden Faktor in der Bildungspolitik darstellt und auch darstellen soll, ist nicht wirklich geklärt. Beispielsweise hat der FHR in seinem Jahresbericht 1997 ein eindeutiges Plädoyer für eine bildungsnachfragegesteuerte Entwicklung mit einer

83 Als Beispiel kann einerseits auf die Studie über die „entrepreneurial universities“ (Clark 1998) verwiesen werden. Andererseits werden in der OECD-Studie als Beispiele zu den verschiedenen Aspekten regionaler Einbindung angeführt: University of Twente, Warwick University, University of Strathclyde, University of Leeds, University of Joensuu, Polytechnic University of Catalonia, Royal Melbourne Institute of Technology, u.a. (OECD 1999, v.a. Ch.5). Im Hinblick auf den Aufbau von breiteren Antizipationssystemen zum Qualifikationsbedarf befindet sich die Entwicklung im Fluss, in verschiedenen Ländern kann auf umfassende Ansätze verwiesen werden, z.B. Deutschland (http://www.frequenz.net/) oder Finnland (Kekkonen 1998) oder in den Niederlanden (vgl. zusammenfassend Steiner et al 2002). 
grundsätzlichen demokratiepolitischen Begründung abgegeben: „Die durch die öffentlichen Finanzierungsbeiträge zwangsgesteuerte Entwicklung müsste nach politischdemokratischen Grundsätzen sich an der vorerwähnten Entscheidung der Studierwilligen orientieren." (FHR 1998, 27) Unsere Interviews haben auch Hinweise ergeben, dass sich die FH-Entwicklung auf politischer Ebene in den Bundesländern teilweise an der Entwicklung der Bildungsnachfrage, konkret an der sich öffnenden Schere zwischen Bewerbungen und Studienplätzen orientiert. Andere Positionen sehen in einer Orientierung an der Bildungsnachfrage vor allem die Gefahr einer Fehllenkung, solange keine entsprechenden Kosten-Nutzen-Relationen bestehen. Für Österreich werden diese Fragen bisher weitgehend im politischen Raum und ohne ausreichend greifbare empirische Basis mehr oder weniger kontrovers abgehandelt.

Die Diskussion spielt sich im Feld zwischen der vorhandenen und zu erwartenden Neigung der Jugendlichen (und zunehmend auch Erwachsenen) zum Erwerb eines Hochschulabschlusses einerseits und dem gesellschaftlich geforderten und erwünschten Ausmaß der Bildungsbeteiligung ab. Hinsichtlich der erwünschten Beteiligung im Hochschulsystem gab es im Beobachtungszeitraum keine expliziten Zielwerte als politische Planungsgrundlagen. Vor dem Hintergrund der Zielsetzung des offenen Hochschulzuganges für Studienberechtigte spielt die Entwicklung der erwarteten Bildungsnachfrage eine zentrale Rolle. In Planungsdokumenten wird für die Universitäten ab 2005 mit keiner weiteren Erhöhung der Neuzugänge gerechnet (BMBWK 2000), dabei wird eine MaturantInnenquote in der Größenordnung von 50\% und eine Studienanfängerlnnenquote in der Größenordnung von $33 \%$ unterstellt. $^{84}$ Die Entwicklung des FH-Sektors wurde in seiner Größenordnung an die Zugänge zu den Universitäten gebunden, für die Universitäten gibt es jedoch keine entsprechenden quantitativen Zielwerte. ${ }^{85}$ Laut dem österreichischen Forschungs- und Technologiebericht soll „.... mittelfristig eine Steigerung der österreichischen Akademikerlnnenquote auf 20\% erreicht werden“ (BMVIT/BMBWK 2002, III). Der Zugang soll für Berechtigte offen sein, insofern gibt die Entwicklung der Zahl der MaturantInnen einen wichtigen Parameter ab. Da das Universitätssystem jedoch aufgrund der nicht spezifizierten Studienplatzbewirtschaftung auch im Hinblick auf die Studienbedingungen „offen“ ist, ist seine quantitative Entwicklung schwer vorhersehbar.

84 Für die Entwicklung der AkademikerInnenquote resultiert nach diesen Erwartungen ein Anstieg von 7\% im Jahr 2000 auf 14\% im Jahr 2025. Der europäische Durchschnitt tertiärer Abschlüsse liegt bei 13\% (Österreich in dieser Berechnung 6\%); aktuell sind bereits 2 Länder über dem erwarteten Wert von 2025, weitere vier Länder sind nahe daran (13\%). Inklusive der postsekundären Abschlüsse (von 2,4\% 2000 auf 3,9\% 2025) ergibt sich ein Anstieg der Gesamtquote von 9,4\% (2000) auf 17,9\% im Jahr 2025 (12 Länder liegen bereits über diesem Wert, der auch im Zieljahr noch 20\% unter dem aktuellen europäischen Durchschnitt liegen würde) (BMBWK 2000, 8, 53-55).

85 Laut Weißbuch zur Hochschulbildung in Österreich (BMWV 1998, 11) sollte „... bis 2010 der Anteil der Fachhochschul-StudienanfängerInnen auf $25 \%$ aller StudienanfängerInnen erhöht werden. Dies bedeutet bei gleichbleibender StudienanfängerInnenquote eine Steigerung der Fachhochschul-Studienplätze auf ca. 27.000. Ein weiterer Ausbau, um auch dem offenen Zugang näher zu kommen, würde einer erheblichen Steigerung der Finanzmittel bedürfen." 
Hinsichtlich der Entwicklung der Bildungsneigung wird in Dokumenten und auch von ExpertInnen die Position vertreten, dass sich die Neigung zur Aufnahme eines Universitätsstudiums einer Sättigung nähere und lediglich die Fachhochschul-Studiengänge einen gewissen weiteren Zuwachs bringen können (BMBWK 2000). Diese Position wird durch die Ergebnisse der Hochschulplanungsprognose (Dell'Mour/Landler 2002) auf Basis langjähriger Erfahrung mit Fortschreibungen und Modellierungen des Bildungs- und Universitätssystems gestützt.

Die Zahl der MaturantInnen wird nach dieser Prognose bis 2012 absolut steigen, unter der Annahme von stagnierenden bis leicht steigenden Übertrittsraten in die höheren Schulen. Die MaturantInnenquote würde nach den Annahmen von gegenwärtig 38\% bis 2010 auf 44\% steigen und dann bis 2020 etwa auf diesem Niveau stagnieren. Ein wesentlicher Aspekt in diesem Zusammenhang ist die demografische Entwicklung. Bis um 2010 steigen die Geburtenjahrgänge für die höheren Schulen noch leicht und werden dann längerfristig drastisch zurückgehen.

Dies bringt erstens Unwägbarkeiten hinsichtlich der Bildungsbeteiligung, indem mit dem demografischen Aufschwung Kapazitäten in den verschiedenen Bereichen des Bildungswesens geschaffen wurden, deren Nutzung mit dem demografischen Abschwung alternative Entwicklungen erlaubt. Die Schwierigkeit, den Effekt von demografischen Umschwüngen auf die Bildungsbeteiligung einzuschätzen, kann man an den MaturantInnenschätzungen der Hochschulplanungsprognose von 1986 sehen, wo auf zehn Jahre eine Fehlschätzung in der Größenordnung zwischen 5.000 und 10.000 MaturantInnen zu verzeichnen war. Auch die Übertrittsraten der Prognose von 1993 für das Jahr 2000 ins höhere Schulwesen zeigen eine von der Realität abweichende Struktur, indem die Übertritte in die AHS über- und die Übertritte in die BHS wesentlich unterschätzt wurden. Einfache Trendprojektionen der Bildungsbeteiligung in einem anderen Projekt (Lassnigg/Nemeth 1999) haben sehr breite mögliche Korridore demonstriert, zwischen der Minimalannahme konstanter Beteiligungsquoten und der Maximalannahme einer Trendfortschreibung der Bildungsbeteiligung. Im letzteren Fall würde es zu einer dramatischen Umschichtung der Bildungsbeteiligung in Richtung der höheren Schulen kommen. Es stellt sich also die Frage, wie sich die Bildungsbeteiligung im Wechselspiel mit bildungspolitischen Weichenstellungen entwickeln wird, wobei mittelfristig große Freiheitsgrade vorhanden sind.

Zweitens stellt sich aus der Sicht der Beschäftigungsentwicklung und des Qualifikationsbedarfes die Frage, welche Auswirkungen diese demografische relative Verknappung der jüngeren Altersgruppen in der Bevölkerung erwarten lässt. Offensichtlich tut sich hier eine Schere auf, auf die vielfach, insbesondere aus dem Wirtschaftssektor, deutlich zu Recht hingewiesen wird: auf der einen Seite wird bisher die Qualifikationserneuerung und die Höherqualifizierung zu einem großen Teil von den jüngeren und neu in Beschäftigung eintretenden Jahrgängen getragen, was durch die demografische Entwicklung gravierend beeinträchtigt wird - auf der anderen Seite wird 
heute von einem Trend zur Höherqualifizierung im Beschäftigungssystem ausgegangen, der sich in Zukunft vermutlich verstärken wird. Damit muss einerseits die Weiterbildung teilweise die frühere Rolle der Erstausbildung übernehmen und andererseits muss aber mit der Verknappung der jüngeren Jahrgänge ihr Qualifikationspotential maximal auf allen Ebenen ausgeschöpft werden. Welche Konsequenzen aus diesen Entwicklungen für das österreichische Bildungswesen abzuleiten wären, wird nur wenig diskutiert. Die Meinungen im Zuge der bildungspolitischen Diskussion tendieren dazu, Qualitätsverbesserung in den bestehenden Strukturen als Strategie zu präferieren und stehen einer nachhaltigen formalen Höherqualifizierung eher skeptisch gegenüber. ${ }^{86}$

Die Übertritte in das Hochschulsystem sind vor diesem Hintergrund mit weiteren Unwägbarkeiten verbunden. Erstens stellt sich die Frage, welche Auswirkungen die Errichtung des FH-Sektors auf die Bildungsbeteiligung im Hochschulsystem hat und erwarten lässt. Die Hochschulplanungsprognose geht davon aus, dass die Zugänge in den FH-Sektor zur Gänze auf eine Entlastung der Universitäten anrechenbar sind. Dies würde einen Rückgang der Übertrittsquote an die Universitäten zu einem beträchtlichen Teil erklären, nähere Untersuchungen lassen aber an dieser Modellannahme berechtigte Zweifel zu (siehe Abschnitt $3.1 .3 \mathrm{c}$ ) zur Entlastung der Universitäten). Die FachhochschulStudiengänge scheinen doch auch $z u$ einem beträchtlichen Teil zusätzliche Bildungsnachfrage an sich gezogen zu haben, die nicht direkt auf den Universitätszugang anrechenbar ist. Weitere Unwägbarkeiten sind mit der nachhaltigen Wirkung der verschiedenen (relativen) Kostenerhöhungen von Universitätsstudien seit den 1990er Jahren verbunden. Beispielsweise könnte der Einbruch der Universitätszugänge im Studienjahr 2001/02 eher eine kurzfristige und vorübergehende Reaktion darstellen (wie auch teilweise in der Literatur nahegelegt wird) als eine langfristige Verhaltensänderung bedeuten. ${ }^{87}$ Ein dritter Faktor betrifft die strategischen Entscheidungen über die weitere quantitative Entwicklung des FH-Sektors. Ein vierter Faktor betrifft schließlich die Frage späterer Zugänge in das Hochschulsystem im Sinne der Entwicklung einer Strategie des lebensbegleitenden Lernens, in Wechselwirkung mit den Wirkungen einer verstärkten Umsetzung des Bologna-Prozesses auf die Bildungsbeteiligung.

Der life-long-learning-Faktor ist bereits im Befund aus der AbsolventInnenstudie (Hoyer/Ziegler 2002) ersichtlich, indem - neben den nicht-traditionellen Zugängen, die immerhin zusammen ein Viertel ausgemacht haben - ein beträchtlicher Teil der Zugänge in den FH-Sektor nicht unmittelbar nach der Matura, sondern nach einer Periode der Berufstätigkeit, oft in Verbindung mit einem begonnenen Universitätsstudium erfolgte. $\mathrm{Ob}$

86 Vgl. das Papier des BMBWK zur Planung des Studienangebotes (BMBWK 2000, 8, 53-55).

87 Gab es im WS 2001/02 etwa 3.000 StudienanfängerInnen an wissenschaftlichen Universitäten weniger als im Vorjahr (rund -15\%), sind es nach vorläufigen Daten im WS 2002/03 wieder um 1.500 mehr als im Jahr zuvor (etwa $+9 \%)$. 
dies weiter und stärker gefördert wird oder nicht, hätte im Wechselspiel mit den quantitativen Entwicklungsvorgaben wesentlichen Einfluss.

Die potentielle Bildungsneigung kann an der Entwicklung der Bewerberlnnenzahlen bis zu einem gewissen Grad abgelesen werden. Bei verstärkter Ausschöpfung könnte der Zugang offensichtlich deutlich erhöht werden. ${ }^{88}$ Ebenso zeigen die Schätzungen der unterschiedlichen regionalen Ausschöpfungsraten für eine Bildungsbeteiligung der 19- bis 25-Jährigen, dass in den dezentralen Regionen noch ein beträchtliches Potential vorhanden sein könnte.

Die Vergleiche mit anderen Ländern zeigen, dass die Beteiligung im Hochschulsystem durch teilweise sehr weitreichende politische Vorgaben gesteuert wird, die teilweise in politische Gesamtstrategien der Verwirklichung der Informationsgesellschaft eingebunden waren. Das weitestgehende Beispiel ist Finnland, wo eine Hochschulbeteiligungsquote von $70 \%$ angestrebt wird. Auch in Deutschland wird von der Notwendigkeit einer deutlichen Erhöhung der Akademisierung ausgegangen und betont, dass "sämtliche der betrachteten Modellrechnungen den Bedarf an Erwerbstätigen mit Hochschulabschluss unterschätzt“ haben (WR 2002, 27).

\subsubsection{Manifeste bildungspolitische Ziele}

Für den FH-Sektor werden klarere und konkretere bildungspolitische Ziele angegeben, als dies ansonsten in der österreichischen Bildungspolitik der Fall ist. Im Vergleich zum Arbeitsprogramm für die Entwicklung gemeinsamer europäischer Ziele (Europäische Kommission 2002; vgl. auch weiter unten den Abschnitt 3.2 zu den Veränderungen im Umfeld) bedürfen auch diese einer weiteren Konkretisierung. Beispielsweise sind die Indikatoren bzw. Informationsgrundlagen zur Erfassung der Ergebnisse im FH-Sektor in vieler Hinsicht unzureichend und vor allem wurde nur in wenigen Aspekten ein zeitlicher Horizont für die Erreichung der Ziele spezifiziert. Die folgenden manifesten Zielsetzungen können im Zusammenhang mit der Errichtung des FH-Sektors aus den einschlägigen Dokumenten herausdestilliert werden:

a) Verbesserung der Durchlässigkeit des Bildungswesens,

b) Förderung der beruflichen Flexibilität

c) Entlastung der Universitäten

d) Förderung der Aus- und Weiterbildung

$88 \mathrm{Da}$ in der FH-Datenbasis keine Zusammenhänge zwischen der Ausschöpfung und den Abbrüchen erkennbar sind (vgl. Abschnitt 2.2.7) und auch nicht zwischen den angegebenen Selektionsverfahren und der Ausschöpfung, können auch keine unmittelbaren Zusammenhänge zwischen Ausschöpfung und Qualität im Sinne der traditionellen elitären Erwartung einer höheren Qualität bei höherer Selektivität angenommen werden. 
e) Abbau regionaler Disparitäten

f) Strukturbereinigung im postsekundären und tertiären Bildungswesen

Die Erreichung dieser Zielsetzungen wird im Folgenden analysiert.

\section{a) Durchlässigkeit}

Das Ziel der Verbesserung der Durchlässigkeit des Bildungswesens wurde ausdrücklich vor allem im Hinblick auf erweiterte Zugangsmöglichkeiten zum Hochschulsektor formuliert. Einerseits sollten zusätzliche Zugangsmöglichkeiten zur Hochschulausbildung ohne Matura im ersten Bildungsweg geschaffen bzw. erweitert werden, andererseits sollten die Zugangsmöglichkeiten für Berufstätige zur Hochschulausbildung erweitert werden. Speziell wurden Zugangsmöglichkeiten nach einschlägiger beruflicher Qualifikation, definiert für AbsolventInnen der Lehrlingsausbildung und der BMS, vorgesehen.

Der zweite Aspekt der Durchlässigkeit betrifft den Zugang zum Doktoratsstudium an den Universitäten. Dieser Zugang wurde ursprünglich im FHStG generell mit einer einjährigen Verlängerung allgemein ermöglicht, dies wurde in der jüngsten Novelle geändert, indem eine Verlängerung nur noch erforderlich ist, wenn das FH-Studium kürzer als das erforderliche Universitätsstudium ist. Der Eintritt in ein Doktoratsstudium wird individuell geregelt, es muss jedoch eine Betreuung gefunden werden und es werden die entsprechenden Bedingungen formuliert.

Ein spezieller Aspekt der Durchlässigkeit betrifft die Frage der Nachqualifizierung von HTLAbsolventInnen in bestimmten Bereichen, insbesondere um EU-Konformität zu erreichen. In diesem Zusammenhang bildet auch die Frage der Anerkennung von (einschlägigen) HTLAbschlüssen auf die Studienzeit der FH-Studiengänge ein Entwicklungsfeld, das aufgrund seiner Komplexität aus der vorliegenden Studie ausgeklammert wurde.

Schließlich ist insgesamt auch die Frage zu stellen, ob und inwieweit die Errichtung des FHSektors die Chancengleichheit im Zugang zum Hochschulstudium in geschlechtsspezifischer und sozialer Hinsicht erweitert hat.

\section{Geschlechtsspezifische und soziale Durchlässigkeit}

Zur Entwicklung der Durchlässigkeit und Chancengleichheit im österreichischen Bildungswesen gibt es leider keine ausreichenden Untersuchungen, teilweise aufgrund der unzureichenden Datenbasis. Daher kann dieser Thematik in der vorliegenden Untersuchung nur sehr ansatzweise nachgegangen werden. 
- Geschlechtsspezifisch liegt der Anteil der Frauen bei den Zugängen im Universitätssektor insgesamt bereits fast bei $60 \%$; wenn man nur die UniversitätsFachrichtungen speziell betrachtet, in denen es FH-Angebote gibt, liegt der Frauenanteil um gut 10 Prozentpunkte niedriger. ${ }^{89} \mathrm{Im}$ FH-Sektor sind die Frauen auch gegenüber dem Überschneidungsbereich an den Universitäten deutlich unterrepräsentiert, der Abstand hat sich jedoch etwas verringert (vgl. Tabelle 6). In den letzten Jahren ist der Frauenanteil unter den FH-Anfängerlnnen v.a. infolge der Errichtung der Studiengänge im Sozialbereich deutlich gestiegen (WS 2002/03: 41\%), liegt aber immer noch unter dem Anteil im Überschneidungsbereich mit den Universitäten.

Tabelle 6: Vergleich der Frauenanteile an StudienanfängerInnen bzw. Studierenden im Universitäts- und FH-Sektor im Zeitvergleich

\begin{tabular}{|l|c|c|c|}
\hline & $1990 / 91$ & $1994 / 95$ & $2001 / 02$ \\
\hline UNI-Anfängerinnen insgesamt & $48 \%$ & $52 \%$ & $57 \%$ \\
\hline UNI-Anfängerinnen im Überschneidungsbereich & $37 \%$ & $39 \%$ & $46 \%$ \\
\hline \hline FH-Anfängerinnen insgesamt & - & $22 \%$ & $37 \%$ \\
FH-Studierende insgesamt & - & $22 \%$ & $33 \%$ \\
\hline
\end{tabular}

Da der Überschneidungsbereich auf der Basis des FH-Sektors definiert wurde, entspricht bis dato nahezu der gesamte FH-Sektor dem Überschneidungsbereich mit den Universitäten.

Quelle: FHR, Statistik Austria, IHS-Berechnungen

- Eine Analyse der Durchlässigkeit aufgrund des Hochschulzuganges nach dem sozialen Hintergrund der Studierenden kann ansatzweise mit Hilfe der Angaben über den Bildungsstand der Väter aus der Hochschulstatistik durchgeführt werden. Es zeigt sich, dass die Hochschulen immer noch eine beträchtliche soziale Selektivität aufweisen. Diese ist beim Universitätszugang deutlich größer als beim Fachhochschulzugang: der Anteil der "Väter" mit Matura oder höherer Ausbildung in der relevanten Bevölkerungsgruppe (40- bis unter 65jährige männliche Wohnbevölkerung) beträgt etwas über 20\%, der Anteil der UniversitätsanfängerInnen mit Vätern dieses Bildungsstandes liegt jedoch mehr als doppelt so hoch (vgl. Tabelle 7). Die Fachhochschul-Studiengänge sind in dieser Hinsicht deutlich weniger selektiv als die Universitäten: Der Anteil an FHAnfängerInnen, deren Väter über keine Matura verfügen, liegt um mehr als 10 Prozentpunkte höher als an den Universitäten. Ob sich durch die Errichtung des $\mathrm{FH}$ Sektors die soziale Selektivität der Hochschulen insgesamt verringert hat, lässt sich aus diesen Vergleichen nicht direkt erschließen. Es könnte auch teilweise eine

89 Folgende Universitätsstudien wurden in den Überschneidungsbereich mit dem FH-Sektor eingerechnet: ISCEDFBR 34 Business Administration, ISCED-FBR 52 Engineering and engineering trade, ISCED-FBR 48 Computing sowie die ISCED-FBR 21 (Arts), 32 (Journalism and Information), 39 (Social sciences, Business, Law-not spec.) , 42 (Life sciences) , 54 (Manufacturing and processing), 58 (Architecture and building), 81 (Personal services). Siehe auch Abschnitt 3.1.3 c) zur Entlastung der Universitäten. 
schichtspezifische Umverteilung von Studierenden mit Vätern ohne Matura aus dem Universitäts- in den FH-Sektor stattgefunden haben.

Tabelle 7: Verteilung der StudienanfängerInnen an Universitäten und FHStudiengängen nach formalem Bildungsniveau der Väter im Zeitvergleich

\begin{tabular}{|c|c|c|c|c|}
\hline & & $1990 / 91$ & $1994 / 95$ & $2000 / 01$ \\
\hline \multirow[t]{3}{*}{ Vätergeneration in Bevölkerung } & Mit Matura & $17 \%$ & $18 \%$ & $22 \%$ \\
\hline & $\begin{array}{l}\text { Ohne } \\
\text { Matura }\end{array}$ & $83 \%$ & $82 \%$ & $78 \%$ \\
\hline & $\begin{array}{l}\mathrm{N} \text { in } 1.000 \\
(100 \%)^{* *}\end{array}$ & $1.127,8$ & $1.193,4$ & $1.302,1$ \\
\hline \multirow[t]{3}{*}{ Hochschulzugänge insges. ${ }^{* *}$} & $\begin{array}{l}\text { Väter mit } \\
\text { Matura }\end{array}$ & $44 \%$ & $43 \%$ & $45 \%$ \\
\hline & $\begin{array}{l}\text { Väter ohne } \\
\text { Matura }\end{array}$ & $56 \%$ & $57 \%$ & $55 \%$ \\
\hline & $\begin{array}{l}\mathrm{N} \text { in } 1.000 \\
(100 \%)^{* *}\end{array}$ & 18,7 & 18,4 & 23,6 \\
\hline \multirow[t]{3}{*}{ Darunter Universitäten } & $\begin{array}{l}\text { Väter mit } \\
\text { Matura }\end{array}$ & $44 \%$ & $43 \%$ & $47 \%$ \\
\hline & $\begin{array}{c}\text { Väter ohne } \\
\text { Matura }\end{array}$ & $56 \%$ & $57 \%$ & $53 \%$ \\
\hline & $\begin{array}{l}\mathrm{N} \text { in } 1.000 \\
(100 \%)^{* *}\end{array}$ & 18,7 & 17,7 & 19,7 \\
\hline \multirow[t]{3}{*}{ Darunter FH-Studiengänge } & $\begin{array}{l}\text { Väter mit } \\
\text { Matura }\end{array}$ & - & $34 \%$ & $35 \%$ \\
\hline & $\begin{array}{l}\text { Väter ohne } \\
\text { Matura }\end{array}$ & - & $66 \%$ & $65 \%$ \\
\hline & $\mathrm{N}(100 \%)$ & - & $1.709^{x \times x \times}$ & 3.773 \\
\hline
\end{tabular}

*Vätergeneration: 40-65-jährige Männer laut Mikrozensus (Durchschnitt der 4 Quartale)

** gerundet

${ }_{* * * *}^{* *}$ Univ.+FH-Studiengänge

Summe aus 1994/95 und 1995/96

Quelle: IHS-Berechnungen auf Basis der Hochschulstatistik

Gleichzeitig hat unter den Studierenden der Anteil mit Vätern im höheren Bildungssegment seit der Mitte der 1990er Jahre zugenommen, aber auch das formale Bildungsniveau der Vätergeneration ist parallel dazu gestiegen. Eine Möglichkeit, um diese parallele Entwicklung zu kontrollieren, besteht in der Berechnung eines Indikators, der die Überrepräsentation der Studierenden mit Vätern aus dem höheren Bildungssegment im Universitätssektor direkt der Überrepräsentation im FH-Sektor - gemessen anhand der jeweiligen Rekrutierungsquoten ${ }^{90}$ - im Zeitverlauf gegenüberstellt (dieser Indikator macht auch die unterschiedlichen Größenordnungen von Universitäts- und FH-Bereich besser vergleichbar). Die Überrepräsentation ist in dieser Darstellung an Universitäten höher als an FH-

90 Dabei wird der Quotient der StudienanfängerInnen pro 1.000 der Väterbevölkerung mit höherer Bildung/StudienanfängerInnen pro 1.000 der Väterbevölkerung ohne höhere Bildung für beide Sektoren verglichen. 
Studiengängen und sie zeigt in beiden Bereichen - abgesehen von einer Schwankung um 1997 - eine sinkende Tendenz (vgl. Abbildung 30). Der Anteil an Studierenden mit Vätern aus dem höheren Bildungssegment im Vergleich zu den Studierenden aus dem niedrigeren Bildungssegment liegt im Universitätssektor in der Größenordnung zwischen dem Vierfachen und dem Dreifachen, im FH-Sektor zwischen dem Zweieinhalbfachen und dem Zweifachen. Der Verlauf des Indikators im Universitätsbereich deutet nicht direkt auf einen „sozialspezifischen Entlastungseffekt“ durch die Errichtung des Fachhochschulsektors hin.

\section{Abbildung 30: Verhältnis der Rekrutierungsquoten (Väter mit versus Väter ohne Matura) an Universitäten und FH-Studiengängen, 1990 bis 2000 \\ Faktor, um den StudienanfängerInnen, deren Vater Matura hat, überrepräsentiert sind, gemessen am Bildungsniveau der Vätergeneration (40 - 65 Jährige) laut Mikrozensen}

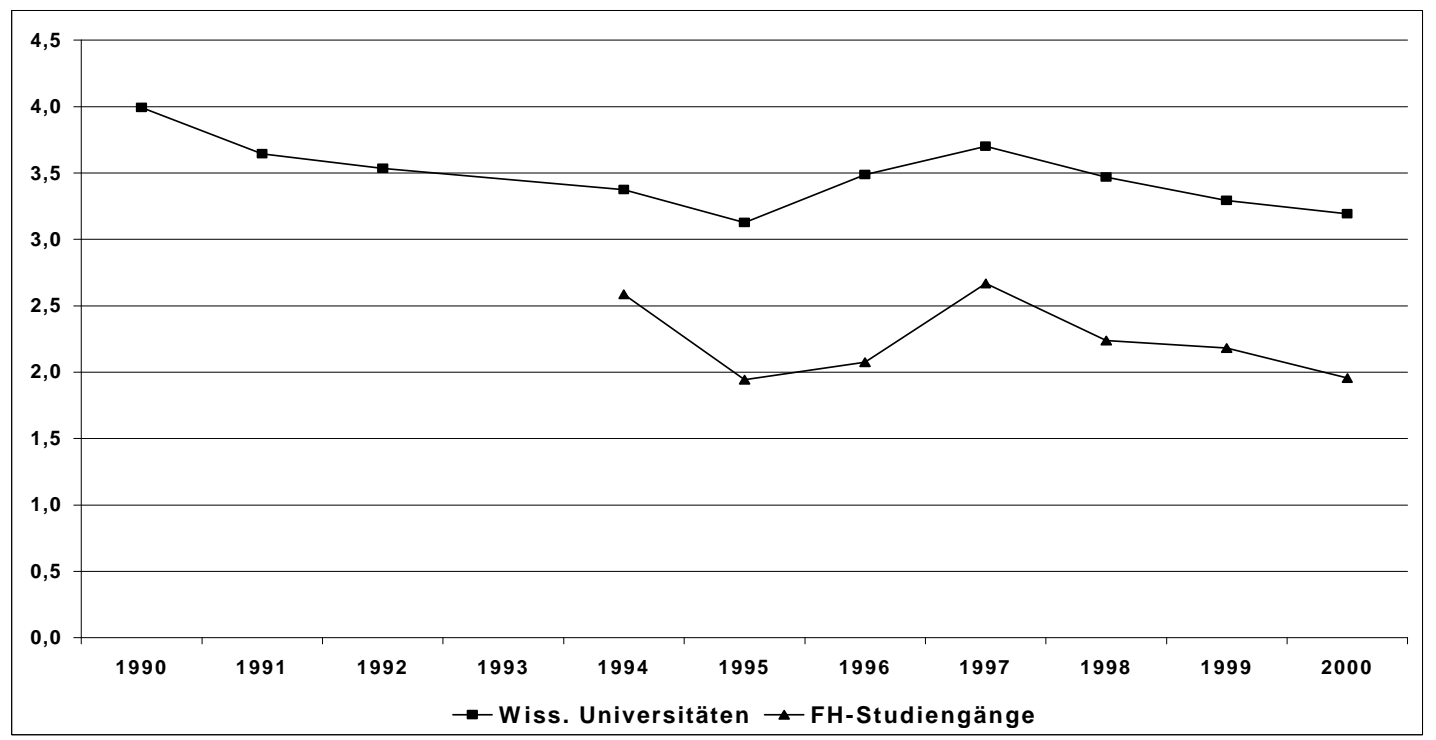

Quelle: FHR, Statistik Austria, IHS-Berechnungen

Viele Erhalter unterstützen die Durchlässigkeit durch eigene Sozialleistungen für die Studierenden. Am häufigsten (fast $70 \%$ ) werden StudentInnenheime und Mensen angeboten, fast ein Drittel haben eigene Stipendiensysteme angegeben und ein kleinerer Anteil leistet Ersatz für die Studienbeiträge bzw. hat dafür Sponsoren gefunden (vgl. Abbildung 31).

Beispiele für spezielle Formen der Förderung sind die folgenden: Landesstipendium, Stipendiensystem des Fördervereines für eine Anzahl von Auslandsstudierenden, Zuschüsse zu Exkursionen, 24-Stunden Zugang zu EDV- und anderen Ressourcen, Essensmarken für sozial bedürftige Studierende, Prämierung von Diplomarbeiten und Preise für soziales Engagement, Veranstaltungen, Alumni-Club, Förderung der Studierendenvertretung bzw. von Studierendenplattformen. 


\section{Abbildung 31: Leistungen der FH-Erhalter für FH-Studierende, 2001}

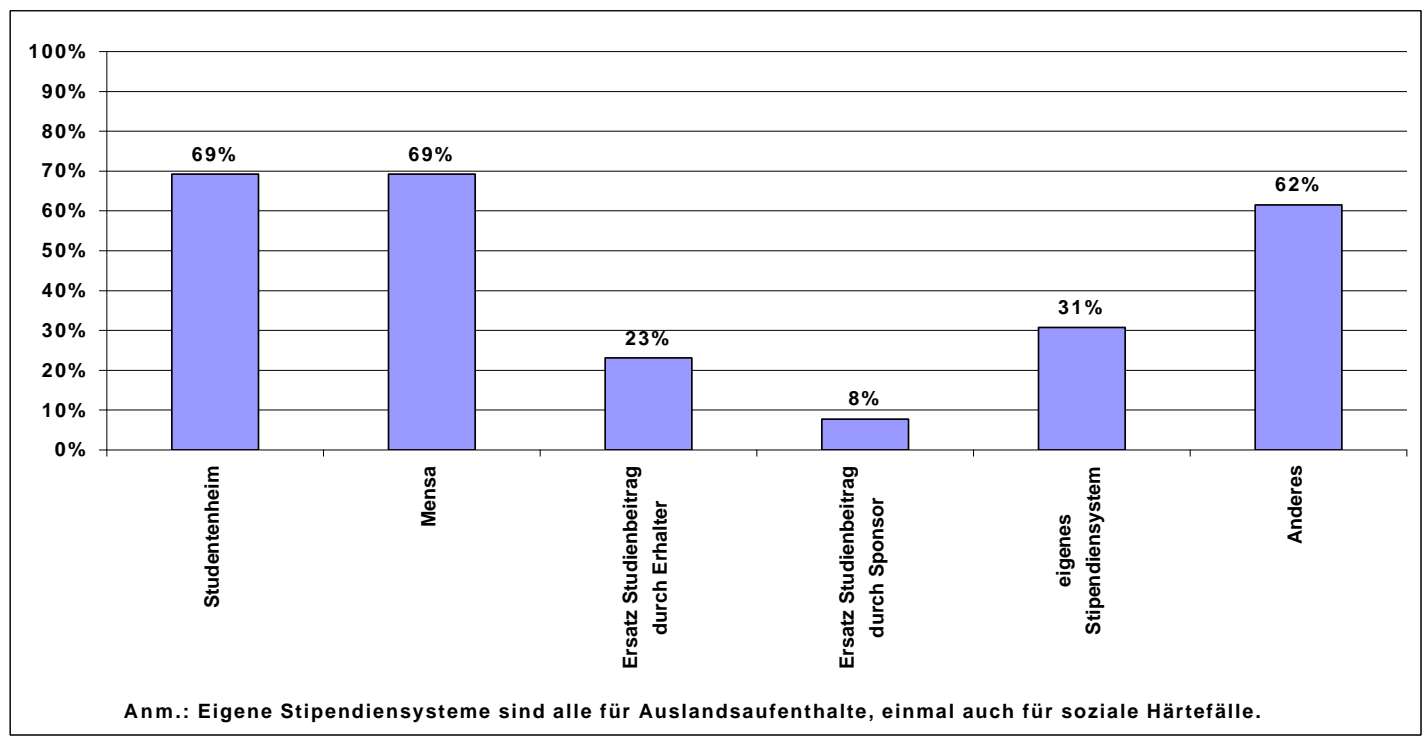

Quelle: Befragung der Erhalter

\section{Durchlässigkeit für nicht-traditionelle Studierende}

Im Zuge der neuen europäischen und internationalen bildungspolitischen Priorität der Entwicklung des lebensbegleitenden Lernens hat die Zugänglichkeit und Durchlässigkeit der Bildungssysteme insgesamt eine neue Bedeutung erlangt. Die Zugänglichkeit durch die Schaffung attraktiver Bildungsmöglichkeiten, unterstützt durch die Anerkennung nicht formal erworbener Kompetenzen und durch entsprechende finanzielle Anreize und Förderungen ist eine der drei grundlegenden Zieldimensionen der neuen europäischen Bildungspolitik seit Lissabon.

In Österreich wurde der „zweite Bildungsweg“ vor allem im formalisierten Schulwesen schon seit langem angeboten und aufgebaut; mit der Studienberechtigungsprüfung und der Berufsreifeprüfung sowie Stipendienregelungen wurden auch Instrumente und Möglichkeiten geschaffen. Diese haben jedoch quantitativ kein besonders hohes Ausmaß erreicht. Eine spezielle Möglichkeit bot der offene Hochschulzugang und die liberale Studienplatzbewirtschaftung für MaturantInnen, indem eine gewisse Vereinbarkeit von Berufstätigkeit und Regelstudium gegeben war. Dies war jedoch erkauft durch niedrige Studienintensität, geringe inhaltliche Bindung an die Studien und insbesondere durch lange Studienzeiten (schon am Anfang der neunziger Jahre wurde geschätzt, dass etwa ein Viertel der „Brutto“-Studiendauer direkt auf Erwerbstätigkeit zurückgeführt werden kann; Lassnigg 1991, vgl. auch Lassnigg et al. 2000, Pechar/Wroblewski 1998). 
Die Anerkennung von nicht formal erworbenen Kenntnissen ist in Österreich zwar für bestimmte Bereiche ziemlich entwickelt, die Anerkennungsregelungen decken jedoch nur einen Teil des gesamten Spektrums an Abschlüssen und Qualifikationen ab und sind meist stark formalisiert und mit Ausbildungsgängen verbunden. ${ }^{91}$

Durch das FHStG wurden ausdrücklich weitere Möglichkeiten zur Verbesserung der Durchlässigkeit ins Auge gefasst, diese wurden auch im Rahmen der Entwicklungsplanung unterstrichen. Insbesondere die Sozialpartner haben den Aspekt der Durchlässigkeit stark betont. Auch der FHR hat diesem Aspekt von Anfang an Bedeutung beigemessen und bereits im Jahr 1996/97 wurde die Thematik der berufsbegleitenden Studiengänge im Schwerpunktprogramm des FHR als einer der vier Jahres-Schwerpunkte in den Vordergrund gerückt.

Die Förderung der Durchlässigkeit ist nach zwei verbundenen, aber unterschiedlichen Aspekten zu differenzieren: (a) Schaffung von alternativen Möglichkeiten der Hochschulberechtigung gegenüber dem Erwerb der Matura im ersten Bildungsweg und (b) Schaffung von Möglichkeiten des Erwerbes von zusätzlichen formalen Bildungsabschlüssen während der Berufstätigkeit.

Beide Aspekte spielen im FH-Sektor eine Rolle. Empirisch ist die Überschneidung der beiden Aspekte nicht sehr groß. Die Ergebnisse der AbsolventInnenstudie (Hoyer/Ziegler 2002) erlauben ein stilisiertes Bild der Größenordnungen: Drei Viertel der AbsolventInnen haben ihr FH-Studium in "Normalform" (also weder berufsbegleitend noch mit nichttraditionellem Zugang abgeschlossen). Immerhin ein Viertel der Studierenden ist einer der beiden Formen von Förderung der Durchlässigkeit zuzurechnen: darunter ist die größte Gruppe jene, die berufsbegleitend mit traditioneller Hochschulberechtigung studiert hat, die zweitgrößte Gruppe hat in Normalform mit nicht-traditionellem Hochschulzugang studiert; bei einer sehr kleinen Gruppe sind die beiden Förderformen kombiniert worden (vgl. Abbildung 32; zur Entwicklung der nicht-traditionellen Zugänge vgl. auch den Abschnitt 2.2.5).

Beim nicht-traditionellen Zugang dominieren klar die formalisierten Formen der Berufsreifeprüfung und der Studienberechtigungsprüfung. Andere, individualisierte Formen haben nur ein sehr geringes Ausmaß.

91 Siehe den österreichischen Länderbericht und den Hintergrundbericht zum EU-Memorandum über das lebenslange Lernen (http://www.lebenslangeslernen.at/ > Ergebnisse) sowie die Protokolle und Berichte zur gesamtösterreichischen ExpertInnentagung und $\mathrm{zu}$ den Koordinationsworkshops (http://www.lebenslangeslernen.at// > Forum \& Workshops). 


\section{Abbildung 32: Kombination von nicht-traditionellem Zugang und berufsbegleitender Studiengangsform bei FH-AbsolventInnen bis 2001}

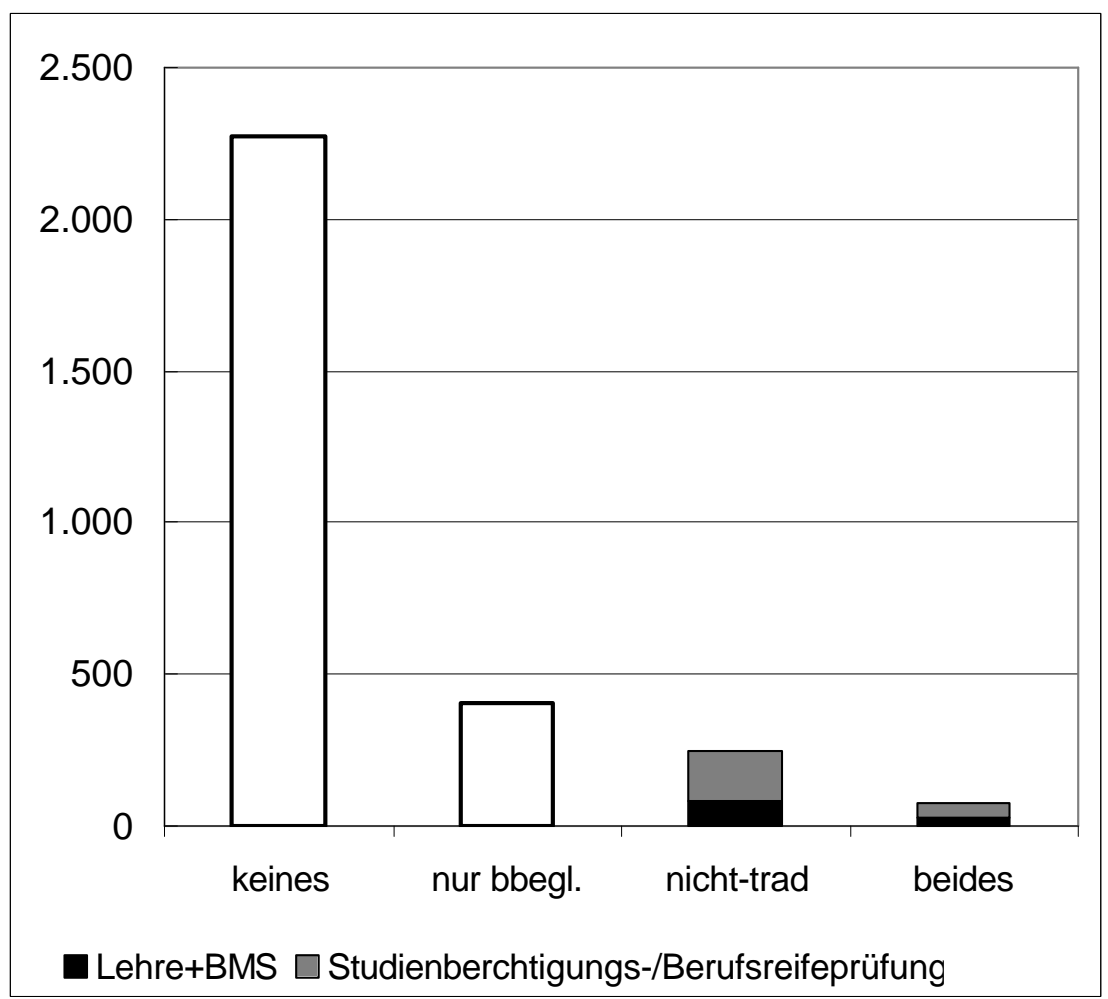

Quelle: Hoyer/Ziegler 2002

Maßnahmen zur Förderung der Zugänglichkeit für nicht-traditionelle Studierende wurden mit der Errichtung des FH-Sektors mehr oder weniger von Anfang an gesetzt (gleich zu Beginn waren 10\% der Studierenden ohne traditionelle Studienberechtigung ${ }^{92}$ und es wurden Vorbereitungslehrgänge errichtet, 1994/95 von vier Erhaltern, 1995/96 bereits von sieben Erhaltern). Parallel wurden zusätzliche formalisierte Möglichkeiten des Zuganges auf gesetzlicher Ebene mit der Berufsreifeprüfung geschaffen. Vor allem letztere scheint zusätzliche Formen der Anerkennung von nicht im formalen Bildungswesen erworbenen Qualifikationen bis zu einem gewissen Grad zu verdrängen. Eine erste Evaluierung hat den raschen Zulauf zu den Vorbereitungslehrgängen dokumentiert (1999 bereits über 4.000 TeilnehmerInnen; Klimmer/Schlögl 1999).

Auch bei den Vorbereitungslehrgängen ist eine Tendenz zur Formalisierung festzustellen. Ein pragmatisches Problem bestand z.B. in der Frage der Koppelung zwischen der Vorbereitung auf die Studienberechtigung und der tatsächlichen Aufnahme in die

92 Der Standort Wels war ein Vorreiter: 1994/95 hat die Hälfte aller österreichischen FH-Anfängerlnnen mit Lehre oder BMS-Vorbildung in Wels begonnen. Diese Gruppe bildete 35\% der Welser Anfängerlnnen, gemeinsam mit $40 \%$ von einer facheinschlägigen BHS. 
Studiengänge im Wettbewerb, vor allem wenn die Vorbereitung in externen Lehrgängen stattgefunden hat. Als Lösung wurde seitens des FHR vorgeschlagen, die Vorbereitungslehrgänge nicht allein dem Ziel der Vorbereitung auf die FH-Zulassung zu widmen, sondern in diesen Lehrgängen gleichzeitig auch verwertbare Qualifikationen zu vermitteln.

Die Frage der Zugänglichkeit für Lehr- oder BMS-AbsolventInnen ist trotz der vorhandenen Ansätze nicht entsprechend den ursprünglichen Erwartungen in dem Sinne gelöst worden, dass für diese Gruppe ein nachhaltiger Zugang zu einem Hochschulstudium geschaffen worden wäre. Dies scheint aber stärker mit Fragen der Gestaltung des Bildungssystems zusammenzuhängen als mit individualisierten Maßnahmen oder Förderungen. Der Kern des Problems besteht vermutlich in den vorhandenen Distanzen zwischen den Ausbildungszielen und Anspruchsniveaus der verschiedenen Bildungsgänge und insbesondere auch in der Dichotomie von Theorie und Praxis zwischen Lehrlingsausbildung und Vollzeitschule bzw. zwischen den mittleren und höheren Schulen. ${ }^{93}$

Die Schweiz hat in dieser Frage ein anderes Modell verfolgt, indem der FH-Sektor in seinem Aufbau grundsätzlich auf der Lehrlingsausbildung aufgesetzt wurde, einerseits mit dem Ziel, weiterführende Möglichkeiten für AbsolventInnen der Berufslehre zu schaffen, andererseits auch mit dem Ziel, das Niveau der Lehre zu heben. Die Berufsmatura sollte daher den „normalen“ Zugang zu einem FH-Studium darstellen. Die Zahl der Berufsmatura-Abschlüsse ist in den 1990er Jahren rasch gestiegen und nahezu die Hälfte der Neu-Eingetretenen in den FH-Sektor hat den Zugang über die Berufsmaturität erlangt (vgl. auch Abschnitt 2.3). ${ }^{94}$ Um mit der allgemeinbildenden Hochschulberechtigung im FH-Sektor aufgenommen werden zu können, ist zumindest ein Praxisjahr erforderlich. Um diese Schnittstelle gibt es Diskussionen, insbesondere werden auch teilweise die Vorkenntnisse als unzureichend kritisiert, aber „Die Berufsmaturität ist und muss der Hauptzubringer für das Studium an einer Fachhochschule bleiben“ (EFHK 2000, 38).

93 Diese Distanzen werden durch die Ergebnisse der PISA-Studie in den Lese-, Mathematik- und Naturwissenschafts-Kompetenzen beim Zugang der 16-Jährigen zu den verschiedenen Schulbereichen ansatzweise illustriert (wo die Mittelwerte der SchülerInnen der Berufschulen und BMS bei $80-90 \%$ der Schülerlnnen der höheren Schulen liegen), gleichzeitig wurde aber auch die Selektivität des Schulwesens demonstriert, indem sehr große individuelle Streu- und Überschneidungsbereiche der Leistungen in den verschiedenen Schultypen bestehen (die Werte der besten 5\% der SchülerInnen der Berufschulen, der BMS und auch der Pflichtschulen liegen in allen drei Testbereichen über dem Mittelwert der höheren Schulen; vgl. Haider/Reiter 2001, Kap.3). In der internationalen Auswertung wird dieser Unterschied zwischen den Schulen unterstrichen: „Österreich und Deutschland sind Länder, in denen vom durchschnittlichen wirtschaftlichen, sozialen und kulturellen Status der Schulen ein erheblicher Einfluss auf die Schülerleistungen ausgeht."(OECD 2001, 238). Eine zentrale Frage wäre, wie sich diese Anfangsdistanzen zwischen den Schulbereichen im Laufe der Jahre weiterentwickeln.

94 EFHK (2000), 37; umgekehrt ist auch etwa die Hälfte der AbsolventInnen mit Berufsmaturität in den FH-Sektor übergetreten. 
In Österreich wurden auch Maßnahmen zum zweiten Aspekt der Durchlässigkeit für nichttraditionelle Studierende, der Förderung der Zugänglichkeit neben Berufstätigkeit bereits sehr bald gesetzt. Die ersten berufsbegleitenden Studiengänge wurden bereits 1996/97 begonnen und ab 1997 wurde auch an einem Projekt zur Nutzung von Fernstudien im Rahmen der berufsbegleitenden Studiengänge gearbeitet. Ab 1997 gab es erste "gemischte“ Lehrgänge, die in Normalform und in berufsbegleitender Form durchgeführt werden.

Im Rahmen der problemzentrierten Interviews ${ }^{95}$ wurden die berufsbegleitenden Studiengänge als einer der ersten Schwerpunkte durch den FHR untersucht. Es wurden im FHR-Jahresbericht 1998 einige Probleme herausgearbeitet (Schwierigkeiten bei der Entwicklung eines stabilen Lehrkörpers; daher gab es teilweise Probleme bei der Koordinierung der Lehrveranstaltungen; die Präsenz am Wochenende schaffte hohe Belastungen für die Studierenden). Es werden vor allem zwei Problemschwerpunkte hervorgehoben: die Organisation und die regionale Zugänglichkeit. Als Lösungen wurden der teilweise Einsatz der gemischten Form von Studiengängen und die Nutzung von Fernstudienelementen empfohlen. Aufgrund der Mobilitätsbarrieren sollten berufsbegleitende Angebote nur in Ballungsgebieten organisiert werden.

Prisching (2002) äußert große Bedenken hinsichtlich der Durchsetzung eines ausreichenden akademischen Niveaus in den berufsbegleitenden Studiengängen. Die AbsolventInnenstudie (Hoyer/Ziegler 2002) zeichnet ein Bild, demzufolge die berufsbegleitenden Studiengänge oft für Berufstätige in gehobenen Positionen die Möglichkeit geschaffen haben, einen akademischen Abschluss zu erlangen und dadurch auch beruflich und einkommensmäßig weiterzukommen. Überwiegend wird das Studium seitens der Arbeitgeber positiv gewertet oder unterstützt, in einem beträchtlichen Teil der Fälle versuchen die Studierenden aber auch, durch das Studium eine Plattform für Mobilität bzw. Arbeitgeberwechsel aufzubauen (etwa ein Fünftel der AbsolventInnen hat eine mehr oder weniger starke Informationszurückhaltung gegenüber ihrem Arbeitgeber geübt).

\section{Durchlässigkeit zum Doktoratsstudium}

Die Durchlässigkeit zum Doktoratsstudium ist im Prinzip gegeben, es werden jedoch in der Realität große Hindernisse festgestellt, die durch die neue vereinfachte Regelung anscheinend abgeschwächt wurden. Laut AbsolventInnenbefragung wird diese Form der Durchlässigkeit nur sehr wenig wahrgenommen (1,7\%, darunter $0,4 \%$ als Vollzeitstudium). Dabei muss jedoch berücksichtigt werden, dass die Zeit seit dem Studienabschluss bei den meisten Antwortenden sehr kurz war. Zukünftige Absichten sind nicht ausdrücklich ausgewiesen, der Weiterbildungsbedarf (worunter auch Doktoratsstudien fallen mögen)

95 Vgl. zu diesem Instrument der Qualitätssicherung die Darstellung in Kapitel 7.1.2). 
wurde sehr hoch angegeben, ist jedoch nicht sehr klar interpretierbar. In der IBWAbsolventInnenbefragung (Wimmer 2000) der ersten 1.500 AbsolventInnen (die jedoch aufgrund eines geringeren Rücklaufes weniger sichere Ergebnisse produziert) haben 38\% die Absicht eines späteren Doktoratsstudiums eindeutig ausgeschlossen, 33\% haben sich diese Option mit einer eher abschlägigen Tendenz („eher nicht“) offengelassen, die übrigen $30 \%$ haben eine positive Antworttendenz, darunter 19\% eher ja und immerhin 11\% der AbsolventInnen haben geantwortet, dass sie das „fix“ vorhätten. ${ }^{96}$

Die Zählung der Studierenden mit FH-Abschluss im Doktoratsstudium weist im Durchschnitt einen stabilen Zuwachs um 40 DoktorandInnen aus, mit einem Schwerpunkt in den Sozialund Wirtschaftswissenschaften (vgl. Tabelle 8). Die Gesamtzahl liegt 2001/02 bei weniger als $3 \%$ der kumulierten FH-AbsolventInnen bis $2000 / 01 .{ }^{97}$

Tabelle 8: Studierende im Doktoratsstudium mit FH-Abschluss, STJ 1997/98 STJ 2001/02

\begin{tabular}{|l|ccccc|}
\hline & $\begin{array}{c}\text { STJ } \\
1997 / 98\end{array}$ & $\begin{array}{c}\text { STJ } \\
1998 / 99\end{array}$ & $\begin{array}{c}\text { STJ } \\
1999 / 00\end{array}$ & $\begin{array}{c}\text { STJ } \\
2000 / 01\end{array}$ & $\begin{array}{c}\text { STJ } \\
2001 / 02\end{array}$ \\
\hline Sozial- und Wirtschaftswiss. & 7 & 27 & 45 & 105 & 76 \\
\hline Philosophie / Naturwiss. & & & & 1 & 5 \\
\hline Technische Wiss. & 3 & 18 & 42 & 68 & 44 \\
\hline Bodenkultur & & & 2 & 2 & 2 \\
\hline Naturwissenschaften & & & & 2 & \\
\hline Philosophie & & & & & 5 \\
\hline Insgesamt & 10 & 44 & 89 & 178 & 132 \\
\hline Zuwachs & & +34 & +45 & +89 & -46 \\
\hline
\end{tabular}

Studienjahr: Jeweils Durchschnitt aus Winter- und Sommersemester.

Studierende: In- und AusländerInnen.

Quelle: BMBWK

Sowohl Hoyer/Ziegler (2002, 72-73) als auch InterviewpartnerInnen der vorliegenden Studie haben über große Probleme der realen Zugänglichkeit von Doktoratsstudien berichtet. Die Probleme reichen von der Suche nach einer Betreuungsperson über die zusätzlichen Erfordernisse (z.B. WU Wien 40 Semesterwochenstunden) bis zu den Schwierigkeiten von

96 Hochgerechnet auf die AbsolventInnenzahl wäre die Absolutzahl der fixen Anwärterlnnen etwas mehr als 150 gewesen, laut der späteren AbsolventInnenstudie haben jedoch absolut nur etwa 50 AbsolventInnen ein derartiges Studium begonnen. Auch nach den Bestandszahlen der DoktoratsstudentInnen liegt der Anteil in der Größenordnung von $5 \%$.

97 Die Zahl der kumulierten AbsolventInnen liegt bei 5.038 bis 2000/01. Wenn man von einem verzögerten Zugang ausgeht, wäre die Rate mit etwa 5\% der 3.020 AbsolventInnen bis 1999/00 immer noch deutlich unter der Erwartung aufgrund der IBW-Befragung Wimmer 2000. 
Anrechnungen. Der Deutsche Wissenschaftsrat hat, nachdem seit 1994 ein Zugang zur Promotion für FH-AbsolventInnen grundsätzlich möglich ist, sehr ähnliche Probleme zwischen Universitäten und Fachhochschulen wahrgenommen. „Die in den Promotionsordnungen vorgesehenen Regelungen bergen die Gefahr, dass hervorragende FachhochschulabsolventInnen auf eine Promotion verzichten." (WR 2000, 127). Als mögliche Quelle von Problemen wird der Mangel an institutionalisierten Beziehungen erwähnt und als Lösungen werden vom Wissenschaftsrat einerseits stärker individualisierte Möglichkeiten der Anrechnung und Feststellung von Defiziten und andererseits das Modell der „kooperativen Promotion“ zwischen Universität und Fachhochschule vorgeschlagen (WR 2000, 54-56, 127-128).

\section{Nachdiplomierung}

Die Nachdiplomierung betrifft eine spezielle Frage der Durchlässigkeit zu bestimmten beruflichen Berechtigungen, die mit der EU-Konformität der Diplome zusammenhängt und vor allem für HTL-Ingenieure in bestimmten Bereichen gilt. Das Problem wurde durch die Zweite Anerkennungsrichtlinie abgeschwächt, bleibt aber in bestimmten Bereichen aufrecht. Durch die zielgruppenspezifischen Studiengänge wird dies gelöst. Vom Umfang her spielen diese jedoch eine kleine Rolle.

Nicht verwechselt werden darf diese Form der Nachdiplomierung mit dem schweizerischen FH-Sektor, wo neben 220 Studiengängen in der Erstausbildung 140 anerkannte Nachdiplomstudien angeboten werden, welche jedoch in der Regel auf einem Hochschuldiplom aufbauen und insbesondere dem Erwerb neuer (und damit zusätzlicher) Qualifikationen dienen. ${ }^{98}$

\section{b) Förderung der beruflichen Flexibilität}

Das Ziel der Förderung beruflicher Flexibilität soll einerseits durch die verbesserte Durchlässigkeit erreicht werden, andererseits ist die geforderte Breite der Studiengangsprofile ein wesentlicher Garant beruflicher Flexibilität.

Etwa die Hälfte der AbsolventInnen berufsbegleitender Studiengänge (Hoyer/Ziegler 2002, Kap. VII) haben diese mit der Erwartung eines Branchenwechsels begonnen, ein Drittel (Mehrfachangaben) äußerte verbesserte Möglichkeiten auf dem Weg zu beruflicher Selbständigkeit, 40\% haben in der Zeit nach dem Abschluss einen Arbeitgeberwechsel versucht und $28 \%$ haben einen solchen in der nicht allzu langen Zeit seit dem Abschluss

98 Vgl. http://www.bbt.admin.ch/d/index.htm. 
bereits realisiert. Neben dem bereits erwähnten Aspekt des formalen „upgradings“ für Aufstiegszwecke spielt der Aspekt der Verbesserung der individuellen beruflichen Flexibilität und Mobilität in den berufsbegleitenden Studiengängen offensichtlich eine wesentliche Rolle.

Die Spezifität der Studiengangsprofile ist ein vieldiskutierter Bereich im FH-Sektor; zu dem auch im Rahmen der Interviews überwiegend kritische Aussagen zu verzeichnen waren. Man kann unterscheiden zwischen der faktischen Ebene (wie breit oder wie schmal sind die vorhandenen Studiengänge einzuschätzen?) und der normativen Ebene (wie breit oder wie schmal sollen die Studiengänge angesichts bestimmter Erwartungen oder Kriterien sein?).

Auf der faktischen Ebene kann man die Breite eines Studienganges ansatzweise an der Vielfalt der verwandten Studienrichtungen der Universitäten bzw. an der Größe des Feldes von Wirtschaftsklassen messen, auf die die Studiengänge bezogen sind. Man muss bei diesen Vergleichen natürlich vorsichtig sein, da auch die Referenzkategorien (Studienrichtungen und Wirtschaftsklassen) unterschiedliche Breite aufweisen. ${ }^{99}$ Eine zweite Möglichkeit besteht darin, die fachlichen Profile der Studiengänge mit denen in anderen Ländern zu vergleichen.

Auf der normativen Ebene hängt die Bewertung von verschiedenen Kriterien ab, wobei - wie in vielen anderen Bildungsbereichen - Unterschiede und Konflikte auftreten, je nachdem, ob man die Frage von der Angebotsseite (Studierende) oder von der Nachfrageseite am Arbeitsmarkt (Unternehmen) her betrachtet. Ein erster Faktor, der oft genannt wird, ist die Erwartung hinsichtlich der unmittelbaren Einsetzbarkeit bzw. der erforderlichen betrieblichen Einschulungszeiten der AbsolventInnen. Dieser Faktor wird oft als „Praxisnähe“ verstanden: Je spezialisierter die Ausbildung, eine umso direktere Einsetzbarkeit oder Verwertbarkeit wird erwartet. Hier besteht jedoch ein „trade-off“ mit der Breite oder Enge des Feldes, in dem die Einsetzbarkeit gegeben ist. Bedarf tritt immer konkret in Form von effektiver Nachfrage auf, gleichzeitig ändert sich diese jedoch auch rasch. Von öffentlicher Ausbildung wird neben dieser Verwertbarkeit aber auch eine gewisse Nachhaltigkeit erwartet, d.h. eine gewisse Resistenz der Abschlüsse gegenüber den dynamischen Veränderungen der Nachfrage. Diese Spannung zwischen Spezialisierung und Flexibilität wird bereits seit einiger Zeit gesehen, aber es hat sich als nicht einfach erwiesen, Lösungen mit nachweisbar besseren oder schlechteren Ergebnissen zu finden. ${ }^{100}$ Dies liegt daran, dass die Zusammenhänge zwischen den Kompetenzen und dem beruflichen Einsatz bisher nur schlecht objektivierbar sind bzw. auch kein Konsens über Mess- und Bewertungsverfahren besteht.

99 Als Indikatoren für die Breite können, wiederum in vorsichtiger Weise, die jeweiligen Studierenden- bzw. Beschäftigtenzahlen herangezogen werden.

100 Die Entwicklungen im Zusammenhang mit dem Kompetenzbegriff haben hier eine viel höhere Komplexität der Problematik aufgedeckt, als sie in der Praxis oft gesehen wird; vgl. Lassnigg/Mayer 2001; Rychen/Sagalnik 2001. 
In der Literatur wie auch in unseren Interviews scheint es hier durchaus nicht unbeträchtliche Widersprüche und unzutreffende Zuschreibungen zwischen den Akteuren zu geben, indem dem Unternehmenssektor aus dem Bildungswesen und aus dem politischen Bereich eine hohe Erwartung auf unmittelbare Einsetzbarkeit zugeschrieben wird, die durch Aussagen aus dem Unternehmenssektor gar nicht gedeckt wird. ${ }^{101}$

Mit der Einsetzbarkeit hängt auch die Erwartung über Einsatzbereiche für die AbsolventInnen zusammen, die bei einem neu errichteten Ausbildungssektor in hohem Maße zunächst offen ist und sich erst im praktischen Wechselspiel zwischen Angebot und Nachfrage im Laufe der Zeit festigt. Der Angebotsseite kommt dabei sicherlich eine hohe Definitionsmacht bei der Positionierung zu, die sich jedoch im Wechselspiel mit Umfeldfaktoren realisiert. Ein wesentlicher Faktor dabei ist der konkrete Mix an Aufgabenzuschreibungen, der den Ausbildungsgängen zugrundegelegt wird (Lehrprofil, F\&E, Dienstleistungen), wie auch die Ausdeutungen derselben. Beispielsweise kann die Verflechtung der Studienaktivitäten mit den anderen Aufgaben (Einbindung der Studierenden in Forschung oder andere Leistungen) sehr unterschiedlich realisiert werden (vgl. dazu die Zusammenfassung und Diskussion bisheriger Befunde zum beruflichen Einsatz der $\mathrm{FH}$ AbsolventInnen und zur Bewertung ihrer Qualifikationen seitens des Unternehmenssektors im Anhang, Kapitel 11.1.1).

Ein weiterer Faktor der beruflichen Flexibilität betrifft die Art und Weise, wie ein FH-Lehrgang in eine individuelle Studien- und Berufskarriere eingebaut ist, auf welchen Vorerfahrungen er aufbaut und in welche weiteren Erfahrungen er mündet. Ein hochspezialisierter Lehrgang wird beispielsweise andere Wirkungen für die berufliche Flexibilität haben, wenn er auf einer breiten Grundausbildung bzw. einer umfassenden Berufserfahrung aufbaut, als wenn er eine bereits spezialisierte Ausbildung fortsetzt. Daher kann die Wirkung der Breite oder Enge der Studiengangsprofile für die berufliche Flexibilität nicht allein von der Gestaltung der Profile her eingeschätzt werden, sondern erfordert auch eine Analyse der Pläne und Erwartungen der Studierenden. Diese Frage wird insbesondere in dem Maße relevant, in dem FH-Studien im Bereich der Weiterbildung absolviert werden.

\section{Abschätzungen zur Spezialisierung}

Die Angaben der Studiengänge darüber, auf welche Wirtschaftsbereiche ${ }^{102}$ ihre Ausbildung bezogen ist, ergab den häufigsten Wert in der Größenordnung von 6 bis 10 der 33 Wirtschaftsbereiche. Bei fast einem Viertel der Studiengänge kann man einen breiten

\footnotetext{
101 Vgl. z.B. Lang/Finder (2000), IBW (1998); Aussagen von Unternehmensvertreterlnnen in Interviews haben immer wieder auf die Bedeutung der überfachlichen Kompetenzen hingewiesen; auch die AbsolventInnen haben hier Diskrepanzen betont (Hoyer/Ziegler 2002).

102 NACE-Zweisteller, etwas zusammengefasst auf 33 Kategorien.
} 
Einsatzbereich annehmen (darunter haben etwas weniger als ein Fünftel alle Wirtschaftsbereiche angegeben), andererseits sind etwa ein Sechstel der Studiengänge auf eine bis fünf NACE-Kategorien spezialisiert. Nach Fachrichtungsgruppen ist dieser Spezialisierungsgrad unterschiedlich verteilt (vgl. Tabelle 9). In den wirtschaftlichen Fachrichtungen liegt eine gewisse Polarisierung vor, indem sowohl die breiten als auch die spezialisierten Studiengänge überrepräsentiert sind; die technisch/ ingenieurwissenschaftlichen Studiengänge liegen zu über $80 \%$ im mittleren Bereich, die naturwissenschaftlichen (IKT) sind auf einen eher breiten Wirtschaftsbereich bezogen, die übrigen (Gesundheit, Soziales, Medien und Design) eher spezialisiert.

Tabelle 9: Breite und Spezialisierung der Studiengänge in Bezug auf Wirtschaftsbereiche, 2001

\begin{tabular}{|l|c|c|c|c|}
\hline & $\begin{array}{c}\text { Breit } \\
\text { (21-33 Wirtschaftsbereiche) }\end{array}$ & $\begin{array}{c}\text { Mittel } \\
(6-20 \text { Wirtschaftbereiche })\end{array}$ & $\begin{array}{c}\text { Spezialisiert } \\
(1-5 \text { Wirtschaftsbereiche })\end{array}$ & N \\
\hline Alle Studiengänge & $24 \%$ & $60 \%$ & $16 \%$ & 67 \\
\hline Wirtschaftliche StG & $\mathbf{5 0 \%}$ & $30 \%$ & $\mathbf{2 0 \%}$ & 20 \\
\hline Technische StG & $7 \%$ & $\mathbf{8 2} \%$ & $11 \%$ & 28 \\
\hline Naturwiss.- IKT StG & $\mathbf{3 3} \%$ & $\mathbf{6 7 \%}$ & $0 \%$ & 12 \\
\hline Andere & $0 \%$ & $43 \%$ & $\mathbf{5 7 \%}$ & 7 \\
\hline
\end{tabular}

Quelle: Online-Befragung der Studiengangsleitungen

Nach den Verwandtschaften zu Studienrichtungen an den Universitäten wurde in einer offenen Frageformulierung nach maximal 5 Studienrichtungen gefragt. 56 Studienrichtungen unterschiedlichen Spezialisierungsgrades wurden genannt, im Durchschnitt pro Studiengang wurden 2,5 Nennungen getätigt (nur 4 Studiengänge haben keine Nennung zu verzeichnen, scheinen sich also als völlig einzigartig einzuschätzen). Darüber hinaus wurde auch die Gewichtung der Fächer erhoben. 12 Universitäts-Studienrichtungen haben einen Indikator von höher als 1 erhalten: ${ }^{103}$ BWL, Internationale BWL, Elektrotechnik, Informatik, Telematik, Wirtschaftsingenieur, Mechatronik, Handelswissenschaft, Wirtschaftsinformatik, Maschinenbau, Bauingenieurwesen und Elektrotechnik-Nachrichtentechnik. Die Breite oder Spezialisierung nach Wirtschaftsbereichen hat keinen Zusammenhang mit der Verwandtschaft zu Universitäts-Studienrichtungen.

Vergleicht man die Bezeichnung der Ausbildungsprofile der österreichischen Studiengänge mit den Studien in den anderen Ländern, so ist zunächst von der „Berufsfeldorientierung“ der österreichischen Studiengänge auszugehen. Die Bezeichnungen sind systematisch schwer einzuordnen. Sie haben gemeinsam, dass sie sich nicht an den meistens gebräuchlichen disziplinären Kategorisierungen der Hochschulsysteme orientieren und auch nicht an den offiziellen Berufskategorien. Am ehesten orientieren sie sich an modernen Bezeichnungen

103 Entspricht mindestens einer Nennung mit $100 \%$ oder 10 Nennungen mit $10 \%$ etc. 
für wirtschaftliche Funktionen. 32 Mal wird „Management“ in irgendwelchen Verbindungen in der Bezeichnung getragen (darunter bei einigen sogar zweifach), achtzehn Mal "Wirtschaft“, siebzehn Mal „Technik“ oder „Technologie“, acht Mal „Engineering“, weitere weniger oft gebrauchte Schlüsselbegriffe sind: Design, Medien, Marketing, Information, Führung.

Die schweizerischen Studiengänge verwenden vorwiegend die allgemeinen Fachbezeichnungen wie Architektur, Bauingenieurwesen, Informatik, Telekommunikation, Informatik, Elektrotechnik, Maschinenbau, Betriebsökonomie, Wirtschaftsinformatik etc. Es gibt auch einige den österreichischen korrespondierende Bezeichnungen, etwa MechatronikIngenieurwesen, Prozess/Anlagentechnik, Systemtechnik, Industrial Design etc. ${ }^{104}$

Die deutschen Fachhochschulen sind disziplinär organisiert und es ist auch eine Annäherung an die Universitäten in der Fächerstruktur in Diskussion. „Zuletzt hat der Wissenschaftsrat in seinen ,Thesen zur künftigen Entwicklung des Wissenschaftssystems in Deutschland' einen Ausbau der Ausbildungskapazität der Fachhochschulen gefordert, die Ausbildung des überwiegenden Teils der Studierenden an Fachhochschulen in solchen Fächern empfohlen, deren Curricula an Universitäten und Fachhochschulen weitgehend identisch sind, erneut auf das Erfordernis hingewiesen, das Fächerspektrum der Fachhochschulen auch auf Teilbereiche traditionell an Universitäten angesiedelter Fächer wie Rechtswissenschaften, Lehramtsstudiengänge und angewandte Naturwissenschaften auszuweiten." (WR 2002, 29f). Die Klassifikation erfolgt nach Fächergruppen und Studienbereichen. Es werden sechs Fächergruppen unterschieden, die klar den wissenschaftlichen Disziplinbereichen entsprechen (Sprach-/ Kulturwissenschaften, Rechts-/ Wirtschafts-/ Gesellschaftswissenschaften, Mathematik/ Naturwissenschaften, Agrar-/ Forst-/ Ernährungswissenschaften, Ingenieurwissenschaften, Kunst/ Kunstwissenschaft). Die Bezeichnungen der Studienbereiche mit den höchsten AnfängerInnenzahlen sind: Wirtschaftswissenschaften, Maschinenbau/Verfahrenstechnik, Sozialwesen, Informatik, Elektrotechnik, Bauingenieurwesen, Wirtschaftsingenieurwesen, Architektur/ Innenarchitektur (in diesen acht Studienbereichen haben 1999 85\% der 81.700 Studienanfängerlnnen begonnen (WR 2002, 216). Die Studiengangsbezeichnungen entsprechen in der großen Mehrheit den Studienbereichen, es gibt aber auch originellere Bezeichnungen, die jedoch oft kürzer und prägnanter und vielleicht auch allgemeiner sind als die österreichischen Bezeichnungen. ${ }^{105}$ Wenn man die Spezialisierung von Studiengängen an der Zahl der Worte

104 Vgl. die Aufstellung im Zwischenbericht über die Schaffung der Fachhochschulen (EFHK 2000, Anhang 2, 73 78).

105 Beispiele für Bezeichnungen in Deutschland: Banking and Finance, internationale Betriebswirtschaft oder Betriebswirtschaft mit Spezialisierungen wie Marketing oder Werbung oder Steuer- und Revisionswesen, Betriebswirtschaftslehre in verschiedenen Verbindungen, Facility Management, Electrical Engineering, Elektrotechnik in verschiedenen Verbindungen wie Energiesysteme, Elektronik, Informatik etc., Energietechnik in vielen Verbindungen, Engineering und Project Management, einige Verbindungen mit European Studies wie Business Administration oder Engineering, Gesundheitsökonomie, Handelsmanagement, Holztechnik, Industrial Design, viele Verbindungen mit Informatik wie technische, angewandte etc., Informationstechnik, 
im Titel festmacht, so haben etwas mehr als $20 \%$ der österreichischen Studiengänge mehr als drei Worte, unter den vom deutschen Akkreditierungsrat akkreditierten Bachelor- und Masterstudiengängen (an Fachhochschulen oder Universitäten) ist dieser Anteil nur wenig geringer (bei Master-Studiengängen höher, bei Bachelor-Studiengängen niedriger). ${ }^{106}$

Die finnischen Polytechnics haben in ihrer Programmstruktur einen zweistufigen Prozess durchlaufen. In der ersten „experimentellen“ Phase waren die einzelnen Polytechnics aufgerufen, neue Programmstrukturen zu entwickeln, die multidisziplinäre Kombinationen von Qualifikationen darstellen konnten und auf die regionale Wirtschaft bezogen sein sollten. „However, one consequence was that the range of variously titled programmes provided by different polytechnics was at risk of becoming too great, with new programmes being added every year ... Though the degree programme structure of individual polytechnics was clear enough, it was no longer clear for the system as a whole. The system was felt to be excessively complex from the point of view of the study and careers guidance given prior to entrance to polytechnics and from the point of view of working life" (MoE o.J., 68). In einer zweiten Stufe wurde in den letzten Jahren in einem Projekt der Rektorenkonferenz an einer Systematisierung der Programmstruktur gearbeitet. Für 2001 wurde eine Bereinigung vorgenommen und für jedes Polytechnic eine unbegrenzt gültige Programmstruktur anerkannt, die auf Antrag verändert werden kann. Die Zahl unterschiedlicher (= unterschiedlich benannter) Programme wurde von 2000 auf 2001 halbiert (von fast 300 auf 149). Die finnischen Programme sind oft modulartig aufgebaut nach der Struktur von Spezialisierungen (Majors) in bestimmten Bereichen (z.B. Informationstechnologie, mit software engineering, information systems etc...). ${ }^{107}$ Die Bezeichnungen der Majors sind oft den österreichischen ähnlich, die Bezeichnungen der Programme sind allgemeiner (vgl. zur Illustration die folgenden beispielhaften Aufstellungen von zwei Polytechnics in Abbildung 33 und Abbildung 34).

Informationswirtschaft, International Business, Kommunikationsdesign, Krankenhausmanagement, Management und Marketing in einigen Verbindungen, viele Verbindungen mit Maschinenbau, Mechatronik, Verbindungen mit Medien wie -informatik, -technik etc., Medizintechnik, Verbindungen mit Pflege, Produktdesign, Software Engineering, Sozialarbeit, Sozialwesen, Sozialpädagogik, Sozialmanagement, Verbindungen mit Verfahrenstechnik, Verkehrswesen, viele Verbindungen mit Wirtschaft wie Recht, Verwaltung, Wirtschaftsinformatik, Verbindungen mit Wirtschaftsingenieurwesen.

106 Die Abfrage von Studien mit Abschluss FH-Diplom ergibt unter Berücksichtigung von Standorten fast 1.700 grundständige Studien von insgesamt etwa 9.200 (http://www.hochschulkompass.hrk.de/); http://www.akkreditierungsrat.de/akkreditierte-studiengaenge.htm.

107 Die Einheiten sind credit units von 40 Stunden, Programme sind oft aus 140 (3 1/2 Jahre) oder 160 cus (4 Jahre) aufgebaut, es gibt aber auch Studien mit höheren Zahlen; eine cu setzt sich in 1,5 ECTS Punkte um. Der Struktur nach besteht z.B. ein Business Programm aus 40 Basic cus, 60 Professional (die dem Major-Bereich entsprechen), 10 optional, 20 Work experience; 10 für thesis. 


\title{
Abbildung 33: Bezeichnung der Studiengänge am Lahti Polytechnic
}

\author{
Betriebswirtschaft \\ Datenverarbeitung \\ International Business Management and Logistics \\ Dienstleistungsmanagement \\ Design und Gestaltung \\ Visuelle Kommunikation \\ Goldschmiedekunst \\ Bildende Kunst \\ Musik \\ Informatik \\ Werkstoff- und Produktionstechnik \\ Umweltwesen \\ Sport \\ Sozialwesen \\ Krankenpflege und Gesundheitsfürsorge \\ Physiotherapie \\ Diakonie \\ Quelle: http://www.lamk.fi/engl/ Lahti
}

\section{Abbildung 34: Programme und Spezialisierungen im Bereich "Business and Administration" am Turku Polytechnic}

International Business (Raisio) 140 credits, 210 ECTS.

- International Marketing

International Business / Internationale Betriebswirtschaft (Salo) 140 credits, 210 ECTS.

- International Technology Marketing

Business InformationTechnology (Salo) 140 credits, 210 ECTS

- Business Information Technology Management

International Business (Turku) 140 credits, 210 ECTS

- International Business Management

Business and Administration 140 credits, 210 ECTS.

- Computer-aided Financial Management (Raisio).

- Electronic Business and Marketing (Salo).

- Entrepreneurship (Turku).

- Financial Management (Turku).

- International Business Operations (Turku).

- Marketing (Turku).

- Marketing for Sports (Turku).

- Marketing for the Tourism Industry (Turku).

- Business Information Technology Management (Loimaa).

-Marketing Communication (Turku).

- International Entrepreneurship (Turku)

Business Information Technology 140 credits, 210 ECTS.

- Business Information Systems (Salo).

- Information Systems Development (Turku).

- Intranet Services (Uusikaupunki).

- Multimedia Production (Loimaa).

- Data communications Systems (Turku)

Business Logistics (Uusikaupunki) 140 credits, 210 ECTS

Library and Information Services (Raisio) 140 credits, 210 ECTS

STRUCTURE OF THE DEGREE OF BUSINESS AND ADMINISTRATION, TRADENOMI, (140 CREDITS)

Common Polytechnic Studies (5 credits)

Common Field of Study Basic Studies ( 22 credits)

Common Degree Programme Basic Studies (8 credits)

Professional Specialisation Studies (65 credits)

Free Choice Studies (10 credits)

Work Placement (20 credits)

Thesis (10 credits).

Quelle: http://www.turkuamk.fi/polytechnic/info.htm; in Klammer der Name des jeweils veranstaltenden Campus'. 


\section{Erwartungen und Bewertungen hinsichtlich der beruflichen Flexibilität}

In unseren Erhebungen wurden von einigen InterviewpartnerInnen Bedenken hinsichtlich einer zu starken Spezialisierung und „Nischenorientierung“ des FH-Sektors geäußert. Als wichtige Weichenstellung in dieser Richtung wurde immer wieder die Interpretation des Kriteriums der "Innovativität" eines Studienganges dadurch gesehen, dass kein gleichlautendes Angebot bestehen durfte.

Ohne näher in die Tiefe zu gehen ist eine Analyse des Spezialisierungsgrades der Studiengänge nicht möglich. Der Vergleich der Studiengangsbezeichnungen mit den anderen Ländern bringt auch kein eindeutiges Ergebnis. Manche Systeme lehnen ihre Studiengänge eher an den wissenschaftlichen Disziplinen an, es gibt jedoch auch in beträchtlichem Ausmaß spezialisierte Bezeichnungen. Andere Systeme folgen eher der Struktur von breiteren Grundstudien in Verbindung mit praxisorientierten Spezialisierungen. Dieser letztere Weg wird in Österreich teilweise gegangen, setzt aber jedenfalls größere Einrichtungen voraus.

Der österreichische FH-Sektor hat im Hinblick auf diese Frage der Flexibilität und Spezialisierung zwei Besonderheiten: erstens die Berufsfeldorientierung und zweitens die Expansion über die Errichtung neuer Studiengänge und weniger über die Vergrößerung bestehender. Beide Besonderheiten verstärken bis zu einem gewissen Grad eine Tendenz zur Spezialisierung. Berufsfelder sind umso greifbarer, je mehr sie in ihrem konkreten Zuschnitt (als bestimmte Gruppen von Arbeitsplätzen) in der Arbeitswelt auffindbar sind und die Einrichtung immer neuer Studiengänge kann ebenfalls durch Auffächerung oder Abspaltung von speziellen Qualifikationsbündeln am leichtesten durchgeführt werden.

In der neueren wissenschaftlichen Diskussion werden diese Fragen in der Spannung zwischen traditioneller und konstruktivistischer Pädagogik noch in abstrakter Weise diskutiert, wobei keine konkreten Lösungen auf der Hand liegen. Zentrale Begriffe in diesem Zusammenhang sind:

- Kompetenzen als Ergebnisse der Lernprozesse (vgl. Lassnigg/Mayer 2001)

- die Dynamik der Wissensformen (kodifiziertes, formalisiertes und stillschweigendes, informelles Wissen; „know why“, „know what“, „know how“, „know who“; vgl. Lassnigg 1998)

- die „community of practice“ und das „learning environment“. Das Konzept der „community of practice" (Lave/Wenger 1991) wurde von Markowitsch (2001) mit dem Konzept der Hochschulpraxisgemeinschaft umfassend in seinen Implikationen für die Hochschulbildung und das österreichische FH-System ausgelotet und aufbauend auf diesem Konzept laufen auch Aktivitäten zur Umsetzung. Beispielsweise ist dieses 
Konzept auch in den Richtlinien des FHR zur Evaluierung (FHR 2002, 19) als ein Kriterium der institutionellen Evaluierung vorgegeben.

Diese Begrifflichkeiten transzendieren in gewisser Weise die traditionellen Dichotomien von disziplinärer und angewandter Orientierung, von Theorie und Praxis, von Lehren und Lernen, in denen die pädagogische Praxis und auch die konzeptionelle Struktur des FHSektors teilweise verhaftet erscheint. An dieser Stelle können diese Fragen nicht vertiefend behandelt werden, sollten aber für die Zukunft im Auge behalten werden.

Im Anhang (Kapitel 11.1.2) wird eine ansatzweise Analyse der in den Anträgen der ersten Jahre (1994-1997) verwendeten Begrifflichkeiten über die Berufsfelder bzw. Berufsprofile aufgrund der Analyse von Lassnigg/Stöger (1999) dokumentiert, die noch sehr wenig Systematik gezeigt haben. Es hat sich ergeben, dass die Berufsfelder in den Anträgen oft in ziemlich komplexer Form beschrieben waren, die man nach den fünf Dimensionen klassifizieren kann:

- Kompetenzen

- Position

- Berufsbezeichnung

- Unternehmensbereich

- Branche

Eine nähere Analyse dieser Berufsfelder und ihrer Bestimmungsmerkmale in ihrer weiteren Entwicklung und auch in ihrem Bezug zu akademischen Klassifizierungen bzw. zum Konzept der Hochschulpraxisgemeinschaft könnte zur Frage der beruflichen Spezialisierung wichtige weitere Aufschlüsse geben.

\section{c) Entlastung der Universitäten}

Eine Entlastung der Universitäten wurde in verschiedenen Dokumenten (z.B. BMWFK/BMUKA 1995, 28) als Zielsetzung formuliert, jedoch nicht weiter konkretisiert. Im Zusammenhang mit der prognostizierten bzw. teilweise erwarteten Stagnation der Zugänge zum Hochschulbereich ändert sich auch bis zu einem gewissen Grad die Konnotation dieses Ziels, indem der Aspekt des Wettbewerbs um (knappe) Studierende mehr betont wird. Teilweise spielen in dieser Diskussion auch Erwartungen hinsichtlich der Qualität eine Rolle, indem die abgewanderten Zugänge (nach dem Motto der Kirschen in Nachbars Garten) als besonders vielversprechend bewertet werden, was die „Entlastung“ in einen „Verlust“ verwandeln würde.

Auf den ersten Blick kann die Entlastung der Universitäten aufgrund der unterschiedlichen Größenordnungen der beiden Sektoren kein besonders hohes Gewicht bekommen. 
Vergleicht man die Gesamtstudierendenzahlen, so macht der FH-Sektor nur einen sehr kleinen Anteil aus. Dieser Vergleich ist jedoch aufgrund der unterschiedlichen Zählweisen und Studienkonzepte (Studienplatz, Studienverlauf, Anwesenheitspflicht etc.) jedenfalls für die Vergangenheit sehr stark verzerrt. Besser vergleichbar sind die Zugänge, wobei man auch hier noch davon ausgehen kann, dass aufgrund der unterschiedlichen Praktiken in den beiden Bereichen die Universitätszahlen weicher $z u$ interpretieren sind als die Fachhochschulzahlen. Insgesamt betragen die Zugänge zu den Universitäten, die im letzten Jahrzehnt in der Größenordnung zwischen 25.000 und über 30.000 liegen, etwas mehr als das Fünffache der Fachhochschulzugänge (gemessen an der aktuellen Größenordnung von etwas über 5.000) (vgl. Abbildung 35). Dieser Vergleich gibt zwar eine grobe Größenordnung für die Entlastung des gesamten Sektors an, lässt jedoch die Frage offen, inwieweit die Zugänge zu den Fachhochschul-Studiengängen in jedem Fall als potentielle Zugänge an die Universitäten zu rechnen sind.

\section{Abbildung 35: Entwicklung der AnfängerInnenzahlen an wiss. Universitäten und FH-} Studiengängen, 1990/91 - 2001/02

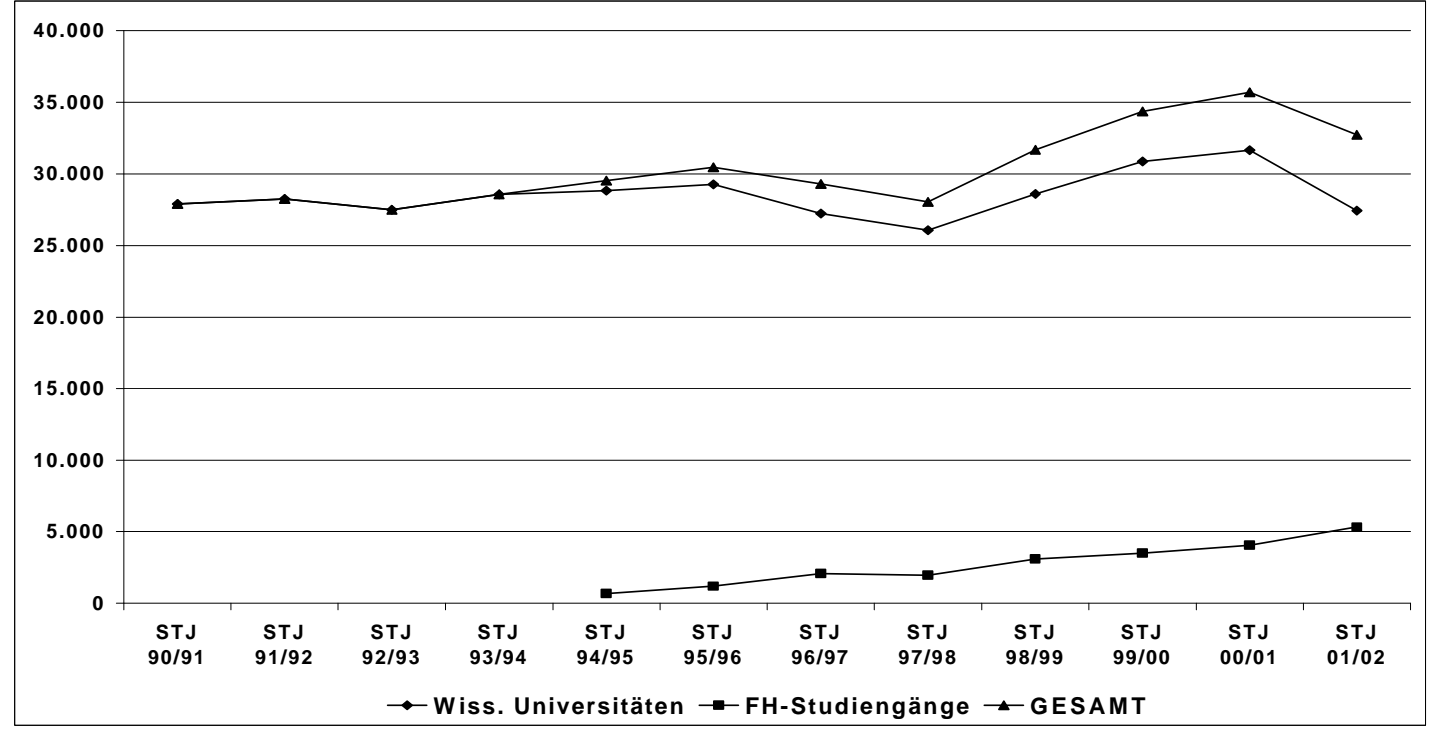

Quelle: FHR, Statistik Austria, IHS-Berechnungen

Stärker operative Bedeutung hat der Vergleich potentiell überschneidender Zugänge nach fachlichen Bereichen (wobei auch hier die Frage offen bleibt, ob die FH-Zugänge alternativ im Universitätssektor die gleichen Bereiche gewählt hätten). Konstruiert man einen Überschneidungsbereich zwischen Universitäten und FH-Studiengängen, so liegen die (konkurrierenden) Zugänge im Universitätssektor in der Größenordnung von 12.000 bis 14.000 bei retrospektiver Betrachtung (vgl. Abbildung 36) bzw. steigen sie von 8.000 bis 
12.000 bei synchroner Betrachtung (vgl. Abbildung 37). ${ }^{108}$ Für etwas weniger als die Hälfte der Universitätszugänge gibt es in den gleichen Fachrichtungen nach der ISCEDKlassifikation Angebote im FH-Sektor (etwa 10\% der FH-Zugänge finden umgekehrt kein fachrichtungsspezifisches Angebot im Universitätssektor). Die Relation der Universitätszugänge zu den $\mathrm{FH}$-Zugängen reduziert sich also in dieser Betrachtung von etwa 5:1 auf 2,5:1. Differenziert man noch weiter nach Fachrichtungen, so ist diese Relation sehr unterschiedlich; man kann sagen, der FH-Sektor hat einen sehr unterschiedlichen „Marktanteil“: Im Bereich der wirtschaftlichen Fachrichtungen (Abbildung 38) liegt die Relation etwa durchschnittlich (2,5:1), im Bereich der technischen Fachrichtungen (Abbildung 39) liegt sie bereits bei 1:1, im sehr dynamischen naturwissenschaftlichen (IKT) Bereich (Abbildung 40) sind die AnfängerInnenzahlen sowohl an den Universitäten als auch in den Fachhochschul-Studiengängen in den letzten Jahren sehr stark gestiegen (aktuell liegt die Relation Universität : Fachhochschul-Studiengang bei 3:1); in den übrigen Bereichen (Abbildung 41) ist der FH-Sektor noch vergleichsweise sehr klein.

Abbildung 36: Entwicklung der AnfängerInnenzahlen an wiss. Universitäten und FHStudiengängen im Überschneidungsbereich (retrospektive Betrachtung), 1990/91 - 2001/02

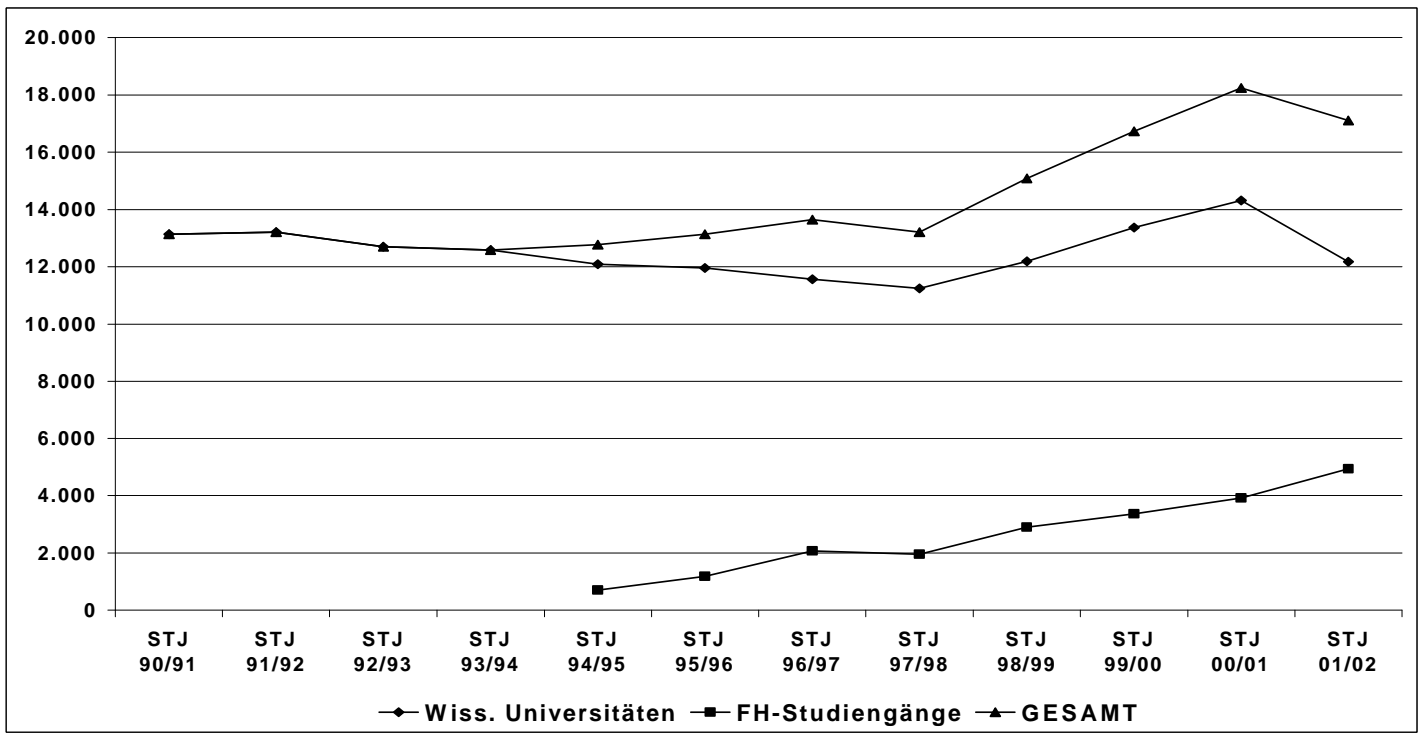

Quelle: FHR, Statistik Austria, IHS-Berechnungen

108 Diese Klassifikation wurde auch bereits beim Vergleich der geschlechtsspezifischen Zugänge zwischen FHSektor und Universitäten verwendet. Die Basis sind die 2-Steller der ISCED-Klassifikation der Fachrichtungen. Im ersten Fall (retrospektiv) wird vom letzten Jahr ausgegangen und der Überschneidungsbereich seit 1990 nach diesem Jahr definiert; im zweiten Fall (synchron) wird die Erweiterung des Fächerspektrums im FH-Sektor bei der Definition des Überschneidungsbereiches berücksichtigt. 
Abbildung 37: Entwicklung der AnfängerInnenzahlen an wiss. Universitäten und FHStudiengängen im Überschneidungsbereich (synchrone Betrachtung), 1990/91 - 2001/02

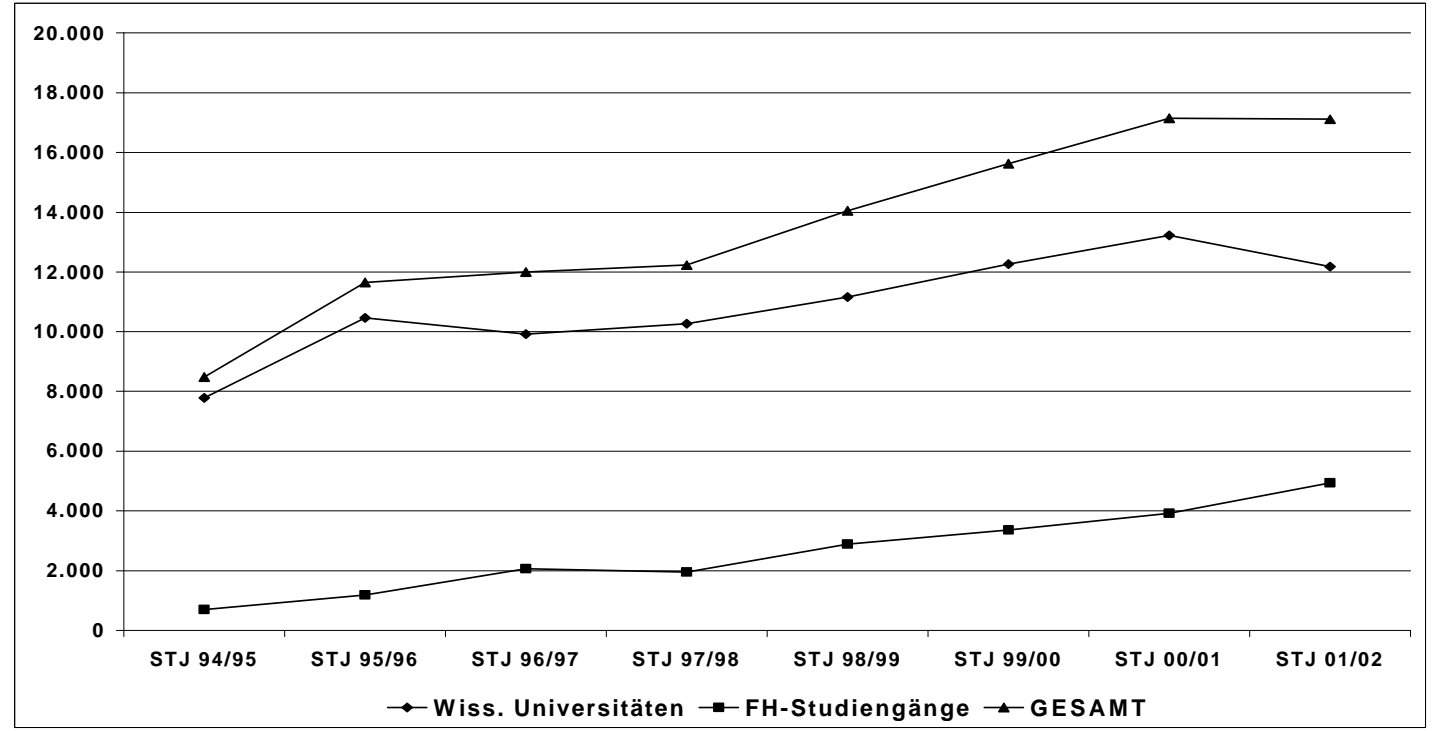

Quelle: FHR, Statistik Austria, IHS-Berechnungen

Im Vergleich der Entwicklung der Zugänge könnte am ehesten im technischen Bereich von einer Entlastung gesprochen werden, da hier die Zugänge an den Universitäten rückläufig sind, während die Zugänge zu Fachhochschul-Studiengängen stark steigen (vgl. Abbildung 39). In den anderen Fachbereichen sind die Zugänge an die Universitäten stabil geblieben oder auch gestiegen. Für den technischen Bereich muss die Aussage der Entlastung insofern eingeschränkt werden, als der Rückgang der AnfängerInnenzahlen an den Universitäten bereits vor der Errichtung des Fachhochschulsektors begonnen hat - die einschlägigen Fachhochschul-Studiengänge könnten also eher zusätzliche Potentiale für diese Studien mobilisiert haben. 
Abbildung 38: Entwicklung der AnfängerInnenzahlen an wiss. Universitäten und FHStudiengängen im Bereich Business and Administration (ISCED 34), 1990/91 - 2001/02

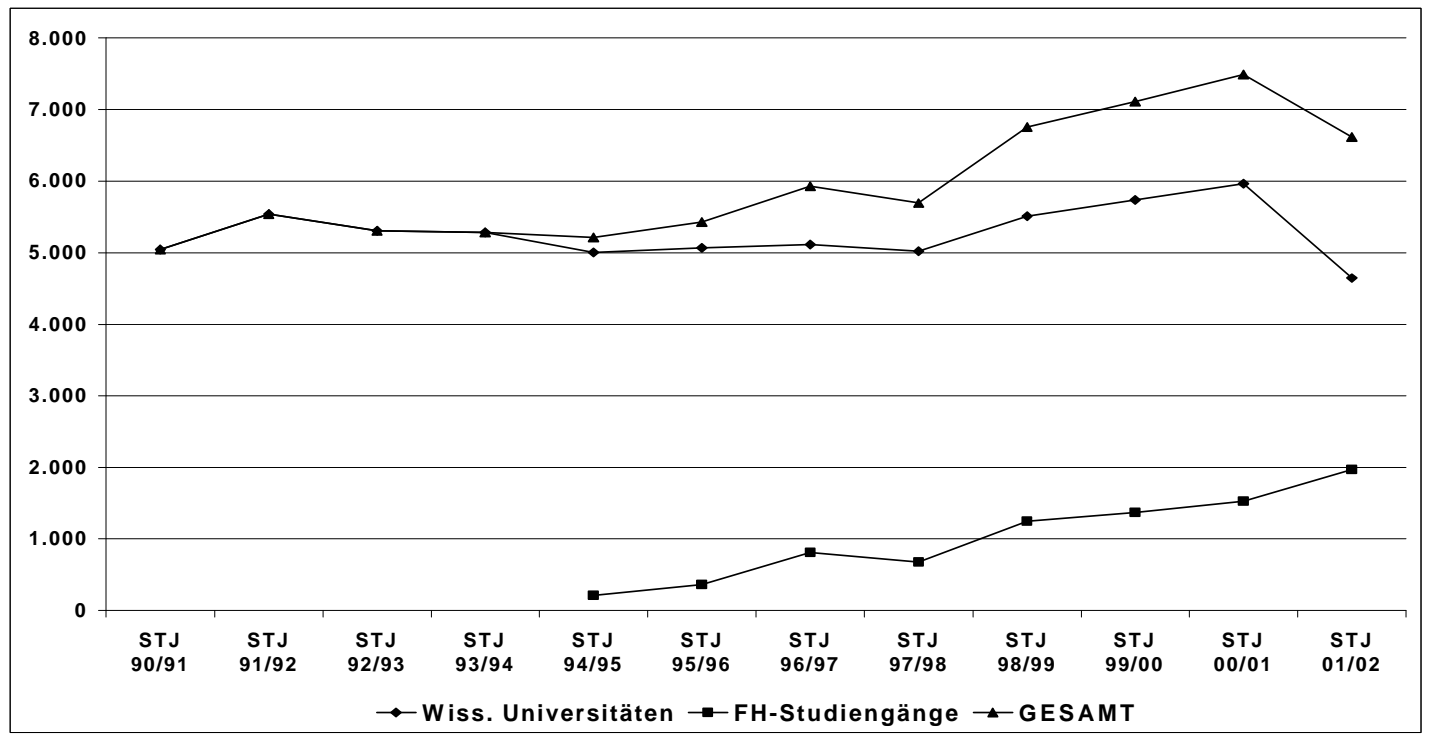

Quelle: FHR, Statistik Austria, IHS-Berechnungen

Abbildung 39: Entwicklung der AnfängerInnenzahlen an wiss. Universitäten und FHStudiengängen im Bereich Engineering and engineering trade (ISCED 2), 1990/91 - 2001/02

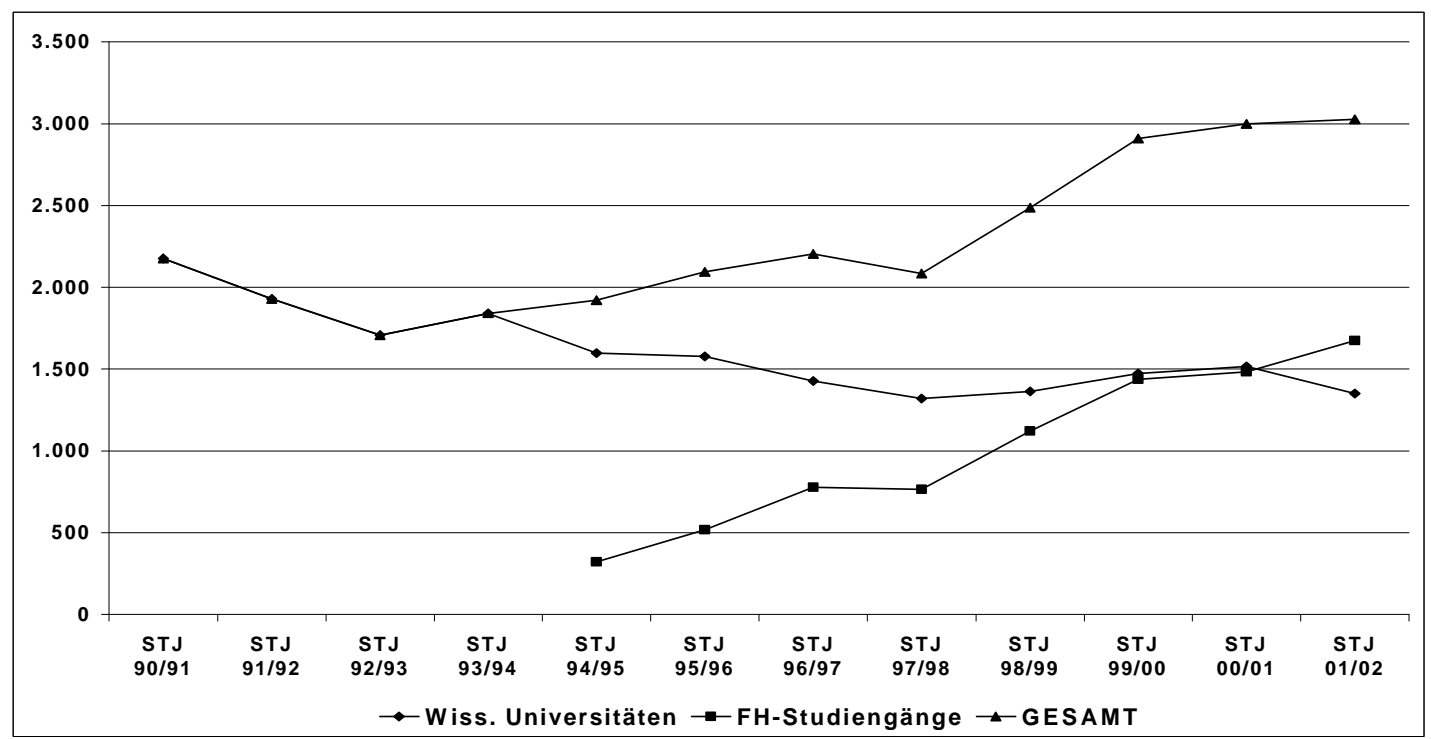

Quelle: FHR, Statistik Austria, IHS-Berechnungen 
Abbildung 40: Entwicklung der AnfängerInnenzahlen an wiss. Universitäten und FHStudiengängen im Bereich Computing (ISCED 48), 1990/91 - 2001/02

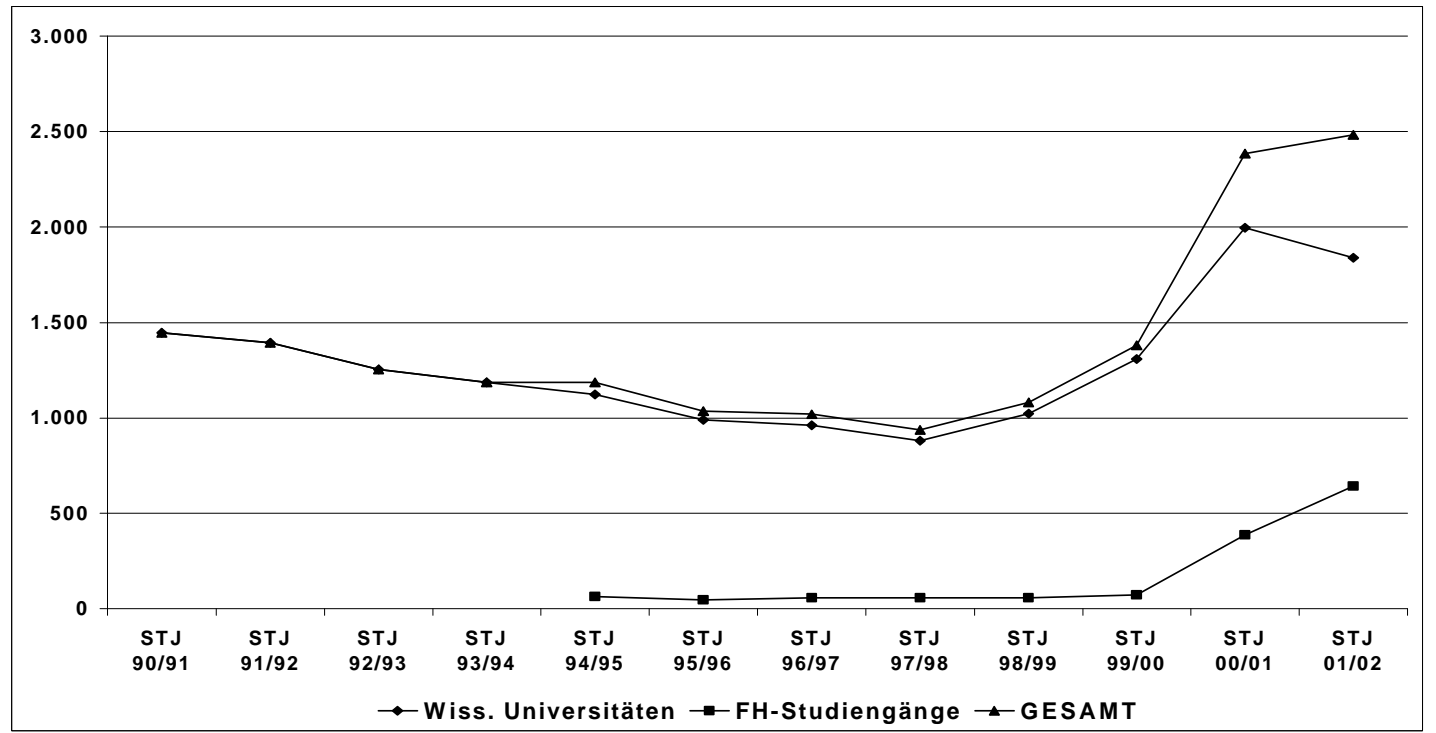

Quelle: FHR, Statistik Austria, IHS-Berechnungen

Abbildung 41: Entwicklung der AnfängerInnenzahlen an wiss. Universitäten und FHStudiengängen im übrigen Überschneidungsbereich (ISCED 21, 32, 39, 42, 54, 58, 81), 1990/91 - 2001/02

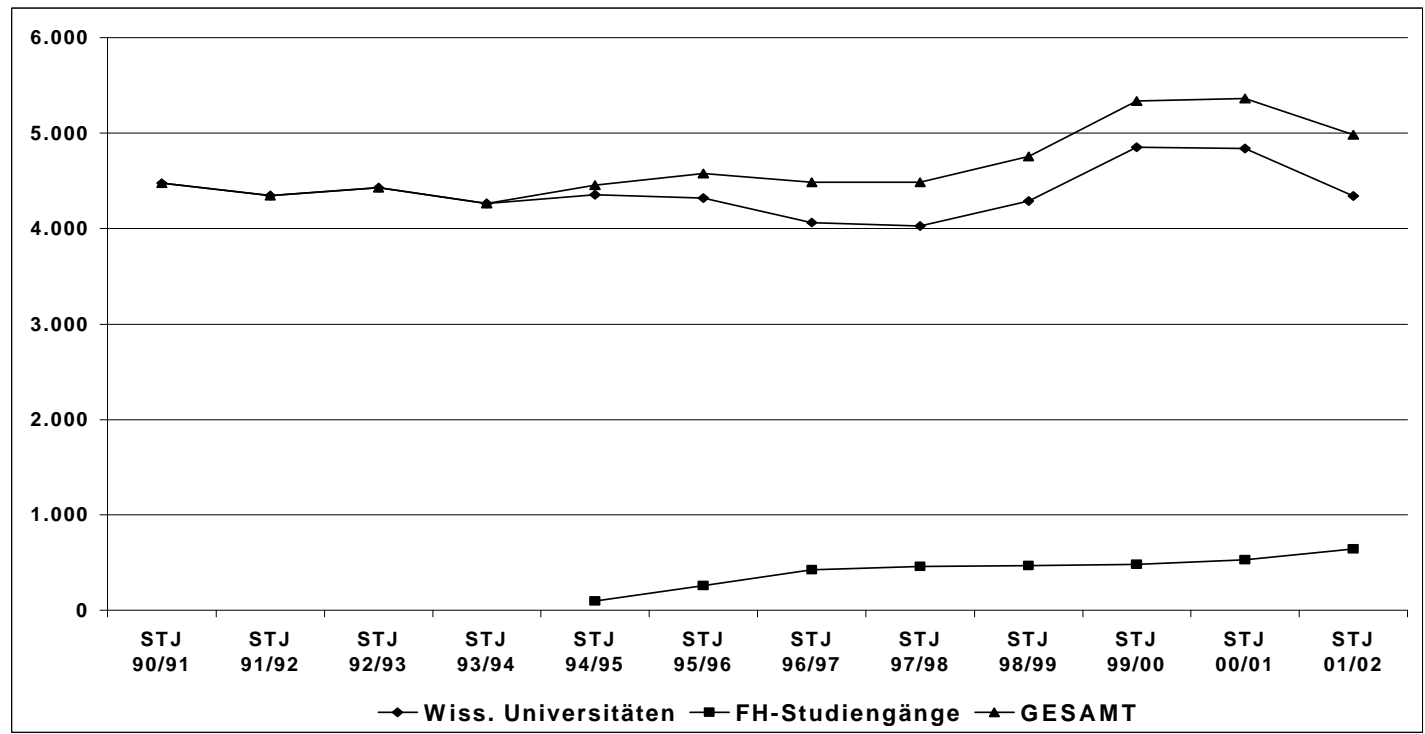

ISCED 21: Arts, 32: Journalism and Information, 39: Social sciences, Business, Law-not spec., 42: Life sciences, 54: Manufacturing and processing, 58: Architecture and building, 81: Personal services.

Quelle: FHR, Statistik Austria, IHS-Berechnungen

Vergleicht man die Zuwachsraten der Universitäts- und Fachhochschulzugänge für drei Perioden, nämlich (1) die Jahre vor der Errichtung der FH-Studiengänge, (2) die frühen 
Jahre 1994 bis 1997 und (3) die späteren Jahre 1998 bis 2001, so wird ersichtlich, dass in der Vorperiode 1990 bis 1993 der Überschneidungsbereich rückläufig war, während der Universitätssektor insgesamt leicht gewachsen ist. In der Gesamtperiode nach Errichtung des FH-Sektors 1994 bis 2001 hat sich diese Entwicklung tendenziell umgekehrt: Die Zugänge an die Universitäten sind im Überschneidungsbereich mit den FachhochschulStudiengängen leicht gewachsen, während der Universitätssektor insgesamt rückläufig war.

Differenziert man weiter nach den drei Perioden, so wird ersichtlich dass die Gesamtzugänge an die Universitäten in den Jahren 1994 bis 1997 deutlicher zurückgegangen sind (-2,5\% im jährlichen Durchschnitt) als im Überschneidungsbereich mit den Fachhochschul-Studiengängen $(-1,8 \%)$. Auch in der folgenden Periode ist die quantitative Entwicklung der gesamten Universitätszugänge noch abgeschwächt rückläufig, während der Überschneidungsbereich mit den Fachhochschul-Studiengängen stagniert. Dieses Muster deutet insgesamt nicht auf einen direkten Entlastungseffekt durch die Fachhochschul-Studiengänge in dem Sinne hin, dass Studierende abgezogen worden wären.

Differenziert man nach Fachbereichen, so sieht man unterschiedliche Muster.

- Im wirtschaftsbezogenen Bereich hat sich an den Universitäten die Entwicklung in der Periode nach Errichtung des FH-Sektors gedreht. Gab es in der Vorperiode eine positive Zuwachsrate $(+1,2 \%)$, so hat sich das Vorzeichen gedreht $(-0,9 \%)$ und man kann auch eine sukzessive Entwicklung in diesen Fachrichtungen in den beiden Perioden 1994-1997 (+0,1\%) und 1998-2001 (-4,2\%) erkennen. Hier könnte man von einer direkten Entlastung sprechen, die Zeitreihe zeigt jedoch, dass diese Entwicklung zur Gänze durch das Jahr 2001/02 geprägt ist (vgl. Tabelle 10).

- In den technischen und naturwissenschaftlichen Bereichen war die Entwicklung im Universitätssektor bereits vor der Errichtung der Fachhochschul-Studiengänge deutlich rückläufig $(-4,1 \%$ im technischen Bereich und $-4,9 \%$ im naturwissenschaftlichen-IKT Bereich), dies hat sich in der ersten Periode 1994-1997 noch leicht verstärkt, während dann ein deutliches Wachstum im naturwissenschaftlichen Bereich $(+15,8 \%)$ und eine Abschwächung des Rückganges im technischen Bereich (-0,2\%) eingesetzt hat (der Einbruch 2001/02 hat sich bei diesen Fachrichtungen weniger stark ausgewirkt.

- In den übrigen Fachrichtungen zusammengenommen ist, wenn auch viel schwächer ausgeprägt, ein ähnliches Muster wie in den technischen und naturwissenschaftlichen Bereichen zu erkennen (leichter Rückgang in der Vorperiode, dann stärkerer Rückgang und in der letzten Periode leichtes Wachstum). 
Tabelle 10: Durchschnittliche jährliche Wachstumsraten der AnfängerInnenzahlen an wissenschaftlichen Universitäten und im Überschneidungsbereich mit den FH-Studiengängen nach Zeitperioden

\begin{tabular}{|c|c|c|c|c|}
\hline Zeitraum & $\begin{array}{c}\text { 90/91-93/94 } \\
4 \text { Jahre }\end{array}$ & $\begin{array}{c}\text { 94/95-97/98 } \\
4 \text { Jahre }\end{array}$ & $\begin{array}{c}\text { 98/99-01/02 } \\
4 \text { Jahre }\end{array}$ & $\begin{array}{c}94 / 95-01 / 02 \\
8 \text { Jahre }\end{array}$ \\
\hline Insgesamt & $0,6 \%$ & $-2,5 \%$ & $-1,0 \%$ & $-0,6 \%$ \\
\hline Überschneidungsbereich mit FH & $-1,1 \%$ & $-1,8 \%$ & $0,0 \%$ & $0,1 \%$ \\
\hline $\begin{array}{l}\text { Business Administration } \\
\text { (ISCED 34) }\end{array}$ & $1,2 \%$ & $0,1 \%$ & $-4,2 \%$ & $-0,9 \%$ \\
\hline $\begin{array}{l}\text { Engineering and engineering trade } \\
\text { (ISCED 52) }\end{array}$ & $-4,1 \%$ & $-4,7 \%$ & $-0,2 \%$ & $-2,1 \%$ \\
\hline Computing (ISCED 48) & $-4,9 \%$ & $-5,9 \%$ & $15,8 \%$ & $6,4 \%$ \\
\hline $\begin{array}{l}\text { Übriger Überschneidungsbereich } \\
\text { (ISCED 21, 32, 39, 42, 54, 58, 81) }\end{array}$ & $-1,2 \%$ & $-2,0 \%$ & $0,3 \%$ & $0,0 \%$ \\
\hline
\end{tabular}

ISCED 21: Arts, 32: Journalism and Information, 39: Social sciences,Business,Law-not spec., 42: Life sciences, 54: Manufacturing and processing, 58: Architecture and building, 81: Personal services.

Quelle: FHR, Statistik Austria, IHS-Berechnungen

Insgesamt deuten die Befunde bis zum Einbruch der Universitätszugänge im Jahr 2001/02 nicht darauf hin, dass das Studienwahlverhalten durch die Fachhochschul-Studiengänge nennenswert beeinflusst worden wäre. Eine direkte Entlastung könnte am ehesten in den wirtschaftlichen Fachbereichen im letzten Jahr stattgefunden haben. In den technischen und naturwissenschaftlichen Fachbereichen kann man aufgrund der vorliegenden Befunde nicht sagen, inwieweit die Zugänge, die durch die FH-Studiengänge mobilisiert wurden, andernfalls an einer Universität begonnen hätten (indirekte Entlastung) oder zusätzlich mobilisiert werden konnten. Aufgrund der Analysen zur regionalen Zugänglichkeit ist es plausibel, von einer zusätzlichen Mobilisierung auszugehen (vgl. das Kapitel 5 zur Regionalisierung und Standortfrage).

\section{d) Aus- und Weiterbildung}

Die Zielsetzung eines Beitrages des FH-Sektors zur Aus- und Weiterbildung kann in zwei unterschiedliche Formen differenziert werden: Erstens, in hoher Überschneidung zur Weiterentwicklung der Durchlässigkeit, indem der Zugang zu Ausbildungsangeboten mit formalen Abschlüssen auf Hochschulniveau für zusätzliche Zielgruppen eröffnet werden sollte (Personen ohne formale Hochschulberechtigung, Berufstätige); zweitens, indem die Fachhochschul-Studiengänge Weiterbildungsangebote im nicht-formalisierten Bereich, außerhalb der Studiengänge, z.B. für die eigenen AbsolventInnen, aber auch darüber hinaus 
z.B. als Angebote im regionalen Kontext anbieten sollten. ${ }^{109}$ Dies wird einerseits als wichtiges Element im Rahmen der Entwicklung des lebensbegleitenden Lernens gesehen, andererseits auch als Chance der Etablierung in einem dynamischen Zukunftsmarkt. Der vorliegende Abschnitt bezieht sich vor allem auf den zweiten Aspekt, das Weiterbildungsangebot im nicht-formalisierten Bereich.

Laut Befragung der Erhalter haben vier der antwortenden Erhalter in ihrem FH-Geschäftsfeld keine Weiterbildungsangebote für externe TeilnehmerInnen entwickelt (darunter ist das bei einem Erhalter für das nächste Studienjahr geplant, bei einem Erhalter ist die Weiterbildung als eigenes Geschäftsfeld außerhalb der FH-Agenden, jedoch mit Synergien zum FHBereich abgegrenzt; bei zwei Erhaltern wurden auch keine geplanten Aktivitäten in dieser Richtung genannt). Vier Erhalter haben daher auch keine Angaben über Teilnahmen an Weiterbildungsangeboten für Externe gemacht. Im Durchschnitt der übrigen Erhalter wurden im Jahr 2001 Weiterbildungsveranstaltungen für etwa 130 Teilnehmerlnnen mit etwa 40 Stunden (also insgesamt im Durchschnitt etwas mehr als 5.000 Teilnehmerlnnenstunden) durchgeführt. Hochgerechnet auf alle Erhalter könnten nach diesen Werten also zwischen 1.000 und 2.500 Teilnahmen an solchen Veranstaltungen im Jahr geschätzt werden. Mit Ausnahme eines Erhalters mit einem kompakten Angebot von 250 Stunden und eines weiteren Erhalters mit sehr umfangreichen Angeboten für 500 Teilnehmerlnnen ist der Großteil der Weiterbildungsveranstaltungen eher kurz (mit zwischen unter 10 und unter 20 Stunden) und wird von 20 bis 100 Teilnehmerlnnen in Anspruch genommen.

Zusätzlich zum Weiterbildungsangebot für Externe werden punktuelle Veranstaltungen für ein Zielpublikum aus der Region im Durchschnitt pro Erhalter etwa 10 mal im Jahr durchgeführt. Vier Erhalter haben keine derartigen Aktivitäten angegeben, die Streubreite reicht von zwei regionalen Veranstaltungen pro Erhalter bis zu 50 Veranstaltungen im Jahr.

Insgesamt setzt die Entwicklung der Weiterbildung eine entsprechende Größenordnung der Einrichtungen voraus. In den Vergleichsländern, etwa in Finnland und der Schweiz hat die Betätigung der Fachhochschulen in der Weiterbildung eine viel größere Dimension als bisher in Österreich. In der Schweiz gehören Weiterbildungsangebote (neben Nachdiplomstudien auch Nachdiplomkurse von kürzerer Dauer und vereinzelte Weiterbildungsveranstaltungen) zum gesetzlichen Auftrag der Fachhochschulen und entsprechend haben die Schweizer Fachhochschulen in diesem Bereich z.T. umfangreiche Veranstaltungsangebote.

109 Die Unterscheidung zwischen formalem, nicht-formalem und informalem Lernen bekommt in der europäischen Bildungspolitik zur Förderung des lebensbegleitenden Lernens zunehmende Bedeutung; zur Definition vgl. EUROSTAT 2001, UNECSO 1996. 


\section{e) Abbau regionaler Disparitäten}

Die Zielsetzung des Abbaus regionaler Disparitäten wurde nicht im FHStG festgelegt; im Entwicklungs- und Finanzierungsplan I ist dieses Ziel eines von zehn Kriterien, die der Bewertung der Anträge für die Entscheidung über eine Bundesfinanzierung zugrundegelegt werden. Gleichzeitig wurden jedoch auch die Kriterien der „überregionalen Abstimmung“ und des "langfristigen Entwicklungskonzeptes" aufgestellt, das auf Vorhaben mit mehreren Studiengängen, einem sinnvollen Fächerspektrum und mindestens 1.000 Studierenden im regionalen Einzugsgebiet oder mit einer Integration in ein „facheinschlägiges, renommiertes Forschungs-, Versuchs- oder Bildungszentrum" ausgerichtet ist. Damit wurde bereits von Anfang an die Spannung zwischen Regionalisierung und einer sinnvollen Mindestgröße der anzustrebenden Einheiten grundgelegt, wobei diese Kriterien die Konturen für die Grundstruktur des Sektors bestimmt haben.

Vor dem Hintergrund des dezentralen Regulierungsmodells und der „bottom-up“-Struktur der Antragstellung wurden im Laufe der Entwicklung zunehmend Anträge für weniger zentrale Standorte gestellt. Die Mitfinanzierung der Länder und Gemeinden spielte eine zusätzliche Rolle. Im Jahresbericht 1999 äußerte der FHR erstmals ausdrückliche Sorge über die „inflationäre Zunahme an neuen Standorten“ und äußerte die Hoffnung auf die Verantwortung der Bundesländer und den Wunsch, die Gebietskörperschaften sollten sich bei ihren Planungen „nicht dem politisch artikulierten Druck lokaler Interessen beugen.“

Heute wird die Frage der Abwägung zwischen Regionalisierung und Konzentration als eine der entscheidenden Entwicklungsfragen des FH-Sektors gesehen. Daher ist diese Frage Gegenstand eines eigenen Schwerpunktes der vorliegenden Evaluierung und wird in Kapitel 5 ausführlich behandelt.

\section{f) Strukturbereinigung}

Auch das Ziel der bildungspolitischen Strukturbereinigung im nicht-universitären Sektor wurde als Kriterium zur Erteilung der Bundesfinanzierung im ersten Entwicklungs- und Finanzierungsplan aufgestellt. Es sollten klare Strukturen und die Beseitigung von Doppelgleisigkeiten bei den Angeboten erreicht werden. Da mit der Strukturbereinigung auch die Frage der Schnittstellen zu den anderen Bereichen des Bildungswesens angesprochen wird, besteht ein enger Zusammenhang zur Durchlässigkeit.

Das nicht-universitäre Angebot besteht vor allem aus den Kollegs an BHS, die für MaturantInnen den berufsbildenden Abschluss ergänzen, aus den Akademien für Lehrerlnnenbildung und für Sozialarbeit sowie den Ausbildungen für die gehobenen Gesundheitsberufe. Die interne Planungsgrundlage des BMWFK für die Finanzierung des FH-Sektors im Jahr 1996/97 demonstriert die Größenordnungen der verschiedenen 
Institutionen auf postsekundärer Ebene in Verbindung mit dem damaligen regionalen Studienverhalten (BMWFK, Abt. I/B/17 1996). Die visualisierte Darstellung (Abbildung 42) demonstriert drei Aspekte:

- Es zeigt sich der geringe quantitative Umfang der nicht-universitären Angebote (inklusive der FH-Studiengänge It. Entwicklungs- und Finanzierungsplan I) zum damaligen Zeitpunkt im Vergleich zu den Universitäten.

- Es zeigt sich die schwache Datenbasis und der Fokus auf die Bundesländer als wesentlicher Fokus regionaler Bildungswanderung.

- Es zeigt sich die studienbedingte Mobilitätsbilanz nach Bundesländern, mit hohen Abwanderungszahlen aus Niederösterreich, Oberösterreich und Kärnten bzw. hohen Zahlen an Zuwanderern v.a. in Wien und Graz. 
Abbildung 42: FH-Planungsgrundlage 1996/97: Studierende an Universitäten und im sonstigen Postsekundarbereich nach Bundesländern und Studium im eigenen Bundesland

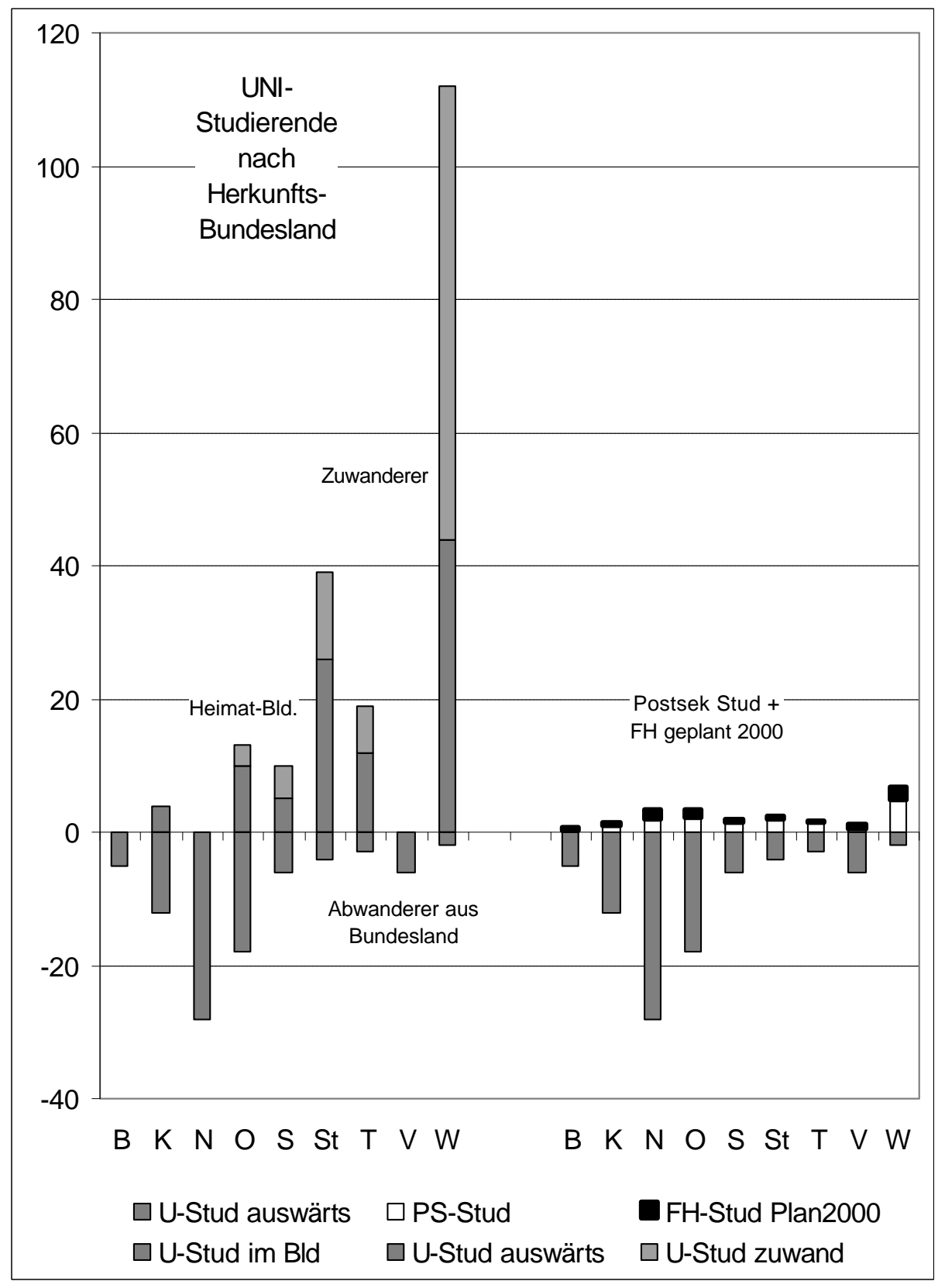

Quelle: BMWFK 1996

In der Frage der Strukturbereinigung gibt es viele Stellungnahmen in der Richtung, dass diese Zielsetzung nicht optimal erreicht wurde. Im Kern geht es dabei um die Grenzen des FH-Sektors zum übrigen Bildungswesen. Folgende Aspekte sind betroffen: erstens das Verhältnis zu den anderen Angeboten im postsekundären Bereich; zweitens die 
Schnittstellen zum berufsbildenden Schulwesen und drittens die Schnittstellen zu den Universitäten.

- Gegenüber den anderen postsekundären Angeboten wurden für die Bereiche der Sozialakademien und der Lehrerlnnenbildung Lösungsansätze gefunden (einerseits teilweise - Anerkennung als FH-Studiengänge, andererseits eigene organisatorische Lösung), die nicht unbedingt den ursprünglichen Vorstellungen zur Strukturbereinigung entsprechen. Die Frage der nichtärztlichen Gesundheitsberufe ist gegenwärtig in Diskussion, wobei vielfältige pragmatische Fragen und Interessenskonflikte zu lösen sind. Im Bereich der Kollegs befindet sich die Lösung der Schnittstellenproblematik in einem Diskussionsprozess über sinnvolle Regelungen der Anerkennung, gleichzeitig entstehen Kooperationen mit anderen Fachhochschuleinrichtungen im europäischen Bildungsraum.

- Die Schnittstelle zur Berufsbildung betrifft einerseits die Möglichkeiten nicht-traditioneller Zugänge, die im entsprechenden Abschnitt dieses Kapitels behandelt werden. Zweitens stellt sich in Verbindung mit den Beziehungen zu den Kollegs auch die Frage der Schnittstellen zu den BHS. Ein beträchtlicher Teil der Zugänge in den FH-Sektor erfolgt über die BHS-Matura, darunter ein hoher Anteil auch über eine einschlägige BHS-Matura (vgl. Abschnitt 2.2.5 und Abbildung 20). Im Hinblick auf eine Strukturbereinigung stellt sich unmittelbar die Frage nach Anerkennungsregelungen. Dies ist wichtig für die individuellen Laufbahnen und damit die Durchlässigkeit des Bildungswesens, aber auch auf einer mehr aggregierten Betrachtungsebene, indem nach Kosten und Nutzen dieser Laufbahnen gefragt werden kann. Diesen Fragen, die aus dem vorliegenden Projekt ausgeklammert wurden, sollte näher nachgegangen werden.

- Die Schnittstelle zur Universität betrifft im Hinblick auf Strukturbereinigung einerseits die Vermeidung von Doppelgleisigkeiten in der Erstausbildung. Dies wurde bisher auf dem Wege der Kohärenzanalyse seitens des FH-Sektors sehr rigoros gehandhabt. Mit der Umsetzung des Bologna-Prozesses und der Entwicklung von Bachelor- und MasterProgrammen in beiden Sektoren wird sich diese Problematik in Zukunft zweifellos verstärkt stellen (vgl. Kapitel 6). Andererseits hat sich mit der Entwicklung der universitären Weiterbildung auch ein Abstimmungsproblem eröffnet, das vom FHR im Zusammenhang mit der Regelung der Universitätslehrgänge im UniStG 1997 immer wieder herausgearbeitet wurde. Bereits im Jahresbericht 1996 wurde betont, dass der FHR ,... mit besonderer Sorgfalt darauf (achtet), dass bestehende und ihre Aufgabe erfüllende Bildungsangebote durch neue fachhochschulische Angebote nicht dupliziert oder in ihrer Existenz bedroht werden“, im UniStG würde demgegenüber bei der Einrichtung von Universitätslehrgängen aber keine derartige Rücksichtnahme vorgeschrieben.

In anderen FH-Systemen sind die Fragen der Strukturbereinigung teilweise in anderer Weise gelöst als in Österreich. Die fachliche Diversifikation ist in den verglichenen Systemen 
breiter, obwohl es andererseits ebenfalls einen Schwerpunkt in den Bereichen Wirtschaft und Technik gibt. Es hat auch den Anschein, dass die Diversifizierung eher als Stärke gesehen wird, während in Österreich - auch aus der Sicht von VertreterInnen des FH-Sektors - eher die Probleme hervorgehoben werden, die mit einer Erweiterung des Fächerspektrums im $\mathrm{FH}$-Sektor verbunden werden. Auch die Rollenteilung mit dem Universitätssystem ist in manchen Systemen (insbesondere in Finnland) insofern anders geregelt, als der FH-Sektor schwerpunktmäßig auf der Bachelor-Ebene angesiedelt ist, während das Universitätssystem eine stark ausgeprägte Forschungsorientierung aufweist.

\subsubsection{Latente bildungspolitische Ziele}

Die latenten bildungspolitischen Ziele betreffen v.a. die Reform des Regulationssystems im österreichischen Bildungswesen, die in Kapitel 4, Abschnitt 4.2.3 näher behandelt werden.

In vielen offiziellen und halboffiziellen Dokumenten wie auch in der wissenschaftlichen und ExpertInnendiskussion wird als ein wichtiges Ziel des FHStG auch die Reform der Regulation und Steuerung im österreichischen Bildungswesen angesprochen. „Bildung ist eine öffentliche Aufgabe. In der österreichischen Tradition hat dies den Staat zu einer möglichst weitgehenden Regelung des Bildungssystems veranlasst. (...) In den letzten Jahren rückten die Nachteile einer Zentralisierung von Entscheidungsstrukturen in den Vordergrund. (...) Vor diesem Hintergrund wurde für den Fachhochschulbereich ein anderer Ansatz gewählt." (Erläuterungen zur Regierungsvorlage des FHStG, 949 Beil. Sten.Prot.NR XVIII. GP, 10). ${ }^{110}$ Im ersten Bericht der zuständigen Ministerien an das Parlament wird von einem „bildungspolitischen Paradigmenwechsel“ gesprochen (BMWF/BMUkA 1995) und John Pratt, der Berichterstatter des OECD-Reviews über die Diversifikation im österreichischen Hochschulsystem, hebt aus internationaler Perspektive diese Aspekte hervor (Pratt/Hackl 1999, 37, 38). „The Fachhochschule policy took an approach that was new to Austrian public administration“ und er bezeichnet diesen Ansatz als „particularly radical in the Austrian context."

Das neue Modell der Regulation und Steuerung ist eingebettet in eine breitere internationale Entwicklung zu sehen, die sich seit den 1980er Jahren im Rahmen der internationalen Organisationen (v.a. Weltbank und OECD) etabliert und seit dem Gipfel von Lissabon auch in der Europäischen Union deutlich an Impetus gewonnen hat. Dieses Modell bezieht sich erstens auf neue Formen der internen Organisation und des Managements der Bildungsanbieter (oft umschrieben mit dem Begriff des New Public Management - NPM) und zweitens, in engem Zusammenhang damit, auf neue Formen der externen politischen

\footnotetext{
110 Vgl. neben vielen anderen auch: BMWFK/BMUkA 1993; Höllinger/Hackl/Brünner 1994; Pratt/Hackl 1999; Prisching 2002.
} 
Steuerung dieser mehr oder weniger „autonomen“ Anbieter (oft umschrieben mit dem Begriff der Ziel-Ergebnis-Steuerung - „management by objectives - MBO“, wobei die Ziele im demokratischen Prozess festgelegt werden sollen) (vgl. auch Abschnitt 4.1). Die Unterschiede zwischen dem traditionellen Modell der Regulation und Steuerung und dem neuen Modell können in vielen Facetten akzentuiert und herausgearbeitet werden. Vor allem in zwei wesentlichen Aspekten unterscheidet sich das neue Modell von der traditionellen Bildungsverwaltung: Erstens befinden sich die Bildungsanbieter nicht mehr direkt im staatlichen Eigentum (der staatliche Einfluss muss also auf indirekte Weise ausgeübt werden); zweitens verlagert sich der Fokus von der Festlegung von mehr oder weniger detaillierten Verfahrensvorschriften („Konditionalsteuerung“, Input-Steuerung) auf die Festlegung und das Monitoring von Zielen und angestrebten Ergebnissen. ${ }^{111}$ Da das österreichische Bildungssystem besonders stark nach der traditionellen Regulierungsform organisiert war, ist mit dem FHStG auch ein besonders deutlicher und in dieser Deutlichkeit auch erster Schritt in eine neue Richtung gegangen worden. ${ }^{112}$

Im zentralisierten Umfeld standen die Schritte in Richtung Dezentralisierung und Devolution im Vordergrund, die Entwicklung des FH-Sektors sollte „bottom-up“ durch Initiativen verschiedenster Akteure vor sich gehen können. In dieser Hinsicht ist die Entwicklung des FH-Sektors ganz klar als Erfolgsgeschichte zu sehen. Es sind bedeutende Energien freigesetzt worden und die nicht wenigen skeptischen Stimmen, die das Modell zunächst als "fremd" für die österreichische Bildungslandschaft gesehen und vielleicht sogar ein Scheitern des Modells erwartet haben, haben offensichtlich nicht recht behalten.

Eine Bewertung sollte jedoch beide Seiten des neuen Regulationsmodells, die Dezentralisierung und Devolution einerseits und die politische Steuerung und Koordination andererseits berücksichtigen. Die zweite Seite wurde von Anfang an kritisch gesehen und die kritischen Stimmen haben sich im Zusammenhang mit dem Wachstum des Sektors verstärkt. In dieser Bewertung kommt der Entwicklungs- und Finanzierungsplanung des Bundes und der Rollenverteilung zwischen öffentlichen Akteuren und FHR eine Schlüsselrolle zu.

Insbesondere der FHR hat von Anfang an eine stärkere politische Steuerung eingemahnt. Vor allem Aussagen über berufliche Schwerpunktbereiche (darunter auch die teilweise bis

111 Die Unterlagen zur OECD-Ministerlnnenkonferenz 1996 (veröffentlicht als OECD 1996) können als paradigmatisches Dokument für dieses neue Modell gesehen werden, wo ganz klar herausgearbeitet wird, dass mit der veränderten Rollenverteilung zwischen den bildungspolitischen Akteuren und der stärkeren direkten Partizipation von verschiedenen Akteuren (Eltern, Regionen, Sozialpartner, Unternehmen, NGOs etc.) die Anforderungen an die politische Steuerung und Koordination nicht geringer werden, sondern aufgrund der steigenden Komplexität der Systeme im Gegenteil noch eher steigen. Vgl. Lassnigg 2000.

112 Auch in den anderen Bildungsbereichen haben parallel und nachfolgend Entwicklungen in eine ähnliche Richtung stattgefunden, wenn auch teilweise viel weniger ausgeprägt, etwa die Ansätze zur Schulautonomie (Posch/Altrichter et al. 1992, Friedrich 1993) oder die aktuelle Universitätsreform. 
heute offenen Fragen der Strukturbereinigung im postsekundären Bildungswesen) und die regionale Verteilung der Angebote wurden bereits im Jahresbericht 1995 als Arbeitserschwernisse hervorgehoben. Im Jahresbericht 1996 wurde festgestellt, dass es „unverantwortlich wäre, den weiteren Ausbau des Fachhochschulbereiches ohne Strukturbereinigungen und Reformen der Universitäten, Kunsthochschulen und des schulischen Postsekundarbereiches zu betreiben“ und es wurde von der „Tendenz der Reformvermeidung durch Neugründung" gesprochen. Im Jahresbericht 1999 wurde die "Sorge" über die schon immer problembehaftete Standortfrage besonders akzentuiert und an die Verantwortung der Gebietskörperschaften (namentlich der Bundesländer) appelliert, die „sich nicht dem politisch artikulierten Druck lokaler Interessen beugen“ sollten.

Im Rahmen der Primärerhebungen zum vorliegenden Bericht war der Wunsch nach klareren politischen Steuerungsvorgaben eine der häufigsten und einhelligsten Äußerungen unserer InterviewpartnerInnen.

Teilweise haben sich in dieser Frage im Laufe der Entwicklung die Gewichte etwas verschoben, indem der FHR durch eine extensive Definition seiner Aufgabe der Qualitätssicherung bis zu einem gewissen Grad auch Steuerungsaufgaben berücksichtigt hat. Der Grundsatzbeschluss (vgl. FHR 2001), der Konsolidierung von Standorten Vorrang vor der Eröffnung neuer Standorte zu geben, kann als Beispiel herangezogen werden. Neuere Analysen der Regulation des FH-Sektors haben herausgearbeitet, dass möglicherweise ein Regelungsdefizit bestehe und der FHR de facto viele Regelungs- und Steuerungsaufgaben übernommen habe, die bestenfalls durch eine sehr extensive Auslegung der Aufgabe der Qualitätssicherung begründet werden können (Prisching 2002).

Die Entwicklung von bildungspolitischen Zielvorgaben und die Festlegung von angestrebten Ergebnissen ist eine Aufgabe, die selbstverständlich nicht vom $\mathrm{FH}$-Sektor oder für den FHSektor allein geleistet werden kann, da dabei die Positionierung seines Stellenwertes im Gesamtsystem zur Diskussion steht. Wie im Folgenden gezeigt wird, gibt es seit der Errichtung des FH-Sektors eine Reihe von Entwicklungen, die die Gesamtarchitektur des Bildungswesens tangieren und eine verstärkte Beachtung bildungspolitischer Steuerungsfunktionen nahe legen. Der erfolgreiche und für Österreich sehr innovative Weg der inkrementalistischen „bottom-up“-Strategie scheint daher nicht allein vom Gesichtspunkt des Größenwachstums, sondern auch von einigen anderen Anforderungsfaktoren im wirtschaftlichen, gesellschaftlichen und politischen Umfeld her an Grenzen zu stoßen. 


\subsection{Veränderungen im Umfeld des FH-Sektors}

\subsubsection{Endogene Entwicklung und Dynamik im internationalen Vergleich}

Die Errichtung des FH-Sektors fand in der Vorbereitungsphase zum EU-Beitritt statt und die EU-Kompatibilität der postsekundären Bildungsebene in Österreich war vielleicht auch der wichtigste treibende Faktor, der zu dieser Errichtung geführt hat. Österreich hat eine radikal innovative Lösung von der Gestaltung des Modells her gewählt, aber gleichzeitig eine sehr vorsichtige Lösung vom quantitativen Wachstumspotential und vom Einfluss auf das Gesamtbildungssystem her gesehen. Vor allem die bereits angesprochenen Vergleiche mit der Entwicklung in Finnland und der Schweiz, wo ebenfalls in den 90er Jahren mit der Errichtung eines $\mathrm{FH}$-Sektors begonnen wurde, machen diesen Unterschied augenfällig: das Wachstum war in diesen Ländern deutlich rascher, da vorhandene Einrichtungen den Ausgangspunkt darstellten (vgl. Abschnitt 2.3). Aus dem gleichen Grund war auch der Einfluss auf die Entwicklung des gesamten Bildungswesens in diesen Ländern viel deutlicher, da wichtige vorhandene Einrichtungen des postsekundären Bildungswesens einem grundlegenden, politisch gesteuerten Reformprozess unterworfen wurden bzw. noch werden.

In der Schweiz ist die Errichtung des FH-Sektors mit drei wesentlichen politischen Steuerungsaufgaben verbunden (EFHK 2000, 3-19): Erstens erfolgt eine Verstärkung der Bundeskompetenz und der interkantonalen Koordination der Hochschulpolitik; zweitens wird bei der Implementation der Fachhochschulen größter Wert auf die Ergänzung der „bottomup“- Prozesse durch strategisches „top-down“- Management im Wege der Errichtung von schlagkräftigen und ergebnisorientierten Führungsstrukturen auf $\mathrm{FH}$-Ebene gelegt; drittens ist der Aufbau der F\&E-Funktion mit dem Ziel des Aufbaus von etwa 10 nationalen Kompetenznetzen verbunden. ${ }^{113}$ In den finnischen Polytechnics wurden im Zuge der Reform wesentliche Weiterentwicklungen gegenüber den Vorläuferorganisationen erreicht, wie im laufenden OECD-Review konstatiert wird (OECD 2002, 14).

Damit ist in diesen beiden Vergleichsländern ein unvergleichlich rascherer Wandel der Struktur des Qualifikationsangebotes und damit mittel- und längerfristig auch der Qualifikationsstruktur der Beschäftigten ausgelöst worden, als dies in Österreich der Fall ist. Ebenso sind die neuen Einrichtungen in diesen Ländern im Durchschnitt deutlich größer konzipiert als die österreichischen $\mathrm{FH}$-Einrichtungen und können damit jedenfalls potentiell ein breiteres Spektrum an Funktionen wahrnehmen. Die größeren Institutionen mit Größenordnungen von durchschnittlich 2.000 bis 3.000 Studierenden sind jedoch in ihrem

${ }^{113}$ Für 1998 -2003 wurden in zwei Etappen zusätzliche Mittel für F\&E im Ausmaß von 110 Mio. SFR veranschlagt. 
Inneren teilweise sehr stark aufgegliedert, in verschiedenen Formen von Teilschulen oder Multicampus-Strukturen, sodass die einzelnen Studiengänge oft sehr klein sind. ${ }^{114}$

Nachdem bisher die Entwicklung des FH-Sektors im Vergleich zu den Zielsetzungen analysiert wurde und auch eine ansatzweise Positionierung im internationalen Vergleich vorgenommen wurde, stellt der folgende Abschnitt diese Entwicklung in einen breiteren Zusammenhang von Veränderungen in seinem Umfeld, die sich vor allem aufgrund der neueren Entwicklungen der europäischen Politik zur Förderung der wissensbasierten Wirtschaft und Gesellschaft ergeben. Damit ergeben sich Verschiebungen der Gewichte im Bildungswesen und neue Anknüpfungspunkte zu anderen gesellschaftlichen Bereichen, wie dem Innovationssystem oder der regionalen Entwicklung, die erst näher ausgelotet werden müssen. Im folgenden Abschnitt wird eine schematische Einordnung des FH-Sektors vorgenommen und vor allem der Zusammenhang zum Bereich der Forschung und Innovation näher betrachtet. Diese Verbindung ergibt sich durch die starke Priorität, die auf die Entwicklung von Forschung und Technologie in jüngerer Zeit auch in Österreich gelegt wurde. ${ }^{115}$

\subsubsection{Wesentliche Veränderungen im Umfeld des FH-Sektors und zentrale Entwicklungsfelder}

Seit der Errichtung des FH-Sektors am Anfang der 90er Jahre sind neben den „endogenen“ Veränderungen aufgrund des Aufbauprozesses, des Größenwachstums sowie der damit verbundenen Verbreitung und Diversifizierung also auch wesentliche Veränderungen im nationalen und transnationalen Umfeld vor sich gegangen bzw. gehen weiterhin vor sich. Eine Synopse dieser Veränderungen wird im Anhang, Abschnitt 11.1.3 präsentiert.

In Stichworten werden die verschiedenen Dimensionen von Umfeld-Veränderungen im folgenden Schema (Abbildung 43) skizziert. Wesentlich an diesen Veränderungen ist, dass sie insgesamt auf das Erfordernis einer stärkeren Koordination der verschiedenen Bereiche des Bildungs- und Hochschulwesens und auch der verschiedenen sich berührenden Politikbereiche hinwirken. Während in der Anfangsphase des FH-Sektors seine Ausdifferenzierung und eigenständige Entwicklung im Vordergrund stand, stellen sich zunehmend Fragen neuer Verbindungslinien und Verknüpfungen, für die auch erst geeignete Strukturen gefunden werden müssen: Verbindung von Erstausbildung und Weiterbildung, Verbindung der traditionellen Lehr- und Forschungsaufgaben mit neuen Dienstleistungen,

\footnotetext{
114 Jährliche Anfängerlnnenzahlen pro spezialisiertem Studiengang von 20 sind an finnischen Polytechnics keine Seltenheit; in der Schweiz wurde mit 15 Anfängerlnnen pro Studiengang als Mindestgröße begonnen, eine Mindestzahl von 20 wird als sinnvoller angesehen, sodass mit der Mindestzahl von drei Studiengängen eine Teilschule mit mindestens etwa 500 Studierenden pro Teilschule entsteht.

115 Siehe die Dokumente des Rates für Forschung und Technologieentwicklung: http://www.rat-fte.at/de.php.
} 
Verbindung von Bildungs- und Innovationssystem, Verbindung von Regionalisierung und Internationalisierung sind einige Stichworte in diesem Zusammenhang.

Neben den Veränderungen, die an den Grenzen des Bildungswesens vor sich gehen, sind mit der Entwicklung des europäischen Forschungsraumes auch Veränderungen in den Hochschulsystemen verbunden, die die Grenzen zwischen Universitäten und Fachhochschuleinrichtungen wie auch deren Arbeitsteilung betreffen. ${ }^{116}$ Die Differenzierungslinien innerhalb der Hochschulsysteme verlaufen in verschiedenen Ländern unterschiedlich und es wird teilweise davon ausgegangen, dass die verstärkte Internationalisierung und die sich verändernden Strukturen der Wissensproduktion zu Umgruppierungen innerhalb der Hochschulsysteme und zu verstärktem Wettbewerb zwischen den verschiedenen Hochschulinstitutionen führen werden (vgl. z.B. Europäische Kommission 2003; vgl. auch das Kapitel 6 zur Internationalisierung). Diese Entwicklungen betreffen insbesondere auch das Verhältnis von Lehre und Forschung in den Institutionen, das im Zusammenhang mit der Entwicklung des Hochschulraumes verstärkte Aufmerksamkeit bekommt. Erstens wird von der europäischen Ebene große Aufmerksamkeit auf die Entwicklung von Spitzenforschung gelegt, die entsprechende Prioritätensetzungen und Kooperationen erfordert, zweitens wird die Entwicklung und Erweiterung des Forschungsnachwuchses stark betont. Das Forschungsprofil der FH-Institutionen wird damit sicher zunehmende Aufmerksamkeit bekommen.

116 Siehe Europäische Kommission 2000 (ftp://ftp.cordis.lu/pub/documents r5/natdir0000001/s 137200520010125143514 C001190de.pdf), vgl. auch: http://www.tip.ac.at/(de)/policy/eu.html, http://www.cordis.lu/rtd2002/era-debate/era.htm. 


\section{Abbildung 43: Übersicht über Umweltveränderungen}

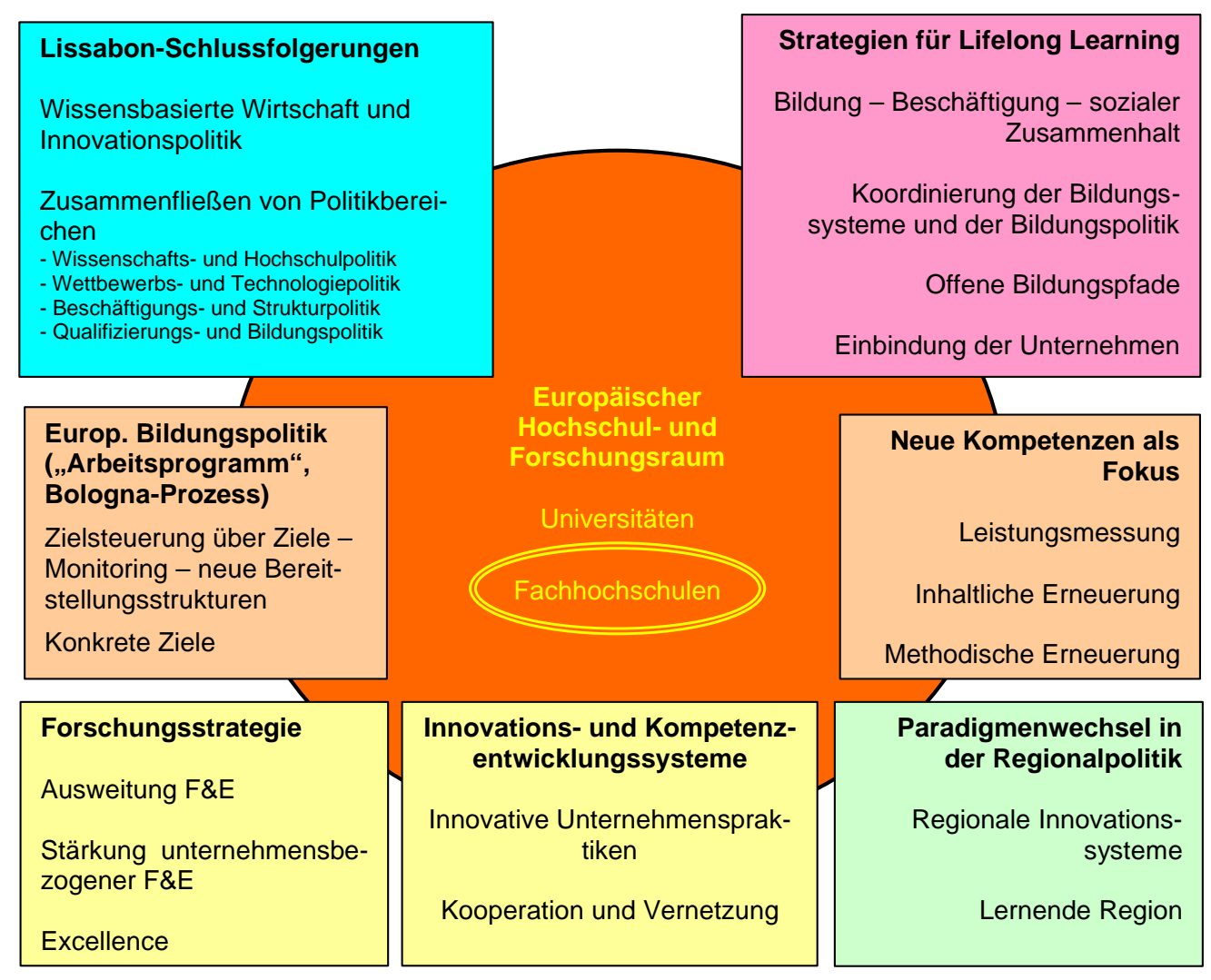

Quelle: IHS-Darstellung

Die in Abbildung 43 skizzierten Veränderungen in den Feldern der Beschäftigungs-, Bildungs-, Innovations- und Regionalpolitik, die durch den Ansatz der Förderung der wissensbasierten Wirtschaft in der Lissabon-Strategie wesentlich beeinflusst sind, unterstreichen und verstärken bestimmte Akzente, die bereits in der Analyse der Entwicklung des FH-Sektors und der Verwirklichung der Zielsetzungen herausgearbeitet wurden: Im Bereich der Bildungspolitik wird eine deutlichere Formulierung der Zielsetzungen und der erwarteten Ergebnisse betont, die die neuen Konzepte der Formulierung und Messung von Kompetenzen und die Erneuerung der pädagogischen Methoden berücksichtigen sollen; im Bereich der Innovationspolitik wird einerseits der Bezug zur Forschung und Entwicklung und andererseits zur Förderung innovativer Unternehmenspraktiken und der Verbindung zu unternehmensbezogenen Kompetenzentwicklungssystemen unterstrichen; im Bereich der Beschäftigungspolitik stellt sich die Herausforderung der Förderung des Systems des lebensbegleitenden Lernens; im Bereich der Regionalpolitik wird die Verstärkung der Innovationsgesichtspunkte gegenüber den Verteilungsgesichtspunkten betont.

Die folgende schematisierte Darstellung (Abbildung 44) bezieht die verschiedenen Dimensionen der skizzierten Umfeld-Veränderungen zusammenfassend auf den FH-Sektor 
bzw. die FH-Institutionen und bündelt diese vor allem in drei entwicklungsbestimmende Felder (Entwicklungsfeld 1 bis 3), die aufgrund dieser Veränderungen an Gewicht gewinnen werden. Die Veränderungen im Umfeld werden in vier Dimensionen zusammengefasst, zwei davon betreffen die Bildungspolitik im engeren Sinne (Umfelddimensionen 1 und 2), zwei weitere (Umfelddimensionen 3 und 4) betreffen die Einbettung der Bildungssysteme in Aspekte ihres Umfeldes:

- Umfelddimension 1: Europäisierung der Bildungspolitik (Bologna-Prozess, Arbeitsprogramm und neuerdings Brügge-Prozess, vgl. Kapitel 6) im Zusammenhang mit der Formulierung von EU-weiten Zielen für die Bildungs- und Ausbildungssysteme und mit den damit verbundenen Methoden der angestrebten Koordinierung der Bildungspolitik (diversifizierte Angebotsstrukturen, Zielsteuerung, Qualitätssicherung und verschiedene Methoden von Erfahrungsaustausch)

- Umfelddimension 2: Umstellung der Bildungs- und Ausbildungssysteme von der Fokussierung auf Erstausbildung in Richtung auf die Öffnung für lebenslanges oder lebensbegleitendes Lernen, was für die verschiedensten Dimensionen der Bildungspolitik neue Herausforderungen mit sich bringt und insbesondere eine verstärkte Integration und Koordinierung der Gesamtsysteme und der damit verbundenen BildungsBeschäftigungslaufbahnen erfordert.

- Umfelddimension 3: Innovationspolitik als Ansatz, um die Verbindung zwischen dem Bildungswesen und den neuen Gewichtungen in der Wirtschafts-, Arbeits- und Lebenswelt aufgrund der Entwicklung der wissensbasierten Wirtschaft sowie der Informations- und Wissensgesellschaft herzustellen. Einerseits bekommen die Wissensproduktion und die damit verbundenen Innovationsprozesse ein neues Gewicht, andererseits steigt die Bedeutung der Investitionen in Bildung, Qualifikation und Kompetenz.

- Umfelddimension 4: Essentielle Bedeutung der Vernetzungen zwischen Akteuren aus Wirtschaft, Politik, Bildung und Zivilgesellschaft auf den verschiedenen gesellschaftlichen und sozialen Aggregationsebenen, von der lokalen und regionalen Ebene über die nationale und transnationale Ebene bis zur globalen Ebene. Diese Vernetzungen betreffen im Zusammenhang mit den FH-Institutionen unmittelbar die Akteure des Innovationssystems (Firmen, F\&E, Intermediäre, Bildung etc.) und die neuen Vernetzungen in den sich verändernden regionalen Räumen im Zusammenhang mit Globalisierung, Internationalisierung und Regionalisierung.

Die daraus resultierenden drei Entwicklungsfelder, in deren Schnittpunkt der FH-Sektor positioniert werden kann, betreffen die folgenden Bündelungen von Einflüssen und Interaktionen zwischen den $\mathrm{FH}$-Einrichtungen und ihrem engeren und weiteren Umfeld: 
- Entwicklungsfeld 1: In diesem Entwicklungsfeld sind die Ansätze der Innovationspolitik und der europäischen Bildungspolitik gebündelt. Die Interaktion zwischen Ausbildung und Innovation betrifft unmittelbar die Gestaltung des Aufgabenportfolios der Fachhochschulen bzw. der FH-Studiengänge. Die zentrale Frage besteht darin, welcher Beitrag von den FH-Institutionen im Innovationssystem erwartet wird. Der erwartete Beitrag kann einerseits größer oder kleiner dimensioniert sein (in Form von Input- oder Output-Vorgaben) und er kann sich auf verschiedene Dimensionen des Aufgabenportfolios beziehen (Ausbildung von Nachwuchs, F\&E-Bereiche zwischen Grundlagenforschung und angewandter Entwicklung, Dienstleistungen etc.) Daraus ergibt sich erstens, inwieweit diese Erwartungen spezifiziert oder offen gelassen werden und zweitens, bis zu welchem Grad diese Erwartungen an die FH-Institutionen generalisiert werden. Diese Spezifikationen sind bisher noch ziemlich offen und liegen in einem Entscheidungsraum, dessen Optionen nicht zwingend aufgrund wissenschaftlicher Argumente eingeschränkt werden können. Ohne Spezifikation wird sich die vorhandene Diversität vermutlich noch verstärken und der Beitrag des FH-Sektors zum Innovationssystem wird vermutlich hinter seinen Möglichkeiten zurückbleiben.

- Entwicklungsfeld 2: Das Entwicklungsfeld der Unternehmenspraktiken betrifft die Positionierung der Fachhochschulen bzw. der FH-Studiengänge im Zusammenspiel zwischen Innovationspolitik einerseits und den Unternehmen als Akteure im Innovationssystem andererseits. Die Wettbewerbsfähigkeit in der wissensbasierten Wirtschaft wird heute nicht nur auf technologische Faktoren zurückgeführt, sondern auch auf organisatorische Entwicklungen und die Orientierung an innovativen Strategien. Die zentralen Fragen bestehen darin, wie die Rolle der FH-Institutionen im Hinblick auf ihren aktiven Beitrag in der österreichischen Innovationspolitik spezifiziert wird und inwieweit von diesen nicht nur Bedarfsdeckung, sondern auch Impulse für das Innovationsverhalten der Unternehmen erwartet werden. ${ }^{117}$ Während das Entwicklungsfeld 1 sich stärker auf den Beitrag zur Produktion und Vermittlung neuen Wissens bezieht, geht es im Entwicklungsfeld 2 stärker um die Verbreitung und Nutzung des Wissens. Im Zusammenhang mit der Entwicklung und Förderung innovativer Unternehmenspraktiken kommt dem Aufbau von Kompetenzentwicklungssystemen im Unternehmenssektor große Bedeutung zu, daher ergibt sich in diesem Entwicklungsfeld

117 Im österreichischen Forschungs- und Technologiebericht 2002 wird der Aspekt der Weiterentwicklung im Unternehmenssektor deutlich unterstrichen: „Die vorhandene Industriestruktur in Österreich kann demnach nach wie vor als eine strukturelle Schwäche auf dem Weg zu einer wissensbasierten Gesellschaft interpretiert werden ... Der Bericht zeigt somit, dass Österreichs ,Technologielücke' nicht durch eine niedrigere Forschungsintensität alleine erklärt werden kann, sondern aufgrund der vorhandenen Industriestruktur sich die Spezialisierung der österreichischen Wirtschaft auf weniger dynamische Sektoren beschränkt."( BMBWK/BMVIT 2002, 1,2). 
auch eine Verbindung zur Förderung des lebensbegleitenden Lernens auf Unternehmensebene. ${ }^{118}$

- Entwicklungsfeld 3: Das Entwicklungsfeld der Positionierung der FH-Institutionen im regionalen Kontext betrifft die Adoption des entwicklungs- und innovationsorientierten Verständnisses von Regionalpolitik (gegenüber dem traditionellen distributiv orientierten Verständnis) und die damit verbundenen Effekte und Anforderungen an die Vernetzungen der Akteure im regionalen Innovationssystem bzw. in lernenden Regionen. Die zentralen Fragen bestehen darin, welche Rolle die Fachhochschulen bzw. der FH-Studiengänge in der Entwicklung von effektiven Kooperations- und Vernetzungsstrukturen in ihrem regionalen Kontext spielen können und inwieweit sie als Mittler zwischen den verschiedenen Aggregationsebenen der regionalen Dimension (von der eher kleinräumigen lokalen und regionalen Dimension zur nationalen und übernationalen Dimension) fungieren können. Im lokalen und regionalen Kontext ergibt sich im Zusammenhang mit dem Konzept der lernenden Region die Frage, welcher Beitrag von den FH-Institutionen bei der Entwicklung einer österreichischen Lifelong-learningStrategie erwartet wird. ${ }^{119}$ Für die Verbindung zwischen den Aggregationsebenen der Regionalisierung und Internationalisierung bildet die Entwicklung von Kooperationen im internationalen Raum und damit auch gewissermaßen die Wettbewerbsposition der $\mathrm{FH}$ Institutionen die Schlüsselfrage.

Aufgrund der Veränderungen im Umfeld des FH-Sektors stellen sich in den drei Entwicklungsfeldern längerfristig Herausforderungen, auf die in unterschiedlicher Weise reagiert werden kann. Bisher sind die erwarteten Beiträge wenig spezifiziert und die vorhandenen Potentiale sind auf diese Herausforderungen wenig ausgerichtet. Die möglichen Beiträge werden einerseits jedenfalls von den Ressourcen abhängen, die investiert werden. Andererseits kann auf unterschiedlichen Ebenen des FH-Sektors mit diesen Entwicklungsfeldern umgegangen werden: Es können die einzelnen Institutionen ihre Strategien bilden und ihre Kräfte aufstellen oder es können breitere Strategien für den Gesamtsektor oder bestimmte Teilbereiche entwickelt werden. Jedenfalls ergibt sich hier ein Spektrum an Optionen, für deren Spezifizierung politische Entscheidungen erforderlich sind. Diese können auf verschiedenen Ebenen getroffen werden, wenn sie nicht getroffen werden, wird es vermutlich zu einer Zersplitterung der Kräfte kommen.

118 Dem Aufbau von unternehmensinternen oder unternehmensnahen Mechanismen der Kompetenzentwicklung wird in den europäischen Ansätzen der Beschäftigungs- und Bildungspolitik großer Wert beigemessen, vgl. European Commission 2002 (http://europa.eu.int/comm/employment_social/news/2002/feb/ap_en.html).

119 Bei der Bewertung der Strategien zur Förderung des lebensbegleitenden Lernens im europäischen Vergleich schneidet Österreich bisher nicht günstig ab (vgl. den Gemeinsamen Beschäftigungsbericht 2001 sowie den Entwurf zum gemeinsamen Beschäftigungsbericht 2002, 25). (http://europa.eu.int/comm/employment social/news/2002/nov/jer2002 draft en.pdf). 
Abbildung 44: Schematische Darstellung der FH-Institutionen im veränderten Umfeld: Umfelddimensionen und Entwicklungsfelder

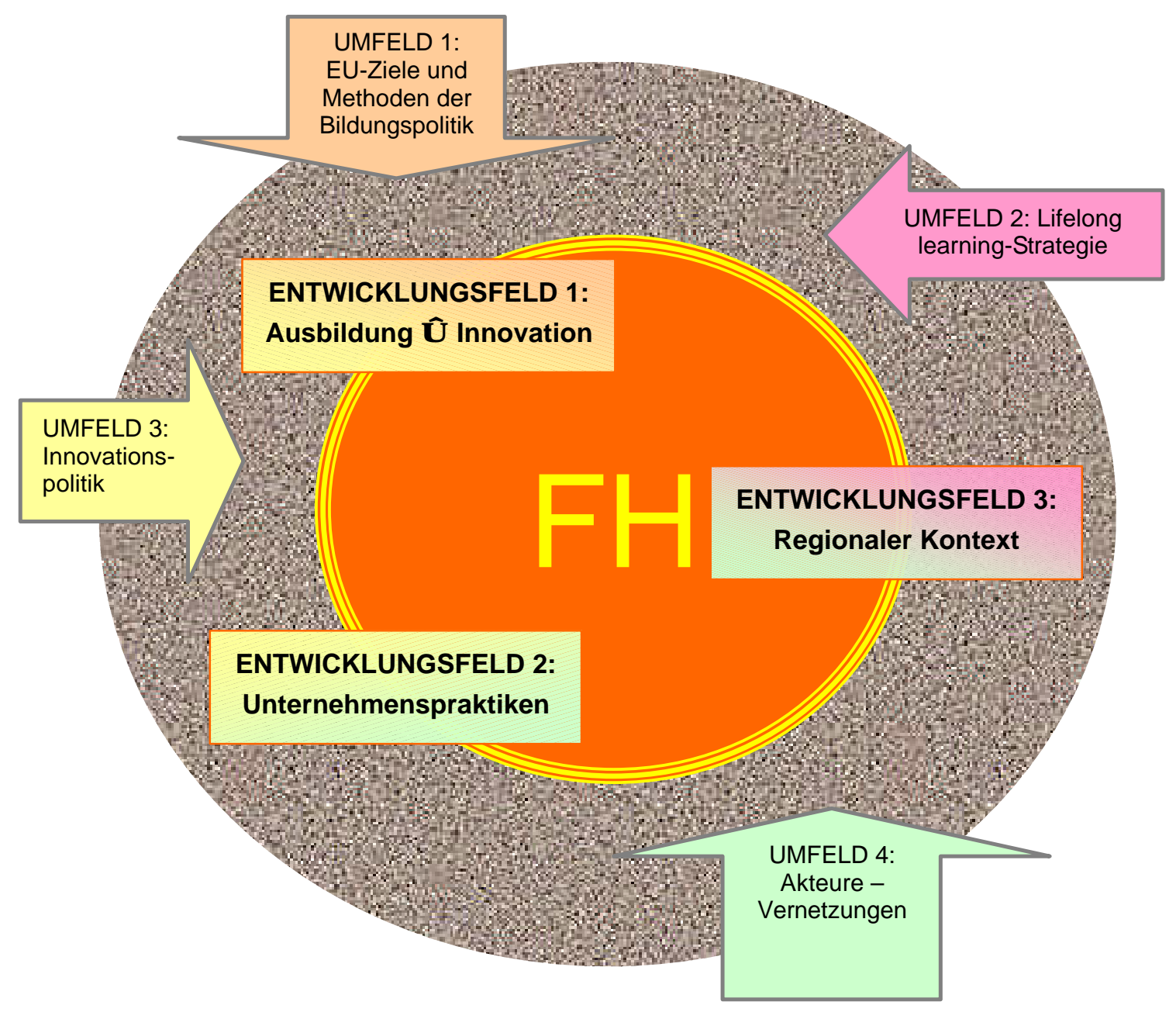

Quelle: eigene Darstellung

\subsubsection{Der FH-Sektor in der Forschungsstrategie}

Der Stellenwert des Fachhochschulbereichs im Hochschulsystem wird davon abhängen, wie sich die $\mathrm{FH}$-Institutionen in diesem veränderten Umfeld positionieren bzw. positioniert werden. Im folgenden wird der Aspekt der Einbindung der FH-Institutionen in die Entwicklung der österreichischen Forschungsstrategie, wie sie im Laufe der letzten Jahre vom Rat für Forschungs- und Technologieentwicklung im Rahmen der Innovationspolitik ausgearbeitet 
wurde, näher diskutiert. ${ }^{120}$ Die Entwicklung der Innovationspolitik betrifft das angestrebte Verhältnis zwischen der Ausbildung für einen gegebenen Bedarf einerseits und der Ausbildung im Wechselspiel mit der Forschung sowie der Unterstützung von Innovationsimpulsen andererseits (vgl. auch Abschnitt 5.2.4).

In der Praxis und Konzeption der Fachhochschulen und Fachhochschul-Studiengänge kann dieses Verhältnis umgesetzt werden in die Frage nach dem angestrebten Aufgaben-Portfolio in Form der Verteilung zwischen den Funktionen Lehre, F\&E und Dienstleistungen. Im Bereich der Innovationspolitik ist seit Jahren ein Handlungsbedarf in Österreich unbestritten und aktuell wird die Frage nach der Beteiligung des FH-Sektors an der österreichischen Forschungsstrategie verstärkt gestellt. Clement et al. (2002) haben die Implikationen der Erhöhung der österreichischen Forschungsquote für verschiedene Zielbereiche des Forschungssystems in Form von strategischen Überlegungen ausgelotet. In diesen Überlegungen wird dem FH-Sektor eine außerordentlich wichtige Rolle zugeordnet.

Den Hintergrund bildet die Überlegung, dass in Österreich vor allem der Anteil des Unternehmenssektors an den F\&E-Aktivitäten vergleichsweise gering ist und erhöht werden sollte, während der Anteil der Universitäten etwa durchschnittlich ist. Die Lücke besteht also vor allem in der unternehmensnahen F\&E (vgl. auch BMBWK/BMVIT 2001, Felderer et al. 2001, Hutschenreiter/Polt/Gassler 2001). Aufgrund der Erhöhung der Forschungsquote als Zielindikator konzentrieren sich die Überlegungen bisher auch stark auf die finanzielle Seite der Aufbringung und Allokation zusätzlicher Mittel. Ein Eckpunkt der Strategie ist ein beabsichtigtes unterschiedliches Wachstumstempo der drei Sektoren: (a) universitäre Forschung, mit einer valorisierten Fortschreibung im Prinzip 1:1, (b) außeruniversitäre Forschung mit besonders dynamischer Entwicklung (im Prinzip 1:3, also etwa eine Verdreifachung der Mittel bis 2005), (c) die Forschung im Unternehmenssektor sollte die Dynamik der vergangenen Jahre fortsetzen und die Aktivitäten bis 2005 verdoppeln (im Prinzip 1:2).

Diese Strategie umfasst also zumindest implizit eine bestimmte Vorstellung über die Entwicklung der Rollen und des Zusammenspiels zwischen den drei Sektoren im Innovationssystem. Der FH-Sektor ist in dem am stärksten wachsenden Bereich der außeruniversitären Forschung angesiedelt. Innerhalb dieses Bereichs werden vier SubBereiche unterschieden: Grundlagenorientierte und staatliche F\&E-Einrichtungen (1), Anwendungsorientierte F\&E-Einrichtungen (2), Temporäre Kompetenzzentren und F\&E\&IProgramme (3) und FH-orientierte F\&E-Zentren bzw. Programme (4), wobei in einer ersten

120 Siehe Austrian Council, Forschungsstrategie Austria „2,5\% + plus“ Wohlstand durch Forschung und Innovation (http://www.rat-fte.at/files/strategie final.pdf) sowie: Vision 2005 (http://www.rat-fte.at/files/vision2005 final.pdf); siehe auch Austrian Council (2002), Nationaler Forschungs- und Innovationsplan (http://www.ratfte.at/files/NFIP 20021203.pdf). 
strategisch orientierten Analyse dem FH-Bereich eine besonders starke Dynamik zugeschrieben wird (vgl. Clement et al. 2002, Detter et al. 2002).

Mit diesem Szenario wird in gewisser Weise eine veränderte Positionierung des FH-Sektors vorgenommen, da bisher die Forschungsorientierung schwach ausgeprägt war (und teilweise auch in ihrer Sinnhaftigkeit bezweifelt wird) und auch die Vorstellungen für die Weiterentwicklung der F\&E-Tätigkeit sehr vorsichtig formuliert wurden (vgl. Grünbacher/Detter 2000, Mayer/Stampfer/Sturn 2001, auch das FH-plus-Programm; vgl. auch Maresch 2002). Abgesehen von der unrealistischen quantitativen Dynamik beruht das Szenario auf bestimmten Annahmen über das Profil der FH-Institutionen und der innen zugeordneten F\&E-Tätigkeit:

- Erstens sollte die Erfüllung der Aufgabenstellungen von Lehre, F\&E und Technologietransfer/Dienstleistungen in integrierter Weise stattfinden, wobei die Lektoren wesentlich als integrierendes Element fungieren sollten (Clement et al 2002, 52: SollStruktur von FH-Einrichtungen).

- Zweitens wird die Entwicklung der F\&E-Tätigkeit der FH-Einrichtungen und die Herausbildung von F\&E-Zentren in starkem Zusammenspiel mit verschiedenen Formen von Impulszentren (Technologietransferzentren, Spezialisierte F\&E-Zentren und clusterbezogene F\&E-Zentren) gesehen (ebd., 60-64). Eine Grundüberlegung dabei ist die Notwendigkeit der Stärkung von F\&E-Funktionen in diesen Zentren und gleichzeitig eine verstärkte Integration von F\&E-Funktionen und Technologietransferaktivitäten (die einerseits an den $\mathrm{FH}$-Institutionen und andererseits in den spezialisierten F\&E-Zentren erfolgen soll).

- Drittens erfolgt eine Positionierung der FH-Institutionen im Vergleich zu den Universitäten und verschiedenen Formen von Forschungs- und Impulszentren in den Aufgabenfeldern zwischen Vorfeldforschung und Dienstleistung, mit der klaren Betonung der Kooperationserfordernisse zwischen FH-Institutionen und Universitäten.

In Anwendung dieses Modells auf die Bedingungen der Umsetzung stellen sich die Fragen nach der Ausgangssituation (Wie stellen sich die F\&E-Aktivitäten dar? Wieweit sind sie entwickelt?), den erforderlichen Rahmenbedingungen für die Verwirklichung (Infrastruktur, Personalstruktur, Aufgabenverteilung zwischen Funktionen, „kritische Größen“ in quantitativer und qualitativer Hinsicht) und nach den Potentialen des FH-Sektors bezüglich seines Beitrages zur Forschungsstrategie (wie rasch können die Funktionen realistischerweise aufgebaut werden? Welcher Aufwand ist dafür erforderlich?). Dabei geht es um die reale Seite der finanziellen Mittelverteilung: Welche Schwerpunkte sollen gesetzt werden? Welche Standards sollen angestrebt werden? Wie viel Personal und welche Infrastruktur ist erforderlich, um die Mittel entsprechend einsetzen zu können? 


\section{Schätzungen zum F\&E-Personal}

Für diese oben angeführten Fragestellungen ist die österreichische Informationsbasis nicht gut geeignet, da nur sehr globale Informationen über das Personal und die Infrastruktur im F\&E-Bereich vorliegen, ${ }^{121}$ die auch nicht mit den Klassifikationen übereinstimmen, die im politischen Bereich herangezogen werden. Man kann überschlagsmäßig vergleichen, welche personellen Konsequenzen die Erhöhung der Finanzmittel bei konstantem Mitteleinsatz haben könnte und diese mit einer einfachen Fortschreibung der personellen Entwicklung der letzten Jahre vergleichen. Wenn man die Entwicklung des wissenschaftlichen Personals zwischen 1993 und 1998 (vgl. BMBWK/BMVIT 2001, 18-23) insgesamt einer Fortschreibung bis 2000 bzw. 2005 zugrundelegt, so ergibt sich ein absoluter jährlicher Zuwachs in der Größenordnung von 1.200 bis 1.700 Vollzeitäquivalenten (je nachdem, ob man die bereits erreichte absolute Größe heranzieht oder den prozentuellen Zuwachs auf die erhöhte Basis bezieht). Für die Fortschreibung wurde ein mittlerer Wert von 1.400 zusätzlichen wissenschaftlichen Vollzeitäquivalenten pro Jahr in der Periode 1993 bis 1998 herangezogen. In der Planungsperiode der Forschungsstrategie zwischen 2000 und 2005 ergibt die Fortschreibung aus der Vergangenheit einen Zuwachs von insgesamt 7.200 VZÄ, die man näherungsweise nach der Verteilung von 1993 auf die drei Sektoren aufteilen kann (vgl. Tabelle 11; diese Berechnungen berücksichtigen noch keinen Ersatzbedarf, der jährlich in der Größenordnung von etwas mehr als 500 VZÄ geschätzt wird; Austrian Council 2002, $61)$.

Die Anwendung der strategischen Prinzipien des FTE-Rates auf das Personal ergibt in Summe eine um fast 10.000 höhere Zahl an wissenschaftlichen Vollzeitäquivalenten in diesem Zeitraum als die Fortschreibung der Entwicklung in der Vergangenheit. Pro Jahr müsste also unter diesen Annahmen eine im Vergleich zum Zuwachs in der Vergangenheit um fast 2.000 höhere Zahl an Vollzeitäquivalenten für wissenschaftliche Tätigkeit zusätzlich rekrutiert werden. ${ }^{122}$

121 Vgl. auch Kap 4 in Austrian Council 2002, 58-63; diese Publikation ist nach Redaktionsschluss unserer Studie erschienen und kann nur teilweise berücksichtigt werden.

122 Im Vergleich zu dieser Zahl an 3.300 zusätzlichen Vollzeitäquivalenten (unter Einschluss der Fortschreibung) für wissenschaftliche Tätigkeit jährlich kann die Zahl der jährlichen Zweitabschlüsse herangezogen werden, die It. dem letzten Hochschulbericht insgesamt in der Größenordnung von 1.600 lagen. Der Austrian Council (2002, 60) schätzt aufgrund der Erstabschlüsse von Universitäten und Fachhochschul-Studiengängen ein „F\&Eorientiertes" jährliches Angebot in allen Bereichen von etwa 2.700 AbsolventInnen, darunter im technischnaturwissenschaftlichen Bereich 1.400 - 1.500 AbsolventInnen. Im Vergleich zur Zahl der Zweitabschlüsse (1.600) geht diese Angebotsschätzung von einem über 40\% höheren F\&E-orientierten Angebot von den Universitäten (2.300) aus. Die Bedarfsschätzung des Austrian Council bezieht auf das F\&E-Personal insgesamt, wenn man aufgrund des Anteils von 1998 (60\%) das wissenschaftliche F\&E-Personal herausrechnet, ergibt sich ein geringerer jährlicher Neubedarf von etwa 1.150 VZÄ; es ergibt sich aus diesen Berechnungen eine jährliche Lücke von etwa 200 wissenschaftlichen VZÄ bzw. von 300 Personen in F\&E; wenn man die Zahl der Zweitabschlüsse als Referenz heranzieht, so müsste deren Zahl um immerhin $20 \%$ erhöht werden; wenn man 
Untergliedert nach Sektoren ergibt sich eine Umschichtung vom Universitätssektor zu den beiden anderen, der Zuwachs im Unternehmenssektor wäre mit 2.500 deutlich größer als im außeruniversitären Sektor mit 800 jährlich. Wenn man nach dem Rekrutierungspotential für diese Zuwächse fragt, so muss diesem Aspekt sicherlich einige Aufmerksamkeit gewidmet werden. Da aufgrund offenbar hoher Teilzeitbeschäftigung die vorhandenen Vollzeitäquivalente die Zahl der Köpfe nur zu etwa 60\% abdecken, kann direkt im Bereich von einem gewissen Potential in Form von "Unterbeschäftigung“ ausgegangen werden andererseits ist in diesem Bereich doch die Spezialisierung hoch und es stellt sich auch die Frage, welche Arten von Qualifikationen kurzfristig benötigt werden. Gleichzeitig sollte bei massiver kurzfristiger Expansion auch die längerfristige Perspektive berücksichtigt werden. Da die 2,5\% Forschungsquote zunehmend eher als untere Grenze gesehen wird (der EUReferenzwert liegt bereits bei 3\%), dürften hier längerfristige Perspektiven gegeben sein. 
Tabelle 11: Bestand an wissenschaftlichem Personal (VZÄ) 1998 und 2000 sowie überschlagsmäßige Schätzungen der personellen Konsequenzen der Forschungsstrategie bis 2005 nach Sektoren

\begin{tabular}{|c|c|c|c|c|c|c|}
\hline & $\begin{array}{c}\text { Bestand an } \\
\text { wissenschaftl. } \\
\text { Personal } \\
\mathbf{1 9 9 8} \\
\text { (in } 1.000 \text { ) } \\
\end{array}$ & \begin{tabular}{|c|} 
Wissenschaftl. \\
Personal \\
Fortschreibung $^{*}$ \\
$\mathbf{2 0 0 0}$ \\
(in 1.000$)$ \\
\end{tabular} & \begin{tabular}{|c|} 
Wissenschaftl. \\
Personal \\
Fortschreibung $^{*}$ \\
$\mathbf{2 0 0 5}$ \\
(in 1.000$)$ \\
\end{tabular} & $\begin{array}{c}\text { Faktoren der } \\
\text { Ausweitung der } \\
\text { Finanzierung It. } \\
\text { FTE-Strategie } \\
2000-2005 \\
\end{array}$ & \begin{tabular}{|c|} 
Wissenschaftl. \\
Personal \\
Hochrechnung \\
$\mathbf{2 0 0 5}$ \\
(in 1.000 ) \\
\end{tabular} & \\
\hline UNI $I^{* \star \star *}$ & 6,0 & 6,9 & 9,2 & 1 & 6,9 & \\
\hline AUF & 1,8 & 2,1 & 2,8 & 3 & 6,3 & \\
\hline UNT & 10,9 & 12,6 & 16,8 & 2 & 25,2 & \\
\hline \multirow[t]{2}{*}{ Sum } & 18,7 & 21,6 & 28,8 & & 38,3 & \\
\hline & & & $\begin{array}{c}\text { Zuwachs } \\
2000-2005 \\
\text { Fortschreibung* }\end{array}$ & & $\begin{array}{c}\text { Zuwachs } \\
2000-2005 \\
\text { Hochrechnung }\end{array}$ & \begin{tabular}{|c|} 
Differenz \\
Zuwachs \\
Hochrechnung \\
- Fortschreibung
\end{tabular} \\
\hline UNI & & & 2,3 & & 0,0 & $-2,3$ \\
\hline AUF & & & 0,7 & & 4,2 & 3,5 \\
\hline UNT & & & 4,2 & & 12,6 & 8,4 \\
\hline \multirow[t]{2}{*}{ Sum } & & & 7,2 & & 16,7 & 9,5 \\
\hline & & & $\begin{array}{l}\text { Jährl Zuwachs* } \\
\text { Fortschreibung }\end{array}$ & & \begin{tabular}{|c|} 
Jährl Zuwachs \\
Hochrechnung
\end{tabular} & $\begin{array}{c}\text { Differenz } \\
\text { jährl. Zuwachs } \\
\text { Hochrechnung } \\
\text { - Fortschreibung }\end{array}$ \\
\hline UNI & & & 0,5 & & 0,0 & $-0,5$ \\
\hline AUF & & & 0,1 & & 0,8 & 0,7 \\
\hline UNT & & & 0,8 & & 2,5 & 1,7 \\
\hline Sum & & & 1,4 & & 3,3 & 1,9 \\
\hline
\end{tabular}

* Lineare Fortschreibung des Zuwachses zwischen 1993-1998

** Forschungsstrategie des Austrian Council

*** Wert Fortschreibung 2000 * Faktoren der FTE-Strategie (Zahl gerundet)

${ }^{* * \star *}$ Da die Aufteilung in der Statistik über das Personal nicht mit der des FTE-Rates übereinstimmt, wurde die Hochschule als UNI klassifiziert, die firmeneigene Forschung als Unternehmensbereich (UNT) und der Rest als außeruniversitär (AUF).

Quelle: IHS-Berechnungen aufgrund verschiedener Quellen (BMBWK/BMVIT 2001, Sturn et al. 1999, Clement et al. 2002).

Überlegungen zum F\&E-Personal im FH-Sektor

Was können diese Überlegungen nun für die FH-Einrichtungen bedeuten? Diese werden als Teil des außeruniversitären Sektors gesehen. Insgesamt ergibt sich für den außeruniversitären Sektor nach der Fortschreibung zwischen 2000 und 2005 ein Zuwachs von 700 VZÄ für wissenschaftliche Tätigkeit (etwas mehr als 100 pro Jahr), nach dem finanziellen Szenario liegt der hochgerechnete Zuwachs bei 4.200 VZÄ (etwa 800 jährlich). In bisherigen Überlegungen werden für die anderen Bereiche des außeruniversitären Sektors keine hohen Zuwächse veranschlagt (die Forschungszentren der Sub-Bereiche 1 und 2 sollen bei der Finanzierung zusammen um einen Faktor von 1,6 wachsen, die temporären Kompetenzzentren (Sub-Bereich 3) vernetzen bestehende Strukturen und die beschäftigten MitarbeiterInnen dürften daher in den beteiligten Organisationen verankert bleiben und nur in geringem Maße neu rekrutiert werden - als Konsequenz würde sich ein starkes Wachstum des FH-Sektors ergeben. In den angestellten Überlegungen würden die 
Forschungszentren der Subbereiche 1 und 2 bei einem Wachstum um den Faktor 1,6 unter der Annahme, dass alle bisherigen Beschäftigten des außeruniversitären Sektors in diesen Subbereichen tätig sind, nur auf 3.400 VZÄ wachsen, d.h. es bleibt etwas weniger als die Hälfte der zusätzlichen Mittel bzw. VZÄ für den FH-Bereich (für 2005 würde das einen Bestand von fast 3.000 VZÄ für wissenschaftliche F\&E im FH-Sektor ergeben).

Bisherige Überlegungen für den $\mathrm{FH}$-Sektor sind jedoch bei weitem vorsichtiger gewesen. Grünbacher/Detter 2000 haben seinerzeit in ihrem Aufbauszenario für 2005 einen Anstieg der Zahl der "forschenden Studiengänge" auf 34 (von insgesamt 140 Studiengängen, also einem Viertel) angenommen, wobei von einer Standard-Forschungsgruppe von vier Personen ausgegangen wurde; daraus ergeben sich weniger als 150 VZÄ für F\&E. Das Ausschöpfen der jährlichen Durchschnittssummen, die für das Programm FH-plus geplant waren, würde nach dem Finanzbedarf der Grünbacher/Detter-Überlegungen die Finanzierung von 16 - 18 Forschungsgruppen pro Jahr ermöglichen (d.h. Personal in der Größenordnung von 60-70 VZÄ). Die durchschnittlichen Projektgrößen würden den Finanzbedarf einer Projektgruppe für etwa zwei Jahre decken, d.h. bei einer Laufzeit von 6 7 Jahren könnten etwa 60 unterschiedliche Projektgruppen für jeweils zwei Jahre gefördert werden (also bei personeller Stabilität Personal in der Größenordnung von kumuliert etwas mehr als 250 Personen in Vollzeitbeschäftigung über zwei Jahre im gesamten Zeitraum; pro Erhalter könnte damit im Durchschnitt eine zweijährige Anschubfinanzierung für F\&E Tätigkeit von etwa 10 - 12 Personen erzielt werden, für jeden zweiten Erhalter 25 Personen, für jeden dritten 40 etc.). Diese Überlegungen berücksichtigen keine Investitionen, keine nichtwissenschaftlichen Kräfte und keine größeren Forschungsgruppen oder -einheiten (da es dies alles jedoch gibt, ist die reale Dimension der Förderpotentiale kleiner als die überschlagsmäßige Schätzung ergibt).

Man kann aber den Impact auf den Sektor in seiner Größenordnung abschätzen und Zielvorstellungen gegenüberstellen. Wenn man etwa die Förderung auf Dauer stellen wollte, wäre der Finanzbedarf zu verdoppeln (250 VZÄ auf vier Jahre) oder zu verdreifachen (auf sechs Jahre), bei stärkerer Konzentration (größere Einheiten) und gleichzeitiger Breitenwirkung erhöht sich der Finanzbedarf wiederum entsprechend. ${ }^{123}$ Nach den Auswertungen der F\&E-Daten durch Mayer/Stampfer/Sturn $(2001,48)$ kann man ableiten, dass etwa 100 VZÄ im Jahr 1999/2000 durch diese gemeldeten F\&E-Tätigkeiten der Lehrenden abgedeckt waren (dazu kommen noch einmal etwas mehr als 100 VZÄ seitens der Studierenden, der Faktor ist 1,26). ${ }^{124}$ Grünbacher/Detter $(2000,12)$ unterstellen in ihren

123500 VZÄ auf vier Jahre, also pro Erhalter Gruppen von 25 Personen, würde in dieser Rechnung kumuliert etwa die vierfache Summe des FH-plus-Programmes erfordern, aber immer noch weit unter der bei Clement et. al veranschlagten Größenordnung eines Wachstums der F\&E-Ausgaben für den Sub-Bereich auf 320 Mio. EUR (4,4 Mrd. ATS) im Jahr 2005 liegen.

124 Im Durchschnitt 1,9 Person-Monate von Lehrenden pro abgeschlossenem Projekt ergeben 1999/2000 bei 212 Projekten kumuliert 460 PM, bei 10,5 Jahresmonaten ergeben sich etwa 44 VZÄ, für die übrigen Projekte kann man dieses Ausmaß verdoppeln; für die Studierenden beträgt der Durchschnitt 2,5 PM. 
Schätzungen für 2001 eine Zahl von 10 forschenden Studiengängen (also nach ihrem Gruppenmodell etwa 40 VZÄ). Nach unseren Schätzungen aufgrund der Befragung wären etwa 250 hauptberufliche VZÄ außerhalb von Lehrtätigkeiten beschäftigt, also im Durchschnitt etwas über 10 pro Erhalter. Aufgrund der verschiedenen Standorte und der verschiedenen Fachrichtungen reduzieren sich jedoch zusammenhängende Gruppengrößen.

\section{Nachwuchsförderung für F\&E durch den FH-Sektor?}

Die verstärkte Einbindung der FH-Institutionen in F\&E-Tätigkeiten ist jedoch nur eine Seite ihres Beitrages zur Forschungsstrategie, ein zweiter Aspekt besteht in ihrem möglichen Beitrag zur Ausbildung für Tätigkeiten im F\&E-Bereich, also im weitestem Sinne zur Nachwuchsförderung für diesen Bereich. Diese Frage betrifft erstens das Bedarfskonzept (und bis zu einem gewissen Grad auch den Fokus der Bedarfserhebungen), zweitens das Profil der angestrebten Qualifikationen und Kompetenzen und drittens das angesprochene Portfolio der verschiedenen Funktionen Lehre, F\&E und Dienstleistungen. Bisher scheint es widersprüchliche Einschätzungen und Auffassungen in diesem Bereich zu geben. Es wurde gezeigt, dass einerseits gerade hinsichtlich der wissenschaftlichen Kompetenzen im engeren Sinn die vorhandenen Erwartungen noch eine ziemlich klare Trennung zwischen Universität und FH-Bereich unterstellen - andererseits wird aber sehr deutlich betont, dass sich die F\&E-Tätigkeit im Unternehmenssektor weiter verbreitern sollte, insbesondere auch im KMUBereich und bezüglich „radikaler Innovationstätigkeit“ (im Unterschied zu inkrementeller Entwicklung), ${ }^{125}$ wobei das Profil der Universitäten hier gleichzeitig als zu stark grundlagenorientiert und theorielastig eingeschätzt wird.

In diesem Bereich sollten weitere Überlegungen angestellt werden, die einerseits die oft zu ausgeprägte Entgegensetzung von FH-Institution und Universität in Frage stellen und andererseits die möglichen unterschiedlichen spezifischen Funktionen besser akzentuieren. ${ }^{126}$ Die oben angestellten Überlegungen zum Personalbedarf im Konnex mit der Verwirklichung der Forschungsstrategie sind in diesem Zusammenhang zu vertiefen: welche Qualifikations- und Kompetenzprofile sind für die Ausweitung der F\&E-Tätigkeit in welchem Zusammenspiel erforderlich? In welchem Ausmaß verändern sich die Prozesse der Wissensproduktion und damit auch die Beziehungen zwischen "traditioneller" disziplinorientierter Wissenschaft (Grundlagenforschung) und stärker anwendungs-

25 Vgl. Urban 2001; auch BMWA-Aussendung v. 9.6.2001 (http://www.bmwa.gv.at/news/2001/08/2dce.htm)

126 Auf theoretischer Ebene werden diese Fragen im Zusammenhang mit der Frage nach dem Niedergang des "linearen Innovationsmodells" und seiner Ersetzung durch systemische und zirkuläre Prozesse und Interaktionen diskutiert, in der Praxis wird jedoch gleichzeitig implizit oft von den Annahmen des linearen Modells ausgegangen. 
orientierten Formen der Wissensproduktion? Welche Konsequenzen haben diese Veränderungen auf die Arbeitsteilung, die Karrieren etc.?

\subsection{Entwicklungspfade für den Stellenwert des FH-Sektors}

Mit Auslaufen des Entwicklungs- und Finanzierungsplans II stellt sich die Frage nach neuen Zielwerten und Prioritäten für die Bundesförderung. Dabei ist die Frage nach der Dimensionierung des FH-Sektors im Hochschulsystem wesentlich. Soll der Sektor weiterhin wachsen? Wenn ja, wie rasch soll der Sektor wachsen? Wie soll er im Hinblick auf die verschiedenen Zielsetzungen und Funktionen profiliert sein? Wie soll er im Vergleich zu den Universitäten positioniert werden? Diese Fragen sind Kernfragen für die Entwicklung des Stellenwertes des FH-Sektors.

Aus dem Material lassen sich unterschiedliche Positionen zur weiteren Entwicklung des FHSektors herausarbeiten und es werden verschiedene Gesichtspunkte angeführt, deren Berücksichtigung gefordert oder nahegelegt wird. Im Folgenden werden diese Positionen und Gesichtspunkte, die von verschiedenen Akteuren in mehr oder weniger expliziter Form verfolgt oder geäußert werden, zu einigen unterschiedlichen Entwicklungspfaden verdichtet, die im Rahmen der Erarbeitung des Entwicklungs- und Finanzierungsplans III weiter ausgelotet werden sollten.

Diese Entwicklungspfade sind jeweils in stilisierter Form aus einigen wenigen (ein bis drei) jeweils bestimmenden Faktoren zusammengesetzt, denen eine gewisse Komplexität zukommt. Teilweise gibt es Verwandtschaften zwischen den Entwicklungspfaden, aber doch auch wesentliche Unterschiede, die einen jeweils eigenen Entwicklungspfad annehmen lassen. Die meisten Faktoren spielen in mehr oder weniger allen Entwicklungspfaden eine gewisse Rolle, die Abgrenzung zwischen den Entwicklungspfaden ergibt sich jedoch aus dem Gewicht, das man den jeweiligen Faktoren zuschreibt. Beispielsweise werden die Bildungsnachfrage oder die wirtschaftliche Nachfrage als Faktoren immer eine gewisse Rolle spielen, es macht jedoch einen Unterschied, ob diese Faktoren nur berücksichtigt werden oder ob sie die Entwicklung bestimmen sollen. Es liegt nicht auf der Hand, wie die weitere Entwicklung gestaltet werden soll. Die folgenden gebündelten Entwicklungspfade können eine Diskussionsgrundlage bieten und auch alternative Optionen deutlicher machen:

- Null-Wachstum. Dieses Szenario ist denkbar, wird jedoch von keinem der Akteure explizit vertreten. Vor dem Hintergrund sowohl der Entwicklung im europäischen und internationalen Umfeld als auch der expansiven Vorstellungen zur österreichischen Entwicklung der Innovationspolitik ergibt sich auch keine sachliche Begründung für diesen Entwicklungspfad. Der wesentliche Faktor wäre in einer derart restriktiven Finanzpolitik zu sehen, dass keine zusätzlichen öffentlichen Investitionen getätigt werden. 
- Fortsetzung einer moderaten und kontrollierten „bottom-up“-Strategie in diversifizierten Strukturen (STATUS-QUO). Dieses Szenario hat nach unseren Befunden eine breite Unterstützung im Sektor selbst. Sie enthält im Wesentlichen eine abgeschwächte Fortsetzung der Wachstumsdynamik der Vergangenheit, weiterhin eher über neue Studiengänge als über Ausweitung bestehender Studiengänge, Konsolidierung der Standorte und Eröffnung neuer Standorte in begrenztem Ausmaß. Die Impulse der letzten beiden Jahre zur verstärkten Ausweitung der Zahl der Studienanfängerlnnen durch neue Studiengänge („Aktion 600+“) werden skeptisch gesehen und es wird für „Qualität statt Quantität“ plädiert. Ein gewisses Dilemma wird darin gesehen, dass die Akquirierung zusätzlicher Mittel an die Gewinnung zusätzlicher Studierender gebunden ist, was teilweise als "Wachstumsfalle“ bezeichnet wird: Es wird auf eine Tendenz hingewiesen, teilweise "wider besseres Wissen“ eine Ausweitung der Studiengänge anzustreben, um den finanziellen Spielraum zu erweitern (teilweise wird von „Wettrüsten“ gesprochen). Zwei Gesichtspunkte sind dabei besonders wichtig: erstens die Erwartungen hinsichtlich der weiteren Entwicklung des Studierendenpotentials im Zusammenhang mit dem demografischen Abschwung und der möglicherweise sich verschärfenden Wettbewerbskonstellation zu den Universitäten (teilweise werden bereits Anzeichen für einen Bewerberlnnenmangel gesehen und die Unwägbarkeiten aufgrund der neuen Studienarchitektur und der Folgen der Universitätsreform werden in diesem Zusammenhang hervorgehoben); der zweite Gesichtspunkt ist der Aufbau der Forschungsfunktion als zweites Standbein und die potentielle Möglichkeit, über diesen Prozess zusätzliche Mittel zu akquirieren, die der gesamten Tätigkeit und der Konsolidierung des Portfolios zugute kommen sollen (Aufbau eines stabilen Personalstocks mit Forschungskompetenz, der auch der Lehre und der Dienstleistungsfunktion zugute kommt).

- Verstärktes Wachstum, bei Regionalisierung und Förderung lokalpolitischer Interessen (EXTENSIVES WACHSTUM). Dieses Szenario wird nicht im sinne einer zusammenhängenden Strategie explizit vertreten, es kommt eher implizit in verschiedenen Befunden und Stellungnahmen zum Ausdruck. Eine wichtige Basis für dieses Szenario ist die Vielzahl an Standortvorschlägen, die in der zunächst sehr „offenen“ Anfangsphase des „bottom-up“-Prozesses entstanden sind. Diese Vorschläge stehen vielfach nach wie vor in der einen oder anderen Form im Raum, werden von Akteuren (z.B. in den Gemeinden oder Landtagen) weiterverfolgt und verbinden sich in verschiedenen Konstellationen mit politischen Lobbying-Aktivitäten, die bis auf die Bundesebene reichen. Viele Stellungnahmen sehen das verstärkte Wachstum der letzten Jahre als einen gewissen Nährboden für dieses Szenario. Ein wesentlicher Gesichtspunkt in diesem Zusammenhang besteht darin, dass das System der Qualitätssicherung im FHR hinsichtlich seiner Verarbeitungskapazität bestimmte Obergrenzen hat, die nach so gut wie einhelliger Einschätzung seit einiger Zeit nennenswert überschritten sind. Es wird eine verstärkte „Politisierung“ festgestellt, die möglicherweise die Standards der Qualitätssicherung in den Hintergrund drängen könnte. 
Dieses Szenario führt mittelfristig mit hoher Wahrscheinlichkeit zur weiteren Ausdehnung von FH-Einrichtungen mit dem Hauptgewicht auf der Lehrtätigkeit und hohem Anteil an nebenberuflicher Lehre sowie vermutlich zu weiterer Vervielfältigung von Studiengängen zur Risikominimierung sowie zu weiterer regionaler Verbreitung des FH-Sektors. In gewisser Weise kann man eine Parallelität herstellen zwischen diesem Szenario und der Phase eines starken „bottom-up“-Wachstums postsekundärer Institutionen in anderen Ländern (z.B. Schweiz und Finnland, auch Niederlande), die der Errichtung des FHSektors vorausgegangen ist und dann für die „merger“-Strategien maßgeblich war. Eine Verstärkung der „top-down“-Komponente (rigorosere Qualitätskriterien für die Anträge, systematische Standortprüfung und explizitere Vorstellungen über das Profil eines „konsolidierten Standortes") wäre erforderlich, wenn diesem Szenario gegengesteuert werden soll.

- Konsolidierung der Standorte, Aufbau von Fachhochschulen, breitere Studiengänge (INTENSIVES WACHSTUM). Dieses Szenario ist in den Zielvorstellungen des FHStG angelegt und wird seit dem Ende der 1990er Jahre vom Fachhochschulrat explizit unterstützt; es gibt aber auch deutliche Signale aus dem Sektor in dieser Richtung. Es verbindet die Gesichtspunkte des quantitativen Wachstums, des Profils hinsichtlich des Aufgabenportfolios und des organisatorischen Modells der Fachhochschule (Mindestgröße und Kollegium). Wesentlich angesprochen ist die Vielfältigkeit und Diversität, die wir bisher im FH-Sektor beobachten können. Das Szenario stellt die Frage nach einer stärkeren Konvergenz und nach einem stärker konturierten Bild dessen, was eine Fachhochschule sein soll. Quantitatives Wachstum ist zumindest erforderlich, um die wenig entwickelten Standorte auf das Niveau einer Fachhochschule zu heben. Das Wachstum soll in den bestehenden Strukturen stattfinden, nach der Entwicklung der Lehre sollen Schritte zur Professionalisierung und personellen Konsolidierung gesetzt werden und die Wahrnehmung der F\&E-Funktion soll im Zuge der Förderprogramme verstärkt und systematisch aufgebaut werden. Für die Dienstleistungsfunktionen bestehen weniger ausgeprägte Vorstellungen. Im Rahmen unseres Interviewmaterials hat die Frage der Abwägung - teilweise auch in der Form alternativer, sich mehr oder weniger ausschließender Optionen - zwischen einem stärkeren Engagement des $\mathrm{FH}$ Sektors im Weiterbildungsmarkt als "Zukunftsmarkt" einerseits und der Entwicklung der F\&E-Funktion andererseits ein nicht unbeträchtliches Gewicht, wobei teilweise deutlich für eine Schwerpunktsetzung auf den Zukunftsmarkt Weiterbildung plädiert wurde. Im Hinblick auf die Verwirklichung des Entwicklungspfades „intensives Wachstum“ scheint eine verstärkte Zuwendung zu zwei Aspekten erforderlich zu sein: erstens zur Frage der Gestaltung des Aufgabenprofils (In welchem Ausmaß sollen die verschiedenen Funktionen wahrgenommen werden? In welcher Weise sollen die Funktionen verbunden werden?) und einer stärker ergebnisbezogenen Formulierung der Aufgaben; zweitens zur Frage der Beziehungen zwischen Studiengängen, Kollegium und Erhalter. Ein zentraler Aspekt der Errichtung einer Fachhochschule betrifft mit der Einrichtung des Kollegiums eine Differenzierung zwischen Kollegium und Erhalter und ein verstärktes Gewicht des 
Kollegiums. Hier scheint ein Ungleichgewicht zwischen der organisatorischen Ebene und der inhaltlichen Ebene zu bestehen, indem die FH-Struktur bisher rein organisatorisch definiert wird, der inhaltliche Mehrwert dieser Ebene jedoch weniger klar ist.

- Befriedigung/Mobilisierung der Bildungsnachfrage (SOCIAL DEMAND). Dieser Entwicklungspfad kann aus der Differenz zwischen Bildungsnachfrage und Studienplätzen abgeleitet werden. Diese Argumentation wird gelegentlich auf regionaler politischer Ebene als Leistung der Politik für die Verbesserung der Aussichten der Jugend, der Qualifikation der Bevölkerung und der regionalen Standortqualität vorgebracht. Obwohl ein Ziel bei der Errichtung des Sektors, wird die potentielle Nachfrage im Bereich der „Zweiten Chancen“ weniger offensiv thematisiert (etwa inwieweit durch massivere Fördermöglichkeiten hier eine zusätzliche latente Nachfrage „effektiv“ würde). ${ }^{127}$ Wie bei der Untersuchung der Akzeptanz gezeigt wurde, besteht hier sicherlich ein Potential, es müssten aber die längerfristigen Entwicklungsaussichten näher ausgelotet werden, wobei im Zusammenhang mit der demografischen Entwicklung, der Universitätsreform und dem Bologna-Prozess sehr starke Unwägbarkeiten berücksichtigt werden müssen. Die Schnittstelle zu den vorangehenden Ausbildungsgängen im Schulwesen und die regionale Ausschöpfung der Bildungspotentiale sind bei diesem Szenario wesentliche Gesichtspunkte. Es besteht auch eine Affinität zum Szenario „extensiven Wachstums“, indem extensives Wachstum mit steigender Bildungsnachfrage begründet werden kann. Eine wichtige Begründung, aufgrund derer die Mobilisierung der Bildungsnachfrage als eigenständiger Entwicklungspfad betrachtet werden kann, betrifft die Möglichkeit der aktiven Mobilisierung von zusätzlicher Bildungsnachfrage für den tertiären Sektor, wie sie etwa in Form von quantitativen Zielwerten in einigen Ländern verfolgt wird. Angesichts der niedrigen Werte der österreichischen Akademisierung auf den verwendeten europäischen Vergleichsindikatoren und der Diskussion um europäische Benchmarks ist diese Frage sicherlich in Betracht zu ziehen.

- Befriedigung des Qualifikationsbedarfs (ECONOMIC DEMAND). Wie bei der Untersuchung der Bedarfsfrage gezeigt wurde, wird in der gegenwärtigen Praxis der Qualifikationsbedarf sehr kleinräumig und eigentlich angebotsorientiert abgetestet. Es sollen nur Angebote entstehen, für die ein Bedarf nachgewiesen ist. Ein bedarfsorientiertes Szenario würde viel breiter ansetzen und die Entwicklung des Qualifikationsbedarfes auf Makro-Ebene zum Ausgangspunkt der Überlegungen machen. Obwohl in der Rhetorik diese Fragen der Befriedigung des Qualifikationsbedarfes im Übergang zur Informationsgesellschaft angesprochen werden, bestehen für die Realisierung dieses Szenarios in Österreich so gut wie keine Voraussetzungen. Die

127 Im Länderbericht zum OECD-Gutachten zur Errichtung des FH-Sektors wurde mit einigen einfachen Annahmen ein sehr großer potentieller Kreis von Nachfragenden für einen Zugang im "recurrent"-Modus demonstriert. 
systematische Beschäftigung mit Fragen des Bedarfes auf Makro-Ebene hat zwar aktuell wieder etwas Aufmerksamkeit gewonnen, aber im Großen und Ganzen ist der Stand der Diskussion in Österreich nicht wesentlich über die 1960er und 1970er Jahre hinausgekommen. Anstatt die Entwicklungen seither mit zu vollziehen und den Bedarfsanalysen und -prognosen den begrenzten, aber dennoch wichtigen Stellenwert zu geben, den sie verdienen, werden diese Aktivitäten geradezu tabuisiert, mit Hinweis auf die "fehlgeschlagenen Ansätze" der 1960er Jahre (vgl. Lassnigg 2002; Wilson 2001). Gegenüber vorhandenen „objektivistischen“ Erwartungen an Bedarfsanalysen ist man sich heute stärker der "gestalterischen" und „konstruktivistischen“ Komponente in diesen Praktiken bewusst, dennoch könnte eine systematische und gepoolte Nutzung der verschiedenen Standardmethoden wesentlich zur Klärung verschiedener Grundfragen der Dimensionierung und Entwicklung des Bildungswesens beitragen (Stichworte: Akademikerquote, Bedeutung der mittleren Qualifikationen, fehlendes Qualifikationsangebot als „bottleneck“, Fachrichtungswahl und Lücke im Bereich Mathematik-Science-Technology sowie IKT etc.). Als Szenario für den FH-Bereich müssten also externe Vorleistungen erbracht werden, die auf den Gesichtspunkt der Koordination der verschiedenen Bereiche im Bildungswesen verweisen. Die unterschiedlichen Dimensionierungen der verschiedenen Bildungsbereiche im internationalen Vergleich verweisen auf hohe Freiheitsgrade für politische Gestaltungsformen und auf die Existenz von komplexen Interaktionen zwischen Bildungsangebot und -nachfrage (vgl. Brown/Green/Lauder 2001, Lynch 1994).

- Mobilisierung des FH-Sektors für die Forschungsstrategie (FTE-STRATEGIE). Dieses Szenario wurde im Rahmen der Entwicklung einer österreichischen Strategie der Forschungs- und Technologiepolitik durch den Rat für Forschungs- und Technologiepolitik in einem Expertengutachten entwickelt (Clement et al. 2002) und in Abschnitt 3.2.3 in Ansätzen skizziert. Dabei setzen die Überlegungen nicht zuerst an der Lehre und am Qualifikationsbedarf insgesamt an, sondern an den Funktionen F\&E und innovationsorientierte Dienstleistungen. Dieses Szenario hat eine Affinität zum Szenario „intensiven Wachstums“ und entwickelt dieses weiter, insbesondere, indem die Schnittstellen im Dreieck zwischen Forschungspolitik, Innovationspolitik und Bildungsbzw. Hochschulpolitik stärker akzentuiert werden. Im Zentrum steht der massive Aufbau einer Infrastruktur für F\&E-Aktivitäten und von Kooperationen mit den Universitäten und den verschiedenen Formen von außeruniversitären Forschungs- und Impulszentren.

Die folgende Darstellung (Abbildung 45) gibt einen Überblick über die verschiedenen Entwicklungspfade und die jeweils bestimmenden Faktoren, die den jeweiligen Pfad definieren. Die Anordnung in der Darstellung soll eine gewisse hierarchische Struktur der Entwicklungspfade aufzeigen, die von links unten (Null-Wachstum) nach rechts oben (FTEStrategie) einen steigenden Entwicklungs- und Komplexitätsgrad impliziert. Ausgehend von der Null-Variante und der Status-quo-Variante, die auf unterschiedlichem Niveau die bestehende Struktur stabilisieren, kommen zusätzliche Aspekte ins Spiel, erstens verstärktes 
Wachstum (in die Breite oder auch in die Tiefe), zweitens verstärkte Ausprägung und Weiterentwicklung der Orientierung an den beiden Marktseiten (Qualifikationsnachfrage bzw. Bildungsnachfrage), drittens die verstärkte Betonung der innovativen Impulse durch den $\mathrm{FH}$ Sektor. Die komplexeren Szenarien setzen nicht unbedingt die vorherigen voraus, die verstärkte Betonung des wirtschaftlichen Bedarfes oder auch der Bildungsnachfrage z.B. im Kontext der Förderung des lebenslangen Lernens kann im Prinzip auch erfolgen, ohne dass das Wachstum wesentlich verstärkt wird.

Es besteht eine gewisse Verwandtschaft zwischen manchen Entwicklungspfaden, indem sie bestimmte Faktoren auf neuer Stufe aufgreifen bzw. betonen, insbesondere zwischen dem Status-quo-Modell, dem intensiven Wachstum und der FTE-Strategie: Das Status-quo-Modell beruht auf inkrementeller Weiterentwicklung im „bottom-up“-Prinzip, ohne genauere Spezifikation eines Zielzustandes; jeder Standort bzw. Erhalter entwickelt sich so weit und so rasch wie es eben seinen Prioritäten entspricht. Übergang zum intensiven Wachstum bedeutet, das Bild einer österreichischen FH-Institution (oder einige verschiedene Bilder) in der inhaltlichen und organisatorischen Konzeption (Größe, Aufgabenprofil, Lehrkörper etc.) deutlicher auszuformulieren und umzusetzen und entsprechende Kriterien zu formulieren (z.B. die vielen kleineren oder schwächeren Standorte systematisch weiterzuentwickeln). Der Übergang zum Modell der FTE-Strategie bedeutet, die F\&E-Aktivitäten zum Fokus der Weiterentwicklung zu machen und von jenen FH-Institutionen auszugehen, die gegenwärtig bereits auf diesem Stand sind. In jedem Fall müssten entsprechende Ziele gesetzt und deren Umsetzung operationalisiert werden.

Der Entwicklungspfad „extensives Wachstum“ kann durch entsprechende Schritte und Maßnahmen in Richtung des economic-demand-Modells weiterentwickelt werden oder in Richtung des social-demand-Modells, indem die entsprechenden Faktoren (im einen Fall z.B. intensivierte Antizipationsstrukturen, im anderen Fall z.B. verstärkte Schritte zur Mobilisierung der Bildungsnachfrage von Erwachsenen) in den Vordergrund gestellt werden.

Eine nähere Überprüfung der Tragfähigkeit der herausgearbeiteten Faktoren und ihrer Bündelung, etwa in Form von Simulationen oder Szenarienmodellen, könnte ein Weg zur weiteren Konkretisierung der Entwicklung des FH-Sektors sein. 


\section{Abbildung 45: Stilisierte Darstellung der möglichen Entwicklungspfade für den FH- Sektor und der jeweils bestimmenden Faktoren}

\begin{tabular}{|c|c|c|}
\hline Innovative Impulse & & $\begin{array}{l}\text { FTE-STRATEGIE } \\
\text { (Mobilisierung des FH-Sektors für die } \\
\text { Forschungsstrategie) } \\
-\quad \text { Aufgabenstellung für FH-Studiengänge } \\
\text { aus der Forschungsstrategie } \\
-\quad \text { Verhältnis zu den Universitäten im } \\
\text { Hinblick auf Förderung des F\&E- } \\
\quad \text { Nachwuchses }\end{array}$ \\
\hline $\begin{array}{l}\text { Fokus der } \\
\text { Entwicklung } \\
\text { auf: }\end{array}$ & $\begin{array}{l}\text { ECONOMIC DEMAND } \\
\text { (Prioritäre Befriedigung des } \\
\text { Qualifikationsbedarfs) } \\
\text { - } \quad \text { Prioritäten bei der Deckung } \\
\text { des Qualifikationsbedarfes } \\
\text { der Wirtschaft } \\
\text { - } \quad \text { Antizipationssystem }\end{array}$ & $\begin{array}{l}\text { SOCIAL DEMAND } \\
\text { (Prioritäre Befriedigung der } \\
\text { Bildungsnachfrage) } \\
-\quad \text { Priorität der Ausschöpfung der } \\
\text { sozialen und regionalen } \\
\text { Bildungspotentiale } \\
-\quad \text { Priorität der Förderung } \\
\quad \text { lebenslangen Lernens (nicht- } \\
\text { traditionelle Studierende) } \\
-\quad \text { Gestaltung der Schnittstellen im } \\
\quad \text { Bildungswesen }\end{array}$ \\
\hline
\end{tabular}

\section{Wachstum verstärkt}

\section{INTENSIVES WACHSTUM}

(Konsolidierung der Standorte, Aufbau von

Fachhochschulen, breitere Studiengänge)

- Profil der Lehrenden

- Aufgabenprofil eines Studienganges

- inhaltliche und organisatorische Konzeption der Fachhochschule

\section{EXTENSIVES WACHSTUM}

(Verstärktes Wachstum, bei Regionalisierung und Förderung lokalpolitischer Interessen)

- Gewicht lokaler und regionaler Interessen

\section{STATUS-QUO}

(Fortsetzung einer moderaten und kontrollierten bottom up-Strategie in diversifizierten Strukturen)

- endogene Potentiale an den Standorten

- Entwicklung des Studierendenpotentials

- Priorität für Forschungsfunktion

\section{NULL-WACHSTUM}

- $\quad$ Aufbringung der Finanzen
Stabilisierung der bestehenden Strukturen 


\subsection{Zusammenfassung und Schlussfolgerungen}

\subsubsection{Zusammenfassung}

Die Frage nach dem Stellenwert des Fachhochschulsektors im Bildungswesen wurde eingeschränkt auf den Stellenwert im Hochschulwesen, die Beziehungen zur Sekundarstufe wurden ausgeklammert. Der Stellenwert im Hochschulsystem wurde aufgrund der wesentlichen Besonderheiten des FH-Sektors (Studienprofil praxisbezogener Hochschulausbildung und Verbindung zu Bedarfs- und Akzeptanzerhebungen) sowie der manifesten Ziele bei seiner Errichtung (Erhöhung der Durchlässigkeit in verschiedenen Aspekten, Förderung der beruflichen Flexibilität, Entlastung der Universitäten, Beitrag zur Weiterbildung, Abbau der regionalen Disparitäten und Strukturbereinigung im postsekundären Bildungswesen) und der eher latenten Zielsetzung einer Reform der Angebots- und Regulierungsstrukturen im Bildungswesen analysiert. Die weitere Entwicklung des Stellenwertes des FH-Sektors wurde in einem politischen Entscheidungsraum, auch in Abhängigkeit von den wesentlichen Umfeldveränderungen seit seiner Gründung, in Form eines Spektrums von möglichen Entwicklungspfaden herausgearbeitet, die in den übergreifenden Schlussfolgerungen dargestellt sind. Es wurde ein breites Bündel an Faktoren herausgearbeitet, die den Gegenstand von Entscheidungen darstellen werden müssen.

In der Analyse des Stellenwertes des FH-Sektors im österreichischen Hochschulwesen wurden in einem ersten Schritt die Zielsetzungen für die Errichtung des FH-Sektors und deren Realisierung unter Berücksichtigung internationaler Vergleiche untersucht. In einem zweiten Schritt wurden die Auswirkungen wichtiger Veränderungen im Umfeld des $\mathrm{FH}$ Sektors herausgearbeitet und in drei Entwicklungsfeldern gebündelt: (1) Interaktion von Ausbildung und Innovation; (2) innovative Impulse für Unternehmenspraktiken; (3) Positionierung im regionalen Umfeld. Die mögliche Rolle des FH-Sektors in der österreichischen Innovationspolitik wurde näher beleuchtet. Abschließend wurden verschiedene mögliche Entwicklungspfade für den $\mathrm{FH}$-Sektor formuliert, die die Wahl zwischen unterschiedlichen Optionen und die dabei wichtigen Faktoren deutlich machen sollten.

\section{Die Zielsetzungen für den FH-Sektor und ihre Realisierung}

Die retrospektive Analyse hat grosso modo gezeigt, dass der Abbau regionaler Disparitäten, die Erhöhung der Durchlässigkeit und die Strukturbereinigung zumindest teilweise gelungen sind. Die berufliche Flexibilität lässt einige Fragen offen und die Entlastung der Universitäten sowie der Beitrag zur Weiterbildung sind eher weniger gelungen. Im Zusammenhang mit den 
verschiedenen Veränderungen im Umfeld (vgl. Abschnitt 3.2) erscheint eine Überprüfung der Zielsetzungen erforderlich, wie in den übergreifenden Schlussfolgerungen ausgeführt wird.

Die Realisierung der Zielsetzungen wurde im Hinblick auf zwei allgemeine Ziele - (A) die Diversifizierung des Hochschulsektors durch eine praxisbezogene Berufsbildung auf Hochschulniveau und (B) die Bindung der Studienangebote an den wirtschaftlichen Bedarf und die Akzeptanz der Studierenden - sowie im Hinblick auf spezifische manifeste und latente Zielsetzungen untersucht. Die folgenden manifesten Zielsetzungen wurden folgendermaßen zusammengefasst:

a) Verbesserung der Durchlässigkeit des Bildungswesens,

b) Förderung der beruflichen Flexibilität

c) Entlastung der Universitäten

d) Förderung der Aus- und Weiterbildung

e) Abbau regionaler Disparitäten

f) Strukturbereinigung im postsekundären und tertiären Bildungswesen

Als latente Zielsetzung wurde der angestrebte Beitrag zur Reform des bildungspolitischen Regulationssystems (v.a. Deregulierung und Dezentralisierung, stärkere Orientierung an Ergebnissen gegenüber Inputs, neue Form der Qualitätskontrolle und -sicherung) gewertet. Die Ergebnisse zur Realisierung können in den folgenden Aussagen zusammengefasst werden:

- Positionierung im Hochschulsystem. Die Aspekte der Praxisnähe, des Hochschulniveaus und des zeitgerechten Studienabschlusses wurden hervorgehoben. Es ist gelungen, den FH-Sektor erfolgreich im Hochschulsystem zu positionieren, insbesondere was die Bildungsnachfrage und die Profilierung gegenüber dem Unternehmenssektor betrifft. Im Verhältnis zu den Universitäten bestehen noch Spannungen, die sich mit der Umsetzung des Bologna-Prozesses eher noch verstärken könnten. Der erwartete Beitrag des FHSektors zur Wissensproduktion ist noch nicht sehr ausgeprägt, im Hinblick auf die akademische Profilierung wurde Entwicklungsbedarf festgestellt. Mit dem Konzept der Hochschulpraxisgemeinschaft wurde ein vielversprechender Ansatz der Profilierung im Hochschulsystem eingeschlagen.

- Bedarf und Akzeptanz. Der möglichst objektivierten Feststellung von Bedarf und Akzeptanz wurde bei der Anerkennung von Studiengängen wesentliche Bedeutung beigemessen. Trotzdem erfolgt die Entwicklung auf dem Wege des „bottom-up“-Prinzips eher angebotsseitig als bedarfsseitig. Eine wesentliche Schwäche besteht darin, dass die Aktivitäten zur Bedarfs- und Akzeptanzerhebung im FH-Sektor nicht in eine entsprechende Praxis auf breiterer Ebene eingebettet sind. Die Aktivitäten zur Analyse und Antizipation des Qualifikationsbedarfes auf der Gesamtebene, gesamtwirtschaftlich und qualifikationsübergreifend, sind bisher in Österreich unzureichend. Es gibt auch 
keine ausreichend begründete Bewertung der vorhandenen Qualifikationsstrukturen. Daher fehlt eine wichtige Informationsbasis für die Dimensionierung des FH-Sektors und die Kleinräumigkeit und Nischenorientierung von Angeboten wird dadurch gefördert.

- Manifeste bildungspolitische Ziele. Die manifesten Ziele unterliegen nur in begrenztem Ausmaß einer operationellen Definition, sie wurden soweit wie möglich operationalisiert und im Hinblick auf ihre Erfüllung einer qualitativen und teilweise auch einer quantitativen Analyse unterzogen. Bei den Zielen Abbau regionaler Disparitäten, Verbesserung der Durchlässigkeit und Strukturbereinigung sind mehr oder weniger deutliche Fortschritte nachweisbar; der Beitrag zur Verbesserung beruflicher Flexibilität ist vor allem aufgrund von Problemen der operationellen Definition fraglich; die Zielsetzungen der Entlastung der Universitäten sowie der Entwicklung von Aus- und Weiterbildung wurden aufgrund der durchgeführten Analysen eher nicht realisiert. Bei den einzelnen Zielsetzungen können die folgenden Aspekte hervorgehoben werden:

- DURCHLÄSSIGKEIT. Die Errichtung des FH-Sektors hat nach den vorliegenden Befunden vermutlich in bestimmtem Ausmaß zur Verbesserung der Durchlässigkeit des Bildungswesens in sozialer Hinsicht beigetragen. Der FH-Sektor ist für Studierende aus bildungsfernem Milieu zugänglicher als der Universitätssektor, dies gilt jedoch vor allem für Männer. Es wurden auch Möglichkeiten der Förderung des Zugangs von nicht-traditionellen Studierenden geschaffen, gegenüber der hohen Priorität dieser Zielsetzung und im Vergleich zu den Ausgangserwartungen jedoch in eher geringem Ausmaß.

- FÖRDERUNG DER BERUFLICHEN FLEXIBILITÄT. Die Förderung der beruflichen Flexibilität ist in objektivierter Hinsicht mit den vorhandenen Mitteln nicht festzustellen. Inwieweit die oft geäußerte Vermutung zutrifft, dass ein Teil der angebotenen Studiengänge angesichts der Zielsetzung beruflicher Flexibilität überspezialisiert ist, sollte näher untersucht werden.

- Entlastung der UniversitÄTEN. Hinsichtlich einer Entlastung der Universitäten haben die Detailauswertungen kein eindeutiges Bild ergeben. Am ehesten lässt sich eine gewisse Entlastung für den wirtschaftlichen Fachbereich feststellen. Insgesamt deuten verschiedene Befunde darauf hin, dass die Errichtung des $\mathrm{FH}$-Sektors in gewissem Ausmaß auch zusätzliche erfolgreiche Bildungsbeteiligung mobilisiert hat.

- AUS- UND WEITERBILDUNG. Manche Einrichtungen haben bisher schon Aktivitäten der Weiterbildung entwickelt, insgesamt im FH-Sektor sind diese Aktivitäten jedoch noch sehr gering ausgeprägt. Um in diesem Bereich, der von vielen Expertlnnen als wichtiger möglicher Zukunftsmarkt gesehen wird, stärker tätig zu werden, sind größere Kapazitäten der Einrichtungen erforderlich. Die Ausweitung der Tätigkeit in diesem Bereich steht möglicherweise in Konkurrenz zur Ausweitung anderer Tätigkeiten (insbesondere F\&E), unter den Expertlnnen bestehen auch divergierende Einschätzungen zur sinnvollen Prioritätensetzung für den FH-Sektor. 
- AbBau Regionaler Disparitäten. Ein Beitrag des FH-Sektors zum Abbau regionaler Disparitäten beim Hochschulzugang wird durch die empirischen Auswertungen in einem eigenen Abschnitt der Untersuchung (vgl. Abschnitt 5.2.1) unterstützt, das Studium an $\mathrm{FH}$-Studiengängen ist regional auch ausgewogener verteilt als das Studium an Universitäten, es bestehen jedoch Unterschiede zwischen den Bundesländern.

- Strukturbereinigung. Ein Beitrag des FH-Sektors zu einer bildungspolitischen Strukturbereinigung konnte bei weitem langsamer erreicht werden, als von vielen beteiligten Akteuren angestrebt wurde. Wesentliche Fragen sind offen geblieben und aktuell in Diskussion, insbesondere die Frage der Angebote im Bereich der Gesundheitsberufe, aber auch die Frage der Abstimmung mit anderen postsekundären Angeboten der Berufsbildung. Im Zusammenhang mit dem Bologna-Prozess werden sich viele Fragen der Abstimmung zwischen FH-Sektor und Universitäten neu stellen.

- Latente bildungspolitische Ziele und Veränderungen im Umfeld. Latente bildungspolitische Ziele bestanden in der Reform des Regulierungssystems im Bildungswesen. Im Hinblick auf die Errichtung dezentralisierter und flexibler Strukturen war der FH-Sektor sehr erfolgreich, wenn auch mit dem Größenwachstum an vielen Punkten die Grenzen dieses Regulierungssystems wahrnehmbar werden (vgl. die näheren Analysen in den Kapiteln zur Entwicklungsplanung und zur Qualitätssicherung; Abschnitte 4 und 7). Eine Schwäche bestand bisher in der politischen Steuerung des FH-Sektors im Rahmen eines Gesamtkonzeptes für den Hochschulsektor. Mit der möglichen demografisch bedingten Verknappung des Studierendenpotentials und mit der stärkeren Überschneidung zwischen FH-Sektor und Universitäten werden Abstimmungsprobleme zunehmend virulent werden. Wenn ein direkter Wettbewerb zwischen den beiden Sektoren des Hochschulsystems entsteht, wird sich die Frage nach einheitlichen Spielregeln für FH-Studiengänge und Universitäten verstärkt stellen, um vergleichbare Wettbewerbsbedingungen herzustellen.

\section{Auswirkungen von Veränderungen im Umfeld des FH-Sektors}

Neben den endogenen Veränderungen aufgrund der Entwicklung und des Wachstums des FH-Sektors wurden auch wesentliche Veränderungen im nationalen, europäischen und internationalen Umfeld diagnostiziert, die bei der weiteren Entwicklung und Konzeption der Politik eine Rolle spielen werden und berücksichtigt werden müssen. Die wesentlichen Veränderungen sind:

- Die Europäisierung der Bildungspolitik in Form verstärkter Koordination mittels Zielsteuerung und Erfahrungsaustausch, mit einem wesentlichen Schwerpunkt auf der 
Entwicklung von Ergebniszielen unter Berücksichtigung erforderlicher neuer Kompetenzen.

- Die Umstellung der Bildungs- und Ausbildungssysteme auf lebenslanges und lebensbegleitendes Lernen, was insbesondere die Gesamtkoordination der Systeme und die Förderung der Einbeziehung von Erwachsenen in reibungslosere Bildungs- und Ausbildungskarrieren impliziert.

- Die Verstärkung und Entwicklung von Innovationspolitik, die ein systematisches Zusammenspiel und eine Integration der Bildungspolitik in andere Politikbereiche wie Wissenschaftspolitik, Technologiepolitik, Struktur- und Industriepolitik, Regionalpolitik etc. erfordert.

- Die steigende Bedeutung des Zusammenspiels der lokalen, regionalen, nationalen und übernationalen Ebenen in der Dynamik von Globalisierung und Regionalisierung und die damit verbundene Notwendigkeit der Vernetzungen der verschiedenen Akteure zur Entwicklung des Innovationssystems (dieser Aspekt wird im Kapitel 5 zur Regionalisierung näher beleuchtet; vgl. Abschnitt 5.2.4).

Aus diesen Veränderungen im Umfeld wurden drei wesentliche Entwicklungsfelder für den FH-Sektor abgeleitet, die als Brennpunkte der weiteren Politikentwicklung dienen können:

- Die Verstärkung und Systematisierung der Interaktion zwischen Ausbildung und Innovation und die Einbindung in die Forschungsstrategie.

- Die Verstärkung von innovativen Impulsen für die Unternehmenspraktiken und die Wirtschaftsstrukturen sowie die Stärkung der Rolle der FHs in diesem Prozess.

- Die Positionierung der FH-Institutionen im regionalen Umfeld einerseits als Mittler zwischen der regionalen Ebene und den verschiedenen Aspekten der überregionalen Ebenen, andererseits als Knotenpunkte in der Vernetzung der regionalen Akteure in regionalen Innovationssystemen bzw. lernenden Regionen.

\section{Rolle des FH-Sektors in der österreichischen Innovationspolitik}

Vor allem in Anknüpfung an das Entwicklungsfeld der Interaktion von Ausbildung und Innovation wurde die Rolle des FH-Sektors in den Formulierungen für eine österreichische Forschungsstrategie näher beleuchtet. Dabei geht es einerseits darum, in welchem Ausmaß der FH-Sektor zur angestrebten Verstärkung der F\&E-Tätigkeit durch den Ausbau der F\&EAktivitäten beitragen kann und andererseits darum, inwieweit die Lehre im FH-Sektor auf F\&E-Tätigkeiten vorbereiten soll. Bisher sind die Vorstellungen dazu noch wenig ausformuliert und es gibt weit divergierende Vorschläge und Ansätze zur Rolle des FHSektors in der Forschungsstrategie. Es werden die quantitativen Größenordnungen der angestrebten Erweiterung der F\&E-Aktivitäten und des damit verbundenen Personalbedarfes verglichen. Ein wesentlicher Aspekt betrifft die Entwicklung der 
Arbeitsteilung und der Kooperationsstrukturen zwischen dem FH-Sektor einerseits und den anderen Institutionen der außeruniversitären F\&E sowie den Universitäten andererseits.

Welcher Entwicklungspfad hier eingeschlagen wird, wird die weitere Profilierung und Positionierung des FH-Sektors wesentlich bestimmen. Da die F\&E-Aktivitäten und kapazitäten bisher noch wenig entwickelt sind, besteht in dieser Frage ein breites Spektrum an Optionen für die weitere Entwicklung.

\section{Entwicklungspfade}

Es wurden sieben unterschiedliche Entwicklungspfade formuliert, die zwischen NullWachstum und voller Einbindung in eine österreichische Strategie der Forschungs- und Technologieentwicklung liegen und deren Eckpunkte unterschiedliche Pfade verstärkten Wachstums sowie ein unterschiedliches $M a ß$ an Betonung des wirtschaftlichen Bedarfes bzw. des Bildungsbedarfes sind. Diese Entwicklungspfade sind in der folgenden Aufstellung (Abbildung 46) in ihren Hauptmerkmalen skizziert.

Sie können als Grundlage für einen weiteren systematischen Entwicklungsprozess dienen, in den möglichst die verschiedenen Akteure einbezogen werden sollten. 
Abbildung 46: Schematisierte Darstellung der Entwicklungspfade sowie der bestimmenden Design-Faktoren und Variablen

Fokus auf

Innovation

FTE-Strategie

Fokus auf Bedarfsart

\section{Social Dem. Economic D}

Verstärktes

Intensiv

Wachstum

\section{Extensiv}

\section{Status-quo}

\section{Null-Wachstum}

\section{Stabilisierung in bestehenden Strukturen}

Design-Faktoren für Entwicklungspfade

FTE-STRATEGIE

\section{F\&E Nachwuchs:}

Innovationsstrategie:

ECONOMIC DEMAND

Qualifikationsbedarf Wirtschaft:

SOCIAL DEMAND

Schnittstellen im Bildungswesen:

Lebenslanges Lernen:

Ausschöpfung Bildungspotenzial:

INTENSIVES WACHSTUM

Konzeption Fachhochschule:

Aufgaben Studiengang:

Profil Lehrende:

EXTENSIVES WACHSTUM

Lokale/regionale Interessen:

STATUS-QUO

Forschungsfunktion:

Studierendenpotential:

Standortpotential:

NULL-WACHSTUM

Finanzen:
Aspekte, Variablen

für Entwicklungspfade

Ausbildungsprofil Fachhochschul-Institution

Ausbau F\&E, reg. Innovationssystem

Antizipationssysteme

nicht-traditionelle Zugänge

Mobilisierung, Förderung?

Zielwerte? Frauen

Aufgabenportfolio, Größe

Zusammenhang Lehre - Forschung

Aufgabenverteilung?

Konkretisierung Impulsfunktion?

Gewicht und Profil Forschung, Mittel?

Wettbewerb mit Universitäten?

Kriterien, Standortprüfung, Aufbau Lehrkörper?

zusätzlicher Finanzbedarf - Sparpolitik 


\subsubsection{Schlussfolgerungen}

Für die weitere Entwicklung wurde als eine zentrale Schlussfolgerung der Evaluierung eine Überprüfung und nähere Spezifikation der Zielformulierung in Form von Ergebniszielen vorgeschlagen.

Im Hinblick auf den Stellenwert des FH-Sektors im Bildungswesen ergeben sich auch Schlussfolgerungen für die Förderung bestimmter Zielgruppen, insbesondere der Chancengleichheit von Frauen und Männern sowie von nicht-traditionellen Studierenden.

In folgenden Themenbereichen kann Entwicklungsbedarf festgestellt werden:

- deutlichere Profilierung der praxisnahen Studien auf Hochschulniveau (Stichworte: Kompetenzentwicklung, Hochschulpraxisgemeinschaften, Spezialisierungsgrad)

- Weiterentwicklung der Bedarfs- und Akzeptanzanalysen (Stichworte: Makroanalysen als Rahmen, interaktive Antizipationssysteme)

- Spielregeln und Rollenverteilung (Stichworte: vergleichbare Wettbewerbsbedingungen im Hochschulsystem, ausgewogene Rollen- und Aufgabenverteilung zwischen Akteuren)

- Fachhochschulen und Fachhochschul-Studiengänge im Innovationssystem (Stichworte: reaktive oder proaktive Funktion, regionale Innovationspolitik)

Einbindung der Akteure in eine vertiefende Diskussion der Dimensionierung des FHSektors im Hochschulsystem

Aus den Analysen wurde ein breites Spektrum an möglichen Entwicklungspfaden für den FH-Sektor herausdestilliert, das als Grundlage für die weitere Diskussion und Systematisierung von Entwicklungsstrategien dienen kann. Zwingend lässt sich keiner der Entwicklungspfade begründen, je nach den allgemeinen Erwartungen und Zielsetzungen, die erfüllt werden sollen, sind unterschiedliche Faktoren und konditionale Zusammenhänge zu beachten.

Es lässt sich beispielsweise feststellen, dass etwa mit der Wahl des NULL-WACHSTUMS- oder des STATUS-QUO-Szenarios nur ein sehr begrenzter Beitrag des FH-Sektors zur österreichischen Forschungs- und Innovationsstrategie erzielt werden kann, da ein hoher Anteil der Standorte für F\&E-Leistungen nicht ausreichend vorbereitet ist. Ebenso wird eine Entwicklung nach dem Modell EXTENSIVEN WACHSTUMS zu einer (weiteren) Zersplitterung der Kräfte führen und ebenfalls die FTE-Strategie nur bedingt unterstützen können. 
Vor allem aufgrund der Veränderungen im Umfeld des FH-Sektors bietet sich für die weitere Entwicklung das Feld zwischen den Modellen des INTENSIVEN WACHSTUMS und der FTESTRATEGIE an, wobei auch eine ausgewogene Beachtung von Qualifikations- und Bildungsnachfrage berücksichtigt werden sollte. Die Wahl der Optionen von ECONOMIC DEMAND oder auch SOCIAL DEMAND würde eine starke Konzentration auf jeweils einen speziellen Aspekt implizieren und zur Vernachlässigung anderer Aspekte führen.

Die näheren Spezifikationen der weiteren Entwicklung sollten in einem systematischen Verfahren vorgenommen werden, in dem die verschiedenen Akteure ihre Positionen einbringen und abstimmen können.

\section{Geschlechtsspezifischer Aspekt der Durchlässigkeit: Förderung und Strukturbereinigung}

Die Befunde zeigen, dass, obwohl der Frauenteil eine etwas steigende Tendenz aufweist, sowohl unter den Studierenden als auch unter den Lehrenden die Frauen deutlich unterrepräsentiert sind. Dies hat teilweise strukturelle Gründe, indem sich durch die fachrichtungsspezifischen Schwerpunkte die generelle geschlechtsspezifische Segmentierung im FH-Sektor reproduziert (Lassnigg/Paseka 1997). Die Erweiterung des Fächerspektrums im Zuge der Strukturbereinigung würde jedenfalls einen Beitrag zur besseren geschlechtsspezifischen Repräsentation leisten. Darüber hinaus bleibt die Frage der fachrichtungsspezifischen Segmentierung, insbesondere in den technischen Bereichen, bestehen. Die Unterrepräsentation der Frauen in diesen Bereichen ist nicht nur eine allgemeine Frage der geschlechtsspezifischen Chancengleichheit, sondern hat auch im Zusammenhang mit der Mobilisierung von zusätzlichen Potentialen insbesondere für die technisch-naturwissenschaftlichen Bereiche Gewicht. Eine bessere Repräsentation der Frauen kann kurz- bis mittelfristig vermutlich nur durch gezielte Förderprogramme erreicht werden. Es wird daher vorgeschlagen, die Entwicklung von spezifischen Förderprogrammen für Frauen, unter Berücksichtigung internationaler Erfahrungen, sowohl auf der Ebene der Lehrenden als auch auf der Ebene der Studierenden ins Auge zu fassen. ${ }^{128}$

${ }^{128}$ Im Schweizerischen FH-System bestehen explizite gesetzliche Zielvorgaben "die Gleichstellung der Geschlechter zu fördern und den Anteil der Frauen zu erhöhen" (EFHK 2000, 39); es wurde 1999 ein Aktionsplan verabschiedet und mit 10 Mio SFR (6,82 Mio EUR, 93,8 Mio ATS) dotiert. Die geschlechtsspezifische Ungleichheit ist stärker ausgeprägt, wenn auch in ähnlichen Grundmustern wie in Österreich und die Wirksamkeit des Aktionsplanes wird sehr vorsichtig bewertet (EFHK 2002, 26-27). 


\section{Durchlässigkeit für nicht-traditionelle Studierende}

Im Bereich der Förderung der Durchlässigkeit sind in begrenztem Ausmaß Erfolge erzielt worden; trotz der starken Betonung dieses Aspektes in den Zielsetzungen ist die Entwicklung gegenüber den hohen ursprünglichen Erwartungen jedoch eher zurückgeblieben. Insbesondere nicht-formalisierte Zugänge sind nur in kleinem Ausmaß realisiert worden. Dies scheint strukturelle Ursachen zu haben, die in der großen Distanz zwischen dem mittleren Ausbildungsniveau und dem FH-Niveau liegen. Eine verstärkte Durchlässigkeit erfordert massivere Formen der Förderung, wobei zwei unterschiedliche Aspekte zu berücksichtigen sind:

- erstens die Vereinbarkeit von Studium und Berufstätigkeit (hier geht es einerseits um Zeitressourcen, andererseits um finanzielle Ressourcen und auch um die Potentiale des Einsatzes von E-learning),

- zweitens der Zugang ohne traditionelle Hochschulberechtigung (hier geht es u.a. um die Anerkennung nicht-formaler Qualifikationen).

Bei beiden Aspekten ist in Abhängigkeit von einem zukünftig möglicherweise zu erwartenden Mangel an Qualifikationen und im Sinne der Umsetzung von Möglichkeiten des lebensbegleitenden Lernens zu erwägen, inwieweit massivere Interventionen eingesetzt werden sollen (insbesondere wenn sich der Zuzug aus der Erstausbildung in Zukunft verknappen wird, wird sich diese Frage in zunehmendem Maße stellen).

\section{Konzentration und Schwerpunktbildung im Bereich F\&E}

Der Aufbau der F\&E-Aktivitäten scheint in ziemlich breitem Rahmen zu erfolgen, es ist zu erwägen, inwieweit eine gezielte Schwerpunktbildung bessere Ergebnisse erbringen könnte.

\section{Fragen zur Diskussion und weiteren Analyse}

In diesem Abschnitt werden einige gezielte Zukunftsfragen angesprochen, die mit den Mitteln des vorliegenden Projektes nicht zureichend geklärt werden können, aber als essentiell für die weitere Entwicklung anzusehen sind. Teilweise übersteigen diese Fragen den unmittelbaren FH-Sektor und betreffen auch die Schnittstellen zu anderen Sektoren des

Bildungswesens bzw. die Positionierung der österreichischen Bildungspolitik im Zusammenhang der Europäisierung und der globalisierten Bildungsmärkte. Aber selbstverständlich gibt es Wechselwirkungen zwischen den Teilsektoren und den Fragen der Entwicklung des Bildungswesens insgesamt. 
- Die Profilierung der FH-Studiengänge im Hochschulsystem. Dieses Thema betrifft den Komplex der Entwicklung der Studiengangsprofile im Hinblick auf die angestrebten Ergebnisse des Lernens (die Breite und Spezialisierung, die zu erwerbenden Kompetenzen, die Verortung im Wissenschaftssystem bzw. Konkretisierung der Wissenschaftsorientierung), die Verbindung der angestrebten Ergebnisse zum didaktischen Konzept (kontextualisiertes und selbstgesteuertes Lernen, E-learning); die angestrebte Beziehung zwischen den Funktionen von Lehre, F\&E und Dienstleistung (erforderliche Qualifikationspotentiale und Formen der Arbeitsteilung und Kooperation, Konsequenzen für die organisatorische Ausgestaltung). Es geht bei der Profilierung um die vertiefende Ausgestaltung und Reflexion der inhaltlichen Komponente in ihrem Zusammenspiel mit den prozeduralen und organisatorischen Erfordernissen zur Erzielung der spezifizierten Ergebnisse. Eine Kernfrage ist die Formulierung der Ausbildungsprofile (In welchen Begriffen findet das statt? Inwieweit ist der Kompetenzbegriff dafür brauchbar und kann stärker akzentuiert werden?). ${ }^{129}$ Eine zweite Kernfrage betrifft die Verbindung der (teilweise verschulten) Lehre mit den praktischen Erfahrungen und das Gewicht der Forschungskompetenz. Dazu gibt es in der AbsolventInnenstudie (Hoyer/Ziegler 2002) bedenkenswerte Ergebnisse, auch Prisching 2002 betont die Bedeutung des Zuganges zu systematischem Wissen und weist explizit auf Probleme z.B. im Bereich berufsbegleitender Studiengänge hin. Einen vertiefenden Ansatz schlägt Markowitsch 2001 vor, indem er das Konzept der "community of practice“ auf praxisbezogenes Hochschulstudium anwendet. In den Expertengutachten für den FTE-Rat (Clement et al. 2002, Detter et al. 2002) werden ebenfalls Vorschläge und Ansatzpunkte zur Profilierung skizziert. Diese Überlegungen sollten weitergeführt werden, um auf die Frage von Manfred Prisching $(2002,20)$ nach „konsistenten Leitvorstellungen darüber, was Fachhochschul-Studiengänge eigentlich sein sollen" vertiefende Antworten zu formulieren und in der vorhandenen Vielfalt einen stärkeren Konsens über akademische Standards herzustellen.

- Weiterentwicklung der Ansätze und Methoden der Antizipation und „Früherkennung“ von Bedarf und Akzeptanz. Dieses Thema betrifft die Anwendung von erweiterten Mechanismen der Dokumentation und Antizipation von wirtschaftlichem und gesellschaftlichem Bedarf nach Kompetenzen wie auch von Potentialen für den Erwerb der Kompetenzen. Es wäre die Entwicklung eines Referenzrahmens auf Makro-Ebene durch die systematische und gezielte Anwendung des in den letzten Jahrzehnten entwickelten methodischen Instrumentariums als Ergänzung der Bedarfs- und Akzeptanzerhebungen erforderlich. Dieses Instrumentarium reicht von quantitativen Projektions- und Prognosemethoden verschiedener Reichweite über die Abschätzung von Kosten-Nutzen-Relationen der Ausbildungen, Analysen von Stellenanzeigen bis zu

129 Eine ansatzweise Analyse der Ausbildungsprofile vor einigen Jahren hat nur wenig konsistente und systematische Begrifflichkeiten erbracht (Lassnigg/Stöger 1999, 29-34). 
qualitativen Mechanismen wie z.B. der Anwendung der Szenariotechnik oder der Analyse von Berufsprofilen und bis zum Aufbau von Netzwerken und ähnlichen Formen des Wissensmanagements unter den beteiligten Akteuren (vgl. Steiner/Steger 2001). Wesentlich wären Schritte zur Verbesserung der verfügbaren Daten- und Informationsgrundlagen in diesem Bereich. Neben diesen FH-externen Anforderungen wäre auch die Praxis der Bedarfs- und Akzeptanzerhebungen der Antragstellenden vertiefend zu überprüfen. Eine erste Evaluation hat für die Periode bis 1998/99 eher problematische Ergebnisse erbracht, was die Standards der Erhebungen betrifft. Ein Follow-up hätte den Rahmen der vorliegenden Untersuchung gesprengt. Es wären auch Fragen nach der Praktikabilität der Vorgangsweise zu stellen, etwa inwieweit die Arbeitsteilung in diesen Erhebungen (Beauftragung durch Antragstellende; studiengangsbezogene kleinräumige Erhebungen, „Ergebnisdruck“ etc.) noch sinnvoll erscheint.

- Spielregeln und Rollenverteilung zwischen den Akteuren und Einrichtungen im Hochschulsektor. Die Beziehung zwischen Fachhochschul- und Universitätssektor im Hochschulsystem steht im Spannungsverhältnis zwischen Konkurrenz und Kooperation. Viele Faktoren wirken in der Richtung, dass sich beide Seiten dieses Spannungsverhältnisses vermutlich intensivieren werden. Damit wird sich die Frage der Abstimmung verstärkt stellen, wenn keine allzu großen Reibungsverluste entstehen sollen. In einer Publikation zur Planung des Studienangebotes wird „ein erhöhter Abstimmungsbedarf bei der Entwicklung des Studienangebotes der verschiedenen Sektoren, vor allem zwischen Universitäten und Fachhochschuleinrichtungen, aber auch zwischen einzelnen Universitäten und Fachhochschuleinrichtungen" hervorgehoben (BMBWK 2000, Vorwort). Mit der Universitätsreform, dem Bologna-Prozess und den in den letzten Jahren immer wieder angezogenen Diskussionen um ein Engagement von Universitäten im FH-Sektor stellen sich auch Fragen nach der Gestaltung der Spielregeln, in denen der Wettbewerb wie auch die Kooperation abläuft. Aus beiden Sektoren werden Aspekte hervorgehoben, unter denen sie sich jeweils gegenüber dem anderen Sektor durch die vorhandenen Spielregeln als "benachteiligt" einschätzen. Insbesondere mit dem Aufbau der F\&E-Funktionen im FH-Sektor werden diese Fragen verstärkt gestellt. Es stellt sich die Frage der Entwicklung eines Gesamtkonzeptes für den Hochschulsektor, das auch die verschiedenen Funktionen der Einrichtungen (Forschung, Lehre, Dienstleistungen) berücksichtigt und in dessen Entwicklung die Akteure eingebunden sind. Leider scheinen gegenwärtig die Bedingungen für ein derartiges Unterfangen nicht sehr günstig zu sein, vermutlich sollten in einen derartigen Prozess externe Beobachter eingebunden sein.

- Verbindung zum Innovationssystem. Dieses Thema bezieht sich auf die explizite Auseinandersetzung mit der Einbeziehung des FH-Sektors in das Innovationssystem in allen ihren Facetten. Die bisher publizierten Überlegungen geben Ansatzpunkte zu 
150 - Lassnigg et al. / FH-Review - Stellenwert des FH-Sektors - I H S / iff / CHEPS

diesem Thema ab, aber sie werfen gleichzeitig noch mehr Fragen auf, als sie Antworten geben. 


\section{Umsetzung der FH-Entwicklungs- und Finanzierungs- planungen}

Im folgenden Kapitel wird die Entwicklungsplanung des Bundes für den Fachhochschulsektor anhand der offiziellen Entwicklungs- und Finanzierungspläne (I und II) analysiert sowie die im Rahmen dieses Reviewprojektes - etwa in den ExpertInneninterviews oder in den projektbezogenen Befragungen - geäußerten Anforderungen an den dritten Entwicklungs- und Finanzierungsplan in den Schlussfolgerungen zusammengefasst.

\subsection{Retrospektive Betrachtung: Entwicklungsplanung und -steuerung im Fachhochschulsektor}

Es gehörte zu den impliziten bildungspolitischen Zielen, mit der Konzeption des Fachhochschulsektors auch ein neues Steuerungsmodell für die Bildungspolitik auszuprobieren. Auf Basis seiner Entwicklungs- und Finanzierungsplanung hat der Bund dabei im Wesentlichen über die Ausbaugeschwindigkeit, die Finanzierung und über inhaltliche Kriterien gesteuert. Im Folgenden wird eine Darstellung und Einschätzung des Grundmodells der Entwicklungssteuerung sowie der einzelnen Steuerungsdimensionen vorgenommen.

\subsubsection{Das Grundmodell der Entwicklungsplanung und -steuerung}

Während die Bildungsreformen der 1960er und 1970er Jahre stark etatistisch geprägt waren, wurde die hohe staatliche Regelungsdichte Anfang der 1990er Jahre in allen Bereichen des österreichischen Bildungswesens als Belastung empfunden. Diese Veränderungen zogen Konsequenzen auf der organisatorischen und finanziellen Ebene nach sich. Das Ziel der Politik war es, Rahmenbedingungen zu schaffen, die ein rasches Reagieren des neuen Hochschultyps auf die Anforderungen des Beschäftigungssystems ermöglichen. Die "Gunst der späten Stunde" hat dazu geführt, dass die fachhochschulischen Bildungseinrichtungen eine neue Grundphilosophie im Bildungsbereich widerspiegeln, die vor allem von der Überzeugung der Vorteile von Vielfalt und unternehmerischem Handeln gekennzeichnet ist. Vielfalt ist untrennbar verbunden mit einer anderen Beziehung zwischen dem Staat und den einzelnen Hochschulen.

Neue Formen der Regulation für die Bildungspolitik zu erproben, gehörte zu den latenten bildungspolitischen Zielen. Der Fachhochschulsektor verkörperte damit auch ein neues Steuerungsmodell in der Bildungspolitik, das insbesondere durch seine veränderte Beziehung zwischen dem Staat auf der einen Seite und den Leistungserbringern, den Studiengängen, auf der anderen Seite, in vielen Aspekten den Prinzipien des New Public 
Management ähnelte, das seit Ende der 1980er Jahre in vielen Industrienationen für den Versuch einer Neuorganisation öffentlicher Aufgaben steht.

Für New Public Management sind zwei Ideen grundlegend. Erstens eine radikale Dezentralisierung des Verwaltungsapparates, kombiniert mit einer umfassenden Verantwortungsdelegation an kleine, teilautonome, marktnah operierende, intensiv vernetzte Einheiten; und zweitens Märkte und Wettbewerb als neue Rahmenbedingungen und Steuerungsprinzipien. Davon verspricht man sich grundsätzlich eine Stärkung der strategischen Führung auf der politischen Ebene. Entlastet von operativen Detailfragen, die den Blick auf das Ganze häufig verstellen, sollen Politikerinnen und Politiker wieder vorausschauend planen, gestalten und steuern können, nicht bloß krisenhaften Entwicklungen hinterher „rudern“ müssen. Und außerdem erhofft man sich eine Erhöhung der Selbstorganisationskraft (vgl. Halblützel et al 1996). Daraus sollen nicht nur Effizienzsteigerungen resultieren, sondern auch eine Öffnung des politisch-administrativen Systems gegenüber der Gesellschaft. New Public Management ist auch aus der Diskussion um die Wirksamkeit staatlichen Handelns und um die Lernfähigkeit des politischadministrativen Systems entstanden, an der sensiblen Schnittstelle von demokratischer Willensbildung und administrativem Vollzug.

In der zweiten Hälfte der 1980er Jahren drehte sich in ganz Europa die Richtung des bildungspolitischen Denkens. Der Marktmechanismus gewann als Alternative zur Bürokratie an Einfluss. Dezentralisierung, Deregulierung und Autonomisierung wurden zu den wichtigsten Gesichtspunkten der Reform der Bildungssysteme in vielen Ländern (vgl. OECD 1996, 132f). Wahlmöglichkeiten für Studierende oder SchülerInnen, erhöhte institutionelle Autonomie durch die Verschiebung von Entscheidungsprozessen, Accountability und Veränderungen der staatlichen Verantwortung wurden wesentliche Aspekte der Politik.

Trotz aller nationalen Variationen findet sich überall eine Kombination der Etablierung von Quasi-Märkten und einer veränderten, nunmehr stärker evaluativen Rolle des Staates. Die Beziehungen zwischen dem Staat und dem Bildungswesen werden auch dadurch reformuliert, dass neue intermediäre Körperschaften errichtet werden. Außerdem wird verstärkt auf Managementmethoden gesetzt. Man hat die Hoffnung, dass dezentralisierte Bildungssysteme eher in der Lage sind, sich an neue Anforderungen anzupassen. Zentralisierte Systeme erwarten sich Anstöße für die Reformdynamik aus Schritten der Dezentralisierung.

Einige dieser Aspekte sind auch in den leitenden Prinzipien für den Aufbau des Fachhochschulsektors in Österreich Anfang der 1990er Jahre zu finden. Die österreichische Bildungstradition war bis dahin durch staatliche Bildungshoheit und zentralistische Steuerung charakterisiert. Das dieser Struktur zugrundeliegende Denkmodell ging davon aus, dass vor allem zentrale staatliche Entscheidungsträger bzw. deren Beamtenstab berufen sind, Bildungsplanung zu betreiben und dass eben fast ausschließlich staatliche Einrichtungen als 
Anbieter auf dem Bildungssektor auftreten sollen, was zur Ausbildung eines relativ homogenen Bildungsangebotes geführt hat, dessen Strukturen sich im Laufe der Zeit zunehmend verfestigt haben. Hohe Regelungsdichte auf Gesetzes- und Verordnungsebene, bürokratische Abläufe, zentrale Planung sind zwar im Sinne von Recht, Sicherheit und Kontinuität zu begrüßen, führen jedoch gleichzeitig zu geringerer Innovationsfähigkeit und zu mangeInder Flexibilität gegenüber dem Beschäftigungssystem und zentrale Planung erweist sich inmitten einer zunehmend komplexen Umwelt vielfach als inadäquates Steuerungsinstrument. Der Fachhochschulsektor ist auch letztlich als eigener Hochschulsektor aufgesetzt worden, weil man sich mehr Reformimpulse aus einem eigenen Sektor für das Gesamtsystem erhoffte und weil bereits institutionalisierte Bildungseinrichtungen internen Reformbestrebungen stets mit der „Tendenz der Einverleibung" begegnen (Hackl/Binder 1995, 78).

\subsection{Die Steuerung durch die Entwicklungs- und Finanzierungs- planung}

Der Entwicklung und Finanzierung des Fachhochschulsektors lagen Planungen des Bundes, der Entwicklungs- und Finanzierungsplan I (1994) und der Entwicklungs- und Finanzierungsplan II (1999) zugrunde. Das Ausbauziel wird in Form der Gesamtzahl von Studienplätzen für den Sektor insgesamt (AnfängerInnenplätze und Gesamtstudienplätze) festgelegt. Der erste Entwicklungs- und Finanzierungsplan wurde 1994 beschlossen und enthielt eine bis ins Jahr 2000 reichende Absichtserklärung über die Höhe und Form der Bundesfinanzierung für den Fachhochschulsektor. Er kündigte bis zum Jahr 200010.000 Studienplätze an. Der zweite Entwicklungs- und Finanzierungsplan für die Zeit 2000 bis 2005 wurde im Frühjahr 1999 beschlossen und gab durch seine Zielwerte das Tempo des weiteren Fachhochschulausbaus vor. Die Zahl der Studienplätze sollte sich bis zum Jahr 2005 auf 21.000 erhöhen.

Auf dieser Grundlage wird eine verbindliche Planung innerhalb eines bestimmten Zeitabschnitts ermöglicht. Es wird der Einsatz von Bundesmitteln geregelt, wobei das vorgesehene Finanzierungskonzept allerdings eine Mischfinanzierung vorsieht. Dadurch soll eine finanzielle Einbindung jener gesellschaftlichen Bereiche geschaffen werden, die einen Bedarf nach Studiengängen bzw. deren AbsolventInnen orten. Die Bundesfinanzierung ist somit auch ein Steuerungsinstrument, da die Entwicklung des neuen Sektors maßgeblich von der Entscheidung des Bundes über Ausmaß und Modalitäten der Fachhochschulfinanzierung abhängt. Weiters enthält die Entwicklungs- und Finanzierungsplanung einen Kriterienkatalog, mit dessen Hilfe die Förderungswürdigkeit von Studiengängen anhand eines Punktesystems beurteilt wird. Seine wesentlichen Schwerpunkte sind die inhaltliche Ergänzung und Strukturbereinigung des Bildungswesens, eine regional ausgewogene Versorgung mit Hochschulangeboten sowie die Verbesserung des Zugangs für neue Zielgruppen. 
Insbesondere mit den Angaben zum quantitativen Engagement des Bundes und zu den von inm angepeilten qualitativen Zielen sollte das Dokument Transparenz über die Rahmenbedingungen schaffen und damit eine Orientierungshilfe für die ansonsten sehr dezentrale Entwicklung sein.

Eine überaus wichtige Aufgabe des Bundes ist die Gesamtkoordination des Fachhochschulsektors, die unter weitgehend dezentralisierten und deregulierten Bedingungen schwieriger ist als in einem zentralistischen System. Durch das „bottom-up“Modell wird die Entscheidung über Standorte, Profile und Größe der Studiengänge nicht auf der Basis einer langfristigen zentralen Planung getroffen. Vielmehr werden die dezentralen Initiativen potentieller Erhalter von Studiengängen im nachhinein koordiniert. Alle Studienpläne müssen erstens vom Fachhochschulrat unter dem Gesichtspunkt akademischer Qualität akkreditiert werden. Um in den Genuss einer Bundesfinanzierung zu gelangen, muss ein Studiengang neben einer positiven Begutachtung durch den Fachhochschulrat auch eine Reihe hochschulpolitischer Kriterien erfüllen, die im Entwicklungs- und Finanzierungsplan angeführt sind (z.B. Abbau regionaler Disparitäten, Förderung von Berufstätigen oder Nicht-Maturanten, Internationalität u.a.). Zweitens ist für jeden einzelnen konkreten Studiengang ein Vertrag zwischen dem zuständigen Bundesministerium (BMBWK) und dem Erhalter erforderlich, in dem die Zahl der Studienplätze und die Höhe des Bundeszuschusses für dieses spezielle Vorhaben festgelegt werden. Der Bund legt dabei Normkosten (laufende Kosten pro Studienplatz; Investitionen sind in diesem Parameter definitionsgemäß nicht enthalten) fest. Als einheitlicher Pauschalbetrag pro Studienplatz waren bei Errichtung des Sektors 88.000 öS $(6.395,30 €)$ für den kaufmännischen und 105.000 öS $(7.630,80 €)$ für den technischen Bereich festgelegt worden (Preise von 1991). Der Bund hatte sich bereiterklärt, 90\% dieser Normkosten zu übernehmen.

Damit hat der Bund auf Basis einer Entwicklungs- und Finanzierungsplanung den Aufbau des Fachhochschulsektors im Wesentlichen über

- die Ausbaugeschwindigkeit

- die Finanzierung

- die inhaltlichen Kriterien

gesteuert. 


\subsubsection{Steuerung des FH-Sektors durch die Ausbaugeschwindigkeit}

Der Ausbaustand des FH-Sektors stellt sich nach dem derzeitigen Kenntnisstand folgendermaßen dar:

- Wintersemester 2002/03: 125 Studiengänge mit 16.838 inländischen Studierenden ${ }^{130}$

- Prognose bis 2005/06: ca. 26.500 Studierende

Die Entwicklungs- und Finanzierungsplanung I (1994) sah seinerzeit folgende Ausbaugeschwindigkeit vor:

Tabelle 12: Wachstum der FH-Studienplätze laut Entwicklungs- und Finanzierungsplan I

\begin{tabular}{|l|c|c|c|}
\hline & Studienplätze It. EFP I & Studierende real & Differenz \\
\hline $1994 / 95$ & bis zu 500 & 695 & +195 \\
$1995 / 96$ & bis zu 2.000 & 1.756 & -240 \\
\hline $1996 / 97$ & bis zu 4.000 & 3.753 & -247 \\
$1997 / 98$ & bis zu 6.000 & 5.771 & -229 \\
\hline $1998 / 99$ & bis zu 8.000 & 7.880 & -120 \\
$1999 / 2000$ & bis zu 10.000 & 9.977 & -23 \\
\hline
\end{tabular}

Quelle: BMWF (1994), FHR.

Die „Entwicklungs- und Finanzierungplanung I" strebte für den Fachhochschulsektor eine Größenordung von 500 Studienplätzen im ersten und 2.000 Studienplätze im zweiten Studienjahr an, dann Steigerungen um jeweils 2.000 pro Jahr, um 1999/2000 10.000 Studienplätze erreicht zu haben. Diese Größenordnungen wurden weitgehend eingehalten.

Der Aufbau des Sektors wurde von Anfang an von der Forderung nach einer stärkeren und schnelleren Expansion des Sektors begleitet. Dagegen gehalten wurde - neben der finanziellen Belastung - immer das Argument, dass es geraume Zeit brauchen würde, bis die Fachhochschul-Studiengänge die erforderliche Qualität erreicht hätten. Mögliche Verschiebungen der Studierendenströme von den Universitäten in den Fachhochschulbereich wurden als quantitativ und zeitlich nur schwer prognostizierbar gesehen.

130 Quelle: Statistik Austria, vorläufige Zahlen. 
Jedenfalls wurde davon ausgegangen, dass die geforderte rasche Expansion eine Entlastung der bestehenden schulischen Postsekundareinrichtungen und Universitäten zur Folge haben müsste (vgl. BMWFK/BMUkA 1995, 28). Daher könne eine Erhöhung der vom Bund geförderten Studienplätze nur in Abstimmung mit der Entwicklung an den Universitäten, den Akademien und den anderen Bildungseinrichtungen erfolgen.

Der Entwicklungs- und Finanzierungsplan I sah jedenfalls nicht nur aus budgetären Gründen ein „mittleres“ Entwicklungstempo vor, sondern auch, weil man davon ausging, dass ein überhastetes Entwicklungstempo die Beobachtung der Implementierung und allfällige Korrekturmaßnahmen eines doch sehr neuen Regelungs- und Finanzierungsrahmens im Fachhochschulsektor erschweren würde.

Die Entwicklungs- und Finanzierungsplanung II (1999) ging davon aus, dass sich bis zum Studienjahr 2004/05 rund 6.000 StudienanfängerInnen für ein Fachhochschulstudium entscheiden werden, was ca. einem Drittel aller österreichischen StudienanfängerInnen entsprechen würde.

Der Ausbauplan für die Phase II legt im einzelnen fest (vgl. BMWV 1999, 4):

„Das angestrebte Ziel, in Österreich bis zum Studienjahr 2004/05 6.000 AnfängerInnenplätze im Fachhochschulbereich zu schaffen, bedeutet, dass in dem genannten Zeitraum jährlich etwa 600 Plätze in neuen Studiengängen oder durch Aufstockung von Plätzen in bestehenden Angeboten zu schaffen sein werden."

Ausgehend von den 3.000 AnfängerInnenstudienplätzen im Studienjahr 1999/2000 laut Entwicklungs- und Finanzierungsplanung I gestaltet sich die weitere Entwicklung der AnfängerInnenstudienplätze wie folgt (vgl. BMBWK 2000, 42):

Tabelle 13: Wachstum der FH-AnfängerInnenstudienplätze laut Entwicklungs- und Finanzierungsplan II

\begin{tabular}{|l|c|c|c|}
\hline & $\begin{array}{c}\text { Anfängerlnnenstudienp } \\
\text { lätze It. EFP II }\end{array}$ & AnfängerInnen real & Differenz \\
\hline $1999 / 2000$ & 3.000 & 3.498 & +498 \\
\hline $2000 / 2001$ & 3.600 & 4.105 & +505 \\
\hline $2001 / 2002$ & 4.200 & 5.320 & +1.120 \\
\hline $\left.2002 / 2003^{*}\right)$ & 4.800 & 6.143 & +1.343 \\
\hline $2003 / 2004$ & 5.400 & & \\
\hline $2002 / 2005$ & 6.000 & & \\
\hline
\end{tabular}

*) AnfängerInnen real 2002/03: nur Wintersemester, nur InländerInnen, vorläufige Zahlen.

Quelle: BMWV (1999), FHR, Statistik Austria. 
Tabelle 14: Wachstum der Gesamtzahl der FH-Studienplätze laut Entwicklungs- und Finanzierungsplan II

\begin{tabular}{|l|c|c|c|}
\hline & Studienplätze It. EFP II & Studierende real & Differenz \\
\hline $1999 / 2000$ & 10.000 & 9.977 & -23 \\
$2000 / 2001$ & 11.800 & 11.766 & -34 \\
\hline $2001 / 2002$ & 13.800 & 14.430 & +630 \\
$2002 / 2003$ & 16.300 & 16.838 & +538 \\
\hline $2003 / 2004$ & 18.700 & & \\
\hline $2004 / 2005$ & 21.000 & & \\
\hline
\end{tabular}

*) Studierende real 2002/03: nur Wintersemester, nur Inländerlnnen, vorläufige Zahlen.

Quelle: BMWV (1999), FHR, Statistik Austria.

Diese Planung ging davon aus (vgl. BMBWK 2000, 42), dass

- die Nachfrage für die bestehenden Studiengänge unverändert anhält,

- etwa im bisherigen Tempo zusätzliche neue Studiengänge geschaffen werden und

- für zwei Bereiche (Sozial- und Pflegebereich) ein sogenanntes „upgrading“ bestehender Ausbildungsgänge vorgenommen wird.

Tabelle 15: Überblick über die Planungsphasen von EFP I und EFP II

\begin{tabular}{|l|c|c|c|}
\hline & Studienjahre & $\begin{array}{c}\text { Studienplätze f. } \\
\text { Anfängerlnnen }\end{array}$ & $\begin{array}{c}\text { Studienplätze } \\
\text { insgesamt }\end{array}$ \\
\hline Planungsphase I: & $1994 / 1995$ bis $1999 / 2000:$ & 3.000 & 10.000 \\
\hline Planungsphase II: & $2000 / 2002$ bis $2004 / 2005$ & 6.000 & 21.000 \\
\hline
\end{tabular}

Quelle: BMWF (1994), BMWV (1999).

Im Jahr 2002 wurde vom BMBWK die „Aktion 600+“ verabschiedet, die zusätzliche 600 Studienplätze für den FH-Sektor vorsah, wodurch eine schnellere Ausbaugeschwindigkeit erreicht wurde als ursprünglich in den Entwicklungs- und Finanzierungsplänen vorgesehen. Der Hochschulbericht 2002 formuliert dazu: „Durchschnittlich bewerben sich nämlich drei Studierende für einen Studienplatz, bei einzelnen Studiengängen sind es über elf Personen. In der Folge wurde für das Studienjahr 2002/03 die Aktion „600+“ initiiert, mit der neben den regulären 600 bundesfinanzierten neuen AnfängerInnenstudienplätzen über 600 weitere AnfängerInnenstudienplätze angeboten werden können, die von den Erhaltern der Studiengänge mit der Unterstützung von Ländern und Standortgemeinden startfinanziert werden." (BMBWK 2002, 63). 
Damit ist man zwar dem seit langem geäußerten Wunsch nach einem stärkeren Ausbau des FH-Sektors nachgekommen, andererseits hat die Strategie, in kurzer Zeit relativ viele neue Studienplätze zu genehmigen, im Sektor selbst einiges an Skepsis ausgelöst, weil

- zusätzliche Finanzen in Form zusätzlicher Studienplätze eher eine weitere unkontrollierte Expansion anstatt der mittlerweile erwünschten, im zweiten Entwicklungs- und Finanzierungsplan auch ausdrücklich festgehaltenen Konsolidierung fördern;

- u.U. zusätzliche Gelder besser in qualitätsfördernde Maßnahmen (etwa im Organisationsund Personalentwicklungsbereich) investiert werden sollten, die auch den Kurs der Konsolidierung unterstützen würden.

- bei einem derartig raschen Ausbau Qualitätsprobleme entstehen würden. ${ }^{131}$

Nach den bisherigen Ausbauplänen werden im Jahr 2005 in Österreich vermutlich 13\% der Studierenden im Fachhochschulsektor studieren. In Finnland sind es $42 \%$, in den Niederlande ca. 65\%, in Deutschland ca. 25\% aller Hochschulstudierenden (vgl. Abschnitt 2.3, Tabelle 5). Ein ähnlicher Ausbau in Österreich ist - als Konsequenz der späten Einrichtung des Sektors sowie der Art seiner Konstruktion (vgl. Abschnitt 2.1) - unrealistisch. Das Thema der weiteren Expansion ist unmittelbar damit verbunden, welches Fächerspektrum in Zukunft im FH-Sektor vertreten sein wird.

\subsubsection{Steuerung des FH-Sektors durch die Finanzierung}

\section{Kontroverse Punkte}

Fragen der Finanzierung haben bei den Kontroversen um das FH-Konzept einen großen Stellenwert eingenommen. Zunächst hat sich die Diskussion auf drei Punkte zugespitzt:

- Gemischte Finanzierung vs. vollständige Kostendeckung durch den Bund. Anfänglich haben die meisten Länder die Forderung des Bundes nach einer Co-Finanzierung nicht ernst genommen bzw. mit Verweis auf das B-VG ${ }^{132}$ vehement abgelehnt. Viele

131 Auch die mangelnde Involvierung des FHR in diese Aktion 600+ wurde in den Expertlnneninterviews kritisch vermerkt.

132 Das B-VG regelt die Kompetenzverteilung hinsichtlich der einfachen Gesetzgebung; eine konkrete Finanzierungsverpflichtung, geschweige denn die Verpflichtung zur vollen Kostendeckung, lässt sich daraus nicht ableiten. Allerdings hätte der Bund keine gesetzliche Finanzierungspflicht der Länder festschreiben dürfen. Das ist auch nicht geschehen; die Zahlungsbereitschaft der Länder folgt einem indirekten, keinem gesetzlichen Zwang. 
Beobachter gingen zunächst davon aus, dass die Länder nie bereit wären, dauerhafte Beiträge in größerem Umfang zu leisten.

- Pauschalbeträge („Normkosten“) vs. „bedarfsorientierte“ Kostenbeiträge des Bundes. Die ursprüngliche Erwartung der meisten Akteure war, dass der Bund den einzelnen Erhaltern eine unterschiedliche, auf ihre Besonderheiten abgestimmte Förderung $z_{\text {zukommen lassen würde }}^{133}$. Das Modell einer Gleichbehandlung ${ }^{134}$ aller Erhalter ohne Berücksichtigung ihrer besonderen Förderungswürdigkeit stieß zunächst auf Kritik.

\section{- Die Höhe der Normkosten}

Die ersten beiden Streitpunkte können mittlerweile als abgeschlossen betrachtet werden.

- Dass die Länder und/oder andere öffentliche Einrichtungen einen Beitrag zur Finanzierung der in ihrem Einflussbereich stehenden Fachhochschuleinrichtungen leisten, wird mittlerweile als selbstverständlich akzeptiert, auch wenn über das notwendige bzw. optimale Ausmaß dieser Co-Finanzierung keine Klarheit besteht. Die ursprüngliche Fundamentalopposition mancher Ländervertreterlnnen ist rasch der pragmatischen Bereitschaft gewichen, Fachhochschuleinrichtungen unter dem Gesichtspunkt regionaler „Standortsicherung" zu fördern.

- Ebenso rasch haben sich die Erhalter und die Vertreterlnnen der Länder damit abgefunden, dass das Finanzierungsmodell des Bundes auf speziellen Förderungsbedarf und sei er noch so gut begründet, keine Rücksicht nimmt. Im allgemeinen wird die Berechenbarkeit und der Autonomiegewinn, die mit diesem distanzierten Verhältnis zwischen Bund und Erhaltern einhergehen, als Vorteil betrachtet. Bei aller grundsätzlichen Akzeptanz des Normkosten-Modells gibt es allerdings zunehmende Kritik an der gängigen Praxis, die Pauschalförderung pro Studienplatz strikt an einzelne Studiengänge zu koppeln. Der Wunsch vieler Erhalter geht dahin, diese Pauschalbeträge nicht dem Studiengang, sondern dem Erhalter zuzuweisen. Das würde den Erhaltern eine höhere Flexibilität geben und innen ermöglichen, Nachfrageschwankungen zwischen unterschiedlichen Studiengängen auszugleichen. In dem Ausmaß, in dem die Erhalter den dadurch gewonnenen Entscheidungsspielraum auch tatsächlich nutzen, käme es freilich zu erhöhten Verteilungskonflikten zwischen den einzelnen Studiengängen bzw. zu Spannungen zwischen den Erhaltervertretern und jenen Studiengängen, die sich benachteiligt fühlen. Auf der institutionellen Ebene müsste eine solche Reform daher mit einer Verbesserung des Konfliktmanagements einhergehen.

133 Das wäre mit der paternalistischen Tradition der österreichischen Bildungspolitik kompatibel gewesen, die gleichermaßen benevolente wie umfassend informierte staatliche Entscheidungsträger unterstellt.

134 Der wichtigste Grund dafür war, dass der Bund mit der Möglichkeit, eine besondere Förderungswürdigkeit zu reklamieren, ein Einfallstor für Interventionen, vor allem von Landesebene, eröffnet hätte. 
Die Kontroverse über die Höhe der Normkosten, d.h. über den Finanzierungsbeitrag des Bundes pro Studienplatz, ist hingegen ein hochschulpolitisches Dauerthema geblieben und daran wird sich wohl auch nichts ändern. Nur bei einer soliden Datenbasis ist eine sachliche Diskussion möglich, die über die Artikulation von Interessen hinausgeht. An dieser Datenbasis fehlt es jedoch.

\section{Transparenz der Finanzdaten}

Eine Grundvoraussetzung des neuen Steuerungsmodells ist die Schaffung von Transparenz, vor allem auch bei den Finanzflüssen. Diese Voraussetzung ist im FH-Bereich derzeit nicht erfüllt. Auch nach einer achtjährigen Aufbau- und Entwicklungsphase ist die Datenlage in Bezug auf die Finanzflüsse äußerst rudimentär und unbefriedigend. Das hat mehrere Ursachen:

- Im Unterschied zu den Universitäten und dem Regelschulwesen zeichnet sich der Fachhochschulbereich durch eine vielfältige Trägerschaft aus, was die Sammlung und Bündelung von Informationen erschwert. Am schwersten wiegt der Umstand, dass einige Erhalter ihre Rechnungsabschlüsse nach Kalenderjahren legen, einige nach Studienjahren, andere nach sachlich nicht nachvollziehbaren Zeiträumen.

- Die Übersicht über die einnahmenseitigen Finanzdaten wird durch die gemischte Finanzierung erschwert; zu den sachlichen Schwierigkeiten einer kohärenten Zusammenführung unterschiedlicher Finanzquellen kommen teilweise interessenbedingte Widerstände gegen Transparenz.

- Hinzu kommt das Problem, dass ein Teil der Förderungen nicht monetär, sondern in Form von Natural- und Sachleistungen („lebende Subventionen“, Raumüberlassung etc.) erfolgt; abgesehen davon, dass diese Naturalsubventionen üblicherweise in keiner Form der Rechnungslegung aufscheinen, wirft ihre Umwandlung in kommensurable Geldwerte erhebliche Probleme auf (Definition ortsüblicher Mieten etc.).

- Die Übersicht über die ausgabenseitigen Finanzdaten wird durch endemische Definitionsunsicherheiten bei der Gliederung nach Kostenarten (Abgrenzung der laufenden Kosten von Ausgaben für Bauten und Investitionen) erschwert; diese Frage ist für die Bewertung der Normkosten des Bundes von Bedeutung. 
Alle diese Probleme wurden durch ein merkwürdiges Verständnis von Deregulierung und Liberalisierung seitens einiger Akteure verschärft ${ }^{135}$. Das zuständige Bundesministerium hat zunächst keine eindeutig definierten Forderungen bezüglich einer finanziellen Rechnungslegung formuliert und die Bundesförderung nicht davon abhängig gemacht, ob diese Anforderung erfüllt wird. Das hat einige Erhalter in der Auffassung bestätigt, die Finanzdaten seien ihre „Privatangelegenheit“. Der Wunsch nach Offenlegung von Finanzströmen wird häufig als bürokratische Verletzung institutioneller Autonomie gesehen. Zwar wird der Fachhochschulsektor von den Rechnungshöfen auf Bundes- und Landesebene geprüft, aber diese Prüfung bezieht sich auf eine allfällige missbräuchliche Verwendung von öffentlichen Geldern, sie stellt keine auch nur minimale Transparenz über die Finanzflüsse im Gesamtsektor sicher.

Der Fachhochschulrat hat sich relativ rasch an diesem "Wildwuchs" in der Datenlage gestoßen und einen Versuch zur Durchsetzung standardisierter Berichtsnormen unternommen. Dieser Ansatz, der noch in die Präsidentschaft von Schelling fällt, war allerdings so detailliert und kompliziert, dass er auf großen Widerstand bei den Erhaltern stieß und sich nicht durchgesetzt hat. Nach Schelling hatte dieses Projekt im Fachhochschulrat keine Priorität.

Parallel dazu hat es im zuständigen Bundesministerium mehrere Anläufe zur Prüfung der Rechnungsabschlüsse gegeben (vgl. Kofler/Urnik 1995, Nadornik/Glander 1997, Nadornik/Glander 1999a). Die bislang umfassendste Analyse gibt es für das Studienjahr 1998/99 (Glander 2001). Aufbauend auf diese Analysen wurde vom BMBWK in Kooperation mit dem Fachhochschulrat und der Fachhochschul-Konferenz ein weiterer Anlauf zur Standardisierung der Rechnungsabschlüsse vollzogen. Seit dem Jahr 2002 (beginnend mit den Voranschlägen für 2002) werden die Finanzdaten in der Datenbank des Fachhochschulrats (BIS - „Bereitstellung von Informationen über den Studienbetrieb“) für das BMBWK erfasst. Grundlage ist ein von allen Beteiligten (BMBWK, FachhochschulKonferenz, Fachhochschulrat) erarbeitetes Finanzdaten-Handbuch (vgl. BMBWK/FHR/FHK o.J.).

Zusammenfassend kann man festhalten: Eine solide Datenbasis, die es erlauben würde, den Verlauf und die Veränderungen der Ressourcenflüsse über die gesamte Zeitspanne des Fachhochschulaufbaus zu dokumentieren, existiert nicht. Für die Vergangenheit gibt es lediglich Momentaufnahmen für einzelne Zeiträume: 1994/95 und 1995/96 (Pechar 1996),

135 In diesem Zusammenhang sei darauf verwiesen, dass die USA, obwohl sich ihr Hochschulsystem durch ein buntscheckiges Muster hinsichtlich Trägerschaft und Finanzierungsmechanismen auszeichnet, vorbildliche Statistiken aller relevanten Parameter besitzt (vermutlich die besten im OECD-Raum, vor allem was die sozialwissenschaftliche Interpretierbarkeit der Daten betrifft). Meldepflicht statistischer Daten ist kein Merkmal hoher bürokratischer Regulierungsdichte. 
1998 und $1999^{136}$ (Glander 2001), die aber, auf Grund unterschiedlicher Gliederungen, nur teilweise vergleichbar sind. Daher wird im Rahmen dieser Expertise auf eine Zeitreihe verzichtet. Erst ab dem Jahr 2002 sollte sich diese Situation grundlegend bessern.

\section{Eigene Erhebungen}

Im Rahmen dieser Studie wurde eine Momentaufnahme der Einnahmen- und Ausgabenstruktur für das Jahr 2002 vorgenommen. Sie gründet sich auf zwei Datenbestände, die beide eine deutliche Verbesserung des Informationsstandes ermöglichen, aber zugleich den Nachteil besitzen, dass sie bei Abschluss des vorliegenden Manuskripts (40. Woche 2002) nur Daten von einem Teil der Erhalter enthalten ${ }^{137}$ :

- In der BIS-Datenbank müssen alle Erhalter ihre Jahresvoranschläge bis ein Monat nach Beginn des Geschäftsjahrs melden. Tatsächlich liegen bislang nur die Meldungen von 12 Erhaltern vor. Ein weiteres Problem dieser Datenquelle ist die sehr grobe Rasterung verschiedener Gliederungen. So sind z.B. die Einnahmengruppen in „Bundesförderungen“, „Förderungen von anderen öffentlichen Stellen“, „sonstige Förderungen“ und „weitere Einnahmen“ unterteilt; in der Kategorie „Förderungen von anderen öffentlichen Stellen" sind laut Handbuch auch die Beiträge von Kammern anzuführen. Nicht-monetäre Subventionen werden von der BIS-Datenbank nicht erfasst.

- Daten aus einer Erhebung bei allen Fachhochschulerhaltern. Diese Erhebung verfolgte den Zweck, bestimmte Informationslücken, die die BIS-Datenbank hinterlässt, zu schließen. So wird einerseits versucht, ein genaueres Bild der Einnahmequellen zu erhalten (die Kategorie „Förderungen von anderen öffentlichen Stellen“ wird nach Land, Kommunen und Kammern differenziert). Zum anderen werden auch die nicht-monetären Förderungen (gegliedert nach Gebäude, Investitionen, Personal) in die Befragung einbezogen.

\section{Sind die Normkosten ausreichend?}

Diese Frage steht nun seit einem knappen Jahrzehnt im Zentrum aller Diskussionen um die Finanzierung des FH-Sektors. Eine sachliche Diskussion wird durch die Schwierigkeit beeinträchtigt, einen konsensualen Bezugspunkt zu finden. „Knappheit“ ist ein sehr relatives Gefühl, es stellt sich auf nahezu jedem beliebigen Finanzierungsniveau ein. Da das

136 Beide Jahre sind lückenhaft.

137 Der Sachverhalt wird zusätzlich dadurch kompliziert, dass die Lücken in den beiden Datenbeständen nicht deckungsgleich sind (d.h. einige Länder sind in beiden Quellen repräsentiert, andere in keinen der beiden, eine dritte Gruppe ist entweder in der BIS-Datenbank oder in unserer eigenen Erhebung repräsentiert). 
wirtschaftliche Verhalten von Bildungseinrichtungen nicht durch den „disziplinierenden Druck" des Marktes gesteuert wird, gibt es für die institutionellen Entscheidungsträger wenig Anlass, die Ausgaben unter das Niveau der Einnahmen zu senken. Sie werden dafür in aller Regel bestraft, weil ihre Subventionen in der Folge gekürzt werden. „The basic principle of college finance is very simple. Institutions raise as much money as they can get and spend it all. Cost per student is therefore determined primarily by the amount of money that can be raised. If more money is raised, cost will go up, if less is raised, costs will go down." (Bowen, zitiert nach Keller 1983, 11).

Daraus kann man andererseits nicht folgern, die Höhe der Förderungen sei bedeutungslos. Zweifellos gibt es ein Minimum, bei dessen Unterschreiten es zu Qualitätseinbußen kommt. Wenn es keine absoluten Bezugspunkte gibt, hilft nur ein Vergleich unterschiedlicher Finanzierungsmuster, um sich der Frage nach einem „angemessenen Förderniveau“ zu nähern. Das kann ein Vergleich zwischen unterschiedlichen Hochschulsektoren sein oder ein Vergleich zwischen den Finanzierungsmustern unterschiedlicher Bundesländer bzw. Regionen.

In der Folge werden die Fördermuster der Bundesländer verglichen:

- Gibt es in der Höhe der Länderbeiträge und in den Mechanismen der Förderung systematische Unterschiede?

- Wenn ja, lassen diese Unterschiede Rückschlüsse auf die Qualität von Lehre und Forschung in den Fachhochschuleinrichtungen der betreffenden Länder zu?

Diese Zuspitzung auf die Rolle der Bundesländer liegt nahe, denn nur bei ihnen kommt es zu markanten, alle Erhalter betreffenden Variationen in der Förderpraxis. Der Förderbeitrag des Bundes ist insgesamt gesehen am wichtigsten ${ }^{138}$, aber auf Grund der strikten Gleichbehandlung aller Erhalter durch den Bund lassen sich hier keine spezifischen Differenzen ausmachen. Die Förderpraxis von Gemeinden und/oder Kammern variiert sehr stark, aber insgesamt ist der aus diesen Quellen kommende Förderbeitrag zu gering, um dies zur Basis einer Gesamttypologie zu machen. Nur die Länder zahlen viel (an die 30\%, vgl. Abb. 49), aber zu sehr unterschiedlichen Konditionen. Es sind also die Unterschiede in der Landespolitik, die eine Schlüsselrolle bei der Erklärung der Finanzierungsmuster im FHSektor spielen.

138 Auf der Basis der gegenwärtigen Datenmeldungen macht der Bundesbeitrag laut BIS $65 \%$ und laut eigener Erhalterbefragung 60\% aus (der Unterschied ergibt sich primär daraus, dass in den beiden Datenquellen unterschiedliche Länder und Erhalter repräsentiert sind). 
Bereits in der 1996 abgeschlossenen ersten Gesamtbetrachtung der Finanzierung des $\mathrm{FH}$ Sektors (Pechar 1996) haben sich - auf naturgemäß noch sehr schmaler empirischer Basis - systematische Unterschiede zwischen den Ländern abgezeichnet. Zum damaligen Zeitpunkt gab es drei Länder, die den Erhaltern ihres Einflussbereichs überdurchschnittlich hohe Förderungen gewährten ${ }^{139}$. Die Förderanteile von zwei Ländern waren unterdurchschnittlich ${ }^{140}$, die übrigen drei Länder ${ }^{141}$ lagen in einer Mittelgruppe, wo der Bund ca. zwei Drittel, Länder (z.T. ergänzt durch Gemeinden) ca. ein Drittel finanzierten. Diese Unterschiede in der Landespolitik hatten zur Folge, dass - im Sinne des obigen Zitats von Bowen - die Ausgaben der Erhalter pro Studienplatz stark schwankten.

Die vorläufigen Daten für 2002 zeigen, dass sich an dem Mitte der 1990er Jahre ermittelten Muster nur wenig geändert hat ( $\mathrm{vgl}$. Abbildung 47 und Abbildung 48). ${ }^{142}$ Es gibt eine aus zwei Ländern bestehende Spitzengruppe, in der der Anteil der Länderförderung zwischen $53 \%$ und $59 \%$ variiert und damit deutlich über dem des Bundes (variiert zwischen $33 \%$ und 43\%) liegt. In der aus drei Ländern bestehenden Mittelgruppe liegt der Anteil der Landesförderung zwischen 27\% und 38\%, der Anteil des Bundes schwankt zwischen 52\% und 64\%. In den beiden Ländern der „Schlussgruppe" hinsichtlich der Landesförderung variiert der Anteil der Landesförderung zwischen 11\% und 3\%, was bedingt, dass der Anteil der Bundesfinanzierung in einem Land den durch das Normkosten-Modell theoretisch unterstellten $90 \%$ nahe kommt.

139 Der Anteil der Länder in dieser Spitzengruppe lag damals zwischen 54\% und 45\%, wurde aber in einem Fall durch einen ungewöhnlich hohen Gemeindeanteil (19\%) ergänzt. Bei allen drei Ländern lag der Anteil des Bundes deutlich unter $50 \%$.

140 Zwischen 17\% und 22\%. Bei beiden Ländern lag der Finanzierungsanteil des Bundes deutlich über 70\%.

141 Tirol hatte zu diesem Zeitpunkt noch keinen Erhalter, daher waren in dieser Studie nur acht Länder repräsentiert.

142 Bei jenen Ländern, die in beiden Abbildungen enthalten sind, kommt es teilweise zu geringfügigen Abweichungen; sie sind durch unterschiedliche Angaben der Erhalter in der BIS-Datenbank bzw. unserer eigenen Erhebung zu erklären. 
Abbildung 47: Einnahmenstruktur der FH-Erhalter nach Bundesländern laut BIS (Voranschlag 2002)

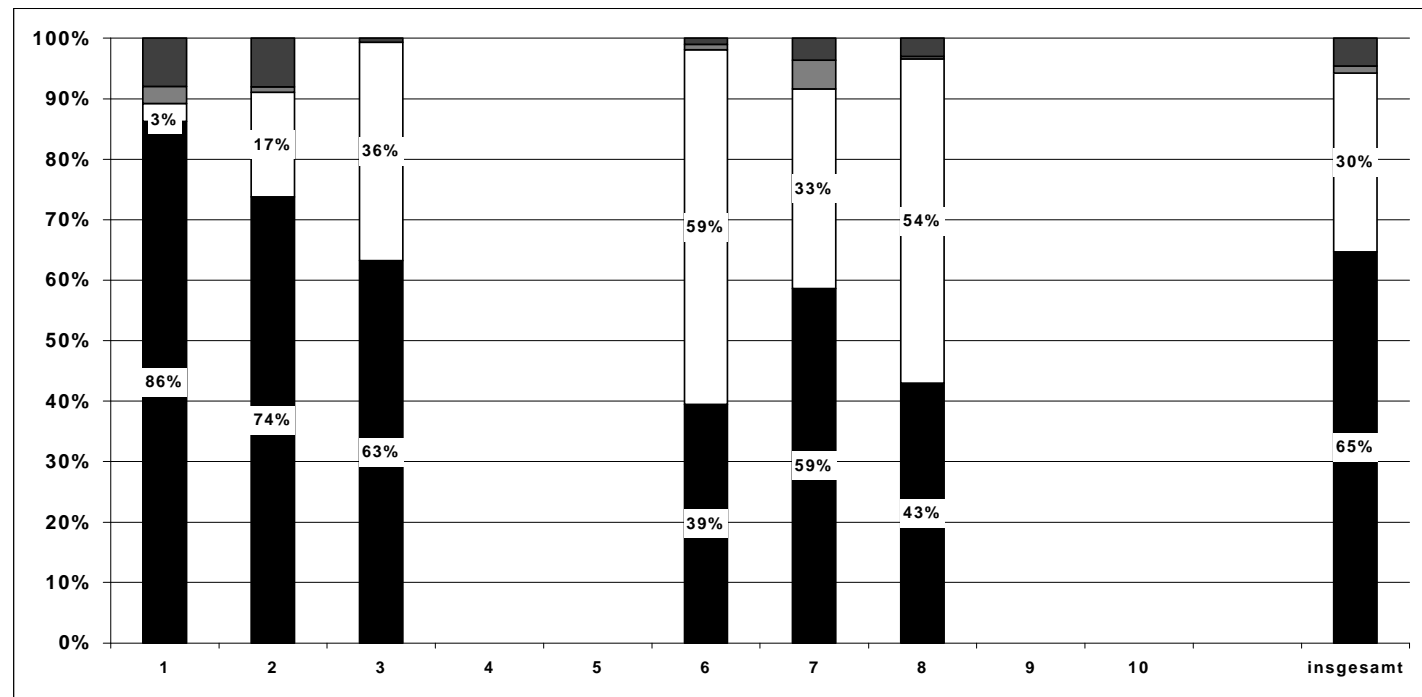

$\square$ Bundesförderungen $\square$ andere öffentliche Stellen $\square$ Sonstige Förderungen $\square$ Weitere Einnahmen

Der Bund als Erhalter wird als eigenständiges, 10. „Bundesland“ betrachtet. Quelle: FHR

\section{Abbildung 48: Einnahmenstruktur der FH-Erhalter nach Bundesländern laut Befragung} der Erhalter 2002 (laufendes Geschäftsjahr)

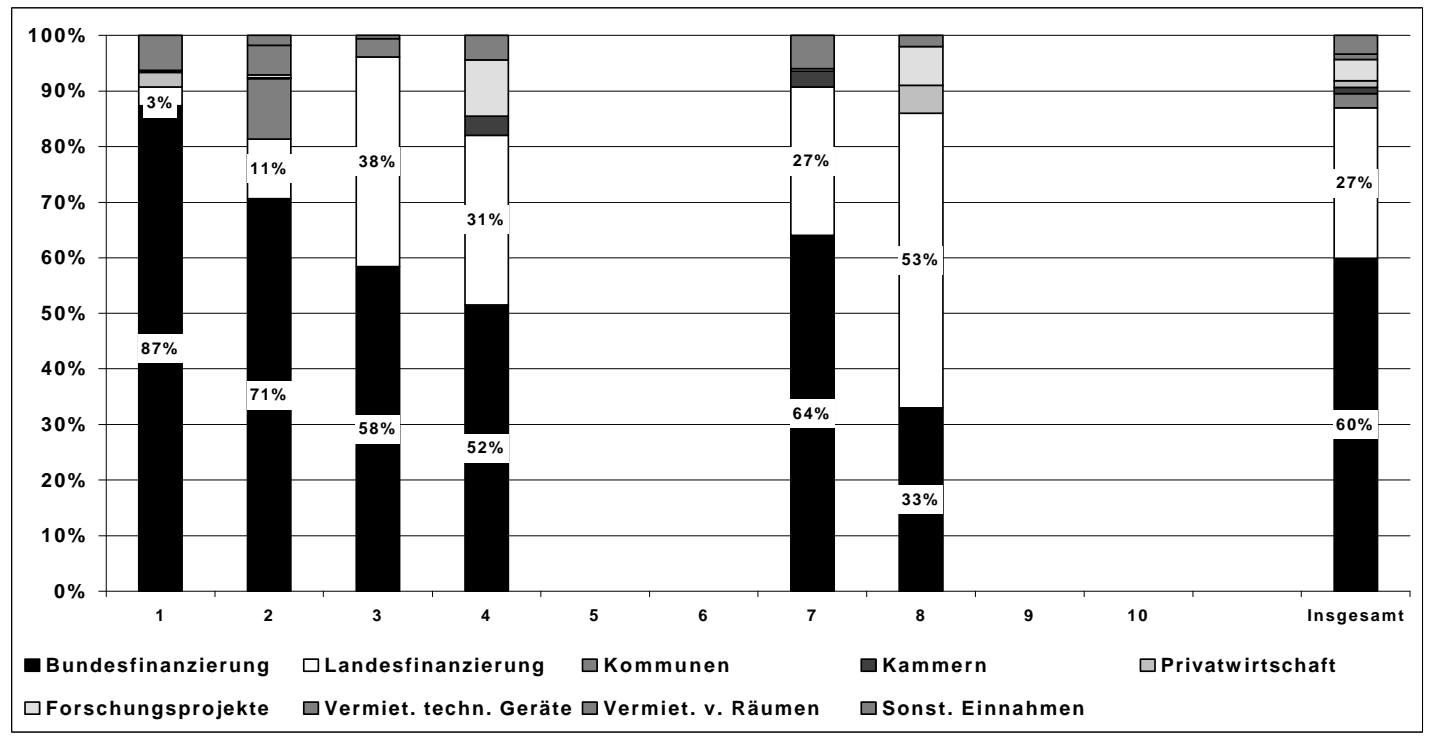

Der Bund als Erhalter wird als eigenständiges, 10. „Bundesland“ betrachtet. Quelle: Befragung der Erhalter 
Wie lassen sich diese - im Zeitverlauf offensichtlich konstanten - Ungleichheiten in den Finanzierungsmustern deuten? Welchen Beitrag können die erhobenen Daten zur Beantwortung der Frage leisten, ob die Höhe der Pauschalförderung durch den Bund ausreichend ist?

Eine mögliche Deutung lautet: wenn die in Bezug auf die Landesförderung „armen“ Erhalter ökonomisch lebensfähig sind und Lehre und Forschung von zufriedenstellender Qualität anbieten, dann haben die Normkosten des Bundes eine realistische Größenordnung. Die großzügige Förderpraxis anderer Länder ist eine glückliche Fügung für die betreffenden Erhalter, die sie vom Druck nach einem effizienten Umgang mit öffentlichen Geldern teilweise entlastet.

Eine gegensätzliche Deutung würde lauten: die Erhalter jener Länder, wo die Landesförderung so gering ist, dass sie fast ausschließlich oder überwiegend auf die Normkosten des Bundes angewiesen sind, müssen auf einer Untergrenze wirtschaften, die sie an bestimmten Entwicklungsoptionen hindert. Diese Erhalter müssen sich in einer Nische positionieren, die ihnen nur sehr kostengünstige Lehre und/oder Forschung ermöglicht. Es ist auch nicht auszuschließen, dass sie Abstriche bei der Qualität machen müssen.

Eine dritte Deutung der unterschiedlichen Finanzierungsmuster könnte sich schließlich darauf beziehen, dass den Erhaltern in den einzelnen Ländern bzw. an den verschiedenen Standorten unterschiedliche Kosten erwachsen (v. a. unterschiedliches Lohnniveau).

Die empirische Basis, um die Stichhaltigkeit der hier überspitzt skizzierten Deutungsalternativen zu überprüfen, ist äußerst schmal und wird überdies durch den Umstand beeinträchtigt, dass bei der Erhalter- und Studiengangsbefragung Anonymität zugesichert wurde. Eine vorsichtige Einschätzung wäre unter folgenden Voraussetzungen möglich:

- Zum einen könnte die Typologie der Standorte nach der Zusammensetzung des Lehrpersonals und der Beteiligung am Impulsprogramm ansatzweise mit den Finanzierungsmustern in Beziehung gesetzt werden $^{143}$. Es ließe sich dann klären, ob Standorte in den Bundesländern mit geringer Landesförderung eher zu den Einrichtungen mit hohem Anteil an nebenberuflichem Personal und keiner Forschungsbeteiligung (in gewissem Sinne also zu den Standorten mit geringerem Potential) zu zählen sind.

143 Die Zusicherung der Anonymität bei den Befragungen erlaubt es nicht, diesen Zusammenhängen im Detail nachzugehen. 
- Zum anderen könnte man einen Zusammenhang zwischen den Finanzierungsmustern der Länder und den Ergebnissen der Evaluierungen der Studiengänge durch den FHR herstellen. Es ist ja eine naheliegende Frage, ob Erhalter mit hohen Ausgaben pro Studienplatz in den Evaluierungen systematisch besser abschneiden als Erhalter mit niedrigen Ausgaben. Derzeit scheitert die Beantwortung dieser Frage daran, dass diese Daten nicht verfügbar sind. Für die Zukunft wäre zu prüfen, ob die Ergebnisse der Evaluierung veröffentlicht und in einer Form aufbereitet werden sollten, die es erlaubt, den oben skizzierten Zusammenhang herzustellen; bzw. ob im Falle des (vorhersehbaren) Widerstandes der Erhalter dagegen nicht zumindest eine anonymisierte Auswertung erfolgen sollte.

\subsubsection{Steuerung des FH-Sektors durch inhaltliche Kriterien}

\section{Entwicklungs- und Finanzierungsplan I - Inhaltliche Kriterien}

Im Entwicklungs- und Finanzierungsplan I wurden insbesondere folgende Motive und Ziele der Bundesregierung für die Einrichtung von Fachhochschul-Studiengängen genannt:

- das Argument, dass die österreichische Wirtschaft dieses Qualifikationssegment benötigt,

- dass viele Jugendliche eine kürzere und berufsbezogene Ausbildung nachfragen würden

- und dadurch auch zu einer Entlastung der Universitäten beigetragen würde.

\section{Erfahrungen mit dem Entwicklungs- und Finanzierungsplan I}

Die angeführten Kriterien sind zwar für die Beurteilung von einzelnen Studiengängen entwickelt worden, aber gleichzeitig kommen durch sie doch auch bildungspolitische Vorstellungen zur Gestaltung des Gesamtsektors zum Ausdruck.

Das Element der Innovation („Innovativer Charakter") scheint durch die Kombination mit dem Kriterium "überregionale Abstimmung" und den Vorgaben durch die Bedarfs- und Akzeptanzanalyse vor allem dazu geführt zu haben, dass eine scharfe Politik der Abgrenzung gegenüber den Universitäten betrieben wurde, indem nur Studienangebote zugelassen wurden, die es in dieser Form noch nicht an Universitäten gab und Innovation vor allem in inhaltlicher Spezialisierung gesehen wurde. Diese im Entwicklungs- und Finanzierungsplan genannten Kriterien wurden durch eine entsprechende Interpretation bei der Akkreditierung verstärkt. 


\section{Kriterien für eine Bundesfinanzierung - Auszug aus dem Entwicklungs- und Finanzierungsplan I}

Bei der hochschulpolitischen Bewertung von Anträgen für Fachhochschul-Studiengänge werden für jedes der folgenden Kriterien 0-10 Punkte vergeben. Um in den Genuss von Bundesmitteln zu kommen, muss ein Antrag auf Durchführung eines Fachhochschul-Studiengangs mindestens 50 Punkte aus der möglichen Höchstzahl von 100 Punkten erreichen. Die Anträge können auch nach der von ihnen erreichten Punktezahl gereiht werden. Dies ist dann notwendig, wenn die Summe der Studienplätze in den förderungswürdigen Anträgen die Zahl jener Studienplätze überschreitet, für die im Budget eine Bundesfinanzierung vorgesehen ist (vgl. 0). Die Anträge werden nach folgenden Kriterien bewertet:

- Innovativer Charakter: Das Vorhaben stellt eine Innovation in organisatorischer Hinsicht und/oder im fachlichen Spektrum des Hochschulbereichs dar.

- Überregionale Abstimmung: Das Vorhaben führt zu keinen Doppelgleisigkeiten, sondern stellt eine sinnvolle Ergänzung anderer Angebote dar.

- Langfristiges Entwicklungskonzept: Das Vorhaben ist Teil eines Aufbaukonzeptes, das mit mindestens 1.000 Studenten aus dem regionalen Einzugsgebiet rechnet und das mehrere sinnvoll aufeinander abgestimmte Studiengänge sowie ein längerfristig lebensfähiges Fächerspektrum vorsieht; ist dies nicht der Fall und beschränkt sich das Vorhaben auf einen Studiengang, so ist dieser in ein facheinschlägiges, renommiertes Forschungs-, Versuchs- oder Bildungszentrum integriert.

- Abbau regionaler Disparitäten: Das Vorhaben ist in einer Region angesiedelt, deren Versorgung mit Hochschulangeboten unter dem Durchschnitt liegt.

- Bildungspolitische Strukturbereinigung: Das Vorhaben befördert klare Strukturen im Bildungsbereich und bereinigt Doppelgleisigkeiten im nichtuniversitären Sektor.

- Öffnung zu neuen Zielgruppen: Das Vorhaben sieht spezielle Fördermaßnahmen für Personen ohne Matura vor.

- Angebot für Berufstätige: Das Vorhaben sieht organisatorische Erleichterungen für Berufstätige vor.

- Nutzung vorhandener Ressourcen: Das Vorhaben bewirkt eine Nutzung brachliegender Räume und Ausstattungen.

- Private Mitfinanzierung: Das Vorhaben wird nicht nur von juristischen Personen des öffentlichen Rechts finanziert, sondern auch durch Beteiligung des privaten Sektors.

- Internationale Kooperation: Das Vorhaben fördert in einer dem Ausbildungsziel entsprechenden Weise die internationale Zusammenarbeit und den internationalen Austausch von Lehrenden und Lernenden.

Der Bericht „2 Jahre FHStG ( BMWFK/BMUkA 1995) - eine erste offizielle Bilanz des ersten Entwicklungs- und Finanzierungsplans - sagt zum Thema „Überregionale Abstimmung“, dass künftig im Zuge der Entscheidungen über die Bundesfinanzierung darauf zu achten ist, unrentable Mehrfacheinrichtungen, wie sie im Schul- und Universitätsbereich bestehen und nur schwer zu beseitigen sind, zu vermeiden. Es wird festgehalten, dass "nach dieser Eingangsphase der Abgrenzung nun Projekte anvisiert werden, die sich mit den Angeboten an den Universitäten überlappen oder mit diesen konkurrieren. Wenngleich sich ein Wettbewerb unterschiedlicher Institutionen positiv auf die Qualität des Angebotes auswirken kann, kann er nicht auf Kosten des Steuerzahlers gehen. Mehrfacheinrichtungen werden nur dort zulässig sein, wo mit den bestehenden Angeboten quantitativ nicht das Auslangen gefunden werden kann. In den übrigen Fällen bedingt jede Neueinrichtung die Auflassung 
eines substituierbaren Angebotes." Jedenfalls haben diese Auflagen eine große Kreativität im Erfinden von Bezeichnungen von Studiengängen und Abschlüssen ausgelöst, wodurch jeweils ein ganz neues Angebot suggeriert und auch ein hoher Grad an Spezialisierung befördert wurde.

Die Frage der kritischen Größenordnungen ist bereits im Entwicklungs- und Finanzierungsplan I enthalten, hat aber angesichts des Aufbaustadiums des Sektors noch nicht den gleichen Stellenwert wie dann im Entwicklungs- und Finanzierungsplan II.

Das Kriterium "Langfristiges Entwicklungskonzept" bezieht sich hier ja auf entsprechend überzeugende Konzepte bei der Antragstellung für einen Studiengang. Für den Sektor insgesamt stellt sich dieses Kriterium im Zusammenhang mit der gesamten Entwicklungsund Finanzierungsplanung - siehe hierzu also die auf den letzten Seiten dieses Kapitels angeführten Überlegungen.

Die Erfüllung des Kriteriums „Abbau regionaler Disparitäten“ gehört zu den Erfolgsgeschichten bei der Etablierung des Fachhochschulsektors, siehe hierzu insbesondere Kapitel 5, Abschnitt 5.2.1.

Das Kriterium der „bildungspolitischen Strukturbereinigung" findet sich nicht nur in den bildungspolitischen Kriterien, die an die einzelnen Studiengänge angelegt werden sollten, sondern begleitet den Aufbau des Sektors von Beginn an. Bereits der Prüferbericht der OECD-Prüfung (BMWF/BMUK 1993) regt die Schaffung eines kohärenten und übersichtlichen Hochschulbereichs sowie die Integration bestehender Postsekundareinrichtungen in den Fachhochschulbereich an. 1995 stellt der Präsident des Fachhochschulrates Schelling fest, dass der Bund eine Aussage über den pädagogischen, sozialberuflichen und medizinpflegerischen Postsekundarbereich tätigen sollte, damit privatrechtlich organisierte Erhalter erkennen können, ob die von innen entwickelten Studiengänge eine Lösung für ein nationales bildungspolitisches Problem darstellen (vgl. Schelling 1995).

Zum Thema "Öffnung zu neuen Zielgruppen“ steht im Bericht der Ministerien von 1995 (BMWFK/BMUkA 1995), dass es eines der wichtigsten Anliegen des Fachhochschulsektors war und ist, die Durchlässigkeit des Bildungssystems zu fördern. Dabei wird das Ziel des Zugangs von Lehrlingen zu einem Fachhochschulstudium unterstrichen. Das FHStG sieht vor, dass man ein Fachhochschulstudium nicht nur mit Reifezeugnis oder Berufsberechtigungsprüfung, sondern auch durch den Nachweis einer einschlägigen beruflichen Qualifikation beginnen kann. Ausdrücklich werden die Zugangsbedingungen für diese Zielgruppe auch als ein Evaluierungskriterium für die Förderungswürdigkeit eines Studiengangs betont (vgl. auch die Ausführungen in Abschnitt 3.1.3). 
Im Sinne einer "second-chance-education“ als auch zwecks beruflicher Fortbildung wurde die Bedeutung von Angeboten für Berufstätige von Anfang an sehr betont. Im Spannungsfeld zwischen dem Aufbau eines neuen Sektors mit akademischer Reputation einerseits und dem Eingehen auf die Anforderungen spezieller Zielgruppen - wie etwa Berufstätiger - scheint am Anfang das erstere Ziel als dringlicher erachtet worden zu sein. Jedenfalls wird bereits 1995 von Seite des Bundes festgestellt, dass man künftig den Angeboten für Berufstätige bei den Finanzierungskriterien eine höhere Priorität einräumen wird müssen.

Die „Nutzung vorhandener Ressourcen“ ist ein wesentlicher Aspekt der Unterstützung durch die Gemeinden, die sich insbesondere durch die Bereitstellung der räumlichen Infrastruktur aktiv an der Fachhochschulentwicklung beteiligten.

Auch „Private Mitfinanzierung“ war ein Thema der Zweijahresbilanz der zuständigen Ministerien: „Das Vorhaben, nicht nur öffentliche, sondern auch private Sponsoren für den neuen Hochschulsektor zu gewinnen, konnte bislang kaum realisiert werden. Die Mittel, die von privatwirtschaftlicher Seite in den neuen Sektor investiert werden, sind im Vergleich zum Beitrag der Gebietskörperschaften höchst bescheiden und beschränken sich auf gewisse Sachleistungen oder einmalige Spenden." (BMWFK/BMUkA 1995, 20)

Im ersten Entwicklungs- und Finanzierungsplan ist die „internationale Kooperation“ noch als ein eigenes Kriterium angeführt; näheres zum Stand der „Internationalisierung“ ist Kapitel 6 zu entnehmen.

Im Bericht „2 Jahre FHStG“ (vgl. BMWKF/BMUkA 1995) heißt es zum Thema Entwicklungsund Finanzierungsplanung, dass der Entwicklungs- und Finanzierungsplan eine wertvolle Orientierungshilfe war, indem er die ungefähren Kosten der Entwicklung des Fachhochschulbereichs für den Bund abzuschätzen erlaubte und durch das Normkostenmodell eine Kalkulationsgrundlage für die Erhalter von FachhochschulStudiengängen darstellte. Die offene Struktur war geeignet, den Rahmen abzustecken, aber nach zweijähriger Aufbauphase würde sich nun abzeichnen, „wo der Bund steuernd eingreifen muss, damit Lücken geschlossen und Doppelgleisigkeiten vermieden werden. Insbesondere hätten die Kriterien nicht die Funktion erfüllt, es einem potentiellen Erhalter zu ermöglichen, die Chancen eines von inm erwogenen Fachhochschul-Studienganges auf Bundesförderung abzuschätzen." Dadurch sollte das Risiko des Antragstellenden, umfangreiche Vorbereitungsarbeiten umsonst geleistet zu haben, minimiert werden.

Eine Interpretation des damaligen Berichts war, dass "eine Tendenz zu Doppelgleisigkeiten, Schwächen bei der regionalen Abstimmung und ein beschränktes Interesse an neuen Zielgruppen bestehen, weil die Kriterien für die Bundesfinanzierung häufig nicht zur Kenntnis genommen wurden bzw. zu wenig spezifiziert wären, um potentiellen Antragstellenden eine Realisierungseinschätzung einzuräumen.“ 
Die Vorgangsweise, zuerst die Beurteilung der Qualität durch den FHR vorzunehmen und dann die Entscheidung über eine Bundesförderung zu fällen, wurde gewählt, um der Qualitätsprüfung durch den FHR den nötigen Stellenwert zu sichern. Nun kann es sein, dass ein Vorhaben vom FHR positiv beurteilt wird, aber keine Bundesfinanzierung erhält, weil es den Förderungskriterien nicht entspricht. Dadurch wurde die Belastung des FHR durch Anträge, die aus finanziellen Gründen nicht realisierbar sind, erhöht. Der FHR hat daher eine stärkere Koordination mit dem zuständigen Bundesministerium (BMBWK) in dieser Frage vorgeschlagen, die - bereits laut dem Bericht von 1995 - „zu diskutieren sei und von einer Arbeitsgruppe geprüft wird“.

Seit 1998/99 wird daher das Verfahren zur Beurteilung der Förderungswürdigkeit eines beantragten Fachhochschul-Studienganges in etwas modifizierter Weise durchgeführt (vgl. BMBWK 2002, 60): Die bis dahin praktizierte strikte zeitliche Trennung zwischen Qualitätsprüfung (Fachhochschulrat) und Finanzierungsentscheidung (Bundesministerium für Bildung, Wissenschaft und Kultur) wurde dahingehend korrigiert, dass der Fachhochschulrat im Rahmen seiner bildungspolitischen Beratungsfunktion in die Beurteilung der Förderungswürdigkeit der Anträge einbezogen wurde.

In den Empfehlungen des Fachhochschulrates (vgl. FHR 1995) - die den Empfehlungen des FHR-Präsidenten Schelling in Rahmen des Informationstages am 12.6.1995 entsprechen findet sich bereits 1995 folgender Passus: „Der Bund möge bei einer grundsätzlichen Aufrechterhaltung des Prinzips der Initiative von unten für die wichtigsten Berufsfelder die Zahl der in den Großregionen Österreichs (Ost, Süd, West) von inm aus nationalen, regionalen, bildungspolitischen und finanziellen Gründen förderbaren Studiengänge/Studienplätze für die Jahre einer Fünfjahresperiode festlegen." Diese Forderung ist bis heute unerfüllt geblieben.

Entwicklungs- und Finanzierungsplan II - Inhaltliche Kriterien

Kriterien für die Bundesförderung im Entwicklungs- und Finanzierungsplan II sind:

- Abbau regionaler Disparitäten im Hochschulzugang

- Erhöhung der Durchlässigkeit oder Erweiterung des Zugangs für AbsolventInnen des dualen Systems und für Berufstätige

- $\quad$ Strukturbereinigung im Postsekundarbereich

- Innovative Studienangebote

Der Kriterienkatalog zur Vergabe einer Bundesförderung wurde dabei im Vergleich zum ersten Entwicklungs- und Finanzierungsplan stärker ausdifferenziert:

Konsolidierung der Standorte und längerfristiges Entwicklungskonzept

Werden von einem Erhalter Studiengänge an mehreren Orten angeboten oder gibt es in einem Bundesland mehr als einen Erhalter, so ist dies ausführlich zu begründen. Betreibt ein Erhalter Studiengänge an dislozierten Standorten, so ist insbesondere nachzuweisen, dass diese durch eine gemeinsame Verwaltung und weitgehende Überlappung der Lehrkörper verbunden sind. Neue Anbieter haben insbesondere nachzuweisen, dass sie mit einer fachlich einschlägigen Bildungs- bzw. F\&E-Einrichtung oder mit einer sozialen Einrichtung ident sind oder mit einer solchen Einrichtung kooperieren. 


\section{Regionaler Ausgleich}

Das Bundesland, in dem der beantragte Studiengang angesiedelt ist, weist eine unterdurchschnittliche Ausstattung mit Postsekundarangeboten auf und/oder der Anteil der StudienanfängerInnen an der gleichaltrigen Wohnbevölkerung liegt unter dem österreichischen Durchschnitt.

\section{Bildungspolitische Strukturbereinigung}

Das Vorhaben befördert klare Strukturen im Bildungsbereich und bereinigt Doppelgleisigkeiten im nichtuniversitären Sektor.

\section{Förderung nicht-traditioneller Studierender}

Der Studiengang sieht Maßnahmen zur Förderung von Studierenden ohne Hochschulreife vor. Dabei ist der Anteil der angestrebten Beteiligung dieser Personengruppe an der Studierendenschaft anzugeben.

\section{Erhöhung des Frauenanteils}

Der Studiengang sieht Maßnahmen zur Erhöhung des Frauenanteils an den Studierenden und/oder Lehrenden vor, wobei auch hier der angestrebte prozentuelle Anteil von Studentinnen bzw. Lehrerinnen anzugeben ist.

\section{Struktur der Programme}

\section{Credit Systeme}

Der Studiengang ist Teil einer Gruppe von Studiengängen, die in einer Weise aufeinander abgestimmt sind, dass den Studierenden (interdisziplinäre) Wahlmöglichkeiten zur Verfügung stehen.

\section{Beschreibung von Kompetenzen}

Der Antrag enthält eine klare Beschreibung jener Kompetenzen, die in einem Fach bzw. einer Fächergruppe oder innerhalb jedes einzelnen Semesters oder Studienjahres erworben werden. Es handelt sich dabei um Kompetenzen, die arbeitsmarktrelevant sind (employability) und/oder um methodische Kompetenzen, die Weiter- oder Umqualifizierungen ermöglichen (transferability).

\section{Einsatz von Fernstudienelementen}

Der Studiengang sieht den Einsatz von Fernstudienelementen unter Verwendung neuer Technologien, des regionalen Rundfunks oder Fernsehens vor.

\section{Innovativer Charakter der Programme}

Das beantragte Curriculum oder seine organisatorische bzw. didaktische Durchführung zeichnen sich durch die Vermittlung von Kenntnissen, Fähigkeiten und Fertigkeiten aus bzw. ist auf ein Berufsfeld zugeschnitten, in denen bzw. in dem aufgrund des gesellschaftlichen und/oder technologischen Wandels besonderer Qualifikationsbedarf zu erwarten ist.

\section{Beschaffenheit des akademischen und sozialen Umfelds}

Der Studiengang findet in einem Umfeld statt, das akademisch anregend ist (Maßnahmen zur Förderung der Qualität der Ausbildung, wie z.B. internationaler Austausch, GastprofessorInnen, F\&E-Kooperationen unter Einbindung von Studierenden, besondere Begleitung der Praxissemester), soziale Bereicherung verspricht (z.B. kulturelle, sportliche Angebote) und so das Lernen und die Erreichung des Studienzieles unterstützt.

\section{Weiterqualifizierungsmaßnahmen für Lehrende}

Die Auswahl der Lehrenden, die ihnen gebotenen Weiterbildungs- und/oder Forschungsmöglichkeiten und das Entlohnungssystem sind so gestaltet, dass sie qualifizierte Lehre und erfolgreiches Lernen stimulieren.

\section{Veränderungen gegenüber dem Entwicklungs- und Finanzierungsplan I}

Im Entwicklungs- und Finanzierungsplan II finden sich damit folgende Veränderungen gegenüber dem ersten Entwicklungs- und Finanzierungsplan :

Verstärkt wird der Hinweis auf das Ziel, nicht-traditionelle Studierende ohne Hochschulreife anzusprechen. Hier sind von den Antragstellenden genauere Angaben zu machen, wodurch man versucht, dieses Ziel besser zu reichen, denn der Zugang von StudienanfängerInnen ohne Hochschulreife, vor allem mit Lehrabschluss, hätte sich wenig dynamisch entwickelt (er 
betrug zu diesem Zeitpunkt weiterhin nur $10 \%$ und ist seitdem auf $8 \%$ gesunken; vgl. Abschnitt 3.1.3). Allerdings - so wird angemerkt - sei dies nicht nur eine Angelegenheit der Bildungspolitik, sondern in Hinblick auf die Frage der Durchlässigkeit von Bildungssystemen käme auch den Unternehmen und Interessenvertretungen eine bedeutende Rolle zu.

Erstmals findet sich auch explizit der Hinweis auf den Frauenanteil, da der Sektor aufgrund seiner immer noch schmalen Fächerstruktur insgesamt einen sehr geringen Frauenanteil aufweist und damit zwar nicht „männlicher" als thematisch vergleichbare Universitätsstudien ist, aber als Sektor insgesamt wesentlich „männlicher“ ist als der Universitätssektor.

Als neuer Bereich, auf den Augenmerk zu legen ist, kommt das Thema Personalentwicklung hinzu, indem nach der Weiterbildung der Lehrenden gefragt wird - ein Umstand, der darauf hinweist, dass man die Frage der Qualitätssicherung in einer breiteren Dimension und unter dem Aspekt der Professionalisierung von Lehrenden an Fachhochschulinstitutionen - und damit im Hinblick auf die Herausbildung eines eigenen, spezifischen Lehrkörpers - sieht. Ausdrücklich betont werden Fernstudienelemente - in dieser Hinsicht hatte sich der an sich innovative Sektor bis dahin nicht wesentlich anders als die Universitäten und damit nicht sehr offensiv verhalten. Das Potential, das der Einsatz neuer Medien gerade im Bereich der Durchlässigkeit des Bildungsbereiches, des Abbaus regionaler Disparitäten, der Erreichbarkeit neuer Bildungsschichten hat, sollte dadurch betont werden.

Die Frage der Innovativität wird im Entwicklungs- und Finanzierungsplan II stärker ausdifferenziert, indem man einen besonderen Qualifikationsbedarf, der aus gesellschaftlichen oder beruflichen Gründen zu erwarten ist, begründen muss. Insbesondere wird auch explizit die Beschreibung von Kompetenzen abverlangt (die insbesondere um die Begriffe der „employability“ einerseits und der „transferability“ andererseits kreisen).

Erstmals wird der ganze Themenkomplex der Konsolidierung der Standorte angesprochen. Es wird festgestellt, dass der Fachhochschulsektor tatsächlich dazu beigetragen hat, regionale Disparitäten im Hochschulzugang abzubauen (vgl. auch Abschnitt 5.2.1). Andererseits wird betont, dass der Regionalisierung von Hochschuleinrichtungen eine Grenze gesetzt ist, da das soziokulturelle und akademische Umfeld auch tatsächlich für einen Studiengang geeignet sein muss. Wenn man diese nicht beachten würde, so würde dies zu Einrichtungen von geringerer Qualität führen durch eine Zersplitterung von Ressourcen (BMWV 1999, 7).

Der Entwicklungs- und Finanzierungsplan II kommt auch in anderen Bereichen zu kritischen Einschätzungen: „Kaum erreicht wurde bislang das Ziel einer Strukturbereinigung im Postsekundarbereich." Auch der FHR hatte wiederholt die Ausklammerung des sozialen und nicht-ärztlichen medizinischen Bereiches kritisiert. Im Entwicklungs- und Finanzierungsplan II wird angemerkt, dass hinsichtlich der nicht-ärztlichen Gesundheitsberufe Gespräche mit dem für die Ausbildung in diesem Bereich zuständigen 
Bundesministerium für Arbeit, Gesundheit und Soziales aufgenommen wurden. „Eine Neustrukturierung der Ausbildung im Bereich der gehobenen medizinisch-technischen Dienste und Hebammen wirft aus Gründen der Finanzierungsverantwortung verschiedener Gebietskörperschaften (Bund, Länder, Gemeinden) komplexere Probleme auf, sodass vorerst Überlegungen über mögliche Reformszenarien anzustellen sind." (ebda, 11).

Mittlerweile hatte sich auch herausgestellt, dass das Anliegen einer Entlastung des Universitätssektors und die Abstimmung mit universitären Angeboten differenzierter betrachtet werden musste als anfangs gedacht. Ein Grund dafür ist etwa, dass der Fachhochschulsektor erstens klein ist und zweites gerade etwa bei den technischen Studiengängen auf ein Fächerspektrum beschränkt ist, das an den meisten Universitäten keine Überlastung aufweist (vgl. auch Abschnitt 3.1.3 c) zur Entlastung der Universitäten). Weiters müsse man sich die Überlastung der Universitäten genauer ansehen, wo sie tatsächlich in welcher Weise gegeben sei und welche Konsequenzen man daraus ziehen würde; so gab es auch Argumente, die betonten, man sollte gerade bei überlaufenen Studienrichtungen nicht noch weitere Angebote - etwa im FH-Sektor - schaffen, weil dann die Studierenden in wenig zukunftsträchtige, überlaufene Berufsfelder gedrängt würden.

Im Gegensatz zum Entwicklungs- und Finanzierungsplan I wird das Kriterium „Private Mitfinanzierung" - vermutlich aus Gründen der nunmehr empirisch feststellbaren und nicht nur vermuteten geringen privaten Beteiligung an der Finanzierung des Sektors - nicht mehr gesondert thematisiert.

Ebenso sind internationale Kooperationen nicht mehr als eigenes Kriterium angeführt.

\subsection{Zusammenfassung und Schlussfolgerungen}

\subsubsection{Zusammenfassung}

Zu den Stärken der beiden bislang erstellten Entwicklungs- und Finanzierungspläne ist zu zählen, dass sie im bildungspolitischen Kontext Österreichs ein Novum dargestellt haben. Die Formulierung eines Rahmenplans, in dem der Bund tatsächlich seine Pläne für diesen Sektor für die nächsten 5 Jahren und vor allem auch die dazugehörigen Ausbau- und Finanzierungspläne offen legt, war ein wichtiges Element für die innovative Entwicklung dieses Sektors und sicher vorbildlich für andere Bildungssektoren. Mittlerweile liegen bereits zwei Entwicklungs- und Finanzierungspläne vor und man kann sagen, dass die darin enthaltenen Finanzierungs- und Ausbaupläne tatsächlich eingehalten, sogar etwas überschritten wurden. Das hat eine erfolgreiche Entwicklung des Fachhochschulsektors ermöglicht. 
Der zweite positive Effekt war, dass die institutionellen Lernprozesse, die der erste Entwicklungs- und Finanzierungsplan noch stark thematisiert hat, schneller erfolgten, als viele zu Beginn der Etablierung des Sektors glaubten. Der Entwicklungs- und Finanzierungsplan I ging davon aus, „dass dieses Reformkonzept eine Kombination wissenschaftlicher, pädagogischer und administrativer Fähigkeiten erfordert, wie sie in Österreich keine Tradition besitzt. Um den gestalterischen Freiraum kreativ zu nutzen, den das Gesetz für die anbietenden Institutionen öffnet, sind Lernprozesse nötig, die nicht über Nacht erwartet werden können“ (BMWF 1994, 308).

Selbst ursprüngliche Skeptikerlnnen gegenüber dieser neuen, marktwirtschaftlich orientierten Bildungsorganisationsphilosophie geben heute zu, dass ein erfolgreicher Einstieg in ein neues Steuerungskonzept gelungen ist und dass neue aktive Akteure im Bildungssystem etabliert werden konnten. Das anfangs umstrittene Normkostenmodell hat ebenfalls zu dieser Ausdifferenzierung der Akteure beigetragen. Die Länder haben erstmals eine aktive Rolle übernommen. Mit den Erhaltern und den Leiterlnnen der Studiengänge sind unternehmerische Persönlichkeiten in den Bereich des Bildungsmanagements eingestiegen, einer Profession, die es bis dahin in Österreich kaum gegeben hatte - es dominierte die Bildungsverwaltung vor dem Bildungsmanagement. Das „bottom-up“-Modell und die damit entfesselte unternehmerische Initiative hat auch ein selbst- und medienbewusstes Auftreten des Sektors mit sich gebracht sowie ein aktives Bemühen um die Studierenden. Der Fachhochschulsektor war von Beginn an durch eine dynamische Grundstimmung - teilweise auch die „Euphorie des Neubeginns“ - gekennzeichnet.

Gleichzeitig war man sich der Innovationsrolle für den gesamten tertiären Sektor bewusst und hat sich auch durchaus selbstkritisch und reflexiv in Form von Tagungen und Studien mit der eigenen Entwicklung auseinandergesetzt. Dieser wichtige Aspekt der Qualitätssicherung des Gesamtsektors ist dabei stark durch die Aktivitäten des Fachhochschulrates, aber auch der Fachhochschul-Konferenz beachtet und eingefordert worden.

Die für den Bildungssektor neue Form der Steuerung über das Steuerungsmittel „Geld“ hat vergleichsweise - gut funktioniert. Bei aller anfänglichen Kontroverse über die Art und Höhe der Finanzierung hat ein klares Finanzierungsmodell einen wesentlichen Beitrag zur Entwicklung des Sektors geleistet. Das im Entwicklungs- und Finanzierungsplan I genannte Ziel, die Finanzierung als bildungspolitischen Lenkungsmechanismus einzusetzen, ist erfolgreich umgesetzt worden.

Die Schwächen der Entwicklungs- und Finanzierungsplanung sind in den folgenden Bereichen zu sehen:

Die bildungspolitische Rolle des Dokuments „Entwicklungs- und Finanzierungsplanung“ ist nicht sehr stark "gepflegt“ worden. Die Formulierung der bildungspolitischen Ziele ist eigentlich nur in relativ vager und nicht wirklich operationalisierter Form erfolgt. Es wurden 
dabei Kriterien festgelegt, die an den einzelnen Studiengang angelegt werden, damit aber nicht automatisch auch Kriterien für die Entwicklung des gesamten Sektors sind. Die "Kleinteiligkeit" der Maßnahmen wie z.B. studiengangsbezogene Bedarfs- und Akzeptanzstudien, Akkreditierungen/Evaluierungen, Finanzierungen und eine gewisse Nischenpolitik ist bei allem Erfolg des „bottom-up“-Modells ein Problem. Wenn man Bedarfsund Akzeptanzanalysen nur pro Studiengang unternimmt, geraten größere Ausschnitte (Region, Land, Fächer- und Quervergleiche, bildungspolitischer Sektor) aus dem Blickwinkel.

Die Überprüfung der Einhaltung der bildungspolitischen Kriterien ist von Beginn an relativ schwach ausgeprägt gewesen und mit den Jahren immer schwächer geworden, wodurch sie in ihrer Steuerungsfunktion stark eingeschränkt waren. Die entscheidende Schwäche war damit die zu gering operationalisierte Zielvorgabe und die mangelnde Überwachung der Zieleinhaltung im Sinne eines Monitoring der Zielvorgaben.

Damit krankt auch der österreichische Fachhochschulsektor an der grundsätzlichen Schwierigkeit des New Public Management (NPM). Die Politik soll sich - laut NPM - von der operativen Ebene verabschieden, um eine strategische Steuerung über Ziele und den Aufbau eines Controllings vorzunehmen. Das politisch-administrative System darf sich nicht darauf beschränken, sich durch einen Rückzug aus der bisherigen Detailsteuerung selbst zu entlasten, sondern muss neue, inm bislang unbekannte Aufgaben in der strategischen Entwicklung des tertiären Sektors übernehmen.

Die Bildungspolitik muss mittel- bis langfristige Entwicklungsziele für den tertiären Bereich erarbeiten: hierzu gehören Themen wie der quantitative Ausbau, die angestrebte AkademikerInnenquote, der offene Hochschulzugang und eine Finanzierung, die das quantitative und qualitative Verhältnis etwa zwischen Fachhochschuleinrichtungen und Universitäten im Blick hat. Die Erreichung der politischen Ziele muss evaluiert werden. Erreichen wir tatsächlich eine Verbesserung in Wirtschaft und Gesellschaft mit den Leistungen der Hochschulen? Worin liegen die Ursachen von Abweichungen? Damit diese politischen Aufgaben erfüllt werden können, muss die Ministerialverwaltung die erforderlichen Vorarbeiten leisten - sie hat eine Art Übersetzerrolle zwischen Politik und Management (vgl. Schedler/Pröller 2000, 53). Und schließlich muss sie ein strategisches Controlling aufbauen; damit wird das laufende Monitoring und das Steuern der Entwicklung ermöglicht.

Die Gestaltung jener Prozesse, die eine Einigung über die Ziele und die grundlegenden Muster ihrer Umsetzung herstellen können, ist in allen Ländern besonders schwierig. Es geht um die Frage, welches Steuerungsmodell die Konsensbildung bei Akteuren der verschiedenen Aggregationsebenen unterstützt, aber gleichzeitig auch autonome Spielräume bestehen lässt, um Dinge auszuprobieren (vgl. Lassnigg 2000, 30). Das ist auch das Thema der "Good Governance“-Debatte, die sich als Weiterentwicklung des New Public 
Management auf europäischer Ebene herauszukristallisieren beginnt. Das Konzept des "Good Governance“ beschreibt die Suche nach neuen Impulsen und Methoden in der Verwaltungsreform und eine Weiterentwicklung des NPM in Richtung eines sozialen und politischen Reforminstruments (vgl. Löffler 2002, 212). Ideen des "Good Governance" gewinnen insbesondere auch auf der Ebene der europäischen Bildungspolitik an Gewicht (vgl. einschlägige OECD-Publikationen der letzten Zeit) und sind dadurch ein wichtiger Kontextfaktor für erfolgreiche nationale Politiken.

Eine besondere Rolle spielen institutionelle Netzwerke und Aushandlungskonzepte zwischen Akteuren unterschiedlicher Bereiche (z.B. aus dem öffentlichen, dem privaten, dem gemeinnützigen Sektor, Medien etc.). Die Entwicklung der kooperativen Beziehungen zwischen den Hochschulen und anderen Organisationen im Unternehmens-, aber auch im Kommunal- und Sozialbereich ist ein wichtiger Aspekt. Die Konstruktion von organisatorischen Formen, etwa entsprechend den Pufferorganisationen des „Evaluative State", ist ein entscheidendes Element in diesem Prozess. Diesen Organisationen Gewicht und Verbindlichkeit zu geben, ohne ihre produktive Kapazität einzuschränken, ist vielleicht das entscheidende Problem für eine funktionsfähige Steuerungsstruktur. Zwischen die politisch Verantwortlichen und die einzelnen Hochschullehrenden schieben sich damit viele neue professionelle Funktionen, die zu einer kooperativen Arbeitsteilung finden müssen: AdministratorInnen, ManagerInnen, ForscherInnen, EvaluatorInnen, BeraterInnen.

Der Aufbau und die Weiterentwicklung einer Informations- und Wissensbasis sowie ein möglichst breiter Zugang zu dieser (Transparenz) ist zur Unterstützung dieser Formen der Steuerung unerlässlich.

Zu den strategischen Zielvorgaben der Bildungspolitik würde insbesondere auch die tatsächliche Verwirklichung der Strukturbereinigung des postsekundären Bereichs, die von Anfang an explizites Ziel der Fachhochschulgesetzgebung war, gehören. Hier sind politische Entscheidungen hinsichtlich des medizinisch-technischen Bereichs erforderlich. Dieser Mangel an ausformulierter Bildungspolitik hat dazu geführt, dass der Fachhochschulrat quasi in dieses Vakuum gesprungen ist, indem er durch die Ablehnung oder Genehmigung von Anträgen implizit bildungspolitische Entscheidungen getroffen hat und in einer weiten Interpretation seiner Aufgabe der Qualitätssicherung auch zum wichtigen bildungspolitischen Akteur im Fachhochschulbereich wurde. Die Weisungsfreiheit des Fachhochschulrates suggeriert dann eine reine Fachentscheidung. Das grundsätzliche Spannungsfeld, das dadurch entsteht, dass die Qualitätskontrolle einer eigens zu diesem Zweck eingerichteten Behörde, dem FHR, übertragen ist, andererseits aber die Entscheidung über die studienplatzbezogene Bundesfinanzierung eines vom FHR positiv beurteilten Antrag dem zuständigen Bundesminister bzw. der zuständigen Bundesministerin unterliegt, ist auf diese Weise verschärft worden. 
Eine neue strategische Rolle des Bundesministeriums und transparente Aussagen der Bildungspolitik sind sicher jene Bereiche, die für die Weiterentwicklung des Fachhochschulsektors von immenser Bedeutung sind. Allerdings ist das wie in allen anderen europäischen Ländern ein äußerst schwieriger Prozess, auch weil die Ebene der nationalen Politik von zwei Seiten unter Druck gerät: zum einen durch die Abgabe von Kompetenzen an die dezentralen Ebenen (institutionelle Autonomie) oder im Fachhochschulbereich auch an neue Mitakteure wie Länder und Gemeinden; zum anderen ist Bildungspolitik auch zunehmend durch supranationale Impulse (Bologna-Erklärung, Europäischer Hochschulraum, Europäischer Forschungsraum etc.) geprägt. Sich hier zu verorten und zwischen diesen beiden Ebenen einen Ausgleich zu finden, einen steuernden Rahmen zu gestalten, ohne in die alte Euphorie der Bildungsplanung und des „man-power-planning“ zu verfallen, ist ein äußerst schwieriges Unterfangen.

\subsubsection{Schlussfolgerungen}

\section{Entwicklung der Zieldimension}

Das drängendste Erfordernis ist die Weiterentwicklung der strategischen bildungspolitischen Zielvorgaben. Gerade in diesem Bereich, der zu den auch im internationalen Vergleich besonders sensiblen Bereichen der modernen Bildungspolitik gehört, könnte der Fachhochschulsektor seine Innovatorenrolle für das Bildungssystem erneut und mit einer gewissen Fokusverschiebung unter Beweis stellen.

Der nächste Entwicklungs- und Finanzierungsplan sollte Annahmen der Politik über die weitere Entwicklung der Partizipationsraten im Sinne des Übertritts in den tertiären Bereich enthalten. Das ist eine wichtige Hintergrundinformation und bringt auch bildungspolitische Zielsetzungen zum Ausdruck, die für die handelnden Akteure im Feld wichtig sind. Der Plan sollte Aussagen enthalten über die Absichten von politischer Seite hinsichtlich Größe, Wachstumsraten und Finanzierung der Fachhochschuleinrichtungen, insbesondere den Beitrag des Bundes zu dieser Finanzierung. Die Bildungspolitik sollte ihre Absichten darlegen, welche Institutionen künftig in den Fachhochschulsektor integriert werden sollen. Ebenso wichtig ist eine bildungspolitische Aussage zur drängenden Frage der Konsolidierung vs. Regionalisierung und auch zum Profil der Fachhochschulinstitutionen, ob es etwa notwendig ist, unterschiedliche Disziplinen in einer Institution zu kombinieren. Einer der wichtigsten Aspekte ist es, der Herausbildung betriebswirtschaftlich lebensfähiger Einrichtungen mehr Gewicht zu verleihen. Schließlich muss das Verhältnis zwischen Fachhochschulsektor und den übrigen nicht-universitären postsekundären Einrichtungen im Sozial-, Schul- und Gesundheitsbereich geklärt werden. 
Die in den ExpertInneninterviews geäußerte zentrale Erwartung an den nächsten Entwicklungs- und Finanzierungsplan ist, dass er die finanziellen Rahmenbedingungen für die weitere Entwicklung des FH-Sektors klar absteckt. Ein Sektor mit derartig viel Bewegung, unternehmerischer Initiative und „bottom-up“-Entwicklung braucht einen stabilen Rahmen. Dazu gehören in erster Linie die vom Bund vorgesehenen finanziellen Mittel und damit indirekt die geplante Ausbaugeschwindigkeit. Es herrscht im Moment eine gewisse Unruhe im Fachhochschulsektor, dass durch einen zu raschen Ausbau das Qualitätsniveau nicht gehalten werden kann und dass durch eine Finanzierung per Studiengang eine zu starke Zersplitterung der $\mathrm{FH}$-Landschaft einsetzt.

Das Thema der weiteren Expansion ist unmittelbar damit verbunden, welches Fächerspektrum in Zukunft im FH-Sektor vertreten sein wird. Im Moment gibt es eine große Dominanz der technischen und wirtschaftlichen Studienrichtungen. Die Auflage für neue Studiengänge, dass es sich um ein neues, noch nicht vorhandenes Studienangebot handeln muss, treibt die Antragstellenden in eine gewisse Nischenpolitik. Es fragt sich, ob man diese Bestimmung etwas öffnet zugunsten breiterer Ausbildungsfelder. Das würde auch die internationalen Entwicklungstendenzen widerspiegeln - so spricht sich etwa auch der deutsche Wissenschaftsrat in seinen Empfehlungen zur Entwicklung der Fachhochschulen (WR 2002) sehr deutlich für eine Erweiterung des Fächerspektrums aus.

Die Rolle des Innovators könnte der FH-Sektor insbesondere auch in der Frage der Erstellung von Weiterbildungsangeboten einnehmen und hier wiederum durch innovative Modelle der Kombination von Präsenz- und Fernlehre hervortreten. Gerade im Bereich der innovativen Regionalentwicklung und der Weiterbildung von Berufstätigen kommt dem FHSektor eine zentrale Rolle zu. Selbst wenn die Universitäten sich hier verstärkt engagieren sollten, könnte die Innovatorenrolle des Fachhochschulsektors besonders deutlich sein.

Der Bund sollte den derzeitigen Kriterienkatalog des Entwicklungs- und Finanzierungsplans um ein mittelfristiges Schwerpunktprogramm für die künftige Förderpolitik ergänzen. Es hat sich etwa am Kriterium der Durchlässigkeit gezeigt, dass Kriterien, die an einzelne Studiengänge angelegt werden und auch nur zu 50\% erfüllt werden müssen, nicht geeignet sind, quasi inkrementalistisch eine einheitliche Gesamtpolitik entstehen zu lassen. Die bildungspolitischen Vorgaben müssen nicht nur operationalisiert und einem genaueren Monitoring unterworfen werden, sondern wahrscheinlich durch eigene Förderprogramme betont werden. Die einzelnen Kriterien sollten auch nach Dringlichkeit gewichtet und Prioritäten formuliert werden. Wenn etwa auch die Antragstellenden Maßnahmenkataloge vorlegen müssen, wie sie diese Prioritäten erreichen wollen, könnten diese eine noch intensivere Steuerungswirkung entfalten. 


\section{Adäquate Organisationsformen zur Zielbildung}

Es sollten vermehrt adäquate Organisations- und Kommunikationsformen, in denen die künftigen bildungspolitischen Rahmenbedingungen für den Fachhochschulsektor konsensuell entwickelt werden können, geschaffen werden.

- Damit das Wissenschaftsressort seine bildungspolitische Rolle im Sinne der Entwicklung strategischer Zielvorstellungen und ihrer Operationalisierung besser entwickeln kann, ist auch dort mehr administrative, professionelle Planungskapazität als derzeit erforderlich. Es könnte sinnvoll sein, wenn der im Universitätssektor im Entstehen begriffene Wissenschaftsrat seine Aktivitäten als strategisch-politisches Beratungsorgan auf den gesamten tertiären Ausbildungsraum erstrecken würde und damit als wissenschaftspolitisches Expertengremium internationale Entwicklungen aufgreifen und nationale Zielvorstellungen bündeln würde.

- Weiters müssen die Länder eine explizite Rolle als bildungspolitische Akteure erhalten. Bislang fehlt eine adäquate Institutionalisierung ihrer wichtigen Rolle. Ihre Einbindung in die Planung der weiteren Entwicklung ist ungeklärt. Es muss einen regelmäßigen Meinungsaustausch zwischen Bund und Ländern geben, damit die jeweiligen Planungen einander nicht konterkarieren, sondern sinnvoll aufeinander abgestimmt werden können.

- Fachhochschulrat und Fachhochschul-Konferenz als Gremien, die viel Know-how und Engagement in die Entwicklung des Fachhochschulsektors investiert haben, müssen genauso in entsprechende Planungsüberlegungen einbezogen werden. Auch das für eine qualitätsvolle Weiterentwicklung des Sektors notwendige gute Zusammenspiel zwischen zuständigem Bundesministerium und Fachhochschulrat bedarf regelmäßiger Foren der Kommunikation, des Austausches und der Absprache.

- Von besonderer Bedeutung ist der Aufbau einer breiten Informations- und Wissensbasis. Der möglichst breite Zugang zu dieser ist im Sinne der Transparenz ein wichtiger Aspekt dieser kooperativen Steuerungsformen. Auch in Bezug auf die Finanzierungsentscheidungen des Bundes und der Länder empfiehlt sich eine transparente Vorgangsweise.

\section{Gesamtbetrachtung des tertiären Sektors}

Der gewichtigste Mangel in der Fachhochschulpolitik des Bundes besteht darin, dass das Instrumentarium zur Gesamtkoordination nur ungenügend ausgebildet ist. Insbesondere erscheint eine bildungspolitische Gesamtkoordination im tertiären Sektor dringend notwendig. 
Der Bund ist vor allem für die Herstellung gleicher Rahmenbedingungen und für die Strukturbereinigung zuständig. Die Verbesserung der Kohärenz des Bildungssystems verlangt einen breiten bildungspolitischen Willensbildungsprozess. Dafür müsste der Bund zunächst bekannt geben, welche Sparten, Berufsfelder und Ausbildungen seiner Ansicht nach in welchen Sektoren (Universität, Fachhochschulbereich, Akademien) angesiedelt sein sollten.

Vor dem Hintergrund einer demnächst wesentlich veränderten Universitätslandschaft stellt sich die Frage, ob man die bildungspolitische Steuerung dieser beiden Bereiche nicht auf ähnliche Grundlagen stellen könnte: im Universitätsbereich fehlt ein ähnliches Instrument wie ein auf 5 Jahre gültiger Entwicklungs- und Finanzierungsplan und für den Fachhochschulbereich erscheint das Instrument der Ziel- und Leistungsvereinbarungen, das nun im Universitätsbereich eingeführt werden soll, durchaus von Interesse. Dieses Steuerungsinstrument betrifft die Beziehung zwischen staatlicher und institutioneller Ebene. Im Fall des Fachhochschulsektors müsste dieses Instrument allerdings im Verhältnis BundLänder-Fachhochschulinstitution eingesetzt werden und es hätte auch nur einen Sinn im Fall des Vorhandenseins von einigermaßen großen Fachhochschuleinrichtungen, die dadurch aber wiederum eine größere institutionelle Bewegungsfreiheit bekommen könnten als derzeit. Im Moment wird - auch wenn die Zahl der Fachhochschulen zugenommen hat nach wie vor vorwiegend auf der Ebene von Studiengängen geplant und gemanagt; es werden auch einzelne Studiengänge akkreditiert. Es stellt sich die Frage, ob man nicht einen Erhalter bzw. eine Institution akkreditieren sollte, um diesem/dieser dann einen gewissen Spielraum zur genauen Ausgestaltung ihrer Studienplätze zu geben.

Der nächste Entwicklungsplan soll - so der Wunsch vieler Interviewpartnerlnnen - stabile Rahmenbedingungen vorgeben und hiefür ist es wichtig, dass man die gesamte Entwicklung des tertiären Sektors in den Blick nimmt. Durch die neue Universitätsgesetzgebung erhalten auch die Universitäten höhere institutionelle Autonomie und werden sich in Zukunft unternehmerischer verhalten. Die Konkurrenz mit den Universitäten wird aus diesem Grund stärker werden. Auch haben die Fachhochschulinstitutionen eine entsprechende Ausbaustufe erreicht, sodass ein weiteres Wachstum wahrscheinlich den Wettbewerb mit den Universitäten stärker thematisiert.

Ein weiteres Moment der Konvergenz zwischen Fachhochschulsektor und Universitäten ist neben dem Organisationsrecht die Tatsache, dass nun sowohl Universitäten als auch Fachhochschuleinrichtungen Bachelor- und Masterstudien anbieten, d.h. aus der Art des Abschlusses erkennt man keine Spezifika des Fachhochschul- oder Universitätssektors und es werden sich spezielle Profile von jeweils einschlägigen Bachelor- und Masterstudien herausbilden müssen. Dafür ist es aber besonders wichtig, dass die Rahmenbedingungen für die Institutionen des gesamten tertiären Sektors (von den Privatuniversitäten über die staatlichen Universitäten bis zu den Fachhochschuleinrichtungen) einigermaßen einheitlich und klar geregelt sind. 
Wenn z.B. universitäre Weiterbildungsangebote anderen Spielregeln unterliegen (z.B. die Pflicht zur Kostendeckung, aber andererseits keine zwingende Vorlage von Bedarfs- und Akzeptanzstudien) als weiterbildende Studienangebote im Fachhochschulbereich, wenn die Fachhochschuleinrichtungen eine Auswahl ihrer Studierenden vornehmen dürfen, Universitäten aber nicht und wenn die Methoden der Qualitätssicherung jeweils ganz anders geregelt sind, so sind dies keine günstigen Ausgangsbedingungen, um ein modernes, differenziertes, durchlässiges und wettbewerbsorientiertes Hochschulsystem entstehen zu lassen.

Die Internationalisierung durch den Bologna-Prozess und die neue Gesetzgebung für die Universitäten könnten zwei Gründe sein, warum die ursprüngliche Intention, den Fachhochschulsektor als ganz eigenen Bereich des tertiären Sektors aufzusetzen, vielleicht nicht mehr zeitgemäß ist und die zukunftsträchtigere Entwicklungsperspektive nun in Richtung Konvergenz weist.

Daher sollte man die Strategie unterstützen, das Fachhochschulmodell auch als Instrument der Weiterentwicklung von bestehenden Bildungseinrichtungen zu nützen und nicht nur auf Innovation durch Neugründung zu setzen. Dies könnte zum Beispiel in der Form erfolgen, dass bestehende Bildungseinrichtungen die Trägerschaft von Fachhochschul-Studiengängen übernehmen.

Es wird also innerhalb eines großen tertiären Sektors zur Differenzierung von unterschiedlichen Profilen kommen und deswegen scheint es notwendig, dass für die weitere Entwicklungsplanung der Gesamtsektor betrachtet wird. 


\section{Konzentration, Regionalisierung und Standorte im FH- Sektor}

\subsection{Der FH-Sektor im Kontext regionaler Strukturen}

\subsubsection{Entwicklung in der Spannung zwischen Regionalisierung und Konzentration}

Die dezentrale Entwicklung von neuen Bildungsangeboten und als eine Dimension der Dezentralisierung die regionale Verteilung der Bildungsstätten, war von Anfang an ein wesentlicher Bestimmungsfaktor im FH-Bereich. Seit der Gründung des FH-Sektors hat die Spannung zwischen Regionalisierung und Konzentration der Standorte eine Rolle gespielt. In den letzten Jahren hat dieses Thema an Bedeutung gewonnen und es wird heute als eine entscheidende Entwicklungsfrage gesehen. Einerseits betonen bestimmte Akteure die Konsolidierung von Standorten und den institutionellen Aufbau von Fachhochschulen, während andererseits vor allem von regionalen und lokalen Akteuren viele Vorschläge für neue dezentralisierte Standorte vorliegen. Viele dieser Vorschläge werden bereits seit Jahren, teilweise seit dem Beginn des Aufbaus des FH-Sektors, mehr oder weniger intensiv verfolgt. Teilweise werden diese Interessen politisch artikuliert, teilweise wurden im Laufe der Zeit auch systematische Verfahren der Standortbewertung entwickelt und eingesetzt.

In den letzten Jahren haben sich in der Standortfrage neue Festlegungen und Entwicklungen ergeben, die bis zu einem gewissen Grad widersprüchlich und konfliktbehaftet sind. Vor allem seitens des FHR, aber auch anderer wichtiger Akteure wird die Notwendigkeit der Konsolidierung von Standorten bzw. Erhaltern stark betont; im Zuge der raschen zusätzlichen Expansion in den Jahren 2001 und 2002 wurde aber auch die weitere Regionalisierung vorangetrieben. Systematische Verfahren der Standortprüfung bzw. planung wurden den Entscheidungen nicht oder bestenfalls teilweise zugrundegelegt bzw. können von der realen Entwicklung überholt werden. Es stellt sich die Frage, in welcher Form die politische Entscheidung über die Standortentwicklung sachlich und durch systematische Verfahren untermauert werden soll bzw. kann.

Das Profil des Sektors hängt mit der Größe und regionalen Verteilung von Fachhochschuleinrichtungen eng zusammen. In der Entwicklung der regionalen Verteilung der Standorte verknüpfen sich die Aspekte

- des weiteren Größenwachstums des Sektors insgesamt (je größer der Sektor, je mehr und größere FH-Einrichtungen kann es im Prinzip geben; mit dem Größenwachstum erweitert sich gleichzeitig der „regionale Entscheidungsraum“, indem eine breitere Streuung möglich wird), 
- mit dem Potential der einzelnen Institutionen für ihre Aufgabenerfüllung innerhalb des nationalen und übernationalen Bildungs- und Forschungssektors und auch gegenüber der regionalen Umgebung (welche „kritische Größe“ wird in welcher Form und mit welchen Aufgabenverteilungen angestrebt?),

- und den Interessen und Einflussmöglichkeiten der verschiedenen Akteure auf den verschiedenen Aggregationsebenen (lokal, regional, überregional, Politik, Administration, Wirtschaft, Bildung, Forschung).

Die Entscheidung, den FH-Sektor in Form der Akkreditierung von neuen Institutionen in einem „bottom-up“-Prozess aufzubauen, hat die Entstehung von neuen dezentralen Anbieterstrukturen im Bildungswesen induziert. In der privatwirtschaftlichen Form haben sich jedoch mit wenigen Ausnahmen öffentliche Akteure, vor allem Gebietskörperschaften und Interessenvertretungen engagiert. ${ }^{144}$ Die gegenwärtige Struktur ist daher in einem dezentralen komplexen Zusammenwirken von Antragstellenden, Förderern auf regionaler bzw. überregionaler Ebene, Qualitätsprüfung und nationaler Förderungsentscheidung entstanden. In diesen verschiedenen Stadien wirken wiederum verschiedene Akteure aus dem Bildungswesen, aus Wirtschaft, Politik und Verwaltung auf den verschiedenen Ebenen zusammen. Diese Zusammenarbeit hat vielfältige Formen angenommen (Planungs- und Entscheidungsvorbereitungsgremien auf verschiedenen Ebenen, Zusammenführung von unterschiedlichen Akteuren in den Erhalterkonstruktionen; unterschiedliche Anbindungen an andere Politikbereiche und strategische Organe wie z.B. im Rahmen von Technologiekonzepten, Innovationsstrategien etc.), teilweise sind auch Forschungsarbeiten involviert.

Die tatsächliche Entwicklung regionaler Strukturen ist also in hohem Maße ungeplant, in emergenten Prozessen verlaufen, wobei bestimmte Vorgaben strukturierend gewirkt haben:

- In der gesetzlichen Basis ist die regionale Dimension indirekt über die Qualität der Fachhochschuleinrichtungen angesprochen. De facto besteht aber auch im Rahmen der Spezifikationen des FHStG ein weiter Spielraum für die nähere Interpretation dessen, was als qualitätsvoller Hochschulstandort anzusehen ist. Auch in Lichte internationaler Vergleiche ist die Streuung der Größe hochschulischer Institutionen ziemlich weit, wenn auch in den meisten Ländern von bedeutend größeren Durchschnittswerten ausgegangen wird als in Österreich. Überdies haben einige Länder systematische Anstrengungen zur Schaffung größerer Institutionen im Laufe der letzten Jahrzehnte gemacht, z.B. über „,merger“-Prozesse in den 1980er Jahren in den Niederlanden und in

144 Dies schließt jedoch nicht aus, dass sich im Zuge der Entwicklung des "world education market" und im Zusammenhang mit der Virtualisierung potente multinationale Anbieter engagieren. Dafür wird die Größe des Marktpotentials in Österreich vermutlich eine Rolle spielen. 
Australien $^{145}$. In den Niederlanden wurde ein solcher Prozess in den 1990er Jahren auch auf der Sekundarebene über die Schaffung Regionaler Bildungszentren (ROCs) durchgeführt und die Schweiz ist gegenwärtig dabei, ein Fachhochschulsystem über die Reorganisation von postsekundären Institutionen aufzubauen (vgl. auch Abschnitt 2.3). Im Hochschulwesen ist aber die Größe der Institution oft nur sehr lose mit der Größe der Einheiten korreliert, in denen tatsächlich die Leistungserstellung stattfindet. Dies lässt sich am Beispiel der österreichischen Universitäten leicht zeigen, die insgesamt hohe Studierendenzahlen haben, wo jedoch die Studienrichtungen an den Standorten meistens sehr klein sind. ${ }^{146}$ Für die deutschen Fachhochschulen werden keine allgemein gültigen Zielgrößen angegeben, indirekt wird jedoch von Größenordnungen mit 3.000 bis 5.000 flächenbezogenen Studienplätzen gesprochen und kritisiert, dass die früher angestrebten Mindestgrößen von 1.000 Studienplätzen vielfach zur Regelgröße geworden sind (WR 2002, 88-89).

- Im ersten Entwicklungs- und Finanzierungsplan wurde ein grundsätzliches Profil einer Fachhochschule konzipiert, mit mindestens 1.000 Studierenden aus dem regionalen Einzugsgebiet, mehreren sinnvoll aufeinander abgestimmten Studienrichtungen, einem längerfristig lebensfähigem Fächerspektrum; es wurden auch einzelne Studiengänge, die in anderen Institutionen integriert sind, in den Kriterienkatalog aufgenommen ( $\mathrm{vgl}$. Abschnitt 4.2.3). Als weitere regionalspezifische Kriterien wurden die überregionale Abstimmung (sinnvolle Ergänzung anderer Angebote und keine Doppelgleisigkeiten) sowie der Abbau regionaler Disparitäten (gemessen an unterdurchschnittlichen Hochschulangeboten) festgelegt. Regionen sind nicht direkt (etwa in Form von Gebietskörperschaften) spezifiziert, als Maßstab für die Förderentscheidung wurden jedoch dann de facto die Bundesländer herangezogen, die sich als die wesentlichen regionalen Einheiten angeboten haben. Im zweiten Entwicklungs- und Finanzierungsplan werden die Grenzen der Regionalisierung stärker betont (genügend große Zahl an StudieninteressentInnen im erpendelbaren Einzugsgebiet für eine akademisch und ökonomisch vitale Einrichtung) und es sind einige Kriterien neu definiert worden, die ein Mindestmaß an Ressourcen erfordern und eher in größeren Einheiten verwirklicht werden können (Weiterbildung der Lehrenden, Credits in der Programmstruktur, Fernstudienelemente) oder ein entsprechendes akademisches und sozio-kulturelles Umfeld erfordern (vgl. Abschnitt 4.2.3). Ein spezieller Aspekt des Profils einer

145 Goedegebuure/Meek (1992) beschreiben diesen Prozess für die Niederlande sehr klar und eindrücklich (vgl. auch Abschnitt 2.3). In Australien wurde beginnend mit 1988 ein „unified system“ aufgebaut, wobei die Finanzierungsregelungen gestuft auf Größenordnungen von 2.000 (Mindesterfordernis für Bundesförderung der Lehre), 5.000 (Lehre und wenig spezialisierte Forschung) und 8.000 (volle Lehre und Forschung) Studierende ausgerichtet wurden. Es wurde davon ausgegangen, dass die große Mehrheit der Institutionen sich dem „merger“-Prozess anschließen würde (die Zahl der ursprünglich 71 Institutionen sollte sich auf etwa 40 reduzieren).

146 "Fast zwei Drittel der Studienstandorte haben bis zu 50 AnfängerInnen ... 65\% aller Standorte haben maximal 20 AbsolventInnen." (BMBWK 2000, 18). 
Fachhochschule bzw. der "kritischen Größe“ besteht in der Berechnung der Normkosten, die aufgrund der Annahme von 1.000 Studierenden erfolgte (bei kleineren Einheiten wären demnach höhere Kosten zu erwarten) und als Anreiz für die Entwicklung von Institutionen in dieser Größenordnung wirken sollte.

Der Jahresbericht des FHR 1999 beschäftigte sich mit der Frage der Konsolidierung der Standorte und arbeitete heraus, dass zu diesem Zeitpunkt die Kriterien für das Profil einer Fachhochschule nur in geringem Maße erfüllt waren: An der Hälfte der Standorte (Studiengangsadressen) wurde nur 1 Studiengang angeboten, zwei Drittel der Standorte waren im Vollausbau auf weniger als 400 Studierende ausgerichtet (nur 1 Standort war auf 1.000 Studierende ausgerichtet). Die Notwendigkeit einer „kritischen Größe“ wurde mit den vier Gesichtspunkten (1) Lernumfeld, (2) Forschungspotential, (3) Qualifikationspotential für Standortqualität und (4) Umfeld für internationale Kooperationen unterstrichen. Gleichzeitig äußerte der FHR seine Sorge über ein Zuviel an Regionalisierung. Im folgenden Jahr wurde für die verbleibende Periode des zweiten Entwicklungs- und Finanzierungsplanes ein Beschluss gefasst, bestehenden Standorten bzw. Erhaltern gegenüber dem Aufbau von neuen Standorten bzw. Erhaltern Vorrang zu geben. ${ }^{147}$ In der Beschreibung und Analyse der Entwicklung des FH-Sektors wird die kleinräumige Struktur in den verschiedenen Merkmalen näher beleuchtet (vgl. Kapitel 2.2). Das rasche Wachstum 2001 und 2002 hat jedenfalls die Konsolidierung nicht gefördert.

\subsubsection{Regionale Strukturen und deren Dynamik}

In der Entwicklung des FH-Sektors haben die Bundesländer eine zentrale Rolle übernommen. Dadurch ergibt sich eine spezielle Art der Einbindung des FH-Sektors in die regionalen politischen und administrativen Strukturen, die auch die Struktur der Regionalisierung bestimmt. Mit der Errichtung des FH-Sektors hat sich der Kreis der Akteure in der Hochschulpolitik erweitert, indem die Monopolstellung des Bundes aufgegeben und das Auftreten anderer Akteure als Antragsteller und Erhalter ermöglicht wurde. Welche Akteure in welcher Form auftreten (sollten) wurde offengelassen, indem vorwiegend prozedurale Regelungen aufgestellt wurden. Diese offene Form war ein wesentlicher Bestandteil des „bottom-up“- Prozesses.

Im Zusammenhang mit dem Ziel eines stärkeren regionalen Ausgleichs der Bildungsangebote hat sich damit auch ein Betätigungsfeld für regionale Akteure ergeben, jedoch ohne dass formale Zuständigkeiten errichtet worden wären. Welche regionalen Akteure in welcher Form hier auftreten würden, war also nicht determiniert, aber es war

147 Im Jahresbericht des FHR 1997 wurde in der Stellungnahme zur geplanten Novelle der FHStG bei den Kriterien für die Verleihung der Bezeichnung Fachhochschule für kleinere Einheiten plädiert, in bestimmten Bereichen (z.B. technische FH-Studiengänge außerhalb von Ballungsgebieten) seien maximal 600 Studierende erreichbar. 
aufgrund der österreichischen föderalen Strukturen absehbar, dass die Länder de-facto eine wesentliche Rolle auf politischer Ebene übernehmen würden. In gewisser Weise haben sich die Bundesländergrenzen als eines der stärksten Gliederungskriterien des FH-Sektors herausgebildet, was auch in Zusammenhang mit den Finanzierungsmechanismen des Sektors steht (vgl. Abschnitt 4.2.2). Teilweise sind die Erhalter direkt nach Bundesländern organisiert und auch die übrigen Erhalter sind vorwiegend innerhalb eines Bundeslandes tätig. Überlegungen hinsichtlich der Entwicklung bundesländerübergreifender oder nationaler FH-Einrichtungen sind im Rahmen unserer Erhebungen bestenfalls vereinzelt und mit größter Vorsicht geäußert worden.

Es muss betont werden, dass das hohe Gewicht der Ländergliederung im FH-Sektor nicht von vorneherein selbstverständlich ist, sondern sich eher aus dem Gewicht der verschiedenen Akteure in den politisch-administrativen Strukturen, im Wechselspiel mit den vorhandenen Praktiken, Prioritäten und Interessen ergibt. Für Überlegungen hinsichtlich alternativer Gliederungsmöglichkeiten sind mehrere Dimensionen ins Kalkül zu ziehen:

- Erstens - aufgrund der konzipierten hohen Wirtschaftsnähe des FH-Sektors wirtschaftliche Strukturen, etwa sektoral oder verschiedene Formen von Kooperationen (Stichwort Cluster). Derartige Strukturen haben sich bis zu einem gewissen Grad im FHSektor entwickelt (Bauwirtschaft, Elektronik, Beteiligung der Wirtschaftskammern bzw. Auftreten von Weiterbildungsinstitutionen, die mit den Sozialpartnern verbunden sind) jedoch im Wesentlichen wiederum innerhalb der Ländergrenzen. Dies hängt damit zusammen, dass sich in der privatwirtschaftlichen Form des Sektors eine fast vollständig öffentliche Finanzierung, wenn auch mit erweitertem Kreis von Financiers, durchgesetzt hat (vgl. Höllinger/Hackl/Brünner 1994, Prisching 2002, 7 sowie Abschnitt 4.2.2).

- Zweitens, horizontale oder vertikale Kooperationen zwischen Gebietskörperschaften, deren wichtige potentielle Rolle im Österreichischen Raumentwicklungskonzept betont wird (ÖROK 2001, 19-20). Die wesentlichen regionalbezogenen Akteure sind in Österreich de facto die Länder und Gemeinden, wobei der Schwerpunkt der Aktivität und Zuständigkeit auf Gemeindeebene angesiedelt ist. Daraus ergeben sich plausiblerweise Spannungen zwischen diesen beiden Akteuren, auf die im FH-Wesen im Zusammenhang mit der Standortfrage immer wieder hingewiesen wird und es ergibt sich eine vergleichsweise Schwäche von Strukturen, die nicht diesen Akteursgrenzen entsprechen (beispielsweise Ostregion). Das Raumentwicklungskonzept betont in diesem Zusammenhang vor allem den durch die EU-Regionalprogramme ausgelösten Kooperationsdruck und die in diesem Zusammenhang entstandenen verschiedenen Formen des Regionalmanagements und die dafür als Erhalter fungierenden Entwicklungsorganisationen (ÖROK 2001, 20). Diese Strukturen sind wiederum im Wesentlichen auf Länderebene angesiedelt und stehen in mehr oder weniger engem Zusammenhang mit der Entwicklung des FH-Sektors. 
- Eine dritte Dimension sind schließlich grenzüberschreitende regionale Strukturen, die sich im Zusammenhang mit der EU-Integration, der Osterweiterung und der Dynamik der wirtschaftlichen Verflechtungen entwickeln. Zwei der sechs wesentlichen Merkmale, die den „Standort Österreich“ in regionaler Hinsicht laut Raumentwicklungskonzept (ÖROK 2001, 30) charakterisieren, beziehen sich auf Österreich als „Grenzland“, indem „mehr als drei Viertel der österreichischen Bevölkerung und Wirtschaftstätigkeit auf grenznahe Gebiete entfallen“. ${ }^{148}$ In diesem Zusammenhang haben sich Kooperationen zwischen Nachbarregionen auf transnationaler Ebene (Arbeitsgemeinschaften Alpenländer, AlpenAdria, Donauländer, Bodenseekonferenz) entwickelt und es werden derartige Kooperationen auch im EU-Kontext gefördert (EUREGIOs). Fragen grenzüberschreitender Mobilität und Kooperation spielen im FH-Sektor in verschiedenen Formen bereits direkt eine Rolle, ${ }^{149}$ und dieser Aspekt wird vermutlich im Zusammenhang mit den Diskussionen um die Einbeziehung von Bildungsaktivitäten in die Regulation des internationalen Dienstleistungshandels (Stichwort: GATS) steigende Bedeutung erlangen.

Auf der anderen Seite wird im Rahmen der Regionalforschung und Regionalpolitik großes Gewicht auf die Entstehung neuer räumlicher Strukturen gelegt. In diesen „neuen Räumen“ (ÖROK 2001, 33) und „multiplen Räumen“ (OECD 1999, Kap. 1,2,4) spielten das Konzept der Standortkonkurrenz und auch die Frage der Übersetzung regionaler Räume in Akteursstrukturen eine wesentliche Rolle. Die Dynamik von Globalisierung und Regionalisierung kommt hinsichtlich der Standortkonkurrenz darin zum Ausdruck, dass sich einerseits infolge der steigenden Mobilität und Verflechtung der Raum erweitert, in dem der Wettbewerb stattfindet. Dies kann in Stufen bis zum globalen Maßstab gehen. Andererseits konzentrieren und verdichten sich die Anforderungen an die regionalen Standorte. „Localities increasingly have to compete with other cities and regions around the world. (...) Basically, communities have to adopt a number of strategies, such as co-operative alliances and leadership programmes to be able to compete in the global economy. (...) What these shifts in the organisation of economic activity point to is the need for localities to embed global capital by fostering a civic culture and collective approaches to regional economic activity." (OECD 1999, 19) Schlüsselbegriffe in diesem konzeptuellen Rahmen sind die Begriffe des „regionalen Innovationssystems“ und der „lernenden Region“, die darauf aufbauen, dass für die wirtschaftliche Entwicklung die Verknüpfungen (Vernetzungen und strukturierten Austauschbeziehungen im Unterschied zu den punktuellen Transaktionen am Markt) zwischen den Akteuren essentiell sind. Ein notwendiges und grundlegendes Element dieser

148 Die anderen vier beziehen sich auf die Merkmale: Binnenland, Gebirgsland, Bedeutung der Länder und föderale Struktur, Grundstruktur der räumlichen Gliederung (überwiegend ländlich-kleinstädtische Prägung, mit nur einer großen Metropole und wenigen, im internationalen Vergleich nur mittelgroßen Agglomerationen).

149 Z.B. Austausch- und Wettbewerbsbeziehungen im Rahmen der Bodenseeregion; Fragen des "Qualifikationsexports" an den Grenzen zu Bayern; Frage der Entwicklung von Kooperationsbeziehungen mit den EU-Erweiterungsländern in Niederösterreich und im Burgenland. 
Verknüpfungen sind interpersonale Beziehungen, die im lokalen und regionalen Kontext am Besten entwickelt werden können.

Diese Veränderungen der räumlichen Strukturen ergeben für die Hochschulen eine neue Rolle, indem diese von ihren Funktionen und Potentialen her eine Schlüsselrolle in der regionalen Entwicklung spielen können. Dass sie dies tun und können, ist jedoch nicht selbstverständlich. Für die Universitäten wird die Wahrnehmung dieser Rolle mit der zunehmenden Bedeutung der Dienstleistungen für die Region als "dritter Funktion“ verbunden, nicht-universitäre Institutionen haben in diesem Kontext von vorneherein eine stärkere Rolle gehabt, aber beide Formen von Hochschulen sind in diesem Zusammenhang essentiell. ${ }^{150}$ Es entstehen Herausforderungen für beide Seiten, für die Hochschulen wie für die Regionen:

- Die Hochschulen müssen sich ihrem regionalen Kontext zuwenden, „to develop a collective understanding of the characteristics of their region, or regions, in order to identify particular opportunities for engagement."(OECD 1999, 31)

- Die Regionen müssen andererseits Strukturen entwickeln, „which will typically include firms, chambers of commerce, government agencies, R\&D laboratories, training and educational institutions including universities", die "regional self-regulation" und die Entstehung von „entrepreneurial municipalities“ ermöglichen (OECD 1999, 18) und insbesondere auch ein Verständnis der potentiellen Leistungen der Hochschulen entwickeln.

Zusammenfassend wird die Notwendigkeit der Entwicklung von kooperativen Strukturen im regionalen Raum herausgearbeitet: „In short, improved integration of HEls (Higher Education Institutions) with regional development will not be readily achieved by top down planning mechanisms at either the institutional or regional level but by insuring that the various stakeholders in the regional development process - education and training providers, employers and employers organisations, trade unions, economic development and labour market agencies and individual teachers and learners - have an understanding of each others roles and the factors encouraging or inhibiting greater regional engagement."(OECD 1999, 42) Als Zielvorstellung sollten kohärente „regional learning systems“ (ebd., 77f)

150 Die OECD-Publikation über den Zusammenhang von Hochschulen und Region (OECD 1999) konzentriert die Überlegungen v. a. auf die Universitäten und arbeitet ihre wichtigen Potentiale, aber auch die notwendigen Veränderungen in diesem Zusammenhang heraus. Die österreichische Diskussion steht in zweierlei Hinsicht in starkem Kontrast zu diesen Überlegungen: Erstens sind wenig Bemühungen aus den Universitäten heraus in Richtung der Entwicklung der "dritten Funktion" zu bemerken, dies wird eher als Zumutung von außen bekämpft, deren Akzeptanz mehr oder weniger zu einer Gefährdung der "Universität als Idee" führen würde. Zweitens, teilweise als Gegenreaktion zu „erstens“, wird im Umfeld des FH-Sektors teilweise eine Abgrenzungsposition zu den Universitäten sichtbar, die ebenfalls in starkem Kontrast zu einer systemischen Sicht steht. 
entwickelt werden. In diesem Zusammenhang wird auch der potentielle Beitrag von Hochschulen zur Entwicklung und Steuerung der "local civil society“ betont (ebd., 93).

Es stellt sich jedoch die grundlegende Frage, auf welche Dimensionalität im Raum sich die Begrifflichkeit dieser regionalen Kontexte bezieht. Was sind die Größenordnungen regionaler Einheiten, auf die im Standortwettbewerb sinnvoll abgestellt werden kann? Diese Frage ist auch für sinnvolle Referenzgrößen im internationalen Vergleich relevant. Berühmte Beispiele aus der einschlägigen Forschung sind die Regionen Boston-Massachusetts oder Kalifornien in den USA, Baden-Württemberg oder Oberitalien um Mailand in Europa. In diesem Kontext wäre ganz Österreich eine Region, das regionale Innovationssystem würde mit dem nationalen Innovationssystem zusammenfallen und der FH-Sektor müsste als Gesamtes in seiner Rolle betrachtet werden.

Aus diesen Diskussionen und Konzepten der Regionalforschung und Regionalpolitik ergibt sich, dass regionale Strukturen weit komplexer sind als die regionalen Gliederungskriterien und politisch-administrativen Einheiten. Wesentlich ist, dass regionale Strukturen durch das Zusammenspiel der Akteure (mit)geschaffen werden und dass mit der Internationalisierung gleichzeitig auch neue Entwicklungen und Herausforderungen auf regionaler Ebene entstehen, in denen Hochschuleinrichtungen eine wichtige Rolle spielen können.

\subsubsection{Regionale Wirkungen von Hochschuleinrichtungen in der Spannung von Regionalisierung und Internationalisierung}

Neben der skizzierten Dynamik regionaler Strukturen sind auch die direkten Effekte von Hochschulen für ihr regionales Umfeld und damit die unmittelbaren regionalpolitischen und -wirtschaftlichen Wirkungen von Hochschuleinrichtungen zu beachten. Diese spielen für die Gebietskörperschaften, die an FH-Einrichtungen interessiert sind, eine zentrale Rolle. Die Regionalpolitik ist eine Querschnittsmaterie, in der viele Akteure auf unterschiedlichen Ebenen zusammenwirken, wobei insbesondere die europäische Ebene stark an Gewicht gewonnen hat. In ihrem Zentrum steht die Raumordnung bzw. Raumentwicklung (ÖROK 2002). Bildungseinrichtungen sind ein wesentlicher Faktor in diesem Zusammenhang, jedoch handelt es sich in großem Maße um getrennte Politikfelder. Ein wichtiger Überschneidungsbereich von Regional- und Bildungspolitik, in dem die FH-Einrichtungen eine zentrale Rolle spielen, sind die verschiedenen Formen von Innovations-, Impuls-, Gründer- und neuerdings Kompetenzzentren. Auf regionaler Ebene wurden seit den 1980er Jahren über verschiedene Formen von Innovationsstrategien sehr wichtige Impulse für die wirtschaftliche und soziale Entwicklung gegeben. ${ }^{151}$ Wenn auch die Bedeutung von Bildung und Qualifizierung in diesen Konzepten immer betont wird, bleibt der reale Zusammenhang

151 Z.B. Steiermark, Oberösterreich, Niederösterreich. 
jedoch meistens eher lose und abstrakt. Die unmittelbare Wirkung von Bildungsinstitutionen für die Qualifizierungsstruktur ist eher gering, bei sehr gezielten massiven Investitionen bestehen Interessenskonflikte und Verteilungsprobleme zwischen verschiedenen Alternativen, die Wirkungen sind mittel- bis langfristig, während sich das Umfeld rasch wandelt. Dennoch kann in der Entwicklung von Regionalstrategien ein wesentlicher Lösungsansatz für die Frage der Positionierung und Weiterentwicklung von Fachhochschulen und Fachhochschul-Studiengängen gesehen werden. Dies würde jedoch abgestimmte Vorgangsweisen erfordern.

Schließlich muss die regionale Entwicklung im Zusammenhang mit den Prozessen steigender Verflechtungen auf überregionaler Ebene im Rahmen des Europäischen Bildungs- und Forschungsraumes und der Globalisierung gesehen werden. Die Entwicklungen in Richtung des Handels mit Bildungsdienstleistungen in einem weltweiten „Bildungsmarkt “ ${ }^{\text {“152 }}$ sind dabei ein wichtiger Faktor. An diesem Punkt bestehen Zusammenhänge zwischen der Untersuchung der Regionalisierung mit den Analysen zur Internationalisierung im FH-Sektor. Die Zusammenhänge zwischen Entwicklungen auf den verschiedenen Aggregationsebenen (regional, überregional und national, trans- und international, global) werden weitgehend in unterschiedlichen Diskursen und Forschungsfeldern behandelt, die bisher nur begrenzt in Verbindung gebracht werden.

Wesentlich ist das Zusammenspiel von Regionalisierung und Internationalisierung. ${ }^{153}$ Der Forschungsstand über diese Zusammenhänge ist jedoch bei weitem noch nicht ausreichend, um klare politische Schlussfolgerungen über Ansatzpunkte und Gewichtungen daraus abzuleiten. ${ }^{154}$ Diese Forschungen stellen den „tradeoff between ,global' efficiency und territorial particularism" (Storper 2000,8), der in den vorherrschenden ökonomischen Modellen stark angenommen wird, in Frage. Jede Aktivität findet an einem Ort statt und Mobilität steigt zwar deutlich, ist aber gleichzeitig nicht grenzenlos. Regionen werden in diesen Konzeptionen als institutionelles Geflecht gesehen, das sich evolutorisch entwickelt hat und eine gewisse Pfadabhängigkeit besitzt. Politik sollte im Wechselspiel zwischen den verschiedenen Ebenen gesehen werden, da die regionale Ebene für sich genommen als Fokus der Politik zum Beispiel für wirtschaftliche Effizienz negative Konsequenzen haben kann, ${ }^{155}$ aber im Sinne der Umsetzung und der Adaptierung überregionaler Ziele als zentrale

152 Siehe die Darstellungen und Analysen im Bereich des "World Education Market" (http://www.wemex.com/App/homepage.cfm?appname=100014\&moduleID=299\&LinkID=5678); siehe auch die entsprechenden Seiten im Rahmen der Weltbank (http://www.ifc.org/edinvest/index.htm).

153 "Dieses Paradoxon - eine verstärkte Bedeutung der Region als Standortraum bei zunehmender Globalisierung der Märkte - führt zu immer höheren Anforderungen an die miteinander konkurrierenden Regionen, eigene Profile zu entwickeln und ihre Stärken entsprechend zur Geltung zu bringen." (Tödtling-Schönhofer et al 1999, 21).

154 OECD 2001; vgl. dazu auch z.B. die Reflexion von Michael Storper über die Regionalprojekte im TSERProgramm der EU (Storper 2000).

155 Die Verstärkung von Besonderheiten ("asset specifities") von bestimmten Regionen kann sehr unterschiedliche Wirkungen haben, die vom Kontext abhängen. Storper $(2000,24)$ drückt dies am Beispiel des Wissens ziemlich 
Dimension angesehen wird. „Nicht jede Maßnahme, die auf regionaler Ebene sinnvoll ist, kann auch im größeren Kontext positiv bewertet werden. (...) Regionalpolitik ist demnach zu wichtig, um sie den individuellen Interessen einzelner Regionen zu überlassen. Regionalpolitik kann demnach auch nicht automatisch mit Standortpolitik gleichgesetzt werden, sondern muss gleichzeitig den Nutzer auf überregionaler Ebene mitberücksichtigen“ (Tödtling-Schönhofer et al 1999, 22).

\section{Regionalpolitik - Innovation - Dienstleistungen als „Dritte Funktion“ von Hochschulen}

Die Förderung von Innovation hat in diesem Sinne als ein Fokus der Regionalpolitik eine Schlüsselrolle bekommen und Hochschulen kommt in diesem Zusammenhang als Institutionen der Wissensproduktion wiederum eine besondere Rolle zu. Hier geht es daher um das Entwicklungsfeld der Positionierung der FH-Institutionen im regionalen Kontext (vgl. Kapitel 5.1.1). Diese besondere Rolle hängt einerseits damit zusammen, dass Hochschulen (insbesondere die Universitäten) zwar lokal situiert sind, aber sich in der Wahrnehmung ihrer Funktionen in einem universalistischen Raum bewegen und andererseits damit, dass in den letzten Jahrzehnten zu den beiden traditionellen Funktionen der Lehre und Forschung eine sogenannte "dritte Funktion“ dazugekommen ist: die Erfüllung von Leistungen für ihre Region.

Dies wird insbesondere im Zusammenhang mit der Diversifizierung des Hochschulwesens betont (vgl. OECD 1999). Daraus ergibt sich für die Hochschulen eine spezielle Rolle auch in der Vermittlung zwischen den verschiedenen Aggregationsebenen. „HEls (Higher education institutions), then, operate within multiple and overlapping territories and usually manage a portfolio of activities ranging from the global to the local. The advantage of the presence of one or more HEls in a region is that expertise from these different scales can be a major asset to the community. The challenge is to manage simultaneously the various territorial portfolios so that they reinforce each other and to establish mechanisms through which the national and international connections of HEls can be mobilised to benefit the region." (OECD 1999, 17)

Seit den 1990er Jahren kann man zumindest auf konzeptueller Ebene eine Konvergenz zwischen einer Reihe von Politikbereichen feststellen, die zunächst weitgehend voneinander getrennt waren: wie schon erwähnt zwischen Innovationspolitik und Regionalpolitik, aber auch zwischen Innovationspolitik und Bildungs- bzw. Hochschulpolitik (vgl. insbesondere Lundvall/Borras 1999).

drastisch aus: "placing the emphasis on local knowledge can be catastrophic if that's not where the essential knowledge is located; by the same token, trying to copy external knowledge can be disastrous if it is only a means to being second-best, i.e. a cost-price competitor." 
Alice Lam und Bengt-Ake Lundvall haben das Konzept des Innovationssystems durch die Einbeziehung des Bildungswesens zum "system of competence building and innovation“ erweitert (Lam/Lundvall 2000). Tatsächlich ist diese Verbindung bisher jedoch eher bloß auf einer konzeptuellen und abstrakten Ebene entwickelt. So weist z.B. auch Clement et al. (2002, 11) zwar auf die "Verknüpfung der Technologiepolitik mit der Qualifikationspolitik“ als einen von acht "innovationspolitischen Trend(s) im internationalen Benchmark" hin, in der Ausarbeitung von Vorschlägen ist diese Verbindung jedoch weniger weit gediehen. Zentrale Punkte in diesem Konzept sind:

- die Entwicklung von F\&E im FH-Sektor,

- Qualitätskriterien im Bereich Qualifikation und

- die Berücksichtigung der Humanressourcen im Rahmen eines „regionalen Kontrollgremiums".

Wenn auch im Zusammenspiel zwischen Regionalpolitik, Innovationspolitik und Hochschuleinrichtungen bereits gewisse Grundkonzepte breite Verwendung gefunden haben und viele Zusammenhänge gut etabliert sind, so ist gleichzeitig auch vieles von der Forschung her noch offen und unklar geblieben. Daher bleibt in der Frage der regionalen Positionierung der Fachhochschuleinrichtungen auch ein breiter Raum zur Gestaltung und zur politischen Entscheidung, wie weit man bei der Positionierung im Rahmen der Innovationspolitik gehen will.

\subsection{Retrospektive Analyse der Regionalisierung im FH-Sektor}

Die Regionalisierung im FH-Sektor wird im Hinblick auf vier Aspekte untersucht: Erstens wird auf Basis von Daten der offiziellen Statistik (Mikrozensus, Volkszählung, Hochschulstatistik) analysiert, ob und inwieweit ein Beitrag zum Abbau regionaler Disparitäten im Hochschulzugang im Zusammenhang mit dem Aufbau des FH-Sektors festzustellen ist. Aufgrund der Daten des FHR und zusätzlicher Informationen aus den eigenen Erhebungen bei den Erhaltern und StudiengangsleiterInnen werden die FH-Einrichtungen zweitens im Hinblick auf ihre Standortpotentiale untersucht und drittens werden die Wirkungen auf regionaler und überregionaler Ebene verglichen. Viertens erfolgt eine Analyse von Ansätzen regionaler Planung und Entwicklung.

\subsubsection{Abbau regionaler Disparitäten}

Die Verringerung regionaler Disparitäten ist zwar eine bildungspolitische Zielsetzung, die Voraussetzungen ihrer direkten Messung sind jedoch in der offiziellen Statistik nicht 
gegeben. Mit vertretbarem Aufwand wurden zwei verschiedene Formen der Berechnung angestellt, ein Vergleich der Entwicklung der Studierendenquoten nach Gemeindegrößen und ein Querschnittsvergleich der Verteilung der StudienanfängerInnen an Universitäten und Fachhochschul-Studiengängen nach ihrer Herkunft aus verschiedenen Regionstypen mit der Verteilung der Bevölkerung auf diese Regionstypen.

In der ersten Berechnung wurde mittels Daten aus dem Mikrozensus eine Schätzung für die Entwicklung der annähernden Zahl der Studienanfängerlnnen nach unterschiedlichen Gemeindegrößen durchgeführt. Dies kann nicht direkt erfolgen, da SchülerInnen und Studierende gemeinsam erfasst werden und es kann auch nicht zwischen Universitäts- und FH-Studierenden unterschieden werden. Es wurden daher die SchülerInnen/Studierenden nach Altersgruppen gruppiert und regionalspezifische Beteiligungsquoten für den Zeitraum 1987 bis 2000 berechnet. Da die Errichtung des FH-Sektors in diesen Zeitraum fällt, kann untersucht werden, ob seit diesem Zeitpunkt eine Steigerung der Quoten in den weniger städtischen Gemeinden erfolgt ist. Diese Auswertung zeigt im Wesentlichen die folgenden Befunde:

- Die regionalspezifischen Beteiligungsquoten unterscheiden sich im Beobachtungszeitraum nach der Gemeindegröße beträchtlich. Die Bildungsbeteiligung der 20- bis 23Jährigen insgesamt ist zwischen 1987 und 2000 von $18 \%$ auf $27 \%$ um fast 10 Prozentpunkte gestiegen. ${ }^{156}$ In den Gemeinden mit über 100.000 Einwohnerlnnen liegt sie durchgängig in der Größenordnung von mehr als 10 Prozentpunkten höher (bei 31\% bis $40 \%)$. In den ländlichen Gemeinden mit bis zu 5.000 EinwohnerInnen liegt die Studierendenquote um etwas weniger als 10 Prozentpunkte unter dem Durchschnitt (bei $11 \%$ bis 20\%). Mit steigender Einwohnerlnnenzahl der Gemeinden steigt die Beteiligung gegenüber den ländlichen Gemeinden systematisch an (um jeweils etwa einen Prozentpunkt, wenn man die Größenklassen auf bis zu 10.000 Einwohnerlnnen bzw. dann wieder auf bis zu 100.000 Einwohnerlnnen erweitert). Insgesamt sind die Quoten etwas zugunsten der städtischen Gemeinden verzerrt, da ein Teil der Studierenden vermutlich nicht mehr am Heimatwohnort gezählt wird (vgl. Abbildung 49).

- Im Zeitverlauf nehmen die Studierendenquoten in den verschiedenen Gemeindegrößen parallel zu, d.h. durch den bloßen Augenschein sind keine deutlichen regionalen Verschiebungen erkennbar (vgl. Abbildung 49).

- Wenn man den möglichen Einfluss der Einrichtung der Fachhochschul-Studiengänge in dieser Entwicklung erfassen will, so kann man einen einfachen Vorher-Nachher-Vergleich

156 Die Absolutzahl liegt 2000 bei 25.600 pro Jahrgang, die Zahl der Erstzugelassenen an den Universitäten liegt bei 21.400 und in den FH-Studiengängen bei 3.900, wobei diese nicht unbedingt aus dieser Altersgruppe stammen. Die Schätzung stimmt erstaunlich gut überein. 
der Entwicklung der regionalen Studierendenquoten anstellen. Dies wird in Abbildung 50 dargestellt. Es wird eine Vorperiode zwischen 1987 und 1995 definiert und für diese wird die durchschnittliche jährliche Veränderung der regionalen Beteiligungsquoten berechnet. Diese liegt zwischen einem halben und einem ganzen Prozentpunkt, mit wenig Unterschieden zwischen den Gemeindegrößen. Zum Vergleich wird für die Nachperiode ebenfalls die jährliche Veränderung dieser Quoten berechnet, wobei mit den durchschnittlichen Quoten 1996 - 2000 begonnen wird und dann sukzessive parallel zur Expansion des FH-Sektors immer ein Jahr weggelassen wird. Man sieht deutlich, dass ab der Periode 1997 - 2000 die Zuwächse in den Gemeinden mit weniger als 100.000 Einwohnerlnnen stärker steigen als die Quoten in den Agglomerationen.

Diese Ergebnisse zeigen, dass in der Beobachtungsperiode ein gewisser regionaler Ausgleich der Bildungsbeteiligung vor sich gegangen ist, der auch die kleinen Gemeinden erreicht hat und der sich in der Periode des Aufbaus des FH-Sektors verstärkt hat. Ein Beitrag des FH-Sektors zur Verringerung der regionalen Disparitäten liegt also nahe. ${ }^{157}$ In Anhang 11.2 sind auch die Ergebnisse für die anderen Altersgruppen ausgewiesen. Es zeigt sich sowohl bei den 23- bis 26-Jährigen als auch bei den über 26-Jährigen ein tendenzieller Ausgleich der regionalen Unterschiede der Bildungsbeteiligung. Die regionalen Unterschiede in der Studienbeteiligung sind in dieser differenzierten Betrachtung nach der Gemeindegröße jedoch immer noch beträchtlich. Dies zeigt die Abbildung 49, wo die Studierendenquote in den Gemeinden mit mehr als 100.000 Einwohnerlnnen immer noch um etwa 20 Prozentpunkte höher liegen als in den Gemeinden mit kleinerer Einwohnerlnnenzahl.

Für die zweite Berechnung wurden Informationen aus der Hochschulstatistik und aus den Volkszählungsergebnissen herangezogen. Die Hochschulstatistik differenziert die StudienanfängerInnen nach regionaler Herkunft, enthält jedoch keine Angaben über die zugehörige Population. Man kann allerdings einen einfachen Querschnittsvergleich der regionalen Herkunft der Universitäts- und FH-Studierenden nach verschiedenen zusammengefassten Gebietstypologien (Zentralität des Bezirkes und Wirtschaftsstruktur ${ }^{158}$ ) mit den entsprechenden Verteilungen der Volkszählung durchführen. Betrachtet man die Verteilung der StudienanfängerInnen nach der Zentralität des Herkunftsbezirkes, die vor allem Agglomerationen mit guter Erreichbarkeit zusammenfasst, so ist die Herkunftsstruktur der FH-Anfängerlnnen (Gesamtösterreich) beinahe perfekt an die Bevölkerung angepasst (vgl. Abbildung 51). Auch im Bundesländervergleich entsprechen sie mit Ausnahme von Niederösterreich besser der Bevölkerung als die Universitäten (vgl. Abbildung 52). In Salzburg, Steiermark und Tirol kommen FH-Anfängerlnnen um mehr als fünf Prozentpunkte

157 Der halbe AnfängerInnenjahrgang von 2000 könnte in den verschiedenen ländlichen Agglomerationen immerhin drei bis vier Prozentpunkte der Beteiligung ausmachen.

158 Definiert nach der im Mikrozensus verwendeten Konzeption. 
häufiger aus zentralen Bezirken, als dies der Bevölkerung entspricht, was auf vergleichsweise stärkere Disparitäten gegenüber den übrigen Bundesländern hinweist.

Auch die Verteilung nach der Wirtschaftsstruktur entspricht bei den Anfängerlnnen des $\mathrm{FH}$ Sektors besser der Bevölkerung als bei denen an Universitäten, wo die großstädtischen Agglomerationen und die Dienstleistungszentren überrepräsentiert sind (vgl. Abbildung 53). In der Steiermark ist die Großstadt-Agglomeration auch unter den FH-Zugängen um etwa zehn Prozentpunkte überrepräsentiert, in Oberösterreich sind die Industriegebiete gegenüber der großstädtischen Agglomeration überrepräsentiert, in Niederösterreich jedoch gegenüber den Agrargebieten (vgl. Abbildung 54). Aus dieser Auswertung ergeben sich also am ehesten für die Steiermark, Tirol und für Niederösterreich und abgeschwächt auch für Salzburg und Vorarlberg Schlussfolgerungen, die es nahe legen, das Augenmerk auf eine weitere Verringerung der regionalen Disparitäten im $\mathrm{FH}$-Zugang zu richten. In diesen Bundesländern sind teilweise sichtbare Vorteile der städtischen Zentren bzw. auch Benachteiligungen der peripheren Regionen und vor allem der Fremdenverkehrs- und Agrarregionen im Zugang zu den FH-Einrichtungen festzustellen.

Abbildung 49: Regionale Studierendenquoten (20 - 23jährige Studierende in der gleichaltrigen Wohnbevölkerung nach Gemeindegrößen), 1987 - 2000

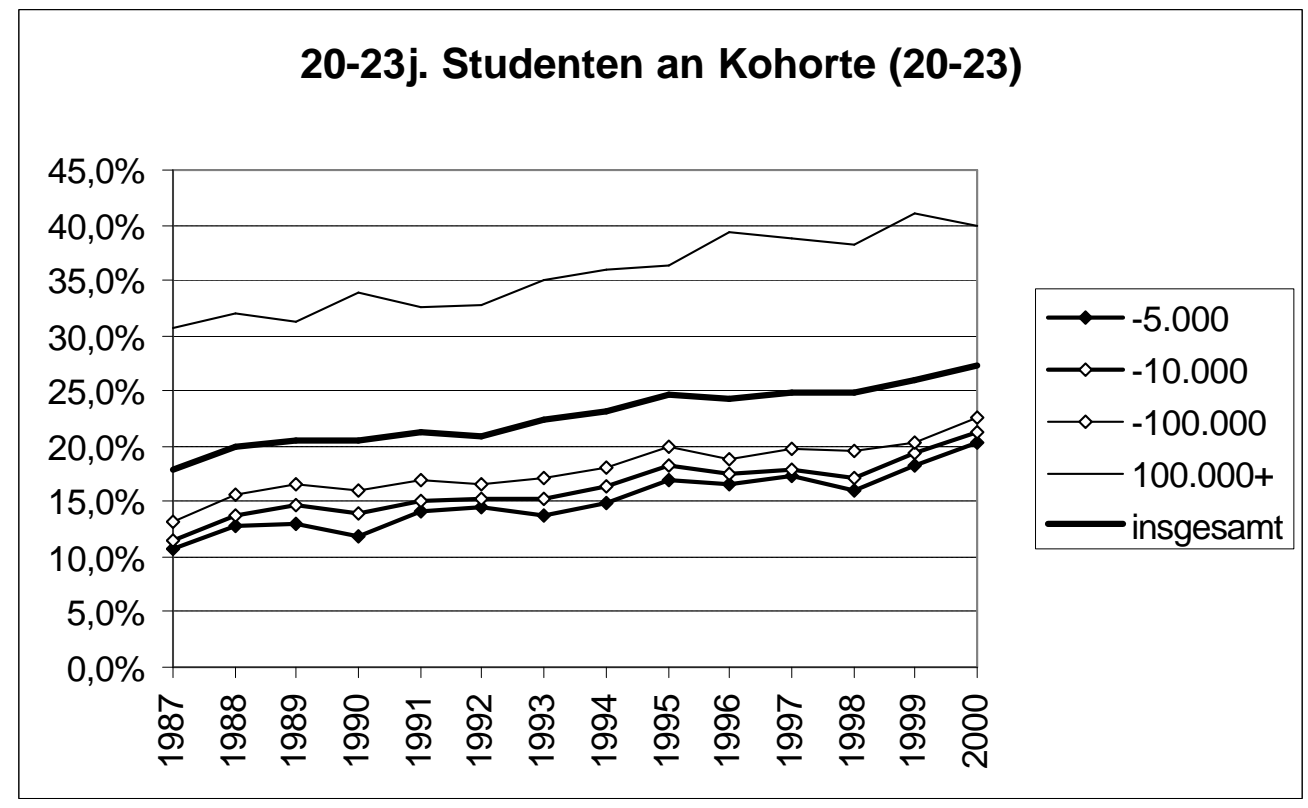

Quelle: Statistik Austria (Mikrozensus), IHS-Berechnungen 
Abbildung 50: Veränderung der regionalen Studierendenquoten (20 - 23jährige Studierende in der gleichaltrigen Wohnbevölkerung nach Gemeindegrößen) nach Zeitperioden

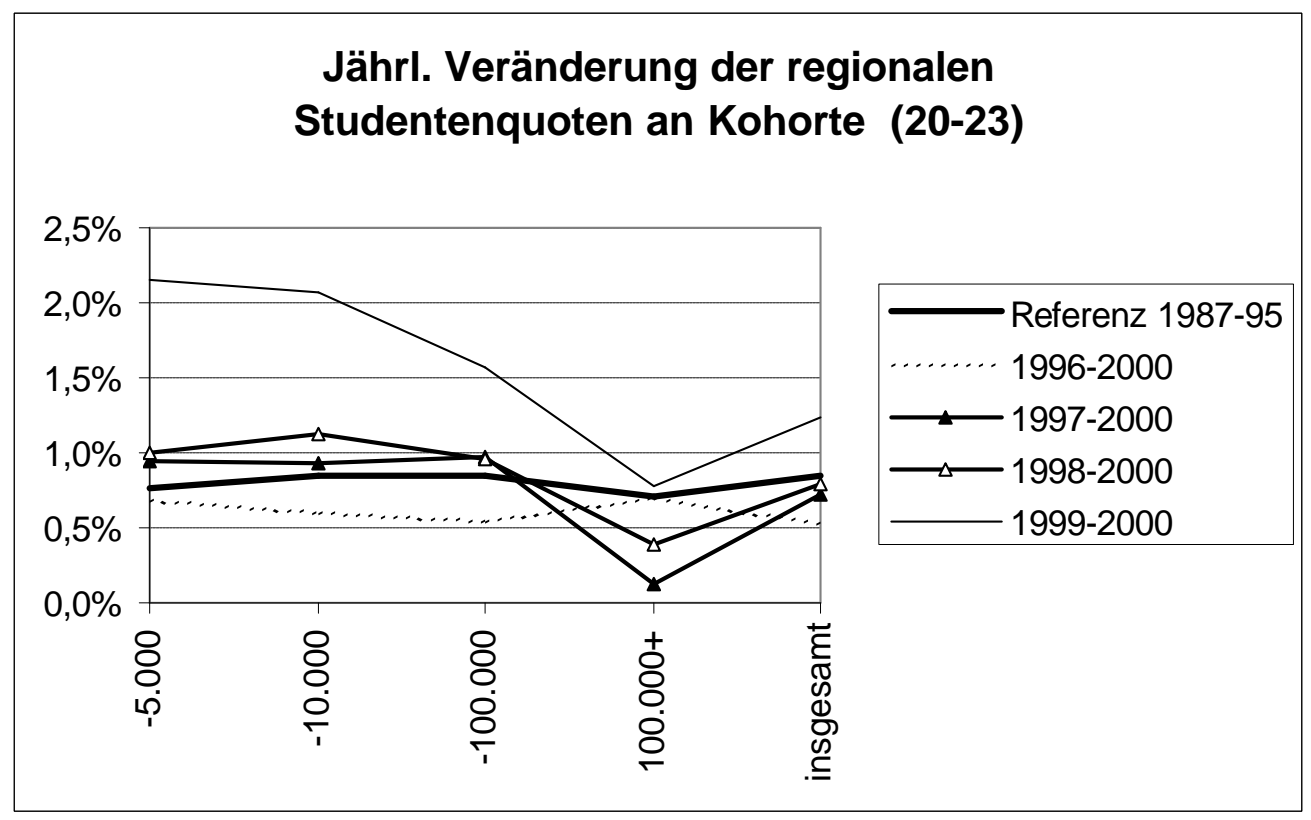

Quelle: Statistik Austria (Mikrozensus), IHS-Berechnungen

Abbildung 51: Wohnbevölkerung und inländische StudienanfängerInnen an Universitäten und FH-Studiengängen nach Zentralität des Herkunftsbezirkes, 2001

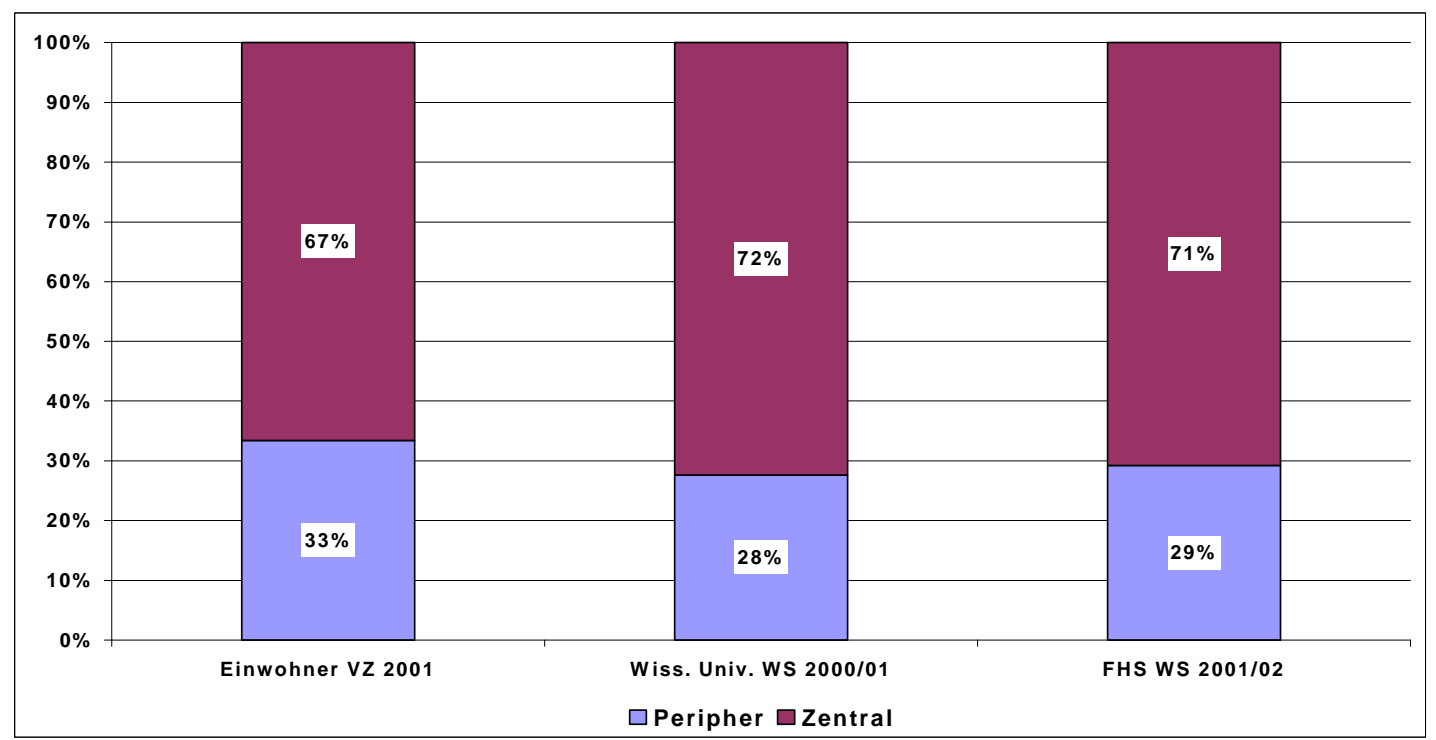

Definition der Zentralität laut Mikrozensus.

Quelle: Statistik Austria, IHS-Berechnungen 
Abbildung 52: Wohnbevölkerung und inländische StudienanfängerInnen an Universitäten und FH-Studiengängen nach Zentralität des Herkunftsbezirkes und Heimatbundesland, 2001

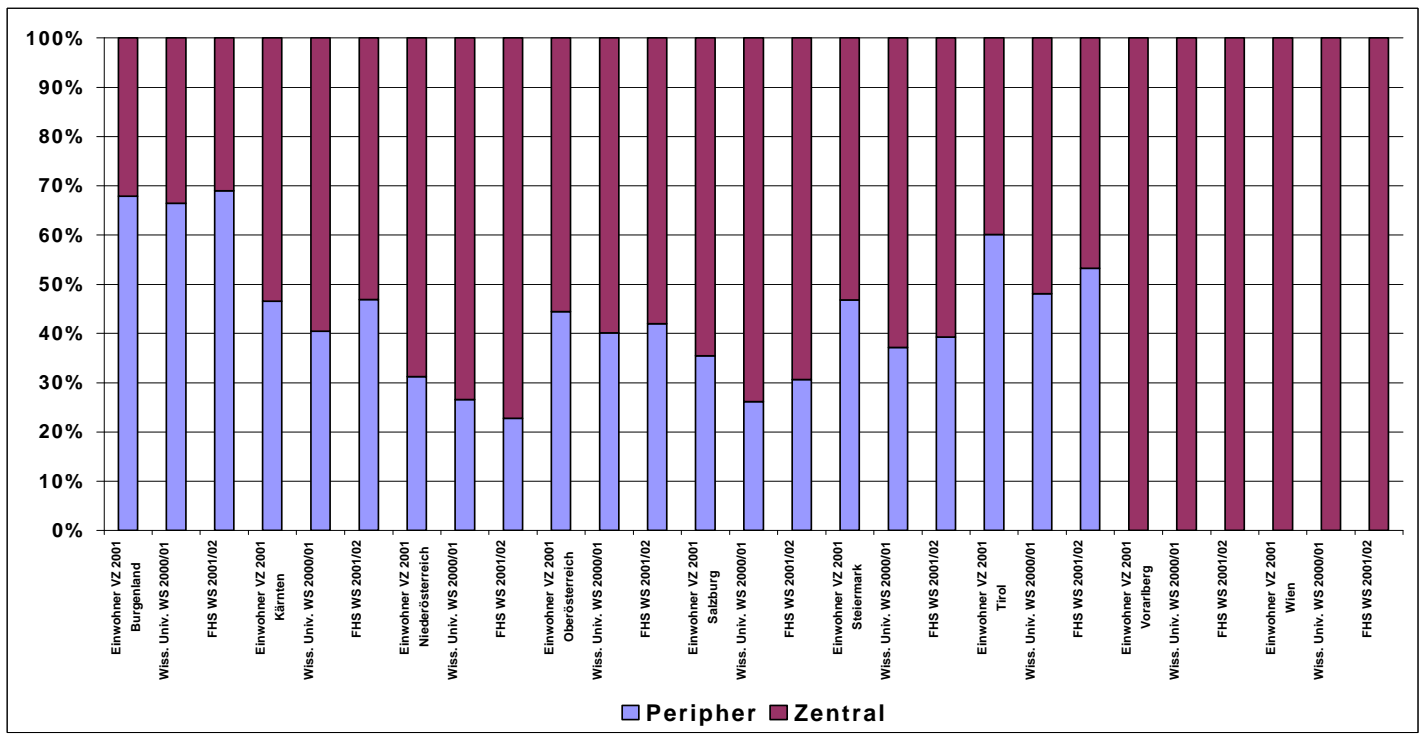

Definition der Zentralität laut Mikrozensus.

Quelle: Statistik Austria, IHS-Berechnungen

Abbildung 53:Wohnbevölkerung und inländische StudienanfängerInnen an Universitäten und FH-Studiengängen nach der Wirtschaftsstruktur des Herkunftsbezirkes, 2001

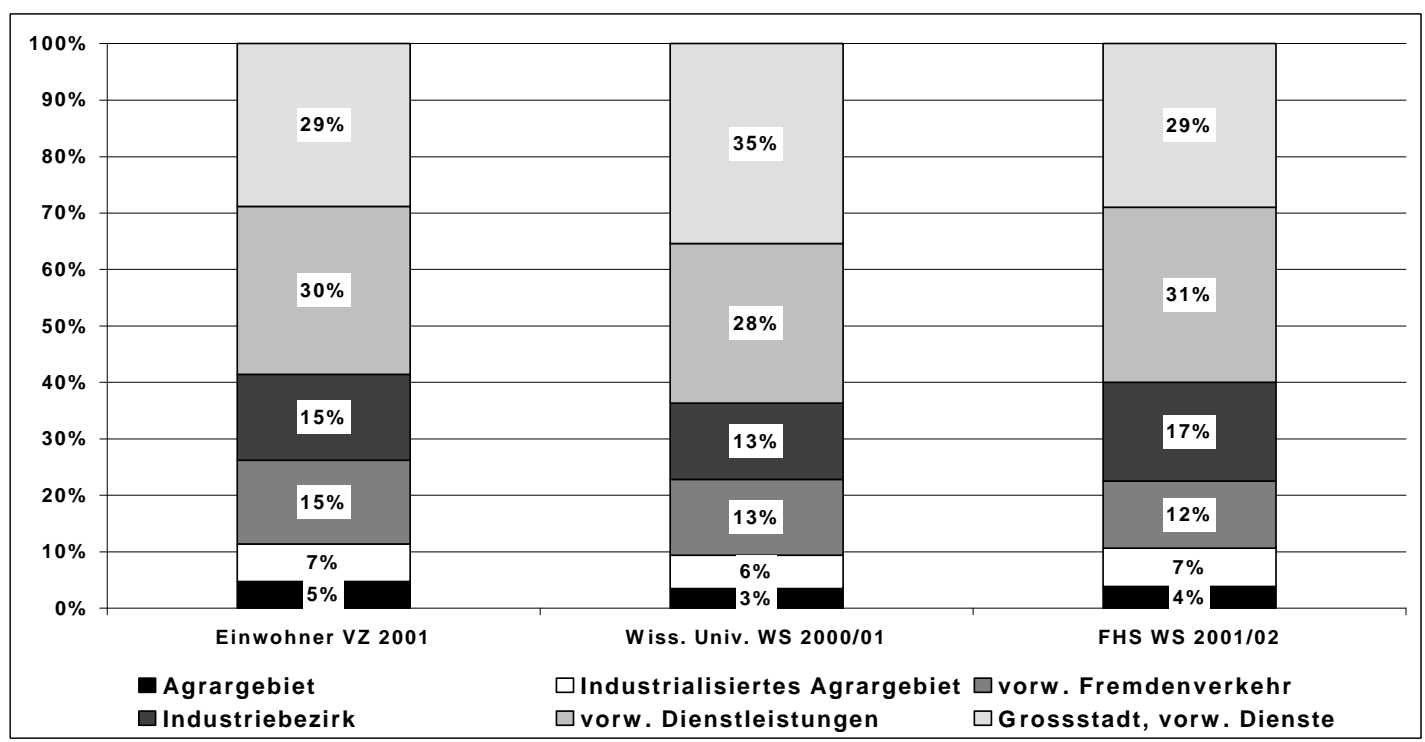

Definition der Zentralität laut Mikrozensus.

Quelle: Statistik Austria, IHS-Berechnungen 
Abbildung 54:Wohnbevölkerung und inländische StudienanfängerInnen an Universitäten und FH-Studiengängen nach der Wirtschaftsstruktur des Herkunftsbezirkes und Heimatbundesland, 2001

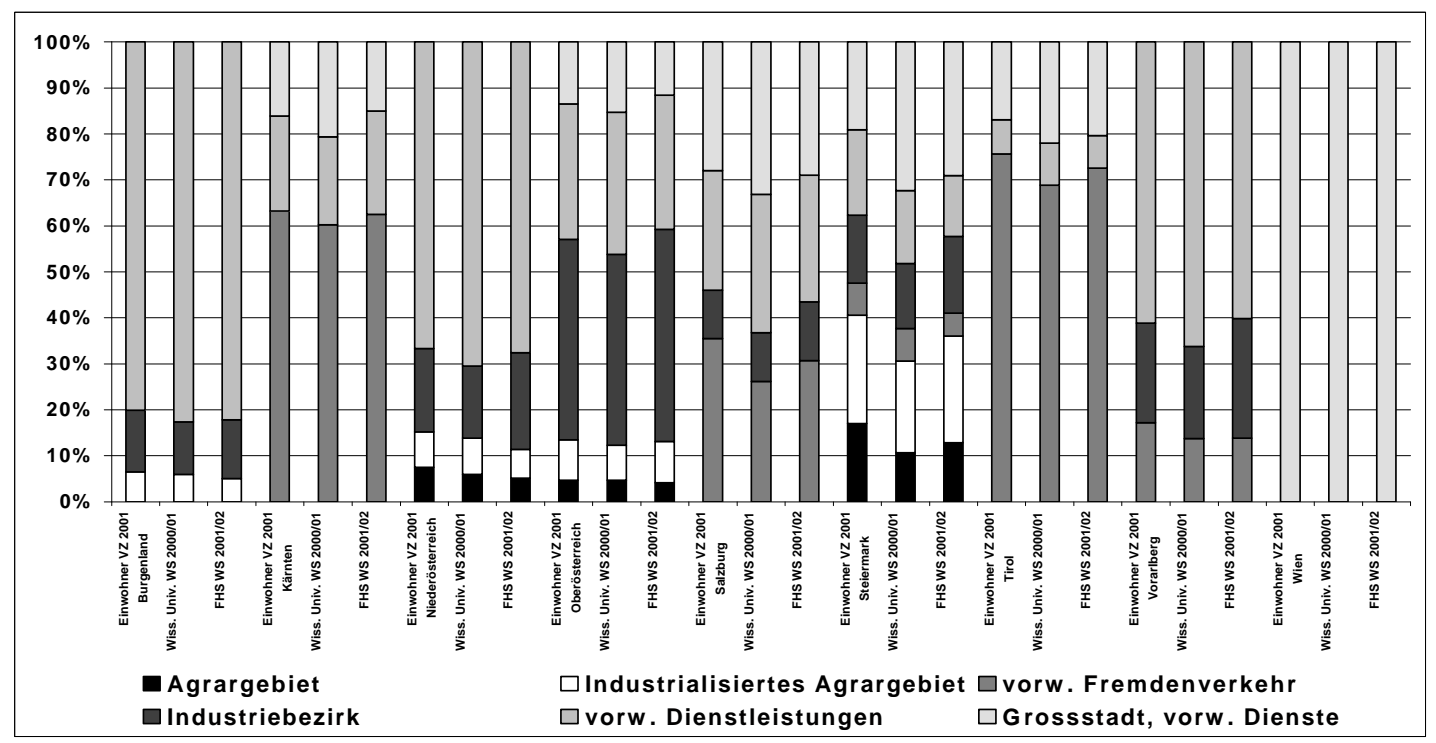

Definition der Zentralität laut Mikrozensus.

Quelle: Statistik Austria, IHS-Berechnungen

\subsubsection{Entwicklung der Potentiale nach Studiengängen, Erhaltern, Standorten}

Das erforderliche Potential eines FH-Standortes ist in Österreich aufgrund der „bottom-up“Strategie nur sehr rudimentär umschrieben. Es gibt einige Mindestkriterien in den gesetzlichen Grundlagen, einige weitere politische Entscheidungskriterien in den Entwicklungsplänen und einzelne, meist taxativ aufzählende Zielbestimmungen mit unterschiedlichem Verbindlichkeitsgrad von diversen Akteuren. ${ }^{159}$ Quantitative Umschreibungen beziehen sich im Wesentlichen auf Input-Faktoren und hier auf einige wenige Aspekte (ein Minimum an Personen für Entwicklungsteam und Lehrende; Zahl der Studienplätze; Zahl Studiengänge und Studierende, Kollegium für die Fachhochschule; Studierendenpotential; einige wenige Hinweise auf das Fächerspektrum; Anforderungen hinsichtlich Umfeld, Weiterbildung, Credits, Fernstudienelemente).

\footnotetext{
159 Vgl. die detaillierte und umfassende Analyse von Zieldimensionen am Beispiel Niederösterreich in Delapina/
} Holzinger/Schausberger 2001, 25. 


\section{Abbildung 55: Vorgaben hinsichtlich des angestrebten Potentials der FH- Einrichtungen in offiziellen Dokumenten}

Gesetz: Minimalanforderungen für Studiengänge und Fachhochschul-Status, aber keine Angaben über den Umfang der erforderlichen Ausstattung, um die Zielerreichung und Qualität zu garantieren.

Hinweise für Studiengänge: Vielfalt wissenschaftlicher Lehrmeinungen und wissenschaftlicher Methoden; Mindeststundenzahl; „erforderliche“ Personal-, Raum- und Sachausstattung; Entwicklungsteam aus mindestens vier Personen, zwei wissenschaftlich und zwei praktisch ausgewiesen, davon müssen mindestens vier Personen in dieser Zusammensetzung dann auch lehren (keine Angabe über das Ausmaß der Lehrtätigkeit).

Hinweise für Status Fachhochschule: mindestens zwei Studiengänge; Erreichung von mindestens 1.000 Studierenden im Vollausbau; Organisation: Einrichtung eines Kollegiums. Indirekt: bei 2 Studiengängen durchschnittliche Zahl von 500 Studierenden pro Studiengang, 125 pro Jahrgang, reduziert sich bei einer höheren Zahl an Studiengängen.

Entwicklungs- u. Finanzierungspläne: Die Entwicklungspläne des Bundes enthalten als Kriterien für die Vergabe der Bundesmittel weitere Hinweise über das angestrebte Potential der Einrichtungen.

EFPI: Das angestrebte Profil für eine Fachhochschule wurde etwas spezifiziert: mindestens 1.000 Studenten aus dem regionalen Einzugsgebiet, mehrere sinnvoll aufeinander abgestimmte Studienrichtungen, längerfristig lebensfähiges Fächerspektrum. Es wurden aber auch einzelne Studiengänge, die in anderen Institutionen integriert sind, genannt.

EFPII: Die regionale Positionierung wurde etwas näher spezifiziert: genügend große Zahl an StudieninteressentInnen im erpendelbaren Einzugsgebiet; es sollte eine akademisch und ökonomisch vitale Einrichtung errichtet werden; es wurden neue Kriterien spezifiziert, die gewisse Anforderungen an das Potential der Einrichtungen stellen: Weiterbildung der Lehrenden; Credits in der Programmstruktur; Fernstudienelemente; ein entsprechendes akademisches und sozio-kulturelles Umfeld wurde verlangt.

FHR-Jahresberichte: Der Fachhochschulrat hat in seinen Jahresberichten verschiedentlich zur Dimensionierung von Fachhochschule und FH-Studiengängen Stellung genommen.

FHR-JB 97: Es wurde vorgeschlagen, die Kriterien für die Verleihung des Status Fachhochschule zu ändern, indem die Zahl der erforderlichen Studiengänge auf 3 erhöht, aber die Zahl der erforderlichen Studierenden auf 500 verringert werden sollte und zusätzlich Kriterien zur Evaluation und Verlängerung von Studiengängen einbezogen werden sollten. Bei 500 Studierenden in drei Studiengängen reduziert sich die durchschnittliche Zahl pro Studiengang auf etwa 180 oder weniger als 50 pro Jahrgang.

FHR-JB 99: Der Jahresbericht hat die Zahl der Studiengänge und der Studierenden im Vollausbau als Indikatoren in den Mittelpunkt gestellt und herausgearbeitet, dass die Erreichung der angestrebten Werte für die Errichtung von Fachhochschulen bei weitem nicht absehbar sei (2 Sudiengänge: die Hälfte der Standorte verfügte nur über einen Studiengang; 1.000 Studierende: zwei Drittel der Standorte strebten im Vollausbau maximal 400 Studierende an). Vier Gesichtspunkte für die „kritische Größe“ wurden herausgearbeitet: (1) Lernumfeld, (2) Forschungspotential, (3) Qualifikationspotential für Standortqualität und (4) Umfeld für internationale Kooperationen.

Bezüglich der angestrebten Ergebnisse gibt es die Vorgaben hinsichtlich der Erfüllung der Studiendauer und damit implizit der Zahl der AbsolventInnen, darüber hinaus im Wesentlichen Benennungen der Ziele, jedoch keine näheren Vorgaben darüber, in welchem Ausmaß und in welcher Form Ergebnisse erwartet werden. Die Sicherung der Ergebnisse erfolgt sozusagen implizit über die Etablierung der verschiedenen Mechanismen der Qualitätssicherung. Hinsichtlich der Lehre gibt es ein Angebotsprofil der Qualifikation der AbsolventInnen, jedoch wegen der Legitimation durch die Bedarfsprüfung keine ausdrücklichen Vorstellungen zu den erwarteten Wirkungen der Ausbildung (Wie sollten diese im Vergleich zu anderen Qualifikationen z.B. einkommensmäßig positioniert sein? Wie sollte die wissenschaftliche Komponente ausgeprägt sein, etwa als Ausbildung von F\&E Personal im Vergleich zu einem Universitätsstudium? Welche individuellen und sozialen Erträge werden erwartet? Welchen ökonomischen Effekt sollte die FH-Ausbildung haben? etc.) 
Noch unbestimmter sind die Erwartungen hinsichtlich der F\&E-Leistungen und der „dritten Funktion" anderer Dienstleistungen für die Region. Im Grundsatz- und Motivpapier zum Impulsprogramm (BMWV o.J.) wurden Eckpunkte dieser Funktionen beschrieben. Der FHSektor sollte zur Lösung bestimmter Probleme in der österreichischen F\&E-Landschaft beitragen, insbesondere im Zusammenhang mit KMUs und im regionalen Kontext (vgl. Maresch 2002, Mayer/Stampfer/Sturn 2001). Die gezielten Förderprogramme (Kooperation Fachhochschulen - Wirtschaft mit drei Ausschreibungsrunden 1997-2000, FH-plus ab 2002) sollten bzw. sollen den Aufbau dieser Funktionen stimulieren. Verschiedene Initiativen aus dem FH-Sektor haben auch Erwartungen formuliert, die Aussagen über „kritische Massen“, Finanz- und Förderbedarf und auch über Entwicklungspotentiale und -geschwindigkeit der F\&E-Aktivitäten enthalten (vgl. Grünbacher/Detter 2000). ${ }^{160}$

Insgesamt folgt aus dieser Konstellation, dass sich das Potential von Studiengängen bzw. Fachhochschulen auf emergentem Wege im Prozess der Umsetzung - eben „bottom-up“ entwickelt hat. Im gegenwärtigen Stand ist das vorhandene Potential das Ergebnis des komplexen Zusammenwirkens vieler Faktoren und nur teilweise die Verwirklichung von angestrebten Ergebnissen. Aufgrund der starken Dynamik im Ausbauprozess v.a. in den letzten beiden Jahren muss auch festgehalten werden, dass jede Feststellung über das gegenwärtige Potential eine Momentaufnahme in einem dynamischen Prozess darstellt. Einige Faktoren wurden im Kapitel 2.2 zum Überblick bereits dokumentiert.

Eine spezielle Frage der Erfassung des Potentials von Einrichtungen besteht darin, auf welcher Betrachtungsebene diese sinnvollerweise erfolgen soll: Studiengänge, Standorte, Studienorte, Erhalter, Bundesland. Nach unseren Erhebungen kommt gegenwärtig der Ebene der Erhalter eine Schlüsselstellung zu, indem die wesentlichen Entscheidungen über die Positionierung und Entwicklung der Einrichtungen auf dieser Ebene getroffen und vor allem auch gegenüber anderen Akteuren vertreten werden.

Für die Erfassung des Potentials kann von den Merkmalen ausgegangen werden, die in den Vorgaben und in der Diskussion über eine sinnvolle Entwicklung des FH-Sektors und seiner Einrichtungen die zentrale Rolle spielen:

- Zahl der Studiengänge und Studierenden als wesentliche Parameter des Anerkennungsverfahrens und der Finanzierung

160 In diesem Papier wird eine Entwicklung skizziert, derzufolge die ersten vier Jahre eines Studiengangs dem Aufbau der Lehre dienen und in den zweiten vier Jahren die F\&E-Funktion ausgehend von punktuellen Aktivitäten entwickelt werden kann. Im Laufe von acht Jahren wird bei entsprechendem Umfeld der Aufbau von F\&E-Kompetenz als realistisch angesehen, wenn entsprechende Finanzierungsbedingungen erfült sind. Grünbacher/Detter (2000) haben ein Entwicklungskonzept formuliert, demzufolge bis 2005 ein Viertel der Studiengänge sich zu "forschenden Studiengängen" in einem konsolidierten Umfeld entwickeln könnten. 
- Zahl der hauptberuflichen Lehrpersonen pro Erhalter

- Relation nebenberufliche Lehrpersonen pro hauptberuflicher Lehrperson

- Profil der nebenberuflichen Lehrpersonen nach inrer Haupttätigkeit (Wirtschaft, Universität, Schule)

- Lehraufwand der hauptberuflichen Lehrpersonen

- Personalressourcen der Erhalter

- finanzielle Ressourcen, insbesondere zusätzliche Finanzquellen zum Bundesbeitrag

- Einnahmen für Leistungen außerhalb der Lehre

Eine umfassende Potentialanalyse der Standorte ist im Rahmen des vorliegenden Projektes nicht möglich und war auch nicht angestrebt. Die folgende Darstellung gibt aufgrund der zwei Kategorien von Lehrpersonal (Zahl der hauptberuflich Lehrenden und Relation nebenberuflich Lehrende pro hauptberuflich Lehrendem) eine Illustration der Verteilung der Standorte und verknüpft diese Parameter mit erstens der „magischen“ Zahl von 1.000 Studierenden am Standort (diese wird nur zwei Mal erreicht) und mit zweitens der Beteiligung am Impulsprogramm für F\&E.

\section{Personelles Potential der Standorte}

Diese einfache Darstellung ergibt ein ziemlich instruktives Bild (vgl. Abbildung 56): Es gibt tendenziell drei bis vier Typen von Standorten: An acht Standorten ist die Zahl der hauptberuflichen Lehrpersonen vergleichsweise mittel (25-35) oder hoch (45-70) und die Relation der nebenberuflichen pro hauptberuflich Lehrendem liegt eher niedrig (bei 2:1 bis $5: 1$ ). Alle diese Standorte haben sich im ersten Jahr bereits am Impulsprogramm beteiligt. An 13 Standorten ${ }^{161}$ liegt die Zahl der hauptberuflich Lehrenden eher niedrig (5-25) und auch die Relation der nebenberuflichen pro hauptberuflich Lehrendem liegt niedrig bis mittel (bis maximal 10:1). Von diesen Standorten hat sich nicht ganz die Hälfte, überwiegend im zweiten oder dritten Jahr am Impulsprogramm beteiligt, die übrigen nicht. Schließlich gibt es fünf Standorte mit einer niedrigen Zahl an hauptberuflich Lehrenden und einer hohen bis sehr hohen Relation der nebenberuflichen pro hauptberuflich Lehrendem (15:1 bis über 40:1). Von diesen Standorten hat sich nur einer am Impulsprogramm beteiligt, dieser hat auch über 1.000 Studierende, die vor allem von nebenberuflichen Lehrkräften unterrichtet

161 Zwei Standorte (Koordinaten: 9,5) liegen fast übereinander und sind daher nur als einer sichtbar. 
werden (Relation 27:1). Umgekehrt formuliert haben alle elf Standorte, die sich nicht am Impulsprogramm beteiligt haben, eine niedrige bis mittlere Zahl an hauptberuflich Lehrenden, die Zusammensetzung der nebenberuflich Lehrenden zeigt an acht dieser zehn Standorte einen Schwerpunkt in der Wirtschaft bzw. einen unterdurchschnittlichen Anteil an akademischen Lehrenden. Es scheint auch zwei Wachstumsstrategien zu geben, eine in Richtung hauptberuflich Lehrender und eine in Richtung nebenberuflich Lehrender (die zweite Entwicklung häuft sich tendenziell in Bundesländern mit geringer Landesförderung).

\section{Abbildung 56: Personelles Potential der FH-Standorte, 2000-2001}

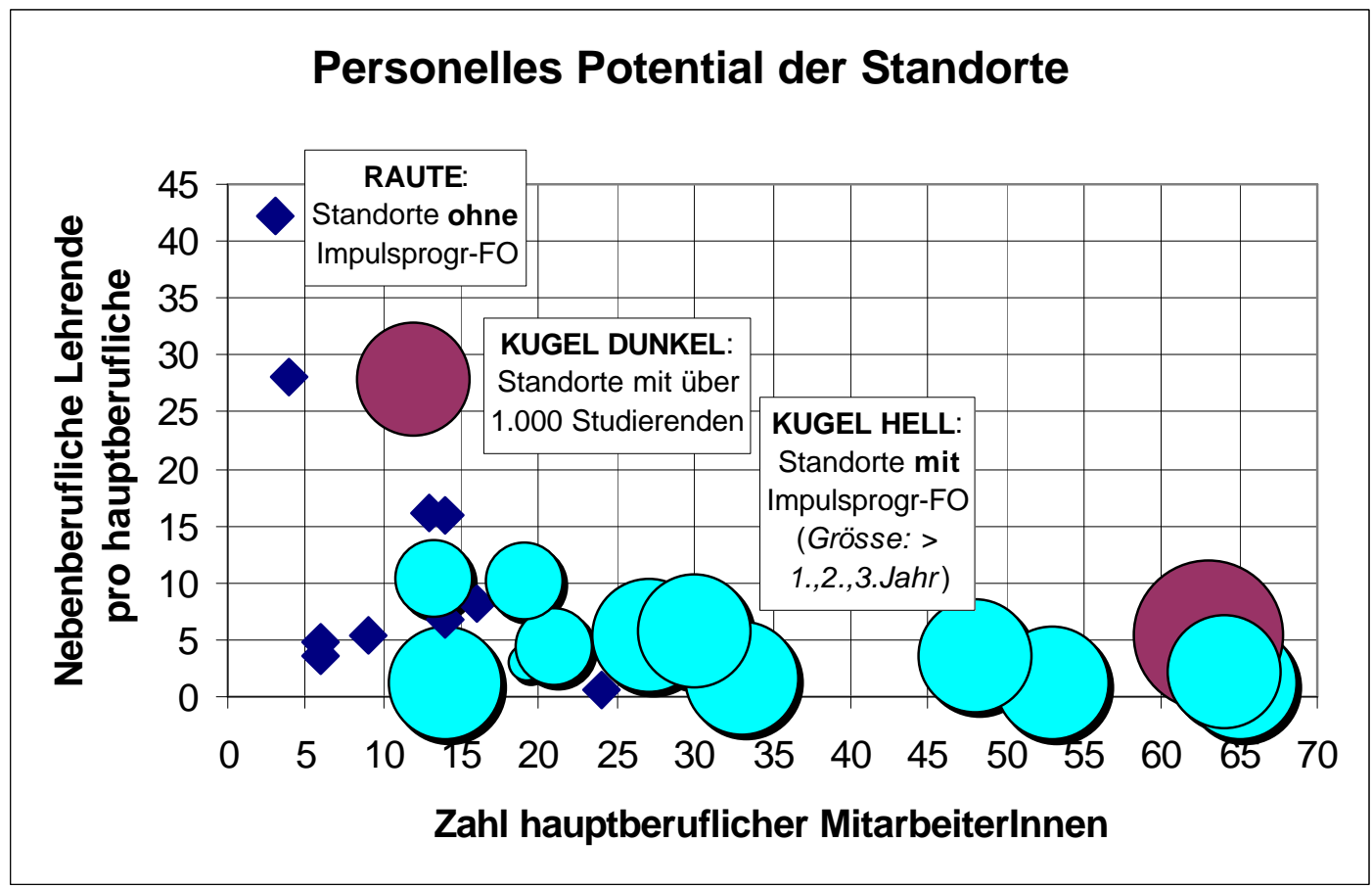

Quelle: Eigene Berechnung aufgrund der Daten und Berichte des FHR und der Erhebungen bei Erhaltern und Studiengangsleitungen

Im Folgenden wird an verschiedenen Voraussetzungen angeknüpft, um der Frage der „kritischen Größen“ näher zu kommen. Vor allem zwei Aspekte erscheinen in diesem Zusammenhang wesentlich zu sein: Erstens die Erwartungen hinsichtlich des Portfolios an Aufgaben, die die Einrichtungen erfüllen sollen, also vor allem die Kombination und Gewichtung von Lehre, F\&E und Dienstleistungen wie z.B. Weiterbildung. Zweitens die Frage nach dem Zusammenspiel der verschiedenen Ebenen und nach dem „Mehrwert“ der Organisationsebene der Fachhochschule. 
„Kritische Größen“ in der Einschätzung der Erhalter

In unserer Befragung der Erhalter haben wir die Vorstellungen über „kritische Größen“ von Standorten und „Filial“-Einrichtungen hinsichtlich der Zahl der erforderlichen Studiengänge und der Zahl der Studierenden erhoben. Es wurde sowohl nach Minima als auch nach Maxima gefragt, um die angestrebte Größenordnung zu umschreiben.

Tabelle 16: „Kritische Größen“ von Standorten bzw. Filial-Einrichtungen in der Einschätzung der Erhalter (Werte gerundet)

\begin{tabular}{|l|c|c|c|}
\hline & Minimum & Maximum & Mittel $^{*}$ \\
\hline Studiengänge (StG) pro Standort (SO) & 4 & 9 & $6-7$ \\
\hline Streubreite der Antworten & $2-5$ & $3-12$ & \\
\hline Studierende pro Standort (SO) & 870 & 2.680 & 1.780 \\
\hline Streubreite der Antworten & $300-1.500$ & $1.000-5.000$ & \\
\hline Studierende pro StG an SO & 220 & 300 & 270 \\
\hline Studierende pro Jgg. an SO & 220 & 670 & 445 \\
\hline Studierende pro StG/Jgg. an SO & 55 & 75 & 70 \\
\hline Fachrichtungen pro Standort (SO) & 2,3 & & \\
\hline Streubreite der Antworten & $2-4$ & & \\
\hline Studiengänge pro Filiale (F) & 2 & 5 & $3-4$ \\
\hline Streubreite der Antworten & $1-3$ & $1-10$ & \\
\hline Studierende pro Filiale (F) & 440 & 1.000 & 720 \\
\hline Streubreite der Antworten & $100-900$ & $400-2.500$ & \\
\hline Studierende pro StG an F & 220 & 200 & 210 \\
\hline Studierende pro Jgg. an F & 110 & 250 & 180 \\
\hline Studierende pro StG/Jgg. an F & 55 & 50 & 50 \\
\hline Fachrichtungen pro Filiale (F) & 1,6 & & \\
\hline Streubreite der Antworten & $1-3$ & & \\
\hline
\end{tabular}

*Alle kursiv gesetzten Werte aus den Angaben abgeleitet Quelle: Befragung der Erhalter

Wenn man die Angaben über die kritischen Größen in Tabelle 16 an der Realität misst, so gibt es auch unter den mindestens vierjährigen Studiengängen eine große Zahl (ca. ein Drittel), die unter der angesetzten Minimalgröße (220 Studierende) liegen, die überwiegende Mehrheit (ca. zwei Drittel) liegt unter dem Mittelwert. Nur vier Standorte erfüllen den errechneten Minimalwert aus der Zahl der Studierenden und der Zahl der Studiengänge von 870 Studierenden, kein Standort erfüllt den aus den Angaben abgeleiteten Mittelwert von 1.780 Studierenden. 


\section{Erfordernisse für F\&E nach ExpertInneneinschätzung}

Verschiedene ExpertInnen geben für das F\&E-Potential bestimmte Größenordnungen und Anhaltspunkte an, um Szenarien für eine F\&E-Wirkstruktur des FH-Sektors zu entwickeln.

Als Minimalbedingung für F\&E-Leistungen in einem speziellen Themenbereich wird angenommen, dass eine Forschungsgruppe „(...) aus mindestens drei bis vier hochqualifizierten, vollzeitäquivalenten MitarbeiterInnen bestehen muss, die auch über eine entsprechende Infrastruktur verfügen“ (Maresch 2002, 169). Unter Berücksichtigung der Lehraufgaben werden sechs vollangestellte Lehrende mit zusätzlichen MitarbeiterInnen in der Forschung angesetzt. ${ }^{162}$ Darüber hinaus wird bereits auf der Ebene der Studiengänge bezweifelt, dass eine einzige Forschungsgruppe die erforderliche Breite abdecken kann. Andere ExpertInnen gehen von Gruppengrößen von etwa sieben bis zehn MitarbeiterInnen pro Thema aus (Clement et al. 2002, Anh. 6.4). Diese müssen über entsprechende Qualifikationen in einem vergleichsweise spezialisierten Gebiet verfügen und über ihre Lehrleistungen hinaus über entsprechende freie Kapazitäten verfügen. Eine grobe Veranschlagung kann also von 5 - 10 erforderlichen Vollzeitäquivalenten als Minimum für nachhaltige F\&E-Leistungen ausgehen. Bei einer angenommenen durchschnittlichen Lehrleistung von $50 \%$ der Kapazität müssten in einem Themenbereich 10 bis 20 Personen verfügbar sein, um 5 bis 10 VZÄ zu erreichen. Als weitere Minimalbedingungen werden einschlägige Kooperationsbeziehungen mit F\&E-Zentren und mit Universitäten genannt. Erforderlich sind auch ein entsprechender organisatorischer Rahmen und ein zukunftsträchtiges Thema hinsichtlich Nachfrage und Entwicklungspotential.

Die Aufgabe der F\&E ist in den gesetzlichen Vorgaben den Studiengängen zugeordnet. Gleichzeitig können F\&E-Themen auch in losem Zusammenhang mit Studiengängen stehen, wenn entsprechende Kooperationen existieren. Wenn man von Studiengängen als Fokus für F\&E-Leistungen ausgeht, so verfügen mindestens vierjährige Studiengänge im Durchschnitt über 10 hauptberufliche Lehrkräfte. ${ }^{163}$ Nur sechs Erhalter verfügen im Durchschnitt über mehr als 10 hauptberufliche Lehrkräfte pro Studiengang. Wenn man 20 hauptberufliche

162 Maresch $(2002,169)$ demonstriert überschlagsmäßig die finanziellen Konsequenzen dieser Größenordnungen in Relation zur Förderung von Studienplätzen und zeigt den zusätzlichen Finanzbedarf, der im Zusammenhang mit der Entwicklung von F\&E entsteht: Eine Forschungsgruppe, die gleichzeitig 40\% der Lehre abdeckt, würde den gesamten Normkostenbeitrag eines Studienganges mit ca. 160 Studierenden kosten, nicht gerechnet Kosten der Infrastruktur etc. $60 \%$ der Kosten der Lehre müssten im Umkehrschluss durch Aufbringung von Mitteln für Forschungspersonal abgedeckt werden, um diese Minimalbedingung für F\&E zu erfüllen. Grünbacher/Detter haben auf der Basis von Forschungsgruppen mit vier MitarbeiterInnen überschlagsmäßig einen Finanzbedarf errechnet, wobei sie von 25\% Lehrverpflichtung dieser Mitarbeiterlnnen ausgegangen sind (Grünbacher/Detter 2000). Clement et al $(2002,51)$ gehen von einem hauptberuflichen F\&E-fähigen Anteil von $70 \%$ bis $80 \%$ des Personals an FH-Studiengängen mit einem Lehranteil von $20-50 \%$ der Wochenarbeitszeit aus, um der F\&E-Aufgabe gerecht werden zu können.

163 Für den Lehrgang Industrielle Elektronik werden 10 hauptberufliche (darunter eine/r in Teilzeit) plus neun weitere MitarbeiterInnen im Transferzentrum angegeben (Maresch 2002, 181-182). 
Lehrkräfte als Minimalwert ansetzt (um 10 freie VZÄ zu erreichen), so reduziert sich diese Zahl auf zwei Erhalter. Auf Erhalterebene, über alle Studiengänge hinweg, verfügen 12 über mindestens 10 hauptberufliche Lehrkräfte und 7 Erhalter verfügen über ein Potential von mindestens 20 hauptberuflichen Lehrkräften. Wenn man jedoch die fachliche Diversifizierung berücksichtigt und auch die Verteilung auf unterschiedliche Standorte, so ergeben sich acht Erhalter mit fach- bzw. standortspezifischen Gruppengrößen von etwa mindestens 10 Personen bzw. nur vier bis fünf Erhalter, die derartig spezifizierte Gruppengrößen von etwa 20 Personen erzielen.

Wenn man in dieser Gruppe weiter die Angaben über Stundendeputate ${ }^{164}$ berücksichtigt bzw. als Gegenprobe die Angaben über die Vollzeitäquivalente an wissenschaftlichem Personal ins Kalkül zieht, so erfüllen acht bis zehn Erhalter das Kriterium, dass mindestens in einem Fachbereich oder Standort eine Gruppengröße von sieben Personen für F\&EAufgaben vorhanden ist. Bei vier bis fünf Erhaltern ist dieses Potential etwas größer, bei drei bis fünf liegt es an der Grenze. Von den Erhaltern mit stärkeren personellen F\&E-Potentialen haben fast alle (eine Ausnahme) eine stärkere personelle Universitätsanbindung, von den schwächeren hat ein Erhalter eine stärkere Universitätsanbindung. Diese groben Vergleiche ergeben also den Schluss, dass etwa die Hälfte der Erhalter nicht über personelle Potentiale verfügt, die die Minimalkriterien für eine nachhaltige Entwicklung von F\&E-Tätigkeit erfüllen würden. Bei etwa einem Viertel der Erhalter sind derartige Potentiale in gewissem Ausmaß vorhanden.

Verfolgt man die Szenarien der Expertlnnen weiter, so können diese Kerngruppen in verschiedenen Formen in größeren Einheiten zusammenwirken:

- Spezialisierte themenspezifische F\&E-Aktivitäten in Verbindung mit Dienstleistungsangeboten im regionalen Umfeld, in Kooperation mit Technologie- oder Forschungszentren und Unternehmen („Stand alone“; Minimum ist eine F\&E-Gruppe, 10 20 Personen, Potentiale dieser Größenordnung sind gegenwärtig an den starken Standorten erreicht).

- Spezialisierte themenspezifische F\&E-Aktivitäten in einem nationalen Netzwerk vor allem mit anderen $\mathrm{FH}$-Einrichtungen und in Kooperation mit anderen Bereichen (Universitäten,

164 Maresch (2002, 178-179) geht von maximal 10 bis 12 Semesterwochenstunden für voll angestellte Fachhochschulprofessorlnnen aus. Mit dieser maximalen Belastung sollte $50 \%$ bis $70 \%$ der gesamten Lehrleistung im Studiengang erbracht werden können. Dies wird als erfahrungsgestützte "kritische Masse" gesehen. Grünbacher/Detter $(2000,10)$ berichten über eine Auslastung der Lehrkräfte im Ausmaß von 16-18 Semesterwochenstunden ("effiziente Forschungsbeiträge ... können von so belasteten wissenschaftsfähigen MitarbeiterInnen nicht erwartet werden"). Auch Grünbacher/Detter (2000, 4) gehen von mindestens 50\% festangestellten MitarbeiterInnen für die Abdeckung des Lehrbetriebs an einer konsolidierten Fachhochschuleinrichtung aus. 
Forschungszentren; Minimum ist ein Netzwerk von 3-6 Forschungsgruppen, $40-80$ Personen)

- F\&E-Zentren, die mehrere spezialisierte Themenbereiche bearbeiten und mit dem nationalen, in einer weiteren Stufe mit dem internationalen F\&E-System vernetzt und verbunden sind. Für derartige Zentren werden Größenordnungen von $30-100$ MitarbeiterInnen angesetzt, die in zusammenhängenden Gebieten arbeiten. Diese Größenordnung ist gegenwärtig für die meisten FH-Einrichtungen bei weitem nicht erreicht, die größten fünf bis sechs Erhalter haben ein Potential an wissenschaftlichem Personal in der Größenordnung zwischen 30 und 60 Vollzeitäquivalenten, aber über alle ihre Aktivitätsgebiete und inklusive der Lehrleistungen.

\subsubsection{Regionale Wirkungen von Hochschuleinrichtungen}

Regionale Wirkungen von Hochschuleinrichtungen können nach zwei großen Kategorien unterschieden werden:

a. Unmittelbare Standortwirkungen, die durch die Stationierung und Aktivität der Einrichtungen (die „Leistungserstellung“) unmittelbar anfallen. Dabei handelt es sich grob um die Investitionen (in gewissem Ausmaß Aufträge für die regionale Wirtschaft), die laufenden Ausgaben für den Betrieb, die Beschäftigung in den Einrichtungen und den Konsum der Beschäftigten und der Studierenden (Wohnung, Transport, Ernährung, Lehrmittel, Freizeit etc.), wie auch mögliche immaterielle Wirkungen der Förderung der Attraktivität oder Bekanntheit der Region. Die unmittelbaren Standortwirkungen werden wesentlich durch die Größe der Einrichtungen im Verhältnis zum regionalen Umfeld bestimmt. Kleine Einrichtungen in einem Agglomerationsgebiet werden kaum messbare Wirkungen erzielen, während in einer peripheren Region eher Wirkungen sichtbar werden können. Vorwiegend ist meist diese Art von Wirkungen gemeint, wenn von "Regionalpolitik“ gesprochen wird; in der Tat bezieht sich diese Gleichsetzung auf ein traditionelles Verständnis von Regionalpolitik, in dem distributive Interventionen vorherrschend sind.

b. Wirkungen durch die Leistungsabgabe der Hochschuleinrichtungen. Diese Wirkungen unterschieden sich von der ersten Kategorie dadurch, dass sie nicht automatisch anfallen, sondern mehr oder weniger expliziter Entscheidungen bedürfen. In der Hochschulforschung der letzten Jahrzehnte spielt diese Frage eine wesentliche Rolle, wobei von einer grundlegenden Veränderung des Leistungsprofils von Hochschulen ausgegangen wird, bei der die Entstehung und Entwicklung des nicht-universitären Sektors eine wichtige Rolle spielt. Im Prinzip wurde ursprünglich davon ausgegangen, dass die Leistungserstellung von Hochschulen - vor allem die akademische Forschung - universell, d.h. nicht an den regionalen Kontext gebunden sei. Die Leistungen finden zwar immer an einem Ort statt, Adressat der Leistungen ist aber die Welt oder zumindest die Nation. „Universities, in 
particular, exist as autonomous institutions which are often characterised by low levels of local territorial embeddedness, regulation at the national level and preoccupation with international and national academic and research communities." (OECD 1999,15). Von verschiedenen Aspekten und Befunden her wurde diese Sicht revidiert, manche Autoren sprechen in diesem Zusammenhang von einer "second academic revolution" und es wurde gezeigt, dass die Beziehungen zwischen Hochschulen und ihrem Umfeld in den letzten Jahrzehnten stark zugenommen haben. Es wird von der Entwicklung einer "dritten Funktion“ gesprochen, die Dienstleistungen der Hochschulen für ein engeres oder weiteres regionales Umfeld neben den beiden traditionellen Funktionen der Forschung und Lehre umschreibt. Folgende Arten von Wirkungen können in dieser Kategorie unterschieden werden (vgl. Benson 2000, 11-12):

- Technologietransfer im Rahmen von F\&E-Projekten

- Wissenstransfer durch Beratungs- und GutachterInnentätigkeit

- Veröffentlichung von Forschungsergebnissen über entsprechende außerwissenschaftliche Medien

- Bereitstellung von Infrastruktur (Geräte, Labors, Bibliotheken, Räumlichkeiten etc..)

- Transfer über Köpfe (Anstellung der AbsolventInnen, wechselseitige Beschäftigung von ArbeitnehmerInnen von Hochschule und Unternehmen)

- Spin-offs, Unternehmensgründungen

- Fort- und Weiterbildung

- Forschungsbedingte Zulieferaufträge (Zwischenprodukte für Forschung oder F\&EProjekte)

Im Rahmen unserer Erhebungen wurde für diese Typen von Leistungen erhoben, inwieweit diese von den befragten FH-Einrichtungen für den regionalen Kontext erstellt werden (vgl. Abbildung 57 und Tabelle 17). ${ }^{165}$ Es ergibt sich nach den drei regionalen Aggregationsebenen (regional, national, EU und international) bei den verschiedenen

165 Tabelle 17 gibt auch grobe Anhaltspunkte über aggregierte Wirkungen des FH-Sektors auf diesen Dimensionen. Der regionale Kontext wurde mit $50 \mathrm{~km}$ Umkreis definiert. Bei vielen dieser Leistungen sind die Werte nach Studiengängen extrem uneinheitlich, was möglicherweise auf unterschiedliche Bewertungen der verschiedenen Aktivitäten zurückgehen könnte. Auch "Marketing"-Überlegungen sind nicht ausgeschlossen. Vermutlich sind die Angaben auch unvollständig, so dass die Angaben und Schätzungen über Volumina insgesamt eher am unteren Rand des tatsächlichen Leistungsvolumens liegen (es ist z.B. nicht immer zwischen Null und keine Angabe unterscheidbar). 
Leistungen der Größenordnung nach eine ziemlich einheitliche Struktur, derzufolge der Schwerpunkt im regionalen Kontext liegt (mit einer Größenordnung von etwa der Hälfte bis zu drei Vierteln der unterschiedlichen Leistungskomponenten). An zweiter Stelle liegt die nationale Ebene (mit Größenordnungen zwischen etwa einem und zwei Fünfteln) und an dritter Stelle die übernationale Ebene (mit einer Größenordnung zwischen über $5 \%$ bis zu 20\%; vgl. Abbildung 57).

\section{Abbildung 57: Leistungen des FH-Sektors 2001/02 nach Aggregationsebenen (Regional, national, international)}

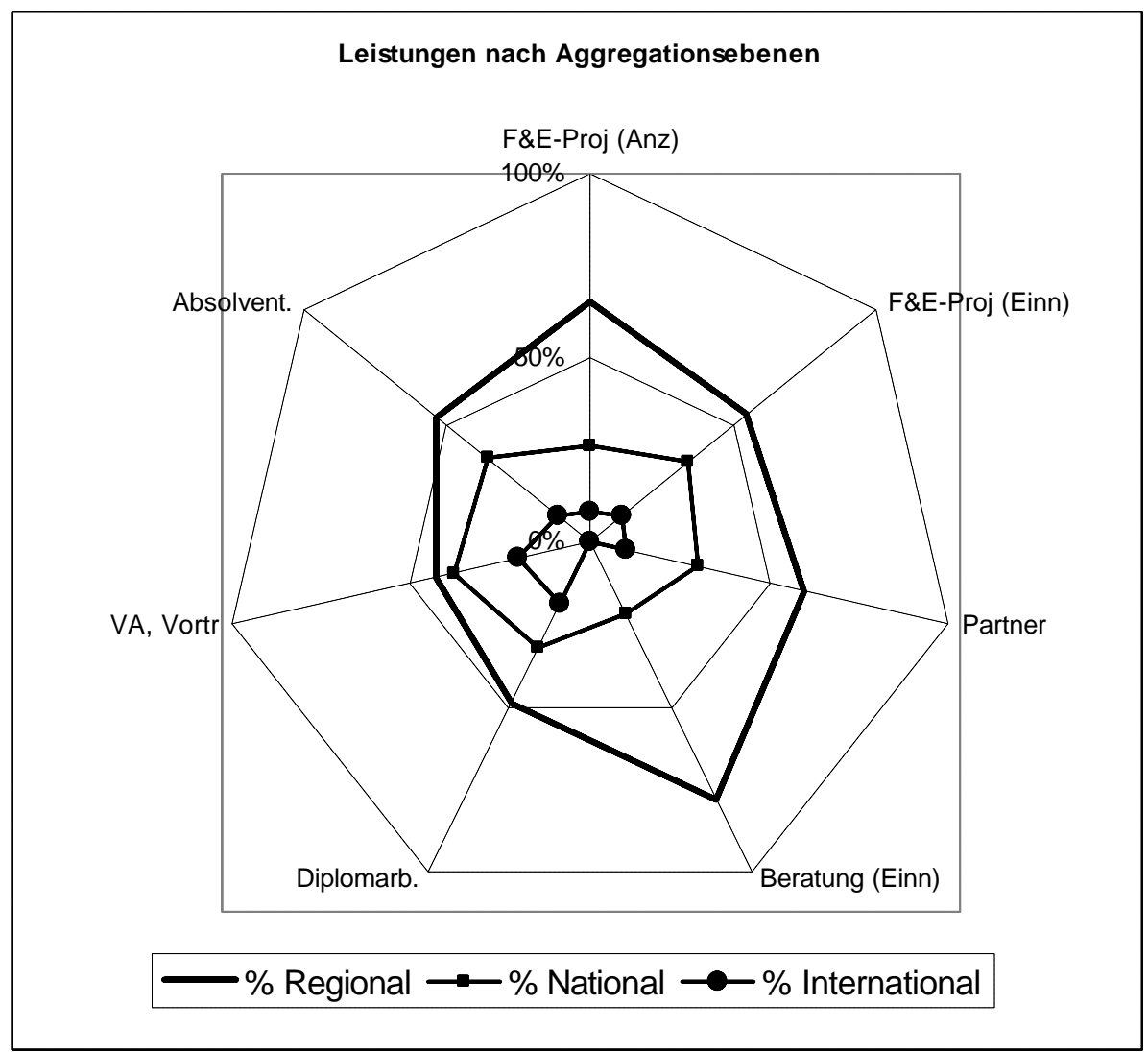

Quelle: Befragungen der Erhalter und Studiengangsleitungen

Eine Reihung der verschiedenen Leistungen nach den drei Aggregationsebenen ergibt ebenfalls eine nachvollziehbare Struktur, derzufolge sich die Beratungsleistungen und Gutachten sowie die F\&E-Projekte im regionalen Kontext konzentrieren, während Vorträge und Veranstaltungen und auch die Leistungen im Zusammenhang mit der Lehre (AbsolventInnenmarkt, bezahlte Diplomarbeiten) höhere Anteile im nationalen und internationalen Kontext aufweisen. Es zeigt sich darin einerseits die regionale Positionierung und andererseits, dass Bemühungen bestehen, sowohl im nationalen als auch im EU- und internationalen Kontext tätig zu werden. 
Tabelle 17: Reihung der Anteile der Leistungen des FH-Sektors 2001/02 auf den Aggregationsebenen regional, national und EU bzw. international

\begin{tabular}{|c|c|c|}
\hline $\begin{array}{l}\text { Reihung der Anteile } \\
\text { Regional }\end{array}$ & $\begin{array}{l}\text { Reihung der Anteile } \\
\text { National }\end{array}$ & $\begin{array}{l}\text { Reihung der Anteile } \\
\text { EU, International }\end{array}$ \\
\hline $\begin{array}{l}\text { Einnahmen für Gutachten-Beratung } \\
(\mathrm{ERH})\end{array}$ & Veranstaltungen, Vorträge & Veranstaltungen, Vorträge \\
\hline Anzahl F\&E-Projekte (ERH) & AbsolventInnenmarkt & Anzahl Diplomarbeiten gegen Entgelt \\
\hline Kooperationspartner F\&E-Projekte & Einnahmen F\&E-Projekte (ERH) & AbsolventInnenmarkt \\
\hline Einnahmen F\&E-Projekte (ERH) & Anzahl Diplomarbeiten gegen Entgelt & Einnahmen F\&E-Projekte (ERH) \\
\hline AbsolventInnenmarkt & Kooperationspartner F\&E-Projekte & Kooperationspartner F\&E-Projekte \\
\hline Anzahl Diplomarbeiten gegen Entgelt & Anzahl F\&E-Projekte (ERH) & Anzahl F\&E-Projekte (ERH) \\
\hline Veranstaltungen, Vorträge & $\begin{array}{l}\text { Einnahmen für Gutachten-Beratung } \\
(\mathrm{ERH})\end{array}$ & $\begin{array}{l}\text { Einnahmen für Gutachten-Beratung } \\
\text { (ERH) }\end{array}$ \\
\hline
\end{tabular}

Quelle: Befragungen der Erhalter und Studiengangsleitungen

Tabelle 18: Leistungen des FH-Sektors 2001/02 nach regionalen Aggregationsebenen (Volumen kumuliert)

\begin{tabular}{|c|c|c|c|c|c|c|}
\hline & Art, Einheit & $\begin{array}{c}\% \\
\text { Regional } \\
\end{array}$ & $\begin{array}{c}\% \\
\text { National }\end{array}$ & $\begin{array}{c}\% \\
\text { EU, Int. }\end{array}$ & $\begin{array}{l}\text { Umfang It. } \\
\text { Erhebung }\end{array}$ & $\begin{array}{l}\text { Umfang hoch- } \\
\text { gerechnet }^{166}\end{array}$ \\
\hline \multirow{3}{*}{$\begin{array}{l}\text { F\&E-Projekte } \\
2001 \text { (Erhalter) }\end{array}$} & Anzahl der Projekte & $65 \%$ & $26 \%$ & $8 \%$ & 185 & 336 \\
\hline & Einnahmen $(€)$ & $55 \%$ & $34 \%$ & $11 \%$ & 2.881.083,- & $5.054 .531,-$ \\
\hline & Kooperationspartner & $60 \%$ & $30 \%$ & $10 \%$ & 150 & 341 \\
\hline \multirow{3}{*}{$\begin{array}{l}\text { Beratung, } \\
\text { Gutachten }\end{array}$} & Einnahmen Erhalter $(€)$ & $78 \%$ & $22 \%$ & $0 \%$ & 172.444,-- & 374.878,-- \\
\hline & $\begin{array}{l}\text { Unternehmenskontakte } \\
\text { F\&E (Studiengänge) }\end{array}$ & - & - & - & 747 & 1.067 \\
\hline & $\begin{array}{l}\text { Diplomarbeiten gegen } \\
\text { Entgelt }\end{array}$ & $49 \%$ & $32 \%$ & $19 \%$ & 568 & 835 \\
\hline \multirow{3}{*}{$\begin{array}{l}\text { Veröffentlich- } \\
\text { ungen, Vorträge, } \\
\text { Veranstaltungen }\end{array}$} & Insgesamt & - & - & - & 1.130 & 1.687 \\
\hline & $\begin{array}{l}\text { Davon Vorträge/ } \\
\text { Veranstaltungen }\end{array}$ & $43 \%$ & $38 \%$ & $20 \%$ & 641 & 957 \\
\hline & $\begin{array}{l}\text { Davon wiss. } \\
\text { Publikationen. }\end{array}$ & - & $60 \%$ & $40 \%$ & 272 & 406 \\
\hline \multirow{3}{*}{ Infrastruktur } & $\begin{array}{l}\text { Bibliotheksbesuche von } \\
\text { Externen pro Monat }\end{array}$ & $100 \%$ & - & - & 388 & 902 \\
\hline & $\begin{array}{l}\text { Vermietung von } \\
\text { Räumen (€/ Jahr) }\end{array}$ & $100 \%$ & - & - & 966.363,- & 1.323.784,- \\
\hline & $\begin{array}{l}\text { Vermietung von Geräten } \\
(€ / \text { Jahr) }\end{array}$ & - & - & - & $0,-$ & $0,-$ \\
\hline \multirow{2}{*}{ AbsolventInnen } & $\begin{array}{l}\text { Marktanteile } \\
\text { Absolventlnnen }\end{array}$ & $54 \%$ & $36 \%$ & $11 \%$ & - & - \\
\hline & $\begin{array}{l}\text { Unternehmenskontakt } \\
\text { Job Placement }\end{array}$ & - & - & - & 950 & 1.357 \\
\hline $\begin{array}{l}\text { Fort- und } \\
\text { Weiterbildung }\end{array}$ & Personen-Stunden & $100 \%$ & - & - & 24.698 & 39.203 \\
\hline
\end{tabular}

Quelle: Befragungen der Erhalter und Studiengangsleitungen

166 Hochrechnung anhand der durch die Antworten repräsentierten Zahl von Studierenden. 
In der Online-Erhebung bei den Studiengängen wurden auch die Unternehmenskontakte näher erhoben. Im Durchschnitt wurden pro Studiengang etwa 60 Unternehmenskontakte angegeben, etwa die Hälfte im Zusammenhang mit der Vorbereitung und Durchführung von Praktika. ${ }^{167}$ Die andere Hälfte der Kontakte bezieht sich in etwas größerem Ausmaß (je etwa ein Sechstel) auf die Dimensionen „Placement der AbsolventInnen“ und „Feedback zur Qualitätssicherung“ und in etwas geringerem Ausmaß (je etwa ein Zehntel) auf die Dimensionen „F\&E“ sowie „Konsultationen zur strategischen Weiterentwicklung der Studiengänge“. Nach Erhaltern gibt es unterschiedliche Muster: bei etwas mehr als einem Drittel dominieren die Praktika-Kontakte (teilweise mit unterschiedlichen Schwerpunkten bei den anderen Aktivitäten; in dieser Gruppe ist die Qualitätssicherung meist ein weiterer Schwerpunkt). Bei einer zweiten Gruppe von etwa einem weiteren Drittel der Erhalter haben die Kontakte zur strategischen Weiterentwicklung - meistens im Zusammenhang mit Qualitätssicherung - ein erhöhtes Gewicht (zusammen $60 \%$ bis $80 \%$ ). Bei einer dritten Gruppe von Erhaltern sind die verschiedenen Dimensionen ausgewogener verteilt, wobei bei den meisten F\&E-Kontakte bzw. teilweise auch Placement-Kontakte höheres Gewicht haben (etwa in der Größenordnung von $20 \%$ bis $30 \%$ der Kontakte). Die unterschiedlichen Gewichtungen hängen nicht mit der Zahl der Unternehmenskontakte zusammen.

Auch die personelle Besetzung der Studiengänge und die regionale Positionierung ist nicht mit der Zahl der Unternehmenskontakte verknüpft. Die Studiengänge in den größeren Agglomerationen haben nicht systematisch mehr Unternehmenskontakte als jene in weniger konzentrierten Regionen. Auch wenn man für das verfügbare hauptberufliche Personal standardisiert, haben beispielsweise die Studiengänge in Wien zwischen 6 und 54 Unternehmenskontakte pro Lehrkraft angegeben.

Es wurde auch erhoben, in welchem Ausmaß sich die Unternehmenskontakte auf die verschiedenen regionalen Aggregationsebenen erstrecken und in welchem Ausmaß $\mathrm{KMUs}^{168}$ einbezogen sind. Dabei zeigt sich, dass regionale Unternehmen besser einbezogen sind (etwa 60\% aller Unternehmenskontakte finden mit Unternehmen aus der Region statt) als kleinere und mittlere Betriebe (diese machen ein Drittel der Kontakte aus). Die Streubreite nach Erhaltern und auch nach Standorten liegt beim Anteil der Unternehmenskontakte in der Region zwischen $24 \%$ und fast $100 \%$, bei den KMU-Kontakten liegt diese im Bereich zwischen etwa 15\% und 75\% bei den Erhaltern bzw. zwischen 0\% und etwa 50\% nach Studienorten. Nach regionaler Zentralität oder dem Agglomerationsgrad ist jedenfalls kein linearer Zusammenhang zum Anteil der regionalen Unternehmenskontakte

167 Die Streubreite der Zahl der durchschnittlichen Unternehmenskontakte pro Studiengang nach Erhaltern liegt vorwiegend in der Größenordnung zwischen unter 10 bis 100, bei zwei Erhaltern liegt diese Zahl bei 200 bis 250. Manche Studiengänge haben vermutlich auch Unternehmensbefragungen als Kontakte gewertet und erreichen dadurch eine hohe Zahl an Kontakten. Der Durchschnitt wird durch den größten Ausreißer um 5 Kontakte erhöht.

168 Es werden verschiedene Maße für die Abgrenzung von kleineren und mittleren Betrieben in der Literatur verwendet, wir haben die KMUs mit 50 Beschäftigten abgegrenzt. 
erkennbar (hohe und niedrige Anteile liegen gleichermaßen eher in nicht so zentral gelegenen Standorten, die großen Agglomerationen haben eher mittlere Werte, aber es gibt auch andere Standorte mit mittleren Werten). Es gibt wenig Zusammenhang zwischen den beiden Dimensionen, am ehesten in der Richtung, dass wenig regionale Kontakte auch nur in Ausnahmefällen mit häufigen KMU-Kontakten verbunden sind. Fünf Erhalter (also etwa ein Viertel) haben einen gewissen Schwerpunkt ihrer Kontakte mit regionalen KMUs, diese konzentrieren sich stark auf die großen Agglomerationen Wien und Graz.

\subsubsection{Regionale Planungen und Strategien}

Für die Untersuchung der regionalen Planungen und Strategien im FH-Sektor ist das Zusammenspiel mit der Entwicklung von regionaler Innovationspolitik von besonderer Bedeutung. „Regionale Innovationssysteme“ bilden daher einen wichtigen Anknüpfungspunkt für die Entwicklung des FH-Sektors auf regionaler Ebene. Die bisherigen Erfahrungen und Analysen über die Entwicklung regionaler Innovationssysteme geben deshalb wichtige Hintergrundinformationen für das Verständnis der regionalen FH-Politik.

\section{Regionale Innovationssysteme}

Ein wesentlicher Aspekt regionaler Planungen und Strategien besteht darin, wieweit die regionalen Akteure auf politischer und administrativer Ebene in der Lage sind, die regionalen Zusammenhänge und Bündelungen auf der wirtschaftlichen und gesellschaftlichen Ebene (die nicht mit den administrativen und politischen Strukturen übereinstimmen müssen) einerseits zu repräsentieren und andererseits zu entwickeln. „Das traditionelle, nach formalen Kompetenzen gegliederte Aufgabenverständnis in der öffentlichen Verwaltung und Politik entspricht immer weniger den Anforderungen der Praxis und ist auch seit längerem in Veränderung begriffen." (ÖROK 2001, 35). Entsprechend wird die Entwicklung von regionalpolitischen Zielen und Strategien, die die verschiedenen regionalen Ebenen auf den Fokus der Entwicklung des "Standortes Österreich in Europa“ bündeln sollen, im Raumentwicklungskonzept stark betont.

In der Praxis liegt der Fokus der Entwicklung von Innovationssystemen aufgrund der spezifischen Bedingungen auf der Ebene der Bundesländer. Auf Bundesebene werden seit einigen Jahren verstärkte Schritte zur Überwindung zersplitterter Strukturen gesetzt, die bisher in der Entwicklung von Strategieelementen zuerst im Rahmen der Technologieberichte und dann im Rahmen des FTE-Rates resultiert haben. Die Zusammenarbeit zwischen Bund und Ländern wurde in der ersten Hälfte der 1990er Jahre durch Regionalabkommen zwischen dem zuständigen Bundesministerium und den Ländern entwickelt. 
Eine Übersicht zur Entwicklung in den Ländern bietet die Analyse der Regionalen Innovationspolitik 2000 (Tödtling-Schönhofer 2000 et al.). Obgleich ein junger Politikbereich, kann die Innovationspolitik in allen Bundesländern bereits als wichtige wirtschaftspolitische Schiene gesehen werden, stärker in den traditionellen Industriebundesländern (Steiermark, Oberösterreich, Niederösterreich) als in den eher touristisch und ländlich geprägten Bundesländern (Salzburg, Tirol, Kärnten). Wesentliche Elemente der Umsetzung sind Entwicklungsgesellschaften, die in verschiedenen Formen in allen Bundesländern bestehen, der Aufbau von Impulszentren ${ }^{169}$ und die Vernetzung dieser Impulszentren untereinander, mit der regionalen Wirtschaft und mit den Ausbildungssystemen.

„Ein wesentliches Umsetzungsproblem ist das Erreichen kritischer Massen vor allem in den ländlichen, wenig industrialisierten Gebieten, um synergetische multiplikative Effekte in Richtung innovatives Klima (bis hin zum mittlerweile äußerst inflationär eingesetzten ClusterBegriff) zu erreichen und nachhaltig zu festigen" (ebd., 69). Eine neuere Studie über die Wirkungen der Technologiezentren (Urban 2001) unterstreicht erstens die Probleme der kleinteiligen lokalen Strukturen und der überregionalen Koordination (,... es gibt zu wenig Abstimmung der Clusteraktivitäten der Länder untereinander und mit dem Bund." Ebd., 6). Zweitens wird die große Bedeutung öffentlicher Trägerschaft und damit die wichtige Rolle der Länder hervorgehoben: ,... überall dort, wo die öffentliche Hand an den Technologie- und Innovationszentren mehr oder weniger beteiligt ist, hat sie Interesse, dass ... durch die Zentren für die Wirtschaft in der Region Impulse gesetzt werden." (ebd., 78) Damit sind vor allem die Bundesländer Steiermark (Steirische Wirtschaftsförderung, Immofinanz), Oberösterreich (TMG), Kärnten (Technologie Land Kärnten GmbH, KWF) und Niederösterreich (RIZ-Holding) angesprochen, in denen das Land in unterschiedlichen Strukturen eine tragende Rolle spielt. „Auf der anderen Seite hat sich in den sogenannten ,alten, etablierten Zentren' wie in Salzburg, Linz, Innsbruck oder auch Götzis gezeigt, dass aufgrund der durchwegs privaten Eigentümerschaft die Impulsfunktion für die Region verloren gegangen ist. Man konzentriert sich in diesen Standorten und im Bundesland Salzburg auch in den sechs regionalen Zentren leider ausschließlich auf das Immobilienmanagement." (ebd., 78). Drittens verweist die Studie auf die steigende Bedeutung von Forschung und Qualifizierung in den Zentren, vor allem aus der Sicht der beteiligten Betriebe: „Die klassischen Förderthemen ,Produktentwicklung' und ,Einführung neuer Produktionstechnologien' rangieren längst nicht mehr an der Spitze des Förderbedarfes und sind verdrängt worden durch den Bedarf an Unterstützung bei den sogenannten ,soft factors': Qualifizierung MitarbeiterInnen, Suche Fachpersonal sowie Marketing- und Kooperationspartner. Die klassische Trennung in Ausbildungs- bzw. Innovations- und Technologieförderung ... sollte daher von einer integralen Innovationspolitik abgelöst werden..." (ebd., 49). Die Firmen bemängelten u.a. „die geringe Ansiedelung von

169 Science Parks, Technologietransferzentren, Gründer- und Technologiezentren, Innovationszentren, Wirtschaftsparks. 
Forschungseinrichtungen in den Zentren“ (ebd., 79) und derartige Einrichtungen sind auch einer der wichtigsten Wünsche für die Zukunft (ebd., 81).

Obwohl die Qualifikation der Beschäftigten als wichtiger Faktor für die Innovationsfähigkeit der Unternehmen und auch für ihre Adoptionsfähigkeit von technologischem Wissen von entscheidender Bedeutung ist und obwohl auch die Bedeutung der Vernetzung von Wissenschaft und Wirtschaft (etwa über Transfereinrichtungen) als wesentliche Faktoren für die Innovationstätigkeit seit langem bekannt sind, besteht über weite Strecken nach wie vor eine Trennung zwischen Innovationspolitik und Qualifizierungspolitik. Erst seit Mitte der 1990er Jahre haben „weiche“ Maßnahmen, wie eben die Förderung der Qualifikation der Beschäftigten, größere Bedeutung in der Förderung von Forschung, technologischer Entwicklung und Innovation bekommen, sodass von einem Paradigmenwechsel gesprochen wird (Tödtling-Schönhofer et al. 2000, 104-106).

Man kann hier von einer Konvergenz zwischen der Entwicklung der regionalen Innovationspolitik einerseits und dem Aufbau des FH-Sektors andererseits in den 1990er Jahren sprechen. Ein Schlüsselpunkt dieser Konvergenz ist die Wahrnehmung der F\&EFunktionen des FH-Sektors. Diese Funktion war zwar von Anfang an als Aufgabe von FHStudiengängen gegeben, wurde aber erst in den späten 1990er Jahren tatsächlich realisiert. Teilweise gibt es immer noch Reservationen. Die F\&E-Funktion ist deshalb ein Schlüsselpunkt, weil die Erstausbildung für die Innovationsentwicklung der Unternehmen nur sehr mittelbar von Bedeutung ist und weil das Spektrum und die Fähigkeit zur Wahrnehmung der Dienstleistungsfunktion letztlich von den F\&E-Kapazitäten abhängt. Beiträge zur Innovation im Unternehmenssektor können nur erwartet werden, wenn die vorhandenen Kompetenzen das bei den Nachfragern vorhandene Niveau übersteigen.

Die Einbindung des FH-Sektors in die Strategie der Entwicklung der österreichischen Forschungsstrategie (vgl. Abschnitt 3.2.3) bringt die Konvergenz zwischen den beiden Politikbereichen gewissermaßen auf den Punkt.

\section{FH-Strategien der Bundesländer}

Eine umfassende Analyse der FH-Strategien der Bundesländer ist im Rahmen des vorliegenden Projektes aufgrund der begrenzten Ressourcen nicht möglich. ${ }^{170}$ Es kann nur versucht werden, aufgrund der Recherchen und ExpertInnengespräche einige Grundzüge dieser Strategien herauszuarbeiten. Drei Aspekte werden näher betrachtet: erstens die Entwicklung von Methoden zur Lösung von Standortfragen, zweitens die Grundmerkmale

170 Vgl. für einen detaillierteren Überblick über die Entwicklung in den Bundesländern Pechar 1999. 
von Entwicklungsstrategien der Länder und drittens das Problem des Zusammenspiels von Bildungspolitik, Wirtschaftspolitik und Regionalpolitik.

Lösung von Standortfragen. Die Form der Entwicklung des FH-Sektors in einem „bottomup"-Prozess hat den Raum für Initiativen vonseiten verschiedenster Akteure eröffnet. Das Interesse, den Aufbau rasch zu beginnen und die Angabe von angestrebten Größenordnungen in den Entwicklungs- und Finanzierungsplänen hat auch eine gewisse Pfadabhängigkeit der Entwicklung erzeugt: Wer zuerst da war, hat eben Tatsachen geschaffen und einen entsprechenden Teil der Ressourcen an sich gezogen. Bei der Standortfrage kommt die Dualität der beiden regionalen Ebenen, Gemeinden und Länder, zum Tragen. Es wurde von Anfang an in verschiedenen Bundesländern versucht, den Prozess der Standortwahl zu systematisieren, wobei in großem Maße auf Beispiele und Praktiken in Deutschland zurückgegriffen wurde.

Als Beispiel kann die Entwicklung in Kärnten herangezogen werden, die gut dokumentiert ist. Dabei wurde die Vorgangsweise in Oberösterreich ausdrücklich als Vorbild genommen. Der Prozess wurde mit einem Ausschreibungsverfahren für Standorte begonnen, das eine umfassende Standortanalyse beinhalten sollte (Pongratz 1993). Es erging seitens des Landes im Februar 1993 eine Aufforderung an 8 ausgewählte Gemeinden (nach einer Regionstypologie), sich zu bewerben. Zwei Gemeinden haben verzichtet, sechs haben eine Bewerbung eingereicht, aber nur eine der sechs Bewerbungen entsprach den Ausschreibungserfordernissen für die Standortanalyse. Diese Analyse hat die in der Übersicht angegebenen Kriterien gut erfüllt, die Analyse des Studierendenpotentials enthält jedoch eine wesentliche Überschneidung (für etwas weniger als die Hälfte des Potentials) mit einem zweiten geplanten Standort (Leitner 1992). Dies macht die Problematik der Koordination deutlich, da aus der Sicht eines Standortes andere (potentielle) Standorte nicht unmittelbar relevant sind. Ein aktuelleres FH-Konzept für Kärnten enthält im Wesentlichen eine Beschreibung des Sektors, mehr oder weniger kursorische Beschreibungen der Studiengänge in den Kategorien laufend, eingereicht und geplant (Technikum Kärnten 1998) und als Plan eine deskriptive quantitative Darstellung der erwarteten Entwicklung der Studiengänge nach der Zahl der jährlichen Studienplätze im Zeitraum 1995/96 - 2004/05, eine grafische Gesamtdarstellung im Vollausbau und eine Verteilung der Studienplätze auf Standorte ohne Angabe des Zeitpunktes (wobei in den Darstellungen unterschiedliche Gesamtzahlen enthalten sind). Die Standorte, die zunächst nicht zum Zuge gekommen sind, sind nach wie vor in Diskussion. Das ursprünglich angestrebte systematisierte Verfahren einer Standortprüfung hat sich offensichtlich nicht durchgesetzt, die Standortfrage hat sich stärker in den Raum politischer Verhandlungen verlagert. Beispiele aus Deutschland zeigen, 
dass diese Problematik bis zu einem gewissen Grad durchaus verallgemeinert werden kann. $^{171}$

\section{Abbildung 58: Kriterien für die Standortanalyse in Kärnten}

- Absorptionsfähigkeit des Arbeitsmarktes

- Transfermöglichkeiten für F\&E (Wirtschaftsstruktur/Probleme; regionalpolitische Entwicklungsstrategien; Zahl und Qualifikationsstruktur der Arbeitsplätze; technologisch-organisatorischer Stand; Innovationspotential; allgemeine Entwicklungsperspektive; Struktur der Arbeitslosigkeit)

- Ausstattungsqualität des Standortes (Verkehr; Infrastruktur; Ressourcen-Gebäude, Ausstattung, Personal)

- Prüfung der Bildungsnachfrage (Bevölkerung nach Alter; Bildungsinfrastruktur; Lehrbetriebe-Qualität; Bildungsverhalten; Mobilität)

Quelle: nach Pongratz 1993.

Nach vielen Auseinandersetzungen um die Errichtung neuer Standorte und mit der sich öffnenden Schere zwischen der Zielsetzung der Konsolidierung einerseits und immer neuen Vorschlägen für neue Standorte stellt sich trotz der Ansiedelung der Entscheidungsbefugnis im politischen Bereich weiterhin die Frage der Systematisierung der Standortwahl.

Für Niederösterreich wurde vom Österreichischen Institut für Raumplanung ein umfassendes Konzept der Standortprüfung entwickelt (Delapina/Holzinger/Schausberger 2001). Dieses Standortprüfungsmodell für NÖ geht von einem innovationspolitischen Ansatz aus, der bildungspolitische und regionalpolitische Aspekte berücksichtigt. Bildungsinstitutionen werden als Teil der „Infrastruktur zur Wissensproduktion und verteilung" gesehen und Wissen als die strategische Ressource für regionale Innovationsfähigkeit: „Die Verteilung der Bildungseinrichtungen im Raum ist daher ausschlaggebend."(ebd., 11) FH-Einrichtungen sind Impulsgeber (aktiv) und Versorger mit Qualifikationen (passiv), wobei Abweichungen von einem idealen Pfad in zwei Richtungen möglich sind: Strukturkonservierung durch zu starke Konzentration auf den bestehenden Bedarf (relative Unterversorgung) oder durch zu stark überfordernde Impulse (relative Überversorgung). Aufgrund der dynamischen Veränderungen der Bedarfsentwicklung wird analog zu den Empfehlungen des deutschen Wissenschaftsrates ein "laufender Diskussionsprozess zwischen den bedarfsbestimmenden Akteuren“ in Verbindung mit Forschung und Monitoring als erforderlich angesehen.

171 Eine Fall-Darstellung über Bayern zeigt, wie sich politische Absichten im Laufe eines Prozesses ändern können: In einem aufwändigen zweistufigen Verfahren ist eine Auswahl von Standorten erfolgt, indem für drei ursprünglich von der Politik angestrebte Standorte aus 25 Bewerberregionen durch das Staatsinstitut drei begründete Dreiervorschläge entwickelt wurden. Letztlich wurden von der Politik nicht die ursprünglich angestrebten drei, sondern acht Standorte realisiert, die jedoch alle aus der Liste der ersten Stufe des Auswahlverfahrens ausgewählt wurden, in der neun Bewerberregionen erfolgreich waren (Schindler 2001). 
Neben den bildungspolitischen Aspekten der Standortplanung (Wechselbeziehungen, Steuerungsmechanismen, Finanzierung, Autonomie) werden drei regionalpolitische Aspekte unterschieden: Regionswirksamkeit, räumliche Nähe-Distanz-Strukturen, Attraktivität. Hinsichtlich der Regionswirksamkeit werden die FH-Einrichtungen als Teil der regionalen Innovationsinfrastruktur konzipiert, wobei Innovationsimpulse durch $\mathrm{FH}$-Institutionen als „Netzwerkknoten zwischen Ausbildungssystem, Wirtschaft und anwendungsbezogener Forschung" (ebd., 13) entstehen sollen. Da im Innovationssystem die Interaktionen und Schnittstellen zwischen Akteuren im Mittelpunkt stehen und nicht-kodifizierbares Wissen eine wesentliche Rolle spielt, muss eine innovationsorientierte Regionalpolitik „sowohl auf die Stärkung unternehmerischer Forschung und Entwicklung ausgerichtet sein, als auch die Vernetzung der Unternehmen mit dem Wissenschaftssystem, dem Bildungssystem und anderen Akteuren fördern." (ebd., 13). In diesem Zusammenhang gewinnt die strukturpolitische Komponente von Aus- und Weiterbildung an Bedeutung, da sie mit den verschiedenen Aspekten des "upgradings“ regionaler Wirtschaft verbunden ist (Steigerung des Technologiegehaltes der Produkte und Verfahren; Gründungstätigkeit; Stärkung der unternehmerischen Funktionen Marketing, Management und F\&E; Steigerung des Potentials für Ansiedlungen). Drei Elemente werden besonders hervorgehoben: bei der Ausbildung die regionale Nähe und Einbindung, bei F\&E die Einbindung von KMUs und bei der Infrastruktur die Berücksichtigung von Weiterbildung im weitesten Sinne. Hinsichtlich der Attraktivität der FH-Einrichtungen wird die Spannung zwischen den positiven Wirkungen großer zentraler Institutionen durch Kostendegression und der Entstehung von „kritischer Masse“ einerseits und den negativen Wirkungen eines "Massenbetriebes“ andererseits betont; als Schlüssel für den Erfolg kleiner regionalisierter Einrichtungen werden die Potentiale der Zusammenarbeit mit regionalen Unternehmen hervorgehoben. In der Spannung zwischen Nähe und Distanz wird die Strategie der Dislozierung als mögliche Überwindung des „scheinbaren Gegensatzes von Dezentralisierung und Konsolidierung“ (ebd., 21) gesehen. Die Vorteile von Filialen sollten klar herausgearbeitet werden, wobei die Anbindung an das regionale Innovationssystem als Ersatz für das „akademische Umfeld“ gewertet wird. Aufgrund des Risikos von Fehlentscheidungen wird bei dislozierten Standorten eine „besonders sorgfältige Prüfung“ als erforderlich erachtet (ebd., 23).

Die Grunddimensionen des ÖIR-Modells der Standortprüfung sind

- die Eignung des Standortes zur Zielerfüllung und räumliche Wirkungen sowie

- die Tragfähigkeit des Standortes.

Für diese Dimensionen wird ein sehr ausdifferenziertes Standardset an 13 Prüfkriterien (7 Kriterien für Eignung und Wirkungen; 6 Kriterien für Tragfähigkeit) und 48 Indikatoren (Eignung und Wirkungen: 28 Indikatoren; Tragfähigkeit: 20 Indikatoren) vorgeschlagen, die noch weiterer Ausarbeitung bedürfen. Es wurde eine Gewichtung der Prüfkriterien nach vier Gestaltungsfaktoren vorgeschlagen, nämlich nach: 
- regional- und bildungspolitischen strategischen Vorentscheidungen, ob die Gründung für das Umfeld kompensatorisch oder additiv/verstärkend wirken soll,

- dem geplanten Angebotstypus für das Studienangebot (Kategorisierung Unikat, neuartig, konkurrierend im FH-Sektor, konventionell),

- dem Status des Standortes hinsichtlich des Grades der Konsolidierung,

- dem regionalen Lagetypus nach NUTS III - Regionen (Ballungsraum, ballungsraumnahes, ballungsraumfernes oder regionales Zentrum; 30).

Die in der Analyse für diese Studie herangezogenen Indikatoren (Delapina/Holzinger/ Schausberger 2001, 41) erlauben einen typologischen Vergleich der FH-Strukturen der Bundesländer zum Stand 1999/2000. Sieben Merkmale wurden für diese Typologie verwendet: Inhalt (zwischen konventionell und Unikat), Art des Studienangebots (Vollzeit oder berufsbegleitend), Lage nach Regionstyp (zentral bis dezentral), Größe der Gemeinde (Einwohnerlnnenzahl), Zahl der Studiengänge, Status hinsichtlich Konsolidierung und Zahl der Studierenden. Diese Merkmale wurden bewertet, wobei als Referenzwert 0 ein konventioneller Studiengang in Vollzeit in zentraler Lage mit Status im Aufbau und niedrige Werte bei den quantitativen Indikatoren (Einwohner, Studiengänge, Studierende) angesetzt wurde. Die Indikatoren wurden auf den österreichischen Durchschnitt mit 1 standardisiert. Je höher der Wert für ein Bundesland, je entwickelter und diversifizierter der Ausbaustand.

Die Bundesländer haben nach diesen Merkmalen sehr unterschiedliche Profile (vgl. Abbildung 59, Abbildung 60, Abbildung 61), es lassen sich aber auch gewisse gemeinsame Typen feststellen:

- Vier Bundesländer (N, B, O, K) haben, wenn auch auf sehr unterschiedlichem Niveau, einen hohen Grad an Regionalisierung, wobei vor allem Kärnten als einziges Bundesland hinsichtlich aller Indikatoren unter dem Referenzwert von 1 liegt (vgl. Abbildung 59).

- Die drei westlichen Bundesländer (S, V, T) haben gemeinsam, dass sie eher zentralisiert sind, einen Schwerpunkt bei den berufsbegleitenden Studiengängen setzen und trotz einer vergleichsweise kleinen Zahl an Studiengängen mit Ausnahme von Tirol einen konsolidierten Status mit einer durchschnittlichen Studierendenzahl und durchschnittlich neuartigen Inhalten haben (vgl. Abbildung 60).

- Wien und die Steiermark haben jeweils eine einzigartige Struktur. Wien sticht durch seine Größe und ein etwas erhöhtes Gewicht von berufsbegleitenden Studiengängen hervor. Die Steiermark sticht durch den Spitzenwert bei inhaltlich neuartigen Studiengängen hervor, bei mit Wien vergleichbarer Zahl an Studiengängen und konsolidiertem Status (vgl. Abbildung 61). 


\section{Abbildung 59: FH-Profile der Bundesländer Niederösterreich, Burgenland, Oberösterreich und Kärnten, Stand 1999/2000}

\section{FH-Profile Bundesländer: N, B, O, K}

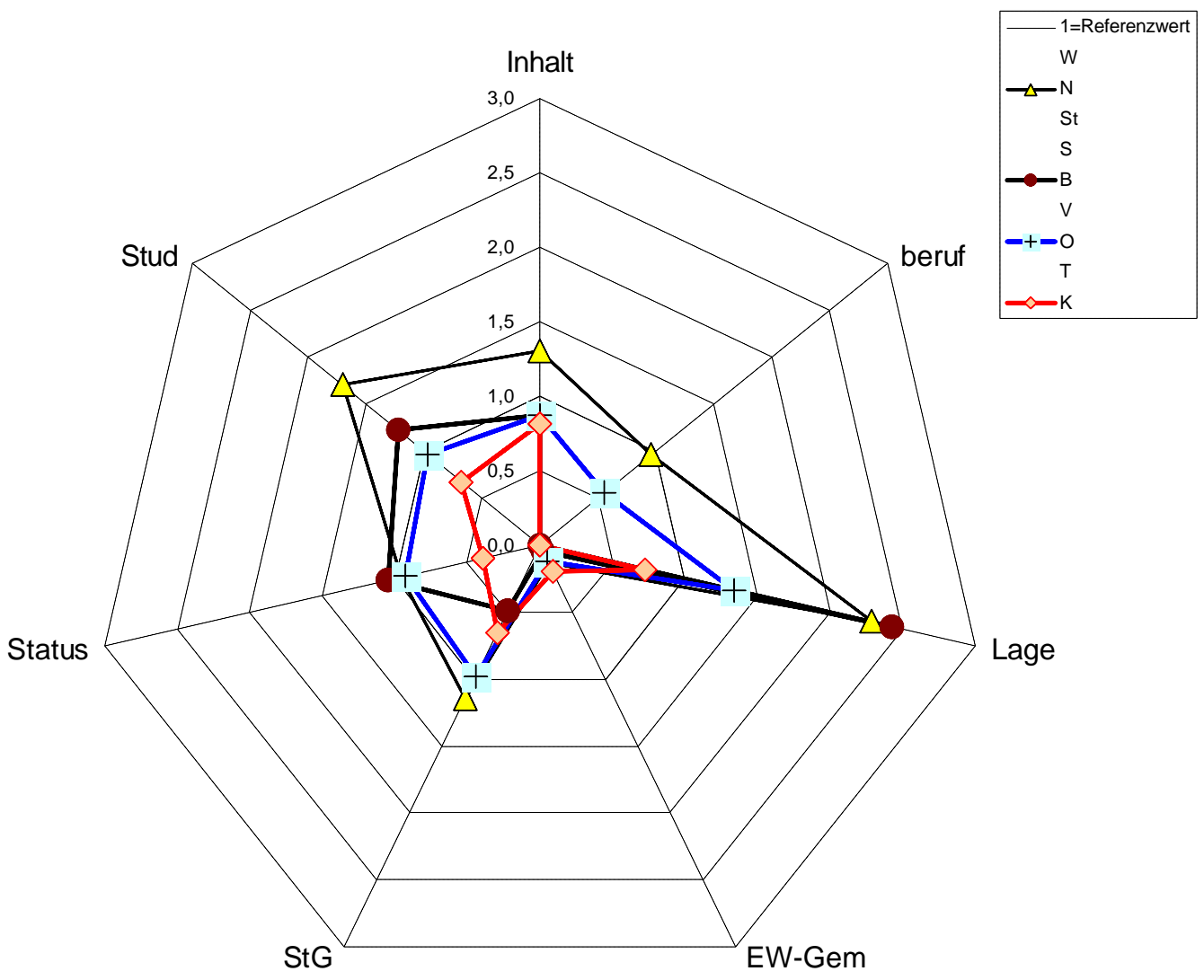

\section{MERKMALE}

Inhalt: Inhalt zwischen konventionell und Unikat

Beruf: Vollzeit oder berufsbegleitend

Lage: Lage nach Regionstyp zentral bis dezentral

EW-Gem: Größe der Gemeinde

StG: Zahl der Studiengänge

Status: Status hinsichtlich Konsolidierung

Stud: Zahl der Studierenden

Referenzwert 0: konventioneller Studiengang in Vollzeit in zentraler Lage mit Status im Aufbau und niedrige Werte bei den quantitativen Indikatoren (EinwohnerInnen, Studiengänge, Studierende)

Die Indikatoren wurden auf den österreichischen Durchschnitt $(=1)$ standardisiert. Je höher der Wert auf einer der Dimensionen für ein Bundesland, je entwickelter und diversifizierter der Ausbaustand. 
Abbildung 60: FH-Profile der Bundesländer Salzburg, Vorarlberg und Tirol, Stand 1999/2000

\section{FH-Profile Bundesländer: S, V, T}

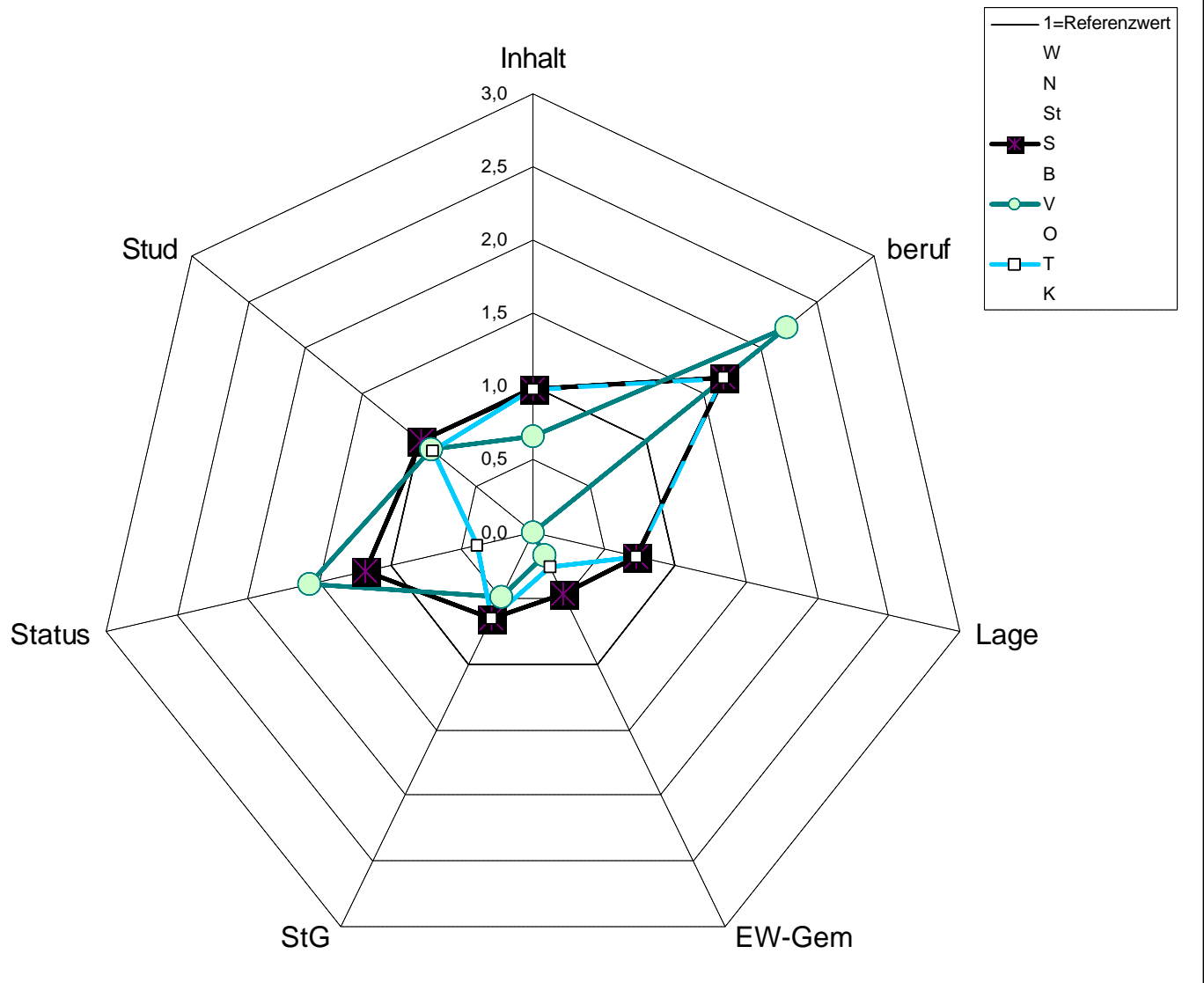

\section{MERKMALE}

Inhalt: Inhalt zwischen konventionell und Unikat

Beruf: Vollzeit oder berufsbegleitend

Lage: Lage nach Regionstyp zentral bis dezentral

EW-Gem: Größe der Gemeinde

StG: Zahl der Studiengänge

Status: Status hinsichtlich Konsolidierung

Stud: Zahl der Studierenden

Referenzwert 0: konventioneller Studiengang in Vollzeit in zentraler Lage mit Status im Aufbau und niedrige Werte bei den quantitativen Indikatoren (EinwohnerInnen, Studiengänge, Studierende)

Die Indikatoren wurden auf den österreichischen Durchschnitt $(=1)$ standardisiert. Je höher der Wert auf einer der Dimensionen für ein Bundesland, je entwickelter und diversifizierter der Ausbaustand.

Quelle: IHS-Berechnungen auf Grundlage von Delapina/Holzinger/Schausberger 2001 


\section{FH-Profile Bundesländer: W, St}

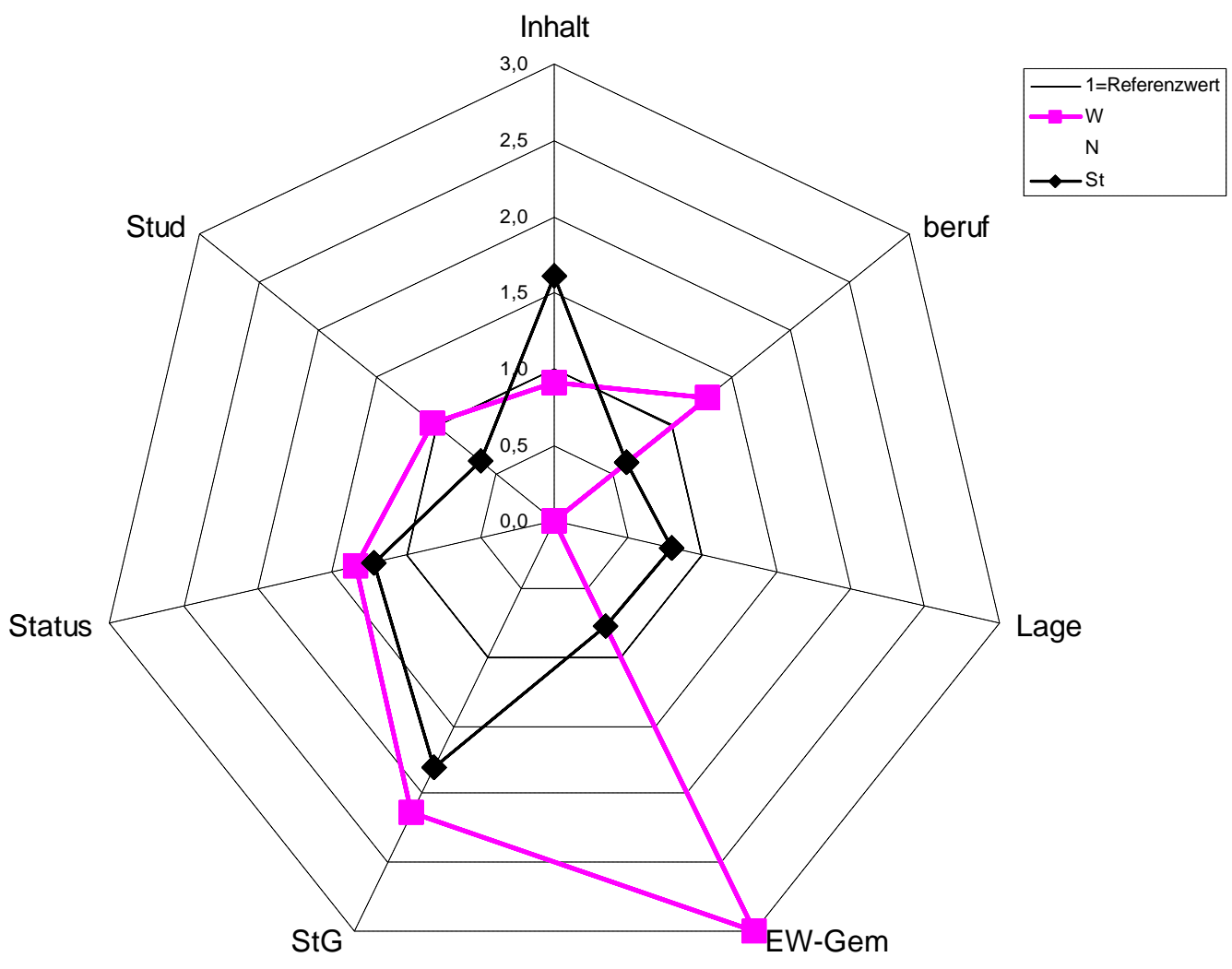

MERKMALE

Inhalt: Inhalt zwischen konventionell und Unikat

Beruf: Vollzeit oder berufsbegleitend

Lage: Lage nach Regionstyp zentral bis dezentral

EW-Gem: Größe der Gemeinde

StG: Zahl der Studiengänge

Status: Status hinsichtlich Konsolidierung

Stud: Zahl der Studierenden

Referenzwert 0: konventioneller Studiengang in Vollzeit in zentraler Lage mit Status im Aufbau und niedrige Werte bei den quantitativen Indikatoren (EinwohnerInnen, Studiengänge, Studierende)

Die Indikatoren wurden auf den österreichischen Durchschnitt $(=1)$ standardisiert. Je höher der Wert auf einer der Dimensionen für ein Bundesland, je entwickelter und diversifizierter der Ausbaustand.

Quelle: IHS-Berechnungen auf Grundlage von Delapina/Holzinger/Schausberger 2001 
Entwicklungskonzepte der Länder. Ähnlich dem bereits skizzierten Konzept des Technikums Kärnten haben die meisten Bundesländer zu verschiedenen Zeitpunkten FHKonzepte entwickelt. Diese Konzepte bringen in der Regel die Resultate von vorausgegangenen mehr oder weniger komplexen und fundierten Konsultationsprozessen in konzentrierter Form zum Ausdruck. Aufgrund der verschiedenen Formen der Verflechtung mit den Erhalterorganisationen finden konkrete Planungen auf verschiedenen Ebenen statt. Diese enthalten meistens eine Beschreibung der wesentlichen Merkmale und Prozeduren des FH-Sektors auf allgemeiner Ebene, eine Beschreibung des Status-quo im jeweiligen Bundesland bis zur Ebene der Studiengänge, Überlegungen und Leitgedanken für die Politik und die wesentlichen Ziele und Eckdaten für die weitere Entwicklung und Finanzierung des Sektors, v.a.:

- geplante Studiengänge

- angestrebte Zahl an Studienplätzen

- Standorte

- Finanzierung

Die Konzepte für den FH-Sektor in den Bundesländern sind auch mit vorhandenen weitergehenden Entwicklungskonzepten der Länder verbunden. Die meisten bisher vorliegenden Konzepte enthalten jedoch keine tiefergehenden Untersuchungen zu den erwarteten Ergebnissen der FH-Einrichtungen, geschweige denn Kosten/NutzenUntersuchungen. Diese Aspekte werden im Prinzip an die Mechanismen der Qualitätsprüfung delegiert. Hinsichtlich der Standorte enthalten die Konzepte teilweise klare Prioritätensetzungen, oft ergänzt durch mehr oder weniger lange Listen von interessierten Bewerberstandorten. Beispielsweise wurden in Rahmenplan 1995 der Steiermark neben den unmittelbar angepeilten Standorten weitere 14 interessierte Orte ausdrücklich genannt.

Eine umfassende Entwicklungsplanung für den FH-Sektor wird in Niederösterreich angepeilt, wo im Zusammenhang der Entwicklungsgesellschaft eine Bildungsges.m.b.H. aufgebaut wurde, deren Rolle in der Koordination der Bildungspolitik des Landes liegt. Ein bisher veröffentlichtes Ergebnis ist das skizzierte Konzept zur Standortprüfung, weitere Vorarbeiten und Überlegungen zum Aufbau eines Monitoring-Systems sind noch im Gange.

Eine spezielle Situation besteht auch in Wien, wo sich das Land vom FH-Sektor lange Zeit weitgehend ferngehalten hat, aber vor einiger Zeit Überlegungen zu einem verstärkten Engagement im Wege der Förderung von Entwicklungsprojekten und Innovationen in Projektform angestellt wurden. Laut den Angaben in der Befragung sind jedoch 2001/02 keine nennenswerten Landesmittel eingesetzt worden (vgl. auch Pechar 1999). 
Insgesamt tragen die Länder einen wesentlichen Anteil an der Finanzierung des Sektors (vgl. Abschnitt 4.2.2), mit der Ausnahme von wenigen Ansätzen haben die Länder aber die angebotsseitige „bottom-up“-Entwicklung im Rahmen der durch die Entwicklungsplanung des Bundes vorgegebenen Eckdaten mitgetragen und keine bis wenig systematische Ansätze zur Koordinierung des Sektors erkennen lassen. Mit der Aktion 600+ haben die Länder ihre Finanzierungsbeiträge verstärkt und so zur jüngsten sprunghaften Expansion beigetragen. Unsere Erhebungen haben auch Anhaltspunkte dafür ergeben, dass die Befriedigung der Bildungsnachfrage, die in der mancherorts hohen Relation „BewerberInnen pro Aufgenommenem/r" zum Ausdruck kommt, eine wichtige Rolle in der Politik der Länder spielt.

Systematische übergreifende Antizipations- oder Früherkennungssysteme werden auf der Länderebene nicht entwickelt und auch Überlegungen zur Dimensionierung der FHEinrichtungen zur Erfüllung eines bestimmten Aufgabenprofils gehen nicht über die Umsetzung der allgemeinen Vorgaben der gesetzlichen Grundlagen und der Entwicklungsplanung hinaus. Die weiter oben skizzierten unterschiedlichen Typologien der Länder sind also eher als emergentes Resultat der Aktivitäten der Erhalter und der FHEinrichtungen zu sehen denn als Resultat bewusster politischer Entwicklungsstrategien und Zielplanungen.

Schnittpunkt von Bildungspolitik, Wirtschaftspolitik und Regionalpolitik. Die Potentiale der FH-Einrichtungen für ihren Beitrag zum Innovationssystem können nur teilweise ausgeschöpft werden, solange nicht für alle Funktionen entsprechende Ziele und Erwartungen formuliert werden. Erst dann kann beurteilt werden, welche Kapazitäten für die Erfüllung dieser Erwartungen erforderlich sind. Wenn festgestellt wird, dass die FHEinrichtungen einen Beitrag zur Entwicklung der F\&E im regionalen Kontext liefern sollen, so ist das zu wenig, um Entscheidungen über die erforderlichen Kapazitäten zu treffen. Welchen Beitrag zu welchen Aufgaben im Rahmen der F\&E? Dies muss näher spezifiziert werden.

Die Tendenz zur Ausgliederung dieser Fragen an die Bedarfsstudien bringt die Gefahr der „relativen Unterversorgung“ mit sich. Auf der anderen Seite müssen innovative Impulse mit dem vorhandenen Umfeld rückgekoppelt werden.

\subsection{Entwicklungsfragen im Bereich der regionalen Standortpolitik}

Die wesentlichen Entwicklungsfragen im Bereich der regionalen Standortpolitik liegen in der Bestimmung der Zielsetzungen von Regionalpolitik und regionaler Entwicklung. Dies ist mit dem „Paradigmenwechsel in der Regionalpolitik“ angesprochen: Nicht mehr die regionale Verteilung von Infrastrukturelementen steht im Mittelpunkt, sondern die Impulsfunktion für regionale Entwicklung in wirtschaftlicher, sozialer und kultureller Hinsicht. Gleichzeitig steigt 
in der Globalisierung die Bedeutung der Region und es entstehen neue räumliche Strukturen, die die traditionellen Gliederungen der Gebietskörperschaften und auch der nationalen Grenzen überschreiten, sowohl in der Größenordnung als auch in ihrer Funktionalität („multiple Räume“). Die Hochschulen bekommen in diesen Veränderungen eine größere Bedeutung und auch neue Funktionen für die Region.

Zum Abbau der regionalen Disparitäten in der Versorgung mit Bildungseinrichtungen kommen damit wesentliche neue Gesichtpunkte hinzu. Im Zentrum steht die Frage nach den Voraussetzungen, die gegeben sein müssen, damit eine $\mathrm{FH}$-Einrichtung ihre regionale Impulsfunktion erfüllen kann. Diese Frage hat mehrere Aspekte:

- erstens die Voraussetzungen hinsichtlich der Funktionserfüllung (Aufgabenportfolio, erforderliche Ressourcen bzw. „kritische Größe“, geeignete organisatorische Strukturen),

- zweitens die Einbindung und Vernetzung in den regionalen Strukturen und mit den regionalen Akteuren,

- drittens das erforderliche Potential zur Überbrückung der verschiedenen regionalen Aggregationsebenen in der Internationalisierung.

Je nach der Bestimmung der Zielsetzungen für FH-Studiengänge können diese Aspekte unterschiedlich abgedeckt werden. Dies wird in den stilisierten Entwicklungspfaden in Kapitel 3.3 herausgearbeitet. Das Aufgabenportfolio kann z.B. im Extremfall auf die Lehre konzentriert werden, die den gegebenen lokal-regionalen Qualifikationsbedarf auf einem gehobenen praktischen Niveau erfüllen soll, mit geringem Grad an nationaler oder internationaler Einbindung. In diesem Fall werden die Voraussetzungen hinsichtlich der erforderlichen Ressourcen eine andere Form annehmen als im anderen Extremfall, wo alle drei Aspekte umfassend erfültt werden sollen: volle Erfüllung des gesamten Aufgabenportfolios (Lehre, F\&E und Dienstleistungen inklusive Weiterbildung), ergebnisorientierte Festlegung von Impulsen für die Region, volle internationale Einbindung zur Erfüllung der Brückenfunktion.

Auf der Ebene der politischen Gestaltung und Steuerung des FH-Sektors schlagen sich diese Entwicklungsfragen in mehreren Aspekten nieder. Erstens stellt sich aus der Sicht der nationalen Steuerung die Frage der Verbindung der Bildungs- und Qualifizierungspolitik mit den anderen Bereichen der regionalen Innovationspolitik, zweitens stellt sich für die regionale Ebene die Aufgabe einer gezielten Einbindung der FH-Einrichtungen in die Entwicklung der regionalen Innovationspolitik, drittens stellt sich die Aufgabe der wirksamen Koordination dieser beiden Ebenen, die auch die Problematik der neuen räumlichen Strukturen berücksichtigt (und damit die Segmentierung durch Ländergrenzen und nationale Grenzen überschreitet). 


\subsubsection{Wachstumsdynamik und Standortpolitik}

Die Standortpolitik - in quantitativer wie in qualitativer Hinsicht - hängt eng mit den Entscheidungen über die weitere Wachstumsdynamik zusammen. Die Entscheidung für den Entwicklungspfad des Null-Wachstums impliziert notwendigerweise, dass eine Vermehrung der Standorte zu einer Verkleinerung der Durchschnittsgrößen führt bzw. dass eine Vergrößerung der Standorte mit einer Verminderung ihrer Zahl einhergeht. Null-Wachstum aus der Sicht der Entwicklungsplanung des Bundes impliziert daher ziemlich sicher Verteilungskonflikte zwischen den bestehenden Institutionen, wenn nicht nennenswerte zusätzliche Finanzierungsquellen auf Dauer mobilisiert werden können.

Ein zweiter Aspekt der Wachstumsdynamik betrifft die Unterscheidung zwischen den Funktionen im Aufgabenportfolio. Solange die Entwicklungsplanung des Bundes an die Zahl der Studierenden gebunden ist, bedeutet Wachstum in erster Linie Wachstum der Studierendenzahl und der damit verbundenen Kapazitäten. Falls aber auch Finanzierungstitel für die anderen Funktionen des Aufgabenportfolios eingerichtet werden, bedeutet Wachstum der Finanzmittel nicht unbedingt auch Wachstum der Studierendenzahl. Eine derartige Umstellung würde aber die bisherige Form der Entwicklung überschreiten und eine qualitative Veränderung des FH-Systems (und auch der Beziehungen im Hochschulsystem) bedeuten.

Im Folgenden werden einige Aspekte, die die Implikationen der verschiedenen Entwicklungspfade betreffen, etwas näher beleuchtet: Expansionspläne der Erhalter, Komponenten der Bildungsnachfrage wie Bewerberlnnen, regionale Potentiale oder Potentiale für Zugänge im Bereich der Weiterbildung. Dies kann nur grob überschlagsmäßig nach Größenordnungen erfolgen, da eine quantitative Projektion die vorhandenen Informationen und die Kapazitäten des vorliegenden Projektes überschreiten würde. Insgesamt ergeben diese Überlegungen einen weiten Spielraum für unterschiedliche Wachstumspfade, deren Realisierung mehr oder weniger inkrementell bzw. mehr oder weniger gesteuert vor sich gehen kann.

\section{Angaben der Erhalter}

Die Angaben der Erhalter im Rahmen der Befragung ergeben ein moderates Wachstum der Studierenden. In den nächsten fünf Jahren sollen die bestehenden Studiengänge um 20\% aufgestockt werden (das entspricht einer Wachstumsrate von 3\%) und 40 neue Studiengänge sollen eingerichtet werden. Bei Anwendung der durchschnittlichen Studierendenzahl pro Studiengang entspricht dies einer Wachstumsrate von 38\%. Die Zahl neuer Plätze beträgt im Zieljahr nach dieser Rechnung etwa 2.700 zusätzliche AnfängerInnenstudienplätze, darunter 1.000 in bestehenden und 1.700 in neuen Studiengängen. Insgesamt ergibt sich eine Wachstumsrate von 7\%. Der größere Teil dieser 
Zuwächse würde bereits im Jahr 2002/03 realisiert. ${ }^{172}$ Diese Wachstumsrate liegt deutlich unter jener der Vergangenheit, die sich bereits in den letzten Jahren abgeschwächt hat (im gesamten Zeitraum beträgt das jährliche Wachstum der Aufgenommenen 29\%, in den letzten vier Jahren mit 15\% nur noch etwa die Hälfte - dies entspricht wohl nicht dem oft bemühten Wort von „exponentiellem Wachstum“).

\section{Entwicklung der BewerberInnenzahlen}

Die Bewerberlnnenzahlen wachsen deutlich stärker als die Zahl der Aufgenommenen. Seit Beginn beträgt das jährliche Wachstum der Bewerberlnnen 41\% (gegenüber 29\% der Aufgenommenen). Auch hier findet eine Abschwächung auf $22 \%$ in den letzten vier Jahren statt (gegenüber $15 \%$ bei den Aufgenommenen).

Neben der Frage nach dem Gewicht von Mehrfachbewerbungen ist die Entwicklung der Wahl von FH-Studien im Vergleich zu Universitätsstudien, wie in Kapitel Stellenwert 3.1.2 bei der Analyse der Akzeptanz gezeigt wurde, gegenwärtig überaus schwer vorherzusagen, nicht zuletzt wegen des in seinen Auswirkungen noch weitgehend offenen BolognaProzesses.

\section{Ausschöpfung des Bildungspotentials Erstausbildung}

Für die mögliche Ausschöpfung des Bildungspotentials kann man auf die immer noch bestehenden regionalen Disparitäten im Hochschulzugang bzw. im Zugang zu tertiären Angeboten zurückgreifen. Es wurde gezeigt, dass die Ausschöpfung einer Kohorte in den städtischen Agglomerationen trotz der Verringerung der Disparitäten immer noch das Doppelte der Ausschöpfung in den regionalen Einheiten mit kleineren Siedlungsgrößen beträgt (für alle tertiären Angebote $40 \%$ gegenüber etwa 20\%; vgl. Abschnitt 5.2.1). Insgesamt stimmt die Schätzung aufgrund des Mikrozensus für 2000 mit den Werten der Hochschulplanungsprognose gut überein ${ }^{173}$. Absolut würde auf Basis der Werte von 2000 eine Erhöhung der Ausschöpfung einer Alterskohorte in allen regionalen Einheiten auf das großstädtische Niveau von $40 \%$ zu einer Erhöhung der Zugänge in das Hochschulsystem um fast 12.000 zusätzliche Studierende in einem Jahr führen. Leider kann hier keine Unterscheidung zwischen den verschiedenen Ausbildungsformen getroffen werden, wenn man noch einen Abschlag von 2.000 für andere tertiäre Angebote macht, so kann man für

172 Wenn man die für 2002/03 bereits umgesetzten Zuwächse in den Sockel einberechnet, ergeben sich Wachstumsraten von ebenfalls $3 \%$ in den bestehenden Studiengängen und von $18 \%$ in den neuen Studiengängen, die gesamte jährliche Rate beträgt in dieser Rechnung $9 \%$.

173 Ein durchschnittlicher Zugangsjahrgang It. Mikrozensus beträgt 2000 etwa 26.000 Personen, die Summe der Zugänge (erstmalig Zugelassene) aus FH- und Universitätssektor liegt It. Hochschulplanungsprognose für 2000 bei etwa 27.000 (wobei sich diese Zugänge nicht nur auf einen Altersjahrgang beziehen). 
die zusätzlichen potentiellen Zugänge zum FH-Sektor verschiedene Annahmen treffen: Die tatsächliche Verteilung im Jahr 2000 würde 1.400 ergeben, ein Wert von 25\% der Zugänge ${ }^{174}$ würde 2.500 ergeben. De-facto ist der Wert von den Zugangspotentialen her nach oben offen bzw. mit 10.000 begrenzt, wenn man (wie in der Hochschulplanungsprognose der Fall) die Potentiale für die Universitäten als ausgeschöpft betrachtet und wenn man für die Zugänge aus den ländlicheren Gebieten außerdem eine gewisse Neigung für den FH-Sektor unterstellt. Im Vergleich zu den realen Zugangszahlen in den FH-Sektor sind das jedenfalls ganz beträchtliche Potentiale (bereits die Minimalannahme erhöht den Zugang für 2000 bereits um 40\%).

\section{Bildungspotential im lebenslangen Lernen}

Bereits im Hintergrundbericht zur OECD-Prüfung der Errichtung des FH-Sektors (BMWF/ BMUK 1993) wurden aufgrund der damaligen Beteiligung an intensiven Weiterbildungsmaßnahmen unter bestimmten Zusatzannahmen Nachfragepotentiale für einen „recurrent“-Zugang in den zu errichtenden FH-Sektor demonstriert. Dabei handelte es sich nicht um ohne weiteres realisierbare Werte, sondern um Potentiale, die bei entsprechender Prioritätensetzung abrufbar (gewesen) wären. Die Werte demonstrieren, dass insbesondere im Bereich der LehrabsolventInnen bereits bei ziemlich restriktiven Annahmen (zwischen MIN: $10 \%$ der halbierten Beteiligung an intensiven ${ }^{175}$ Weiterbildungskursen und MAX: $30 \%$ der tatsächlichen Beteiligung an intensiven Weiterbildungskursen) beträchtliche quantitative Potentiale entstehen. Die damaligen Schätzungen sind Tabelle 19 wiedergegeben.

Tabelle 19: Potentiale für Fachhochschulzugang im lebenslangen Lernen

\begin{tabular}{|l|c|c|}
\hline & MIN-Schätzung & MAX-Schätzung \\
\hline traditionell Studienberechtigte & 2.400 & 9.900 \\
\hline traditionell nicht Studienberechtigte & & \\
- mit Lehrausbildung & 2.850 & 17.400 \\
- mit mittlerer Schule & 750 & 4.800 \\
Zusammen & 6.000 & 32.100 \\
\hline
\end{tabular}

Quelle: BMWF 1992a

174 „In der unmittelbar anschließenden Ausbauphase soll bis 2010 der Anteil der FachhochschulStudienanfängerInnen auf $25 \%$ aller StudienanfängerInnen erhöht werden. Dies bedeutet bei gleichbleibender StudienanfängerInnenquote eine Steigerung der Fachhochschul-Studienplätze auf ca. 27.000. Ein weiterer Ausbau, um auch dem offenen Zugang näher zu kommen, würde einer erheblichen Steigerung der Finanzmittel bedürfen." (BMWVK 1998, 11).

175 Intensiv: Kurse mit mehr als 1 Monat Vollzeitausbildung. 
Demografische Prognosen, MaturantInnenprognosen, Hochschulplanungsprognose

Die demografischen Prognosen gehen von einem langfristigen Rückgang der Geburtsjahrgänge und auch des Anteils der jungen Jahrgänge in der Bevölkerung in Zukunft aus; bei den MaturantInnen wirkt sich der demografische Umschwung in der Hochschulplanungsprognose in den Jahren um 2012 aus, in den nächsten 10 Jahren ist also auch unter den eher restriktiven Annahmen noch mit einer Steigerung des Potentials zu rechnen. Unter expansiven Annahmen (Fortschreibung des Trends im Zugang zu den höheren Schulen der Periode 1983-1993) ist für den gesamten Zeitraum bis 2030 bei entsprechenden Umschichtungen in Richtung der höheren Schulen (AHS und BHS) ein Halten des Maximalniveaus möglich.

Es ist klar, dass sich damit ein sehr breiter Korridor ergibt, der wesentlich durch bildungspolitische Entscheidungen geprägt wird: erstens, welche weitere Entwicklung ist im Schulwesen bzw. zwischen Schulwesen und Lehrlingsausbildung angestrebt bzw. realisierbar; zweitens, welche Entwicklung ist im Zugang zur tertiären Ebene bzw. zum Hochschulwesen angestrebt; drittens, welche Verteilungen sind innerhalb der tertiären Ebene angestrebt?

\subsubsection{Leistungen von Hochschulen und regionale Innovationssysteme}

Es wurde gezeigt, dass die Beiträge von Hochschulen für die Entwicklung von regionalen Innovationssystemen weit über die Funktion der Ausbildung von entsprechend qualifizierten Arbeitskräften hinausgehen (vgl. Abschnitt 5.2.3). Es geht auch um das Potential für F\&E, um die Entwicklung von Dienstleistungen und Weiterbildungsangeboten, um essentielle Beiträge in der Vernetzung der Akteure und der Förderung der Kooperation zwischen Unternehmen und um die Überbrückung zur überregionalen Ebene.

Im Zusammenhang mit den Leistungen des FH-Sektors für die Entwicklung der regionalen Innovationssysteme ist nicht nur - und auch nicht in erster Linie - die Entwicklung der Studierenden betroffen, sondern vor allem die Entwicklung der Potentiale an den Standorten. Die Analysen haben in vielfältiger Weise gezeigt, dass derzeit nur in wenigen Standorten die Potentiale für einen nennenswerten Beitrag zum regionalen Innovationssystem in diesem umfassenden Sinne bestehen. Um die entsprechenden Wirkungen zu erzielen, müsste in die Funktionen, die über die Lehre hinausgehen, wesentlich investiert werden. Auch die bisherigen Programme zur Förderung von F\&E können aufgrund der vorsichtigen Dimensionierung nur geringe Effekte erzielen.

Eine wichtige Entwicklungsfrage ist die Gestaltung des Zusammenspiels zwischen der Lehre und den anderen Funktionen im Aufgabenportfolio: sollen diese eher parallel und mehr oder weniger unabhängig entwickelt werden oder in engem Zusammenspiel, wie dies dem 
Anspruch nach an den Universitäten der Fall sein sollte. Falls ein Zusammenwirken der Funktionen angestrebt wird, sollte dies jedenfalls in expliziten Strukturen erfolgen, die nicht nur das Zusammenspiel, sondern auch die Zurechenbarkeit der Funktionen ermöglichen.

\subsubsection{Szenarien der Erhöhung der Forschungsquote}

In den Überlegungen des FTE-Rates kommt den F\&E-Aktivitäten im FH-Sektor eine wesentliche Rolle zu (vgl. Abschnitt 5.2.2). Die Überlegungen sind jedoch bisher stark auf die finanzielle Seite bezogen, die personelle Seite wurde nur sehr ansatzweise berücksichtigt. In einschlägigen Gutachten werden dem FH-Sektor sehr große Potentiale zugeschrieben, die vermutlich jedoch den Status-quo nicht realistisch einschätzen.

Im Hinblick auf das Zusammenspiel der Funktionen wird in diesen Überlegungen eine enge Zusammenarbeit zwischen Lehre, F\&E und Dienstleistungen unterstellt. Inwieweit die Qualifizierung im FH-Sektor auch in bestimmtem Ausmaß auf den Nachwuchs in F\&E ausgerichtet sein soll, wird nicht näher thematisiert.

\subsection{Zusammenfassung und Schlussfolgerungen}

\subsubsection{Zusammenfassung}

Die Regionalisierung wurde nach den folgenden Dimensionen untersucht: Abbau regionaler Disparitäten beim Hochschulzugang, Entwicklung der Potentiale nach Standorten, Erwartungen an „kritische Größen“ aus dem Sektor, erforderliche Potentiale für F\&ELeistungen, regionale Wirkungen von Fachhochschul-Studiengängen im Vergleich zur nationalen und internationalen Dimension und bisherige Fachhochschulstrategien der Länder, insbesondere im Hinblick auf Standortprüfung und Aspekte der Entwicklungsplanung.

Es gibt empirische Anhaltspunkte dafür, dass die regionale Zugänglichkeit von Bildungsangeboten nach der Sekundarstufe durch die Errichtung des FH-Sektors erhöht wurde, auch wenn noch immer beträchtliche Unterschiede bestehen. Im Querschnittsvergleich ist die regionale Verteilung der FH-Studierenden der Bevölkerung besser angepasst als die Verteilung der Universitätsstudierenden. In einigen Bundesländern (vor allem in der Steiermark und in Niederösterreich, aber auch in Tirol und Salzburg) gibt es Anzeichen für größere Abweichungen von einer ausgewogenen Verteilung als in den anderen. 
Die Frage der kritischen Größe ist in mehrfacher Hinsicht schwer zu beantworten. Es gibt keine allgemeinen Kriterien, da diese von der Aufgabenstellung und dem Leistungspotential der Einrichtungen abhängen. Beides ist für den FH-Sektor unzureichend festgelegt, um daraus entsprechende Größen abzuleiten. Die Aufgabenstellungen sind zu allgemein definiert und es gibt nur wenig Anhaltspunkte und Vorgaben zu den erwarteten Leistungen. Das Leistungspotential der Einrichtungen ist aufgrund der Komplexität und Vielfältigkeit im Sektor und aufgrund teilweise weitmaschiger und schwer in ihrer Aussagekraft zu beurteilender Datengrundlagen nur ansatzweise einzuschätzen. Es kann gesagt werden, dass zum gegenwärtigen Ausbaustand nur ein Teil der Einrichtungen über ausreichende Potentiale verfügt, um das gesamte Spektrum der Aufgaben mit einer gewissen merkbaren Wirkung zu erfüllen.

Das Leistungsspektrum des Sektors (AbsolventInnen, F\&E-Projekte, Vorträge und Veranstaltungen, Diplomarbeiten auf Auftrag, Beratungsleistungen) wird schwerpunktmäßig für den regionalen Kontext abgegeben, gleichzeitig bestehen auch Leistungen im nationalen und internationalen Bereich.

Hinsichtlich der Entwicklungsplanung hat die regionale Ebene bisher bestenfalls in Teilbereichen und in Ansätzen die insgesamt schwache übergreifende Koordination und Steuerung unterstützt. Überprüfbare Strategien der Einbindung in regionale Innovationssysteme sind bisher nicht entwickelt worden.

\subsubsection{Schlussfolgerungen}

Der Aspekt der Regionalisierung und Standortwahl im FH-Sektor wurde auf dem Hintergrund der Dynamik der Globalisierung - Regionalisierung und im Licht der neueren Konzepte der (regionalen) Innovationspolitik analysiert. Je nach dem Spektrum der Erwartungen in regionale Wirkungen von Fachhochschul-Studiengängen ergeben sich unterschiedliche Schlussfolgerungen für die erforderlichen Standortpotentiale. Die groben überschlagsmäßigen Abschätzungen der Standortpotentiale haben zu dem Schluss geführt, dass etwa die Hälfte der Erhalter nicht über personelle Potentiale verfügt, die bestimmte Minimalkriterien für eine nachhaltige Entwicklung von F\&E-Tätigkeiten erfüllen würden, lediglich bei einem Viertel der Erhalter sind derartige Potentiale vorhanden. Bisher hat sich jedoch noch nicht klar herausgestellt, welche Rolle der FH-Sektor im Hinblick auf die Entwicklung der (regionalen) Innovationspolitik spielen soll. Weitgehende Erwartungen wurden im Rahmen der Forschungsstrategie formuliert, jedoch sind diese bisher nicht konsensuell verankert und auch noch sehr weit von der Umsetzung entfernt.

Die Eckpunkte für eine weitere regionale Verteilung der Standorte werden wesentlich von der quantitativen Entwicklung beeinflusst (je stärker das Wachstum, desto größer auch der Spielraum für stärkere regionale Streuung von Standorten). Für die weitere quantitative 
Entwicklung des $\mathrm{FH}$-Sektors ergibt sich ein breites Spektrum an möglichen quantitativen Entwicklungspfaden, das nur mit großer Unsicherheit eingrenzbar ist und wesentlich von politischen Entscheidungen beeinflusst werden kann. Als wichtige Faktoren wurden die Demografie, die Bildungsbeteiligung in höheren Schulen auf der Sekundarstufe, die angestrebte Ausschöpfung der Potentiale im Bereich der Erstausbildung für die Bildungsbeteiligung im tertiären Sektor, die Wahl zwischen Fachhochschul- und Universitätsausbildung sowie die angestrebte Ausschöpfung der Potentiale für nichttraditionelle Zugänge in den FH-Sektor im Wege des lebenslangen Lernens identifiziert. Neben den Entscheidungen über die weitere quantitative Entwicklung wird die Standortplanung davon bestimmt, inwieweit sich ein bestimmtes "Bild" einer österreichischen Fachhochschuleinrichtung herausbildet, welche Variationsbreite bzw. Konturiertheit dieses Bild bekommt. Die beiden Merkmale, das erwartete Aufgabenportfolio einer Fachhochschuleinrichtung und die als notwendig angesehene Mindestausstattung ("kritische Masse") für seine Erfüllung, bestimmen dieses Bild wesentlich.

Wenn der FH-Sektor eine sichtbare Impulsfunktion für die Entwicklung regionaler Innovationssysteme ausüben soll, dann ist erstens die Konsolidierung weiter voranzutreiben. Ein wesentliches Element dabei sollte die Konkretisierung der erwarteten Leistungen der $\mathrm{FH}$-Einrichtungen im gesamten Spektrum sein. Größere Einrichtungen, die die Ansprüche an Regionalisierung möglicherweise mit multi-campus-Strukturen erfüllen, sind erforderlich, um das gesamte Leistungsspektrum an Funktionen abzudecken. Die bisher zentrale Größe der Studierendenzahl zur Spezifikation der "kritischen Masse" einer fachhochschulischen Einrichtung bezieht sich vor allem auf die Lehre. Die Evaluierung hat gezeigt, dass eine entsprechende Gewichtung der Forschungs- und der Dienstleistungsfunktion für einen großen Teil der Standorte ein beträchtliches Wachstum der personellen und sachlichen Ressourcen erfordern würde. Für die weitere Entwicklung stellt sich also erstens die Frage der Konsolidierung bestehender Standorte (welche vor allem bei einer geringeren Wachstumsvariante in den Vordergrund tritt) und zweitens die Frage der Eröffnung weiterer Standorte (welche vor allem bei stärkerem Wachstum relevant wird).

Die Standortplanung ist eine der zentralen Fragen für die weitere Entwicklung des $\mathrm{FH}$ Sektors. Bisher ist die bemerkenswert große Diversität der Standorte ein wesentliches Kennzeichen des FH-Sektors. Von diesem Ausgangspunkt sind sehr verschiedene weitere Entwicklungen denkbar und möglich. Diese Optionen werden in den übergreifenden Schlussfolgerungen zur Frage des Aufgabenportfolios angesprochen (vgl. Abschnitt 8.3.3). In der Frage der Standortwahl sollten systematische Verfahren angewendet werden. Im Prinzip könnte eine übergreifende Standortprüfung für Österreich durchgeführt werden, die das gesamt Spektrum an möglichen Standorten systematisch auslotet. Dadurch könnte eine gewisse Erwartungssicherheit im Hinblick auf lokale Interessen geschaffen werden. In Österreich wurden, vor dem Hintergrund deutscher Erfahrungen, im Laufe der Jahre bereits Verfahren der systematischen Standortprüfung und -planung entwickelt, die im Prinzip auf die in Frage kommenden Orte und Regionen in Österreich (die ja alle schon vorhanden sind) 
angewendet werden könnten. Eine derartige generelle Standortprüfung könnte den potentiell möglichen Raum für die weitere regionale Entwicklung des $\mathrm{FH}$-Sektors ausloten und es könnten Größenordnungen und Potentiale in verschiedenen Varianten herausgearbeitet werden. Damit könnten gleichzeitig auch die Entscheidungsgründe und die erwarteten Aufgabenprofile der potentiellen $\mathrm{FH}$-Standorte transparent gemacht werden und die immer wieder auftauchenden Auseinandersetzungen um eine sinnvolle Standortwahl besser fundiert werden.

Es sollten systematischere Überlegungen über die Konkretisierung der Funktionen im Kontext von regionalen Innovationssystemen und der Entwicklung von lernenden Regionen angestellt werden. Eine erste Grundlage sind die Szenarien für den FTE-Rat. Diese entwickeln Vorschläge, wie die FHs in Strategien der Erhöhung der österreichischen Forschungsquote eingebaut werden können, sind jedoch noch bei weitem zu wenig konkretisiert. Im Hinblick auf die Funktionen von Fachhochschul-Studiengängen wurden bisher v.a. die Standortvoraussetzungen für die Lehre herausgearbeitet, für die Forschung gibt es ansatzweise Vorstellungen, die Wahrnehmung der Dienstleistungsfunktion und der Weiterbildung ist noch weitestgehend unbestimmt. Bei der Dienstleistungsfunktion verbindet sich die Standortfrage am deutlichsten mit der Frage nach Impulsen für die regionale Entwicklung. Diese Aufgabenstellung könnte im Akkreditierungsprozess im Einklang mit den Erfahrungen aus internationalen Analysen bei der Errichtung neuer Standorte stärker betont werden (etwa indem die Antragsteller bereits ex ante entsprechende Vorstellungen über die geplanten bzw. erwarteten Impulse sowie über die Beziehungen mit potentiellen Kooperationspartnern entwickeln).

Die Bedarfsanalysen sollten stärker in den Aufbau von Antizipationsmodellen eingebaut werden, die auf die Vernetzung und Kommunikation der regionalen Akteure zurückgreifen können. Das heißt, das Entwicklungsteam müsste nicht nur den Bedarf nachweisen, sondern stärker auf die angestrebten Ergebnisse der gesamten Tätigkeit und ihre Vernetzung in einem regionalen Raum (der sehr unterschiedlich ausgedehnt sein kann) abstellen.

Ansatzpunkte für die weitere Entwicklung könnten sein, erstens eine vertiefende Bearbeitung der Überlegungen zu den Standortwirkungen der verschiedenen Entwicklungspfade. Beispielsweise könnte eine systematische bundesweite quantitativ gestützte Szenarienanalyse durchgeführt werden, um einerseits die alternativen Optionen weiter zu klären und in ihren Implikationen und Entwicklungspotentialen zu konkretisieren und um andererseits zur Konsensbildung unter den Akteuren beizutragen. 


\section{Die Internationalisierung der Hochschulen und das Entstehen eines „Europäischen Hochschulraums“}

Seit der Gründung des österreichischen Fachhochschulsektors hat sich die Internationalisierung des Hochschulwesens, die in Österreich primär als „Europäisierung“ in Erscheinung tritt, massiv beschleunigt. Zwar haben Universitäten seit ihren mittelalterlichen Ursprüngen eine internationale, über die Landesgrenzen hinausweisende Orientierung. Auch im 19. Jahrhundert, als die nationalstaatliche Prägung der Hochschulsysteme ihren Höhepunkt erreichte, gab es - jedenfalls was die Forschungsaktivitäten betrifft internationale Kommunikation und Mobilität. In der 2. Hälfte des 20. Jahrhunderts hat es zunehmende organisatorische Anstrengungen gegeben, den „kosmopolitischen“ Charakter der Universitäten zu stärken. In den späten 1980er und 1990er Jahren sind diese Aktivitäten sprunghaft angewachsen und haben sich zugleich qualitativ verändert; auch in anderen Teilen des Bildungswesens, vor allem im nicht-universitären Sektor des Hochschulbereichs, hat sich eine internationale Orientierung entwickelt.

Der österreichische Fachhochschulsektor nimmt die Herausforderungen, die sich durch diese Entwicklung ergeben, sehr ernst. In einer Erhebung bei FH-Studiengängen, die im Rahmen eines noch nicht abgeschlossenen Forschungsprojekts durchgeführt wurde ${ }^{176}$, wird die Wichtigkeit dieses Themas durchgängig betont. Bei der Gewichtung der einzelnen Aspekte der Internationalisierung wurde vor allem die Mobilität der Studierenden (Studierendenaustausch, Auslandspraktika), die Durchführung von Tagungen und Kongressen sowie der Lehrendenaustausch hervorgehoben. Internationale Forschungskooperationen wurden an vierter Stelle genannt. Deutlich abgeschlagen in der Prioritätensetzung sind Summerschools und länderübergreifend organisierte Ausbildungslehrgänge. Hervorzuheben ist, dass nahezu alle Aspekte der Internationalisierung in den nächsten beiden Jahren verstärkt werden sollen; am stärksten ausgeweitet werden soll der Lehrendenaustausch und die internationale Forschungskooperation. Gemäß dieser Einschätzung wird der Lehrendenaustausch in zwei Jahren annähernd denselben Stellenwert besitzen wie der Studierendenaustausch.

\subsection{Die Mobilitätsprogramme der EU}

Aus österreichischer Perspektive bedeutet die Verstärkung internationaler Aktivitäten primär „Europäisierung“ (mit Schwerpunkt EU) oder anders formuliert: die Teilnahme an einem und Integration in einen Europäischen Hochschulraum. Die treibende Kraft dieser Entwicklung ist

176 Es handelt sich um ein vom FHR in Auftrag gegebenes Projekt zur „Internationalisierung im österreichischen FH-Sektor". Die Erhebung wurde im Mai 2002 bei allen 93 zu diesem Zeitpunkt bestehenden Studiengängen durchgeführt, die Rücklaufquote betrug zwei Drittel. 
die Europäische Kommission (vgl. Hackl 2001). Seit Mitte der 1980er Jahre hat die Europäische Kommission (zunächst gegen den Widerstand der nationalen Bildungsministerlnnen) mit großem Erfolg Mobilitätsprogramme entwickelt. Ihr Ziel ist es, in der gesamten Bevölkerung die Kenntnis anderer Gemeinschaftssprachen zu verbessern und auf diesem Weg den kulturellen Austausch und die Bildung einer europäischen Identität zu fördern. Die Europäische Kommission formulierte als Zielquote, dass sich $10 \%$ aller in den Mitgliedstaaten inskribierten StudentInnen an den Austauschprogrammen beteiligen sollten.

Die Mobilitätsprogramme der EU sind vor allem deshalb so erfolgreich, weil die politischen Ziele und die individuellen Motive der Studierenden in diesem Fall perfekt harmonieren. Immer mehr StudentInnen erkennen die hohe Bedeutung von verbesserten Fremdsprachenkenntnissen und Auslandserfahrungen für ihre Karrierechancen am Arbeitsmarkt. Im Grunde ist die Mobilitätsbereitschaft der Studierenden mittlerweile größer als die zur Unterstützung von Austauschprogrammen bereitgestellten Stipendien. Eine wachsende Zahl von Studierenden (die freilich statistisch kaum erfasst ist) wählt eine noch viel radikalere Lösung und absolviert das gesamte Studium im Ausland. Das Diskriminierungsverbot (EU-BürgerInnen müssen beim Hochschulzugang nach den gleichen Kriterien behandelt werden wie die eigenen BürgerInnen eines Landes) hat diese Option innerhalb stark Europas vereinfacht.

\subsubsection{Daten zur akademischen Mobilität: Vergleich zwischen Fachhochschul- und Universitätssektor}

Die Auslandsmobilität österreichischer Studierender ist seit Mitte der 1980er Jahre sprunghaft angewachsen, vor allem in den späten 1980er und frühen 1990er Jahren gab es sehr hohe Wachstumsraten, danach war die Entwicklung weniger spektakulär ${ }^{177}$. Allerdings gab es bis Mitte der 1990er Jahre keine verlässliche Erfassung der Mobilitätsdaten; somit ist gerade die Phase der stürmischen Veränderungen nicht gut dokumentiert. Eine ungefähre Vorstellung von der Dynamik dieser Entwicklung kann man gewinnen, wenn man die Budgetposten für die staatliche Förderung der Auslandsmobilität vor und nach dieser sprunghaften Expansion vergleicht ${ }^{178}$. Im Jahr 1983 betrug die Summe aller Budgetansätze, die man der Förderung von Auslandsaufenthalten zurechnen kann, 13 Mio. öS (rund $945.000 €$ ). Der Vergleichswert aus dem Jahr 1994 beträgt 216 Mio. öS (rund 1,57 Mio. €). Aus diesen Zahlen lässt sich die Vermutung ableiten, dass die Auslandsmobilität im dazwischen liegenden Zeitraum mindestens um den Faktor 10 gestiegen ist.

177 In den Jahren 1996 und 1997 gab es - bedingt durch Budgetkürzungen - leichte Rückgänge, seither wieder moderates Wachstum.

178 Ein solcher wurde von Pechar et al $(1998,90)$ vorgenommen; es handelt sich allerdings nur um eine Schätzung, denn im Laufe der beobachteten Zeitspanne wurde die Gliederung der Budgetansätze mehrfach verändert; bei den zitierten Summen handelt es sich um unbereinigte Nominalwerte. 
Seit Mitte der 1990er Jahre ist die akademische Mobilität statistisch zufriedenstellend dokumentiert. Seit dem Beitritt Österreichs zur EU werden die Auslandsaufenthalte, die aus Mitteln des BMBWK gefördert werden, systematisch erhoben. Zusätzlich gibt es die von Statistik Österreich gewartete AbsolventInnenstatistik, in deren Rahmen auch studienbezogene Auslandsaufenthalte erhoben werden. Die im folgenden erläuterten Daten stammen aus dieser letzteren Quelle. Diese Daten der amtlichen Statistik werden um Ergebnisse der Erhebung im Rahmen des Projekts „Internationalisierung“ ergänzt.

Ende der 1990er Jahre gibt mehr als ein Viertel aller UniversitätsabsolventInnen einen Auslandsaufenthalt im Rahmen der Studientätigkeit an (vgl. Tabelle 20). Dieser Wert liegt deutlich über der Zielquote von 10\%, die das Europäische Parlament gefordert hat und der sich auch der österreichische Nationalrat angeschlossen hat (14.3.1997). Über die Entwicklung der Auslandsaufenthalte im Fachhochschulsektor liegen bislang nur Daten der Studienjahre 1999/2000 und 2000/01 vor (vgl. Tabelle 21). Trotz seiner Jugend ist der Durchschnittswert für den gesamten Sektor gleich hoch wie für die Universitäten und deutlich höher als für die Kunstuniversitäten (vgl. Tabelle 22).

Tabelle 20: AbsolventInnen von Universitäten mit Auslandsaufenthalt, STJ 1996/97 - STJ 1999/2000

\begin{tabular}{|cccc|}
\hline & AbsolventInnen insges. & mit Auslandsaufenthalt & $\%$-Anteil \\
\hline $1996 / 1997$ & 13.987 & 2.670 & $19,09 \%$ \\
\hline $1997 / 1998$ & 13.740 & 3.576 & $26,03 \%$ \\
\hline $1998 / 1999$ & 13.591 & 3.671 & $27,01 \%$ \\
$1999 / 2000$ & 13.303 & 3.682 & $27,68 \%$ \\
\hline
\end{tabular}

Quelle: Statistik Austria 
Tabelle 21: Prozentueller Anteil von Fachhochschul-AbsolventInnen mit Auslandsaufenthalt nach Studiengängen, STJ 1999/2000 und STJ 2001/01

\begin{tabular}{|c|c|c|}
\hline & STJ $1999 / 00$ & STJ 2000/01 \\
\hline Internat. Wirtschaft / Management Kufstein & & $100,0 \%$ \\
\hline Internat. Wirtschaftsbeziehungen Eisenstadt & $96,9 \%$ & $100,0 \%$ \\
\hline Tourismusmanagement / Freizeitwirt. Krems & $97,3 \%$ & $100,0 \%$ \\
\hline Telematik / Netzwerktechnik Klagenfurt & & $85,7 \%$ \\
\hline Industrial Design Graz & $64,3 \%$ & $80,0 \%$ \\
\hline Fahrzeugtechnik Graz & $42,1 \%$ & $75,0 \%$ \\
\hline MultiMediaArt Salzburg & $40,4 \%$ & $65,5 \%$ \\
\hline InterMedia Dornbirn & $42,1 \%$ & $60,0 \%$ \\
\hline Betriebl. Prozess-/ Projektmanag. Dornbirn & $50,0 \%$ & $59,6 \%$ \\
\hline Tourismus & $59,5 \%$ & $55,7 \%$ \\
\hline Software-Engineering Hagenberg & $43,2 \%$ & $55,6 \%$ \\
\hline Technisches Produktionsmanagement Dornbirn & $57,1 \%$ & $47,4 \%$ \\
\hline Produktion und Management Steyr & $37,8 \%$ & $46,9 \%$ \\
\hline Facility Management Kufstein & & $43,3 \%$ \\
\hline Medien & $33,1 \%$ & $40,1 \%$ \\
\hline Militärische Führung Wr.Neustadt & $57,7 \%$ & $37,5 \%$ \\
\hline Sonstiges & $57,7 \%$ & $37,5 \%$ \\
\hline Europ. Wirt.- und Unternehmensführung Wien & $32,4 \%$ & $33,3 \%$ \\
\hline Industriewirtschaft Kapfenberg & $18,4 \%$ & $31,8 \%$ \\
\hline INSGESAMT & $28,0 \%$ & $30,4 \%$ \\
\hline Wirtschaft & $25,1 \%$ & $29,7 \%$ \\
\hline Telekommunikationstech./ -systeme Salzburg & $34,0 \%$ & $28,0 \%$ \\
\hline Automatisierte Anlagen / Prozesstechnik Wels & $26,9 \%$ & $27,8 \%$ \\
\hline Technik & $22,7 \%$ & $25,6 \%$ \\
\hline Gebäudetechnik Pinkafeld & $17,9 \%$ & $24,1 \%$ \\
\hline Medientechnik und -design Hagenberg & $40,0 \%$ & $23,3 \%$ \\
\hline Informationsberufe Eisenstadt & & $23,1 \%$ \\
\hline Bauing.wesen / Projektmanag. Spittal/Drau & $25,0 \%$ & $22,7 \%$ \\
\hline Tourismus-Management Wien & $26,2 \%$ & $20,5 \%$ \\
\hline Holztechnik / Holzwirtschaft Kuchl & $10,3 \%$ & $18,5 \%$ \\
\hline Telekommunikation und Medien St. Pölten & $18,4 \%$ & $18,2 \%$ \\
\hline Bauplanung / Baumanagement Graz & $10,0 \%$ & $15,8 \%$ \\
\hline Präzisions-, System- u. InfoTech. Wr.Neustadt & $9,6 \%$ & $14,9 \%$ \\
\hline Produktions-/ Automatisierungstechnik Wien & $7,7 \%$ & $14,3 \%$ \\
\hline Elektronik Wien & $9,2 \%$ & $13,9 \%$ \\
\hline Kommunales Management Spittal/Drau & & $13,8 \%$ \\
\hline Wirtschaft und Management Innsbruck & $16,4 \%$ & $13,5 \%$ \\
\hline Elektronik Villach & $6,1 \%$ & $13,0 \%$ \\
\hline Wirtschaftsberatende Berufe Wr.Neustadt & $9,3 \%$ & $11,5 \%$ \\
\hline Unternehmensführung / mittelst. Wirt. Wien & $0,0 \%$ & $8,8 \%$ \\
\hline Marketing Graz & $29,3 \%$ & $8,7 \%$ \\
\hline Finanz-, Rechnungs- und Steuerwesen Wien & $4,9 \%$ & $7,7 \%$ \\
\hline Industrielle Elektronik Kapfenberg & $5,9 \%$ & $5,3 \%$ \\
\hline Marketing \& Sales Wien & & $3,7 \%$ \\
\hline Automatisierungstechnik Graz & $0,0 \%$ & $2,9 \%$ \\
\hline Mechatronik / Wirtschaft Wels & & $2,9 \%$ \\
\hline Bauingenieurwesen / Baumanagement Wien & $0,0 \%$ & $2,8 \%$ \\
\hline INSGESAMT & $28,0 \%$ & $30,4 \%$ \\
\hline
\end{tabular}

Quoten berechnet ohne AbsolventInnen, die keine Angaben bzgl. Auslandsaufenthalt machen. Quelle: Statistik Austria 
Tabelle 22: AbsolventInnen von Kunstuniversitäten mit Auslandsaufenthalt, STJ 1998/99 und STJ 1999/2000

\begin{tabular}{|lccc|}
\hline & AbsolventInnen insg. & mit Auslandsaufenthalt & \%-Anteil \\
\hline $1998 / 1999$ & 452 & 77 & $17,04 \%$ \\
\hline $1999 / 2000$ & 451 & 95 & $21,06 \%$ \\
\hline
\end{tabular}

Quelle: Statistik Austria

Auch die Befragung zur Internationalisierung bestätigt den Befund, dass ein Auslandsaufenthalt für Studierende im FH-Sektor einen hohen Stellenwert besitzt. Auf die Frage: „Planen Sie, im Rahmen Ihres Fachhochschulstudiums ein Auslandssemester zu absolvieren?" antworten 75\% der Studierenden mit ,ja“.

Der hohe Durchschnittswert bei Auslandsaufenthalten von Fachhochschülerlnnen geht allerdings mit einer höheren Streuung als an Universitäten und Kunstuniversitäten einher. Ein weiterer Unterschied zwischen den Sektoren besteht darin, dass mehr als die Hälfte der Auslandsaufenthalte von Fachhochschul-AbsolventInnen zum Zweck des Praxissemesters erfolgt $^{179}$, während an den Universitäten studienbezogene Auslandsaufenthalte dominieren (vgl. Tabelle 23). Der hohe Stellenwert von Auslandsaufenthalten für das Praktikum wird auch durch die Befragung der StudiengangsleiterInnen bestätigt (vgl. Abbildung 62). Bei acht Studiengängen absolvieren zwischen 50\% und 100\% der Studierenden ihr Praktikum im Ausland; nur bei berufsbegleitenden oder zielgruppenspezifischen Studiengängen wurden keine Auslandspraktika angegeben.

Tabelle 23: Zweck des Auslandsaufenthalts von AbsolventInnen der Universitäten/Kunstuniversitäten und von FachhochschulabsolventInnen, STJ 1999/2000

\begin{tabular}{|lcc|}
\hline & Fachhochschulabsolv. & Univ./Kunstuniv.absolv. \\
\hline Praxis & $56,0 \%$ & $23,7 \%$ \\
\hline Sprachkurs & $14,1 \%$ & $18,3 \%$ \\
Fachstudium & $13,3 \%$ & $31,9 \%$ \\
Diplomarbeit/Dissertation & $13,2 \%$ & $20,9 \%$ \\
Unterrichts-/Lehrtätigkeit & $2,7 \%$ & $3,2 \%$ \\
Keine Angabe & $0,7 \%$ & $2,1 \%$ \\
\hline
\end{tabular}

Quelle: Statistik Austria

179 Eine andere Gewichtung als die AbsolventInnen nehmen die Studiengangsleiterlnnen vor, die im Rahmen des Projekts „Internationalisierung“ befragt wurden: hier wird zu 35\% das Fachstudium und nur zu $31 \%$ das Praktikum als Zweck eines Auslandsaufenthalts genannt. 
Abbildung 62: Anteil der FH-Studierenden, die ihr Praktikum im Ausland verbringen, nach Studiengängen, die mind. 3 Jahre bestehen, 2002

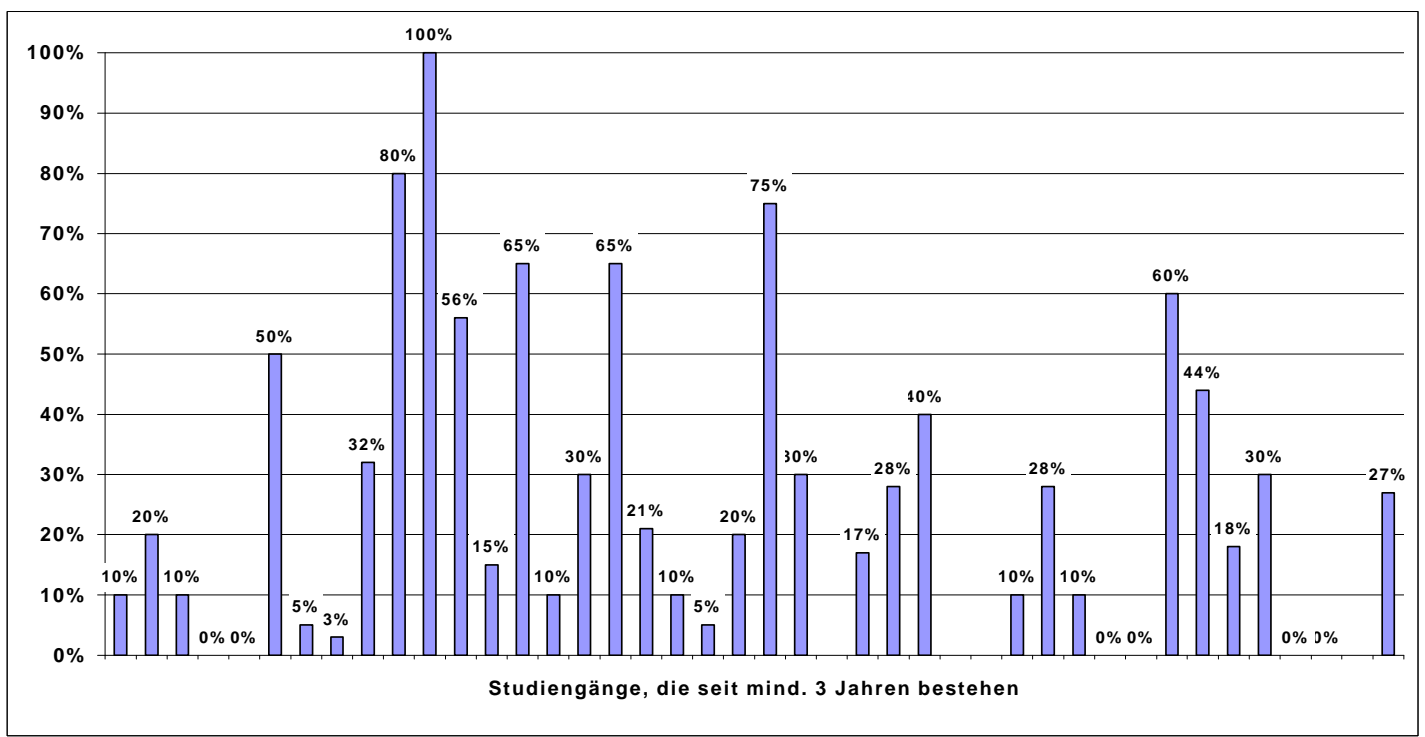

Anmerkung: Studiengänge mit $0 \%$ sind berufsbegleitend oder zielgruppenspezifisch.

Quelle: Befragung der Studiengangsleitungen

Unterschiede zwischen den Sektoren gibt es auch hinsichtlich der Art der Förderung. Fast die Hälfte der Auslandsaufenthalte von FachhochschulabsolventInnen erfolgte ohne Förderung, was vor allem auf die geringen Anteile des FH-Sektors bei den Mitteln aus der Studienförderung und der hochschuleigenen Förderung zurückzuführen ist. Bei den Mitteln aus EU-Mobilitätsprogrammen liegen die FachhochschulabsolventInnen hingegen vor denen von Universitäten (vgl. Tabelle 24). Aus der Befragung im Rahmen des Projekts "Internationalisierung" geht hervor, dass die Austauschprogramme LEONARDO und SOKRATES annähernd die gleiche Bedeutung haben. CEEPUS und bilaterale Abkommen spielen hingegen nur eine marginale Rolle.

Tabelle 24: Art der Förderung der Auslandsaufenthalte von FachhochschulabsolventInnen und von AbsolventInnen der Universitäten /Kunstuniversitäten, 1999/2000

\begin{tabular}{|lcc|}
\hline & Fachhochschulabsolv. Univ./Kunstuniv.absolv. \\
\hline Keine Förderung & $48,1 \%$ & $35,2 \%$ \\
\hline EU-Mobilitätsprogramm & $25,3 \%$ & $20,2 \%$ \\
\hline Andere Förderung & $8,5 \%$ & $9,2 \%$ \\
Beihilfe gem. Studienförderungsgesetz & $7,4 \%$ & $19,9 \%$ \\
Keine Angabe & $6,4 \%$ & $4,6 \%$ \\
Staatliches oder hochschulisches Programm & $4,3 \%$ & $11,1 \%$ \\
\hline
\end{tabular}

Quelle: Statistik Austria 
Keine starken Unterschiede gibt es hingegen in Bezug auf die Zielländer. Bei FachhochschulabsolventInnen findet sich ein höherer Anteil an europäischen Ländern außerhalb der EU, was mit geringeren Anteilen an außereuropäischen Ländern einhergeht.

Tabelle 25: Auslandsaufenthalte von FachhochschulabsolventInnen und von AbsolventInnen der Universitäten /Kunstuniversitäten nach Region, 1999/2000

\begin{tabular}{|lcc|}
\hline & Fachhochschulabsolv. & Univ./Kunstuniv.absolv. \\
\hline EUROPÄISCHE UNION & $66,6 \%$ & $66,2 \%$ \\
\hline EUROPA, ohne EU & $17,7 \%$ & $12,0 \%$ \\
NORDAMERIKA & $12,3 \%$ & $14,4 \%$ \\
MITTEL-SÜDAMERIKA (2) & & $2,0 \%$ \\
\hline AFRIKA & $0,9 \%$ & $1,2 \%$ \\
\hline ASIEN & $0,7 \%$ & $2,2 \%$ \\
AUSTRALIEN /PAZIFIK & $1,8 \%$ & $1,9 \%$ \\
\hline
\end{tabular}

(1) bei Universitäten/Kunstuniversitäten: Durchschnitt der Jahre 1995-2001, bei FH-Studiengängen: 1999/2000

(2) bei FH-Studiengängen: nur Gesamtwert für "Amerikanische Staaten" verfügbar

Quelle: Statistik Österreich

Weitere Informationen, die aus der Befragung im Rahmen des Projekts „Internationalisierung“ hervorgehen, sind:

- Die Auswahl der Outgoing-Studierenden erfolgt überwiegend auf Basis des Studienerfolgs, auch der Zeitpunkt im Studium und sprachliche Fähigkeiten spielen eine Rolle. In vielen Fällen ist aber keine Selektion nötig, da die Nachfrage die verfügbaren Austauschplätze nicht übersteigt.

- Die Dauer des Auslandsaufenthalts beträgt bei mehr als $80 \%$ aller Studierenden bis zu 6 Monaten. In ca. $25 \%$ liegt sie bei 3 Monaten oder darunter.

- In der großen Mehrzahl der Fälle (ca. 90\%) werden die im Ausland absolvierten Lehrveranstaltungen bei der Rückkehr nach Österreich anerkannt. Wo dies nicht möglich ist, werden die nicht angerechneten Leistungen in der Regel im Laufe des nächsten Semesters nachgeholt.

Aus der Befragung der Studiengangsleiterlnnen geht hervor, dass es ein deutliches Ungleichgewicht zwischen Outgoing- und Incoming-Studierenden gibt: im Durchschnitt gehen mehr als vier Mal soviel österreichische FH-Studierende an Fachhochschulen ins 
Ausland (9\% aller Studierenden) als ausländische Studierende an österreichische Fachhochschuleinrichtungen kommen (2\%). Von den 26 Studiengängen, die Mobilitätsdaten melden, haben 6 einen Überhang an Incoming-Studierenden, bei 4 Studiengängen gibt es ein Gleichgewicht, aber bei 16 Studiengängen gibt es einen z.T. deutlichen Überhang von Outgoing-Studierenden (vgl. Abbildung 63). Das lässt den Schluss zu, dass ausländische Hochschulen für österreichische FH-Studierende attraktiver sind als die österreichischen Fachhochschulen und Fachhochschul-Studiengänge für ausländische Studierende. Eine mögliche Erklärung dafür könnte in den Sprachbarrieren liegen. Es könnte sein, dass die Attraktivität von Deutsch als Fremdsprache bei ausländischen Studierenden geringer ist, als es der Anreiz für österreichische Studierende ist, die Sprache ihres Ziellandes zu lernen (abgesehen von Englisch, das im „Sprachenwettbewerb“ einen uneinholbaren Vorsprung hat, haben auch einige romanische Sprachgemeinschaften eine hohe Anziehungskraft). Ein weiteres Problem besteht in der mangelhaften Wahrnehmbarkeit des österreichischen FHSektors im Ausland. In der Erhebung im Rahmen des Projekts „Internationalisierung“ werden als Hindernisse für Incoming-Studierende nach den mangelnden Deutschkenntnissen an zweiter Stelle Informationsdefizite im Ausland genannt.

Abbildung 63: Outgoing- und Incoming-Studierende im STJ 2001/02 in \%aller Studierenden nach Alter der Studiengänge (mind. 2jährige Studiengänge), 2002

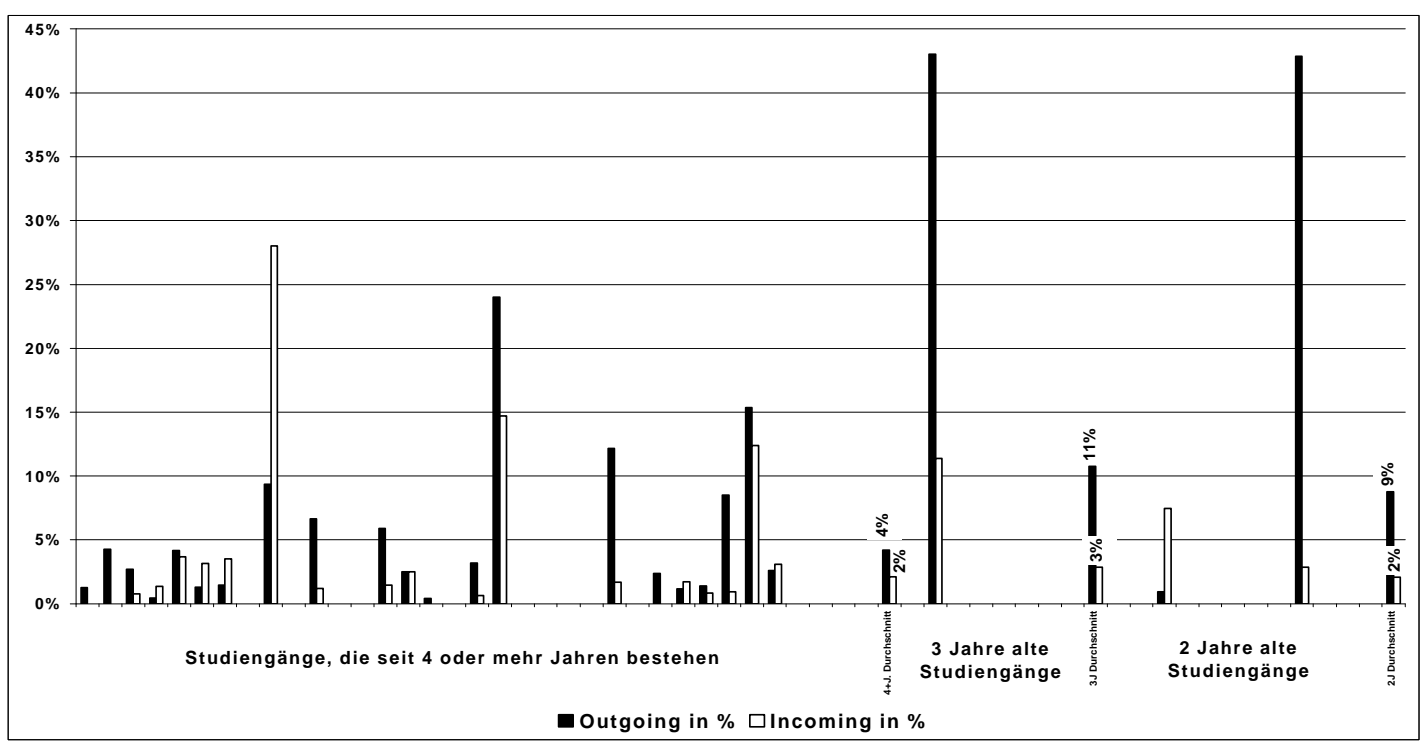

Quelle: Befragung der Studiengangsleitungen

Die Befragung im Rahmen des Projekts „Internationalisierung“ zeigt, dass die Lehrendenmobilität gegenwärtig einen deutlich geringeren Stellenwert einnimmt als die Studierendenmobilität. Dennoch gibt es bei etwa zwei Fünfteln der befragten Studiengänge Outgoing-Lehrende und bei etwa drei Fünfteln der Studiengänge Incoming-Lehrende. Das 
Verhältnis von „outgoing“ und "incoming“ ist also bei den Lehrenden genau umgekehrt als bei den Studierenden. Während das Defizit bei den Incoming-Studierenden in der (noch) zu geringen Sichtbarkeit und Attraktivität des österreichischen FH-Sektors im Ausland liegen dürfte, scheint das Defizit bei den Outgoing-Lehrenden hauptsächlich mit den durch das geringe Stammpersonal verursachten Kapazitätsengpässen zu erklären zu sein. Als Haupthindernisse für eine Ausweitung der Mobilität des eigenen Lehrpersonals werden von den StudiengangsleiterInnen genannt: das Problem, eine geeignete Vertretung für den eigenen Studiengang zu finden und die finanzielle Mehrbelastung für den Erhalter. Ungeachtet dessen planen die meisten Studiengänge, die Auslandsmobilität des eigenen Lehrpersonals zu steigern und sie wollen ihren Lehrkräften auch finanzielle und organisatorische Unterstützung dabei anbieten. Eine weitere Mobilitätsbarriere dürfte aber auch bei Lehrenden die Sprache sein. Von ausländischen Lektorlnnen wird in der Regel Englisch als Unterrichtssprache erwartet, was eine hohe aktive Sprachkompetenz voraussetzt.

\subsubsection{Perspektiven der weiteren Entwicklung}

Wie wirken sich die allgemeinen Merkmale des Sektors (Organisation, Finanzierung, Größe), die sich von den Unis unterscheiden, auf die Internationalisierungsbestrebungen aus? Der Fachhochschulsektor ist ein vergleichsweise kleines Segment in der österreichischen Hochschullandschaft, das aus einer Vielzahl sehr kleiner Einheiten mit einem fachlich begrenzten Studienangebot besteht, die überwiegend eine starke Verbindung zur Landes-, teilweise auch zur Gemeindepolitik aufweisen. Alle angeführten Faktoren haben in der Pionierphase zum Erfolg des neuen Hochschulsektors beigetragen. Es ist aber wahrscheinlich, dass einige dieser Faktoren eine „lokale“ Orientierung des Fachhochschulsektors bedingen, die in einem Spannungsverhältnis zum Ziel der Internationalisierung steht. Wenn dies zutrifft, könnte es für Fachhochschuleinrichtungen beim Versuch, an Mobilitätsnetzwerken teilzunehmen, Probleme geben. Ein mögliches Problem besteht darin, dass für kleine Einheiten der administrative Overhead, der mit Internationalisierungsprogrammen einhergeht, viel schwerer zu bewältigen sein dürfte als für Universitäten. Zweitens könnte es für Fachhochschuleinrichtungen beim Versuch, in attraktive Netzwerke aufgenommen zu werden, (Prestige-) Probleme geben. Mit anderen Worten: einige der Merkmale, die sich bisher positiv auf die Entwicklung des Fachhochschulsektors ausgewirkt haben, könnten die Bemühungen um verstärkte Internationalisierung beeinträchtigen.

Generell ist es ein Problem, dass Erfolg in der Internationalisierung ein sehr hohes Engagement (einzelner Personen bzw. Einheiten) voraussetzt, es aber nicht immer klar ist, ob und in welcher Weise sich dieser Einsatz bezahlt macht. Niemand kann verlässlich sagen, wann das internationale Wettbewerbsregime tatsächlich wirksam wird, wann der nationale Schonraum stark genug durchlöchert ist, um Anreize und Sanktionen von der 
gesamteuropäischen Ebene durchzulassen. Wahrscheinlich kann man das gar nicht für alle Segmente (Niveaus, Disziplinen) gleich beantworten. Man kann jedenfalls davon ausgehen, dass langfristig die aktive Beteiligung an der Schaffung des Europäischen Hochschulraums belohnt wird, die Nichtbeteiligung Nachteile mit sich bringt. Aber was ist die Skala, auf der lang-, mittel- und kurzfristige Effekte gemessen werden?

Für den Augenblick, bei einer auf kurze Zeiträume beschränkten Sichtweise, wird Engagement für Internationalisierung nicht belohnt. Das ist ein „Rückkopplungs-Dilemma": um die Internationalisierung der österreichischen Hochschulen voranzutreiben, sind zeitliche "Investitionen" erforderlich, aus denen diejenigen, die die Arbeit tun, nur selten unmittelbare Vorteile ziehen können; sie erhalten in der Regel weder materielle noch symbolische Gratifikationen (z.B. höheres Ansehen). Nach dem Kriterium der Maximierung individueller Vorteile wäre Passivität zweckrational. Nötig ist entweder Altruismus (Engagement für kollektive Ziele) und/oder intrinsische Motivation (Freude an Kooperation mit internationalen Kollegen). Der wirksamste Belohnungsmechanismus wäre akademische Reputation (Personen mit internationalen Kontakten haben mehr Ansehen), aber es ist unklar, wann dies zu wirken beginnt. „Investitionen“ in Internationalisierung erfordern einen langfristigen Planungshorizont und der kann von großen Einheiten leichter entwickelt werden als von kleinen.

\section{Größe des Fachhochschulsektors und der Fachhochschuleinrichtungen}

Es wird mit hoher Wahrscheinlichkeit eine österreichische Besonderheit bleiben, dass der Fachhochschulsektor deutlich kleiner als der universitäre Sektor ist. Das liegt an den irreversiblen Folgen seiner späten Einrichtung (vgl. Kapitel 3). Die Größe des gesamten Sektors hängt eng mit der Größe der einzelnen Fachhochschuleinrichtungen bzw. der von innen angebotenen Studiengänge zusammen. In der Pionierphase des Fachhochschulaufbaus wurden seitens der staatlichen Hochschulpolitik die Vorteile der „,bottom-up"-Strategie (die Mobilisierung dezentraler Initiativen) wichtiger eingeschätzt als ihre Nachteile (hohe Fixkosten, schwache akademische Infrastruktur an den einzelnen Standorten). Seit den späten 1990er Jahren versuchen die Bundespolitik und der Fachhochschulrat, das Entstehen kritischer Größen zu fördern.

Die Festlegung auf eine Politik der Schaffung kritischer Größen und der Konsolidierung der bestehenden Standorte wird seitens der Politik überwiegend mit Kostenargumenten (Fixkostendegression) und seitens des Fachhochschulrats überwiegend mit Qualitätsargumenten ${ }^{180}$ argumentiert. Zusätzliche Argumente für diese Option kann man

180 Unterhalb einer bestimmten Größe ist es kaum möglich, eine ausreichende akademische Infrastruktur (z.B. Bibliothek) zu schaffen; im übrigen schränken kleine Institutionen mit geringer fachlicher Streuung sowohl für 
aber aus dem Bereich der Internationalisierung ableiten. Kleine Einheiten werden bei ihren Bemühungen um die Herstellung internationaler Kooperationen größere Probleme haben als jene Fachhochschuleinrichtungen, die einen kritischen Schwellenwert erreicht haben. Auch bei der Internationalisierung bringt die kritische Größe sowohl einen Kosten- wie einen Qualitätsvorteil:

- Ohne eine unterstützende administrative Infrastruktur ist ein Erfolg in der Internationalisierungspolitik kaum denkbar. An den Universitäten wurden seit Beginn der 1990er Jahre „Auslandsbüros“ eingerichtet, die wesentlich zum Erfolg der Internationalisierung beigetragen haben. Auch an den kleinen Universitäten verfügen diese Einrichtungen über zwei oder drei Planstellen. Nur auf diesem Weg konnte die für diese Aufgabe erforderliche Professionalisierung des Verwaltungspersonals erreicht werden. Fachhochschuleinrichtungen werden erst ab einer kritischen Mindestgröße in der Lage sein, das für Internationalisierung verantwortliche Personal von anderen Funktionen zu entlasten.

- Die Größe der Fachhochschuleinrichtungen wirkt sich auch auf ihre Fähigkeit aus, in internationale Netzwerke aufgenommen zu werden. Gerade im Fachhochschulbereich findet akademische Mobilität fast ausschließlich im Rahmen von Austauschprogrammen statt, die die Einbindung aller teilnehmenden Institutionen in Mobilitätsnetzwerke zur Voraussetzung hat.

\section{Regionalbezug}

Bei der Einrichtung des Fachhochschulsektors hat der Bund (als die gemäß B-VG für Gesetzgebung und Vollzug verantwortliche Gebietskörperschaft) von vorneherein Einflussmöglichkeiten für andere Gebietskörperschaften geschaffen. Damit wurde dem Umstand Rechnung getragen, dass Fachhochschulen und Fachhochschul-Studiengänge in einem engeren Verhältnis zu regionalen Interessen stehen. In fast allen Fällen haben Politiker auf Landes- und Gemeindeebene damit zugleich Einfluss auf die Entscheidungen der Erhalterorganisation genommen, wobei das Ausmaß dieses Einflusses den aus dieser Quelle kommenden Anteil an der Finanzierung der Fachhochschuleinrichtungen häufig übersteigt.

Fachhochschulische Einrichtungen unterliegen damit aber einem Spannungsverhältnis, von dem Universitäten weitgehend entlastet sind. Aus anderen Hochschulsystemen ist der Unterschied zwischen "localism" and „nationalism“ gut bekannt (vgl. Jencks/Riesman 1968, 
155 -198). Regionale Gebietskörperschaften erwarten von den von ihnen unterstützten Hochschulen, dass sie „responsive to local conditions“ (ebda, 155) sind, was ab dem Augenblick ein Problem werden kann, wenn sich diese Institutionen an universalistischen professionellen und wissenschaftlichen Kriterien orientieren und sich auf nationaler oder internationaler Ebene um akademische Anerkennung bemühen. In diesem Sinn kann auch eine zu enge Bindung an die Bedürfnisse der Region für den Ausbau der internationalen Aktivitäten des Fachhochschulsektors hinderlich sein. Vermutlich werden die lokalen politischen Entscheidungsträger internationale Aktivitäten "ihrer" Fachhochschuleinrichtungen grundsätzlich unterstützen; aber die Frage ist, ob sie ihnen jenes $\mathrm{Ma}$ an akademischer Autonomie gewähren, das Voraussetzung für internationale Anerkennung ist. Die bisherigen Erfahrungen geben zu einer gewissen Skepsis Anlass (vgl. Prisching 2002).

Im Rahmen der Erhebung bei den Erhaltern haben vier Fünftel der ErhaltervertreterInnen angegeben, keinen Zielkonflikt zwischen Regionalbezug und Internationalisierung zu sehen. Darin könnte eine Unterschätzung des Aufgabenspektrums der Internationalisierung und des dafür erforderlichen Aufwands zum Ausdruck kommen. Wenn man in der Fragestellung einen Zielkonflikt unterstellt, der eine Entscheidung (im Sinne einer Schwerpunktsetzung) erforderlich macht, entscheidet sich etwa ein Drittel für Internationalisierung, ein anderes Drittel für Regionalbezug, das restliche Drittel beharrt darauf, dass die Frage keinen Sinn ergibt oder nicht pauschal beantwortet werden kann.

Ein Aspekt des Regionalbezugs besteht darin, dass einige fachhochschulische Einrichtungen bewusst abseits großräumiger Ballungszentren errichtet wurden. Institutionen an solchen Standorten haben erhebliche Nachteile bei der Rekrutierung ausländischer Studierender. Generell ist es ein wichtiges Motiv akademischer Mobilität, auch die Kultur des besuchten Landes kennen zu lernen. In dieser Hinsicht haben große Städte einen uneinholbaren Vorteil gegenüber kleinen und entlegenen Orten ${ }^{181}$.

Eine wichtige Aufgabe für den Fachhochschulsektor wird darin bestehen, eine Balance zwischen Regionalbezug (der Landesförderung auch künftig sichert) und einer nationalen und internationalen Orientierung zu finden.

\section{Fächerspektrum}

Der Fachhochschulsektor wurde in bewusster Abgrenzung zum universitären Sektor gegründet, dessen Traditionen eine Fokussierung auf den Bedarf des

181 Das geht auch daraus hervor, dass in Hinblick auf die innerösterreichische Mobilität Wien der einzige Universitätsstandort mit eindeutig überregionaler Bedeutung ist; die Universitäten in anderen Städten sind „Landesuniversitäten“, deren Einzugsgebiet die Landesgrenzen nur geringfügig überschreitet. 
Beschäftigungssystems erschweren. Da es primär die Wirtschaft war, die auf die Etablierung eines Fachhochschulsektors gedrängt hat, war es naheliegend, dass sich das Fachhochschulangebot in der Anfangsphase auf wirtschaftsnahe Studienrichtungen der Technik und Betriebswirtschaft konzentrierte.

Die praktische Umsetzung dieser Ziele hat zu einer gewissen fachlichen Verengung des Studienangebots von Fachhochschul-Studiengängen geführt, an der man zwei Aspekte unterscheiden kann:

- Auf der einen Seite gibt es eine Begrenzung des Studienangebots auf technische, wirtschaftliche und touristische Berufsfelder, die erst seit 2001/02 durch die Inklusion von Teilen der ehemaligen Sozialakademien partiell erweitert wurde.

- Zum anderen hat der Versuch, sich hochschulpolitische Legitimation durch Abgrenzung von den Universitäten zu verschaffen, zu einer extremen Spezialisierung und Nischenbildung im Studienangebot geführt. Diese Strategie wurde von den Fachhochschul-Studiengängen nicht freiwillig gewählt, sie wurde hauptsächlich durch Auflagen bei der Akkreditierung bedingt.

Beide Aspekte der Verengung des Studienangebots sind auch im Zusammenhang mit den Internationalisierungsbestrebungen des Fachhochschulbereichs problematisch. Die daraus resultierende fachliche Monostruktur kann sich auf die überregionale und vor allem internationale Attraktivität hemmend auswirken. Auch die Tendenz zur Überspezialisierung kann die akademische Anerkennung und die Akzeptanz in Mobilitätsnetzwerken erschweren.

Gegen eine thematische Erweiterung des Studienangebots gibt es politische Widerstände, aber zum Teil auch Einwände von Seiten der Fachhochschuleinrichtungen, die von der Sorge motiviert sind, der Sektor könnte sein klares Profil verlieren. Inhaltliche Heterogenität muss jedoch keinesfalls mit Profilverlust einhergehen. Eine produktive Mischung unterschiedlicher Fachkulturen ist eine Voraussetzung für wechselseitige Anregungen, aus denen unvorhersehbare neue Entwicklungen entstehen können. Sie ist kein zwingendes, aber doch ein weit verbreitetes Merkmal akademischer Institutionen und könnte auch bei den fachhochschulischen Bildungseinrichtungen erhöhte akademische Anerkennung nach sich ziehen.

Einbindung der Fachhochschuleinrichtungen in die unterstützende Infrastruktur auf nationaler Ebene

Eine unabdingbare Voraussetzung für die guten Fortschritte bei der Internationalisierung der Universitäten war der Ausbau einer unterstützenden Infrastruktur auf nationaler Ebene. Hier wird einerseits ein Teil der Fördermittel kanalisiert, es findet ein inter-institutioneller 
Erfahrungsaustausch statt, es werden Hilfestellungen bei der Abwicklung der bürokratischen Verfahren mit der EU angeboten etc. Bei der Befragung der Studiengänge im Rahmen des Projekts „Internationalisierung“ wird die Unterstützung durch diese Einrichtungen als sehr hilfreich beschrieben. Am besten bewertet werden die Serviceleistungen des Sokratesbüros, gefolgt von ÖAD und LEONARDO-Büro; die Unterstützung durch das BMBWK wird weniger gut bewertet; am wenigsten hilfreich wird die Unterstützung durch die FH-spezifischen Organisationen, die Fachhochschul-Konferenz und den FHR bewertet.

Allerdings sind die Serviceleistungen der unterstützenden Infrastruktur weitgehend auf die Bedürfnisse der Universitäten zugeschnitten. In vielen Fällen kann dieses Angebot problemlos auf den Fachhochschulbereich ausgeweitet werden; teilweise gibt es aber hier Reibungsverluste, die in der Regel zu Lasten des Fachhochschulsektors gehen:

- Das früher für Kulturabkommen budgetierte Geld wird nun zum einen Teil den Universitäten zugeteilt und zum anderen Teil vom ÖAD vergeben. Dieses vom ÖAD verwaltete Stipendienprogramm ist ganz auf die Universitäten zugeschnitten: es handelt sich um Unterstützung für individuelle Mobilität, um einseitige Stipendien, mit deren Hilfe gute ausländische Studierende nach Österreich geholt werden sollen. Die Fachhochschuleinrichtungen können von diesen Programmen nur wenig profitieren, dort gibt es primär bilaterale Abkommen zwischen Institutionen, wobei ganze Jahrgangsklassen im Mittelpunkt stehen.

- Fachhochschulen und Fachhochschul-Studiengänge kommen an bestimmte Fördergelder für postgraduale Mobilität nicht heran, denn für diese Zwecke müsste die Fachhochschuleinrichtung Anträge ihrer Absolventlnnen an das zuständige Bundesministerium weiterleiten. Die Fachhochschuleinrichtungen stehen aber auf dem Standpunkt, dass die AbsolventInnen bereits aus der Institution ausgeschieden sind, sie für diese daher weder Verantwortung tragen noch übernehmen können. Das traditionelle Konzept der "Mitgliedschaft" in der Universität, die auch mit Studienabschluss nicht erlischt, hat hier keine Gültigkeit. Im Fachhochschulsektor dominiert eher ein „konsumeristisches“ Verständnis vom Verhältnis zwischen Studierenden und Hochschule, das während des Studiums eine hohe Serviceorientierung garantiert, aber mit dem Studienabschluss eine klare Trennlinie zieht.

\subsection{Der Bologna-Prozess: eine neue Studienarchitektur für den „Europäischen Hochschulraum“"}

Die Unterzeichnung der Bologna-Erklärung durch 29 europäische Bildungsministerlnnen hat eine neue Phase der Hochschulpolitik in Europa eingeleitet. Nie zuvor hat es eine so weitreichende hochschulpolitische Maßnahme auf supranationaler Ebene gegeben. Die Bologna-Erklärung kann als erster genuiner Schritt einer europäischen Hochschulpolitik 
betrachtet werden, ihr Ziel ist die Schaffung eines Europäischen Hochschulraums, als dessen wichtigste Voraussetzungen die Harmonisierung der Studienarchitektur und der Abbau aller Hindernisse bei der akademischen Mobilität betrachtet werden.

In unserem Zusammenhang ist der Bologna-Prozess deshalb von Bedeutung, weil er die Grenzziehungen zwischen Universitäts- und Fachhochschulsektor, die in Österreich durch das FHStG und die bisherige Akkreditierungspraxis des Fachhochschulrats relativ klar definiert waren, neu zur Disposition stellt. Die Universitäten begeben sich nun - durch die Einrichtung von Bachelor-Studien - auf das Terrain der kurzen und berufsorientierten Ausbildung, die bislang die Domäne des Fachhochschulbereichs war. Zugleich wird die Gleichwertigkeit der Abschlüsse beider Sektoren außer Frage gestellt, was den Legitimationsdruck für den Fachhochschulsektor mindert. Diese Relativierung eindeutiger sektoraler Profile eröffnet für beide Seiten Chancen und Risken.

\subsubsection{Ein Ziel: Abbau von Mobilitätshemmnissen}

Trotz der beeindruckenden Steigerung bei der akademischen Mobilität seit den späten 1980er Jahren (vgl. Abschnitt 6.1) gibt es weiterhin Spannungen zwischen den nationalen Systemen und der Internationalisierung bzw. Europäisierung von Hochschulbildung. Hier setzt die "Bologna-Erklärung“ an, die diese Reibungsverluste durch die Harmonisierung der Studienarchitektur in Europa beseitigen will (vgl. Haug 2000). Die Bologna-Erklärung wurde 1999 von den Bildungsministerlnnen von 29 europäischen Staaten unterzeichnet. Diese haben sich darin verpflichtet, bis zum Jahr 2010 eine gemeinsame Architektur des europäischen Hochschulraums zu errichten.

Auch wenn dieses Dokument keine präzise völkerrechtliche Relevanz besitzt, reicht seine Bedeutung über eine unverbindliche Absichtserklärung hinaus. Es handelt sich um eine freiwillig eingegangene Verpflichtung zu einem Arbeitsprogramm mit präzise definierten Zielen, Terminen und Arbeitsschritten. Die gemeinsame Architektur des europäischen Hochschulraums soll bis 2010 abgeschlossen sein. Um diesen ambitionierten Terminplan zu ermöglichen, gibt es auf nationaler und supranationaler Ebene laufende Follow-ups, die diesen Prozess im Detail planen und seinen Fortschritt überprüfen sollen.

Die Eckpfeiler des Vorhabens sind:

- Eine Vereinfachung der über die Landesgrenzen hinausreichenden Anerkennung akademischer Abschlüsse. In dem Maß, in dem ein europäischer Arbeitsmarkt Realität wird, verstärkt sich die Notwendigkeit, die akademischen Titel jedes Landes für internationale Arbeitsmärkte verständlich und transparent zu machen. Daher strebt die Bologna-Erklärung ein "System leicht verständlicher und vergleichbarer Abschlüsse" an. Dem dient zum einen das Diploma Supplement, das eine standardisierte Information als 
Ergänzung zu den Hochschuldiplomen darstellt. Zum anderen will man sich auch in Europa an einem zweiphasigen Studiensystem orientieren, das sich weltweit immer klarer durchsetzt: ein drei- oder vierjähriges Undergraduate-Studium schließt mit einem Bachelor ab; darauf folgt eine Graduate-Phase mit einem ein- oder zweijährigen MasterProgramm und einem Doktoratsstudium, dessen Dauer sich nicht mehr so klar definieren lässt.

- Eine Vereinfachung der Anrechnung von im Ausland verbrachten Studienzeiten. Das ist nach wie vor eine aufwändige Prozedur, die viel Energie bindet und die Lust auf Mobilität dämpft. Nicht einmal innerhalb Österreichs ist es immer einfach, von einer Universität auf eine andere zu wechseln und die erworbenen Teilqualifikationen „mitzunehmen“. Diesen Problemen liegt ein anachronistisches Verständnis von akademischer Anerkennung zu Grunde, das auf inhaltliche Identität abzielt. Der europäische Hochschulraum benötigt ein abstrakteres Verfahren, das gleichsam eine „akademische Währung“ definiert, die man von einem Studienort auf den anderen übertragen kann. Im amerikanischen Hochschulsystem ist ein solcher credit transfer selbstverständlich. Auch in Europa gibt es Ansätze zu einem European Credit Transfer System (ECTS), das aber an den Universitäten noch häufig auf Ablehnung stößt. Die Bologna-Erklärung will die Durchsetzung eines solchen Verfahrens beschleunigen.

- Andere Maßnahmen, die die Bologna-Erklärung anstrebt, sind eine europäische Zusammenarbeit bei der Qualitätssicherung (vgl. Kapitel 7) sowie eine Beseitigung von Mobilitätshemmnissen bei der Studienförderung.

Die Realisierung eines Zielkatalogs, der von allen wichtigen europäischen BildungsministerInnen unterzeichnet wird, sollte eigentlich kein Problem sein. Ganz so einfach ist es freilich nicht, denn an den Hochschulen, auf deren Reformbereitschaft es letztlich ankommt, stoßen diese Ziele teilweise auf Skepsis und Ablehnung. Es ist ein offenes Geheimnis, dass der Druck zur Schaffung eines Hochschulraums weniger von den für Kultur und Bildung verantwortlichen Ministerlnnen als vielmehr ihren für Wirtschaft und Arbeitsmärkte zuständigen KollegInnen ausgeht. Aus ökonomischer Perspektive ist akademische Mobilität ein Mittel zur Stärkung der Wettbewerbsfähigkeit Europas gegenüber Nordamerika und Ostasien.

\subsubsection{Umstellung auf ein „two-tier“-System (angelsächsisches Modell)}

Im angelsächsischen System ist das Studium in zwei eigenständige Phasen geteilt, eine Undergraduate- und eine Graduate-Phase. Erstere bietet ein sehr klar strukturiertes (d.h. in unserem Sprachgebrauch: verschultes) Studium, bei dem bestimmte Formen akademischer 
Freiheit und die Erwartung einer Involvierung in Forschung reduziert sind ${ }^{182}$. Diese Phase schließt mit einem Bachelor ab, der vom Arbeitsmarkt voll akzeptiert wird. Demgegenüber bietet das Graduiertenstudium eine Vertiefung und Spezialisierung, an der nur noch eine Minderheit der Studierenden partizipieren. Für gehobene Karrieren sind die entsprechenden Abschlüsse des Master's Degree bzw. PhD ein Startvorteil oder unbedingt erforderlich.

In den meisten europäischen Ländern hat sich historisch eine andere Struktur der Abschlüsse durchgesetzt. Die tertiäre Bildungsphase beginnt hier (jedenfalls in ihrer traditionellen, universitären Form) bereits mit einem Graduiertenstudium. Demgemäss entspricht die traditionelle Gliederung österreichischer Studien in Diplom- und Doktoratsstudien nicht der Unterscheidung einer Undergraduate- und einer Graduate-Phase, sondern muss als interne Abstufung der Graduiertenphase betrachtet werden.

Bei der Diskussion dieser Unterschiede gibt es eine gewisse terminologische Konfusion.

- Auf der einen Seite spricht man von einem "two-tier system" im Gegensatz zum "one-tier system" europäischer Prägung (vgl. Clark 1983, 49ff). Diese Unterscheidung ein- bzw. zweiphasiger Studiensysteme betont die unterschiedliche Logik von Undergraduateversus Graduate-Studium. Die Unterschiede in Didaktik und Curriculum wurden bereits angedeutet: die Undergraduate-Phase ist von den emphatischen Ansprüchen der "Einheit von Forschung und Lehre" entlastet, in der Graduiertenphase, vor allem in den Doktoratsstudien, wird dieser Anspruch hingegen ernst genommen. Das ist ein scharfer Kontrast zur Mehrheit der einphasigen Studiensysteme, in denen die Einheit von Forschung und Lehre theoretisch vom ersten Semester an gilt; auf Grund der offenkundigen Überforderung der Massenhochschulen mit diesem Anspruch wird er dann insgesamt nicht wirklich ernst genommen.

- Man spricht aber auch von "dreigliedrigen" im Gegensatz zu "zweigliedrigen" Studiensystemen. Dieser Sprachgebrauch, der in Österreich weit verbreitet ist, betont den quantitativen Aspekt (ein Abschluss, der Bachelor, ist hinzugekommen), was mit der Gefahr einhergeht, dass die qualitative Differenz der beiden Studienphasen aus dem Blick gerät. Ein Indiz dafür, dass dies in Österreich tatsächlich der Fall ist, ist die verbreitete Redeweise vom "Zwischenabschluss", durch die nicht nur die Eigenlogik, sondern auch die Eigenständigkeit des Bachelors entwertet wird.

182 Ein sehr entscheidender Aspekt ist die obligatorische Prüfung. In den meisten europäischen Systemen melden sich die StudentInnen nach eigenem Ermessen zur Prüfung an; es ist also möglich, die Prüfung hinauszuzögern und die langen Studienzeiten sind ein Indiz dafür, dass von dieser Möglichkeit reichlich Gebrauch gemacht wird. In den angelsächsischen Systemen gibt es diese Option in der Regel gar nicht, hier schließt die Anmeldung für ein Fach automatisch die Anmeldung für die Prüfung mit ein; Nichterscheinen gilt als "nicht bestanden". 


\subsubsection{Gründe für die „Harmonisierung der Studienarchitektur“}

Von gemeinsamen mittelalterlichen Ursprüngen ausgehend haben sich in der Neuzeit unterschiedliche nationale Hochschulsysteme entwickelt, die sich hinsichtlich der Einbettung der Hochschulen in die Gesellschaft und deren Interaktion mit anderen sozialen Subsystemen, aber auch hinsichtlich der Gliederung des Lehrangebots und der Struktur der Abschlüsse deutlich voneinander unterscheiden. Solange sich das akademische Leben fast ausschließlich innerhalb der nationalen Grenzen abspielte, waren die Heterogenität und Intransparenz der Abschlüsse kein ernstes Problem. In den seltenen Fällen akademischer Mobilität konnten Anrechnungs- und Anerkennungsfragen auf der Basis von Einzelfallentscheidungen gelöst werden. Durch das drastische Anwachsen der akademischen Mobilität seit den 1980er Jahren hat sich diese Situation rasch geändert. Für die verschiedenen Akteure ergeben sich nun neue Aufgaben und Probleme:

- Hochschulen und staatliche Behörden wurden durch das quantitative Anwachsen von Anrechnungs- und Anerkennungsverfahren vor neue organisatorische Aufgaben gestellt. Mit Einzelfallentscheidungen ließen sich diese Probleme nicht mehr lösen. Vielmehr wurden auf bi- und multilateraler Basis neue Netzwerke ${ }^{183}$ geschaffen, die im Wesentlichen zwei Ziele verfolgen. Einerseits will man durch verbesserten Informationsaustausch die Transparenz und damit die Vergleichbarkeit der einzelnen Systeme erhöhen. Zum anderen gibt es zunehmend Versuche zur Harmonisierung der Bildungssysteme. Eine inhaltliche Harmonisierung, also die Schaffung gleicher Standards in allen Ländern, hat sich als unmöglich erwiesen. Daher orientierte man sich an formalen Gesichtspunkten, in erster Linie an der zeitlichen Dauer der Studiengänge. In diesem Zusammenhang gewann die "International Standard Classification of Education" (ISCED) $^{184}$ an Bedeutung ${ }^{185}$.

- Aus der Perspektive der Studiennachfrage stellt sich das Problem der Anerkennung etwas anders dar. Wer in einem anderen Land studiert, möchte die Sicherheit, dass der erworbene Abschluss auch über die engeren Landesgrenzen hinaus anerkannt wird und hohe Reputation genießt. Für die Nachfrage ausländischer Studierender nach einem Studiengang ist ein „gängiger" Abschluss daher nicht minder wichtig als hohe Qualität. Der Abschluss hat eine ähnliche Signalwirkung wie das prestigereiche Label eines Konsumgutes. Die gängigen Studienabschlüsse der angelsächsischen Hochschulsysteme, der Bachelor und der Master, haben hierbei einen uneinholbaren

183 Z.B. das National Academic Recognition Centre (NARIC).

184 ISCED wird in bestimmten Zeitabschnitten revidiert; die jüngste Version ist ISCED-97 (vgl. OECD 2000a, 362f).

185 Der Vergleich von Bildungsquoten auf ISCED-Basis spielte z.B. bei der Diskussion um die Einführung der Fachhochschul-Studiengänge eine wichtige Rolle. Am Vorabend des EU-Beitritts wurde klar, dass der BHSAbschluss im internationalen Vergleich als Sekundärabschluss gewertet wird, während eine funktional äquivalente Ausbildung im nicht-universitären Postsekundarbereich als Hochschulabschluss gilt. 
Vorteil; ihre starke Verbreitung ${ }^{186}$ wirkt wie ein positiver Verstärkungseffekt. Weil diese Abschlüsse fast überall bekannt und anerkannt sind, haben sie auch den meisten Zulauf ausländischer StudentInnen. Mittlerweile werden diese Abschlüsse von vielen ExpertInnen als allgemein anerkannte „Mobilitätsschienen" zwischen den Hochschulsystemen unterschiedlicher Länder betrachtet. Hochschulen, die diese Abschlüsse nicht vergeben können, haben bei der Rekrutierung ausländischer Studierender ernste Wettbewerbsnachteile. In einigen Ländern haben die Hochschulen schon frühzeitig auf diese Phänomene reagiert und ihre traditionellen akademischen Grade durch Bachelor- und Master-Abschlüsse ergänzt oder ersetzt.

\subsubsection{Zum „Europäischen Leistungspunktesystem“}

Die Diskussion über Credit-Systeme hat in Europa in den 1990er Jahren - von unterschiedlichen Motiven angetrieben - an Intensität stark zugenommen. Entstanden sind diese Beurteilungssysteme fast ein Jahrhundert zuvor in den USA, als dort das Hochschulsystem expandierte und an Komplexität gewann. Der pragmatische amerikanische Ansatz, um die so entstehende Unübersichtlichkeit auf ein erträgliches Maß zu reduzieren, bestand in der Einführung einer universellen „Währung“, die sich sowohl zur Messung studentischer Leistungen als auch zur Berechnung des Arbeitsaufwands der Lehrkräfte eignete (vgl. Altbach 2000, 79ff). Das so entstandene Credit-System geht von zwei zentralen Voraussetzungen aus:

- Die akademisch relevante Leistung lässt sich durch die investierte Zeit darstellen (Umwandlung von Studienaktivitäten in Mengenmaße).

- Jede beliebige Gesamtleistung lässt sich in Einzelschritte zerlegen; der Nachweis des Gesamterfolgs besteht in der Akkumulation von Teilerfolgen.

In Europa hat das Interesse an einem Credit-System mit der verstärkten Internationalisierung der Hochschulen zugenommen. Das European Credit Transfer System (ECTS) wurde entwickelt, um die Anrechnung von im Ausland erbrachten Studienleistungen zu erleichtern. Das ist möglich, weil - bei einem ausreichenden Konsens über Mindeststandards - die einzelnen Währungseinheiten getauscht werden können. ECTS setzt eine Gesamtjahresleistung von 60 Credits voraus. Damit hat zugleich die implizite Grundphilosophie der Modularisierung und Standardisierung von Curricula auch in Europa eine größere Verbreitung erfahren. In vielen Ländern gibt es Ansätze, das Credit-System zur

186 Neben den angelsächsischen Ländern auch überall dort, wo das Hochschulsystem entweder im Einflussbereich des britischen Kolonialreichs aufgebaut oder - wie z.B. in Japan - nach 1945 unter US-Einfluss restrukturiert wurde. 
Reform der Studiensysteme zu nutzen. Vielfach verbindet sich damit die Erwartung einer erhöhten Flexibilität von Curricula.

Als Kern eines Credit-Systems kann folgende Definition gelten: „Unter Credit-Systemen werden Systeme der Leistungsbewertung an Hochschulen verstanden, in denen das gesamte Studium im Rahmen eines Studiengangs in einzelne - gewöhnlich nach zeitlichem Aufwand gemessene - Einheiten gegliedert wird, diese Einheiten getrennt bewertet werden und diese Teil-Bewertungen in die Bewertung der gesamten Studienleistung eingehen." (Schwarz/Teichler 2000, 5).

Ein Ziel des Bologna-Prozesses ist es, auf der Basis der Erfahrungen mit ECTS ein "Leistungspunktesystem“ zum Zweck erhöhter Mobilität und verbesserter Beschäftigungsfähigkeit in ganz Europa einzuführen. In der Praxis dürfte das weitgehend darauf hinauslaufen, den Geltungsbereich von ECTS auszuweiten. Der europäische Rahmen ist als „nichtinvasives“ System konzipiert (ähnlich wie die Mobilitätsprogramme), das nicht in die Souveränität der nationalen Regierungen eingreift, sondern auf freiwillige Kooperation abzielt.

Eine Hauptsorge von Hochschulangehörigen besteht darin, dass Credit-Systeme zu einer Niveausenkung führen könnten, indem sie Studierenden erlauben, ohne Einschränkungen ihr eigenes "Studienmenü“ zusammenzustellen. Diese Befürchtung beruht auf einem Missverständnis. Jede Institution bzw. jedes System hat die Freiheit, Leistungspunkte anzuerkennen oder nicht. Auf diese Weise soll verhindert werden, dass Credits unterschiedlichen Niveaus miteinander vermischt werden. Die Entscheidung, einen akademischen Grad/einen Abschluss zuzuerkennen, liegt weiterhin bei der wissenschaftlichen Einrichtung, dem entsprechenden nationalen Ausschuss oder der für die Vergabe zuständigen Behörde. Die Vergabe von Anrechnungspunkten für den Prozess des Lernens bedeutet nicht die automatische Anerkennung andernorts. Akademisch unabhängige Einrichtungen und autonome Staaten legen fest, wie ihre Bildungsprogramme zu bewerten sind und wie sie aufgebaut sind.

\subsubsection{Zum Stand der Implementierung des Bologna-Prozesses in Europa}

Im Gegensatz zur weitverbreiteten Meinung, der Bologna-Prozess würde zur europaweiten Uniformierung der Hochschulstrukturen führen, kann man bei den Ländern, die nun erst auf eine zweiphasige Studienarchitektur umstellen, eine erhebliche Varianz beobachten. Unterschiede gibt es in der Länge und im Profil des Undergraduate-Studiums sowie im Stellenwert, im Tempo und in der Form der Umstellung. Das zeigt ein exemplarischer Blick auf Deutschland und die Niederlande. 


\section{Deutschland}

In Deutschland hat die Diskussion über die Vor- und Nachteile der neuen Studienarchitektur schon relativ früh eingesetzt (vgl. Jahn/Olbertz 1998), unter anderem deshalb, weil unter den politischen und ökonomischen Eliten dieses Landes die Sorge um die Attraktivität des „Hochschulstandorts Deutschland" sehr viel größer war und ist als z.B. in Österreich (vgl. Pinkau 1998). Das kommt auch darin zum Ausdruck, dass Deutschland zu den Unterzeichnern der Sorbonne-Erklärung und zu den treibenden Kräften bei der Gestaltung von Rahmenbedingungen für einen europäischen Hochschulraum gehört. Schon 1997 hat die Hochschulrektorenkonferenz (HRK) eine Positionsbestimmung zur Einführung von Bachelor- und Master-Studiengängen abgegeben, in die auch Fachhochschulen einbezogen waren. 1998 wurde durch eine Änderung des Hochschulrahmengesetzes die Möglichkeit geschaffen, zur Erprobung Studiengänge einzurichten, die zu einem Bachelor- oder MasterGrad führen. Die Regelstudiendauer für den Bachelor wurde mit mindestens drei und höchstens vier Jahren, die für den Master mit mindestens einem und höchstens zwei Jahren festgelegt. Entsprechend der Bildungshoheit der Länder bedarf es zur Einrichtung solcher Studiengänge einer Genehmigung des entsprechenden Landes. Um die Gleichwertigkeit der Abschlüsse sicherzustellen, wurde von der HRK und der Kultusministerkonferenz ein bundesländerübergreifender Akkreditierungsrat eingerichtet, dem die Koordination und das Monitoring der Qualitätssicherungsverfahren obliegt.

Eine im Sommer 2002 abgeschlossene Studie (CHE/CHEPS 2002) kommt zu folgenden Ergebnissen: Die Umstellung auf die neue Studienarchitektur befindet sich in Deutschland in einer Pionierphase, noch wird keine komplette Umstellung angestrebt; andererseits haben immerhin 41\% der Fachhochschulen, 25\% der Technischen Universitäten und 18\% der Universitäten mit einer flächendeckenden Einführung der neuen Abschlüsse begonnen. Für die Zukunft erwarten die meisten Hochschulleitungen ein massives Wachstum.

Der gegenwärtige Pioniercharakter kommt in mehreren Merkmalen zum Ausdruck:

- Es gibt eine mittlere Innovationsbereitschaft der Hochschulen (immerhin 10\% des gesamten Studienangebots sind Bachelor/Master-Studiengänge), aber nur wenig Breitenwirkung ( $1 \%$ der Studierenden); daraus folgt: es gibt gute Betreuungsrelationen, aber hohe Kosten.

- Die Einführung neuer Studiengänge erfolgt zumeist dezentral, nur selten auf Initiative der Hochschulleitung.

- Die neuen Abschlüsse sind überwiegend als zusätzliches Angebot eingerichtet (das bestehende Angebot wird nicht ersetzt). 
- Es überwiegt das „unabhängige Modell“ (v.a. unabhängige Master-Programme), das „konsekutive Modell“ (Master folgt auf einschlägigen Bachelor) ist selten.

- Die Akkreditierung ist sehr aufwändig: es gibt lange Wartezeiten und hohe Kosten.

Weitere Befunde der Studie:

- In Relation zu den Studierendenzahlen sind die Fachhochschulen besonders aktiv (40\% der Bachelor/Master-Studiengänge bei ca. einem Viertel der Studierenden). Für Fachhochschulen sind die neuen Abschlüsse sehr attraktiv, da sie sich formell nun nicht mehr von den Abschlüssen der Universitäten unterscheiden. Generell führt die neue Studienarchitektur zu einer Aufweichung der sektoralen Grenzen zwischen Universitäten und Fachhochschulen: Universitäten bieten in höherem Ausmaß anwendungsorientierte Studiengänge an, im Gegenzug können sich Fachhochschulen im Bereich theorie- und forschungsorientierter Studiengänge profilieren.

- Bei den Motiven für die Einführung neuer Abschlüsse dominiert an den Hochschulen sehr stark das Ziel einer Verbesserung der Rahmenbedingungen für Internationalisierung; dementsprechend sind ausländische Studierende die attraktivste Zielgruppe (v.a. für Master-Studiengänge). Das von der Hochschulpolitik ebenfalls stark betonte Ziel der Verbesserung der Studienstrukturen (Senkung der Abbruchquoten, Verkürzung der Studienzeiten) steht an den Hochschulen deutlich im Hintergrund. Dementsprechend gibt es nur in wenigen Fällen neue Angebotsformen (Teilzeit- oder Fernstudium).

- Ungeklärt ist der - vom HRG geforderte - berufsqualifizierende Charakter des BachelorAbschlusses. Ob es möglich und sinnvoll ist, mit diesem Abschluss in den Beruf einzutreten, ist weiterhin umstritten.

\section{Die Niederlande}

Die Niederlande hatten keine akuten nationalen Probleme, die mit Hilfe der BolognaDeklaration ,gelöst' werden mussten. Diese Deklaration war aber eine gute Möglichkeit, um die internationale Anerkennung von niederländischen Hochschulstudien zu vergrößern. Sowohl die Diplomstruktur, mit im Ausland unbekannten Graden, als auch die nicht immer transparenten Qualitätsbewertungsprozeduren wurden bemängelt. Letzteres wurde gerade im FH-Sektor als nationales Problem betrachtet; 1999 wurde ein Pilotprojekt zur Akkreditierung angefangen (evaluiert in Goedegebuure et al. 2002; Inspectie van het Onderwijs 2002). Was die Diplomstruktur anbelangt, erklärte bereits das Hochschulgesetz („WHW“) von 1993, dass HochschulabsolventInnen das Recht hätten, die Abschlüsse „Master" oder „Bachelor" als äquivalent zum niederländischen Titel zu verwenden. Der Abschluss eines universitären Studienprogramms wurde mit dem „Master“ gleichgestellt; ein 
FH-Abschluss wurde im Hochschulgesetz 1993 mit einem „Bachelor“ gleichgestellt. Aber diese Gesetzeserklärung besaß keine ausreichende Glaubwürdigkeit in den Augen von Ausländern, sodass die Bologna-Deklaration ein Anstoß war, um beide Probleme neu zu lösen: künftig werden alle Hochschulstudienprogramme auf das zweistufige Bachelor/Master-Modell umstrukturiert. Für die Diplome werden die englischen Bezeichnungen verwendet und die Qualität soll mittels Akkreditierung abgesichert werden. Die benötigten Gesetzesänderungen wurden 2002 vom Parlament verabschiedet. Die neue Studienstruktur sollte von der Mehrzahl der Hochschulen ab September 2002 eingeführt werden, die übrigen werden im September 2003 folgen.

Der Bologna-Prozess wird für einen großen Teil der FH-Ausbildung wenig ändern: die bestehenden Studienprogramme waren schon zuvor auf dem Niveau des Bachelors und bleiben das. ${ }^{187}$ Die Fachhochschulen haben aber außerdem schon in den neunziger Jahren, meist mit Hilfe von britischen Universitäten (in der Regel durch Doppeldiplome mit ehemaligen polytechnics) viele Master-Programme eingeführt, die mit der generellen Einführung der Bachelor/Master-Struktur an den niederländischen Hochschulen eine eigene, offizielle niederländische Akkreditierung bekommen werden. ${ }^{188}$ Die Finanzierung dieser Master-Programme ist bis jetzt umstritten: Bachelor-Programme werden vom Staat an allen Hochschulen finanziert (wenn auch unterschiedlich für Universitäten und hogescholen), Master-Programme voraussichtlich nur an Universitäten. Die Fachhochschulen müssen für ihre Master-Programme bis jetzt Marktpreise verlangen.

Die Akkreditierung soll 2003 beginnen. Vorbereitungen dazu erfolgten in den Jahren 2000 bis 2001 durch eine "Quartiermachergruppe" (Commissie Accreditatie Hoger Onderwijs 2001). Der Vorschlag dieser Kommission, der zum größten Teil in die Novelle des Hochschulgesetzes von 2002 übernommen wurde, war es, alle Studienprogramme alle fünf Jahre zu akkreditieren. „Alle Studienprogramme“ heißt nicht nur die von den öffentlichen Universitäten und Fachhochschulen, sondern auch die von privaten und ausländischen Anbietern, die ein niederländisches Bachelor- oder Masterdiplom anbieten möchten.

Dabei wird mehr oder weniger das auch in Deutschland angewendete Modell des „open accreditation system" verfolgt. Die Nationale Akkreditierungsorganisation (NAO) wird nicht selbst alle Studienprogramme beurteilen, sondern überlässt diese Tätigkeit Agenturen zur Qualitätsbeurteilung, die freien Zutritt zu den niederländischen Hochschulen haben werden. Die Hochschulen können sich die Agentur, von der sie eine Akkreditierung wünschen, aussuchen. Die heutigen Qualitätsbeurteiler, die Vereine der Hochschulen (VSNU und HBORaad), können auch im neuen System ihre Arbeit fortsetzen - wenn auch vielleicht in

187 Größere Änderungen sind wohl an den Universitäten nötig, die bis jetzt praktisch keine Zwischenstufung kannten - aber darauf geht dieser Bericht weiter nicht ein.

188 Auch diese Akkreditierung wurde schon vom FH-Sektor „vorbereitet“, indem aus dem FH-Rat (HBO-Raad) der Dutch Validation Council (DVC) hervorging und bereits Ende der neunziger Jahre einsatzfähig war. 
anderer Form als bisher -, aber sie werden nicht mehr wie bisher eine Monopolstellung besitzen. Neue und ausländische Akkreditierungsorganisationen können ebenso auftreten; Studienprogramme können somit eine ihrem Qualitätsprofil gerecht werdende Beurteilung wählen (van Vught 1994; Westerheijden 2003 forthcoming). Die NAO wird alle Berichte mit Qualitätsurteilen darauf überprüfen, ob sie den methodischen Standards entsprechen; Selbstevaluationsberichte und Reviews unabhängiger Peers gehören dabei zu den methodischen Mindestbedingungen.

Der Unterschied zwischen universitären und $\mathrm{FH}$-Studienprogrammen wird im neuen Akkreditierungsverfahren mehr oder weniger erhalten bleiben. Studienprogramme werden wählen können, ob sie als „akademisches“ oder „professionelles“ Studienprogramm akkreditiert werden wollen. Sowohl auf Bachelor- als auch auf Masterebene wird dieser Unterschied gemacht, mit entsprechenden Unterschieden in den Zielsetzungen der Programme und in den Kompetenzen, die von AbsolventInnen erwartet werden. Im Titel soll dieser Unterschied sichtbar werden; so werden die allgemeinen Bezeichnungen wie „B.A.“ und „M.Sc." nur von „akademischen“ Programmen verliehen; „professionelle" Programme führen zu Titeln mit Bezeichnung des Fachbereichs, z.B. „M.Eng.“, „B.Ed.“. Zu beachten ist, dass dieser Unterschied nur auf der Ebene der Studienprogramme definiert ist; Universitäten und Hochschulen können, theoretisch gleichermaßen, sowohl "akademische“ als auch „professionelle“ Programme anbieten. Eine Folge dieser „Deinstitutionalisierung“ der curricularen Profile scheint zu sein, dass Universitäten und Fachhochschulen noch mehr als zuvor zur Zusammenarbeit geneigt sind, was in manchen Fällen bis zu Fusionsplänen reicht. ${ }^{189}$

$\mathrm{Ob}$ es zu einer Akkreditierung kommt oder nicht wird mehrere wichtige Folgen haben. Erstens wird Akkreditierung eine Bedingung werden, um Bachelor- oder Masterdiplome nach niederländischem Recht anbieten zu können (andere Abschlüsse werden nicht durch Akkreditierung geschützt). Zweitens wird sie eine Bedingung für finanzielle Unterstützung vom Staat. Wie erwähnt werden aber Masterprogramme an Fachhochschulen nicht vom Staat finanziert werden, ${ }^{190}$ und auch private Anbieter können keine öffentliche Finanzierung bekommen. Drittens - und dies gilt auch für Studienprogramme von privaten Anbietern - ist Akkreditierung eine Voraussetzung dafür, dass Studierende staatliche Stipendien und sonstige öffentliche Förderung bekommen können.

Die NAO sollte ab 2003 aktiv werden, ist aber gegenwärtig noch im Aufbau begriffen. Die NAO wird auch die Akkreditierungsbehörde für Flandern sein, denn die flämische Behörde findet das eigene Hochschulsystem zu klein, um eine eigene Organisation aufzubauen. Das

\footnotetext{
189 Der Grad von Zusammenarbeit war durch den deutlichen Unterschied zwischen den Zielsetzungen der beiden Sektoren immer schon international gesehen hoch (Huisman/Kaiser 2001).

190 Vorläufig werden diese denn auch nicht akkreditiert, sondern nur registriert werden.
} 
ist ein interessantes Argument, denn das flämische Hochschulsystem ist mit acht Universitäten und vielen $\mathrm{FH}$-artigen Institutionen größer als manch andere, die eine eigene Akkreditierung aufbauen (Westerheijden 2002).

\subsection{Die Implementierung des Bologna-Prozesses in Österreich}

\subsubsection{Die Rezeption des Bologna-Prozesses in der österreichischen Hochschulpolitik}

Die österreichische Hochschulpolitik hat sehr rasch auf die Harmonisierungsbestrebungen der europäischen Politik reagiert. Im Juli 1999, nur knapp ein Jahr nach der SorbonneErklärung, wurden in Österreich die gesetzlichen Grundlagen für die neue Studienarchitektur geschaffen. Seitens der Politik wurde ein sehr rasches Vorgehen gewählt, um Doppelgleisigkeiten bei der Studienreform zu vermeiden (an allen österreichischen Universitäten hatten die Studienkommissionen gerade die Arbeit aufgenommen, um das 1997 verabschiedete UniStG zu implementieren). Diese Eile hatte andererseits zur Folge, dass die neue Studienarchitektur, die tief in die Logik des akademischen Lebens eingreift, nicht in ihrer vollen Tragweite diskutiert wurde. Dazu einige Anmerkungen:

- Häufig wird der Bachelor als "Zwischenabschluss" bezeichnet. Das führt in die Irre. Ein Zwischenabschluss verlangt nach einer Fortsetzung in Richtung "eigentlicher" Abschluss. Der Bachelor aber ist im angelsächsischen Bereich keine halbe Sache, sondern ein kompletter, vom Arbeitsmarkt akzeptierter Erstabschluss. Die meisten Studierenden verlassen die Universität nach dem Erststudium und kehren nur noch zum Zwecke der Weiterbildung (die zu keinem formellen Abschluss führt) an sie zurück. Nur noch eine Minderheit setzt mit einem Graduiertenstudium (Master, Doktor) fort. Hier wird eine auf die Bedingungen der Massenuniversität zugeschnittene Logik erkennbar: ein sehr breiter Zugang in der ersten Phase, der sich dann in den späteren Stufen der akademischen Ausbildung verengt. Bei den postgradualen Studien werden entsprechend weniger Studienplätze angeboten, die Anforderungen sind hoch, die Zulassung selektiv. Auf dieser Basis gelingt es vor allem dem amerikanischen, aber auch anderen angelsächsischen Systemen, eine sehr weitgehende Öffnung und Partizipation mit höchster Qualität zu verbinden. Das Erststudium erfüllt nicht nur eine breite Qualifizierungsfunktion, es ist zugleich ein Testfeld, auf dem sich die viel selteneren Talente bewähren müssen, die den anspruchsvolleren Teil der akademischen Ausbildung fortsetzen wollen.

- Es gibt einen weitgehenden Konsens, dass die Studiendauer verkürzt werden sollte. Nun wird ein dreijähriges Studium in Aussicht gestellt. Aber ein Abschluss namens Bachelor bewirkt gar nichts, wenn er nicht mit einer entsprechenden Kultur der akademischen Lehre korrespondiert. Im angelsächsischen System wird die vorgesehene Studiendauer 
tatsächlich eingehalten, die Zahl der Jahre bezeichnet dort nämlich keine unverbindliche Mindestdauer, sondern einen Normwert, von dem sehr klare Erwartungen in Richtung der StudentInnen, aber auch ihrer LehrerInnen ausgehen. Das Curriculum ist übersichtlich angeordnet, die Anforderungen an die Studierenden sind klar formuliert und müssen erfüllt werden. Umgekehrt sind auch die Aufgaben des akademischen Personals präziser festgelegt und ihre unterstützende Funktion (gute didaktische Fähigkeiten, Betreuungsaufgaben) wird stärker betont als bei uns. Das Studium im angelsächsischen Bereich ist, wie man bei uns abwertend sagt, "verschult". Jedenfalls gilt das für das Erststudium, die Undergraduate-Phase. Im anspruchsvolleren Graduiertenstudium sind dann auch die Freiheiten (und Risken) höher.

- Entscheidend ist schließlich die Schnittstelle zum Arbeitsmarkt. Ursprünglich stand die Frage, ob der Bachelor für A-wertige Stellen im öffentlichen Dienst berechtigt, im Vordergrund. Diese Frage hat durch die Dienstrechtsreform im öffentlichen Dienst, in deren Folge Neueinstellungen auf der Basis des Vertragsbedienstetenrechts erfolgen, seine ursprüngliche Brisanz verloren. Wichtiger ist es, ob der Bachelor vom Arbeitsmarkt als berufsqualifizierender Abschluss akzeptiert wird. Das wird zum einen vom Curriculum abhängen. Auch aus dieser Perspektive wäre das Konzept eines "Zwischenabschlusses" verfehlt. Zum anderen sind die Erwartungen der Arbeitgeber entscheidend. Im angelsächsischen Raum funktioniert das zweiphasige Studiensystem nicht zuletzt deshalb, weil die Unternehmen darauf eingestellt sind, dass die AbsolventInnen eine gewisse "Anlernphase" im Unternehmen benötigen. Hier gibt es ein derzeit noch ungelöstes Spannungsverhältnis zwischen der Eigenlogik eines UndergraduateAbschlusses und den in Österreich etablierten Traditionen bezüglich der Schnittstelle zwischen Hochschule und Arbeitsmarkt.

\subsubsection{Reservierte Haltung der Universitäten}

Welchen Fortschritt macht die Implementierung ${ }^{191}$ der neuen Studienarchitektur bzw. des Bologna-Prozesses an den Universitäten? Bis zum Studienjahr 2001/02 wurden von den wissenschaftlichen Universitäten 14 Bachelor-Studienrichtungen mit insgesamt 26 Studienzweigen angeboten; im Studienjahr 2002/03 kommen an wissenschaftlichen

191 Im BMBWK findet ein Monitoring der Implementierung der in der Bologna-Erklärung angeführten Ziele statt. Dieses Monitoring beschränkt sich allerdings auf sehr hochaggregierte Ergebnisse der Umsetzung, nicht auf den Prozess selbst. Die ProjektmitarbeiterInnen führen die dem Bundesministerium bekannten Daten über die Einführung von Bachelor-Graden oder die Beteiligung an ECTS-Netzwerken zusammen und erstellen so Gesamtübersichten, aus denen erkennbar ist, welche Anstrengung die einzelnen Universitäten bei der Umsetzung der Bologna-Erklärung auf sich nehmen. Der Prozess der Implementierung ist eine "black box", die nicht beobachtet wird. Hier zeigt sich schon die neue (distanzierte) Beziehung zwischen Bundesministerium und Universitäten: ersteres hat sich aus der operativen Ebene (und auch aus dem Monitoring der operativen Ebene) weitgehend zurückgezogen, das liegt in der Verantwortung der Universitäten. 
Universitäten 21 Bachelor-Studienrichtungen mit 26 Studienzweigen hinzu, an Universitäten der Künste 25 Studienrichtungen mit insgesamt 28 Studienzweigen. Die Implementierung der neuen Studienarchitektur kommt also langsam in Gang; aber es ist bislang nur ein sehr geringer Prozentsatz der Studien, der von der neuen Möglichkeit Gebrauch macht.

Diese faktische Zurückhaltung (die nicht auf die österreichischen Universitäten beschränkt ist), steht in einem gewissen Widerspruch zu den offiziösen Dokumenten der europäischen Politik, die den Eindruck erwecken, die Mehrzahl der Akteure in fast allen Ländern sei mit großem Ernst und Eifer dabei, ihre Systemarchitekturen und Curricula vollständig umzugestalten. Sieht man näher hin, dann relativiert sich diese Reformeuphorie. Viele der genannten Maßnahmen sind de facto legistische Maßnahmen, die Möglichkeiten schaffen, welche von den Universitäten kaum genutzt werden (oft nicht einmal bekannt sind) und die manchmal selbst auf der politisch-administrativen Ebene nur halbherzig unterstützt werden.

Handelt es sich also nur um politische Propaganda ohne Substanz? Nicht ganz: Es ist zweifellos so, dass die Proponenten der Reform einen gewissen Zweckoptimismus verbreiten; man will bei den Adressaten den Eindruck vermitteln, ganz Europa sei auf den Beinen, wer nicht ebenso emsig mitmache, sei ein Nachzügler, gerate unaufholbar ins Hintertreffen. Man hofft gewissermaßen eine „sich selbst erfüllende Übertreibung" in die Welt zu setzen, welche die zögerlichen bzw. bremsenden ${ }^{192}$ Akteure unter Zugzwang setzt.

Allerdings könnten die seit 2001/02 fälligen Studienbeiträge die weitere Entwicklung beschleunigen. Es ist anzunehmen, dass ein nicht vernachlässigbarer Teil der StudentInnen den kürzeren und damit billigeren Abschluss bevorzugen werden. In dem Ausmaß, in dem die Universitäten den Präferenzen der Studierenden Rechnung tragen bzw. - durch allfällige Änderungen in der Hochschulfinanzierung - diese Präferenzen zu spüren bekommen, wird auch die Opposition gegen die neue Studienarchitektur abnehmen.

192 Es gibt legistische Schlupflöcher, die es Einzelnen ermöglichen, den Prozess zu blockieren. Z.B. ECTS: es ist ein Vorausbescheid nötig, der mobilen Studierenden sagt, welche an fremden Universitäten gemachten Lehrveranstaltungen daheim anerkannt werden. Es hat schon Fälle gegeben, wo nach Rückkehr des Studierenden ein/e neue/r Vorsitzende/r der Studienkommission im Amt war, der/die den Bescheid des/r Vorgängers/in nicht anerkannt hat. In diesem Fall ist derzeit nur der Gang zum VWGH möglich. 


\subsection{Konsequenzen der neuen Studienarchitektur für den Fachhochschulsektor}

\subsubsection{Implementierung der neuen Studienarchitektur im Fachhochschulbereich}

Bei der Novellierung des UniStG zur Neugestaltung der Studienarchitektur (1999) wurde der Fachhochschulsektor zunächst nicht berücksichtigt. Eine explizite Begründung gab es nicht, aber unter der Hand konnte man die Argumente hören, eine Inklusion des Fachhochschulsektors in den Bologna-Prozess sei einerseits nicht nötig und zugleich potentiell gefährlich. Nicht nötig, weil die wichtigsten Reformziele, die man an den Universitäten anstrebe (Verkürzung der Studienzeiten, Anbindung der österreichischen Universitäten an den europäischen Hochschulraum) für den Fachhochschulsektor nicht aktuell seien: da es keine exzessive Studiendauer gibt, bedarf es auch keiner Verkürzung. Und da Fachhochschul-Studiengänge primär regionale Bedeutung hätten und die akademische Mobilität nur eine geringe Rolle spiele, seien international gängige Studienabschlüsse nicht nötig. Darüber hinaus wurde es als riskant betrachtet, ein ohnehin erfolgreiches Modell durch Reformhektik unter Druck zu setzen und den noch im Aufbau begriffenen Sektor durch eine Veränderung der Rahmenbedingungen in Turbulenzen zu bringen.

Gegen diesen faktischen Ausschluss des FH-Sektors aus dem Bologna-Prozess hat sich vor allem die FHK gewandt. Als Argument wurde genannt, dass damit die Äquivalenz von Magister- und Master-Abschlüssen gefährdet sein könnte: beim Anrechnungsverfahren könnten ausländische Partner darauf verweisen, dass der Mag. (FH) ein Erstabschluss, also dem Bachelor vergleichbar sei. Von einer solchen Interpretation wären natürlich in erster Linie die Fachhochschuleinrichtungen selbst betroffen; die Universitäten könnten argumentieren, dass der von innen vergebene Diplomabschluss ein wirklicher Zweitabschluss sei.

Die FHK hat sich mit diesen Argumenten durchgesetzt und eine Novellierung des FHStG erreicht. Es ist aber unklar, in welchem Zeitrahmen und welchem Umfang die Erhalter eine faktische Implementierung der neuen Studienarchitektur anstreben; in einigen Fällen dürfte es den Erhaltern eher um die rechtliche Möglichkeit gehen (um den Wert des Mag. (FH) abzusichern), weniger um die tatsächliche Nutzung der neuen Abschlüsse.

Die Befragung der Erhalter hat ein sehr disparates Bild ergeben. Für ein knappes Drittel der Erhalter ist diese Frage noch offen. Die meisten Erhalter wollen einige Studiengänge in der neuen Struktur anbieten, aber zugleich ihre Diplomstudiengänge beibehalten. Eine definitive Ablehnung der neuen Abschlüsse stellt eine Minderheitenposition dar. Einige Erhalter haben sich bereits dazu entschlossen, alle Studiengänge auf die Bachelor/Master-Struktur umzustellen. 


\subsubsection{Wie verändern sich die Profile der beiden Sektoren?}

Eine weit verbreitete Kritik lautet, dass durch die Einführung von Bachelor/MasterStudiengängen die Grenzen zwischen Universitäts- und Fachhochschulsektor unscharf werden: Die Universitäten dringen in ein Terrain ein, in dem sich bislang die fachhochschulischen Bildungseinrichtungen erfolgreich profiliert hatten - in das Feld kurzer, berufsorientierter Studienangebote. Das gilt jedenfalls dann, wenn man den Bachelor nicht als "Zwischenabschluss", sondern als vollwertigen berufsqualifizierenden Erstabschluss betrachtet. Einwände dieser Art kamen sowohl von Universitäts- wie von Fachhochschulseite. Bei ersteren mit dem Tenor: unter den neuen Voraussetzungen hätten wir uns die Einrichtung von Fachhochschul-Studiengängen sparen können. Bei letzteren schwingt zuweilen die Befürchtung mit, die Universitäten könnten dem Fachhochschulsektor nun jenes Feld, auf dem er sich bewährt hat, streitig machen.

Gegenüber diesen Einwänden ist festzuhalten, dass es in Wahrheit langfristig keine ernsthafte Alternative gegen eine Inklusion der Fachhochschuleinrichtungen in den BolognaProzess gegeben hat. Sie auszuschließen hätte bedeutet, sie auf ein Nebengleis zu stellen. Der Einwand, dass die Grenzen zwischen dem universitären und dem nicht-universitären Sektor an Trennschärfe verlieren, ist richtig, aber er geht von falschen Voraussetzungen aus. Nur solange die einzelnen Staaten die uneingeschränkte Souveränität hatten, innerhalb ihres Territoriums in autonomer Weise die Hochschulpolitik zu gestalten, konnten sie auch die Grenzen zwischen allfälligen Sektoren nach selbstgewählten Kriterien definieren ${ }^{193}$. In der „postnationalen Konstellation“ (Habermas) büßt die nationale Hochschulpolitik diese Fähigkeit weitgehend ein. Der „Europäische Hochschulraum“ gehört dieser postnationalen Konstellation an. Das Verständnis dieser Situation wird dadurch erschwert, dass sie sich durch viele Ambivalenzen auszeichnet: die Machtverhältnisse sind fragmentiert, weder auf supranationaler, noch auf nationaler Ebene gibt es Akteure, die aus eigener Kraft das Feld der Hochschulpolitik dominieren.

Die konkrete Auswirkung in Bezug auf das Verhältnis von Universitäten und Fachhochschuleinrichtungen ist: das Feld wird neu "aufgemischt“; die klare sektorale Differenzierung verschwimmt. Auszuschließen ist, dass es zu einer neuen Homogenisierung kommt, dass Universitäts- und Fachhochschulsektor sich in umfassender Weise angleichen, also alle Fachhochschuleinrichtungen und alle Universitäten zu gleichartigen Institutionen werden. Im Gegenteil, die Differenzierung wird insgesamt vermutlich zunehmen.

- Erstens wird die sektorale Differenzierung schwächer, aber nicht völlig aufgehoben. Niemand denkt in Österreich (derzeit) an eine Aufhebung des „binary system“. Daher

\footnotetext{
193 So konnte Österreich etwa mehr als zwei Jahrzehnte lang gegen den internationalen Trend keine sektorale Differenzierung seines Hochschulsystems vornehmen.
} 
werden die neuen Rahmenbedingungen zu einem schärferen Wettbewerb zwischen den Sektoren führen, denn ihre Aufgabenfelder überlappen sich nun stärker als zuvor. Unter der nicht unplausiblen Annahme nur schwach wachsender, im ungünstigsten Fall vielleicht sogar schrumpfender Gesamtressourcen wird es zu einem Verdrängungswettbewerb kommen.

- Zweitens wird es innerhalb der Sektoren zu einer verstärkten institutionellen Differenzierung kommen. Dies ist für die Universitäten eine weit stärkere Herausforderung als für den Fachhochschulbereich. Der Fachhochschulsektor hat de facto nie jene homogene Gestalt gehabt wie die Universitäten der Ära vor dem UOG 1993. Es war immer schon eine unsachgemäße Übertragung des universitären Musters auf den Fachhochschulbereich, wenn man - was doch das dominante Wahrnehmungsmuster war - auch dort eine weitgehende Gleichartigkeit der unterschiedlichen Einrichtungen unterstellte. Nun werden auch die Unterschiede zwischen den Universitäten zunehmen. Es ist leicht denkbar, dass von den neuen Rahmenbedingungen einzelne Universitäten und Fachhochschuleinrichtungen profitieren, während andere Institutionen aus beiden Sektoren zu den Verlierern zählen.

Anstelle der (oder besser: in Ergänzung zur) formellen, deutlich sichtbaren Differenzierung werden in verstärktem Maß informelle Mechanismen der Differenzierung treten. Die Situation wird dann wesentlich komplexer und wird von allen Akteuren erhöhte Orientierungsleistungen verlangen:

- Für die Anbieterseite ergibt sich die Notwendigkeit verstärkter strategischer Profilierung: wo liegen die Stärken der eigenen Institution, was sind realistische Ziele, die man anstreben sollte?

- Für die Nachfrager nach Ausbildungsangeboten (Studierende) und qualifiziertem Personal (Unternehmen) verschärft sich das Informationsproblem, da die herkömmliche Terminologie an Aussagekraft verliert, die traditionellen Abschlüsse ihre Signalfunktion partiell einbüßen. Daher werden zusätzliche Maßnahmen zur Erhöhung der Transparenz erforderlich (Akkreditierung, Ranking).

Betrachtet man die neue Situation unter dem Gesichtspunkt des Wettbewerbs zweier Sektoren, dann kann man aus der Perspektive des Fachhochschulsektors folgende Chancen identifizieren:

- Im Bereich der Undergraduate-Ausbildung, welche die quantitativ stärkste Bedeutung spielt, haben die Fachhochschulen und Fachhochschul-Studiengänge eine Art „Heimvorteil“; sie haben ein knappes Jahrzehnt Erfahrung als Anbieter einer praxisorientierten und vergleichsweise kurzen Ausbildung und gute Erfolge erzielt. Viele hochschulpolitische Akteure bezweifeln, ob auch die Universitäten dazu fähig sind und 
diese Einschätzung korrespondiert mit der Selbsteinschätzung eines großen Teils der Universitätsangehörigen, die genau dieses Ausbildungsprofil nicht als Aufgabe der Universitäten betrachten.

- Der Fachhochschulsektor hat auch eine neuartige Lehr- und Lernkultur entwickelt, die auf das oben beschriebene Ausbildungsprofil abgestimmt ist. In quantitativer und qualitativer Hinsicht ist Lehre in den Mittelpunkt gerückt; durch eine adressatenspezifische Didaktik und eine klare Strukturierung des Curriculums unterscheidet sie sich von den meisten Lehrangeboten der Universitäten, an denen diese Merkmale als „Verschulung“ abgewertet werden.

- Auf der formalen Ebene ist die Gleichwertigkeit der Abschlüsse, die grundsätzlich schon im FHStG vorgesehen ist, aber immer wieder in Frage gestellt wurde, bestätigt worden. Die nähere Beschreibung der vermittelten Qualifikationen in Form eines Diploma Supplements und der Hinweis auf die den Abschluss vergebende Hochschule immer wichtiger werden. Aber das gilt auch für die Universitäten, ist also keine einseitige Informationsverpflichtung für den Fachhochschulsektor, die von vorneherein ein hierarchisches Verhältnis konstituiert.

Auf der anderen Seite entstehen für den Fachhochschulsektor einige Risken:

- Das Eindringen der Universitäten in den Kernbereich des Fachhochschulsektors kann auch als Bedrohung betrachtet werden. Es ist nicht ausgeschlossen, dass die Universitäten erfolgreich sind, wenn sie auf Grund der veränderten Rahmenbedingungen gezwungen sind, das bislang vom Fachhochschulsektor besetzte Ausbildungsprofil ernst zu nehmen. Ohne das im Fachhochschulbereich vorhandene Potential an Kreativität zu schmälern, kann man vermuten, dass ein Teil ihres Erfolges darauf beruht, dass innen die Universitäten ein bestimmtes Aufgabenfeld "kampflos“ überlassen haben (nicht zuletzt deshalb, weil es einem großen Teil des wissenschaftlichen Personals an den Universitäten nicht attraktiv genug erschienen ist). Es bleibt abzuwarten, ob sich dies in naher Zukunft tatsächlich spürbar ändern wird; und es ist auch eine offene Frage, wie schnell und erfolgreich die Universitäten das für sie neue Aufgabenspektrum besetzen können.

- Die neuen Rahmenbedingungen führen zu einer „Ermächtigung“ der Studierenden im Sinne einer erweiterten Konsumentensouveränität, die vor allem erhöhte Mobilitätschancen zwischen den Sektoren eröffnet. Als Konsequenz dieser Entwicklung reduziert sich die Definitionsmacht der Anbieterseite über den Bildungsprozess. Den Studierenden bieten sich nun „exit-Optionen“, sie können Anforderungen, die innen unattraktiv oder überflüssig erscheinen, vermeiden, indem sie auf andere Angebote ausweichen. Das könnte nur durch ein „Anbieterkartell“ verhindert werden, das über kollektive Absprachen die Definitionsmacht der Bildungseinrichtungen vor der Erosion 
durch Konsumentenwünsche schützt ${ }^{194}$. Es ist aber sehr unwahrscheinlich, dass es zu wirkungsvollen, alle Anbieter bindenden Absprachen dieser Art kommt. Bildungseinrichtungen werden daher verstärkt „Zugeständnisse“ an die Nachfrager machen müssen. ${ }^{195}$ Das kann positive und negative Konsequenzen haben.

- Bei den Erhaltern gibt es eine Unsicherheit über die Finanzierung von MasterStudiengängen; es ist nicht klar, in welchem Umfang der Bund diese Studiengänge im Fachhochschulsektor finanzieren wird.

\subsection{Zusammenfassung und Schlussfolgerungen}

\subsubsection{Zusammenfassung}

Die gesamtgesellschaftlichen Globalisierungsprozesse haben ihren Niederschlag in unterschiedlichen Formen der Internationalisierung im Hochschulbereich gefunden. Die nationalstaatlich definierten Systemarchitekturen und Praktiken bleiben erhalten und bilden weiterhin einen entscheidenden Bezugsrahmen für die Hochschulen, ihr wissenschaftliches Personal und ihre Studierenden. Aber neben dieser nationalen gewinnt eine supranationale Ebene, auf der Hochschulen miteinander kooperieren und zueinander in Konkurrenz treten, an Bedeutung. Der Begriff „Internationalisierung“ verweist auf eine neue Qualität der grenzüberschreitenden Verflechtung und Vernetzung von Hochschulsystemen. Der wechselseitige Einfluss beschränkt sich nicht mehr darauf, sich im Zuge internationaler Vergleiche Anregungen bzw. Legitimationen für die eigene Politik zu holen. Vielmehr kommt es verstärkt zu Interdependenzen, die auch für die einzelne Hochschule unmittelbar handlungsrelevant sind.

Sowohl die nationale Hochschulpolitik als auch die strategischen Positionierungen der einzelnen Institutionen stehen vor der Notwendigkeit, diese beiden Ebenen aufeinander abzustimmen. Da im FH-Sektor auch die regionale Politik auf der Ebene der Länder und teilweise auch der Gemeinden eine wichtige Rolle spielt, könnte die Komplexität dieser Abstimmung sowie das Ausmaß der potentiellen Spannungsfelder hier noch größer sein als bei den Universitäten.

194 Der einzige wirkungsvolle Schutz gegenüber dem Zwang, qualitätsmindernde Zugeständnisse an die Nachfrageseite machen zu müssen, besteht in den Auflagen der Agenturen zur Qualitätssicherung. Zunehmend konzentriert sich die Definitionsmacht über Standards auf diese Orte. Allerdings stehen auch diese Einrichtungen unter massivem Legitimationsdruck, sie müssen ihre Anforderungen und Auflagen politischen und wirtschaftlichen Interessen gegenüber rechtfertigen, was immer wieder Veränderungen nach sich zieht (z.B. hat erst eine Neuorientierung der Akkreditierungsagenturen die Akzeptanz gewinnorientierter Hochschulen in den USA ermöglicht).

195 Wie die meisten hochschulpolitischen Trends war auch diese Entwicklung in den USA früher zu spüren und wurde dort von David Riesman bereits 1980 thematisiert (Riesman 1980). 
In diesem Rahmen wurden primär jene Aspekte der Internationalisierung thematisiert, die für den österreichischen FH-Sektor gegenwärtig von Bedeutung sind. Internationalisierung bedeutet überwiegend akademische Mobilität, derzeit vor allem Austausch von Studierenden, zunehmend aber auch Mobilität des akademischen Personals. Das geographische Zentrum dieser Mobilität sind die Länder der Europäischen Union, deren Mobilitätsprogramme die wichtigsten Beziehungsnetzwerke und Förderschienen eröffnen. So gesehen ist die gewachsene akademische Mobilität ein Ausdruck der Entstehung eines Europäischen Hochschul- und Forschungsraums.

Die Auslandsaufenthalte von FH-AbsolventInnen liegen in der gleichen Größenordnung wie die der Universitäts-AbsolventInnen und über jener der AbsolventInnen von Kunstuniversitäten. Angesichts des geringen Alters des FH-Sektors kann das als beachtlicher Erfolg gewertet werden. Der Studierendenaustausch an fachhochschulischen Einrichtungen zeichnet sich durch einige Besonderheiten aus, auf die bei der weiteren Entwicklung geachtet werden muss:

- Die Auslandspraktika nehmen - im Vergleich mit Fachstudium und wissenschaftlichen Abschlussarbeiten - einen sehr hohen Stellenwert ein, was darauf verweist, dass das akademische Profil der Austauschbeziehungen ungenügend entwickelt ist. Bemerkenswert ist, dass es eine Diskrepanz zwischen den Angaben der AbsolventInnen (betonen die Bedeutung des Praktikums) und jenen der Studiengangsleiterlnnen (betonen das Fachstudium) gibt.

- Es gibt ein Ungleichgewicht zwischen „incoming“ und „outgoing“. Bei den Studierenden gehen ca. vier Mal soviel Inländerlnnen hinaus als ausländische Studierende an österreichische FH-Einrichtungen kommen. Der österreichische Fachhochschulsektor wird von ausländischen Studierenden also nur ungenügend wahrgenommen und/oder nicht als ausreichend attraktiv eingeschätzt. Bei den Lehrenden geht das Ungleichgewicht in die entgegengesetzte Richtung. Ausschlaggebend dürfte sein, dass die österreichischen Fachhochschuleinrichtungen den Ausfall der eigenen Lehrkräfte auf Grund des geringen Stammpersonals nur schlecht verkraften können.

Da das geographische Zentrum der Internationalisierung für alle österreichischen Hochschulen, im besonderen aber für die fachhochschulischen Institutionen Europa ist, besitzt der Bologna-Prozess eine so große Bedeutung. Es ist offenkundig, dass die Universitäten der neuen Studienarchitektur mehrheitlich mit großen Vorbehalten gegenüberstehen. Der FH-Sektor hat sich lange darum bemüht, in diesen Prozess als ein den Universitäten gleichwertiger Partner eingebunden zu werden und sie haben dieses Ziel erreicht. Es gibt derzeit aber keine Anzeichen dafür, dass die Mehrheit der Erhalter offensiv von dieser Möglichkeit Gebrauch machen möchte. 
Ein Grund für diese Vorsicht scheint zu sein, dass mit der neuen Studienarchitektur zugleich die Grenzen zwischen dem Universitäts- und dem Fachhochschulsektor durchlässiger werden. In der „postnationalen Konstellation“, die sich hochschulpolitisch als „Europäischer Hochschulraum" geltend macht, wird die Fähigkeit und Bereitschaft des Staates zur sektoralen Differenzierung des Hochschulsystems schwächer. Mit den kurzen Bakkalaureats-Abschlüssen übernehmen die Universitäten z.T. Aufgaben, die bislang die Basis für den Erfolg des FH-Sektors gebildet haben, auf der anderen Seite wird der FHSektor verstärkt als Ort von Forschung und Entwicklung wahrgenommen. Man kann derzeit nicht abschätzen, ob für den FH-Sektor bzw. die einzelnen Institutionen eher die Chancen oder die Risken dieses Prozesses spürbar werden.

\subsubsection{Schlussfolgerungen}

Damit verweist eine Bestandsaufnahme im Bereich der Internationalisierung auf ähnliche Problemlagen, wie sie sich auch aus der innerösterreichischen Diskussion ergeben: aus beiden Blickwinkeln ist eine Konsolidierung bestehender Standorte und Studiengänge sowie eine thematische Verbreiterung des Studienangebots $z u$ empfehlen. Ob und in welchem Ausmaß sich Erhalter bzw. Studiengänge international positionieren wollen, ist eine strategische Entscheidung, für die mehrere Optionen offen stehen. Es gibt derzeit keine Anhaltspunkte dafür, dass für Fachhochschuleinrichtungen, die sich weitgehend auf nationale und regionale Zielsetzungen beschränken und der Internationalisierung einen sehr geringen Stellenwert zuweisen, kein Platz wäre. Aber es ist zu vermuten, dass eine solche Entscheidung die weiteren Entwicklungsmöglichkeiten stark einschränkt; mittel- und langfristig könnte das zu einem Reputationsverlust und zu einer gewissen Marginalisierung führen.

Umgekehrt sind bei einer offensiven Strategie im Bereich der Internationalisierung Synergieeffekte für die Entwicklung im nationalen Rahmen zu erwarten.

- Die Konsolidierung von Standorten schafft bessere Möglichkeiten, um die für Internationalisierung nötige Infrastruktur aufzubauen.

- Die thematische Erweiterung des Studienangebots ist ein wichtiger Schritt zu verbesserter internationaler Sichtbarkeit des FH-Sektors.

- Ein Ausbau der Forschungskapazität und die Einbindung in internationale Netzwerke verstärken sich wechselseitig.

Eine defensive Haltung bei der Implementierung der neuen Studienarchitektur ist der weiteren Entwicklung des FH-Sektors nicht förderlich. Zweifellos ist es möglich, die Einführung der neuen Abschlüsse zu verzögern bzw. eine gewisse Zeit lang eine 
Doppelstruktur aufrechtzuerhalten (sowohl Bachelor/Master-Abschlüsse als auch Diplomabschlüsse). Langfristig wird sich die neue Architektur europaweit durchsetzen. Der Fachhochschulsektor kann sich das Image der Reformfreudigkeit erhalten, indem er diesen Prozess offensiv mitgestaltet.

Auf der Ebene der nationalen Hochschulpolitik sollte dieser Prozess durch folgende Maßnahmen unterstützt werden:

- Fachhochschul- und Universitätssektor sollen hinsichtlich der finanziellen Förderung akademischer Mobilität gleichgestellt werden. Die gegenwärtigen Förderpraktiken sind nur aus ihrem historischen Kontext heraus zu verstehen, können aber nicht mit dem spezifischen Profil der beiden Sektoren begründet werden. Eine Ungleichbehandlung würde die Botschaft an den $\mathrm{FH}-$ Sektor senden, dass Internationalisierung keine hohe Priorität hat.

- Der Bund sollte Klarheit über die Finanzierung von Master-Studiengängen im Fachhochschulsektor schaffen, um der Verunsicherung im Sektor entgegenzuwirken. Eine nahe liegende Form, Transparenz herzustellen, wäre es, die Zahl der auf Normkostenbasis finanzierten Plätze in Master-Studiengängen im Entwicklungs- und Finanzierungsplan festzulegen. 



\section{Qualitätssicherung}

Die Etablierung neuer Formen der Qualitätssicherung war ein essentieller Aspekt des neuen bildungspolitischen Steuerungsmodells im Fachhochschulsektor. Im folgenden werden Akkreditierung, Monitoring, Evaluierung und die interne Qualitätssicherungsmaßnahmen der Studiengänge und Fachhochschulen als wichtige Bestandteile einer integralen Qualitätssicherung im Fachhochschulsektor, die in dieser Form neu und zukunftsweisend für den gesamten tertiären Bildungssektor ist, kurz beschrieben sowie das Qualitätssicherungsmodell überblicksartig in einen internationalen Rahmen gestellt. Perspektiven und ausgewählte Aspekte der Weiterentwicklung des Qualitätssicherungsmodells bilden den Abschluss des Kapitels.

\subsection{Das Qualitätssicherungsmodell des FH-Sektors}

Im Fachhochschulbereich wurde erstmals ein Weg zur Deregulierung der Qualitätskontrolle im Hochschulsystem in Österreich beschritten. Die fachhochschulischen Bildungseinrichtungen wurden selbst für die Entwicklung ihres akademischen Angebotes verantwortlich gemacht und es wurde eine eigene Einrichtung für die Qualitätskontrolle geschaffen, die einen Weg zur Dezentralisierung der Qualitätssicherung darstellt. Das neue Steuerungsmodell, das der Fachhochschulsektor im österreichischen Bildungssystem Anfang der 1990er Jahre verkörpert hat, ist auch durch eine hohe Sensibilität Qualitätsfragen gegenüber gekennzeichnet. Deregulierung, institutionelle Autonomie, Dezentralisierung gehen Hand in Hand mit einer auch stärkeren Eigenverantwortung für die Qualität der zu entwickelnden Ausbildungsangebote. Die Verantwortung für pädagogische Entscheidungen wird dem FHR und den fachhochschulischen Bildungsinstitutionen übertragen. Hatte bis dahin das zuständige Bundesministerium zentral zumeist noch in einem mehrstufigen, äußerst komplizierten Prozess durch Gesetze und Verordnungen versucht, Qualitätssicherung im Hochschulbereich zu betreiben, so wurde im FH-Sektor zum ersten Mal das neue Element einer Akkreditierung in Kombination mit einer Ex-postEvaluierung festgelegt. Die die Akkreditierung durchführende Behörde, der Fachhochschulrat, ist dabei ein ExpertInnengremium, das überwiegend aus Personen mit wissenschaftlich-pädagogischer bzw. berufsfeldspezifischer Kompetenz und nicht aus InteressensvertreterInnen oder politischen RepräsentantInnen zusammengesetzt ist und das insbesondere die Qualität der Anträge zu prüfen hat. ${ }^{196}$ Akkreditierung und Evaluierung sind

196 Der Fachhochschulrat besteht aus 16 Mitgliedern, wobei die Hälfte wissenschaftlich durch eine Habilitation (oder eine dieser gleichwertigen Qualifikation) ausgewiesen sein muss, die Hälfte der Mitglieder über mehrjährige Tätigkeit in einem relevanten Berufsfeld verfügen muss. Die Mitglieder werden vom/von der zuständigen Bundesministerln ernannt. Sie sind in der Ausübung ihrer Tätigkeiten an keine ministeriellen Weisungen gebunden. 
dabei miteinander verknüpft: bei der Verlängerung der Anerkennung (Re-Akkreditierung) ist dem Fachhochschulrat ein entsprechender Evaluationsbericht vorzulegen.

Der Fachhochschulrat hat aber nicht nur Aufgaben in Bezug auf die Evaluation der einzelnen Studiengänge, sondern auch in Bezug auf den Sektor als solchen, indem er diesen hinsichtlich seiner Kohärenz mit dem übrigen Bildungssystem sowie hinsichtlich seiner Akzeptanz durch Beschäftigungssystem und Bildungsnachfrage zu überprüfen hat. Im Wesentlichen kontrolliert der Fachhochschulrat im Bereich der Anerkennung anhand von Input-Merkmalen ex-ante die Qualität des Angebots. Die Ex-post-Kontrolle nimmt er nicht selbst vor, sondern überprüft ein von den Erhaltern vorgeschlagenes Evaluationsvorhaben auf seine Tauglichkeit.

Zunehmend hat sich aber doch der Wunsch nach einem vergleichbaren Verfahren eingestellt und der Fachhochschulrat hat einen Evaluationsausschuss eingesetzt. Dieser hat Richtlinien für die Durchführung der Evaluierungsverfahren und die Erstellung eines Evaluationsberichtes entworfen. Für die laufende Evaluation der Studiengänge hat der Fachhochschulrat einmal jährlich die Bereitstellung von Informationen zu folgenden Bereichen verordnet:

Quantitative Angaben (Studierendenpopulation, Lehrende etc.) und qualitative Angaben (Maßnahmen zur pädagogisch-didaktischen Weiterbildung, Erfahrungsberichte über das Aufnahmeverfahren, über interne Evaluationsmaßnahmen etc.).

Man kann somit zwei Regelkreise der Qualitätssicherung an Fachhochschul-Studiengängen unterscheiden (vgl. Hackl et al 1997, 150). Der erste langfristige Regelkreis ergibt sich aus den im Akkreditierungsverfahren und im Rahmen des Fördervertrages festgelegten Bestimmungen durch Vorgabe der Finanziers. Der zweite Regelkreis, der innere Regelkreis, liegt im autonomen Gestaltungsbereich des Studienganges und ermöglicht eine unmittelbare Umsetzung qualitätssichernder Maßnahmen. Die Elemente beider Regelkreise werden in den folgenden Ausführungen näher beschrieben.

\subsubsection{Die Akkreditierung}

Ganz generell geht es bei der Akkreditierung als einem Instrument der Qualitätssicherung um eine inhaltliche fachliche ExpertInnenmeinung über die Qualität eines neu anzubietenden Studienganges, die als eine Art "Verbraucherschutz“ funktioniert und die Abkehr von ministerieller Studienplanprüfung ermöglicht. Akkreditierung bedeutet im internationalen Kontext daher eine Gewährleistung von Mindeststandards.

Die Akkreditierung im österreichischen Fachhochschulsystem erfolgt durch eine eigens dafür geschaffene intermediäre Einrichtung - dem Fachhochschulrat - und in Form professioneller 
Expertise. Unter Akkreditierung versteht die Geschäftsstelle des österreichischen Fachhochschulrates „ein Verfahren zur Überprüfung der Einhaltung der vorgegebenen Anforderungen an die Erstellung, das mit einer Ja- oder Nein-Entscheidung endet, wobei qualitätssteigernde Vorgaben des FHR nicht ausgeschlossen sind“ (Sohm 2002, 87).

Ein Akkreditierungsverfahren besteht im Wesentlichen aus zwei Abschnitten. In einem ersten Schritt wird der vorgelegte Antrag formal hinsichtlich seiner Übereinstimmung mit den gesetzlichen Bestimmungen geprüft. Grundlage der Prüfung sind die im FHStG festgelegten Voraussetzungen für die Anerkennung als Fachhochschul-Studiengang. Hier ergibt sich meist ein erster Mängelbehebungsauftrag; so werden z.B. Unvollständigkeiten, eventuelle Fehler u.ä. aufgelistet. Diese Liste geht an den Antragstellenden mit der Aufforderung, innerhalb einer Frist die Mängel zu beheben. Die korrigierte Fassung wird in einem zweiten Schritt auf die inhaltliche Qualität durch den Fachhochschulrat in seiner Funktion als Expertlnnengremium geprüft. Speziell eingerichtete Arbeitskreise befassen sich mit einzelnen Aspekten des Antrages. Jedenfalls kann die inhaltliche Prüfung des Antrages zu einem weiteren Mängelbehebungsauftrag führen. Das Akkreditierungsverfahren gilt als abgeschlossen, wenn alle gesetzlichen Voraussetzungen erfüllt sind. Die bescheidmäßige Anerkennung wird jedoch erst ausgesprochen, wenn der Antragstellende entsprechende Verträge für die Finanzierung vorlegen kann. Ein Fachhochschul-Studiengang kann vom Fachhochschulrat nur für maximal 5 Jahre anerkannt werden. Es muss jedoch allen Studierenden, die innerhalb dieses Zeitraumes aufgenommen werden, die Garantie gegeben werden, das Studium im Fall einer Nichtverlängerung der Anerkennung regulär beenden zu können. Daher werden nur Finanzierungsverträge akzeptiert, die eine entsprechende Haftung beinhalten. Kann der Antragstellende schließlich einen Finanzierungsvertrag in Form eines Fördervertrags mit dem zuständigen Bundesministerium, also die sogenannte Bundesförderung, als Basisfinanzierung vorlegen, so wird der Fachhochschul-Studiengang vom Fachhochschulrat mit Bescheid anerkannt.

Jeder Erhalter muss vor Ablauf des Genehmigungszeitraumes eines FachhochschulStudienganges um Verlängerung der Anerkennung ansuchen. Die Re-Akkreditierung ist spätestens sechs Monate vor Ablauf des Akkreditierungszeitraumes zu beantragen. Erneut muss ein vollständiger Antrag inklusive Finanzierungszusagen eingereicht werden. Nach etwaigen Mängelbehebungsaufträgen und deren Erfüllung kann die Fortführung des Studienbetriebes für weitere höchstens 5 Jahre genehmigt werden.

Der Antrag eines Fachhochschul-Studienganges übernimmt die Funktion eines individualisierten Organisations- und Studiengesetzes. Bei der Akkreditierung handelt es sich um eine Art Input-Kontrolle, wie sie auch die Grundlage der Sicherung einheitlicher Standards im universitären Bereich bildet. Da das FHStG jedoch als Rahmengesetz nur grobe Richtlinien vorgibt, die unterschiedlichen Antragstellenden aber das Recht auf Gleichbehandlung ihrer Anträge haben, geriet der FHR in folgende Schwierigkeiten (vgl. Kozar 1999, 65): Um die geforderte Gleichbehandlung zu gewährleisten, aber auch trotz der 
Fülle von Anträgen eine bewältigbare Bearbeitung zu sichern, sah sich der Fachhochschulrat gezwungen, die Vorgaben des FHStG zu operationalisieren. Insbesondere die unerwartete Fülle von Anträgen, verbunden mit der personellen Unterbesetzung der Geschäftsstelle und eines nur nebenberuflich tätigen Rates, machte rasch zumindest eine Teilstandardisierung unumgänglich. Damit erhöhte sich auch die Regelungsdichte und der auf Deregulierung basierende Rahmencharakter des FHStG wurde durch eine Fülle von (notwendig gewordenen) Verordnungen partiell unterlaufen.

\subsubsection{Monitoring}

Der Aufbau einer möglichst transparenten Informations- und Wissensbasis ist ein wichtiger Aspekt der qualitätsvollen Entwicklung des Fachhochschulsystems. Folgende Instrumente dazu sind als Minimalinformationen vorgesehen.

\section{Jährliche statistische Erhebungen}

Der FHR gab eine Statistikverordnung heraus, aufgrund dieser Verordnung sind alle Erhalter verpflichtet, einmal jährlich eine Reihe von Daten an den Fachhochschulrat zu übermitteln. Dazu zählen quantitative Angaben wie Bewerberlnnen, Aufgenommene, Studierende, DropOuts, AbsolventInnen, Lehrende und qualitative Angaben wie Maßnahmen zur pädagogischdidaktischen Weiterbildung der Lehrenden, Forschungs- und Entwicklungsarbeiten der Lehrenden, Erfahrungsbericht über das Aufnahmeverfahren, Bericht über interne Evaluierungsmaßnahmen des Studienganges.

Dieses Berichtswesen bezieht sich auf die Ziele und leitenden Grundsätze von Fachhochschul-Studiengängen, die im FHStG geregelt sind. Kozar (1999) hält nun fest, dass die Statistikverordnung evaluierungstechnisch gesehen zwei wichtige Aspekte hat. Einerseits erfüllt der FHR mit diesen Erhebungen nicht nur die gesetzlichen Vorgaben, weil er detailliertere Angaben verlangt. Mit der Festlegung signalisiert der FHR implizit auch, welche Bereiche als relevant für die Beobachtung und Beurteilung eines $\mathrm{FH}$-Studienganges angesehen werden. Tatsächlich wurden durch diese Verordnung Indikatoren und einfache Kennzahlen festgelegt, die erst in nachträglichen Bearbeitungen in Relationen übersetzt werden. Andererseits bedingt eine derartige Festlegung eine mehr oder weniger erwünschte Orientierung der betroffenen Einrichtungen an eben diesen Indikatoren. Als Beispiel führt Kozar an, dass etwa die Statistikverordnung den Zustand der Bibliotheken nicht erfasst, der aber etwa bei Peer-Review-Verfahren als äußerst dürftig bezeichnet wurde. Neben den positiven Effekten der Statistikverordnung, die vor allem darin zu sehen sind, dass ein Satz von Eckdaten ständig erhoben und dieser von den Studiengängen auch beobachtet wird, sind die negativen Effekte nicht zu vernachlässigen, die vor allem in der ausschließlich quantitativen Erhebung sowie im Fehlen von Leistungsindikatoren liegen. Der FHR 
versuchte jedenfalls, so meint Kozar, die erhobenen Kennzahlen als das einzusetzen, was sie tatsächlich sind: als Anzeigen und Hinweise.

\section{Problemzentrierte Interviews}

Nicht interpretierbare Rückmeldungen bei den statistischen Erhebungen z.B. führen oft zum Einsatz von eintägigen Vorort-Besuchen, die vom FHR in Auftrag gegeben werden. Unabhängige WissenschafterInnen versuchen, anhand der Unterlagen Problembereiche zu identifizieren, bereiten qualitative Interviews mit Akteuren vor. Nach dem eintägigen Besuch werden dann Interviews und Beobachtungen protokolliert und ein Endbericht sowohl dem FHR als auch dem Erhalter des evaluierten Studienganges vorgelegt. In fast allen Fällen kommt es dann zu einem Gespräch zwischen dem FHR und dem Erhalter, in dem die eruierten Problembereiche besprochen werden. Diese Methode wurde als „problemzentriertes Interview“ (PZI) bezeichnet und ist eigentlich von den meisten Fachhochschul-Studiengängen (vgl. Kozar 1999, 68) eher positiv empfunden worden. Dieses Instrument ermöglicht zum einen eine spontane Reaktion des Fachhochschulrates im Falle bedenklicher Entwicklungen; diese Methode der qualitativen Sozialforschung wurde aber auch erfolgreich angewandt, um Erfahrungen über beispiellose Innovationen zu gewinnen (vgl. Schelling 1999, 83). Berichte über PZI bilden Informationen an die Peers im Zuge der wissenschaftlichen Evaluierung. Feststellungen der Peers im Endbericht, die auf Mängel hinweisen, die bereits zum Zeitpunkt eines vorangegangenen PZI bestanden und offenbar nicht behoben wurden, kommt ein besonderes Gewicht zu (vgl. Schelling 1999, 83).

\section{Beobachtung der Abschlussprüfungen}

Weiters gibt es noch das Instrument der Beobachtung von Abschlussprüfungen. Derartige Beobachtungen wurden zwar schon mehrmals von Mitgliedern des FHR vorgenommen und diese würden das - so kam es in den ExpertInneninterviews zum Ausdruck - gerne öfter tun, schätzen dies aber aufgrund begrenzter Kapazitäten skeptisch ein. Die Berichte darüber dienten lange vor allem der ratsinternen Information, was in einem gewissen Sinne schade ist - eine Systematisierung der Beobachtung und eine Einbindung der Protokolle wäre sicher sinnvoll (vgl. Kozar 1999, 71). Im Fall jener fachhochschulischen Einrichtungen, die den Status einer Fachhochschule haben, liegen hier auch entsprechende Verpflichtungen bei der Leitung des Kollegiums. 


\subsubsection{Evaluation}

Das strategische Ziel von Evaluierung als einem unabdingbaren Qualitätssicherungsinstrument ist die Integration valider Rückkoppelungsmechanismen in die Entscheidungs- und Handlungsprozesse von Organisationseinheiten.

Die Geschäftsstelle des Fachhochschulrates versteht unter Evaluierung den Versuch, „durch Beurteilen, Analysieren, Einschätzen, Kritisieren, Bewerten etc. den Vorzug, Wert bzw. Nutzen von etwas im Hinblick auf die geforderte Qualität zu bestimmen... Das Evaluierungsverfahren beruht auf dem Qualitätskonzept „Fitness for Purpose“, d.h. die Qualität einer fachhochschulischen Bildungseinrichtung wird im Grad der Erfüllung der vorgegebenen und selbstgesteckten Zielsetzungen gesehen“ (Sohm 2002, 88).

Der österreichische Fachhochschulrat signalisierte recht bald, dass er an einer ausschließlich auf Kontrolle ausgerichteten Evaluierung nicht interessiert sei, sondern zur Förderung von wissenschaftlicher Kompetenz und Selbstorganisation des Fachhochschulbereiches auf diesem Gebiet beitragen wolle. Evaluierungen in allen Varianten sollten als ein integrativer Bestandteil des Fachhochschulwesens verstanden und etabliert werden. Vor diesem Hintergrund hat der Evaluierungsausschuss des FHR gemeinsam mit der Fachhochschul-Konferenz - also dem Zusammenschluss von Erhaltern und Studiengangsleiterlnnen der österreichischen Fachhochschul-Studiengänge - ein System der Qualitätssicherung im FH-Bereich entwickelt. Die für eine Verlängerung der Anerkennung eines Studienganges notwendige wissenschaftliche externe Evaluierung in Form des Peer-Review steht dabei im Zentrum. Sie hat einen zweistufigen Aufbau und basiert als externe Evaluierung auf einer vorherigen internen Evaluierung. Das Qualitätssicherungssystem der FHK/FHR (QS) gibt 15 Gliederungspunkte vor, nach denen sowohl die Berichte der Selbstevaluierung als auch die Berichte der externen Evaluierung strukturiert sein müssen. 


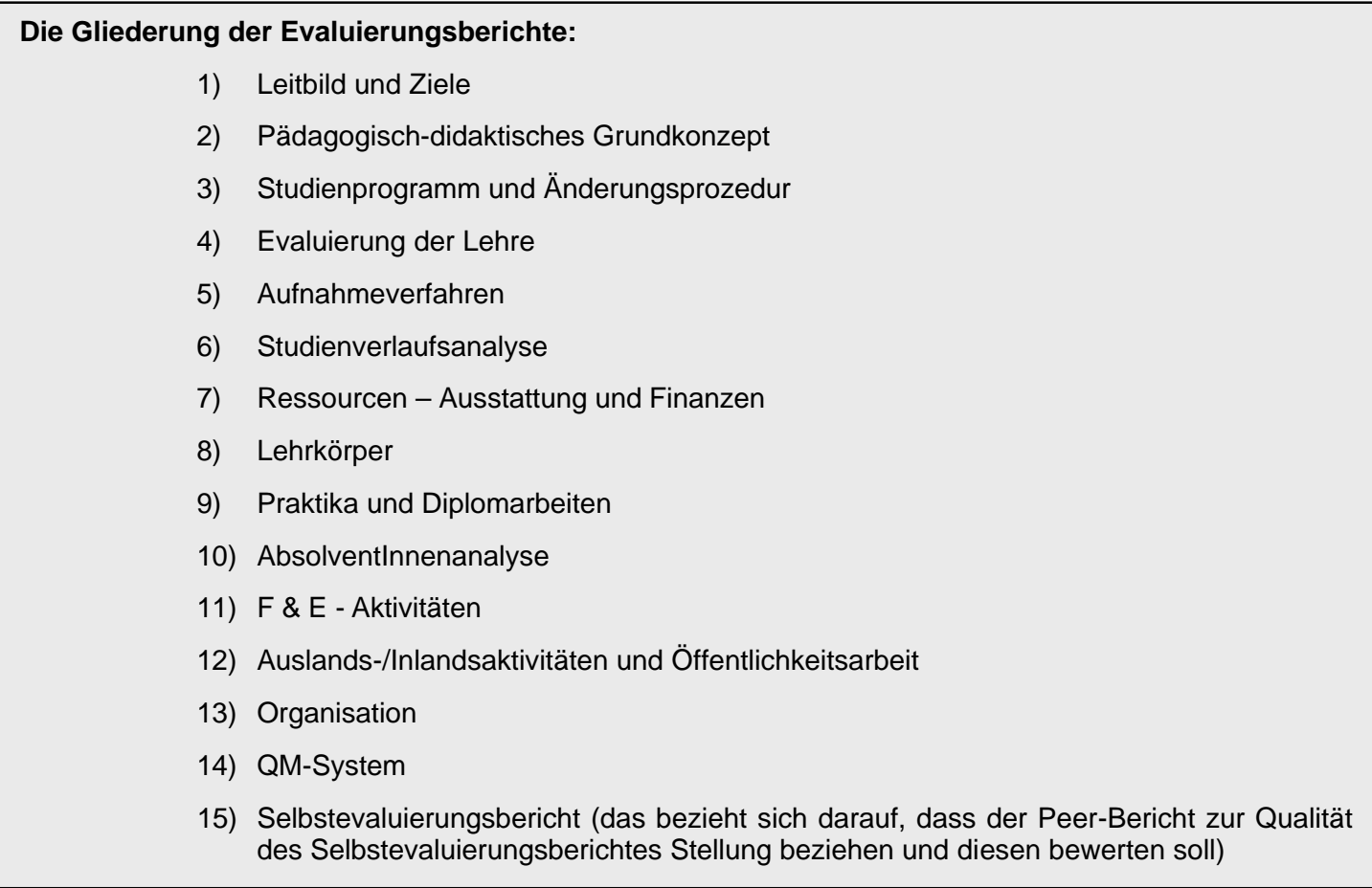

Die Basis des Modells bildet eine umfassende Selbstevaluierung, deren Ergebnisse wiederum Ausgangspunkt einer mehrtägigen Vorort-Untersuchung durch sogenannte Peers, also facheinschlägige oder fachverwandte KollegInnen sind. Der evaluierten Einheit steht schließlich noch das Recht zu, eine Stellungnahme zum Endbericht abzugeben. Der Endbericht der Peers inkl. der Stellungnahme der FH-Studiengänge geht schließlich an den FHR, für den dieser Bericht, gemeinsam mit dem Antrag auf Verlängerung der Anerkennung die Entscheidungsgrundlage im Re-Akkreditierungsverfahren zur Verlängerung bildet.

Das Grundmodell der Fachhochschulevaluierung entspricht damit jenem der Hochschulevaluierung in den meisten europäischen Ländern. Es gibt vielleicht in einigen Details Unterschiede, aber auch gleiche Probleme.

Kozar fasst die folgenden kritischen Bereiche der Peer-Reviews zusammen, denen man besonderes Augenmerk widmen sollte (vgl. Kozar 1999, 131):

1) Wichtig ist es, bei der Nominierung der Peers auf deren Qualifikation und nicht so sehr auf deren Funktion zu achten. Durch eine Schulung etwa in qualitativen Interviewtechniken und die Betonung des Forschungs- gegenüber dem Kontrollcharakter wäre auch ein Schritt zur Intensivierung des Reviewgeschehens und Vergleichbarkeit der Endberichte gegeben.

2) Man muss in die Arbeitsfähigkeit der Peer-Gruppe investieren. Eine Vorbereitungsphase der Peers wäre unter diesem Gesichtspunkt zu überdenken. 
3) Peer-Review beruht darauf, dass etablierte ExpertInnen zu Rate gezogen werden. Diese aber sind zumeist Repräsentanten der bestehenden Normen, deren Einsatz an Fachhochschul-Studiengängen, die als innovative Einheiten eingerichtet wurden, zumindest diskussionswürdig wäre. Das problematisiert wiederum die Nominierung der Peers. Neben den einschlägigen Qualifikationen und der Teamfähigkeit wird somit die Offenheit gegenüber Neuerungen und neuen Wegen eine wichtige Rolle bei der Suche nach geeigneten ExpertInnen für ein Peer-Review-Verfahren spielen müssen (Kozar 1999, 133).

\section{Die Einführung der Institutionellen Evaluierung}

Derzeit wird das Verfahren der Evaluierung umgestellt. Nach einer inoffiziellen Begutachtungsphase bei inländischen und ausländischen Begutachterlnnen wurde es Ende Juli 2002 im Fachhochschulrat beschlossen. Es gibt künftig (ab 2003) institutionelle Evaluierungen, die eher die Institution Fachhochschule im Blick haben und damit wahrscheinlich zum rechten Zeitpunkt die Institutionswerdung unterstützen; sie reduzieren auch den Arbeitsaufwand für den Fachhochschulrat, weil ansonsten eine Vielzahl von Evaluierungen und Re-Akkreditierungen von einzelnen Studiengängen anstehen würde.

Ein zweites Element wird die studiengangsbezogene Evaluierung im Quervergleich sein, also themenspezifische Studiengänge werden parallel miteinander verglichen, unabhängig von der Genehmigungsdauer. Das heißt, es werden im Jahr 2004 nicht 25 Studiengänge, sondern eben nur 12 Erhalter institutionell evaluiert. Insofern ist diese Umstellung im Evaluierungsverfahren auch ein Beitrag zur organisatorischen Weiterentwicklung.

Auszug aus dem im Juli 2002 vom FHR beschlossenen Konzept „Evaluierung im österreichischen Fachhochschulsektor":

Unter Evaluierung versteht der FHR also einerseits ein Verfahren zur Erhebung und Steigerung der professionellen Selbstorganisation der fachhochschulischen Institution, andererseits ein Verfahren zur Steigerung der Qualität der einzelnen Bildungsangebote. Maßnahmen zur Qualitätssicherung des Bildungsangebotes setzen die Selbst-Organisation der fachhochschulischen Institutionen voraus.

In einem übergeordneten Zusammenhang betrachtet stellt die Evaluierung auch ein Instrument zur Aufrechterhaltung und Steigerung der Wettbewerbs- und Veränderungsfähigkeit der fachhochschulischen Bildungseinrichtungen dar. Vor dem Hintergrund, dass die Wettbewerbs- und Konkurrenzfähigkeit der fachhochschulischen Bildungseinrichtungen durch deren Veränderungsfähigkeit bestimmt wird, sind sie als "Lernende Organisationen“ zu begreifen.

Evaluierung im österreichischen FH-Sektor entspricht internationalen Standards und setzt sich aus den folgenden Elementen zusammen:

- Interne Evaluierung durch die fachhochschulische Einrichtung

- $\quad$ Externe Evaluierung durch ein Review-Team

- Stellungnahme der evaluierten fachhochschulischen Einrichtungen zum Evaluierungsbericht des Review-Teams

- Follow-up Verfahren

- $\quad$ Veröffentlichung der Ergebnisse der Evaluierung 
Die Aufgabe der Institutionellen Evaluierung besteht in der Analyse und Bewertung der professionellen Selbstorganisation der fachhochschulischen Institutionen in den Bereichen Lehre, Angewandte Forschung $u$. Entwicklung und Verwaltung.

Das Verfahren der Institutionellen Evaluierung geht von der Voraussetzung aus, dass die Verantwortung für die Sicherung und Verbesserung der Qualität letztlich bei den fachhochschulischen Institutionen liegt. Die Zielsetzung besteht in der (Weiter-) Entwicklung der Selbststeuerungskompetenzen der fachhochschulischen Institutionen. Sie werden im 5- bis 7-Jahres-Rhythmus evaluiert.

Bereiche der Institutionellen Evaluierung :

1. Strategische Ausrichtung, Profilbildung, Entwicklungskonzept, Kommunikationsstrukturen

2. Aufbau- und Ablauforganisation

3. Ressourcen und Infrastruktur

4. Personal und Personalentwicklung

5. Angewandte Forschung \& Entwicklung sowie Dienstleistungen

6. Organisation der Lehre / Pädagogisch-didaktisches Gesamtkonzept

7. Aufnahmeverfahren und Anerkennung nachgewiesener Kenntnisse

8. Qualitätsmanagement und Evaluierung

9. Internationalisierung

10. Öffentlichkeitsarbeit

11. Qualität des Selbst-Evaluierungsberichtes

Jede Re-Akkreditierung eines FH-Studienganges erfordert die Vorlage eines Evaluierungsberichtes (vgl. § 13 Abs. 2 FHStG). Die Vorlage des Berichts über das Ergebnis der Institutionellen Evaluierung im Zuge der Re-Akkreditierung eines FH-Studienganges gilt als Erfüllung dieser gesetzlichen Voraussetzung.

Die Aufgabe der studiengangsbezogenen Evaluierung besteht in der Analyse und Bewertung des Zusammenhangs zwischen Berufsfeld, Qualifikationsprofil und Curriculum. Die Zielsetzung der studiengangsbezogenen Evaluierung besteht in der Überprüfung der Plausibilität dieses Zusammenhanges und der inhaltlichen Adäquanz und Aktualität des Curriculums.

\subsubsection{Interne Qualitätssicherung der Studiengänge und Fachhochschulen}

Ein wichtiges Element der Qualitätssicherung im Fachhochschulbereich war auch immer die eigenständige Wahrnehmung der Aufgabe der internen Qualitätssicherung durch die Studiengänge bzw. Fachhochschulen selbst. Die Studiengänge sind verpflichtet, Lehrveranstaltungsbewertungen durchzuführen und für die Weiterbildung ihrer Lehrenden Sorge zu tragen, in der Wahl der genauen Methoden und im Einsatz weiterer Instrumente sind sie allerdings frei. Für den Fachhochschulrat war es auch immer wichtig - trotz aller Bemühungen, etwa gemeinsame Richtlinien für die Evaluierung zu formulieren -, dass jeder Fachhochschul-Studiengang bei der Auswahl bzw. der Gestaltung seines internen Qualitätsmanagementsystems frei ist (vgl. Schelling 1999, 74). Um die Fähigkeit des Fachhochschulsektors abzuschätzen, auf neue Anforderungen adäquat zu reagieren - und das ist ein wichtiger Aspekt aller Formen der Qualitätssicherung - wäre eine VorortUntersuchung der eingesetzten Qualitätsmanagementsysteme ein adäquater Weg gewesen; eine solche Untersuchung hätte allerdings den Rahmen der vorliegenden Studie gesprengt. So wurde das Thema der Qualitätssicherung als (kleiner) Fragenkomplex in die Befragungen 
der Studiengangsleitungen und Erhalter aufgenommen, die im Rahmen des vorliegenden Projektes im Juli 2002 durchgeführt wurde. Im folgenden werden die Ergebnisse kurz wieder gegeben.

Die Ergebnisse der Befragungen zeigen, dass das Qualitätssicherungsbewusstsein unter den Studiengangsleiterlnnen und den Erhaltern im allgemeinen recht hoch ist ( $\mathrm{vgl}$. Abbildungen 63 und 64).

Abbildung 64: Qualitätssicherung im FH-Sektor: Anteile der FH-Studiengänge nach Art der Maßnahmen zur Qualitätssicherung, 2002

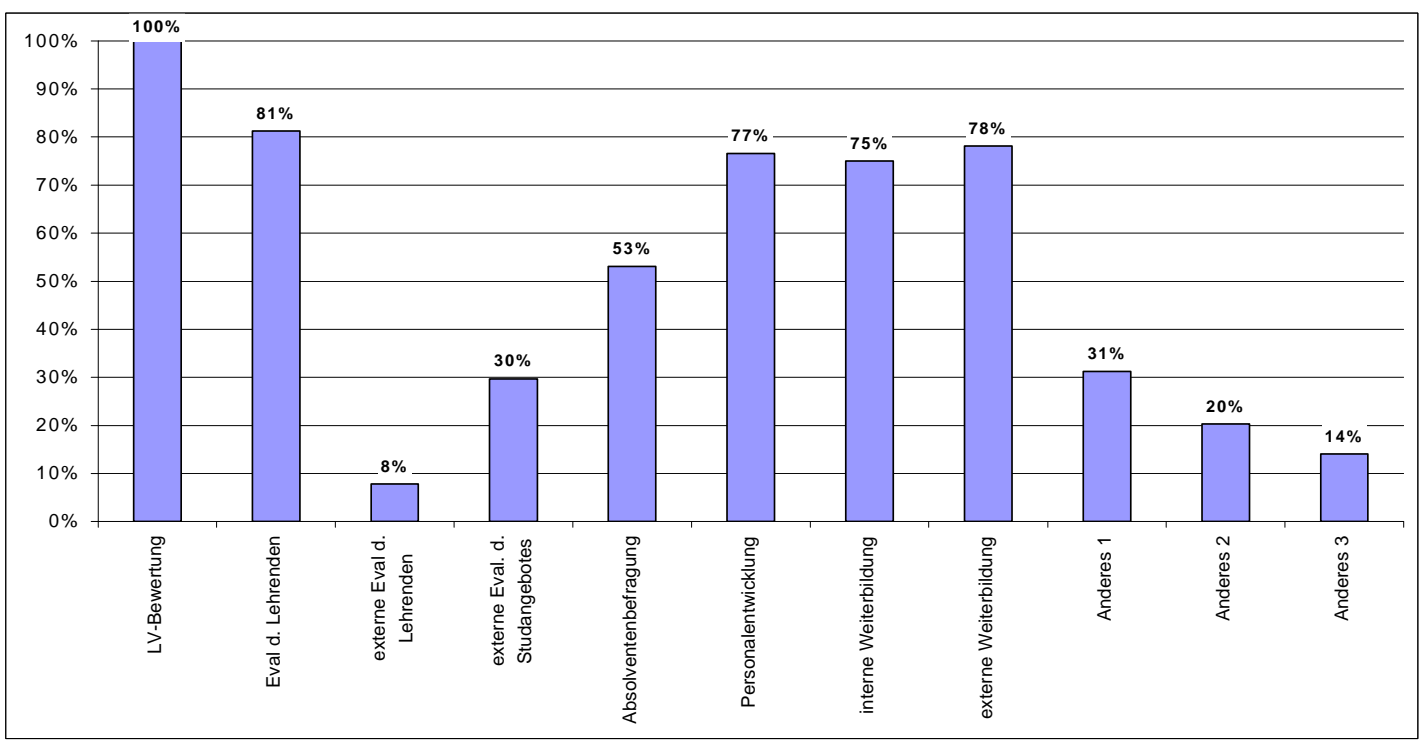

Quelle: Befragung der Studiengangsleitungen 
Abbildung 65: Qualitätssicherung im FH-Sektor: Anteile der FH-Studiengänge (nur Studiengänge, die 4 oder mehr Jahrgänge umfassen) nach Art der Maßnahmen zur Qualitätssicherung, 2002

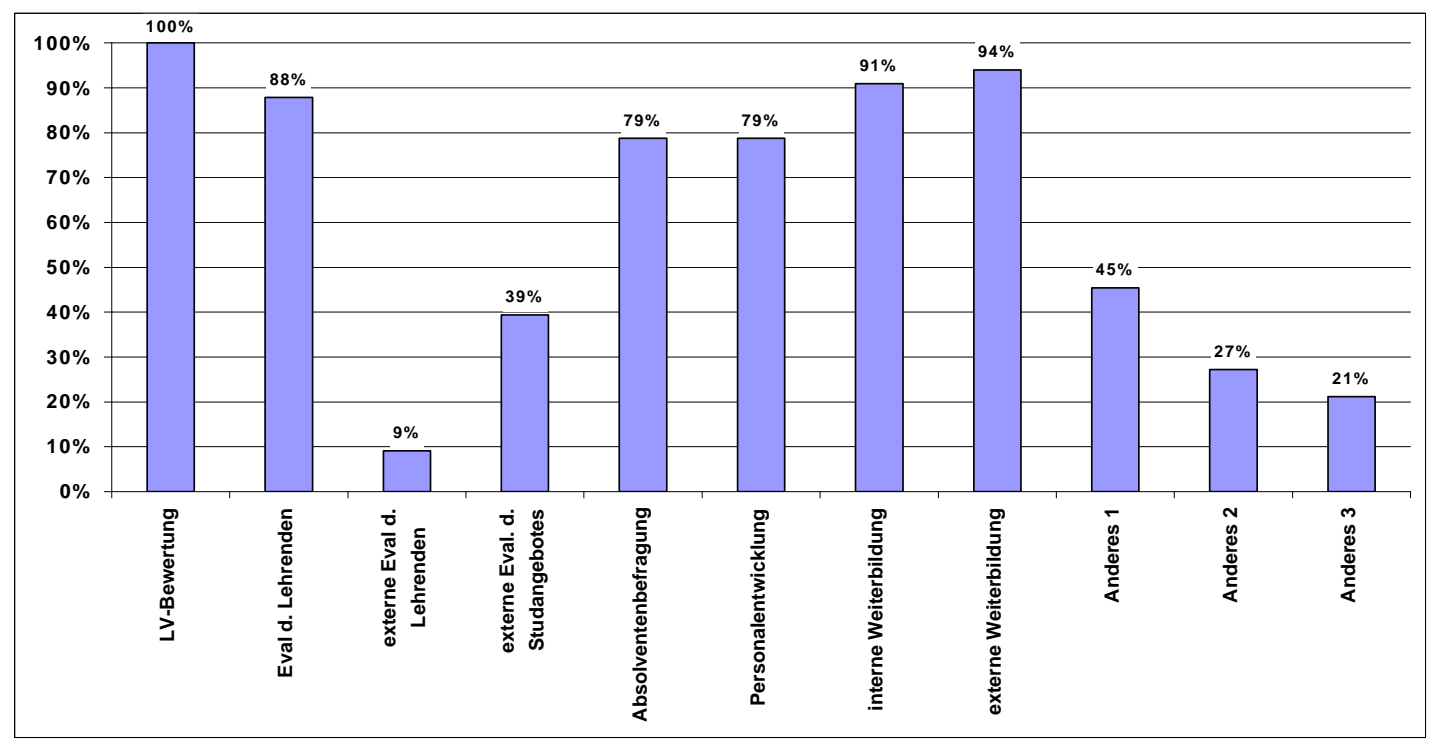

Quelle: Befragung der Studiengangsleitungen

Eine gesetzlich verankerte Evaluierung ist die studentische Lehrevaluation (daher zeigt die Befragung an dieser Stelle auch 100\%-Wert; vgl. Abb. 63 und 64). Das FHStG hatte dazu festgelegt: „Die Lehrveranstaltungen sind einer Bewertung durch die Studierenden zu unterziehen, die Bewertungsergebnisse dienen der Qualitätssicherung und sind für die pädagogisch-didaktische Weiterbildung der Lehrenden heranzuziehen."

Der FHR hat für die studentischen Lehrveranstaltungsbewertungen einen Leitfaden ausgearbeitet, in dem allgemeine als auch spezielle Mindestanforderungen an dieses Instrument enthalten sind. Der Leitfaden enthält auch eine grundsätzliche Positionierung, in der festgehalten wird, dass die Lehrevaluation vor allem eine breite Diskussion über die Qualität der Lehre auslösen und eine Maßnahme mit anregendem Charakter sein soll und dass die Verknüpfung negativer Evaluationsergebnisse mit materiellen und personellen Konsequenzen eher abzulehnen sei (vgl. Kozar 1999). Dem steht die Erfahrung entgegen (vgl. Hackl et al 1997, 49), dass die Studiengänge sich bei der Verwendung der Bewertungsergebnisse ähnlich verhalten wie kommerzielle Weiterbildungseinrichtungen, d.h. sie arbeiten mit Sanktionen und Anreizen für die Lehrenden. Es scheint innen leichter zu fallen, Konsequenzen aus Lehrveranstaltungsbewertungen zu ziehen, als etwa den Universitäten.

Damit steht auch die studentische Lehrevaluation an Fachhochschul-Studiengängen in dem grundsätzlichen Spannungsfeld, einerseits ein Instrument der Entwicklung zu sein, also eine eher formative Ausrichtung zu haben, aber dadurch die Gefahr in sich zu bergen, sanktions- 
und konsequenzenlos zu sein. Andererseits muss man aber auch vermeiden, dass sie sich zu sehr in Richtung Kontrolle-Sanktion-Verknüpfung mit Ressourcenentscheidung entwickelt, weil es dadurch oft zu einer rein symbolischen Rechtfertigungsübung kommt, die einen Umgang mit tatsächlichen Schwächen eigentlich verunmöglicht, weil es eine „Politik der Vertuschung" gibt. Die Lehrveranstaltungsbewertung wurde im Fachhochschulsektor von Anfang an flächendeckend eingesetzt und das zu einem Zeitpunkt, zu dem an österreichischen Universitäten noch darum „gerungen“ wurde, dieses Instrument tatsächlich in allen Lehrveranstaltungen einzusetzen.

Eine Evaluation der Lehrenden ist auch bei $81 \%$ der Studiengänge ein Pflichtprogramm, wobei eine externe Evaluation der Lehrenden die große Ausnahme darstellt. Auch eine externe Evaluation des Studienangebots wird nur in einem knappen Drittel vorgenommen, dafür ist der Anteil jener Studiengänge, die bereits AbsolventInnen-Befragungen einsetzen, mit $53 \%$ relativ hoch (vgl. Abb. 63).

Im Vergleich zu anderen Sektoren des tertiären Bildungsbereichs (wie etwa den Universitäten) fällt auf, dass auch wesentlich mehr Offenheit gegenüber der Evaluation des Studienangebots durch VertreterInnen der Praxis gegeben ist. Die meisten Studiengänge bemühen sich um eine aktive Einbindung, die ja nicht nur in den Entwicklungsteams in Form einer Mischung von Theorie- und PraxisvertreterInnen vorgeschrieben ist, sondern auch in jenen Bereichen, in denen sie selbst Maßnahmen setzen können. Sie versuchen, ExpertInnen aus der Wirtschaft, der Verwaltung oder der Bildung miteinzubeziehen, sie führen regelmäßige Gespräche mit Unternehmervertreterlnnen durch oder befragen Unternehmen, holen sich PraktikerInnen zu Gastvorlesungen in den Studiengang oder in Entwicklungskommissionen. Sie lassen umgekehrt aber auch Praktikumunternehmen durch Studierende evaluieren oder führen auch regelmäßige Jour-Fixes mit Studierenden durch. Verstärkt werden auch "quality circles" oder "circles of excellence“ unter den Lehrenden im Sinne von LektorInnentreffen eingesetzt oder Team-Teaching mit externen ReferentInnen.

Hohen Stellenwert - gemäß der projektinternen Befragung - hat auch die Personalentwicklung; didaktische und pädagogische Weiterbildung scheint mittlerweile doch an den meisten Standorten eine Selbstverständlichkeit zu sein. Interne und externe Schulungen im inhaltlichen, methodischen und didaktischen Bereich werden von vielen durchgeführt. Fast drei Viertel aller Studiengänge setzen hier ganz konkrete Maßnahmen. Die Prozentsätze unter den mindestens 4-jährigen Studiengängen sind noch wesentlich höher (vgl. Abb. 64). Hier zeigt sich, dass interne und externe Weiterbildung in über $90 \%$ der Studiengänge eingesetzt wird. Das zeugt von einem zunehmenden Qualitätsbewusstsein mit zunehmendem „Alter“ eines Studienganges. Personalentwicklung erfolgt dabei zumeist nicht studiengangsbezogen, sondern studiengangsübergreifend in den Fachbereichen.

Auffällig ist auch, dass an den meisten Studiengängen eine Diskussion in Gang ist, wie man das bestehende Evaluierungssystem im Sinne eines prozessorientierten Qualitäts- 
managementsystems unter Einbeziehung der MitarbeiterInnen, der nebenberuflichen Lehrbeauftragten und auch der Studierenden weiterentwickelt.

Auch Weiterbildung des Lehrkörpers in Quality-Management-Fragen wird als wichtig erachtet, ebenso wie die Umstellung von Quality-Management-Systemen auf Prozessorientierung und die gemeinsame Entwicklung eines praktikablen Qualitätssicherungssystems.

MitarbeiterInnengespräche, Mentoringsysteme, Job enrichment wird auch von einer Reihe von Studiengängen genannt als aktive und bereits stattfindende Maßnahmen der Personalentwicklung. Zusätzlich finden sich auch im Fachhochschulsektor spezifische Formen akademischer Personalentwicklung, die insbesondere in der Unterstützung von Promotionen und Habilitationen bestehen oder in der Teilnahme an internationalen Tagungen, Projekten und Kooperationen.

Den stärksten Stellenwert in der Weiterbildung hat die Weiterbildung in didaktischpädagogischer Hinsicht, aber zunehmend findet sich auch interne Weiterbildung in Richtung von Management-Trainings, PR-Management für die Studiengangsassistenz etwa oder Office Management.

Zunehmend professionalisiert sich auch das Aufnahmeverfahren von Professoren und Professorinnen, in mehreren Studiengängen werden Berufungskommissionen eingesetzt, es gibt eine internationale Ausschreibung und neben der Bewerbung auch einen Probevortrag. Gleichzeitig wird in diesem Zusammenhang gefordert, dass die Richtlinien zu Verleihung einer Fachhochschulprofessur stärker vereinheitlicht werden.

Als größtes Hindernis für weitere Personalentwicklung wird der Mangel an hauptberuflich angestellten LektorInnen genannt. Zu jenen Maßnahmen, die die meisten Studiengänge gerne verstärkt im Bereich Personalentwicklung setzen würden, gehört es, Freiräume zu schaffen für Projekte und wissenschaftliche Arbeit, Anreizsysteme für Forschung und Entwicklung, z.B. Sabbaticals, internationaler Professorlnnen-Austausch in Austauschprogrammen, auch internationale Weiterbildungsprojekte. Gemeinsame Forschungsprojekte, Herausgabe einer wissenschaftlichen Zeitschrift, Aufbau einer wissenschaftlichen Community gehören zu den Desiderata akademischer Personalentwicklung. Sabbaticals, visiting professors, Praxisjahre, das sind die meistgewünschten Formen in der Personalentwicklung, für die es aber derzeit zu wenig Kapazitäten in personeller, zeitlicher und finanzieller Hinsicht zu geben scheint. 


\section{Empfehlungen der Erhalter zur Weiterentwicklung der Qualitätssicherung:}

- Einrichtung von Akkreditierungsagenturen

- permanente Weiterbildung des Lehrkörpers auf dem Qualitätssicherungssektor

- Heranbildung von Spezialisten für die Qualitätssicherung

- stärkerer Einbezug der AbsolventInnen und deren Dienstgeber in die Evaluierung

- zeitliche Abstimmung der Aufnahmeverfahren aller Erhalter (ein „Zeitfenstermodell“)

- starke Abgrenzung und Positionierung der inländischen Fachhochschulangebote gegenüber ausländischen, in Österreich angebotenen Fachhochschul-Studiengängen

- Aufbau eines prozessorientierten Qualitätsmanagements unter Einbeziehung sowohl der nebenberuflichen Lehrbeauftragten als auch der Studierenden

- Aufbau eines Kennzahlen- und Berichtssystems

\section{Empfehlungen der StudiengangsleiterInnen zur Qualitätssicherung des gesamten} Fachhochschulsektors:

- Verleihung einer Fachhochschulprofessur über den Fachhochschulrat nach exakten Richtlinien

- Stärkung der Fachhochschulen vor allem hinsichtlich der Qualität und nicht der Quantität

- Ein Finanzierungsmodell, das nicht nur von der Kopfzahl der Studierenden abhängig ist und daher der derzeitigen Inflation an Studiengängen entgegenwirkt.

- fachhochschulübergreifende und fachhochschulspezifische Didaktikzirkel

- verstärkte Förderung von Organisations- und Personalentwicklung auf einer österreichweiten Basis, insb. Weiterbildung der Studiengangsleiterlnnen

- gemeinsame Qualitätssicherungsveranstaltungen für den gesamten Sektor

- die Fortführung der Qualitätssicherungsgruppe in der FachhochschulKonferenz

- stärkere internationale Positionierung

- Verankerung von Forschung und Entwicklung als explizite Evaluierungskriterien

- Vereinbarung eines vernünftigen Maßes an Standardisierung der Aufnahme

- Verhinderung qualitätsmindernder „Nischenorientierung“ von FachhochschulStudiengängen

- Veröffentlichung der Evaluierungsergebnisse und Weiterentwicklung im Sinne eines Rankings

- Akkreditierung nach internationalen Richtlinien

Manche wünschen sich auch verstärkt Leistungsprämien oder klassische Formen der Beförderung, der aber die für den Fachhochschulbereich typischen flachen Hierarchien entgegenstehen würden.

Insgesamt haben die Befragungen gezeigt, dass die aktive Beschäftigung mit Qualitätssicherungsfragen ein vitales Eigeninteresse der meisten Studiengänge ist. 
Viele würden das Fachhochschulevaluierungs- und Akkreditierungssystem gerne in Richtung eines transparenten, objektiven Rankingsystems weiterentwickeln, das alle Fachhochschuleinrichtungen umfasst und sich auf die Qualität der Ausbildung bezieht. Oft werden auch verpflichtende Pädagogik- und Didaktikseminare für Lehrende genannt.

\subsection{Internationale Einbettung des Qualitätssicherungsmodells}

Im Rahmen der Hochschulreformen der europäischen Länder spielt die Reorganisation der Qualitätssicherung eine wichtige Rolle. In der Frage der Entwicklung eines Europäischen Hochschulraums gewinnt auch der Aspekt der europaweiten Harmonisierung der Qualitätssicherungssysteme an Bedeutung. Die Bologna-Erklärung von 1999 nennt als eines ihrer Ziele „die Förderung der europäischen Zusammenarbeit bei der Qualitätssicherung im Hinblick auf die Erarbeitung vergleichbarer Methoden und Kriterien." In Prag 2001 - als einem nächsten wichtigen Schritt auf dem Weg zum Europäischen Hochschulraum - wurde schon eine wesentlich längere Passage zum Bereich Qualitätssicherung formuliert, vor allem wurde die Notwendigkeit einer wechselseitigen Akzeptanz der nationalen Qualitätssicherungssysteme und der Zusammenarbeit im Rahmen etwa innerhalb des European Network of Quality Assurance in Higher Education (ENQA) sowie der Austausch von „best practices“ betont (vgl. Westerheijden 2002, 23). Für viele bildungspolitische Entscheidungsträger hat dabei Akkreditierung eine besonders hohe Glaubwürdigkeit, da sie als Ja-/Nein-Entscheidung eine transparente und zugleich kompakte Form der Qualitätsinformation darstellt. Im folgenden wird kurz beleuchtet, welchen Weg einige ausgewählte europäische Länder derzeit bei der Etablierung der für den kontinentaleuropäischen (im Gegensatz zum anglo-amerikanischen) Raum neuen Akkreditierungskultur einschlagen.

\subsubsection{Deutschland}

In Deutschland gibt es für die neustrukturierten Studienangebote seit Ende 1998 den Akkreditierungsrat, der bundesweit die Anerkennung der neuen Programme koordiniert, indem er die Aufgabe wahrnimmt, „Agenturen zu begutachten bzw. zu akkreditieren, die ihrerseits wiederum die zunächst probeweise eingeführten Studiengänge Bachelor/Bakkalaureus und Master/Magister akkreditieren. Die Agenturen wie die von innen akkreditierten Studiengänge tragen im Falle einer erfolgreichen Begutachtung das Qualitätssiegel des Akkreditierungsrates. In begründeten Fällen, so sieht es der Beschluss der Kultusministerkonferenz KMK vor, kann der Akkreditierungsrat auch selbst Studiengänge akkreditieren. ${ }^{197}$ Die letzte Möglichkeit muss jedoch als Ausnahme gesehen werden. Nach

197 Vgl. http://www.akkreditierungsrat.de. 
einer dreijährigen Probephase und anschließender Evaluation ist der Akkreditierungsrat im Jahr 2002 dauerhaft von der Kultusministerkonferenz (KMK) eingerichtet worden.

Das System von Akkreditierungsagenturen ist trotz des frühen Starts noch im Aufbau. Bis August 2002 sind sieben Akkreditierungsagenturen vom Akkreditierungsrat anerkannt. Davon sind vier spezialisiert in der Akkreditierung von Programmen in bestimmten Fachbereichen (business studies, Ingenieurwissenschaften, Chemie, Pflege), die anderen drei sind regional gegründet, arbeiten aber im ganzen Spektrum von Programmen und im ganzen Bundesgebiet. Zur Anerkennung von Agenturen hat der Akkreditierungsrat Mindeststandards und Kriterien vorausgesetzt, die zum größten Teil als organisatorische und methodische Kriterien zu sehen sind (Unabhängigkeit der Agentur, Transparenz des Verfahrens usw.), nicht aber inhaltlich sind.

Gleichfalls bis August 2002 haben insgesamt 151 Programme, 57 Bachelor-Programme und 94 Master-Programme ihr Qualitätssiegel bekommen. Das sind ungefähr 13\% aller Hochschulprogramme in Deutschland. Bemerkenswert ist, dass die Akkreditierung der Programme von den anerkannten Agenturen selbständig durchgeführt wird; nach der Anerkennung der Agenturen ist der Akkreditierungsrat im Normalfall nicht direkt an der Akkreditierung beteiligt.

Im KMK-Beschluss „Künftige Entwicklung der länder- und hochschulübergreifenden Qualitätssicherung in Deutschland“ vom 1. März 2002 wird die „funktionale Trennung zwischen staatlicher Genehmigung und Akkreditierung" betont. Auch bei den neuen Bachelor/Master-Studiengängen bezieht sich die staatliche Genehmigung auf „die Gewährleistung der Ressourcenbasis des einzurichtenden Studiengangs, die Einbindung des Studiengangs in die Hochschulplanung des jeweiligen Landes sowie auf die Einhaltung von Strukturvorgaben” und "die staatliche Verantwortung für die Einrichtung von Studiengängen kann nicht durch die Akkreditierung ersetzt werden." Daraus kann man schließen, dass Akkreditierung und Genehmigung parallel sind. Akkreditierung ist deshalb, selbst für Bachelor- und Master-Programme, keine bundesweite Bedingung für staatliche Genehmigung (d.h. unter anderem für die Finanzierung und die Anerkennung der Diplome). Die jeweiligen Bundesländer können jedoch derartige Bestimmungen setzen (wie z.B. in Nordrhein-Westfalen).

In Deutschland ist die Akkreditierung zur Zeit eine Ex-ante-Beurteilung von Studienprogrammen, die zu einem ganz bestimmten Bachelor- oder Masterabschluss führt. Die Akkreditierung erfolgt auf Antrag einer Hochschule, die einen Bachelor- oder Masterstudiengang einrichten oder verlängern möchte.

Evaluierung und Akkreditierung sind derzeit streng getrennt, was aber von manchen Seiten kritisch kommentiert wird, da dadurch nicht nur der Aufwand für die Institutionen und Fächer beträchtlich ist, sondern auch, weil aus der Perspektive eines strategischen 
Qualitätsmanagements beide Perspektiven integriert bzw. aufeinander bezogen werden müssten (vgl. Reuke 2002, 103).

\subsubsection{Niederlande}

Die Niederlande haben vor, ab 2003 alle Hochschulprogramme zu akkreditieren. Die Gesetzesnovellierung wurde zusammen mit dem Umstieg auf Bachelor- und Masterstudien in der ersten Jahreshälfte 2002 vom Parlament bewilligt; die von der nationalen Regierung ernannte Organisation ist im August 2002 im Aufbau. Beim Entwurf der neuen nationalen Akkreditionsorganisation („NAO“) sind einige Prinzipien vom deutschen Beispiel „geliehen“. So werden auch in den Niederlanden die Evaluationsaktivitäten, die per Studienprogramm stattfinden, nicht von der nationalen Organisation ausgeführt, sondern von mehreren „visitierenden und validierenden Instituten“ (,VVI“), die - auch wie in Deutschland - vom Nationalorgan anerkannt werden. Das System wird offen sein für VVI aus dem In- und Ausland. Im Unterschied zu Deutschland jedoch wird das Qualitätssiegel direkt vom Nationalorgan abgegeben. Die Qualitätsurteile der VVI sind als Empfehlungen für das Nationalorgan zu sehen. Die Entscheidungen des nationalen Organs wiederum sind zu sehen als Empfehlungen für den/die Bildungsministerln, um das begutachtete Programm, die Studierenden zu finanzieren und das Diplom offiziell anzuerkennen. Akkreditierung wird dazu eine Bedingung sein, nicht nur - wie bis jetzt - für die öffentlichen Hochschulen, sondern auch für private und ausländische Institutionen, die Bachelor- oder MasterProgramme anbieten wollen. ${ }^{198}$

Der mehr als zehnjährigen Tradition der externen Qualitätsbewertung durch die Pufferorganisationen der Hochschulen (VSNU für die Universitäten, HBO-raad für die Fachhochschulen), die im neuen System zu „VVI“ werden, soll dadurch entsprochen werden, dass die VVI nicht nur kontrollierende, sondern auch qualitätsfördernde Aufgaben wahrnehmen können.

Zur gleichen Zeit ist das niederländische Bildungsministerium daran interessiert, internationale Übereinstimmung zu erreichen über die Kernkompetenzen, die die Bachelorbzw. Master-Ebene ausmachen. Dazu arbeitet es mit VertreterInnen in verschiedenen anderen europäischen Ländern zusammen, was u.a. aufbauend auf Vorschlägen für die NAO-Kriterien zu einem Konsens geführt hat. ${ }^{199}$

198 Die Finanzierung von Programme ist beschränkt auf öffentliche Hochschulen; Diplomanerkennung und Stipendien sind Konsequenzen für alle akkreditierten Programme.

199 Siehe: www.jointquality.org. 


\subsubsection{Schweiz}

Qualitätssicherung ist in der Schweiz auf hochschulübergreifender Ebene noch jung und unterschiedlich für den Fachhochschul- und den Universitätssektor.

Der erste Jahresbericht vom „Organ für Akkreditierung und Qualitätssicherung der Schweizerischen Hochschulen“ (OAQ) wurde 2002 veröffentlicht, denn das Organ besteht erst seit 1. Oktober 2001, es wurde parallel zur Einführung eines neuen Finanzierungsmodus für die universitären Hochschulen errichtet. Diese Organisation hat bisher noch keine Akkreditierungen unternommen und beschränkt sich vorläufig auf Universitäten, obwohl letztendlich die Integration der Fachhochschuleinrichtungen im Akkreditierungssystem vorgesehen ist (OAQ, 2002).

Bis dahin ist für die Qualitätsförderung im FH-Sektor die Eidgenössische Fachhochschulkommission (EFHK) zuständig. Die Erneuerung der Genehmigungen in 2003 erfolgt aufgrund von Qualitätsurteilen, die in einem Prozess entstanden sind, der dem allgemeinen Modell mit Selbst- und Fremdevaluation (peer review) entspricht. Diese Evaluation hat 2001 bis 2002 stattgefunden. Darauf aufbauend soll 2002 ein zusammenfassender Bericht über die Aufbauphase der Fachhochschulen vom EFHK erstellt werden. Dieser Bericht „bildet eine wesentliche Grundlage für die Genehmigungserneuerung". ${ }^{200}$

Die hochschulinternen Qualitätssicherungssysteme werden in den kommenden Jahren unter der Verantwortung der Hochschulen weitergeführt. Das neu etablierte nationale „Organ für Akkreditierung und Qualitätssicherung" soll auf übergeordneter Ebene die hochschulinternen Qualitätssicherungsmaßnahmen unterstützen und zu einer erhöhten Harmonisierung der Systeme beitragen. Dem Organ für Akkreditierung und Qualitätssicherung wurde „ins Pflichtenheft geschrieben, bei den von den Universitäten in eigener Regie durchgeführten Evaluationen koordinierend und unterstützend zu wirken." (Schencker-Wicki 2002, 96)

Die Schweiz hat einen etwas anderen Weg als etwa Deutschland und die Niederlande gewählt, indem ein wichtiger Aspekt darin gesehen wird, institutionelle Einheiten insbesondere hinsichtlich ihres Qualitätsmanagements zu evaluieren und anzuerkennen. Deutschland und die Niederlande spiegeln in ihrem Akkreditierungszugang eine im Vergleich dazu distanziertere Position des Staates wider - wie immer Institutionen sich organisieren, untersucht wird die Qualität des „Bildungsgutes“. Der Schweizer Zugang weicht in seinem Verständnis vom deutschen oder niederländischen Verständnis der Aufgaben von Akkreditierungseinrichtungen ab. Gleichzeitig wird - anders etwa als derzeit noch in Deutschland - sehr darauf geachtet, dass "die von den Universitäten in eigener Regie

200 Website BBT: http://www.bbt.admin.ch. 
durchgeführten Evaluationen unterstützt, koordiniert und wenn immer möglich als Basis für die Selbstevaluation im Akkreditierungsverfahren benutzt werden..., um die gefürchtete Evaluationsbürokratie auf ein Minimum zu beschränken, besser noch zu vermeiden.“ (Schencker-Wicki 2002, 97)

\subsubsection{Finnland}

Der finnische Hochschulevaluationsrat (in Englisch abgekürzt zu „FINHEEC“) besteht seit 1996. Der Rat, vierjährlich von der nationalen Regierung ernannt aus VertreterInnen der Hochschulen, Wirtschaft und Studierenden, hat als Zweck die langfristige Förderung des Hochschulsystems durch Evaluation. „The approach adopted by the Council is based on the principle of improving the quality of teaching and is based on nationally tailored assessments of sectors of study, ${ }^{201}$ degree programmes and subjects, and of various individual themes ${ }^{202}$. A further principle followed is that, whenever technically possible, education and theme assessments are made simultaneously in both the university and the polytechnic sectors" (Opetusministeriö, 2002).

FINHEEC's Akkreditierungsarbeit (als besondere Form der Evaluation) ist beschränkt auf "continuing education and specialist programmes comprising at least 20 credits offered by the universities and polytechnics to supplement basic degrees" (Opetusministeriö, 2002). Freiwillig können Hochschulen ihr internes Qualitätsmanagement vom FINHEEC evaluieren lassen.

FINHEEC's Aufgabe im Bereich der Fachhochschulen war die Beratung des Bildungsministeriums über die Anträge zur Errichtung der permanenten Fachhochschulen ab 1995. Die wichtigsten Kriterien für Neugründungen sind meist dem FH-Gesetz entnommen:

„1) the operating principle (mission), 2) the topicality and need for the planned degree programmes, 3) how well the sectors of study fit together, 4) the main area of strength, 5) adequate size relative to educational function, 6) the qualifications of the teaching staff, 7) library and information services, 8) relations with working life, 9) cooperation with universities/other polytechnics, and with other educational institutions, 10) international cooperation, 11) educational and service function in the region, 12) arrangement of evaluation, 13) the learning environment, and 14) the working environment." (Opetusministeriö, 2002)

201 Z.B. Gesundheitswesen und Berufslehrerbildung.

202 Z.B. Internationalisierung und Zusammenarbeit mit der Wirtschaft. 
Die FINHEEC-Evaluationen sind mitbestimmend für einen Teil der leistungsbezogenen Finanzierung der finnischen Fachhochschulen (nicht jedoch für die Basisfinanzierung):

"Some funding is allocated on a performance basis according to general criteria such as efficiency and cost-effectiveness, impact, international activities, equal opportunity, and capacity for future functioning and renewal. Some performance-based funding has also been allocated to polytechnics on the basis of evaluations made by the Finnish Higher Education Evaluation Council. In 1999 these evaluations were made at centres of excellence in teaching, and in 2000 at centres of excellence in regional impact. In 2001, EUR 1.7 million was allocated on the basis of general performance criteria and EUR 1.7 million went to centres of excellence selected on the basis of the evaluations." (Opetusministeriö, 2002)

FINHEEC verfolgt auch einen institutionellen Ansatz und ist in dieser Hinsicht dem Schweizer OAQ ähnlich, gleichzeitig ist FINHEEC auch beratend in hochschulpolitische Entscheidungen (etwa durch die Verbindung zur Finanzierung), die durch das Bildungsministerium getroffen werden, eingebunden.

\subsubsection{Einbettung des Qualitätssicherungsmodells des österreichischen Fachhoch- schulsektors}

Durch das nun seit fast 10 Jahren erprobte Akkreditierungsmodell ist der Fachhochschulsektor wesentlich besser auf die internationale Kooperation im Bereich der Qualitätssicherung im Zuge des Bologna-Prozesses vorbereitet als der Universitätssektor. Hinsichtlich der Begutachtung von Studienprogrammen gleicht das österreichische Modell dem deutschen oder niederländischen Akkreditierungsmodell.

Auch die Überzeugung, dass Qualitätsförderung weitere, über die Akkreditierung hinausgehende Evaluationsaktivitäten benötigt, stimmt mit internationalen Erfahrungen, etwa in Finnland oder den Niederlanden, überein.

Die grundsätzlichen Verfahren sowohl der Akkreditierung als auch der Evaluierung gleichen den mittlerweile international üblichen Verfahren der Qualitätssicherung. Besonders ist lediglich das Element der Beobachtung von Abschlussprüfungen (das ohnehin auch in Österreich nur selten durchgeführt wird), durch das externe ExpertInnen aber einen sehr unmittelbaren, direkten Qualitätseindruck bekommen, das aber in den meisten anderen Ländern als zu intervenierend empfunden wurde. Nur in Großbritannien war die Beobachtung von Vorlesungen ein vom „Her Majesty`s Inspectorate“ übernommener Teil des Evaluierungsverfahrens (vgl. Westerheijden 1994, 243).

Der Bologna-Prozess fokussiert die Ebene der Studienprogramme bzw. der Studienabschlüsse - die Entwicklung in Deutschland und den Niederlanden spiegelt diese 
Fokussierung wider. In diesem Sinn ist die neue Entwicklung im Fachhochschulsektor in Richtung institutioneller Evaluierung eine Abweichung vom europäischen Trend. Andererseits gibt es dafür gute Gründe: die Institutionelle Evaluierung ist ein wichtiger Beitrag zur institutionellen Weiterentwicklung und sie hilft auch, den Arbeitsaufwand der Evaluierungen zu reduzieren bzw. zu bündeln. Vermutlich wird diesem Zugang daher auch in den Niederlanden und in Deutschland größeres Gewicht als derzeit beigemessen werden, da etwa schon in Deutschland bei den Akkreditierungen auf Programmebene immer öfter Überlastungssituationen entstehen. Finnland verfolgt ja bereits jetzt das Prinzip der institutionellen Evaluierung.

Die adäquaten Verknüpfungsformen von Akkreditierung und Evaluierung sind ein letztlich in allen Ländern bislang nicht wirklich gelöstes Problem, wenngleich die Verknüpfung in der Schweiz und in Finnland unter Umständen besser gelöst ist als etwa in Deutschland, wo diese beiden Stränge am stärksten voneinander getrennt sind.

\subsection{Zusammenfassung und Schlussfolgerungen}

\subsubsection{Zusammenfassung}

Das Qualitätssicherungsmodell des Fachhochschulsektors ist als für den tertiären Bereich vorbildlich einzustufen. Es ist ein guter Methodenmix gegeben, insbesondere sind Akkreditierung und Evaluierung gut miteinander verflochten und haben sich nicht zu arbeitsaufwändigen „Parallelaktionen“ entwickelt, was - wie man derzeit im deutschsprachigen Raum teilweise beobachten kann - aber eine reale Gefahr darstellt. Weil die Evaluierungsergebnisse die Basis für die Re-Akkreditierung sind, sind beide Verfahren aufeinander bezogen und komplementär. Vor allem wird dem „bottom-up“-Approach des Sektors folgend die institutionelle Ebene der Qualitätssicherung sehr betont. Der vorliegende Bericht kann nicht auf ausführliche Vor-Ort-Untersuchungen der einzelnen Studiengänge und Fachhochschulen verweisen. Um eine fundierte Aussage über die Qualität des institutionellen Qualitätsmanagements zu machen, wäre das vonnöten. Die Hinweise aus der projektbezogenen Online-Befragung legen aber nahe, dass das Qualitätssicherungsbewusstsein in den Studiengängen recht ausgeprägt ist. Auf der staatlichen Ebene kann eigentlich nur ein „Meta-Qualitätsmanagement" erfolgen, das zum Ziel hat, die entstandenen Qualitätssicherungssysteme vor Ort von Zeit zu Zeit auf ihre Qualität hin zu überprüfen und den Erfahrungsaustausch unter den Akteuren auch in Bezug auf Fragen der Qualitätssicherung zu stimulieren. Veränderungsnotwendigkeiten des an sich gut funktionierenden Qualitätssicherungsmodells des Sektors ergeben sich u. E. aus der nun erreichten Größe des Sektors, den sich entwickelnden Konturen der Qualitätssicherung im europäischen Hochschulraum und der sich verändernden universitären Landschaft. 
Das Akkreditierungssystem des FHStG sieht vor, dass die inhaltlich-qualitative Prüfung der Studienangebote durch ein dafür eingerichtetes ExpertInnengremium vorgenommen werden soll. Dieses Modell der Akkreditierung stammt aus Rechtssystemen, die den Bildungsbereich weitgehend der professionellen Selbstkontrolle unterwerfen und die hoheitliche Tätigkeit darauf beschränken, diese sicherzustellen (vgl. BMWFK/BMUkA, 1995). Damit ergibt sich für den Fachhochschulrat ein Spannungsverhältnis aus seiner Funktion als Behörde (§ 6 FHStG) und seiner Funktion als Expertlnnengremium. Als Behörde ist er Organ im Rahmen der Hoheitsverwaltung und sollte für jeden Sachverhalt eine Lösung formulieren; ein ExpertInnengremium wird hingegen eher mit Empfehlungscharakter verbunden. Der Behördencharakter erzeugt Druck in Richtung exakter Vorgaben für und Anleitung von Antragstellenden. Das mag zwar anfangs angenehm sein, birgt jedoch die Gefahr einer Rücknahme der an sich intendierten Dezentralisierung und behindert u.U. die Kreativität. Generell kann aus dem Behördenstatus eine zu große Betonung formaler Kriterien bei der Prüfung der Anträge entstehen.

In der Pionierphase des Aufbaus der FH-Studiengänge war die starke und umfassende Rolle des Fachhochschulrats wichtig für einen qualitätsvollen Einstieg des Sektors, mittlerweile geht es um die Phase der Konsolidierung des Sektors, diese wird nur bei einer klareren Ausdifferenzierung der Funktionen und einer stärkeren Arbeitsteiligkeit auch im Bereich der Qualitätssicherung möglich sein. Jedenfalls erfordert der Übergang von der Pionierphase in die Konsolidierungsphase auch eine kritischere Selbstbetrachtung. Noch ist der Neuheitswert, ein gewisses Interesse der Wirtschaft, ein hohes Interesse der Medien am Fachhochschulsektor festzustellen. Im Inneren mehren sich schon selbstkritische Stimmen. Gerade die Initiativen zur Förderung von Organisations- und Personalentwicklung erscheinen wichtig und zeugen auch von der selbstkritischen Selbstbeobachtungsfähigkeit des Sektors.

Die Umstellung der Evaluierung auf institutionelle Evaluierungen zeigt, dass auch die Qualitätssicherung als solche wirklich eingesetzt wird, um den Sektor in seinem jeweiligen Zustand weiterzuentwickeln. Folgerichtig geht die Fachhochschul-Konferenz auch stark in die Richtung der Betonung von Organisations- und Personalentwicklung - das ist ein wichtiger Aspekt der Konsolidierungsphase und begleitet die Einrichtung größerer Einheiten oder die Zusammenführung von Standorten mit mehreren Filialbetrieben.

Da nun der Universitätssektor in Österreich auch stärker durch die bislang für den Fachhochschulsektor gültigen Prinzipien wie institutionelle Autonomie und Wettbewerb gekennzeichnet sein wird und es dadurch, insbesondere aber durch die für beide Sektoren verstärkte Internationalisierung zu Konvergenzerscheinungen in zentralen Bereichen wie etwa der Studienarchitektur kommt, ist vermutlich auch mit Auswirkungen auf die Organisation der Qualitätssicherung zu rechnen. 


\subsubsection{Schlussfolgerungen}

\section{Funktionsdifferenzierung der Institutionen}

\section{Arbeitsweise des FHR und der Geschäftsstelle}

Sowohl die nebenamtlichen Mitglieder des Fachhochschulrates als auch die sieben angestellten Mitglieder der Geschäftsstelle sind angesichts des massiven quantitativen Ausbaus des FH-Sektors stark überlastet. Damit der FHR rund um die Aufgabe der Qualitätssicherung klar konturiert werden kann, ist erstens eine administrative Aufstockung der Geschäftsstelle und eine differenzierte Arbeitsteilung in den verschiedenen Bereichen der Qualitätssicherung erforderlich.

Für die Entlastung der nebenamtlichen Mitglieder des FHR muss überlegt werden, ob sie die inhaltliche Prüfung tatsächlich immer selbst durchführen müssen. Es hat sich hier schon eine Änderung ergeben. Ursprünglich, in der ersten Aufbauphase, haben immer alle 16 Mitglieder des Fachhochschulrates alle Anträge gelesen. Das lässt sich bei der Fülle der Anträge heutzutage nicht aufrechterhalten und daher geht man arbeitsteilig vor. Der Rat bzw. die Geschäftsstelle hätte dann eher eine Qualitätsfeststellungen organisierende Rolle als selbst die Aufgabe, inhaltliche Prüfungen durchzuführen. Bei der Fülle der Anträge ist auch eine arbeitsteilige Vorgehensweise in dem Sinn anzustreben, dass verstärkt Fachexpertise zugekauft und von der inhaltlichen Beurteilung der einzelnen Studienpläne bis ins Detail abgerückt wird, um so mehr Kapazitäten für die Qualitätsentwicklungsfragen des Gesamtsektors freizubekommen. Die Geschäftsstelle muss verstärkt als eigenständige Einrichtung geführt werden. Wenn der Fachhochschulrat öfter mit Delegationen und Einbeziehung von externen ExpertInnen arbeitet, ist die Geschäftsstelle auch eine wichtige Organisatorin der Kommunikation und der Betreuung von Sachverständigen.

Für die Qualitätssicherung im Sektor ist, angesichts des sich doch sehr stark wandelnden Umfelds (Universitätsreformen, Bologna-Prozess mit Bachelor-/Masterabschlüssen), mehr Platz für Strategieüberlegungen und für die Betreuung von Forschungsprojekten zu schaffen.

Dafür ist es auch wichtig, das von den Mitgliedern des FHR angesammelte Know-how (vor allem die Pioniere der ersten Bestellungsjahre haben hier ganz wichtige Aufbauarbeit geleistet) zu sichern. Bei der Zusammensetzung des Fachhochschulrates sollten insbesondere Personen mit innovativen didaktischen Ideen sowie Lehrende des Fachhochschulsektors vertreten sein und das Gremium selbst muss sich immer auch zum Team entwickeln. 
Weiters ist darauf zu achten, dass jene Kriterien, die leicht formal überprüfbar sind, nicht die Überhand gewinnen vor jenen, die mehr Auseinandersetzung und Kommunikation mit den Antragstellenden erfordern, weil sie im qualitativ-weicheren, aber nichtsdestotrotz kaum weniger wichtigen Bereich zu finden sind. Ebenso ist darauf zu achten, dass Vorgaben, die zwar in der ersten Phase für viele Antragstellende hilfreich waren, nicht zu Vorschriften erstarren, sondern stattdessen verstärkt prozessuale Sicherungen wie Beschwerderechte, punktuelle Überprüfungen oder Evaluationen eingesetzt werden. Dieses Vorgehen würde dem Vorhaben einer Deregulierung im Hochschulwesen eher entsprechen.

Die Geschäftsstelle hat die wichtige Aufgabe, die Kontinuität des Betriebes sicherzustellen und quasi als kollektives Gedächtnis der doch wechselnden akademischen Mitglieder des Fachhochschulrates zu agieren. Sie hat einen komplex gewordenen Sektor zu verwalten mit vielen Neuanträgen, Wiederbeantragungen, Evaluierungen, Verlängerungen etc. Empfehlenswert ist der (weitere) Ausbau juristischer Kompetenz, als Teil der Qualitätssicherung des Gesamtsektors. Der FHR trifft immer wieder bindende Aussagen durch seine Entscheidungen und Publikationen, aber dennoch „semioffiziell“, wodurch Entscheidungen nicht mehr nachlesbar, argumentierbar udgl. sind - Prisching bezeichnet dies als „Management bei Gerücht“ (Prisching 2002). Mittlerweile gibt es eine Fülle von Regeln, Rundbriefen, Newslettern. Diese haben nicht den verbindlichen Charakter von Verordnungen oder Bescheiden und somit tut sich eine große Grauzone auf. Der Stolz auf schlanke Regulierungen sollte nicht zu rechtsfreien Räumen führen, die dann mit informellen, aber doch bindenden Beschlüssen des FHR gefüllt werden.

\section{Funktionsprofil des FHR und ergänzende Einrichtungen}

Die ursprüngliche Konstruktion des Fachhochschulrates als weisungsfreies ExpertInnengremium und Behörde war zum Zeitpunkt der Einführung eine kreative Lösung, um Elemente eines anderen bildungsorganisatorischen Modells in die bestehende stark rechtlich determinierte Bildungsorganisation in Österreich einzuführen. Fast zehn Jahre später sind die Bildungssektoren in allen europäischen Ländern enormen Veränderungen unterworfen und auch der gesamte tertiäre Bildungssektor in Österreich ist stark in Bewegung geraten. Angesichts der veränderten Umwelten sollten „spezifisch österreichische Lösungen" in der Fachhochschullandschaft noch einmal überdacht werden. Das eine Spannungsfeld ist bedingt durch die Konstruktion des Fachhochschulrates als Behörde einerseits und ExpertInnengremium andererseits, beides Organisationsformen, die unterschiedlichen Struktur- und Entscheidungslogiken folgen.

Die ursprünglich geplante Trennung von Qualitätsentscheidung einerseits und Finanzierungsentscheidung andererseits, die das Zusammenspiel zwischen dem zuständigen Bundesministerium und dem Fachhochschulrat kennzeichnet, ist ebenso „spannungsgeladen“ wie das Verhältnis von bildungspolitischen Kriterien, die im FHStG 
sowie in den Entwicklungsplänen enthalten sind und den Kriterien, die der Fachhochschulrat in seiner Qualitätsprüfung anlegt. Qualität ist immer relational festzustellen und damit abhängig von den gesetzten Zielen. Insofern ist sie schlecht abstrakt und unabhängig von den bildungspolitischen Kriterien und Erwartungen an den Fachhochschulsektor festzulegen.

Wenn auch die Trennung zwischen Qualitätsprüfung einerseits und Finanzierung andererseits sehr sinnvoll erscheint, sind diesbezügliche Entscheidungen im praktischen Vollzug stark miteinander verflochten. Bei der jetzigen Arbeitsteilung zwischen Fachhochschulrat und zuständigem Bundesministerium erscheinen uns auf Seiten des BMBWK auch zuwenig Kapazitäten für die Beschäftigung mit den strategischen bildungspolitischen Fragen vorhanden. Derzeit erfüllt der FHR die Rolle eines zentralen bildungspolitischen Akteurs als auch des „zentralen Qualitätsprüfungsorgans“.

Angesichts der mittlerweile erreichten Größe des Fachhochschulsektors und der Entwicklung in den europäischen Bildungssystemen erscheinen folgende Szenarien zu Funktionsdifferenzierungen zwischen bildungspolitischen Vorgaben und akademischer Qualitätsprüfung denkbar:

Der FHR konzentriert sich auf seine Rolle als eine Einrichtung, die die akademische Qualitätssicherung durchführt. Dann ist der Fachhochschulrat eine Akkreditierungs- und Evaluierungsagentur. Für die Besetzung dieses Expertlnnengremiums würde dies aber bedeuten, dass die ExpertInnen neben einer fachspezifischen Expertise insbesondere Know-how in den Bereichen Lehrprofilierung, Curriculumentwicklung und Berufsfeldforschung aufweisen müssten, Kriterien der bildungspolitischen Orientierung oder Interessenvertretungen dürften keine wichtige Rolle spielen.

Dann bräuchte es ergänzend mehr strategisches bildungspolitisches Know-How außerhalb des Fachhochschulrates, in zentraler Beratungsfunktion für das zuständige Bundesministerium. Das könnte - eventuell als Teilbereich des für den Universitätsbereich nunmehr vorgesehenen Wissenschaftsrates - ein Rat/Beirat sein, der sich insbesondere mit den bildungspolitischen Vorgaben des FH-Sektors in Relation zu den anderen Sektoren des tertiären Bildungssystems beschäftigt, die Entwicklung des Gesamtsektors begleitet und darauf achtet, dass die Voraussetzungen geschaffen werden, die notwendig sind, um neue Anforderungen (wie etwa den Forschungsauftrag, den verstärkten Internationalisierungsdruck etc.) bewältigen zu können. Eine solche Einrichtung scheint notwendig, weil einzelne Abteilungen eines Ministeriums, die personell zumeist knapp besetzt sind, überfordert wären, ohne zusätzliche Expertise die doch gewichtigen bildungspolitischen Entscheidungen (wie Expansionsrate, benötigte Finanzierung, Verhältnis zu anderen Sektoren, Themenspektrum, inhaltliche Felder etc.) vorzubereiten.

Ein mögliches Szenario wäre es auch, dass der bestehende Fachschulrat ein stärkeres Profil als bildungspolitisches Beratungsorgan entwickelt mit hoher Autonomie, aber letztlich 
bildungspolitisch entscheidungsvorbereitend. Dann müsste nicht so sehr das zuständige Bundesministerium, sondern der Fachhochschulrat mehr Kapazitäten für die Behandlung wichtiger strategischer bildungspolitischer Fragen und die Begleitung der Entwicklung des Sektors haben. Die Frage der akademischen Qualitätssicherung müsste dann stärker über die vermehrt entstehenden Akkreditierungs- und Evaluierungsagenturen organisiert werden. Diese hätten dann eine „Zulieferfunktion“ für den Fachhochschulrat. Der internationale Trend, Akkreditierungsagenturen von staatlicher Seite (etwa über den FHR) zu akkreditieren, aber darüber hinaus nicht selbst akkreditierend tätig zu sein, würde den Institutionen mehr Wahlmöglichkeiten lassen und dem FHR mehr Kapazitäten für strategische Überlegungen.

\section{Institutionelle Organisationsformen und stabile Kommunikationsorte}

Unabhängig davon, in welcher institutionellen Rollenverteilung Aspekte der bildungspolitischen Beratung und der Qualitätssicherung wahrgenommen werden, bedarf es zwischen diesen unterschiedlichen Akteuren - insbesondere aber in der Beziehung zwischen Fachhochschulrat und Bundesministerium - einer regelmäßigen offiziellen Information und gegenseitigen Beratung.

Weiters ist die Frage der adäquaten Kommunikation mit den Ländern offen, die wichtige bildungspolitische Akteure im Fachhochschulbereich geworden sind. Die Wissenschafts- und Bildungspolitik des Bundes und der Länder in diesem doch überschaubaren Bildungssektor muss koordiniert erfolgen. Zum einen bezieht sich das auf die Koordination der Länder untereinander, zum anderen auf die Abstimmung mit dem Bund.

Eine wichtige institutionelle Organisationsform für das Thema Qualitätssicherung ist auch die Fachhochschul-Konferenz als Zusammenschluss der Erhalter und LeiterInnen von Fachhochschul-Studiengängen. Der Fachhochschulrat wäre als alleiniger Garant für die Qualitätssicherung wohl überfordert. Die Erhalter sind sozusagen auch eine Art Gegenpol oder Kontrollinstanz für die Qualität der Entscheidungen des zuständigen Bundesministeriums und des Fachhochschulrats.

Die Förderung einer professionellen Diskussion zwischen den Lehrenden der Fachhochschul-Studiengänge hat eine wichtige Stellung in der Qualitätsentwicklung. Die Unterstützung nicht nur der Entwicklungsteams, sondern auch einer FachhochschulCommunity wird zukünftig ein ganz wichtiges Qualitätssicherungsmoment sein, wobei man die Ebene der Erhalter von Fachhochschul-Studiengängen (die sich über organisatorische Aspekte der Lehre, Forschung, Qualitätssicherung, Weiterbildung etc. unterhalten müssen), die Ebene der Lehrenden selbst und die Ebene der Studierenden stärker unterscheiden sollte. 


\section{Differenzierung der Qualitätssicherungsdimensionen}

Der Fachhochschulrat als wichtiger Promotor von Qualität im Fachhochschulsektor hat immer sowohl summative (abschließend-bewertende) Funktionen von Qualitätssicherung etwa in Form der Akkreditierung - betrieben als auch formative (im Sinne von externbegleitenden) Funktionen der Qualitätssicherung - etwa in Form des gewählten Evaluierungsmodells. Dieses Evaluationsmodell ist zudem recht konsensuell mit den Erhaltern der Studiengänge und Fachhochschulbetreibern entwickelt worden. In letzter Zeit erfolgte eine stärkere Fokussierung in dem Sinne, dass der Fachhochschulrat seine Zuständigkeit für Evaluation stark betonte und auch eine Veröffentlichung der Evaluationsergebnisse ab dem nächsten Jahr beschlossen wurde. Damit wird die Accountability-Funktion der Qualitätssicherung unterstrichen.

Zunehmend lässt sich auch im deutschsprachigen Raum der hochschulpolitische Trend der Zunahme der Bedeutung von Rankings im Sinne eines Instruments der „Kundeninformation“ beobachten.

Auf die österreichische Situation übertragen, könnte sich die Qualitätssicherung folgendermaßen weiterentwickeln:

Qualitätssicherung und Transparenz: Der summativ-bewertende Aspekt der Evaluierung sollte transparenter gestaltet werden - etwa durch adäquate Formen der Veröffentlichung. Zusätzlich könnten in methodisch abgesicherter Form vergleichende Bewertungen vorgenommen werden, die dann eine wichtige Form der „Kundeninformation“ darstellen.

Qualitätssicherung und Entwicklung: Der formativ-institutionell unterstützende Aspekt wird stärker mit Aktivitäten der Personal- und Organisationsentwicklung verbunden.

\section{Qualitätssicherung und Transparenz}

Der Mangel an ausformulierter Bildungspolitik führt dazu, dass Regulierungsbehörden dort Sachentscheidungen vorspiegeln, wo in Wahrheit Politik gemacht wird (vgl. Prisching 2002). So fällt dann der Fachhochschulrat mangels einer anderen Instanz bildungspolitische Entscheidungen. Angesichts des Mangels ausformulierter Paradigmen des Fachhochschulsektors, sowohl was die Konfiguration des gesamten Sektors als auch was die inhaltliche Beschreibung betrifft, entwickeln sich die Beziehungen des Fachhochschulrats, des zuständigen Bundesministeriums, der Fachhochschulträger und der sonstigen Interessenten in einem besonderen Spannungsfeld. Das Problem einer strukturellen Unterversorgung mit Informationen ist dabei ein fundamentales Defizit. Es gibt wenig Informationen, welche Kriterien der Fachhochschulrat anlegt, wenig Wissen über längerfristige Strategien, Schwerpunktsetzungen, Absichten. Die Entwicklung transparenter 
Regulierungskompetenz bedeutet auch, Hochschulsteuerung als einen sozialen Prozess zu organisieren, in dem es im Wesentlichen um die Herstellung von Dialogsituationen zwischen den Systemmitgliedern geht (vgl. Prisching 2002). Prisching ist auch der Meinung, dass der in der politischen Öffentlichkeit geäußerte Stolz auf die „Regelungsschlankheit“ des Sektors eine Illusion sei. Regulierungsbehörden seien zudem mit einem Minimum an administrativen Ressourcen ausgestattet und daher in kürzester Zeit überfordert (vgl. Prisching 2002).

\section{Explizite Unterschiede}

Die hohe Diversität des österreichischen Fachhochschulsektors hinsichtlich der Größenordnung der Studiengänge und der Standorte, in Hinblick auf Studierende, Lehrende, Ressourcen, Personalstruktur und Merkmale der Leistungserstellung hat auch entsprechende Qualitätsunterschiede zur Folge, die in den ExpertInneninterviews immer wieder thematisiert wurden, die aber nur informell geäußert und inoffiziell vorhanden sind. Die in den vorangegangen Kapiteln angesprochene Frage nach der Gestaltung des Aufgabenprofils und der Spezifikation der verschiedenen Funktionen bzw. deren Gewichtung hat enge Auswirkungen auf die Methoden und Ergebnisse der Qualitätssicherung. Ein weiterer Aspekt des Qualitätsthemas ist daher vermutlich eine notwendige stärkere Differenzierung zwischen verschiedenen Typen von Studiengängen.

In den Interviews häufig angesprochen wurde eine Differenzierung zwischen berufsbegleitenden und Vollzeitstudiengängen. Beide schließen jetzt in der gleichen Zeit ab, was eine gewisse "Schieflage“ bedeutet. Gerade zwischen diesen beiden Angeboten scheinen oft besondere Qualitätsunterschiede zu bestehen und hier muss ein Ausgleich geschaffen werden, sei es durch Ranking, durch Profile unterschiedlicher FachhochschulStudiengänge oder durch die Kombination von Vollstudiengängen mit berufsbegleitenden an einem Standort, so dass man sich auf nicht nur ein Segment davon konzentrieren kann. Gerade auch die Weiterbildungsaufgabe sollte ja von den Fachhochschuleinrichtungen stark wahrgenommen werden und sie sind in dieses Segment erst partiell eingestiegen.

Da in Österreich die Vorstellung verbreitet zu sein scheint, dass auch ein Berufstätiger nicht länger studieren müsse als ein Vollzeitstudent (vgl. Prisching 2002), entwickeln sich innerhalb des Fachhochschulbereichs unterschiedliche Qualitäten.

Dazu kommt das qualitative Dumping von außen: HTL-Abgängerlnnen, die mithilfe deutscher Fachhochschulen binnen kürzester Zeit einen Titel erlangen. Diese „Dumpinggefahr" war ein wichtiges Thema in den ExpertInnen-Interviews, ein Mangel an Qualitätssicherung dadurch, dass HTL-Abschlüsse von manchen deutschen Fachhochschulen für ein erstes, manchmal sogar auch zweites Studienjahr anerkannt werden, was noch kombiniert wird mit einem hohen Fernstudienanteil und wenig Präsenz, sodass man dann nach oft nur zwei Jahren einen Abschluss als FH-Ingenieur einer 
deutschen FH „in der Tasche haben“ kann. Dies wäre eine Entwicklung, wo man Qualitätsmängel befürchtet, wenn der Bund nicht einschreitet - indem er etwa genauer auf die Nebenbeschäftigungen, Rahmenbedingungen der Arbeit an BHS achtet, die zu Konkurrenten der Fachhochschul-Studiengänge heranreifen, aber nicht die entsprechende Qualität aufweisen. Es sollte daher in der Öffentlichkeit ein stärkeres Bewusstsein für Qualitätsunterschiede erzeugt werden.

\section{Vergleichende Bewertungen}

Eine bislang nicht angewendete, aber im Sinne von klaren Kundeninformationen wichtige Form der Qualitätssicherung könnten veröffentlichte, vergleichende Bewertungen im Sinne von Rankings sein, wodurch auch Qualitätsunterschiede im Sektor klarer zum Ausdruck kommen und damit auch wettbewerbsfördernde Elemente und eine gewisse Transparenz in die Qualitätssicherung gebracht werden. Aus der Literatur gibt es jedoch auch deutliche Hinweise auf mögliche nachteilige Wirkungen von Rankings.

In Bildungssystemen, in denen Wettbewerb zwischen den Institutionen als Steuerungsmittel eingesetzt wird, spielen Verfahren, durch die Unterscheidungen in den Leistungen transparent gemacht werden, eine wichtige Rolle. Solche Rankings haben auch das Ziel, die Profilbildung zu stärken und vor allem eine Orientierung für die Studienwahl zu geben.

Die fortschreitende Differenzierung der österreichischen Hochschullandschaft wird bislang noch wenig offiziell wahrgenommen. Vergleichende Bewertungen, also Rankings, sollten stärker in Erwägung gezogen werden, um dieser Differenzierung der Landschaft auch ein Informationsinstrument in die Hand zu geben. Dabei sollte darauf geachtet werden (vgl. Markowitsch 2001, 153), dass es

- ein Ranking gibt, welches in den verwendeten Kriterien ein möglichst umfangreiches Spektrum abdeckt,

- es zu einer Kritik dieser Rankings kommt, sodass auch diese entsprechend beurteilt werden können und

- dass es die Bewertungskriterien erlauben, verschiedene Stärken und Schwächen stärker hervortreten zu lassen. Für die Studienbewerber würde sich dadurch die Transparenz erhöhen und die Lücke zwischen Image und Wirklichkeit reduzieren. Rankings dieser Art könnten die Profilbildung der österreichischen Hochschulinstitutionen stärker unterstützen und der andauernden Differenzierung des Hochschulsystems entgegenkommen. 
Aussagekräftige Rankings müssen dabei nicht nur den methodischen Standards genügen, sie sollten auch fachbezogen sein und ein breites Indikatorenset einbeziehen, das Einschätzungen und Fakten umfasst. Vor allem dürfen nicht verschiedene Formen zu einem Gesamtwert verrechnet werden, denn nur dann ergibt sich eine einigermaßen realistische Stärken- und Schwächenbetrachtung. Sorgfältig durchgeführt ergeben sie aber wesentlich seriösere „Kundeninformationen“ als die derzeitigen, im Wesentlichen unter Marketinggesichtspunkten erstellten Informationen.

Für den Universitätssektor gibt es ab dem Studienjahr 2003/04 ein jeweils auf bestimmte Fächerbündel bezogenes Ranking. ${ }^{203}$ Wenn Rankings im Universitätssektor eingesetzt werden, könnte auch der Zeitpunkt gekommen sein, diese im Fachhochsektor ernsthaft zu diskutieren.

\section{Qualitätssicherung und Entwicklung}

Das formativ ausgerichtete Evaluierungsverständnis, das die erste Phase der Evaluierung im Fachhochschulsektor begleitet hat und das kooperativ zwischen FHR und FHK entwickelt wurde, könnte dadurch weiterentwickelt werden, dass die Akkreditierung im Sinne einer Exante-Bewertung gegenüber der Evaluierung im Sinne einer Ex-post-Bewertung eingeschränkt wird (vgl. Markowitsch 2001 sowie Fischer/Melchior 1995). Wenn man Ressourcen in der Akkreditierungsphase einspart (oder an Agenturen auslagert) zugunsten der Evaluierung und der begleitenden Beobachtung, könnten u.U. die Akkreditierungsphasen verkürzt werden. Interessant wäre es auch, die schriftlichen Anträge durch teilnehmende Beobachtungen in Entwicklungsteamsitzungen bzw. durch entsprechend ausführliche Dialogsituationen mit den Antragstellenden zu ergänzen. Auf der einen Seite ginge es um mehr persönlichen Kontakt, wohlwissend, dass dies natürlich eine Kapazitäts- und Ressourcenausstattungsfrage ist (siehe Aufstockung der Geschäftsstelle) und auf der anderen Seite ist dies für eine Weiterentwicklung im Sinne einer stärkeren Klassifizierung (siehe Thema Ranking) eine wichtige „Hintergrundinformation“.

Darüber hinaus sollte der eher formative Bereich der Qualitätssicherung in Richtung Organisations- und Personalentwicklung weiterentwickelt werden. Bereits Mitte der 1990er Jahre hat es erste Initiativen gegeben, den Weiterbildungsbedarf zu erheben. In der projektinternen Befragung wurde von vielen rückgemeldet, dass sie gerade in diesem didaktische Bereich ihren Lehrenden gerne mehr anbieten würden. Dies wäre sicher auch wie im Monitoringprojekt von 1995 (vgl. Fischer/Melchior 1995) bereits angeregt - eine

${ }^{203} \mathrm{Ab}$ dem nächsten Jahr sollen einzelne universitäre Fächer in das vom Deutschen Zentrum für Hochschulentwicklung (CHE) in Gütersloh organisierten Rankingverfahren eingebunden werden. 
Möglichkeit, Berufsfeldforschung und Lernforschung im Fachhochschulbereich zu entwickeln.

Auseinandersetzung mit dem didaktischen Grundmodell

Zum Thema Qualitätssicherung gehört auch die Auseinandersetzung mit der eigenen „Spezialität“ und der eigenen Identität, auch als Voraussetzung für einen funktionierenden Wettbewerb zwischen Institutionen. Der Weiterentwicklung eines fachhochschulspezifischen Didaktikmodells sollten in den nächsten Jahren spezielle Bemühungen gewidmet werden.

Aus den AbsolventInnenbefragungen geht klar hervor, dass die Studierenden zwar sehr zufrieden mit der fachlichen Kompetenz ihrer Lehrenden sind, aber doch bei der didaktischen Kompetenz einige Verbesserungswünsche haben. Zur Qualitätssicherung gehören der Begriff des Hochschulniveaus und der Berufsfeldorientierung ebenso wie die zukünftig immer wichtiger werdenden Überlegungen, welche Rolle Forschung und Entwicklung in Fachhochschul-Studiengängen haben und wie man sicherstellen kann, dass Bedarfsstudien quasi eine permanente Auseinandersetzung mit dem jeweils dazugehörigen Berufsfeld sind (Bedarfsstudien als eine Art Forschungsaufgabe) (vgl. auch Abschnitte 4.1.2 und 4.2.2).

Die Bedeutung der Auseinandersetzung mit der Besonderheit des didaktischen Modells der Fachhochschulausbildung stellt sich auch dadurch, dass in Hinkunft sowohl Universitäten als auch Fachhochschuleinrichtungen Bakkalaureats- und Masterstudien anbieten können und dass daher der Abschluss als solcher noch kein Indikator für die jeweilige Besonderheit eines Studienangebotes ist. Man wird anschaulich vermitteln müssen, worin nun das Spezifische eines FH-Bakkalaureats im Gegensatz zu einem universitären Bakkalaureat besteht. Es kann auch von Nutzen sein, innerhalb der Angebote zu differenzieren. All das macht jedoch eine intensivere Beschäftigung mit der Curriculumentwicklung und didaktischen Grundfragen notwendig.

Die Qualität im Fachhochschulbereich manifestiert sich im Ausprägungsgrad einer „praxisbezogenen Ausbildung auf Hochschulniveau“. An diesen beiden Begriffen - und damit implizit am Qualitätsbegriff - muss bei der Auseinandersetzung mit den eigenen Besonderheiten zuerst Arbeit geleistet werden. Was Hochschulniveau, so schreibt Sohm (1999), im spezifischen Kontext der fachhochschulischen Berufsausbildung und unter der Bedingung der Einhaltung der Regelstudienzeit konkret bedeuten würde/könnte, dürfte für die FH-Studiengänge noch nicht klar sein. Im Sinne der Positionierung der FH-Studiengänge innerhalb der österreichischen Bildungslandschaft scheint aber die Entwicklung eines eigenständigen Profils hinsichtlich der Definition des theoretischen Niveaus auch insofern vonnöten, als davon auch die Frage der Durchlässigkeit betroffen ist. Denn Niveau und Dauer definieren die Zugangsbedingungen, die wiederum bedeutsam sind, um den 
AbsolventInnen der dualen Berufsausbildung einen Zugang zu den FH-Studiengängen zu eröffnen und andererseits bestimmt die Differenz zur universitären Ausbildung die Rahmenbedingungen bezüglich eines Doktoratsstudiums, das den AbsolventInnen von Fachhochschul-Studiengängen offen steht (vgl. Abschnitte 4.1.1 und 4.1.3).

Die Formen des Praxisbezugs sollten untersucht und verglichen werden, weil diese ihre Qualität erst unter besonderen Bedingungen entfalten. Altrichter /Posch kamen bereits 1995 zu folgenden, immer noch aktuellen Vorschlägen: Man sollte StudentInnenteams im Rahmen eines Projektstudiums an Aufgaben arbeiten lassen, die von kooperierenden Praxisbetrieben gestellt werden oder über Juniorfirmen, in denen Lehrende und Studierende zusammenarbeiten, Beratungen für Wirtschaftsbetriebe anbieten. Praxisbüros könnten die Zusammenarbeit mit interessierten Firmen organisieren. Praxiskontakte entfalten jedenfalls erst durch entsprechende Vorbereitung, Begleitung und forschungsmäßige Reflexion ihre didaktische Qualität.

Zunehmend erhält etwa der kreative Einsatz von Fernstudienelementen und ihre Kombination mit Präsenzphasen mehr Aufmerksamkeit; einzelne FachhochschulStudiengänge wie etwa in Hagenberg, am Wifi Wien oder am Zentrum für multimediales Lernen/Joanneum haben hier Vorreiterfunktionen. Der Einsatz neuer Medien in der Lehre ist für die Bildungsträger eine große Herausforderung. In diesem Zusammenhang ist eine Kooperation der Bildungsträger, also der Universitäten und Fachhochschuleinrichtungen, von großer Relevanz (Pauschenwein et al 2002, 104). Die vom BMBWK Ende 1999 durch ein Förderprogramm eingeleitete Initiative „Neue Medien in der Lehre an Universitäten und Fachhochschulen" hat die bis dahin nur vereinzelt vorhandenen hochschulischen Aktivitäten sehr unterstützt und die Kooperation zwischen Fachhochschuleinrichtungen und Universitäten vorangetrieben.

Personal- und Organisationsentwicklung

John Pratt schreibt bereits 1994 in seinem Artikel „Lehren aus der Geschichte: Die österreichische Entwicklung aus britischer Sicht“" (vgl. Höllinger et al 1994), dass der Erfolg der Fachhochschulreform von der Schaffung eines selbstbewussten und eigeninitiativen Lehrkörpers abhängen wird. Nur wenn der Lehrkörper fähig ist, als Antwort auf veränderte Umstände Reformen und Innovationen einzuleiten, kann ein eigenständiges Bildungsprofil entstehen, das den Universitäten nicht unterlegen ist. Dafür wird es einer erheblichen Unterstützung für Personalentwicklung bedürfen.

Organisations- und Personalentwicklung (OE/PE) werden vor allem aus den folgenden Gründen zu drängenden Themen im österreichischen FH-Sektor: 
Die Pionierphase und die ersten Aufbaujahre haben die fachhochschulischen Institutionen selbst sowie der gesamte Bildungssektor hinter sich. Nunmehr setzt eine Phase der Konsolidierung ein, bei der das Unternehmertum der ersten Jahre in arbeitsteilige Organisationswerdung überführt werden muss. Das setzt eine behutsame Weiterentwicklung der einzelnen Institutionen im Sinne eines „institution building“ voraus. Geeignete Verfahren der Organisationsentwicklung können diese institutionelle Weiterentwicklung gut unterstützen. Strategiegeleitete Personal- und Organisationsentwicklung leistet einen wichtigen Beitrag zur Steigerung der professionellen Selbstorganisation der einzelnen Institutionen und damit zur Qualität ihres Bildungsanbotes.

Der Übergang in die Konsolidierungsphase hat aber auch Auswirkungen auf die notwendigen Kompetenzen und Qualifikationen der Lehrerlnnen, ForscherInnen und ManagerInnen der fachhochschulischen Institutionen. Ebenso müssen sich neue Herausforderungen - verstärkte Internationalisierung, verstärkter Forschungsauftrag - auch in Konzepten und Strategien der Personalentwicklung niederschlagen, damit sie tatsächlich angenommen und gelebt werden.

Durch die Entstehung des europäischen Hochschulraumes und durch die Vollrechtsfähigkeit der österreichischen Universitäten wird der Wettbewerb um MitarbeiterInnen, Studierende und Ressourcen sowie der Druck zur institutionellen Profilbildung steigen.

Veränderungsprozesse in Organisationen müssen auf jeden Fall Veränderungen auf drei verschiedenen Ebenen - Individuen, Gruppen und Organisation - umfassen. Daher sind Organisations- und Personalentwicklung eng miteinander verflochten. Fachhochschuleinrichtungen sind als Bildungsinstitutionen sehr personenzentrierte Systeme. Probleme haben aber oft nicht nur mit Personen zu tun, sondern mit wenig durchdachten Abläufen und Strukturen. Jedenfalls müssen sie in einem strukturellen Kontext betrachtet werden. Auch lässt sich eine "exzellente" Organisation dadurch kennzeichnen, dass sich die Individuen für organisatorische Prozesse und Ziele engagieren. Organisationsentwicklungsmaßnahmen wiederum müssen vereinbar sein mit Auswahl, Beförderungs-, Entlohnungs- und Beurteilungssystemen. Die Verbindung von OE und PE im Sinne einer Rückkoppelung von Wissen und Dokumentation von Erfahrung sind ein entscheidendes Charakteristikum einer lernenden Organisation. Lernfördernde Systeme sind gekennzeichnet durch die Verankerung von Kommunikations-, Zielvereinbarungs- und Feedback-Systemen.

Dabei ist davon auszugehen, dass insbesondere ein spezifisches Lehr-/Lernverständnis (didaktische Umsetzung des fachhochschulischen Bildungsauftrages) ein wichtiges Thema der Personalentwicklung ist. Es fragt sich jedoch, ob bei einem Lehrkörper mit typischerweise hohem Anteil an nebenberuflich Lehrenden (vgl. Abschnitt 2.2.8) die personalpolitischen Voraussetzungen vorliegen, um die mit einem hohen Aufwand an Kooperation verbundenen ambitiösen didaktischen Zielsetzungen verfolgen zu können. Das 
würde eigentlich voraussetzen, dass der größere Teil der Lehrkörpers hauptamtlich beschäftigt ist. Der oft geringe Anteil an hauptberuflich Lehrenden dürfte auch in der Betreuung von Berufspraktika und Diplomarbeiten zu Problemen führen (vgl. Sohm 1999, 94).

Das Konzept der Lehrveranstaltungsbewertung sollte zur pädagogisch-didaktischen Weiterbildung der Lehrenden in Beziehung gesetzt werden. Es geht um die Beteiligung aller relevanten betroffenen Gruppen eines Studiengangs, also Studierende, Lehrende und VertreterInnen der einschlägigen Berufsfelder. Interessant sind jene Ansätze, die Qualitätszirkel mit Studierenden und Lehrenden bzw. Studiengangsleitungen durchführen und die schon einige Studiengänge implementiert haben.

Aber auch ein spezifisches Forschungsverständnis (Auftrag der angewandten Forschungsorientierung) gehört $\mathrm{zu}$ den zentralen Herausforderungen der Personalentwicklung und damit auch Qualitätssicherung. Hier ist entscheidend, ob es gelingt, den Auftrag der Forschungsförderung auch tatsächlich in entsprechende Rahmenbedingungen für das Personal umzusetzen. Dazu gehört es, Freiräume für Projekte und wissenschaftliche Arbeit, Anreizsysteme für F\&E, Sabbaticals in Kombination mit internationalem Professorlnnenaustausch, gemeinsame Forschungsprojekte und den Aufbau einer Community zu schaffen. Es geht auch darum, diesem Tätigkeitsfeld die entsprechende Zeit und Organisation zukommen zu lassen, etwa durch Forschungsfreisemester, Ausschreibung von Sabbaticals, Bereitstellung von Geldern für Ersatzlehrkräfte, spezielle Forschungsaufträge für Fachhochschullehrerlnnen etc.

Ebenso wird ein adäquates Managementverständnis (angepasst an die Besonderheiten von Fachschuleinrichtungen als Organisationen bzw. die spezifischen Anforderungen der Entwicklung des FH-Sektors) ein wichtiges Feld der Personalentwicklung sein.

Die Qualität eines Studienangebotes ist stark abhängig von einem engagierten Kernteam; daher sind wichtige Fragen der Qualitätssicherung - wie man dieses Team bekommt, wie man eine FH-Karriere attraktiv genug macht, wie man die nun vorgesehene Forschungsorientierung wirklich zum Leben erwecken kann - sowohl von den personellen als auch von den finanziellen Kapazitäten abhängig. Die Komponente der Organisationsentwicklung wiederum verweist auf den Aufbau begleitender, stützender Strukturen für die nun größer gewordenen Einheiten, ihre „Institutionenwerdung“ und Entwicklung hin zur Fachhochschule. Die gesetzlich festgeschriebene Kollegiengröße trägt dazu bei, dass es bislang nur begrenzt attraktiv ist, tatsächlich eine Fachhochschule zu werden. Hier erscheint eine Gesetzesänderung notwendig. Es scheint günstiger, Subeinheiten zu ermöglichen, die dann diese kollegialen Strukturen ausbilden.

Das Thema Personalentwicklung wird auch stark vor dem Hintergrund des Verhältnisses der fixangestellten oder hauptberuflichen versus nebenberuflichen Mitarbeiterlnnen diskutiert. 
Sicher kann man aus der Perspektive der Qualitätssicherung zustimmen, dass eine weitere institutionelle Konsolidierung der Fachhochschul-Studiengänge hin zu Fachhochschulen ohne hauptberuflich Beschäftigte kaum vorstellbar erscheint. Ein frühzeitiger Aufbau eines Teams von hauptberuflich Beschäftigten ist auch notwendig, um das Image zu korrigieren, es handle sich hier um Aufgaben, die man problemlos nebenberuflich erledigen kann (vgl. Altrichter/Posch 1995). Umgekehrt, wenn man stärker in Richtung hauptberufliches Lehrendenprofil geht, müssen auch den Lehrenden Profilierungsmöglichkeiten eröffnet werden, die sich nicht nur aus der Beteiligung an der Aufbauphase, sondern eben etwa über die Qualität der Lehre als zentralen Entwicklungsbereich ergeben. Es muss potenzielle längerfristige Anreize geben wie Forschungs- und Entwicklungsmöglichkeiten, mit denen auch finanzielle Anreize verbunden sind, interessante Möglichkeiten im Weiterbildungsbereich, wodurch qualifizierte Lehrende mit interessanten Gruppen von Berufstätigen in der Region in Kontakt gebracht werden und die Entwicklung eines attraktiven Berufsbildes vorangetrieben wird. Eine Möglichkeit wäre es, die Profilierung des Fachhochschullehrberufs über eine Profilierung der Standorte zu betreiben. Verstärktes Engagement im Weiterbildungsbereich für Berufstätige gibt nicht nur für die einzelnen Standorte große Chancen für die Erweiterung des Angebotsprofils, sondern auch für die Erschließung zusätzlicher Einnahmequellen und damit auch attraktive und marktfähige Profilierungsmöglichkeiten für die einzelnen FachhochschullehrerInnen.

Ein Problem der Personal- und Organisationsentwicklung ist es auch, rechtzeitig eine stützende Infrastruktur für die massiver werdende Internationalisierung oder die Aufgabe der angewandten Forschung und Entwicklung aufzubauen. Das setzt zum einen kritische Größen voraus, aber eben zum anderen auch gewisse Finanzierungsmöglichkeiten und OE/PE stützende Infrastrukturen. Das sind zumeist Querschnittsmaterien, die sich ein Standort erst dann leisten kann, wenn er eine bestimmte Größe hat, in die Kostendegression kommt und damit auch in die Lage, Querschnittsmaterien zu finanzieren. Gleichzeitig ist das eine Angelegenheit, die im Verbund der fachhochschulischen Einrichtungen - insbesondere über die FHK - vorangetrieben werden kann.

Ein wesentliches Qualitätssicherungsmoment ist die (Organisations-)Entwicklung hin zu einer akademisch-autonomen, selbstbestimmten Einrichtung. Während bei Universitäten vielleicht die starke Praxisferne und die starke Ausrichtung an der innerwissenschaftlichen Entwicklung ein Problem ist, so ist es im FH-Sektor die starke Politiknähe. Hier muss ein besonderer Schutzwall an selbstbewusster Autonomie aufgebaut werden. Dabei helfen kollegiale Strukturen, die im Inneren die akademische Entwicklung thematisieren und natürlich bedarf es auch eines gewissen Erkenntnisprozesses der Erhalter (etwa der Länder), die ja bislang Schulen, Schwimmbäder, Thermen verwaltet haben. Wenn man, wie Prisching sagt, eine Fachhochschule wie eine Therme sehr direktiv, intervenierend behandelt, dann wird man auch nur lauwarmes Wasser, wie bei einer Therme, bekommen (Prisching 2002). Eine gewisse Politikdistanz und der Aufbau eines selbstbewussten akademischen Eigenbildes ist ein besonders Merkmal der Qualitätssicherung. Der 
Fachhochschulsektor simuliert eine Nähe zum privatwirtschaftlichen Sektor, so Prisching, er bleibt aber auf Grund der Trägerschaft und der Finanzierung dem politischen Bereich eng verbunden. Hier sind Körperschaften wie Landesregierungen und Gemeinden in eine für sie neue bildungspolitische Rolle gekommen. Dazu bedarf es des Respekts vor hochschulischer, akademischer Autonomie und einer selbstbewussten Institutionenkultur, die auf ihr Selbstverständnis als wissenschaftliche Einrichtung pocht. Diesen notwendigen Respekt haben einige der neuen bildungspolitischen Akteure zuweilen vermissen lassen. 


\section{Zusammenfassung und übergreifende Schlussfolgerungen}

\subsection{Zweck und Methodik}

Die vorliegende Evaluierung der bisherigen Entwicklung des FH-Sektors soll zur Konzeption des dritten Entwicklungs- und Finanzierungsplanes beitragen. Gemäß der Aufgabenstellung wurden die Arbeiten auf die folgenden Aspekte konzentriert:

- den Stellenwert des FH-Sektors im Hochschulsystem

- die Funktionsfähigkeit und Wirksamkeit der Finanzierungs- und Entwicklungsplanung

- die Entwicklung und Allokation der Standorte im Raum

- die internationale Positionierung angesichts der Entwicklung des Europäischen Hochschulraumes

- die Funktionsfähigkeit und Wirksamkeit der Qualitätssicherung

Da für die Evaluierung ein enger zeitlicher Rahmen vorgegeben war, wurde sie methodisch in Form eines Review-Verfahrens durchgeführt. Eine breite und vielfältige Informationsbasis wurde für die Erstellung des Rohberichtes verarbeitet (Literaturanalyse, Sonderauswertungen der FHR-Datenbasis und einschlägiger Statistiken, ExpertInneninterviews, schriftliche Erhebungen bei den FH-Erhaltern und den StudiengangsleiterInnen). Nach Abschluss des Rohberichtes konnten in einem mehrschichtigen Feedback-Prozess Rückmeldungen aus dem $\mathrm{FH}$-Sektor sowie von internationalen und nationalen ExpertInnen eingebracht werden.

Im Rahmen dieser Zusammenfassung werden zunächst einzelne Ergebnisse der jeweils untersuchten Aspekte dargestellt. Daran schließen sich themenübergreifende Zusammenfassungen und Schlussfolgerungen an. Am Ende werden die zentralen Empfehlungen nochmals gebündelt veranschaulicht.

\subsection{Einzelergebnisse der spezifischen Untersuchungsaspekte}

\subsubsection{Stellenwert des FH-Sektors in der Bildungs- und Hochschullandschaft}

Die Frage nach dem Stellenwert des Fachhochschulsektors im Bildungswesen wurde eingeschränkt auf den Stellenwert im Hochschulwesen, die Beziehungen zur Sekundarstufe 
wurden ausgeklammert. Der Stellenwert im Hochschulsystem wurde aufgrund der wesentlichen Besonderheiten des FH-Sektors (Studienprofil praxisbezogener Hochschulausbildung und Verbindung zu Bedarfs- und Akzeptanzerhebungen) sowie der manifesten Ziele bei seiner Errichtung (Erhöhung der Durchlässigkeit in verschiedenen Aspekten, Förderung der beruflichen Flexibilität, Entlastung der Universitäten, Beitrag zur Weiterbildung, Abbau der regionalen Disparitäten und Strukturbereinigung im postsekundären Bildungswesen) und der eher latenten Zielsetzung einer Reform der Angebots- und Regulierungsstrukturen im Bildungswesen analysiert. In einem zweiten Schritt wurden die Auswirkungen wichtiger Veränderungen im Umfeld des FH-Sektors herausgearbeitet und in drei Entwicklungsfeldern gebündelt: (1) Interaktion von Ausbildung und Innovation; (2) innovative Impulse für Unternehmenspraktiken; (3) Positionierung im regionalen Umfeld. Die mögliche Rolle des FH-Sektors in der österreichischen Innovationspolitik wurde näher beleuchtet. Abschließend wurden verschiedene mögliche Entwicklungspfade für den FH-Sektor formuliert, die die Wahl zwischen unterschiedlichen Optionen und die dabei wichtigen Faktoren deutlich machen sollten. Es wurde ein breites Bündel an Faktoren herausgearbeitet, die den Gegenstand von Entscheidungen darstellen werden müssen. Diese möglichen Entwicklungspfade sind in den übergreifenden Schlussfolgerungen (Abschnitt 8.3.4) dargestellt.

Die Zielsetzungen für den FH-Sektor und ihre Realisierung

Die Realisierung der Zielsetzungen wurde im Hinblick auf zwei allgemeine Ziele - (A) die Diversifizierung des Hochschulsektors durch eine praxisbezogene Berufsbildung auf Hochschulniveau und (B) die Bindung der Studienangebote an den wirtschaftlichen Bedarf und die Akzeptanz der Studierenden - sowie im Hinblick auf spezifische manifeste und latente Zielsetzungen untersucht. Die Ergebnisse zur Realisierung können in den folgenden Aussagen zusammengefasst werden:

- Positionierung im Hochschulsystem. Die Aspekte der Praxisnähe, des Hochschulniveaus und des zeitgerechten Studienabschlusses wurden hervorgehoben. Es ist gelungen, den FH-Sektor erfolgreich im Hochschulsystem zu positionieren, insbesondere was die Bildungsnachfrage und die Profilierung gegenüber dem Unternehmenssektor betrifft. Im Verhältnis zu den Universitäten bestehen noch Spannungen, die sich mit der Umsetzung des Bologna-Prozesses eher noch verstärken könnten. Der erwartete Beitrag des FHSektors zur Wissensproduktion ist noch nicht sehr ausgeprägt, im Hinblick auf die akademische Profilierung wurde Entwicklungsbedarf festgestellt. Mit dem Konzept der Hochschulpraxisgemeinschaft wurde ein vielversprechender Ansatz der Profilierung im Hochschulsystem eingeschlagen.

- Bedarf und Akzeptanz. Der möglichst objektivierten Feststellung von Bedarf und Akzeptanz wurde bei der Anerkennung von Studiengängen wesentliche Bedeutung 
beigemessen. Trotzdem erfolgt die Entwicklung auf dem Wege des „bottom-up“-Prinzips eher angebotsseitig als bedarfsseitig. Eine wesentliche Schwäche besteht darin, dass die Aktivitäten zur Bedarfs- und Akzeptanzerhebung im FH-Sektor nicht in eine entsprechende Praxis auf breiterer Ebene eingebettet sind. Die Aktivitäten zur Analyse und Antizipation des Qualifikationsbedarfes auf der Gesamtebene, gesamtwirtschaftlich und qualifikationsübergreifend, sind bisher in Österreich unzureichend. Es gibt auch keine ausreichend begründete Bewertung der vorhandenen Qualifikationsstrukturen. Daher fehlt eine wichtige Informationsbasis für die Dimensionierung des $\mathrm{FH}$-Sektors und die Kleinräumigkeit und Nischenorientierung von Angeboten wird dadurch gefördert.

- Manifeste bildungspolitische Ziele. Die manifesten Ziele unterliegen nur in begrenztem Ausmaß einer operationalen Definition, sie wurden soweit wie möglich operationalisiert und im Hinblick auf ihre Erfüllung einer qualitativen und teilweise auch einer quantitativen Analyse unterzogen. Bei den Zielen Abbau regionaler Disparitäten, Verbesserung der Durchlässigkeit und Strukturbereinigung sind mehr oder weniger deutliche Fortschritte nachweisbar; der Beitrag zur Verbesserung beruflicher Flexibilität ist vor allem aufgrund von Problemen der operationalen Definition fraglich; die Zielsetzungen der Entlastung der Universitäten sowie der Entwicklung von Aus- und Weiterbildung wurden aufgrund der durchgeführten Analysen eher nicht realisiert. Bei den einzelnen Zielsetzungen können die folgenden Aspekte hervorgehoben werden:

- DURCHLÄSSIGKEIT. Die Errichtung des FH-Sektors hat nach den vorliegenden Befunden vermutlich in bestimmtem Ausmaß zur Verbesserung der Durchlässigkeit des Bildungswesens in sozialer Hinsicht beigetragen. Der FH-Sektor ist für Studierende aus bildungsfernem Milieu zugänglicher als der Universitätssektor, dies gilt jedoch vor allem für Männer. Es wurden auch Möglichkeiten der Förderung des Zugangs von nicht-traditionellen Studierenden geschaffen, gegenüber der hohen Priorität dieser Zielsetzung und im Vergleich zu den Ausgangserwartungen jedoch in eher geringem Ausmaß.

- FördeRUNG DER BERUFLICHEN FLEXIBILITÄT. Die Förderung der beruflichen Flexibilität ist in objektivierter Hinsicht mit den vorhandenen Mitteln nicht festzustellen. Inwieweit die oft geäußerte Vermutung zutrifft, dass ein Teil der angebotenen Studiengänge angesichts der Zielsetzung beruflicher Flexibilität überspezialisiert ist, sollte näher untersucht werden.

- ENTLASTUNG DER UNIVERSITÄTEN. Hinsichtlich einer Entlastung der Universitäten haben die Detailauswertungen kein eindeutiges Bild ergeben. Am ehesten lässt sich eine gewisse Entlastung für den wirtschaftlichen Fachbereich feststellen. Insgesamt deuten verschiedene Befunde darauf hin, dass die Errichtung des FH-Sektors in gewissem Ausmaß auch zusätzliche erfolgreiche Bildungsbeteiligung mobilisiert hat.

- AUS- UND WEITERBILDUNG. Manche Einrichtungen haben bisher schon Aktivitäten der Weiterbildung entwickelt, insgesamt im $\mathrm{FH}$-Sektor sind diese Aktivitäten jedoch noch sehr gering ausgeprägt. Um in diesem Bereich, der von vielen Expertlnnen als 
wichtiger möglicher Zukunftsmarkt gesehen wird, stärker tätig zu werden, sind größere Kapazitäten der Einrichtungen erforderlich. Die Ausweitung der Tätigkeit in diesem Bereich steht möglicherweise in Konkurrenz zur Ausweitung anderer Tätigkeiten (insbesondere F\&E), unter den Expertlnnen bestehen auch divergierende Einschätzungen zur sinnvollen Prioritätensetzung für den $\mathrm{FH}$-Sektor.

- AbBau REgIOnAler DiSPARITÄTEN. Ein Beitrag des FH-Sektors zum Abbau regionaler Disparitäten beim Hochschulzugang wird durch die empirischen Auswertungen in einem eigenen Abschnitt der Untersuchung (siehe das Kapitel zu Regionalisierung bzw. Abschnitt 8.2.3) unterstützt, das Studium an FH-Studiengängen ist regional auch ausgewogener verteilt als das Studium an Universitäten, es bestehen jedoch Unterschiede zwischen den Bundesländern.

- Strukturbereinigung. Ein Beitrag des FH-Sektors zu einer bildungspolitischen Strukturbereinigung konnte bei weitem langsamer erreicht werden, als von vielen beteiligten Akteuren angestrebt wurde. Wesentliche Fragen sind offen geblieben und aktuell in Diskussion, insbesondere die Frage der Angebote im Bereich der Gesundheitsberufe, aber auch die Frage der Abstimmung mit anderen postsekundären Angeboten der Berufsbildung. Im Zusammenhang mit dem Bologna-Prozess werden sich viele Fragen der Abstimmung zwischen FH-Sektor und Universitäten neu stellen.

- Latente bildungspolitische Ziele und Veränderungen im Umfeld. Latente bildungspolitische Ziele bestanden in der Reform des Regulierungssystems im Bildungswesen. Im Hinblick auf die Errichtung dezentralisierter und flexibler Strukturen war der FH-Sektor sehr erfolgreich, wenn auch mit dem Größenwachstum an vielen Punkten die Grenzen dieses Regulierungssystems wahrnehmbar werden (vgl. die näheren Analysen in den Kapiteln zur Entwicklungsplanung und zur Qualitätssicherung; bzw. die Abschnitte 0 und 8.2.5). Eine Schwäche bestand bisher in der politischen Steuerung des FH-Sektors im Rahmen eines Gesamtkonzeptes für den Hochschulsektor. Mit der möglichen demografisch bedingten Verknappung des Studierendenpotentials und mit der stärkeren Überschneidung zwischen FH-Sektor und Universitäten werden Abstimmungsprobleme zunehmend virulent werden. Wenn ein direkter Wettbewerb zwischen den beiden Sektoren des Hochschulsystems entsteht, wird sich die Frage nach einheitlichen Spielregeln für FH-Studiengänge und Universitäten verstärkt stellen, um vergleichbare Wettbewerbsbedingungen herzustellen. Im Zusammenhang mit den verschiedenen Veränderungen im Umfeld erscheint eine Überprüfung der Zielsetzungen erforderlich, wie in den übergreifenden Schlussfolgerungen (Abschnitt 8.3.2) ausgeführt wird.

Die retrospektive Analyse hat grosso modo gezeigt, dass der Abbau regionaler Disparitäten, die Erhöhung der Durchlässigkeit und die Strukturbereinigung zumindest teilweise gelungen sind. Die berufliche Flexibilität lässt einige Fragen offen und die Entlastung der Universitäten sowie der Beitrag zur Weiterbildung sind eher weniger gelungen. Diese Befunde bilden die 
Grundlage für die übergreifenden Schlussfolgerungen (Abschnitt 8.3). Darüber hinaus wurden bei der Analyse des Stellenwertes des FH-Sektors Schlussfolgerungen zu drei weiteren Themen (geschlechtsspezifische Segmentierung, Durchlässigkeit für nichttraditionelle Studierende und der FH-Sektor in der Innovationspolitik) gezogen:

\section{Geschlechtsspezifischer Aspekt der Durchlässigkeit: Förderung und Strukturbereinigung}

Die Befunde zeigen, dass, obwohl der Frauenteil eine etwas steigende Tendenz aufweist, sowohl unter den Studierenden als auch unter den Lehrenden die Frauen deutlich unterrepräsentiert sind. Dies hat teilweise strukturelle Gründe, indem sich durch die fachrichtungsspezifischen Schwerpunkte die generelle geschlechtsspezifische Segmentierung im FH-Sektor reproduziert (Lassnigg/Paseka 1997). Die Erweiterung des Fächerspektrums im Zuge der Strukturbereinigung würde jedenfalls einen Beitrag zur besseren geschlechtsspezifischen Repräsentation leisten. Darüber hinaus bleibt die Frage der fachrichtungsspezifischen Segmentierung, insbesondere in den technischen Bereichen, bestehen. Die Unterrepräsentation der Frauen in diesen Bereichen ist nicht nur eine allgemeine Frage der geschlechtsspezifischen Chancengleichheit, sondern hat auch im Zusammenhang mit der Mobilisierung von zusätzlichen Potentialen insbesondere für die technisch-naturwissenschaftlichen Bereiche Gewicht. Eine bessere Repräsentation der Frauen kann kurz- bis mittelfristig vermutlich nur durch gezielte Förderprogramme erreicht werden. Es wird daher vorgeschlagen, die Entwicklung von spezifischen Förderprogrammen für Frauen, unter Berücksichtigung internationaler Erfahrungen, sowohl auf der Ebene der Lehrenden als auch auf der Ebene der Studierenden ins Auge zu fassen. ${ }^{204}$

\section{Durchlässigkeit für nicht-traditionelle Studierende}

Im Bereich der Förderung der Durchlässigkeit sind in begrenztem Ausmaß Erfolge erzielt worden; trotz der starken Betonung dieses Aspektes in den Zielsetzungen ist die Entwicklung gegenüber den hohen ursprünglichen Erwartungen jedoch eher zurückgeblieben. Insbesondere nicht-formalisierte Zugänge sind nur in kleinem Ausmaß realisiert worden. Dies scheint strukturelle Ursachen zu haben, die in der großen Distanz zwischen dem mittleren Ausbildungsniveau und dem FH-Niveau liegen. Eine verstärkte Durchlässigkeit erfordert massivere Formen der Förderung, wobei zwei unterschiedliche Aspekte zu berücksichtigen sind:

204 Im Schweizerischen FH-System bestehen explizite gesetzliche Zielvorgaben "die Gleichstellung der Geschlechter zu fördern und den Anteil der Frauen zu erhöhen" ( EFHK 2000, 39); es wurde 1999 ein Aktionsplan verabschiedet und mit 10 Mio SFR (6,82 Mio EUR, 93,8 Mio ATS) dotiert. Die geschlechtsspezifische Ungleichheit ist stärker ausgeprägt, wenn auch in ähnlichen Grundmustern wie in Österreich und die Wirksamkeit des Aktionsplanes wird sehr vorsichtig bewertet (EFHK 2002, 26-27). 
- erstens die Vereinbarkeit von Studium und Berufstätigkeit (hier geht es einerseits um Zeitressourcen, andererseits um finanzielle Ressourcen und auch um die Potentiale des Einsatzes von E-learning),

- zweitens der Zugang ohne traditionelle Hochschulberechtigung (hier geht es u.a. um die Anerkennung nicht-formaler Qualifikationen).

Bei beiden Aspekten ist in Abhängigkeit von einem zukünftig möglicherweise zu erwartenden Mangel an Qualifikationen und im Sinne der Umsetzung von Möglichkeiten des lebensbegleitenden Lernens zu erwägen, inwieweit massivere Interventionen eingesetzt werden sollen (insbesondere wenn sich der Zuzug aus der Erstausbildung in Zukunft verknappen wird, wird sich diese Frage in zunehmendem Maße stellen).

Rolle des FH-Sektors in der österreichischen Innovationspolitik

Vor allem in Anknüpfung an das Entwicklungsfeld der Interaktion von Ausbildung und Innovation wurde die Rolle des FH-Sektors in den Formulierungen für eine österreichische Forschungsstrategie näher beleuchtet. Dabei geht es einerseits darum, in welchem Ausmaß der FH-Sektor zur angestrebten Verstärkung der F\&E-Tätigkeit durch den Ausbau der F\&EAktivitäten beitragen kann und andererseits darum, inwieweit die Lehre im FH-Sektor auf F\&E-Tätigkeiten vorbereiten soll. Bisher sind die Vorstellungen dazu noch wenig ausformuliert und es gibt weit divergierende Vorschläge und Ansätze zur Rolle des FHSektors in der Forschungsstrategie. Es werden die quantitativen Größenordnungen der angestrebten Erweiterung der F\&E-Aktivitäten und des damit verbundenen Personalbedarfes verglichen. Ein wesentlicher Aspekt betrifft die Entwicklung der Arbeitsteilung und der Kooperationsstrukturen zwischen dem FH-Sektor einerseits und den anderen Institutionen der außeruniversitären F\&E sowie den Universitäten andererseits.

Welcher Entwicklungspfad hier eingeschlagen wird, wird die weitere Profilierung und Positionierung des FH-Sektors wesentlich bestimmen. Da die F\&E-Aktivitäten und kapazitäten bisher noch wenig entwickelt sind, besteht in dieser Frage ein breites Spektrum an Optionen für die weitere Entwicklung. Insgesamt scheint der Aufbau der F\&E-Aktivitäten in ziemlich breitem Rahmen zu erfolgen, es ist zu erwägen, inwieweit eine gezielte Schwerpunktbildung bessere Ergebnisse erbringen könnte.

\section{Fragen zur Diskussion und weiteren Analyse}

In diesem Abschnitt werden abschließend zwei weitere gezielte Zukunftsfragen angesprochen, die mit den Mitteln des vorliegenden Projektes nicht zureichend geklärt werden können, aber als essentiell für die weitere Entwicklung anzusehen sind. 
- Die Profilierung der FH-Studiengänge im Hochschulsystem. Dieses Thema betrifft den Komplex der Entwicklung der Studiengangsprofile im Hinblick auf die angestrebten Ergebnisse des Lernens (die Breite und Spezialisierung, die zu erwerbenden Kompetenzen, die Verortung im Wissenschaftssystem bzw. Konkretisierung der Wissenschaftsorientierung), die Verbindung der angestrebten Ergebnisse zum didaktischen Konzept (kontextualisiertes und selbstgesteuertes Lernen, E-learning); die angestrebte Beziehung zwischen den Funktionen von Lehre, F\&E und Dienstleistung (erforderliche Qualifikationspotentiale und Formen der Arbeitsteilung und Kooperation, Konsequenzen für die organisatorische Ausgestaltung). Es geht bei der Profilierung um die vertiefende Ausgestaltung und Reflexion der inhaltlichen Komponente in ihrem Zusammenspiel mit den prozeduralen und organisatorischen Erfordernissen zur Erzielung der spezifizierten Ergebnisse. Eine Kernfrage ist die Formulierung der Ausbildungsprofile (In welchen Begriffen findet das statt? Inwieweit ist der Kompetenzbegriff dafür brauchbar und kann stärker akzentuiert werden?). ${ }^{205}$ Eine zweite Kernfrage betrifft die Verbindung der (teilweise verschulten) Lehre mit den praktischen Erfahrungen und das Gewicht der Forschungskompetenz. Dazu gibt es in der AbsolventInnenstudie (Hoyer/Ziegler 2002) bedenkenswerte Ergebnisse, auch Prisching 2002 betont die Bedeutung des Zuganges zu systematischem Wissen und weist explizit auf Probleme z.B. im Bereich berufsbegleitender Studiengänge hin. Einen vertiefenden Ansatz schlägt Markowitsch 2001 vor, indem er das Konzept der "community of practice“ auf praxisbezogenes Hochschulstudium anwendet. In den Expertengutachten für den FTE-Rat (Clement et al. 2002, Detter et al. 2002) werden ebenfalls Vorschläge und Ansatzpunkte zur Profilierung skizziert. Diese Überlegungen sollten weitergeführt werden, um auf die Frage von Manfred Prisching $(2002,20)$ nach „konsistenten Leitvorstellungen darüber, was Fachhochschul-Studiengänge eigentlich sein sollen" vertiefende Antworten zu formulieren und in der vorhandenen Vielfalt einen stärkeren Konsens über akademische Standards herzustellen.

- Weiterentwicklung der Ansätze und Methoden der Antizipation und „Früherkennung" von Bedarf und Akzeptanz. Dieses Thema betrifft die Anwendung von erweiterten Mechanismen der Dokumentation und Antizipation von wirtschaftlichem und gesellschaftlichem Bedarf nach Kompetenzen wie auch von Potentialen für den Erwerb der Kompetenzen. Es wäre die Entwicklung eines Referenzrahmens auf Makro-Ebene durch die systematische und gezielte Anwendung des in den letzten Jahrzehnten entwickelten methodischen Instrumentariums als Ergänzung der Bedarfs- und Akzeptanzerhebungen erforderlich. Dieses Instrumentarium reicht von quantitativen Projektions- und Prognosemethoden verschiedener Reichweite über die Abschätzung von Kosten-Nutzen-Relationen der Ausbildungen, Analysen von Stellenanzeigen bis zu

205 Eine ansatzweise Analyse der Ausbildungsprofile vor einigen Jahren hat nur wenig konsistente und systematische Begrifflichkeiten erbracht (Lassnigg/Stöger 1999, 29-34). 
qualitativen Mechanismen wie z.B. der Anwendung der Szenariotechnik oder der Analyse von Berufsprofilen und bis zum Aufbau von Netzwerken und ähnlichen Formen des Wissensmanagements unter den beteiligten Akteuren (vgl. Steiner/Steger 2001). Wesentlich wären Schritte zur Verbesserung der verfügbaren Daten- und Informationsgrundlagen in diesem Bereich. Neben diesen FH-externen Anforderungen wäre auch die Praxis der Bedarfs- und Akzeptanzerhebungen der Antragstellenden vertiefend zu überprüfen. Eine erste Evaluation hat für die Periode bis 1998/99 eher problematische Ergebnisse erbracht, was die Standards der Erhebungen betrifft. Ein Follow-up hätte den Rahmen der vorliegenden Untersuchung gesprengt. Es wären auch Fragen nach der Praktikabilität der Vorgangsweise zu stellen, etwa inwieweit die Arbeitsteilung in diesen Erhebungen (Beauftragung durch Antragstellende; studiengangsbezogene kleinräumige Erhebungen, „Ergebnisdruck“ etc.) noch sinnvoll erscheint.

\subsubsection{Umsetzung der Entwicklungs- und Finanzierungsplanungen}

Der Aufbau des Fachhochschulbereichs erfolgte auf Basis zweier Entwicklungspläne, die das Tempo des Fachhochschul-Ausbaus - vor allem durch die Zahl der vom Bund zu finanzierenden Studienplätze - sowie einen Kriterienkatalog enthalten, mit dessen Hilfe die Förderungswürdigkeit von Studiengängen anhand eines Punktesystems beurteilt wird. Die derart vorgegebenen Zeitreihen und Budgets sind eingehalten worden, die Vorgaben hinsichtlich des Ausbaus hat man sogar übertroffen. Die Steuerung über das Steuerungsmittel "Geld“ hat dabei gut funktioniert, die bildungspolitische Rolle des Dokuments „Entwicklungs- und Finanzierungsplanung“ ist hingegen nicht sehr stark "gepflegt" worden.

Es wurden dabei Kriterien festgelegt, die vorwiegend an den einzelnen Studiengang angelegt werden, die damit aber nicht automatisch Kriterien für die Entwicklung des gesamten Sektors ergeben. Die „Kleinteiligkeit“ der Maßnahmen wie z.B. studiengangsbezogene Bedarfs- und Akzeptanzstudien, Akkreditierungen/Evaluierungen, Finanzierungen und eine gewisse Nischenpolitik ist bei allem Erfolg des Bottom-Up-Modells ein Problem. Wenn man Bedarfs- und Akzeptanzanalysen nur pro Studiengang unternimmt, geraten größere Ausschnitte (Region, Land, Fächer- und Quervergleiche, bildungspolitischer Sektor) aus dem Blickwinkel.

Die Überprüfung der Einhaltung der bildungspolitischen Kriterien ist - von Beginn an bereits relativ schwach ausgeprägt - mit den Jahren immer schwächer geworden, wodurch sie in ihrer Steuerungsfunktion stark eingeschränkt waren. Die entscheidenden Schwächen waren damit die zu gering operationalisierte Zielvorgaben und die mangelnde Überwachung der Zieleinhaltung im Sinne eines Monitoring der Zielvorgaben. 
Zentrales Element der Entwicklungsplanung ist also ein System von quantitativen Zielen und Vorgaben über Mengen (d.s. die Studienplätze) und Preise (d.s. die Normkosten), in Verbindung mit einem Katalog bildungspolitischer Kriterien. Die Zielsteuerung auf der Ebene dieses einfachen Mengengerüstes war erfolgreich. Die Umsetzung der bildungspolitischen Kriterien war nur teilweise erfolgreich.

Der Informationsstand über die finanziellen Aspekte ist bei weitem unzureichend und die getrennte Bearbeitung von Finanz- und Mengendaten vermindert die Transparenz (die bisher jedoch auch in anderen Bereichen des Bildungswesens nicht ausreichend gegeben ist, um eine wirksame Planung und Bewertung vorzunehmen). Daher ist das MonitoringSystem vor allem im Bereich der Finanzdaten zu verbessern. Wie in anderen Ländern sollte ein einheitliches System des Controlling entwickelt werden, wobei im Zusammenhang mit der laufenden Universitätsreform die Etablierung eines vergleichbaren Rahmens möglich und wünschenswert wäre (was beispielsweise in der Schweiz gelungen ist). Die gegenwärtige Trennung von Mengen- und Finanzinformationen, die sich aus den unterschiedlichen Zuständigkeiten von FHR und BMBWK ergibt, erschwert die Auswertung der Informationen im Hinblick auf wichtige Fragestellungen zur Wirksamkeit und Effizienz des FH-Sektors (Kosten-Nutzenanalysen, Ertragsraten).

Die wichtigste Erwartung an den nächsten Entwicklungs- und Finanzierungsplan ist, dass er die finanziellen Rahmenbedingungen für die weitere Entwicklung des FH-Sektors klar absteckt. Dazu gehören in erster Linie die vom Bund vorgesehenen finanziellen Mittel und damit indirekt die geplante Ausbaugeschwindigkeit. Das Thema der weiteren Expansion ist unmittelbar damit verbunden, für welchen der möglichen Entwicklungspfade man sich entscheidet. Eine wichtige Frage wird auch sein, welche inhaltlichen Bereiche in Zukunft im FH-Sektor vertreten sein werden und inwieweit eine Erweiterung des Fächerspektrumspektrums und der Funktionen stattfindet. Dieser Aspekt sowie Überlegungen zum Steuerungsmodell des $\mathrm{FH}$-Sektors werden in den übergreifenden Schlussfolgerungen thematisiert (siehe Abschnitte 8.3.3 und 8.3.5).

\subsubsection{Konzentration, Regionalisierung und Standorte im FH-Sektor}

Die Regionalisierung wurde nach den folgenden Dimensionen untersucht: Abbau regionaler Disparitäten beim Hochschulzugang, Entwicklung der Potentiale nach Standorten, Erwartungen an „kritische Größen“ aus dem Sektor, erforderliche Potentiale für F\&ELeistungen, regionale Wirkungen von Fachhochschul-Studiengängen im Vergleich zur nationalen und internationalen Dimension und bisherige Fachhochschulstrategien der Länder, insbesondere im Hinblick auf Standortprüfung und Aspekte der Entwicklungsplanung. 
Es gibt empirische Anhaltspunkte dafür, dass die regionale Zugänglichkeit von Bildungsangeboten nach der Sekundarstufe durch die Errichtung des FH-Sektors erhöht wurde, auch wenn noch immer beträchtliche Unterschiede bestehen. Im Querschnittsvergleich ist die regionale Verteilung der FH-Studierenden der Bevölkerung besser angepasst als die Verteilung der Universitätsstudierenden. In einigen Bundesländern (vor allem in der Steiermark und in Niederösterreich, aber auch in Tirol und Salzburg) gibt es Anzeichen für größere Abweichungen von einer ausgewogenen Verteilung als in den anderen.

Das Leistungsspektrum des Sektors (AbsolventInnen, F\&E-Projekte, Vorträge und Veranstaltungen, Diplomarbeiten auf Auftrag, Beratungsleistungen) wird schwerpunktmäßig für den regionalen Kontext abgegeben, gleichzeitig bestehen aber auch Leistungen im nationalen und internationalen Bereich. Hinsichtlich der Entwicklungsplanung hat die regionale Ebene bisher bestenfalls in Teilbereichen und in Ansätzen die insgesamt schwache übergreifende Koordination und Steuerung unterstützt. Überprüfbare Strategien der Einbindung in regionale Innovationssysteme sind bisher nicht entwickelt worden.

Der Aspekt der Regionalisierung und Standortwahl im FH-Sektor wurde auf dem Hintergrund der Dynamik der Globalisierung - Regionalisierung und im Licht der neueren Konzepte der (regionalen) Innovationspolitik analysiert. Je nach dem Spektrum der Erwartungen in regionale Wirkungen von Fachhochschul-Studiengängen ergeben sich unterschiedliche Schlussfolgerungen für die erforderlichen Standortpotentiale. Die groben überschlagsmäßigen Abschätzungen der Standortpotentiale haben zu dem Schluss geführt, dass etwa die Hälfte der Erhalter nicht über personelle Potentiale verfügt, die bestimmte Minimalkriterien für eine nachhaltige Entwicklung von F\&E-Tätigkeiten erfüllen würden, lediglich bei einem Viertel der Erhalter sind derartige Potentiale vorhanden. Bisher hat sich jedoch noch nicht klar herausgestellt, welche Rolle der FH-Sektor im Hinblick auf die Entwicklung der (regionalen) Innovationspolitik spielen soll. Weitgehende Erwartungen wurden im Rahmen der Forschungsstrategie formuliert, jedoch sind diese bisher nicht konsensuell verankert und auch noch sehr weit von der Umsetzung entfernt.

Die Eckpunkte für eine weitere regionale Verteilung der Standorte werden wesentlich von der quantitativen Entwicklung beeinflusst (je stärker das Wachstum, desto größer auch der Spielraum für stärkere regionale Streuung von Standorten). Für die weitere quantitative Entwicklung des FH-Sektors ergibt sich ein breites Spektrum an möglichen quantitativen Entwicklungspfaden, das nur mit großer Unsicherheit eingrenzbar ist und wesentlich von politischen Entscheidungen beeinflusst werden kann. Als wichtige Faktoren wurden die Demografie, die Bildungsbeteiligung in höheren Schulen auf der Sekundarstufe, die angestrebte Ausschöpfung der Potentiale im Bereich der Erstausbildung für die Bildungsbeteiligung im tertiären Sektor, die Wahl zwischen Fachhochschul- und Universitätsausbildung sowie die angestrebte Ausschöpfung der Potentiale für nichttraditionelle Zugänge in den FH-Sektor im Wege des lebenslangen Lernens identifiziert. 
Wenn der FH-Sektor eine sichtbare Impulsfunktion für die Entwicklung regionaler Innovationssysteme ausüben soll, dann ist die Konsolidierung weiter voranzutreiben. Ein wesentliches Element dabei sollte die Konkretisierung der erwarteten Leistungen der FHEinrichtungen im gesamten Spektrum sein (siehe hierzu die übergreifenden Schlussfolgerungen Abschnitt 8.3.3). Größere Einrichtungen, die die Ansprüche an Regionalisierung möglicherweise mit multi-campus-Strukturen erfüllen, sind erforderlich, um das gesamte Leistungsspektrum an Funktionen abzudecken. Die bisher zentrale Größe der Studierendenzahl zur Spezifikation der "kritischen Masse" einer fachhochschulischen Einrichtung bezieht sich vor allem auf die Lehre. Die Evaluierung hat gezeigt, dass eine entsprechende Gewichtung der Forschungs- und der Dienstleistungsfunktion für einen großen Teil der Standorte ein beträchtliches Wachstum der personellen und sachlichen Ressourcen erfordern würde. Für die weitere Entwicklung stellt sich also erstens die Frage der Konsolidierung bestehender Standorte (welche vor allem bei einer geringeren Wachstumsvariante in den Vordergrund tritt) und zweitens die Frage der Eröffnung weiterer Standorte (welche vor allem bei stärkerem Wachstum relevant wird).

In der Frage der Standortwahl sollten jedenfalls systematische Verfahren angewendet werden. Im Prinzip könnte eine übergreifende Standortprüfung für Österreich durchgeführt werden, die das gesamte Spektrum an möglichen Standorten systematisch auslotet. Dadurch könnte eine gewisse Erwartungssicherheit im Hinblick auf lokale Interessen geschaffen werden. In Österreich wurden, vor dem Hintergrund deutscher Erfahrungen, im Laufe der Jahre bereits Verfahren der systematischen Standortprüfung und -planung entwickelt, die im Prinzip auf die in Frage kommenden Orte und Regionen in Österreich (die ja alle schon vorhanden sind) angewendet werden könnten. Eine derartige generelle Standortprüfung könnte den potentiell möglichen Raum für die weitere regionale Entwicklung des FH-Sektors ausloten und es könnten Größenordnungen und Potentiale in verschiedenen Varianten herausgearbeitet werden. Damit könnten gleichzeitig auch die Entscheidungsgründe und die erwarteten Aufgabenprofile der potentiellen FH-Standorte transparent gemacht werden und die immer wieder auftauchenden Auseinandersetzungen um eine sinnvolle Standortwahl besser fundiert werden.

Zudem sollten systematischere Überlegungen über die Konkretisierung der Funktionen im Kontext von regionalen Innovationssystemen und der Entwicklung von lernenden Regionen angestellt werden. Eine erste Grundlage sind die Szenarien für den FTE-Rat. Diese entwickeln Vorschläge, wie die FHs in Strategien der Erhöhung der österreichischen Forschungsquote eingebaut werden können, sind jedoch noch bei weitem zu wenig konkretisiert. Im Hinblick auf die Funktionen von Fachhochschul-Studiengängen wurden bisher v.a. die Standortvoraussetzungen für die Lehre herausgearbeitet, für die Forschung gibt es ansatzweise Vorstellungen, die Wahrnehmung der Dienstleistungsfunktion und der Weiterbildung ist noch weitestgehend unbestimmt. Bei der Dienstleistungsfunktion verbindet sich die Standortfrage am deutlichsten mit der Frage nach Impulsen für die regionale Entwicklung. Diese Aufgabenstellung könnte im Akkreditierungsprozess im Einklang mit den 
Erfahrungen aus internationalen Analysen bei der Errichtung neuer Standorte stärker betont werden (etwa indem die Antragsteller bereits ex ante entsprechende Vorstellungen über die geplanten bzw. erwarteten Impulse sowie über die Beziehungen mit potentiellen Kooperationspartnern entwickeln).

Die Bedarfsanalysen sollten stärker in den Aufbau von Antizipationsmodellen eingebaut werden, die auf die Vernetzung und Kommunikation der regionalen Akteure zurückgreifen können. Das heißt, das Entwicklungsteam müsste nicht nur den Bedarf nachweisen, sondern stärker auf die angestrebten Ergebnisse der gesamten Tätigkeit und ihre Vernetzung in einem regionalen Raum (der sehr unterschiedlich ausgedehnt sein kann) abstellen.

\subsubsection{Die Internationalisierung der Hochschulen und das Entstehen eines „Europäischen Hochschulraums“}

Die gesamtgesellschaftlichen Globalisierungsprozesse haben ihren Niederschlag in unterschiedlichen Formen der Internationalisierung im Hochschulbereich gefunden. Die nationalstaatlich definierten Systemarchitekturen und Praktiken bleiben erhalten und bilden weiterhin einen entscheidenden Bezugsrahmen für die Hochschulen, ihr wissenschaftliches Personal und ihre Studierenden. Aber neben dieser nationalen gewinnt eine supranationale Ebene, auf der Hochschulen miteinander kooperieren und zueinander in Konkurrenz treten, an Bedeutung. Der Begriff „Internationalisierung“ verweist auf eine neue Qualität der grenzüberschreitenden Verflechtung und Vernetzung von Hochschulsystemen. Der wechselseitige Einfluss beschränkt sich nicht mehr darauf, sich im Zuge internationaler Vergleiche Anregungen bzw. Legitimationen für die eigene Politik zu holen. Vielmehr kommt es verstärkt zu Interdependenzen, die auch für die einzelne Hochschule unmittelbar handlungsrelevant sind.

Sowohl die nationale Hochschulpolitik als auch die strategischen Positionierungen der einzelnen Institutionen stehen vor der Notwendigkeit, diese beiden Ebenen aufeinander abzustimmen. Da im FH-Sektor auch die regionale Politik auf der Ebene der Länder und teilweise auch der Gemeinden eine wichtige Rolle spielt, könnte die Komplexität dieser Abstimmung sowie das Ausmaß der potentiellen Spannungsfelder hier noch größer sein als bei den Universitäten.

In diesem Rahmen wurden primär jene Aspekte der Internationalisierung thematisiert, die für den österreichischen FH-Sektor gegenwärtig von Bedeutung sind. Internationalisierung bedeutet überwiegend akademische Mobilität, derzeit vor allem Austausch von Studierenden, zunehmend aber auch Mobilität des akademischen Personals. Das geographische Zentrum dieser Mobilität sind die Länder der Europäischen Union, deren Mobilitätsprogramme die wichtigsten Beziehungsnetzwerke und Förderschienen eröffnen. 
So gesehen ist die gewachsene akademische Mobilität ein Ausdruck der Entstehung eines Europäischen Hochschul- und Forschungsraums.

Die Auslandsaufenthalte von FH-AbsolventInnen liegen in der gleichen Größenordnung wie die der Universitäts-AbsolventInnen und über jener der AbsolventInnen von Kunstuniversitäten. Angesichts des geringen Alters des FH-Sektors kann das als beachtlicher Erfolg gewertet werden. Der Studierendenaustausch an fachhochschulischen Einrichtungen zeichnet sich durch einige Besonderheiten aus, auf die bei der weiteren Entwicklung geachtet werden muss:

- Die Auslandspraktika nehmen - im Vergleich mit Fachstudium und wissenschaftlichen Abschlussarbeiten - einen sehr hohen Stellenwert ein, was darauf verweist, dass das akademische Profil der Austauschbeziehungen ungenügend entwickelt ist. Bemerkenswert ist, dass es eine Diskrepanz zwischen den Angaben der AbsolventInnen (betonen die Bedeutung des Praktikums) und jenen der StudiengangsleiterInnen (betonen das Fachstudium) gibt.

- Es gibt ein Ungleichgewicht zwischen „incoming“ und „outgoing“. Bei den Studierenden gehen ca. vier Mal soviel InländerInnen hinaus als ausländische Studierende an österreichische FH-Einrichtungen kommen. Der österreichische Fachhochschulsektor wird von ausländischen Studierenden also nur ungenügend wahrgenommen und/oder nicht als ausreichend attraktiv eingeschätzt. Bei den Lehrenden geht das Ungleichgewicht in die entgegengesetzte Richtung. Ausschlaggebend dürfte sein, dass die österreichischen Fachhochschuleinrichtungen den Ausfall der eigenen Lehrkräfte auf Grund des geringen Stammpersonals nur schlecht verkraften können.

Da das geographische Zentrum der Internationalisierung für alle österreichischen Hochschulen, im besonderen aber für die fachhochschulischen Institutionen Europa ist, besitzt der Bologna-Prozess eine so große Bedeutung. Der FH-Sektor hat sich lange darum bemüht, in diesen Prozess als ein den Universitäten gleichwertiger Partner eingebunden zu werden und sie haben dieses Ziel erreicht. Es gibt derzeit aber keine Anzeichen dafür, dass die Mehrheit der Erhalter offensiv von dieser Möglichkeit Gebrauch machen möchte.

Ein Grund für diese Vorsicht scheint zu sein, dass mit der neuen Studienarchitektur zugleich die Grenzen zwischen dem Universitäts- und dem Fachhochschulsektor durchlässiger werden. In der „postnationalen Konstellation“, die sich hochschulpolitisch als „Europäischer Hochschulraum" geltend macht, wird die Fähigkeit und Bereitschaft des Staates zur sektoralen Differenzierung des Hochschulsystems schwächer. Mit den kurzen Bakkalaureats-Abschlüssen übernehmen die Universitäten z.T. Aufgaben, die bislang die Basis für den Erfolg des FH-Sektors gebildet haben, auf der anderen Seite wird der FHSektor verstärkt als Ort von Forschung und Entwicklung wahrgenommen. Man kann derzeit 
nicht abschätzen, ob für den FH-Sektor bzw. die einzelnen Institutionen eher die Chancen oder die Risken dieses Prozesses spürbar werden.

Damit verweist eine Bestandsaufnahme im Bereich der Internationalisierung auf ähnliche Problemlagen, wie sie sich auch aus der innerösterreichischen Diskussion ergeben: aus beiden Blickwinkeln ist eine Konsolidierung bestehender Standorte und Studiengänge sowie eine thematische Verbreiterung des Studienangebots $z u$ empfehlen. Ob und in welchem Ausmaß sich Erhalter bzw. Studiengänge international positionieren wollen, ist eine strategische Entscheidung, für die mehrere Optionen offen stehen. Es gibt derzeit keine Anhaltspunkte dafür, dass für Fachhochschuleinrichtungen, die sich weitgehend auf nationale und regionale Zielsetzungen beschränken und der Internationalisierung einen sehr geringen Stellenwert zuweisen, kein Platz wäre. Aber es ist zu vermuten, dass eine solche Entscheidung die weiteren Entwicklungsmöglichkeiten stark einschränkt; mittel- und langfristig könnte das zu einem Reputationsverlust und zu einer gewissen Marginalisierung führen.

Umgekehrt sind bei einer offensiven Strategie im Bereich der Internationalisierung Synergieeffekte für die Entwicklung im nationalen Rahmen zu erwarten.

- Die Konsolidierung von Standorten schafft bessere Möglichkeiten, um die für Internationalisierung nötige Infrastruktur aufzubauen.

- Die thematische Erweiterung des Studienangebots ist ein wichtiger Schritt zu verbesserter internationaler Sichtbarkeit des FH-Sektors.

- Ein Ausbau der Forschungskapazität und die Einbindung in internationale Netzwerke verstärken sich wechselseitig.

Eine defensive Haltung bei der Implementierung der neuen Studienarchitektur ist der weiteren Entwicklung des FH-Sektors nicht förderlich. Zweifellos ist es möglich, die Einführung der neuen Abschlüsse zu verzögern bzw. eine gewisse Zeit lang eine Doppelstruktur aufrechtzuerhalten (sowohl Bachelor/Master-Abschlüsse als auch Diplomabschlüsse). Langfristig wird sich die neue Architektur europaweit durchsetzen. Der Fachhochschulsektor kann sich das Image der Reformfreudigkeit erhalten, indem er diesen Prozess offensiv mitgestaltet.

Auf der Ebene der nationalen Hochschulpolitik sollte dieser Prozess durch folgende Maßnahmen unterstützt werden:

- Fachhochschul- und Universitätssektor sollen hinsichtlich der finanziellen Förderung akademischer Mobilität gleichgestellt werden. Die gegenwärtigen Förderpraktiken sind nur aus ihrem historischen Kontext heraus zu verstehen, können aber nicht mit dem 
spezifischen Profil der beiden Sektoren begründet werden. Eine Ungleichbehandlung würde die Botschaft an den $\mathrm{FH}$-Sektor senden, dass Internationalisierung keine hohe Priorität hat.

- Der Bund sollte Klarheit über die Finanzierung von Master-Studiengängen im Fachhochschulsektor schaffen, um der Verunsicherung im Sektor entgegenzuwirken. Eine nahe liegende Form, Transparenz herzustellen, wäre es, die Zahl der auf Normkostenbasis finanzierten Plätze in Master-Studiengängen im Entwicklungs- und Finanzierungsplan festzulegen.

\subsubsection{Qualitätssicherung}

Das Qualitätssicherungsmodell des Fachhochschulsektors ist insgesamt als für den tertiären Bereich vorbildlich einzustufen. Es ist ein guter Methodenmix gegeben, insbesondere sind Akkreditierung und Evaluierung gut miteinander verflochten und haben sich nicht zu arbeitsaufwändigen „Parallelaktionen“ entwickelt, was - wie man derzeit im deutschsprachigen Raum teilweise beobachten kann - aber eine reale Gefahr darstellt. Weil die Evaluierungsergebnisse die Basis für die Re-Akkreditierung sind, sind beide Verfahren aufeinander bezogen und komplementär. Vor allem wird dem „bottom-up“-Approach des Sektors folgend die institutionelle Ebene der Qualitätssicherung sehr betont. Der vorliegende Bericht kann nicht auf ausführliche Vor-Ort-Untersuchungen der einzelnen Studiengänge und Fachhochschulen verweisen. Um eine fundierte Aussage über die Qualität des institutionellen Qualitätsmanagements zu machen, wäre das vonnöten. Die Hinweise aus der projektbezogenen Online-Befragung legen aber nahe, dass das Qualitätssicherungsbewusstsein in den Studiengängen recht ausgeprägt ist. Auf der staatlichen Ebene kann eigentlich nur ein "Meta-Qualitätsmanagement" erfolgen, das zum Ziel hat, die entstandenen Qualitätssicherungssysteme vor Ort von Zeit zu Zeit auf ihre Qualität hin zu überprüfen und den Erfahrungsaustausch unter den Akteuren auch in Bezug auf Fragen der Qualitätssicherung zu stimulieren.

Im Bereich der Akkreditierung hat der Fachhochschulrat in den Aufbaujahren Pionierarbeit geleistet und ein umfassendes Verfahren entwickelt und umgesetzt, das jedoch in den letzten Jahren - mit dem Größenwachstum des Sektors und der steigenden Zahl von Studiengängen und Anträgen - an seine Grenzen gestoßen ist. In diesem Zusammenhang gibt es verschiedene Reformvorschläge, die in ihrer Praktikabilität weiter ausgelotet werden sollten, die wiederum teilweise von den weiteren Entwicklungspfaden abhängt (siehe übergreifende Schlussfolgerungen Abschnitt 8.3.4). Wenn beispielsweise das Wachstum des Sektors in Form einer weiteren Vervielfältigung von Studiengängen (extensives Wachstum) fortgesetzt wird, werden andere Anforderungen und Schwerpunkte an die Akkreditierung bestehen, als wenn sich bei intensivem Wachstum der Schwerpunkt der Aktivitäten von der Akkreditierung neuer Studiengänge zur Evaluierung und Re-Akkreditierung verlagert. 
Im Bereich der Evaluierung wurde bereits ein neues Verfahren entwickelt, das die institutionelle Ebene stärker betont. Dieses Verfahren könnte zur Stärkung der Fachhochschule als Institution beitragen. Die Weiterentwicklung der Qualitätssicherung wird im Zusammenhang mit den Überlegungen zum Steuerungssystem im Rahmen der übergreifenden Schlussfolgerungen thematisiert (Abschnitt 8.3.5).

Der Fachhochschulrat als wichtiger Promotor von Qualität im Fachhochschulsektor hat immer sowohl summative (abschließend-bewertende) Funktionen von Qualitätssicherung etwa in Form der Akkreditierung - betrieben als auch formative (im Sinne von externbegleitenden) Funktionen der Qualitätssicherung - etwa in Form des gewählten Evaluierungsmodells. Dieses Evaluationsmodell ist zudem recht konsensuell mit den Erhaltern der Studiengänge und Fachhochschulbetreibern entwickelt worden. In letzter Zeit erfolgte eine stärkere Fokussierung in dem Sinne, dass der Fachhochschulrat seine Zuständigkeit für Evaluation stark betonte und auch eine Veröffentlichung der Evaluationsergebnisse ab dem nächsten Jahr beschlossen wurde. Damit wird die Accountability-Funktion der Qualitätssicherung unterstrichen. Zunehmend lässt sich auch im deutschsprachigen Raum der hochschulpolitische Trend der Zunahme der Bedeutung von Rankings im Sinne eines Instruments der „Kundeninformation“ beobachten.

Auf die österreichische Situation übertragen, könnte sich die Qualitätssicherung folgendermaßen weiterentwickeln:

Qualitätssicherung und Transparenz: Der summativ-bewertende Aspekt der Evaluierung sollte transparenter gestaltet werden - etwa durch adäquate Formen der Veröffentlichung. Zusätzlich könnten in methodisch abgesicherter Form vergleichende Bewertungen vorgenommen werden, die dann eine wichtige Form der „Kundeninformation“ darstellen.

Qualitätssicherung und Entwicklung: Der formativ-institutionell unterstützende Aspekt wird stärker mit Aktivitäten der Personal- und Organisationsentwicklung verbunden. Ein wesentliches Qualitätssicherungsmoment ist die (Organisations-)Entwicklung hin zu akademisch- autonomen, selbstbestimmten Einrichtungen.

Der Bologna-Prozess fokussiert die Ebene der Studienprogramme bzw. der Studienabschlüsse - die Entwicklung in Deutschland und den Niederlanden spiegelt diese Fokussierung wider. In diesem Sinn ist die neue Entwicklung im Fachhochschulsektor in Richtung von institutioneller Evaluierung eine Abweichung vom europäischen Trend. Andererseits gibt es dafür gute Gründe: die institutionelle Evaluierung ist ein wichtiger Beitrag zur institutionellen Weiterentwicklung und sie hilft auch, den Arbeitsaufwand der Evaluierungen zu reduzieren bzw. zu bündeln. Vermutlich wird diesem Zugang daher auch in den Niederlanden und in Deutschland größeres Gewicht als derzeit beigemessen werden, da etwa schon in Deutschland bei den Akkreditierungen auf Programmebene immer öfter 
Überlastungssituationen entstehen. Finnland etwa verfolgt ja bereits jetzt das Prinzip der institutionellen Evaluierung.

Im Feedback der internationalen Experten wurde ebenfalls die große Bedeutung eines ausgewogenen Verhältnisses zwischen Entwicklung und Kontrolle (Accountability, öffentliche Rechenschaftspflicht und Transparenz) in der Qualitätssicherung besonders herausgearbeitet. Überwiegt die Kontrollfunktion zu sehr, besteht die Gefahr, dass die Wirksamkeit für die Weiterentwicklung schwer beeinträchtigt wird. Auch das Verhältnis von Aufwand und Ertrag sollte in einem vernünttigen Rahmen stehen („Wie viel Qualitätssicherung verträgt ein System?").

\subsubsection{Besonderheiten im internationalen Vergleich}

Der österreichische FH-Sektor liegt innerhalb des europäischen Bezugsrahmens. Allerdings befindet er sich, sowohl was die Größe als auch was die Relation zum universitären Sektor betrifft, am unteren Rande des Spektrums. Das liegt nicht nur an der späten Gründung, denn in zwei der Vergleichsländer (Finnland, Schweiz) wurde der FH-Sektor ebenfalls erst in den 1990er Jahren aufgebaut. Einzigartig in diesem Vergleich ist, dass der österreichische FHSektor nicht auf Vorläuferinstitutionen aufbaut, sondern von Grund auf neu errichtet wurde. Ein wichtiger Aspekt ist auch die Breite oder Enge des von Fachhochschul-Studiengängen angebotenen Fächerspektrums; in Österreich wird es vergleichsweise eng definiert. Eine Sonderstellung nehmen die österreichischen Fachhochschul-Studiengänge auch hinsichtlich der geringen Größe der einzelnen Standorte ein. Allerdings weist Österreich (nach Finnland) von den Vergleichsländern die geringste Bevölkerungsdichte auf, was neben institutionellen Aspekten (Geographie, Alter des Sektors etc.) auch längerfristig zu einer breit gestreuten, aber kleinteiligeren Fachhochschuldichte führen könnte. Auch in den anderen Ländern hat sich kein „Standard-Modell“ für Fachhochschulen herauskristallisiert, sondern es wurden unterschiedliche Möglichkeiten genutzt. Elemente dieses Spektrums können für die weitere Entwicklung des sich noch immer im Aufbau befindlichen österreichischen FH-Sektors interessant sein. Entscheidend sind dabei die politischen Präferenzen für die zukünftige Position des FH-Sektors im österreichischen Hochschulsystem. 


\section{3 Übergreifende Schlussfolgerungen}

\subsubsection{Stärken und Schwächen des FH-Sektors}

Insgesamt ist der Aufbau des FH-Sektors überaus erfolgreich verlaufen. Was sind die wesentlichen, hervorzuhebenden Erfolge?

- Der neue Bildungssektor konnte sich in der Bildungslandschaft erfolgreich positionieren, die Bildungsnachfrage überschreitet deutlich die Zielwerte, die AbsolventInnen haben sich im Beschäftigungssystem durchgesetzt und werden in breiten Bereichen als den UniversitätsabsolventInnen ebenbürtig bewertet.

- Das wesentlich von den umgebenden Strukturen abweichende Reformmodell des neuen Bildungssektors (schlankes Gesetz, Dezentralisierung, Vielfalt von privatrechtlichen Anbietern, Akkreditierung durch ExpertInnengremium, öffentliche Beteiligung mittels Zielsteuerung etc.) konnte mit Hilfe des großen Engagements der Beteiligten wirksam realisiert werden.

- Mit dem Normkostenmodell wurde eine neue Form der öffentlichen Finanzierung etabliert, die stärker als die traditionellen Methoden eine rationale Bearbeitung von Interessenskonflikten ermöglicht.

- Neue Akteure wurden in neuen Funktionen für das System des Bildungsangebotes auf Hochschulebene mobilisiert, insbesondere die Länder und viele Gemeinden, aber auch die Sozialpartner, andere Bildungsorganisationen, Firmen etc. Damit wurden vielfältige institutionelle Lernprozesse ausgelöst, die teilweise ein großes Maß an Engagement und Ressourcen von den Beteiligten erforderten und mobilisierten. Durch die Mischfinanzierung, die neben dem Bund auch andere Akteure in die Finanzierung einbindet, reduziert sich die Abhängigkeit der Erhalter von einer einzigen Finanzquelle.

- Eine neue Studienstruktur konnte etabliert werden, die einen im Vergleich zu den Universitäten rascheren Studienfortgang ermöglicht, eine neue Form der Verbindung von Ausbildung auf Hochschulniveau und Praxis verwirklicht und den Unternehmenssektor stärker einbezieht.

- In vielen dynamischen Berufs- und Beschäftigungsfeldern wurden in Verbindung mit Bedarfserhebungen neue Qualifikationsprofile entwickelt, die neben den fachlichen Kompetenzen auch auf die Vermittlung der neuen Kompetenzen Wert legen. 
- Ein System der Qualitätssicherung auf mehreren Ebenen sorgt dafür, dass die Verwirklichung der Zielsetzungen überprüft wird und dass im Falle von Problemen rasch Korrekturmaßnahmen getroffen werden können.

- Die regionale Chancengleichheit im Zugang zu Hochschulstudien konnte verbessert werden und die neuen Einrichtungen konzentrieren ihre Leistungserstellung auf das regionale Umfeld.

- In gewissem Maße wurde der Zugang von nicht-traditionellen Studierenden zu Studien auf Hochschulniveau erweitert.

- Die Fachhochschul-Studierenden nehmen erfolgreich an den europäischen Mobilitätsprogrammen teil.

Die verschiedenen Zielsetzungen und Funktionen wurden in diesem Aufbauprozess sukzessive und entsprechend dem jeweiligen Entwicklungsstand vorangetrieben. Mit der Etablierung der Prozeduren zur Akkreditierung, Re-Akkreditierung und Evaluierung sowie mit der verstärkten Betonung der F\&E-Funktion und der Verleihung der ersten „Fachhochschule"-Bezeichnungen wurden die Aufgabenstellungen in beträchtlichem Maße abgedeckt. Mit dem Auslaufen des zweiten Entwicklungs- und Finanzierungsplanes kann man den Aufbauprozess im Hinblick auf die Lehre in wesentlichen Schritten als abgeschlossen betrachten, wenn auch das Bild einer Fachhochschule in Österreich noch nicht sehr deutliche Konturen hat und durch die institutionelle Weiterentwicklung (Etablierung von Fachhochschulen, institutionelle Evaluierung) noch wichtige Schritte folgen werden. Im Hinblick auf die übrigen Funktionen (F\&E, Dienstleistungen, Weiterbildung) besteht noch beträchtlicher Klärungs- und Entwicklungsbedarf.

In einigen Bereichen wurden weniger deutliche Fortschritte erzielt, sei es, dass weniger Priorität darauf gelegt wurde oder, dass die gesetzten Maßnahmen bisher weniger erfolgreich waren:

- Das Ziel der Strukturbereinigung im postsekundären Sektor wurde nur sehr langsam und in manchen Bereichen bis heute nicht erfolgreich umgesetzt.

- Die Eröffnung von nicht-traditionellen Wegen zum Hochschulzugang blieb in verschiedener Hinsicht hinter den Erwartungen zurück.

- Die Chancengleichheit zwischen Frauen und Männern ist bei weitem nicht verwirklicht.

- Die Aufgabenbereiche der anwendungsorientierten Forschung und Entwicklung wie auch der Dienstleistungen wurden in den letzten Jahren verstärkt wahrgenommen, sind jedoch keinesfalls als gleichwertige Funktionen mit der Lehre etabliert. 
- Die Betätigung im Weiterbildungsmarkt erfolgt nur in geringem Ausmaß.

- Die Transparenz der Leistungserstellung könnte in einigen Bereichen wesentlich verbessert werden.

- Für das Zusammenwirken der Akteure der verschiedenen Politikbereiche und die Einbindung der neuen Akteure wurde trotz der Entwicklung von Lösungsansätzen noch keine ausreichende Plattform entwickelt.

Auch die Erwartungen, die in die Rollen der jeweiligen (für den Tertiärsektor zum Teil neuen) Akteure gesetzt wurden, sind großteils erfüllt worden, jedoch zeigen sich auch hier noch einige Schwächen in der Umsetzung:

- Die Mitglieder des Fachhochschulrates mit verschiedenem beruflichen Hintergrund (Universitäten, Forschung, Bildungswesen, Unternehmen, Sozialpartner etc.) hatten in inrem Status als ExpertInnen insbesondere in den ersten Funktionsperioden die Aufgabe, die Bestimmungen des schlanken Gesetzes im Bereich der Akkreditierung umzusetzen und auch geeignete Verwaltungsstrukturen aufzubauen. Damit sind Vernetzungen und Lernprozesse verbunden, die in vielfältiger Weise auch in andere Bereiche des Bildungswesens und des gesellschaftlichen Umfeldes hineinwirken. Es ist aber auch ein umfangreicher Apparat von Praktiken, Regeln und Bestimmungen entstanden, der als Ergänzung des "schlanken Gesetzes" fungiert.

- Die Länder und eine Reihe von Gemeinden, unter Einbeziehung eines weiteren oder engeren Kreises von anderen Akteuren, haben sich direkt als Erhalter konstituiert oder in anderen Formen an der Förderung des FH-Sektors beteiligt. Damit ist oft eine neue bildungspolitische Dimension für diese Akteure eröffnet worden, die sie auch vor neue Herausforderungen der Planung, Konzeption und Steuerung gestellt hat. Die bisherigen Praktiken in diesem Feld sind trotz gewisser Ansätze noch nicht sehr weit in Richtung einer fundierten strategischen Planung und Steuerung entwickelt.

- Der Bund ist durch die neue Form der Gesetzgebung in eine neue Rolle gekommen, die von der traditionellen Bildungsverwaltung deutlich abweicht. Anstelle detaillierter operativer Vorgaben und der kameralistischen Budgetierung stellte sich die Aufgabe, nach bestimmten Entscheidungskriterien, Zielen und Bedingungen in bestimmtem Ausmaß quasi Studienplätze "zu kaufen". Damit wurde die Gelegenheit zu einer viel direkter wirksamen Zielsteuerung eröffnet, die jedoch in der Bildungspolitik und verwaltung bislang nicht üblich ist und bei der Umsetzung Schwächen erkennen lässt.

- Schließlich wurden im neuen Sektor Beschäftigungsmöglichkeiten für Lehrende und sonstiges Personal eröffnet, die von den verschiedensten Bereichen (Universitäten, Schulen, Bildungseinrichtungen, Unternehmen etc.) rekrutiert werden konnten bzw. 
mussten und die teilweise über die Mitarbeit in den Entwicklungsteams auch zunächst beträchtliche Gestaltungsmöglichkeiten für die Konzeption und den Aufbau der Studien hatten. Auch auf dieser Ebene bestehen vielfältige Verflechtungen und Vernetzungen zwischen den $\mathrm{FH}$-Einrichtungen und vielen anderen Institutionen und Organisationen, die eine wesentliche Bereicherung des Hochschulsystems darstellen.

- Insbesondere über die Praxisphasen und über die Vielzahl an nebenberuflich Lehrenden, aber auch über die Beteiligung an anderen Aktivitäten (Sitze im FHR, in Erhaltervereinen, als Auftraggeber für Forschungs- oder Dienstleistungen etc.) sind auch Unternehmen als Akteure in den FH-Sektor eingebunden. Insbesondere für klein- und mittelbetriebliche Unternehmen (KMUs) sollten durch den FH-Sektor bessere Zugangsmöglichkeiten zur Hochschulebene eröffnet werden. In der Anfangsphase gab es teilweise auch die Erwartung, dass sich Unternehmen als Financiers für Studiengänge betätigen würden, was jedoch nicht eingetreten ist.

Der FH-Sektor ist bis vor kurzem im Einklang mit den konzipierten Zielgrößen gewachsen, zuletzt wurde das Wachstum kurzfristig durch besondere Initiativen (Aktion 600+) zusätzlich stimuliert. Mit diesem Wachstum hat der Sektor eine Größenordnung erreicht, die eine Hinterfragung der vorhandenen Strukturen sowie der bisher etablierten und eingespielten Prozeduren rechtfertigt.

\subsubsection{Veränderungen im Umfeld}

Neben den endogenen Veränderungen aufgrund der Entwicklung und des Wachstums des FH-Sektors wurden auch wesentliche Veränderungen im nationalen, europäischen und internationalen Umfeld diagnostiziert, die bei der weiteren Entwicklung und Konzeption der Politik eine Rolle spielen werden und berücksichtigt werden müssen. Diese Veränderungen sind (vgl. Abbildung 66):

- Die Europäisierung der Bildungspolitik (Bologna-Prozess, Arbeitsprogramm und neuerdings Brügge-Prozess) in Form verstärkter Koordination mittels Zielsteuerung, Qualitätssicherung und Erfahrungsaustausch, mit einem wesentlichen Schwerpunkt auf der Entwicklung von Ergebniszielen unter Berücksichtigung erforderlicher neuer Kompetenzen.

- Die Umstellung der Bildungs- und Ausbildungssysteme auf lebenslanges und lebensbegleitendes Lernen, was insbesondere die Gesamtkoordination der Systeme und die Förderung der Einbeziehung von Erwachsenen in reibungslosere Bildungs- und Ausbildungskarrieren impliziert.

- Die Verstärkung und Entwicklung von Innovationspolitik, die ein systematisches Zusammenspiel und eine Integration der Bildungspolitik in andere Politikbereiche wie 
Wissenschaftspolitik, Technologiepolitik, Struktur- und Industriepolitik, Regionalpolitik etc. erfordert.

- Die steigende Bedeutung des Zusammenspiels der lokalen, regionalen, nationalen und übernationalen Ebenen in der Dynamik von Globalisierung und Regionalisierung und die damit verbundene Notwendigkeit der Vernetzung der verschiedenen Akteure zur Entwicklung des Innovationssystems.

\section{Abbildung 66: Übersicht über Umweltveränderungen}

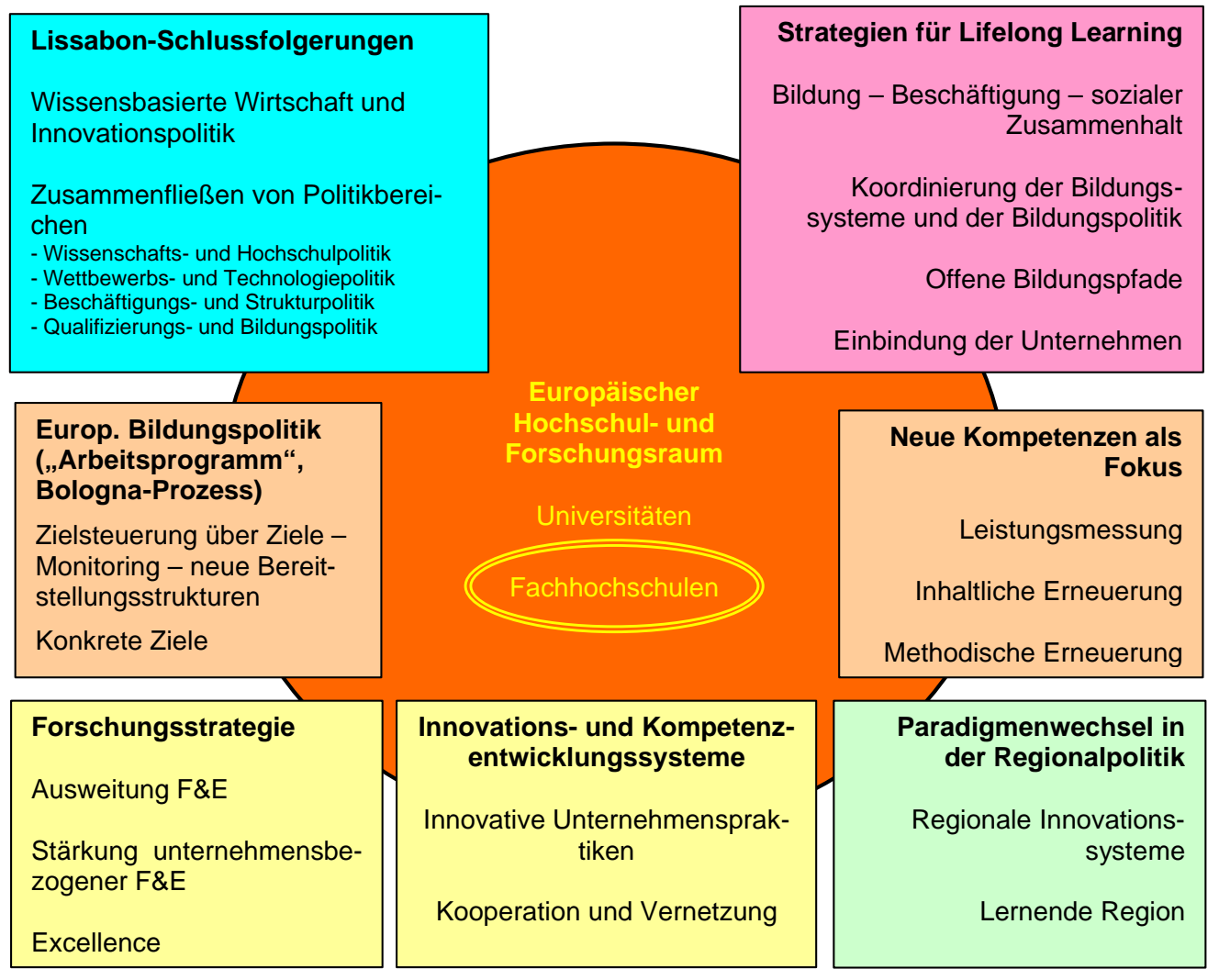

Quelle: IHS-Darstellung

Aus diesen Veränderungen im Umfeld wurden drei wesentliche Entwicklungsfelder für den FH-Sektor abgeleitet, die als Brennpunkte der weiteren Politikentwicklung dienen können:

- Die Verstärkung und Systematisierung der Interaktion zwischen Ausbildung und Innovation sowie die Einbindung in die österreichische Forschungsstrategie.

- Die Verstärkung von innovativen Impulsen für Unternehmenspraktiken und Wirtschaftsstrukturen sowie die Stärkung der Rolle der Fachhochschuleinrichtungen in diesem Prozess.

- Die Positionierung der Fachhochschulen und Fachhochschul-Studiengänge im regionalen Umfeld einerseits als Mittler zwischen der regionalen Ebene und den verschiedenen 
Aspekten der überregionalen Ebenen, andererseits als Knotenpunkte in der Vernetzung der regionalen Akteure in regionalen Innovationssystemen bzw. lernenden Regionen.

Diese Entwicklungsfelder wurden in stilisierter Form in der folgenden grafischen Darstellung zusammengefasst (Abbildung 67).

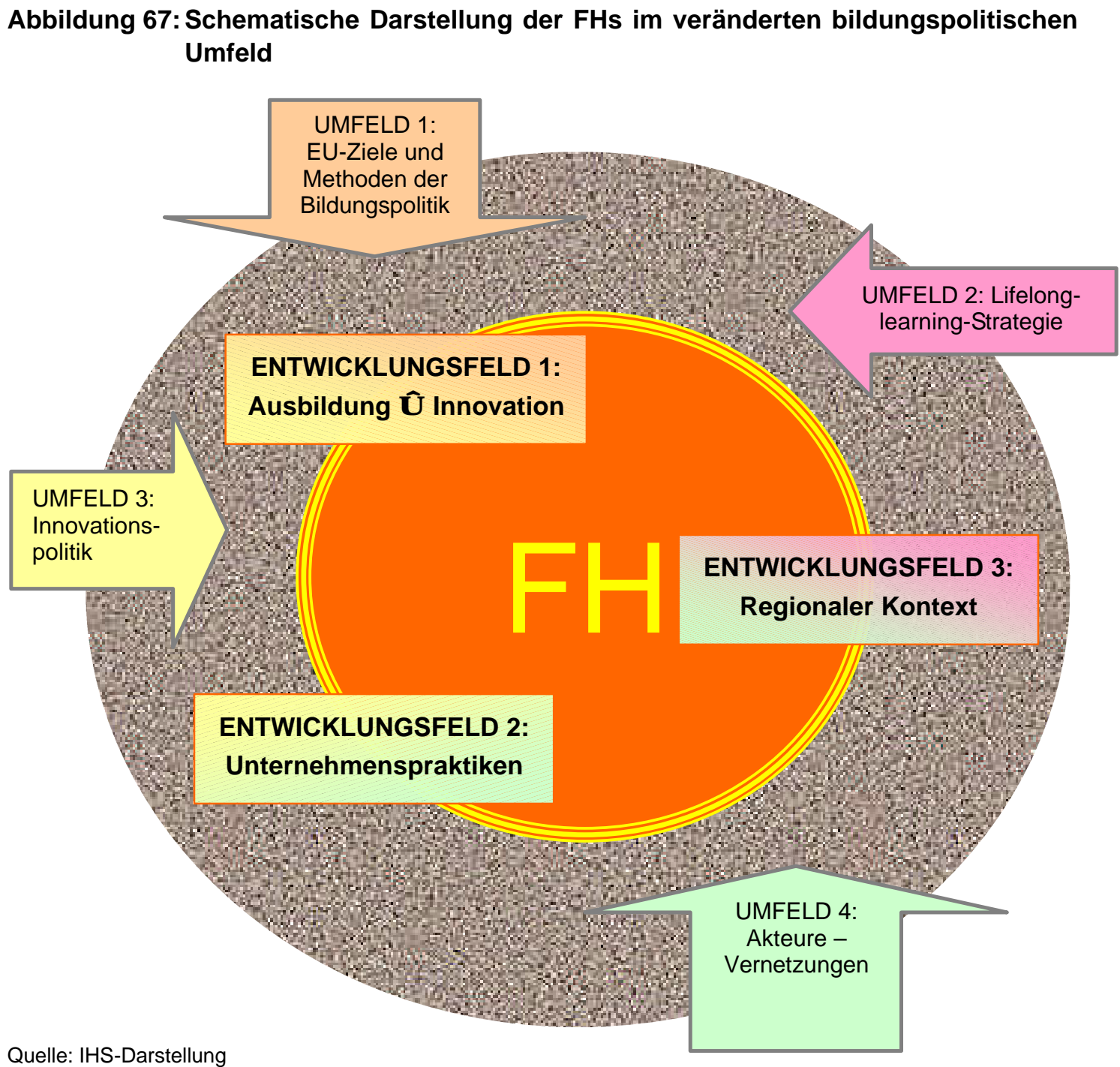

\subsubsection{Diversität und Aufgabenportfolio}

Einer der hervorstechendsten übergreifenden Befunde aus unseren Analysen ist die hohe Diversität, die so gut wie alle untersuchten Aspekte und die meisten Merkmale des FHSektors betrifft:

- die Größenordnungen der Studiengänge, Standorte etc. im Hinblick auf Studierende, Lehrende, Ressourcen etc. 
- die Aufgabenstruktur („Aufgabenportfolio“: Profil der Lehrgänge, thematische Breite des Lehrangebots, Forschungsorientierung, Dienstleistungs- und Weiterbildungsangebot)

- Personalstruktur und Personaleinsatz (Verhältnis von externem und Stammpersonal, Lehrverpflichtungen)

- die Merkmale der Leistungserstellung (Regulierung des Zuganges, Selektivität, Gruppengrößen, Abbruchquoten).

In dieser Diversität drückt sich die große Vielfalt der Art und Weise der Aufgabenerfüllung durch die FH-Studiengänge bzw. Fachhochschulen im Rahmen der einzuhaltenden Vorgaben aus. Obwohl die Entwicklung von diversifizierten Anbieterstrukturen ein wesentliches Ziel des FH-Sektors ist, ergibt sich aufgrund der dargestellten Vielfältigkeit die Frage, ob ein derart großes Ausmaß an Diversität angestrebt war und sinnvollerweise weiterhin anzustreben ist.

Diese Frage steht im Zusammenhang mit der Art und Weise der Formulierung von Zielen und Aufgabenstellungen für den FH-Sektor. Im Vergleich zu anderen Bereichen des Bildungswesens erfolgte in den regulatorischen Vorgaben eine stärkere Betonung der Ergebnisdimension im Verhältnis zu Input- und Prozessmerkmalen. Insbesondere wurde die Regulierung der Input- und Prozessdimension im Vergleich zu anderen Bereichen des Bildungswesens deutlich zurückgenommen, die erwarteten Ergebnisse wurden jedoch nicht in gleichem Verhältnis spezifiziert. Im Wesentlichen werden die angestrebten Ergebniskategorien vorgegeben und die Prozeduren für die Überprüfung (Akkreditierung, Evaluation, laufende Qualitätssicherung) bestimmt. Für die Etablierung einer wirksamen Zielsteuerung reicht dies jedoch nicht aus, da dafür Ergebnisziele im Zeitverlauf erforderlich sind. Dieses Problem betrifft insbesondere die Umsetzung des Aufgabenportfolios der FHEinrichtungen:

- In den regulatorischen Grundlagen wird zwar spezifiziert, welche Aufgaben erfüllt werden sollen und welche Nebenziele zu berücksichtigen sind, es wird aber nur teilweise spezifiziert, in welcher Gewichtung und in welchem Zeitraum die verschiedenen Aufgaben erfüllt werden sollen. Für die Lehre wurden die Mengengerüste und bestimmte Nebenbedingungen (wie die Erreichung eines zeitgerechten Studienabschlusses) vergleichsweise strikt vorgegeben. Es bestehen jedoch keine Formulierungen über die angestrebte Gewichtung von Lehre, Forschung und Dienstleistungen im Aufgabenportfolio einer FH-Einrichtung.

- Die Formulierung der Aufgaben ist stark auf die Ebene der Studiengänge fokussiert, sodass die Fachhochschulen, mit bestimmten Spezifikationen, gewissermaßen Konglomerate von Studiengängen ohne deutliche eigene Funktion darstellen. Möglicherweise resultiert aus dieser Gewichtung eine schwache Motivation zur 
Errichtung von Fachhochschulen und auch eine gewisse Schwächung der Entwicklung eines ausgeprägten akademischen Portfolios. Die Erhalter, deren Aufgaben eigentlich in den Bereichen der Infrastruktur und der wirtschaftlichen Belange liegen, haben dadurch eine starke Stellung in den strategischen Belangen bekommen, die mehr oder weniger stark in die akademischen Bereiche hineinwirken.

- Für die akademische Profilierung (besonders im Bereich der Forschung und der Internationalisierung) wird das Thema einer ausreichenden Größenordnung der Einheiten virulent. Ebenso erscheint erst ab einer gewissen Komplexität und Größe die für das akademische Leben wichtige institutionelle Selbstorganisation und Selbstverwaltung durchführbar und ein entsprechendes Hochschulmanagement sinnvoll.

- Die Personalpolitik ist ein Bereich, der im FH-Sektor im Vergleich zum übrigen Bildungswesen besonders wenig reguliert ist. Dieser Bereich war kein besonderer Schwerpunkt in der vorliegenden Studie, aber viele Befunde und Hinweise deuten auf einen starken Entwicklungsbedarf im Personalwesen hin. Die vermehrte Aufmerksamkeit der Fachhochschul-Konferenz und des Fachhochschulrates für den Bereich der Personal- und Organisationsentwicklung zeigt, dass die große Bedeutung dieses Themas bei wichtigen Akteuren des FH-Sektors zunehmend gesehen wird.

Es ist zu hinterfragen, inwiefern diese Merkmale der Definition und Umsetzung des Aufgabenportfolios einer Modifikation bedürfen. Insbesondere, inwieweit die Erfüllung der Ziele des FH-Sektors eine Umsetzung der Zielvorgaben in den verschiedenen Aufgabenbereichen eher gleichgewichtig verlangt oder ob die Gewichtung der Aufgabenbereiche eben der Diversität überlassen bleiben soll. Mit anderen Worten, ob Fachhochschulen oder FH-Studiengänge sich letztlich auf das gesamte Portfolio konzentrieren müssen oder ob hier eben Spezialisierungen, mit einer gewissen minimalen Erfüllung der jeweils anderen Aufgabenstellungen, zulässig sind bzw. angestrebt werden (d.h. es gibt "forschende Studiengänge" und solche, die nur minimal forschen; manche Studiengänge oder Fachhochschulen konzentrieren sich auf Dienstleistungen, manche auf die Lehre, manche auf die Forschung, andere präferieren ein mehr gemischtes Portfolio etc.). Wenn die Diversität und Spezialisierung im Vordergrund steht, dann ist klar, dass bestimmte übergeordnete Ziele für den gesamten Sektor aufgrund der dezentralisierten Struktur nur unter dem Einsatz zusätzlicher Steuerungsmittel (wie z.B. spezifischer Anreize oder gezielter Förderprogramme, mit den entsprechenden Unsicherheiten der Wirksamkeit) verwirklicht werden können.

Die Beantwortung dieser Fragen kann mit den Mitteln der Forschung nicht hinreichend erfolgen, da aus verschiedenen Paradigmen unterschiedliche Antworten resultieren. Die Implikationen verschiedener Zugänge wurden herausgearbeitet und zu den Filterungen in der aktuellen europäischen Politik und den Ansätzen der österreichischen Innovationspolitik (Forschungsstrategie, Regionalentwicklung) in Beziehung gesetzt. Die weitere Entwicklung 
des Fachhochschulsektors kann in engerem oder weniger engem Zusammenhang mit den Ansätzen der Innovationspolitik sowie den Entwicklungen auf europäischer Ebene konzipiert werden. Je nachdem, welcher Pfad eingeschlagen wird, können einerseits unterschiedliche Wirkungen auf das Innovations- und Wirtschaftsgeschehen erwartet werden und sind andererseits aber auch (aufgrund bleibender Unsicherheiten der voraussagbaren Wirkungen) mehr oder weniger starke bzw. risikoreiche politische Entscheidungen zu treffen.

Insbesondere wären für den Dritten Entwicklungs- und Finanzierungsplan die folgenden Fragestellungen zu stellen und zu beantworten. Von den Antworten hinsichtlich der Zielstruktur und des angestrebten Aufgabenportfolios hängen viele weitere Aspekte ab:

- Die Profilierung der Lehre ist bis zu einem gewissen Grad damit verbunden, ob die anderen Funktionen in einem Studiengang oder an einem Standort auch mit großem Gewicht verfolgt werden oder ob sich Studiengänge mehr oder weniger rein auf die Lehre spezialisieren können.

- Die Verbindung zu Bedarf und Akzeptanz erhält eine zusätzliche Facette, wenn Forschung und Dienstleistungen in einem Studiengang neben der Lehre bzw. in Verbindung zu ihr ein starkes Profil haben.

- In der Entwicklungs- und Finanzierungsplanung ergibt sich, je nach Lösung, die Aufgabe der Formulierung von Ergebniszielen bzw. Mindeststandards für die verschiedenen Funktionen sowie die Aufgabe der Allokation der erforderlichen Mittel für die Erfüllung der verschiedenen Funktionen.

- Bezüglich der Entwicklung der Standorte ist das angestrebte Aufgabenportfolio der zentrale determinierende Faktor insbesondere für die Definition von Mindestgrößen, aber auch für die Standortwahl und die regionale Einbindung. Je nach angestrebtem Profil ergeben sich unterschiedliche Dimensionen von "kritischen Massen" und unterschiedliche Anforderungen bzw. Möglichkeiten im Verhältnis zum regionalen Umfeld.

- Die Erfordernisse für die Qualitätssicherung und die Bedingungen der Internationalisierung hängen selbstverständlich direkt mit dem angestrebten Aufgabenprofil zusammen.

\subsubsection{Entwicklungspfade}

Die Frage nach dem Aufgabenprofil von Fachhochschuleinrichtungen hängt auch eng mit den Entscheidungen über die beiden Hauptaspekte der weiteren quantitativen Entwicklung des Sektors zusammen, nämlich a) dem Entwicklungspfad für den Sektor insgesamt (Zahl der Studienplätze und Finanzbedarf aggregiert) und b) der regionalen Verbreitung der Fachhochschuleinrichtungen und dem Profil der Standorte. 
Die Analyse der Standortprofile zum bisherigen Stand zeigt deutlich, dass eine große Zahl der Standorte mit dem gegenwärtigen Profil ein voll entwickeltes Aufgabenportfolio bei weitem nicht erfüllen kann. Die Formulierung entsprechender Ergebnisziele würde Entscheidungen über die Entwicklung der Standortprofile erfordern (etwa ein systematisches Nachziehen der schwächeren Standorte oder entsprechende Umverteilungen der Gewichte und Schwerpunktsetzungen).

Die Analyse der Umsetzung des Bedarfskonzeptes zeigt eine doch eher reaktive und lokal orientierte Perspektive und eine eher schwache bzw. indirekte Betonung der Verbindung zu unternehmensnaher F\&E. Eine stärkere und direktere Betonung der impulsgebenden Beteiligung am Innovationssystem würde die Formulierung entsprechender Ergebnisziele erfordern und in weiterer Folge stärkere und gezieltere Investitionen, vor allem in die F\&Eund die Dienstleistungsfunktion sowie eine entsprechende Spezifikation der Positionierung im Verhältnis zu den Universitäten notwendig machen.

Die mögliche Rolle des Fachhochschulsektors (im Verhältnis zu den Universitäten) bei der Heranbildung des personellen Potentials für die angestrebte Verstärkung der F\&ETätigkeiten müsste im Falle der stärkeren Einbindung in die Forschungsstrategie tiefer ausgelotet werden, als dies im vorliegenden Projekt möglich ist.

Mit Auslaufen des Entwicklungs- und Finanzierungsplans II stellt sich die Frage nach neuen Zielwerten und Prioritäten für die Bundesförderung. Wesentlich dabei ist die Frage nach der Dimensionierung des FH-Sektors im Hochschulsystem. Soll der Sektor weiterhin wachsen? Wenn ja, wie rasch soll der Sektor wachsen? Wie soll er im Hinblick auf die verschiedenen Zielsetzungen und Funktionen profiliert sein? Wie soll er im Vergleich zu den Universitäten positioniert werden? Diese Fragen betreffen die Entwicklung des Stellenwertes des FHSektors.

Aus dem vorliegenden Material lassen sich unterschiedliche Positionen zu diesen Fragen herausarbeiten und es werden einige Gesichtspunkte angeführt, deren Berücksichtigung gefordert oder nahegelegt wird. Diese Positionen und Gesichtspunkte, die von verschiedenen Akteuren in mehr oder weniger expliziter Form verfolgt oder geäußert werden, wurden zu einigen unterschiedlichen Entwicklungspfaden verdichtet, die im Rahmen der Erarbeitung des Entwicklungs- und Finanzierungsplans III weiter ausgelotet werden sollten (siehe Abbildung 68). 


\section{Abbildung 68:Stilisierte Darstellung der Entwicklungspfade des FH-Sektors, Hauptaspekte}

- NULL-WACHSTUM. Der maximale Ausbaustand wurde mit dem zweiten Entwicklungsplan erreicht, es wird seitens der öffentlichen Hand kein weiteres Wachstum angestrebt. Dieses Szenario ist denkbar, wird jedoch nicht in wesentlichem Maße vertreten.

- Fortsetzung einer moderaten und kontrollierten „bottom-up“-Strategie in diversifizierten Strukturen (STATUS-QUO). Dieses Szenario enthält im Wesentlichen eine abgeschwächte Fortsetzung der Wachstumsdynamik der Vergangenheit, weiterhin eher über neue Studiengänge als über die Ausweitung bestehender, eine Konsolidierung der Standorte und die Eröffnung neuer Standorte in begrenztem Ausmaß. „Qualität statt Quantität“; Gefahr der „Wachstumsfalle“, da die Akquirierung zusätzlicher Mittel an die Gewinnung zusätzlicher Studierender gebunden ist. Zwei wichtige Gesichtspunkte: Entwicklung des Studierendenpotentials und Aufbau der Forschungsfunktion als potentielle Möglichkeit, zusätzliche Mittel zu akquirieren.

- Verstärktes Wachstum, bei Regionalisierung und Förderung lokalpolitischer Interessen (EXTENSIVES WACHSTUM). Eher implizites Szenario auf Basis der Vielzahl an Standortvorschlägen aus der Anfangsphase des „bottom-up“-Prozesses, in Verbindung mit politischen Lobbying-Aktivitäten und einer festgestellten Tendenz zur „Politisierung“ in gewissem Konflikt mit der Qualitätssicherung. Weitere Ausdehnung von Einrichtungen mit Hauptgewicht auf Lehrtätigkeit und hohem Anteil an nebenberuflicher Lehre, tendenziell weitere Vervielfältigung von Studiengängen zur Risikominimierung und zu weiterer regionaler Verbreitung. Parallelität zu „bottom-up“-Wachstum postsekundärer Institutionen in anderen Ländern (z.B. Schweiz und Finnland, auch Niederlande) vor der Errichtung des FH-Sektors als Ausgangspunkt für „merger"-Strategien.

- Konsolidierung der Standorte, Aufbau von Fachhochschulen, breitere Studiengänge (INTENSIVES WACHSTUM). Verbindet quantitatives Wachstum, Aufgabenportfolios und das organisatorische Modell der Fachhochschule (Mindestgröße und Kollegium). Gegenüber der vorhandenen Vielfältigkeit und Diversität stärkere Konvergenz und stärker konturiertes Bild dessen, was eine Fachhochschule sein soll. Wachstum in den bestehenden Strukturen, Schritte zur Professionalisierung und personellen Konsolidierung, Verstärkung und systematischer Aufbau der F\&E-Funktion, teilweise in Abwägung zur Dienstleistungsfunktion und insbesondere stärkerem Engagement im Weiterbildungsmarkt. Zwei Aspekte zentral: Gestaltung des Aufgabenprofils und Beziehungen zwischen Studiengängen, Kollegium und Erhalter ("inhaltlicher Mehrwert" der Ebene „Fachhochschule").

- Befriedigung der Bildungsnachfrage (SOCIAL DEMAND). Abgeleitet aus der Differenz zwischen Bildungsnachfrage und Studienplätzen, (regionale) politische Zielsetzung der Verbesserung der Aussichten der Jugend, der Qualifikation der Bevölkerung und der regionalen Standortqualität. Verbindung zu lifelong-learning-Strategie und potentieller Nachfrage im Bereich der „Zweiten Chancen“. Starke Unwägbarkeiten im Zusammenhang mit der demografischen Entwicklung, der Universitätsreform und dem Bologna-Prozess. Wichtige Aspekte: Schnittstelle zu vorangehenden Ausbildungsgängen im Schulwesen, regionale Ausschöpfung der Bildungspotentiale. Affinität zum Szenario „extensiven Wachstums".

- Befriedigung des Qualifikationsbedarfs (ECONOMIC DEMAND). Abgeleitet aus Bedarfsanalysen, entsprechend der gegenwärtigen Praxis (Bedarfstestung kleinräumig und überwiegend angebotsorientiert) oder breiter angesetzt unter Berücksichtigung von Bedarfsanalysen auf Makro-Ebene und möglicherweise unter Berücksichtigung der "gestalterischen“ und „konstruktivistischen“ Komponente von umfassenderen Antizipationsmechanismen. Wichtige Aspekte: Erforderliche Vorleistungen zur Weiterentwicklung der Bedarfsprognostik in Form systematischer und gepoolter Nutzung der verschiedenen Standardmethoden, Koordination der verschiedenen Bereiche im Bildungswesen, Klärung verschiedener Grundfragen der Dimensionierung und Entwicklung des Bildungswesens (Stichworte: AkademikerInnenquote, Bedeutung der mittleren Qualifikationen, fehlendes Qualifikationsangebot als „bottleneck“, Fachrichtungswahl und Lücke im Bereich Mathematik-Science-Technology sowie IKT etc.).

- Mobilisierung des FH-Sektors für die Forschungsstrategie (FTE-STRATEGIE). Abgeleitet von den Funktionen von F\&E und innovationsorientierten Dienstleistungen, Bestimmung der Rolle des FH-Sektors in einer österreichischen Innovationsstrategie, Berücksichtigung der Entwicklung des F\&E-Nachwuchses. Spezifikation der Schnittstellen im Dreieck zwischen Forschungspolitik, Innovationspolitik und Bildungsbzw. Hochschulpolitik. Affinität zum Szenario „intensiven Wachstums“.

Diese Entwicklungspfade sind jeweils in stilisierter Form aus einigen wenigen (ein bis drei) jeweils bestimmenden Faktoren zusammengesetzt, teilweise gibt es Verwandtschaften, aber doch auch wesentliche Unterschiede, die einen jeweils eigenen Entwicklungspfad annehmen lassen. Die meisten Faktoren spielen in mehr oder weniger allen Entwicklungspfaden eine gewisse Rolle, die Abgrenzung zwischen den Entwicklungspfaden ergibt sich jedoch aus dem Gewicht, das man den jeweiligen Faktoren zuschreibt. Beispielsweise wird die 
Bildungsnachfrage oder die wirtschaftliche Nachfrage als Faktor immer eine gewisse Rolle spielen, es macht jedoch einen Unterschied, ob diese Faktoren nur - neben anderen wichtigeren - berücksichtigt werden oder ob sie die Entwicklung bestimmen sollen. Es liegt nicht auf der Hand, wie die weitere Entwicklung gestaltet werden soll. Die in der folgenden Darstellung gebündelten Entwicklungspfade können eine Diskussionsgrundlage für die Gestaltung bieten.

Abbildung 69 gibt einen Überblick über die verschiedenen Entwicklungspfade und die jeweils bestimmenden Faktoren, die den jeweiligen Pfad definieren. Die Anordnung in der Darstellung soll eine gewisse hierarchische Struktur der Entwicklungspfade aufzeigen, die von links unten (Null-Wachstum) nach rechts oben (FTE-Strategie) einen steigenden Entwicklungs- und Komplexitätsgrad impliziert. Ausgehend von der Null-Variante und der Status-quo-Variante, die auf unterschiedlichem Niveau die bestehende Struktur stabilisieren, kommen zusätzliche Aspekte ins Spiel, erstens verstärktes Wachstum (in die Breite oder auch in die Tiefe), zweitens verstärkte Ausprägung und Weiterentwicklung der Orientierung an den beiden Marktseiten (Qualifikationsnachfrage bzw. Bildungsnachfrage), drittens die verstärkte Betonung der innovativen Impulse durch den FH-Sektor. Die komplexeren Szenarien setzen nicht unbedingt die vorherigen voraus, die verstärkte Betonung des wirtschaftlichen Bedarfes oder auch der Bildungsnachfrage, z.B. im Kontext der Förderung des lebenslangen Lernens, kann im Prinzip auch erfolgen, ohne dass das Wachstum wesentlich verstärkt wird.

Es besteht eine gewisse Verwandtschaft zwischen manchen Entwicklungspfaden, indem sie bestimmte Faktoren auf neuer Stufe aufgreifen bzw. betonen, insbesondere zwischen dem StATUS-QUO-Modell, dem INTENSIVEN WACHSTUM und der FTE-STRATEGIE: Das Status-quoModell beruht auf inkrementeller Weiterentwicklung im „bottom-up“-Prinzip, ohne genauere Spezifikation eines Zielzustandes; jeder Standort bzw. Erhalter entwickelt sich so weit und so rasch, wie es eben seinen Prioritäten entspricht. Übergang zum intensiven Wachstum bedeutet, das Bild einer österreichischen FH-Institution (oder einige verschiedene Bilder) in der inhaltlichen und organisatorischen Konzeption (Größe, Aufgabenprofil, Lehrkörper etc.) deutlicher auszuformulieren und umzusetzen und entsprechende Kriterien zu formulieren (z.B. die vielen kleineren oder schwächeren Standorte systematisch weiterzuentwickeln). Der Übergang zum Modell der FTE-Strategie bedeutet, die F\&E-Aktivitäten zum Fokus der Weiterentwicklung zu machen und von jenen $\mathrm{FH}$-Institutionen auszugehen, die gegenwärtig bereits auf diesem Stand sind. In jedem Fall müssten entsprechende Ziele gesetzt und deren Umsetzung operationalisiert werden.

Der Entwicklungspfad EXTENSIVES WACHSTUM kann durch entsprechende Schritte und Maßnahmen in Richtung des ECONOMIC-DEMAND-Modells weiterentwickelt werden oder in Richtung des SOCIAL-DEMAND-Modells, indem die entsprechenden Faktoren (im einen Fall z.B. intensivierte Antizipationsstrukturen, im anderen Fall z.B. verstärkte Schritte zur Mobilisierung der Bildungsnachfrage von Erwachsenen) in den Vordergrund gestellt werden. 


\section{Abbildung 69: Stilisierte Darstellung der möglichen Entwicklungspfade für den FH- Sektor und der jeweils bestimmenden Faktoren}

\begin{tabular}{|c|c|c|}
\hline Innovative Impulse & & $\begin{array}{l}\text { FTE-STRATEGIE } \\
\text { (Mobilisierung des FH-Sektors für die } \\
\text { Forschungsstrategie) } \\
-\quad \text { Aufgabenstellung für FH-Studiengänge } \\
\text { aus der Forschungsstrategie } \\
-\quad \text { Verhältnis zu den Universitäten im } \\
\text { Hinblick auf Förderung des F\&E- } \\
\quad \text { Nachwuchses }\end{array}$ \\
\hline $\begin{array}{l}\text { Fokus der } \\
\text { Entwicklung } \\
\text { auf: }\end{array}$ & $\begin{array}{l}\text { ECONOMIC DEMAND } \\
\text { (Prioritäre Befriedigung des } \\
\text { Qualifikationsbedarfs) } \\
\text { - } \quad \text { Prioritäten bei der Deckung } \\
\text { des Qualifikationsbedarfes } \\
\text { der Wirtschaft } \\
-\quad \text { Antizipationssystem }\end{array}$ & $\begin{array}{l}\text { SOCIAL DEMAND } \\
\text { (Prioritäre Befriedigung der } \\
\text { Bildungsnachfrage) } \\
\text { - } \quad \text { Priorität der Ausschöpfung der } \\
\quad \text { sozialen und regionalen } \\
\quad \text { Bildungspotentiale } \\
-\quad \text { Priorität der Förderung } \\
\quad \text { lebenslangen Lernens (nicht- } \\
\quad \text { traditionelle Studierende) } \\
\text { - } \quad \text { Gestaltung der Schnittstellen im } \\
\quad \text { Bildungswesen }\end{array}$ \\
\hline
\end{tabular}

\section{Wachstum verstärkt}

\section{INTENSIVES WACHSTUM}

(Konsolidierung der Standorte, Aufbau von

Fachhochschulen, breitere Studiengänge)

- Profil der Lehrenden

- Aufgabenprofil eines Studienganges

- inhaltliche und organisatorische Konzeption der Fachhochschule

EXTENSIVES WACHSTUM

(Verstärktes Wachstum, bei Regionalisierung

und Förderung lokalpolitischer Interessen)

- Gewicht lokaler und regionaler Interessen

\section{STATUS-QUO}

(Fortsetzung einer moderaten und kontrollierten

bottom up-Strategie in diversifizierten Strukturen)

- $\quad$ endogene Potentiale an den Standorten

- Entwicklung des Studierendenpotentials

- Priorität für Forschungsfunktion

NULL-WACHSTUM

- $\quad$ Aufbringung der Finanzen

\section{Stabilisierung der bestehenden Strukturen}

\section{Quelle: eigene Darstellung}

Eine nähere Überprüfung der Tragfähigkeit der herausgearbeiteten Faktoren und ihrer Bündelung, etwa in Form von Simulationen oder Szenarienmodellen, könnte ein Weg zur weiteren Konkretisierung der Entwicklung des FH-Sektors sein. 


\subsubsection{Steuerungsmodell: Kooperation und Funktionendifferenzierung}

Der Fachhochschulsektor verkörpert ein neues Steuerungsmodell in der Bildungspolitik, insbesondere durch seine veränderte Beziehung zwischen dem Staat auf der einen Seite und den wichtigsten Leistungserbringern, den Studiengängen, auf der anderen Seite.

Zu den Stärken des Steuerungsmodells über Entwicklungs- und Finanzierungspläne ist zunächst zu zählen, dass diese als Novum im bildungspolitischen Kontext Österreichs erfolgreich umgesetzt werden konnten. Dies ist nicht selbstverständlich, da die einschlägige Implementationsforschung eher davon ausgeht, dass sehr weit von der hergebrachten Praxis abweichende Reformmodelle mit hoher Wahrscheinlichkeit scheitern werden. Die Formulierung eines Rahmenplans, in dem der Bund seine Pläne für diesen Sektor in den nächsten 5 Jahren und vor allem auch die dazugehörigen Ausbau- und Finanzierungspläne offen legt, war ein wichtiges Element für die innovative Entwicklung dieses Sektors. Die Steuerung über das Steuerungsmittel „Geld“ hat dabei gut funktioniert. Die Formulierung der bildungspolitischen Ziele und Kriterien hingegen ist nur in wenig operationalisierter Form erfolgt. Zudem war die Überprüfung der Einhaltung der bildungspolitischen Kriterien von Beginn an relativ schwach ausgeprägt gewesen und ist mit den Jahren immer schwächer geworden, wodurch sie in ihrer Steuerungsfunktion stark eingeschränkt waren.

Neben einer Weiterentwicklung der strategisch-bildungspolitischen Dimension der Zielvorgaben, einer stärkeren Operationalisierung der Vorgaben und einer konsequenteren Überwachung der Zieleinhaltung im Sinne eines Monitoring bedarf es adäquater Organisations- und Kommunikationsformen, in denen die künftigen bildungspolitischen Rahmenbedingungen für den Fachhochschulsektor konsensuell entwickelt werden können. Hiefür können - in der Weiterentwicklung des bildungspolitischen Steuerungskonzeptes institutionelle Netzwerke, in denen sich die beteiligten Akteure aus den unterschiedlichen Bereichen organisieren, eine besondere Rolle spielen. Solche Ideen des "Good Governance" gewinnen auch auf der Ebene der europäischen Bildungspolitik und im internationalen Rahmen seit einigen Jahren an Gewicht, wie man beispielsweise einschlägigen OECD-Publikationen entnehmen kann. Sie sind jedenfalls ein nicht zu unterschätzender Kontextfaktor für erfolgreiche nationale Politiken. Es wäre wünschenswert, wenn der Fachhochschulsektor in dieser Hinsicht seine Innovatorenrolle für das Bildungssystem erneut und mit einer gewissen Fokusverschiebung unter Beweis stellen würde.

So sollten die Länder neben der Weiterentwicklung ihrer internen Steuerungs- und Planungsaktivitäten auch eine explizite Rolle als bildungspolitische Akteure erhalten. Bislang fehlt eine adäquate Institutionalisierung ihrer Rolle. Ihre Einbindung in die Planung der weiteren Entwicklung ist ungeklärt. Es muss einen regelmäßigen, organisierten Meinungsaustausch zwischen Bund und Ländern und auch zwischen den Ländern geben, 
damit die jeweiligen Planungen einander nicht konterkarieren, sondern sinnvoll aufeinander abgestimmt werden können.

Ebenso müssen der Fachhochschulrat und die Fachhochschul-Konferenz als Gremien, die viel Engagement in die Entwicklung des Fachhochschulsektors investiert und entsprechendes Know-how aufgebaut haben, in solche Planungsüberlegungen einbezogen werden. Auch das für eine qualitätsvolle Weiterentwicklung des Sektors notwendige gute Zusammenspiel zwischen zuständigem Bundesministerium und Fachhochschulrat bedarf regelmäßiger Foren der Kommunikation, des Austausches und der Absprache.

Von besonderer Bedeutung ist der Aufbau einer breiten Informations- und Wissensbasis, die von den verschiedenen Akteuren genutzt und weiterentwickelt werden kann. Der möglichst breite Zugang zu dieser ist im Sinne der Transparenz ein wichtiger Aspekt dieser kooperativen Steuerungsformen. Auch in Bezug auf die Finanzierungsentscheidungen des Bundes und der Länder empfiehlt sich eine transparente Vorgangsweise, die umgekehrt auch von der Funktionsfähigkeit und Transparenz des Informations- und Monitoring-Systems im FH-Sektor abhängig ist.

Die Konstruktion des Fachhochschulrates als weisungsfreies ExpertInnengremium und Behörde war zum Zeitpunkt der Einführung eine kreative Lösung, um Elemente eines neuen bildungsorganisatorischen Modells in die bestehende stark rechtlich determinierte Bildungsorganisation in Österreich einzuführen. Fast zehn Jahre später sind die Bildungssektoren in allen europäischen Ländern enormen Veränderungen unterworfen und auch der tertiäre Bildungssektor in Österreich ist stark in Bewegung geraten. Angesichts der veränderten Umwelten sollten „spezifisch österreichische Lösungen“ überdacht werden. In der vorhandenen Konstruktion sind einige Spannungsfelder festzustellen, die auch nicht zufällig in den Überlegungen und Diskussionen zur Funktionsteilung im Zusammenhang mit der jüngsten Novelle zum FHStG zum Ausdruck gekommen sind :

- Ein wesentliches Spannungsfeld ergibt sich durch die Konstruktion des Fachhochschulrates als Behörde einerseits und ExpertInnengremium andererseits, beides Organisationsformen, die unterschiedlichen Struktur- und Entscheidungslogiken folgen.

- Die ursprünglich geplante Trennung von Qualitätsentscheidung einerseits und Finanzierungsentscheidung andererseits, die das Zusammenspiel zwischen zuständigen Bundesministerium und Fachhochschulrat kennzeichnet, ist ebenso „spannungsgeladen“ wie das Verhältnis von bildungspolitischen Kriterien im FHStG und den Entwicklungsplänen und den Kriterien, die der Fachhochschulrat in seiner Qualitätsprüfung anlegt. Qualität ist immer nur relational festzustellen und damit abhängig von den gesetzten Zielen. Insofern kann Qualität nicht unabhängig von den 
bildungspolitischen Kriterien und Erwartungen an den Fachhochschulsektor festgelegt werden.

- Wenn auch die Trennung zwischen Qualität einerseits und Finanzierung aufgrund politischer Prioritäten andererseits als sehr sinnvoll erscheint, so sind diese Entscheidungen im praktischen Vollzug stark miteinander verflochten, was wiederum immer wieder Spannungen im Ablauf der Entscheidungsprozesse und der Umsetzung mit sich gebracht hat.

In der gegenwärtigen Aufgabenteilung zwischen Fachhochschulrat und zuständigem Bundesministerium erscheinen nicht nur die Kapazitäten des Fachhochschulrats unzureichend, sondern auch auf Seite des BMBWK zu wenig Kapazitäten für die Beschäftigung mit den strategischen bildungspolitischen Fragen im FH-Sektor vorhanden zu sein.

Zunehmend drängend wird auch eine bildungspolitische Gesamtkoordination im tertiären Sektor. Der Bund ist vor allem für die Herstellung gleicher Rahmenbedingungen und für die Strukturbereinigung zuständig. Durch die neue Universitätsgesetzgebung werden sich auch die Universitäten in Zukunft vermutlich unternehmerischer verhalten; die Konkurrenz des FHSektors mit den Universitäten wird aus diesem Grund stärker werden. Auch hat der Fachhochschulsektor eine Ausbaustufe erreicht, die im Zuge eines weiteren Wachstums aufgrund der sich überschneidenden Rekrutierungsbasis vermutlich zu verstärktem Wettbewerb mit den Universitäten führen wird, zumindest in bestimmten Studienbereichen. Das zweite Moment der Konvergenz zwischen Fachhochschul- und Universitätssektor besteht im nunmehr möglichen Angebot entsprechender formaler Abschlüsse (Bachelor/Master).

Die verschiedenen Teilbereiche des tertiären Sektors benötigen die gleichen Spielregeln, um sinnvoll miteinander sowie komplementär wirken zu können und Reibungsverluste zu vermeiden. Die Verbesserung der Kohärenz des Bildungssystems verlangt zudem einen breiten bildungspolitischen Willensbildungsprozess.

Angesichts der mittlerweile erreichten Größe des Fachhochschulsektors und der Entwicklungen in den europäischen Bildungssystemen erscheinen die folgenden Überlegungen zur Funktionsdifferenzierung zwischen bildungspolitischen Vorgaben und akademischer Qualitätsprüfung angebracht, die auch die internationalen Experten in ihrem Feedback deutlich betont haben:

Es könnte - zum Beispiel als Teilbereich eines für den Universitätsbereich geplanten Wissenschaftsrates - ein Gremium entstehen, das sich insbesondere mit den bildungspolitischen Vorgaben für den FH-Sektor in Relation zu den anderen Sektoren des tertiären Bildungssystems beschäftigt, das die Entwicklung des Gesamtsektors begleitet und 
darauf achtet, dass die Voraussetzungen gegeben sind, die notwendig sind, um neue Anforderungen (wie etwa der Forschungsauftrag, der verstärkte Internationalisierungsdruck etc) bewältigen zu können. Eine solche Einrichtung scheint notwendig, weil einzelne Abteilungen eines Ministeriums, die personell zumeist knapp besetzt sind, überfordert wären, die doch gewichtigen bildungspolitischen Entscheidungen ohne zusätzliche Expertise vorzubereiten (Expansionsrate, benötigte Finanzierung, Standorte, Verhältnis zu anderen Sektoren, Themenspektrum, inhaltliche Felder etc.).

In Ergänzung dazu braucht es eine Einrichtung, die die akademische Qualitätssicherung zu organisieren hätte. Das kann der bestehende Fachhochschulrat im Sinne einer Akkreditierungs- und Evaluierungsagentur sein, wobei es hier vor allem darum geht, die Aufgaben mit Hilfe von weiteren ExpertInnen und einer Geschäftsstelle zu organisieren. Für die Besetzung dieses Expertengremiums würde dies aber bedeuten, dass die Kompetenz der Expertlnnen neben einer fachspezifischen Expertise insbesondere auch Know-how in den Bereichen der Gestaltung der Lehr/Lernprozesse, der Curriculumentwicklung, der berufsfeldbezogenen F\&E etc. aufweisen müssten. Kriterien der bildungspolitischen Orientierung oder Interessenvertretungen dürften in diesem Rahmen keine Rolle spielen, weil dieser ebenfalls wichtige Aspekt im Wissenschaftsrat abgebildet ist.

Eine andere Überlegung wäre dass der Fachhochschulrat mehr Kapazitäten für die Behandlung wichtiger strategischer bildungspolitischer Fragen und für die Begleitung der Entwicklung des Sektors bekommt. Die Frage der akademischen Qualitätssicherung müsste dann stärker über Akkreditierungs- und Evaluierungsagenturen organisiert werden. Diese hätten dann „Zulieferfunktion“ für den Fachhochschulrat. Der Trend, Akkreditierungsagenturen von staatlicher Seite lediglich zu akkreditieren und damit auch den Institutionen Wahlmöglichkeiten zu lassen, scheint sich in vielen Ländern Europas stärker durchzusetzen (es sind jedoch auch Überlegungen der Größenordnung der Länder und der Effizienz zu berücksichtigen).

Bei der Weiterentwicklung des Steuerungsmodells, das im Zusammenspiel zwischen politischer Steuerung und Qualitätssicherung erfolgen muss, ist die dynamische Entwicklung von Kooperationen und Vernetzungen auf europäischer und internationaler Ebene zu berücksichtigen, welche eine zunehmend wichtige Quelle für die Entwicklung der Hochschulsysteme darstellen. Die Organisationsformen der Steuerungsstruktur sollten jedenfalls sicherstellen, dass ein möglichst wirksamer Austausch zwischen der nationalen und der internationalen Ebene erfolgen kann. 


\subsection{Zusammenfassung der Empfehlungen}

Im folgenden Abschnitt werden aus den Analysen und Schlussfolgerungen noch einmal die wichtigsten Vorschläge und Empfehlungen zusammengefasst, die das Projektteam für die weitere Entwicklung des FH-Sektors als Teil des österreichischen Hochschulsystems sowie des europäischen Hochschul- und Forschungsraumes als bedeutsam hervorheben möchte. Diese Vorschläge und Empfehlungen beziehen sich auf wichtige Weichenstellungen, die im Zuge der anstehenden Festlegungen für die weitere Entwicklung berücksichtigt werden sollten.

\subsubsection{Bereich Entwicklungs- und Finanzierungsplanung: Entwicklung der Zieldimension}

Für die weitere Entwicklung wurde als eine zentrale Schlussfolgerung des Reviews eine Überprüfung und nähere Spezifikation der Zielformulierungen in Form von Ergebniszielen vorgeschlagen. Der nächste Entwicklungs- und Finanzierungsplan sollte Aussagen zu folgenden Bereichen enthalten:

- Die Annahmen der Politik über die weitere Entwicklung der Partizipationsraten beim Übertritt in den tertiären Bereich und die angestrebte Verteilung zwischen dem FH-Sektor und den Universitäten.

- Die Absichten der Bildungspolitik hinsichtlich der Größe der Wachstumsraten und der Finanzierung der Fachhochschuleinrichtungen.

- Aussagen zum Profil der Fachhochschulinstitutionen (Aufgabenportfolio, Diversifizierung nach Disziplinen) - als Aspekte der Herausbildung kritischer Größenordnungen in inhaltlicher Hinsicht.

- Eine Klärung, welche Institutionen künftig in den Fachhochschulsektor integriert werden sollen (bzw. wie das Verhältnis zwischen Fachhochschulsektor und den übrigen nichtuniversitären postsekundären Einrichtungen gestaltet werden soll) sowie eine Aussage über das Fächerspektrum, das in Zukunft im FH-Sektor vertreten sein sollte (die Dominanz des technisch-wirtschaftlichen Bereiches wäre zugunsten einer Öffnung gegenüber anderen Bereichen, wie z. B. dem Sozial- und Gesundheitsbereich zu hinterfragen und auch die Strukturbereinigung mit anderen nicht-universitären postsekundären Einrichtungen sollte gelöst werden).

- Der Kriterienkatalog des Entwicklungsplanes sollte um ein mittelfristiges Schwerpunktprogramm ergänzt werden, das die Prioritäten für die weitere Entwicklung 
formuliert, d.h. die Prioritäten sollten stärker operationalisiert und einem genaueren Monitoring unterworfen werden und darüber hinaus auch durch eigene Förderprogramme betont werden. Dazu bedarf es einer Gewichtung von Prioritäten, für deren Erreichung entsprechende Fördermittel- oder -programme vorgesehen werden sollen (dies betrifft zum Beispiel die Gewichtung zwischen den verschiedenen Aufgabenstellungen). Aus den Analysen ergibt sich auch Bedarf für die Förderung bestimmter Zielgruppen, insbesondere der Chancengleichheit von Frauen und Männern sowie von nichttraditionellen Studierenden.

- Die Kleinteiligkeit bisheriger Maßnahmen, etwa in Form studiengangsbezogener Bedarfsund Akzeptanzstudien, Akkreditierungen, Evaluierungen und Finanzierungen, sollte zugunsten einer verstärkten Einbettung auf ein Makroniveau verlagert werden. Das sollte auch ein Beitrag sein, die Nischenpolitik zugunsten breiterer Ausbildungsfelder zu verlassen.

\subsubsection{Wahl der Entwicklungspfade für die weitere quantitative Dimensionierung und qualitative Profilierung des FH-Sektors}

Eine wichtige Frage betrifft die Festlegungen über die weitere quantitative Entwicklung, die in engem Zusammenhang mit dem angestrebten qualitativen Profil der FH-Institutionen und den allgemeinen Zielsetzungen für den $\mathrm{FH}$-Sektor getroffen werden müssen. Es wurden sieben unterschiedliche Entwicklungspfade formuliert, die zwischen Null-Wachstum und voller Einbindung in eine österreichische Strategie der Forschungs- und Technologieentwicklung liegen und deren Eckpunkte unterschiedliche Pfade verstärkten Wachstums sowie ein unterschiedliches $M a ß$ an Betonung des wirtschaftlichen Bedarfes bzw. des Bildungsbedarfes sind.

- Es wird vorgeschlagen, dieses Spektrum an möglichen Entwicklungspfaden für den FHSektor als Grundlage für die weitere Diskussion und Systematisierung von Entwicklungsstrategien heranzuziehen.

- Vor allem aufgrund der Veränderungen im Umfeld des FH-Sektors bietet sich für die weitere Entwicklung das Feld zwischen den Modellen des INTENSIVEN WACHSTUMS und der FTE-STRATEGIE an, wobei auch eine ausgewogene Beachtung von Qualifikationsund Bildungsnachfrage berücksichtigt werden sollte.

- Die näheren Spezifikationen der weiteren Entwicklung sollten in einem systematischen Verfahren vorgenommen werden, in dem die verschiedenen Akteure ihre Positionen einbringen und abstimmen können. 


\subsubsection{Die Gestaltung des Aufgabenportfolios und nähere Spezifikation der Aufgaben der FH-Institutionen}

Auf dem Hintergrund von wesentlichen Umfeldveränderungen wurden drei Entwicklungsfelder für die $\mathrm{FH}$-Institutionen herausgearbeitet: Thematisierung der Interaktion zwischen Ausbildung und Innovation; Verstärkung von innovativen Impulsen für die Unternehmenspraktiken und Wirtschaftsstrukturen; Positionierung im regionalen Umfeld. Vor allem die Rolle, die die Fachhochschuleinrichtungen in der österreichischen Forschungsstrategie spielen sollen und können, betrifft eine zentrale Entwicklungsfrage in diesem Zusammenhang.

- Für die in letzter Zeit verstärkt angedachte akademische Profilierung etwa im Bereich der Forschung und der Internationalisierung wird das Thema der ausreichenden Größenordnung virulent; auch ist erst ab einer gewissen Komplexität und Größe institutionelle Selbstorganisation möglich. Während bislang die Aufgaben auf die Lehre konzentriert sind, sollten auch klarere Festlegungen über Zielvorgaben in den Bereichen der Forschung, Dienstleistung und Weiterbildung getroffen werden, etwa inwieweit alle Fachhochschuleinrichtungen das gesamte Portfolio abdecken müssen oder ob hier Spezialisierungen der einen oder anderen Aufgabe möglich sind, mit einer gewissen minimalen Erfüllung der jeweils anderen Aufgabenstellungen.

- Zum gegenwärtigen Zeitpunkt kann eine große Zahl von Standorten ein voll entwickeltes Aufgabenportfolio bei weitem nicht erfüllen und es fragt sich, ob ein systematisches Nachziehen der schwächeren Standorte erfolgen soll oder eine entsprechende Umverteilung der Schwerpunktsetzungen. Die Rolle des Aufgabenprofils (Einbindung in die FTE-Strategie, Verbindung zum Unternehmenssektor etc.) steht auch in Zusammenhang mit der Frage, wie der FH-Sektor im Hochschulsystem insgesamt dimensioniert wird.

- Wenn Forschung und Dienstleistung neben der Lehre oder in Verbindung zu ihr ein starkes Profil haben sollen, dann hat das Auswirkungen auf die Erhebung von Bedarf, von Akzeptanz und die Methoden der Qualitätssicherung.

\subsubsection{Adäquate Organisationsformen zur Zielbildung und Koordination}

Es müssen auf verschiedenen Ebenen verstärkt adäquate und transparente Kommunikationsforen geschaffen werden, um den Prozess der Zielbildung unter Einbindung der beteiligten Akteure partnerschaftlich zu organisieren:

- Die administrative professionelle Planungskapazität des zuständigen Bundesministeriums muss verstärkt werden. 
- Unter Umständen ist auch der entstehende Wissenschaftsrat, wenn er seine Aktivitäten auf den gesamten tertiären Ausbildungsraum erstrecken würde, ein auch für den Fachhochschulsektor sinnvolles strategisch-politisches Beratungsorgan.

- Die Rolle der Länder als bildungspolitische Akteure muss stärker institutionalisiert und ihre Einbindung in die Planung gewährleistet werden, damit die Planungen einander nicht konterkarieren.

- Weiters bedarf es regelmäßiger Kommunikationsforen zwischen dem Fachhochschulrat, der Fachhochschul-Konferenz und dem zuständigen Bundesministerium, die derzeit eher auf informeller Basis geführt werden.

- Besonders wichtig ist die Transparenz einer breiten Informations- und Wissensbasis. Insbesondere in Bezug auf Finanzierungsentscheidungen von Bund und Ländern empfiehlt sich eine transparentere Vorgangsweise. Die gegenwärtige Trennung von Mengen- und Finanzinformationen, die sich aus den unterschiedlichen Zuständigkeiten von Fachhochschulrat und Bundesministerium ergibt, erschwert die Auswertung der Informationen. Hier ist ein einheitliches System des Controllings und der Berichtslegung (womöglich in Abstimmung zu den Universitäten) zu entwickeln, damit ein modernes Monitoringsystem entsteht.

\subsection{5 Überprüfung der Funktionen und Aufgaben des FHR}

Durch den Übergang von der Pionier- zur Konsolidierungsphase als auch durch veränderte bildungspolitische, nationale und europäische Rahmenbedingungen sollten die Funktionsprofile der einzelnen Einrichtungen überprüft werden. Damit einher geht u. U. ein Profilwandel des Fachhochschulrates, der im Moment sowohl zentraler bildungspolitischer Akteur als auch zentrales „Organ der Qualitätssicherung“ ist. Die derzeitige Kombination von selbständiger Qualitätsprüfung (quasi Arbeit als Akkreditierungs-Evaluierungsagentur in einer bestimmten Form) plus Behörde plus (da Vakuumsituation) bildungspolitisches Beratungsorgan erscheint zu komplex, diese Funktionen müssten daher stärker ausdifferenziert werden. Hier sind verschiedene Ausdifferenzierungen denkbar:

- Der Fachhochschulrat zieht sich zurück auf die reine Funktion der akademischen Qualitätssicherung, hat also das Profil einer Akkreditierungs- und Evaluierungsagentur. Dann muss aber die strategische Kompetenz des Bundesministeriums (in Fragen der bildungspolitischen Rahmenbedingungen etwa) verstärkt werden.

- Oder der Fachhochschulrat entwickelt sich selbst zu einem stärker bildungspolitischen Beratungsorgan und man lagert die Frage der Qualitätssicherung und der Akkreditierung an Agenturen aus. Die Studiengänge und die Fachhochschulen können sich dann 
bestimmter Agenturen bedienen und der Fachhochschulrat nimmt dann nicht mehr selber die Qualitätsprüfung vor, sondern diese wird quasi bei der Antragstellung mitgeliefert. So würde der Fachhochschulrat an Spielraum für die strategische Ebene der Qualitätssicherung gewinnen.

\subsubsection{Gesamtbetrachtung des tertiären Sektors:}

Es gibt verschiedene Gründe, warum die ursprüngliche Intention, den Fachhochschulsektor als eigenen Bereich aufzusetzen, nicht mehr zeitgemäß ist und die zukunftsträchtigere Entwicklungsperspektive nun in Richtung Konvergenz weist: Durch die neue Universitätsgesetzgebung erhalten die Universitäten höhere institutionelle Autonomie und die Konkurrenz zwischen Fachhochschuleinrichtungen und Universitäten wird sich auch auf Grund des erreichten Größenstands der Fachhochschuleinrichtungen verstärken. Zukünftig werden sowohl Universitäten als auch Fachhochschuleinrichtungen Bachelor- und Masterstudien anbieten, es werden sich daher spezielle Profile von jeweils einschlägigen Bachelor- und Masterstudien herausbilden müssen. Zur Gesamtbetrachtung des tertiären Sektors gehört auch die Bestimmung der Größenordnungen der einzelnen Bereiche zueinander.

- Die Verbesserung der Kohärenz im Bildungssystem verlangt eine Bekanntgabe durch den Bund, welche Sparten, Berufsfelder und Ausbildungen in welchen Sektoren (Universitäten, Fachhochschuleinrichtungen) angesiedelt sein sollen.

- Gleiche Spielregeln sind innerhalb eines kohärenten, tertiären Bildungssystems anzustreben. Innerhalb eines großen tertiären Sektors sollte es zur Differenzierung von unterschiedlichen Profilen kommen, anstelle einer Sektoralisierung mit jeweils unterschiedlichen Spielregeln.

- Zum Beispiel sollte es Ziel- und Leistungsvereinbarungen nicht nur im Universitätsbereich, sondern auch im Fachhochschulbereich geben. das setzt aber das Vorhandensein von einigermaßen großen Fachhochschuleinrichtungen voraus, die dann auch eine größere institutionelle Bewegungsfreiheit bekommen würden.

- Umgekehrt braucht der Universitätsbereich etwa einen ähnlichen fünfjährigen Entwicklungs- und Finanzierungsplan wie der Fachhochschulbereich.

- Auch die Erstellung von Weiterbildungsangeboten muss den gleichen Spielregeln unterworfen sein.

- Oder die Auswahl der Studierenden, die nur der Fachhochschulsektor vornehmen darf und die Universität nicht. 
- Auch die Methoden der Qualitätssicherung sollten á la longue ähnlich geregelt sein.

\subsubsection{Regionalisierung und Standortplanung}

Die Spielräume für eine weitere Regionalisierung ergeben sich aus einer weiteren quantitativen Expansion. Zum Abbau regionaler Disparitäten erscheint eine weitere Regionalisierung wenn überhaupt nur noch begrenzt argumentierbar. Ansonsten ist eine solche abhängig von der Festlegung über das Aufgabenportfolio.

- Es sollten systematischere Überlegungen über die Konkretisierung der Funktionen im Kontext von regionalen Innovationssystemen und der Entwicklung von lernenden Regionen angestellt werden. Eine erste Grundlage sind die Szenarien für den FTE-Rat. Diese entwickeln Vorschläge, wie die $\mathrm{FHs}$ in Strategien der Erhöhung der österreichischen Forschungsquote eingebaut werden können, sind jedoch noch bei weitem zu wenig konkretisiert.

- Wenn der FH-Sektor eine sichtbare Impulsfunktion für die Entwicklung regionaler Innovationssysteme ausüben soll, dann ist die Konsolidierung weiter voranzutreiben. Ein wesentliches Element dabei sollte die Konkretisierung der erwarteten Leistungen der $\mathrm{FH}$ Einrichtungen im gesamten Spektrum sein.

- In der Frage der Standortwahl sollten systematische Verfahren angewendet werden. Im Prinzip könnte eine übergreifende Standortprüfung für Österreich durchgeführt werden, die das gesamt Spektrum an möglichen Standorten systematisch auslotet. Dadurch könnte eine gewisse Erwartungssicherheit im Hinblick auf lokale Interessen geschaffen werden.

- Die Bedarfsanalysen sollten stärker in den Aufbau von Antizipationsmodellen eingebaut werden, die auf die Vernetzung und Kommunikation der regionalen Akteure zurückgreifen können. Das heißt, das Entwicklungsteam müsste nicht nur den Bedarf nachweisen, sondern stärker auf die angestrebten Ergebnisse der gesamten Tätigkeit und ihre Vernetzung in einem regionalen Raum (der sehr unterschiedlich ausgedehnt sein kann) abstellen.

\subsubsection{Internationalisierung}

Hinsichtlich der Internationalisierung scheinen die Anforderungen und die erforderlichen Investitionen durch den Sektor noch etwas unterschätzt zu werden. 
- Auch aus der Perspektive günstiger Rahmenbedingungen für eine offensive Internationalisierungsstrategie ergibt sich die Empfehlung einer Konsolidierung der Standorte, einer thematischen Erweiterung des Studienangebots und eines Ausbaus der Forschungskapazität. Die Erwartungen hinsichtlich der Internationalisierung sollten stärker in den Zielkatalog aufgenommen und spezifiziert werden.

- Hinsichtlich der Implementierung der neuen Studienarchitektur sollte der FH-Sektor eine offensive Strategie wählen. Diese neue Architektur wird sich europaweit durchsetzen und der FH-Sektor hat die Chance, seine Rolle als Reformmotor aufrecht zu erhalten.

- Alle Ungleichbehandlungen des FH-Sektors bei der Förderung akademischer Mobilität im Vergleich zu den Universitäten sollten eliminiert werden.

\subsubsection{Qualitätssicherung}

Das System der Qualitätssicherung ist insgesamt ein Erfolgsmodell im Fachhochschulsektor. Die Weiterentwicklung der Qualitätssicherung kann sich vor allem in zwei Richtungen bewegen:

- Es wird dem Aspekt der Accountability und der Informationsfunktion von Qualitätssicherungsmaßnahmen größeres Gewicht gegeben. Der Sektor selbst, der Fachhochschulrat, hat eine Veröffentlichung der Evaluationsergebnisse beschlossen. Weiters wird angeregt, das Hochschulranking des CHE, das ab kommendem Wintersemester für die österreichischen Universitäten durchgeführt wird (das ein ausgewogenes Verfahren von Gruppenranking bestimmter Fachgruppen ist, in einem ausgewogenen Methodenmix) eventuell in angepasster Form auch auf den Fachhochschulsektor auszudehnen. Die Informationsfunktion der Qualitätssicherung ist ein wichtiger Aspekt in einem System, das stark auf Kundenzufriedenheit, Reaktion auf Bedarf und Nachfrage beruht. Hier soll man die Kunden- und Nachfrageseite auch mit den entsprechenden Informationen versorgen. Bevor das wie derzeit hauptsächlich unter Marketinggesichtspunkten der FH-Institutionen erfolgt, sollten Informationen auch in einem methodisch sauberen Verfahren ermittelt werden.

- Der formativ unterstützende Aspekt der Qualitätssicherung wird stärker mit Personal- und Organisationsentwicklung verbunden. Hier gibt es schon erste unterstützende Aktivitäten im $\mathrm{FH}-S e k t o r$ und die fachhochschulischen Einrichtungen selbst werden zunehmend sensibel für die Aspekte der Personal- und Organisationsentwicklung. 



\section{Zusammenfassung des nationalen und internationalen Feedback-Prozesses}

Der Rohbericht wurde von internationalen und nationalen Expertlnnen kommentiert und an alle Erhalter und StudiengangsleiterInnen sowie die InterviewpartnerInnen mit dem Ersuchen um Kommentare ausgesendet. Dieses Verfahren war bis zu einem gewissen Grad ein Wagnis, da nicht vorhersehbar war, wie viele wie ausführliche Stellungnahmen wir bekommen würden und ob diese noch überblickbar sein würden - das Feedback war jedoch nicht sehr lebhaft. Neben den Stellungnahmen der FHK und der Vertreter der Bundesländer, ${ }^{206}$ die sich auf den Bericht insgesamt bezogen, sind einige individuelle Stellungnahmen zu spezifischen Aspekten eingelangt. ${ }^{207}$ Vereinzelt fand sich auch "die Lust, Fehler zu erkennen und anzuzeichnen". Außerdem wurde der Rohbericht im Rahmen des Projektbeirates diskutiert, wo auch VertreterInnen des FHR und der Geschäftstelle die Gelegenheit für kritische Kommentare genützt haben. Im Anschluss an den FeedbackProzess wurde der Bericht, insbesondere Schlussfolgerungen und Empfehlungen, nochmals überarbeitet.

\subsection{Ausgewählte Themen}

Im folgenden werden einige zentrale Themen, Kritikpunkte und Anregungen aus dem Feedback-Verfahren zusammengefasst und aus der Sicht des Projektteams diskutiert.

\subsubsection{Strategische Planung und Steuerung, politisches Gesamtkonzept für den tertiären Sektor}

Ein wesentlicher Schwerpunkt der Stellungnahmen im Feedback bezieht sich auf die Fragen der Gesamtsteuerung des FH-Sektors. Die Analysen und Schlussfolgerungen des Berichtes wurden unterstützt bzw. verstärkt. Die folgenden Aspekte wurden besonders unterstrichen:

- Die strategische Planung und Steuerung des FH-Sektors sollte in den Rahmen eines Gesamtkonzeptes für die Entwicklung des tertiären Sektors gestellt werden, das

206 Die formellen Stellungnahmen sowie eine Zusammenfassung der Diskussion mit den internationalen Experten werden im Anhang dokumentiert.

207 Angesprochene Punkte waren: Erschwernisse im FH-Bereich gegenüber den Universitäten im Bereich der Internationalisierung (z.B. bei der Anbahnung von Kontakten, bei außereuropäischen Joint-study-Programmen); das Verhältnis zwischen den Erhaltern und dem FH-Kollegium; bildungspolitische Koordination im Verhältnis zwischen FH-Sektor und Universitäten. 
einerseits Leitlinien für wichtige Eckdaten für den tertiären Sektor und andererseits eine klare Abgrenzung der Profile und Aufgaben von Universitäten und FH-Sektor sowie eine Konzeption der Arbeitsteilung und Zusammenarbeit enthalten sollte. Insbesondere die Notwendigkeit von produktiven Lösungsansätzen für die widersprüchliche Relation von Wettbewerb und Kooperation im Gesamtsystem wird betont. In die Entwicklung dieses Gesamtkonzeptes sollten die verschiedenen Akteure (Länder, Sozialpartner, Vertreter der Bildungsinstitutionen etc..) eingebunden sein und der Prozess sollte transparent ablaufen. Hinsichtlich geeigneter Strukturen für diesen Prozess wurden unterschiedliche Aspekte betont, vor allem die Funktionen von Meinungsbildung und Beratung einerseits und Entscheidung andererseits wurden angesprochen. Ersteres sollte in einem möglichst breiten Rahmen erfolgen, während für die Entscheidungen ein „schlankes und effektives Organ" erforderlich ist.

- Die strategische Planung und Steuerung für den FH-Sektor selbst sollte intensiviert und verbessert werden. ${ }^{208}$ Als wichtiger Aspekt wurde herausgearbeitet, dass die Funktionen der politischen Steuerung und der Qualitätssicherung deutlicher unterschieden werden sollten und klarere und effektivere Entscheidungsstrukturen für die politische Steuerung entwickelt werden sollten. Es wurde geäußert, dass der Ort der politischen Entscheidungen nicht klar sichtbar sei und dass keine ausreichenden Kapazitäten dafür vorhanden seien. Auch wurde der Punkt einer Verstärkung der Zielsteuerung unterstrichen. Dies erfordert die Formulierung und Konkretisierung von Ergebniszielen für die verschiedenen Aufgabenbereiche und die Etablierung von Mechanismen zur Überprüfung der Zielerfüllung auf Sektorebene, nicht nur auf Ebene der einzelnen Institutionen. Eine strategische Planung des Studienangebotes wurde eingefordert, die stärker als bisher ihre Überlegungen auf eine übergreifende Koordination für Gesamtösterreich beziehen und die Internationalisierung entsprechend berücksichtigen sollte.

- Die Verwirklichung einer strategischen Konzeption erfordert Rahmenbedingungen, die entsprechende Rechts- und Planungssicherheit gewährleisten. Für die umfangreichen regulatorischen Materialien, die neben dem „schlanken“ Gesetz seitens des FHR entstanden sind, wird eine übersichtliche Re-regulierung eingefordert und es wird die Frage nach "gleichen Spielregeln“ für den gesamten tertiären Sektor gestellt. und Finanzierungsplan III) im Sinne erweiterter strategischer bildungspolitischer Zielvorgaben (S.189 ff. der Rohfassung; Abschnitt 4.3.2 ab Seite 178 der Endfassung) bedeuten eine wesentliche Verbesserung des 
- Man muss sich bewusst sein, dass kein Steuerungsmodell perfekt funktioniert und dass der wesentliche Faktor für eine wirksame Steuerung in der Tragfähigkeit der Beziehungen zwischen den Institutionen und der politischen Ebene liegt - „time and again the modern state stumbles over the academic system" (Burton Clark).

\subsubsection{Verhältnis von politischer Steuerung und Qualitätssicherung, organisatorische Steuerungs- und Behördenstruktur, Funktion und Konstruktion des FHR}

Auf organisatorischer Ebene wurden verschiedene Aspekte der Beziehungen zwischen den beteiligten Akteuren (Bund, Länder, FHR, Erhalter, FH-Studiengänge und FH-Kollegien) sowie die Struktur und Arbeitsweise des FHR und die Bedeutung der Anerkennung von Fachhochschulen in diesem Akteursgeflecht angesprochen. Teilweise wurde kritisiert, dass die Analyse der Rollen der Akteure und ihrer Tätigkeitsfelder sowie der Stärken und Schwächen ihrer Aktivitäten zu unscharf geblieben ist.

- Einen besonderen Diskussionspunkt bildet das Verhältnis zwischen politischer Steuerung („das Richtige tun“, Frage was? Festlegung der Ziele) und Qualitätssicherung („es richtig tun, Frage wie? Überprüfung der Leistungen zur Zielerfüllung). Der Befund unzureichend konkretisierter Zielformulierungen von der politischen Ebene wurde im Feedback mehrfach bekräftigt und auch als zentrales Ergebnis hervorgehoben. ${ }^{209}$ Die Effektivität der Zielsteuerung wird dadurch beeinträchtigt und es entstehen Unschärfen in der Zielbildung (Wie entstehen die Ziele? Wer formuliert sie?), der Aufgabenerfüllung (welche Aufgaben muss eine $\mathrm{FH}$ oder ein Studiengang erfüllen?) und Schwierigkeiten der Überprüfung der Zielerfüllung (Wie wird die Zielerfüllung gemessen?). Wenn keine ausreichende Zielformulierung gegeben ist, verschiebt sich die politische Steuerung auf die Qualitätssicherung (da Qualität nur gegen Ziele erfasst werden kann). Es werden für den neuen Entwicklungsplan deutlichere politische Festlegungen seitens des Bundes moniert.

- Zwischen den Akteuren sei eine klarere Aufgabenabgrenzung erforderlich, insbesondere der FHR habe ein spannungsreiches Aufgabenprofil entwickelt, in dem sich die Funktionen der Qualitätssicherung und der politischen Steuerung vermischen. Die starke

Steuerungs-, Koordinations- und Kontrollsystems für den FH-Sektor im Rahmen des gesamten tertiären Bildungsbereiches; sie stellen zugleich den Kern des Ertrags der Untersuchung dar" (Feedback Bodenhöfer).

209 „Interessant fand ich die Überlegungen zur Gestaltung des Aufgabenprofils künftiger FH's. Die Frage nach dem Umfang der Leistungen bzw. des Portfolios der FH's wurde aus meiner Sicht in dieser Direktheit noch nie 
Vertretung des Universitätssektors im FHR ohne entsprechende Reziprozität wird kritisiert. Die Bundesländer haben in ihrem Bereich einen bestimmenden Einfluß auf die Entwicklung im FH-Sektor, sind jedoch in das Steuerungssystem formell nicht klar einbezogen. Die Erhalter haben teilweise sehr komplexe Strukturen und sind in unterschiedlicher Weise mit den Bundesländern verflochten. Es wurde auch von verschiedenen Aspekten her betont, dass die akademische Vertretung in diesem Geflecht auf verschiedenen Ebene zu schwach erscheint (in den FHK vermischen sich die Interessen der Erhalter mit den akademischen Interessen; im Verhältnis zwischen Erhaltern und Kollegien wird eine Stärkung der Kollegien moniert).

- Über einige Probleme in der Struktur und Aufgabenstellung des FHR besteht in den Stellungnahmen Einigkeit, nicht jedoch über die angesprochenen Lösungsansätze für die Auflösung der Spannung zwischen politischer Entscheidung und Qualitätssicherung. Insbesondere auf zwei Probleme wurde hingewiesen: Erstens, dass Strukturen die für eine breite und inklusive Meinungsbildung geeignet sind, nicht unbedingt auch für das Treffen von Entscheidungen geeignet sind; zweitens, dass zwar einerseits eine Trennung zwischen politischer Steuerung und Qualitätssicherung nötig ist, dass aber andererseits diese beiden Funktionen auch zusammenspielen müssen, damit nicht eine Situation entsteht, wo „das Falsche richtig gemacht wird“.

- Im Bereich der Qualitätssicherung wird die Befürwortung von Rankings, bzw. die Veröffentlichung von Ergebnissen von Evaluierungen teilweise sehr kritisch gesehen, da diese in den (als ungleich eingeschätzten) Wettbewerbsbedingungen missbraucht werden können. Entsprechende Regelungen müssten jedenfalls für alle Marktteilnehmer gleichermaßen gelten.

\subsubsection{Entwicklungspfade, „Bild“ und Aufgabenstruktur der Fachhochschule}

Die Rückmeldungen zu den verschiedenen Entwicklungspfaden haben diese als Ausgangspunkt für weitere Überlegungen begrüßt und eine klare Verbindung zur Bestimmung des Aufgabenportfolios der FHs hergestellt. Verschiedentlich wurde eine intensivere Herausarbeitung der Implikationen gefordert. Hinsichtlich der formulierten Entwicklungspfade wurden im Feedback unterschiedliche Positionen entwickelt: 
- Seitens der FHK wurde für ein verbessertes STATUS-QUO-Modell votiert, wobei vor allem ein Abgehen von der Nischenpolitik und ein erweitertes Leistungsangebot als ergänzende Kriterien angeführt werden. Die Gestaltung des Aufgabenportfolios wird als zu klärende Vorfrage hervorgehoben, wobei eine Erweiterung des Aufgabenspektrums mit entsprechenden Revisionen des Finanzierungsmodells verbunden sein muss.

- Seitens der ExpertInnen wurde für einen Entwicklungspfad im Bereich zwischen dem Modell INTENSIVEN WACHSTUMS und der FTE-STRATEGIE votiert, wobei die Konsolidierung und die Erreichung kritischer Größen besonders hervorgehoben wurde.

- Keine Unterstützung wurde geäußert für die Modelle des NULL-WACHSTUMS und des EXTENSIVEN WACHSTUMS mit weiterer Regionalisierung; aber auch gegenüber einer massiven Stärkung des FH-Sektors im Sinne der Forschungsstrategie wurde in den österreichischen Stellungnahmen eine eher vorsichtige Stellung eingenommen (die internationalen Experten haben hier eine deutlich offensivere Haltung vertreten).

- Zu den Entwicklungspfaden, die sich auf die Bildungs- und Qualifikationsnachfrage beziehen (SOCIAL DEMAND, ECONOMIC DEMAND) gibt es weniger Bezugnahmen.

Welcher Entwicklungspfad eingeschlagen wird bestimmt sich wesentlich dadurch, wie sich das Aufgabenprofil für die FH-Institutionen entwickelt. Die Frage, ob die große vorhandene Diversität durch ein stärker kontouriertes "Bild" einer FH abgelöst werden soll, hat im Feedback wenig ausdrückliche Resonanz gefunden. Aus dem Sektor wurde eher betont, dass die Aufgabenbestimmung mit den Möglichkeiten und Ressourcen in Einklang stehen müsse, und es wurde die Gefahr der Überforderung betont - die ExpertInnen haben stärker eine weitergehende Aufgabenbestimmung befürwortet, in Richtung international wettbewerbsfähiger Hochschulinstitutionen.

An der Frage der näheren Umschreibung des „Bildes“ und der Aufgabenstruktur einer FH bündeln sich also viele wesentliche Entwicklungsfragen in inhaltlicher und organisatorischer Hinsicht. Es stellt sich jedoch nicht nur die Frage, wie das Bild einer FH aussehen soll, es stellt sich auch die Frage, wie darüber bestimmt werden soll. Auf welcher Ebene soll das festgelegt werden, welche Rolle soll die FH als akademische Institution bei der Festlegung ihres Aufgabenprofils spielen? Dazu gab es indirekte und auch ausdrückliche Stellungnahmen. Bisher ist dies ein hybrider und emergenter Prozess, in dem die verschiedenen Akteure, teilweise in unterschiedlichen Zusammenhängen und in mehrfachen Rollen zusammenwirken. Die starke Betonung der Top-down Komponente von politischer Planung und Steuerung, insbesondere der Wunsch nach der Aufgabenabgrenzung zwischen Universitäten und $\mathrm{FH}$-Sektor könnte implizit nahe legen, dass eine nähere Aufgabenbestimmung auf dieser Ebene erfolgen sollte. Insbesondere die internationalen 
Experten haben jedoch eher die Autonomie der Fachhochschulen betont. Die Kollegien sollten gestärkt werden und über entsprechende Mechanismen wie beispielsweise mittelfristige Aufgaben- und Investitionspläne in den von innen angestrebten Entwicklungen gefördert werden.

\subsubsection{Finanzierung und Kostenstrukturen}

Nähere Analysen zur Finanzierung und zu den Kostenstrukturen im FH-Sektor waren aus dem Auftrag ausgeklammert bzw. konnten aufgrund der fehlenden Transparenz der Informationsbasis nicht durchgeführt werden, wurden aber im Feedback nachdrücklich eingefordert.

Die Verlegung der Finanzierung von den Studiengängen auf die institutionelle Ebene wurde im Prinzip befürwortet. Bei einer Erweiterung des Aufgabenportfolios müssen entsprechende Vorkehrungen für die Finanzierung getroffen werden. Ebenso wurde unterstützt, dass für die Erreichung spezieller Ziele entsprechende finanzielle Förderungen und Anreize entwickelt werden sollten.

Seitens der internationalen Experten wurde für eine komplexere Formel votiert, bzw. für die Möglichkeit der Finanzierung von Investitionsplänen. Von österreichischer Seite wurde zumindest eine Indexierung der Bundesbeiträge moniert.

\subsubsection{Anregungen für den EFP III, Standortplanung}

Für den nächsten Entwicklungs- und Finanzierungsplan wurden im Feedback verschiedene politische Festlegungen eingemahnt, insbesondere eine überregional orientierte strategische Planung des Studienangebotes und Klarstellungen über das Verhältnis zu Universitäten und BHS, klare Festlegungen hinsichtlich der Prioritäten für Konsolidierung oder Regionalisierung sowie Definition von Standortkriterien.

\subsection{Wertung des Berichtes und seiner Rolle für die weitere Entwicklung des Sektors}

Das Feedback hat unterschiedliche Wertungen des Berichtes hervorgebracht, die teilweise mit unterschiedlichen Erwartungen zusammenhängen. 
- Einerseits wird in vielen Punkten Enttäuschung darüber geäußert, dass der Bericht nicht zu definitiveren Empfehlungen geführt hat, insbesondere im Hinblick auf die Wahl zwischen den verschiedenen Entwicklungspfaden. Diese Kritik ist in verschiedener Hinsicht zu relativieren. Erstens, im Hinblick auf die Möglichkeiten, die der methodische Ansatz eines Reviews grundsätzlich bietet. Dieser Ansatz spiegelt im Prinzip - in durch das ExpertInnenwissen der ReviewerInnen gefilterter bzw. angereicherter Weise - die verschiedenen vorhandenen Positionen und Ansätze zurück, ordnet diese systematisch an usw. Vorhandenes „implizites Wissen“ wird „explizit und gebündelt einer breiteren Öffentlichkeit als Entscheidungsgrundlage für die Weiterentwicklung des FH-Sektors zugänglich gemacht“, wie es in einem Beitrag zum Feedback heißt. Zweitens im Hinblick auf die grundsätzliche Möglichkeit, in einem derart komplexen System mit diffusen Zielbestimmungen und unterschiedlichen Optionen der Einbindung in das Umfeld „wissenschaftlich gesicherte“ Empfehlungen abzugeben. Es ist klar, dass die Wahl einer Option von weitergehenden politischen Entscheidungen im Umfeld abhängt. Der FHSektor kann beispielsweise nur sinnvoll in eine offensive Innovationsstrategie eingebunden werden, wenn diese insgesamt gefahren wird - ebenso kann nicht ausgeschlossen werden, dass im Falle einer kleinräumigen Regionalisierungsstrategie in bestimmtem Rahmen positive Effekte erzielt werden können, die die (vergleichsweise kleineren) Investitionen mit entsprechenden Effekten rechtfertigen. Es werden insgesamt andere Effekte zu erwarten sein und andere Zielsetzungen bedient. Drittens, ist die Enttäuschung insofern berechtigt, als die Implikationen der Entwicklungspfade im Prinzip rigoroser herausgearbeitet werden können. Aufgrund der vielfältigen Aufgabenstellung bei begrenztem Budget und Zeitrahmen sowie der teilweise völlig unzureichenden Datenbasis war dies im vorliegenden Projekt nicht möglich.

- Andererseits wird die breite Präsentation der vielfältigen Ergebnisse und die Offenheit der Optionen aber auch als Stärke gewertet, indem das Material im FH-Sektor selbst und in der bildungspolitischen Öffentlichkeit als Grundlage für eine weitere Diskussion über die Entwicklungsmöglichkeiten des österreichischen Fachhochschulsystems genutzt werden kann. Viele der angesprochenen Fragen betreffen Weichenstellungen, die weiterer Überlegungen und vor allem auch weiterer Prozesse der Konsensfindung bedürfen. Der Entwicklungs- und Finanzierungsplan III sollte entsprechende Entwicklungsmöglichkeiten jedenfalls nicht verschließen. 


\subsection{Zusammenfassung kontroverser Punkte}

Insgesamt wurden die Kernaussagen insbesondere $\mathrm{zu}$ den übergreifenden Schlussfolgerungen im Feedbackprozess in geringerem Masse in Frage gestellt, als wir erwartet haben. Insbesondere in folgenden Punkten sind unterschiedliche oder kontroverse Einschätzungen zu verzeichnen:

- Teilweise wurde angemerkt, dass den Analysen und Schlussfolgerungen zu hohe Erwartungen in Planungs- und Steuerungsaktivitäten zugrunde liegen.

- Es wurde insbesondere von den internationalen Experten angemerkt, dass ein sehr enger Ansatz der Analyse der Internationalisierung, konzentriert auf den Austausch von Studierenden gewählt wurde. Es sollte jedoch auch dem Austausch von Personal und der Kooperation in F\&E gleichermaßen Gewicht beigemessen werden. Auch wurde die Frage aufgeworfen, inwieweit die Internationalisierung der Qualitätssicherung neben der Vereinheitlichung der Studienstruktur im Bologna-Prozess multiplikative Wirkungen der Dynamisierung von Internationalisierungsprozessen entfalten wird. Strategien der Internationalisierung sollten daher auch im Akkreditierungs-Prozess berücksichtigt sein.

- Es bestehen kontroverse Positionen zur Rolle und Gewichtung der Bundesländer in den Steuerungsstrukturen. Einerseits haben sie sich als wesentliche Akteure in diesem System etabliert, andererseits wird befürchtet, dass lokalen und kleinräumigen Interessen gegenüber übergreifenden Gesichtspunkten ein zu hohes Gewicht zukommt. Die Notwendigkeit einer Systematisierung der Entwicklungsplanung auf Bundesländerebene wird auch im Feedbackprozess bekräftigt.

- Es bestehen unterschiedliche Positionen über die präferierten Entwicklungspfade, insbesondere darüber mit welchem Nachdruck die Konsolidierung betrieben werden soll und in welchem Ausmaß sie mit weiterem Wachstum und einer Erweiterung des Aufgabenspektrums verbunden werden soll.

\subsection{Offene Punkte, die dringend näher behandelt werden sollten}

Im Feedbackprozess wurden einige wichtige offene Punkte bzw. Problembereiche angesprochen, die teilweise aus der vorliegenden Untersuchung bewusst ausgeklammert waren und teilweise aufgrund der thematischen Schwerpunktsetzung nicht oder nicht mit entsprechendem Gewicht behandelt wurden. Teilweise wurden damit im Feedback auch 
Fragen verstärkt, die bereits als Entwicklungsbedarf herausgearbeitet wurden. Diese Punkte werden kurz angeführt:

- Die Analysen des Lehrpersonals und der personellen Potentiale haben bestimmte Grundparameter herausgearbeitet, es sind aber bestenfalls grobe Umrisse des Personaleinsatzes sichtbar geworden.

- Weiterentwicklung der Studienprofile, z.B. Deutlichere Profilierung der praxisnahen Studien auf Hochschulniveau, Breite der Ausbildungsprofile, Verhältnis von Grundlagen und Spezialisierung, Flexibilität in Bezug auf die Dynamik der Arbeitswelt.

- Weiterentwicklung der Bedarfs- und Akzeptanzanalysen, intensivere Analysen von Karrierepfaden und Erträgen aus der Ausbildung.

- Im Bereich der Kosten und Finanzierung sind viele Fragen offen geblieben, insbesondere die Höhe der Normkosten, aber auch insgesamt der Herstellung von Transparenz des Mitteleinsatzes.

- Die Beziehung zur Sekundarstufe, insbesondere zum Bereich der BHS, sowohl auf der Seite der Ausbildungsprofile, als auch auf der Seite des Qualifikationsbedarfes ist offen geblieben.

- Zur Frage der „kritischen Größe“ sind nur ansatzweise Ergebnisse entwickelt worden, nähere Standortanalysen könnten hier wichtige weitere Aufschlüsse erbringen. 



\section{Literaturverzeichnis}

Altbach Philip (2000), Die Messung von Lehr- und Lernleistungen, in: Schwarz Stefanie, Teichler Ulrich (2000), Credits an deutschen Hochschulen. Kleine Einheiten - große Wirkungen, Luchterhand, Neuwied, 79-89

Altrichter Herbert, Posch Peter (1995), Begleitende Evaluation der Implementation des FHStG - Teil 3: Profile und Entwicklungsprozesse der neuen Studienprogramme. Kurzfassung von Teil III: Kritische Felder für die Weiterentwicklung des Fachhochschulsektors

Altrichter Herbert, Posch Peter, Thaler Michaela (1995), Begleitende Evaluation der Implementation des FHStG Teil 3: Profile und Entwicklungsprozesse der neuen Studienprogramme. Projektbericht 1 (Inhaltsanalyse der Anträge für Fachhochschul-Studiengänge), Studie im Auftrag des BMWFK, Innsbruck/Klagenfurt

Altrichter Herbert, Posch Peter, Thaler Michaela (1995), Begleitende Evaluation der Implementation des FHStG Teil 3: Profile und Entwicklungsprozesse der neuen Studienprogramme. Abschlußbericht (Teil II: Porträts zweier Studiengänge, Teil III: Kritische Felder für die Weiterentwicklung des Fachhochschulsektors), Studie im Auftrag des BMWFK, Innsbruck/Klagenfurt

Altrichter Herbert, Schratz Michael (Hrsg.) (1992), Qualität der Universitäten, Österreichischer StudienVerlag, Innsbruck

Amt der Tiroler Landesregierung (2002), Fachhochschulen und Fachhochschul-Studiengänge in Tirol - Bericht und Konzept, Stand 1. März 2002

Astleitner Hermann, Sindler Alexandra (1999), Pädagogische Grundlagen virtueller Ausbildung - Telelernen im Fachhochschulbereich, WUV-Verlag, Wien

Austrian Council (2002), Nationaler Forschungs- und Innovationsplan, Wien (http://www.ratfte.at/files/NFIP_20021203.pdf)

Badelt Christoph, Leichsenring Kai (1998), Analyse und mögliche Neustrukturierung der Ausbildungen im Sozialbereich, Studie im Auftrag des BMUK und des BMWV, Wien

Benson L. (2000), Regionalwirtschaftliche Effekte von Hochschulen während ihrer Leistungsabgabe. Theoretische Analyse und methodische Überlegungen zur Empirie. TAURUS Materialien Nr. 7, Trier

Berka Walter (1998), Autonomie im Bildungswesen - Eine Untersuchung zu den juristischen Konsequenzen des Autonomiebegriffs im Bildungswesen, Forschungsbericht im Auftrag des BMWV, Salzburg

Berryman S. E., Bailey T. R. (1992), The Double Helix of Education and the Economy, Institute on Education and the Economy, Teachers College (Columbia), New York

Blumberger Walter, Poller Bettina (1992), Brennpunkte der bildungspolitischen Reformdiskussion. Teil II Fachhochschulen in Österreich - Bildungspolitische Positionen und kritisches Resümee, Studie im Auftrag der Kammer für Arbeiter und Angestellte für Oberösterreich, Linz

BMAGS (Bundesministerium für Arbeit, Gesundheit und Soziales) (1999), Endbericht der Unterarbeitsgruppe "Gesundheitsberufe“ im Rahmen der Arbeitsgruppe zur Entwicklung neuer Berufsbilder im Bereich von Sozial-, Gesundheits- und Freizeitberufen, Wien

BMBF (Bundesministerium für Bildung und Forschung) (2001), Grund- und Strukturdaten 2000/2001, Bonn

BMBWK (1996), Disfunktionalitäten des Studienangebots der Universitäten; (Unvollständige) Kopie eines internen Arbeitspapiers

BMBWK (2000), Entwicklung, Struktur und Planung des Studienangebots der österreichischen Universitäten und Fachhochschulen, Wien

BMBWK (2001), Regionalisierung im Fachhochschulbereich - Tagungsbericht, Wien

BMBWK (2001a), Erkennung und Erhebung des Qualifikationsbedarfes. Ergebnisprotokoll des Koordinationsworkshops, Wien (http://www.lebenslangeslernen.at/ > Forum \& Workshops > Workshops >)

BMBWK (2001b): Österreichischer Länderbericht zum Memorandum über Lebenslanges Lernen der Europäischen Kommission, Wien (download: http://www.lebenslangeslernen.at/ > Ergebnisse)

BMBWK (2001c): Hintergrundbericht zum Österreichischen Länderbericht. Memorandum über Lebenslanges Lernen der Europäischen Kommission, Wien (download: http://www.lebenslangeslernen.at/ > Ergebnisse)

BMBWK (2002), Hochschulbericht 2002, Wien

BMBWK, Fachhochschulrat und Österreichische Fachhochschul-Konferenz (Hrsg.) (o.J.), FachhochschulStudiengänge in Österreich - Studiengänge 2002, Wien

BMBWK, Fachhochschulrat und Österreichische Fachhochschul-Konferenz (Hrsg.) (2001), Handbuch Finanzdatenerfassung, Wien 
BMBWK, BMVIT (2001), Forschungs- und Technologiebericht 2001, Wien (http://www.bmbwk.gv.at/medien/3358_fb2001.pdf).

BMBWK, BMVIT (2002), Österreichischer Forschungs- und Technologiebericht 2002 (http://www.bmbwk.gv.at/medien/7246_ftb02.pdf)

BMU (Bundesministerium für Unterricht, Hrsg.) (1967), Bildungsplanung in Österreich - Band 1 Erziehungsplanung und Wirtschaftswachstum, Bericht an die OECD, Wien

BMWF (1992), Fachhochschule als Alternative zur Universität. Beiträge zum Seminar vom 25./26. November 1991 in Wien, Materialien zur Bildungspolitik 1, Wien

BMWF (1992a), Das österreichische Bildungssystem in Veränderung. Bericht an die OECD über die geplante Diversifikation des Postsekundarsektors, Wien

BMWF (1994), Entwicklungs- und Finanzierungsplanung für den Fachhochschulbereich („FHS-Entwicklungsplan I“)

BMWF, BMUK (Hrsg.) (1993), Reform des Österreichischen Postsekundarbereiches. OECD-Länderprüfung: Bericht der Prüfer

BMWFK (1995), Follow-up to the Review of Austria, Vienna

BMWFK, Abt. I/B/17 (1995), Anträge auf Anerkennung von Studiengängen als Fachhochschul-Studiengänge (Beginn WS 1995/96); aktenmäßige Erledigung GZ 51.002/63-1/B/17/95 vom 28.3. 1995

BMWFK, Abt. I/B/17 (1996), Anträge auf Anerkennung von Studiengängen als Fachhochschul-Studiengänge, 1996/97 und Stellungnahme zur Förderungsentscheidung

BMWFK, BMUkA (Hrsg.) (1995), Zwei Jahre FHStG. Bericht über Stand und Perspektiven des Fachhochschulsektors sowie über die Möglichkeiten einer Nachqualifizierung von AbsolventInnen berufsbildender Schulen, Wien

BMWV (Hrsg.) (1998), Die Beschäftigung von Hochschulabsolventinnen und Hochschulabsolventen in Österreich. Österreichischer Beitrag zur Tagung der Generaldirektoren für Hochschulwesen und Vorsitzenden der Rektorenkonferenzen am 28. und 29. Oktober 1998 in Wien

BMWV (1998), Weißbuch zur Hochschulbildung in Österreich (http://www.bmbwk.gv.at/medien/5150_wbw2.pdf)

BMWV (1999), Entwicklungs- und Finanzierungsplanung für den Fachhochschulbereich II 2000/01-2004/05 („FHS-Entwicklungsplan II“)

BMWV (1999a), Hochschulbericht 1999, Wien

BMWV, Forschungsförderungsfonds (o.J.), Impulsaktion „Kooperation Fachhochschulen Wirtschaft“ des BMWV und des Forschungsförderungsfonds, Homepage-Info (www.bmwf.gv.at)

Booth A. L., Snower D. J. (Eds) (1996), Acquiring Skills. Market Failures, their Symptoms and Policy Responces, Cambridge University Press, Cambridge

Braczyk H-J. , Cooke P., Heidenreich M. (Eds) (1998), Regional innovation systems, UCL Press, London

Breinbauer Ines M. (1996), Fachhochschulen - Elitesegment des österreichischen Hochschulsystems? in: Österreichisches Jahrbuch für Politik 1995 (S. 637 - 650), Wien

Brinek Gertrude, Schirlbauer Alfred (Hrsg.) (1996), Vom Sinn und Unsinn der Hochschuldidaktik, WUVUniversitätsverlag, Wien

Brown Phillip, Green Andy, Lauder Hugh (2001), High Skills, Globalization, Competitiveness, and Skill Formation. Oxford University Press, Oxford

CEDEFOP (2000), Towards the learning region. Education and regional innovation in the European Union and the United States, OOP-EC, Luxembourg

CHE (Centrum für Hochschulentwicklung), CHEPS (Center for Higher Education Policy Studies) (2002), Die Einführung von Bachelor- und Masterprogrammen an deutschen Hochschulen (Zusammenfassung der Ergebnisse), Studie im Auftrag des DAAD

Cerych Ladislav, Sabatier Paul (1986), Great Expectations and Mixed Performance. The Implementation of Higher Education Reforms in Europe, Trentham Books, Stoke-on-Trent

Clark Burton R. (1983), The Higher Education System. Academic Organization in Cross-National Perspective, Berkeley

Clark Burton R. (1998), Creating Entrepreneural Universities. Organizational Pathways of Transformation, Pergamon, Oxford

Clement Werner (1991), Towards non-university institutions in Austria. In: BMWF (1992), Fachhochschule als Alternative zur Universität. Beiträge zum Seminar vom 25./26.November 1991. Materialien zur Bildungspolitik 1, Wien 
Clement Werner (2002), Innovationspolitische Gestaltungsempfehlungen für Regionen. Vortragsmaterialien zur innoregio-Styria-Veranstaltung „Regionale Innovationspolitik auf dem Prüfstand“, Graz

Clement Werner, Detter Helmut, Fröhlich Josef, Turnheim Georg, et al. (2002), Basiskonzept zur Umsetzung des Zieles „Wachstumsschub in der angewandten Forschung und Entwicklung durch Stärkung der außeruniversitären Forschung und Entwicklung“, Endbericht einer Studie im Auftrag des Rates für Forschung und Technologieentwicklung und des BMVIT, Wien

Commissie Accreditatie Hoger Onderwijs (2001), Prikkelen presteren profileren: Eindrapport, Van de Bunt, Amsterdam

La politique de l'éducation tertiaire suisse. Rapport national de la Suisse, remis à l'OCDE dans le cadre du projet , L'examen de la politique tertiaire suisse 2001/2002 par l'OCDE“" (http://www.edk.ch/ PDF_Downloads/ oecd_tert)

Delapina Franz, Holzinger Elisabeth, Schausberger Bernhard (2001), Verfahren der Standortprüfung für Fachhochschulstudiengänge; Forschungsbericht des Österreichischen Instituts für Raumplanung, Wien

Dell'mour Rene, Landler Frank (2002), Hochschulplanungsprognose 2002, Endbericht des Instituts für Demographie der Österreichischen Akademie der Wissenschaften, im Auftrag des BMBWK, Wien

Detter Helmut et al. (2002), „Fachhochschulsektor und FH-orientierte F\&E-Zentren / Programme“ (Teilstudie Bereich 4 zur Studie „Basiskonzept zur Umsetzung des Zieles ,Wachstumsschub in der angewandten Forschung und Entwicklung durch Stärkung der außeruniversitären Forschung und Entwicklung“" des Rates für Forschung und Technologieentwicklung und des BMVIT), Endbericht, Wr. Neustadt

DETYA (Department of Education, Training and Youth Affairs) Australia (2000), Learning for the knowledge society: An education and training action plan for the information economy (http://www.dest.gov.au/edu/edactplan.htm).

Dorninger Christian, Koller Ernst (Hrsg.) (1995), Fachhochschulen als Innovationspotential in der österreichischen Bildungslandschaft? Erste Erfahrungen mit einem neuen Ausbildungssektor; Heft 3-4/1995 der Zeitschrift für Hochschuldidaktik

Dubs Rolf (1995), Konstruktivismus. Einige Überlegungen aus der Sicht der Unterrichtsgestaltung, in: Zeitschrift für Pädagogik 41(1995), 889 - 903

Eder Ferdinand et. al (Hrsg.) (2002), Qualitätsentwicklung und Qualitätssicherung im österreichischen Schulwesen. Bildungsforschung 17 des BMBWK, StudienVerlag, Innsbruck

EFHK (Eidgenössische Fachhochschulkommission) (2000), Zwischenbericht über die Schaffung der Fachhochschulen - Stand der Aufbauarbeiten und Erfüllungsgrad der Auflagen des Bundesrates

EFHK (Eidgenössische Fachhochschulkommission) (2002), Fachhochschulen 2002. Bericht über die Schaffung der Schweizer Fachhochschulen, Bern (http://www.bbt.admin.ch/fachhoch/publi/d/bericht170602.pdf)

Europäische Kommission (2000) Hin zu einem europäischen Forschungsraum, Mitteilung der Kommission, $\operatorname{KOM}(2000) 6$ (ftp://ftp.cordis.lu/pub/documents_r5/natdir0000001/s_1372005_20010125_143514_C001190de.pdf)

Europäische Kommission (2002), Allgemeine und berufliche Bildung in Europa. Unterschiedliche Systeme, gemeinsame Ziele für 2010. Arbeitsprogramm, AAV-EU, Luxemburg (http://europa.eu.int/comm/dgs/education_culture/publ/pdf/educ-training/en.pdf)

Europäische Kommission (2003), Die Rolle der Universitäten im Europa des Wissens, Mitteilung der Kommission, KOM(2003)58, Brüssel

European Commission (2002), Commission's Action Plan for skills and mobility, 13.2.2002 COM(2002)72, Brussels (http://europa.eu.int/comm/employment_social/news/2002/feb/ap_en.html)

European Commission - Enterprise (2002), European Competitiveness Report 2002, Commission staff working document SEC(2002)528, OOP-EC, Luxembourg (http://europa.eu.int/comm/enterprise/enterprise policy/competitiveness/index.htm)

EUROSTAT (2001), Report of the Eurostat task force on measuring lifelong learning, Brussels

Fachhochschul-Konferenz (2002), Konzept für Podiumsdiskussion „Zukunft des FH-Sektors im Hinblick auf die aktuellen Entwicklungen", Generalversammlung am 13./14. Mai 2002

Fachhochschul-Konferenz (2002a), Präsentation des Ausschusses für Internationale Angelegenheiten der Fachhochschul-Konferenz auf der Generalversammlung der Fachhochschulkonferenz 13./14. Mai 2002 in St.Pölten

Fachhochschulrat (1995), Bericht des Fachhochschulrates über die Tätigkeit des Fachhochschulrates im Jahre 1994 (FHR-Jahresbericht 1994), Wien

Fachhochschulrat (1996), Bericht des Fachhochschulrates über die Tätigkeit des Fachhochschulrates im Jahre 1995 (FHR-Jahresbericht 1995), Wien 
Fachhochschulrat (1997), Bericht des Fachhochschulrates über die Tätigkeit des Fachhochschulrates im Jahre 1996 (FHR-Jahresbericht 1996), Wien

Fachhochschulrat (1998), Bericht des Fachhochschulrates über die Tätigkeit des Fachhochschulrates im Jahre 1997 (FHR-Jahresbericht 1997), Wien

Fachhochschulrat (1999), Bericht des Fachhochschulrates über die Tätigkeit des Fachhochschulrates im Jahre 1998 (FHR-Jahresbericht 1998), Wien

Fachhochschulrat (2000), Bericht des Fachhochschulrates über die Tätigkeit des Fachhochschulrates im Jahre 1999 (FHR-Jahresbericht 1999), Wien

Fachhochschulrat (2001), Bericht des Fachhochschulrates über die Tätigkeit des Fachhochschulrates im Jahre 2000 (FHR-Jahresbericht 2000), Wien

Fachhochschulrat (2002), Evaluierung im österreichischen Fachhochschulsektor (Entwurf, beschlossen am 28.6.2002) (http://www.fhr.ac.at/3_erhalt/pdfs/EvalFHSektor_280602.pdf)

Fachhochschulrat (2002a), Präsentation des FHR zu den Themen „Neues Akkreditierungsverfahren“, „Bachelorund Master-Studiengänge" und „Evaluierung" auf der Generalversammlung der Fachhochschul-Konferenz 13./14. Mai 2002 in St.Pölten

Fachhochschul-Studiengänge der Wiener Wirtschaft m.b.H. (1999), Unterstützungsbedarf durch die Stadt Wien für die Studiengänge der Wiener Wirtschaft

Felderer Bernhard et al. (2001), Forschungsfinanzierung in Österreich, IHS-Kurzbericht, Wien (http://www.ratfte.at/de.php).

FHR-INFO Nr. 1/ Juni 1998

FHR-INFO Nr. 10 / 1998

FHR-INFO Nr. 11 / Juli 1998

FHR-INFO Nr. 12 / 1998

FHR-INFO Nr. 13 / November 1998

FHR-INFO Nr. 14 / September 1999

FHR-INFO Nr. 15 / Oktober 1999

FHR-INFO Nr. 16 / Oktober 2000

FHR-INFO Nr. 17 / Oktober 2000

FHR-INFO Nr. 2 / 1995

FHR-INFO Nr. 3 / August 1995

FHR-INFO Nr. 4 / Januar 1996

FHR-INFO Nr. 5 / Mai

FHR-INFO Nr. 6 / Juli 1996

FHR-INFO Nr. 7 / Sept. 1996

FHR-INFO Nr. 8 / Juli 1997

FHR-INFO Nr. 9 / Oktober 1997

Fischer Roland, Melchior Josef (1994), Qualitätssicherungsmechanismen im Fachhochschulbereich - Interviews mit Mitgliedern des Fachhochschulrates; Bericht im Rahmen der Studie im Auftrag des BMWFK, Forschungsbericht des IHS, Wien

Fischer Roland, Melchior Josef (1995), Monitoring des Fachhochschulsektors - Qualitätssicherungsmechanismen; Zwischenbericht im Rahmen der Studie im Auftrag des BMWFK, Forschungsbericht des IHS, Wien

Fischer Roland, Melchior Josef (1995a), Qualitätssicherungsmechanismen im Fachhochschulbereich, Studie im Auftrag des BMWFK, Endbericht, Forschungsbericht des IHS, Wien

Fischer Roland, Melchior Josef (1995b), Qualitätssicherungsmechanismen im Fachhochschulbereich - Interviews und Ergebnisse einer schriftlichen Befragung; Bericht im Rahmen der Studie im Auftrag des BMWFK, Forschungsbericht des IHS, Wien

Fischer Roland, Melchior Josef (1995c), Qualitätssicherungsmechanismen im Fachhochschulbereich - Interviews mit VertreterInnen des BMWF, des BMUK, FH-Betreibern und den Geschäftsführern der Geschäftsstelle des FHR; Bericht im Rahmen der Studie im Auftrag des BMWFK, Forschungsbericht des IHS, Wien 
Fischer Roland, Melchior Josef (1995d), Qualitätssicherungsmechanismen im Fachhochschulbereich Projektpräsentation

Forum Wissenschaft (2002), Reformbedarf bei Fachhochschulen; Ausdruck aus der Homepage www.forum.wissenschaft.at am 10.6.2002

Friedrich Gerhard (1993), Autonomie der Schule. Ein Organisationsentwicklungskonzept, BMUK Bildungsforschung 3, Wien

Gemeinsame Erklärung der Europäischen Bildungsministerlnnen (1999), Bologna-Erklärung vom 19. Juni 1999 über den Europäischen Hochschulraum, Bologna

Gemünd Wolfgang (2001),Verzweifelt gesucht! Techniker-Mangel in der heimischen Wirtschaft; in: Profil Spezial Uni Extra vom Herbst 2001

Glander Anabel (2001), Analyse der Rechnungsabschlüsse der Fachhochschul-Studiengänge, Endbericht im Auftrag des BMBWK, Wien

Goedegebuure L.C.J., Jeliazkova M., Pothof F., Weusthof P. (2002), Alle begin is moeilijk : Evaluatie van de proefaccreditering HBO, CHEPS, University of Twente, Enschede

Goedegebuure L.C.J. (1992), Mergers in Higher Education: A Comparative Perspective, Utrecht

Goedegebuure Leo C.J., Lynn Meek V. (1992), The restructuring of higher education. A comparison of Australian and Dutch developments, in: Frackmann Edgar, Maassen Peter (eds) (1992), Towards excellence in European higher education in the 90's, Lemma, Utrecht, 201-233

Götschl Johann, Track Karl, Leopold Hans, Hochegger Peter (1993), Bericht des Beratungsgremiums für die Steiermärkische Landesregierung zur Errichtung von Fachhochschul-Studiengängen in der Steiermark

Grünbacher Herbert (2001), Forschung und Entwicklung an Fachhochschulen, in: Kärntner Jahrbuch (2001)

Grünbacher Herbert, Detter Helmut (2000), Forschung und Entwicklung an Fachhochschulen, Positionspapier, im Auftrag der Fachhochschul-Konferenz ( http://www.cti.ac.at/F\&E_FH/F\&E_FH_1-0.pdf )

Hackl Elsa (2001), Towards a European Area of Higher Education: Change and Convergence in European Higher Education, EUI Working Papers 2001/9

Hackl Elsa, Binder Regina (1995), Fachhochschulen - Anspruch und Realität. Eine Zwischenbilanz, in: Dorninger Christian, Koller Ernst (Hrsg.) (1995), Fachhochschulen als Innovationspotential in der österreichischen Bildungslandschaft? Erste Erfahrungen mit einem neuen Ausbildungssektor, Zeitschrift für Hochschuldidaktik, 19. Jahrgang, Heft 3-4/1995, Österreichischer StudienVerlag , Innsbruck, 76 - 88

Haider Günther, Reiter Claudia (2001), PISA 2000. Nationaler Bericht, Studienverlag, Innsbruck

Halblützel Peter, Haldemann Theo, Schedler Klaus, Schwaar Karl (Hrsg.) (1996), Umbruch in Politik und Verwaltung. Ansichten und Erfahrungen zum New Public Management in der Schweiz, Verlag Paul Haupt, Bern-Stuttgart-Wien

Hannan Michael T., Freeman John (1984), Structural Inertia and Organizational Change, in: American Sociological Review 49, 149-164

Hartmann C., Koubek A., Kremshofer A., Renner P., Theissel K. (2002), Marktunvollkommenheiten im Weiterbildungssektor. Analyse am Beispiel der steirischen Automobil- und Zulieferindustrie. FH-Joanneum \& Joanneum Research Arbeitspapier 98/002, Graz (download: http://www.fhjoanneum.at/fhj/material/publikationen/arbeitspapier98_002.pdf )

Haug Guy (2000), Trends and Issues in Learning Structures in Higher Education in Europe. Beiträge zur Hochschulpolitik 1/2000 der HRK, Bonn

Hauser Werner (1998), Die Regionalisierung der Hochschulen - Rechtliche Prämissen und Voraussetzungen, in : ÖBZ 11/1998

Hauser Werner (1999), Das Wesen des Fachhochschulbereiches. In: Hauser Werner, Maresch Helfried, Reininghaus Peter (1999), Voraussetzungen für den optimierten Betrieb von Fachhochschul$\begin{array}{llrlll}\text { Studiengängen, } & \text { Verlag } & \text { Österreich, } & \text { Wien } & 1999 & \text { (http://www.fh- }\end{array}$ joanneum.at/fhj/material/publikationen/Arbeitspapier00_009.pdf)

Hauser Werner (2001), Das österreichische Fachhochschul-Recht; in: Flämig Christian et al. (Hrsg.) (2001), Wissenschaftsrecht Band 34, Heft 3, Mohr Siebeck, S.231 - 258

Hauser Werner (2002), Die 4. Novelle zum Fachhochschul-Studiengesetz - Regelungsinhalte und Auswirkungen; in: Zeitschrift für Hochschulrecht 2002/1, Springer-Verlag, 115 - 118

Hauser Werner (2002a), Fachhochschulen in Österreich - vom Reden zum Handeln; in: Kyrer Alfred (Hrsg.) (2002), Integratives Management für Universitäten und Fachhochschulen oder : Governance und Synergie im Bildungswesen in Österreich, Deutschland und der Schweiz, neuer wissenschaftlicher Verlag, 29 - S. 44 
Heublein Ulrich, Schmelzer Robert, Sommer Dieter, Spangenberg Heike (2002), Studienabbruchstudie 2002 - Die StudienabbrecherInnenquoten in den Fächergruppen und Studienbereichen der Universitäten und Fachhochschulen; HIS Kurzinformation Hannover (http://www.his.de/Abt2/Foerderung/pdf/Kia/kia200205.pdf)

Heurlin M. (2001), How Finland became a successful high tech country. Presentation. WWRF Second Working Session, May 2001, Helsinki (Hintergrundbericht zum Memorandum)

Hofstätter Maria, Eickhoff Volker, Finder Ruth (1997), Beschäftigungschancen für Hochschulabsolventlnnen Unternehmensbefragung; Forschungsprojekt des Arbeitsmarktservice Österreich, Berufs- und Qualifikationsforschung, Wien

Hood Ch. (1998), The art of the state. Culture, rhetoric, and public management, Clarendon Press, Oxford

Hoyer Andrea, Ziegler Judith (2002), Das Fachhochschulstudium aus der Sicht der AbsolventInnen - Eine österreichweite Studie zur beruflichen Situation und zur Bewertung des FH-Studiums aus der Sicht der AbsolventInnen, Studie im Auftrag des FHR, Wien

Höllinger Sigurd, Hackl Elsa, Brünner Christian (Hrsg.) (1994), Fachhochschulstudien - unbürokratisch, brauchbar und kurz, Passagen Verlag, Wien

Huisman J., Kaiser F. (Eds) (2001), Fixed and Fuzzy Boundaries in Higher Education. A comparative study of (binary) structures in nine countries (report AS 19), Adviesraad voor het Wetenschaps- en Technologiebeleid (AWT), Den Haag

Hutschenreiter G., Polt W., Gassler H. (2001), Möglichkeiten zur Erhöhung der österreichischen Forschungsquote Abschätzung der Effekte öffentlicher auf private F\&E-Ausgaben, WIFO-Joanneum-Gutachten, Wien (http://www.rat-fte.at/de.php)

Industriewissenschaftliches Institut (IWI) (o.J.), AbsolventInnenstudie des FHS-Studiengangs Fertigungsautomatisierung, im Auftrag des Technikums Vorarlberg.

Industriewissenschaftliches Institut (IWI) (2000), IWI-MaturantInnenbefragung Herbst 2000

Industriewissenschaftliches Institut (IWI) (2001), Fachhochschul-Führer, Wien

Industriewissenschaftliches Institut (IWI), Institut 3s (o.J.), Befragung von 205 FH-Absolventlnnen aus den Bereichen Technik und Wirtschaft

Inspectie van het Onderwijs (2002), Accreditatie beproefd, Inspectie van het Onderwijs, Utrecht

Institut 3s (2002), Regionalwirtschaftliche Implikationen von Fachhochschul-Studiengängen in Österreich; vorläufige, unveröffentlichte Ergebnisse einer Studie im Auftrag des Jubiläumsfonds der Österreichischen Nationalbank, Wien

Institut für Bildungsforschung der Wirtschaft (ibw) (1998), Ergebnisse einer Befragung österreichischer Unternehmen, die FachhochschulabsolventInnen beschäftigen (,Berufliche Perspektiven von FachhochschulabsolventInnen“), im Auftrag der Wirtschaftskammer und der Industriellenvereinigung, Wien

Jahn H., Olbertz J.H. (1998), Neue Stufen - alte Hürden? Flexible Hochschulabschlüsse in der Studienreformdebatte, Beltz, Weinheim

Jencks, Riesman David (1968), The Academic Revolution, New York

Joint declaration of the European Ministers of Education Convened in Bologna on the 19th of June 1999 (1999), Bologna

Keber Christian (1992), Zur sukzessiven Einrichtung neugestalteter nichtuniversitärer Institutionen Finanzbedarfsanalyse, Studie im Auftrag des BMWF, Wien

Kekkonen K. (1998), Instruments, Tools, and Policies to Anticipate the Effects of Industrial Training on Employment and Vocational Qualification. Country Report Finland, ESF-Publication Series 20/98, Helsinki

Keller George (1983), Academic Strategy. The Management Revolution in American Higher Education, The Johns Hopkins University Press, Baltimore/London

Klimmer Susanne, Schlögl Peter (1999), Die Berufsreifeprüfung. Eine erste Evaluierung im Auftrag des Bundesministeriums für Unterricht und kulturelle Angelegenheiten, ibw, ÖIBF, Wien

KMK-Beschluss „Künftige Entwicklung der länder- und hochschulübergreifenden Qualitätssicherung in Deutschland“ vom 1. März 2002

Kofler Herbert, Urnik Sabine (1995), Kalkulation von Fachhochschulen, März 1994; Prüfungsbericht über die Prüfung der Kalkulation und Kostenentwicklung bei den Fachhochschulen Wels und Wr. Neustadt

Kozar Gerhard (1999), Hochschul-Evaluierung - Aspekte der Qualitätssicherung im tertiären Bildungsbereich, WUVVerlag, Wien 
Krugman P. (1996), Wettbewerbsfähigkeit: Eine gefährliche Wahnvorstellung. In: Fricke W., Fricke E. (Hrsg.) (1996), Zukunft der Industriegesellschaft. Jahrbuch Arbeit + Technik 1996, Verl. Dietz Nachf., Bonn, 37 - 49

L\&R Sozialforschung (1995), Tagung „Evaluation der Implementierung des FHStG“ - Dokumentation Ergebnisse der Arbeitsgruppen, Stellungnahme BMWF und Diskussion

Lam Alice, Lundvall Bengt-Ake (2000), Innovation Policy and Knowledge Management in the Learning Economy the Interplay Between Firm Strategies and National Systems of Competence Building and Innovation. Paper to the OECD Conference "Knowledge Management: the New Challenge for Firms and Organisations", September 2000, Ottawa, Canada

Landesrechnungshof Salzburg (2000), Bericht des Landesrechnungshofes über die „Widmungsgemäße Verwendung von Landesförderungen im Techno-Z Verbund“"

Landesrechnungshof Vorarlberg (2001), Prüfbericht über die Fachhochschule Vorarlberg GmbH, Bregenz

Lang Barbara, Finder Ruth (2000), Arbeitsmarkt- und Beschäftigungssituation von Fachhochschulabsolventlnnen, Endbericht, Wien

Lassnigg Lorenz (1990), Akademikerbeschäftigung in den achtziger Jahren, Forschungsbericht des IHS, im Auftrag des BMWF, Wien

Lassnigg Lorenz (1991), Längerfristige Entwicklungstendenzen der Akademikerbeschäftigung: Befunde - Probleme - Perspektiven Forschungsbericht des IHS, Wien

Lassnigg Lorenz (1998), Qualifizierungspolitik, Innovationssystem und Beschäftigung - Herkömmliche und neue Perspektiven. In: Zukunfts- und Kulturwerkstätte (Hrsg.) (1998), Re-engineering der österreichischen Industriepolitik, Wien, 76-132

Lassnigg Lorenz (2000), Zentrale Steuerung in „autonomisierten” Bildungssystemen, in: Schweizerische Konferenz der kantonalen Erziehungsdirektoren et al. (Hrsg.) (2000), Die Vielfalt orchestrieren, OECD/CERI Seminarbericht, StudienVerlag, Innsbruck, 107-141

Lassnigg Lorenz (2000a), Lifelong Learning: Österreich im Kontext internationaler Strategien und Forschungen, IHS-Reihenpaper Soziologie Nr. 45, Wien

Lassnigg Lorenz (2002), Projections of Qualifications and Occupations in Austria - Short-term Approaches, Macro Perspective and Emphasis on the Supply Side, in: Neugart M., Schömann K. (Eds) (2002), Forecasting Labour Markets in OECD Countries. Measuring and tackling mismatches, Elgar, Cheltenham, 240 - 282

Lassnigg Lorenz, Loudon Susanne, Schramm Brigitte, Steiner Peter (2000), Der Berufseinstieg von HochschulabsolventInnen, in: Mitterauer Lukas, Reiter Walter (Hrsg.) (2000), Der Arbeitsmarkt für AkademikerInnen in Österreich, Wissenschaftsverlag, Wien, 129 - 207

Lassnigg Lorenz, Mayer Kurt (2001), Definition und Auswahl von Kompetenzen in Österreich. Länderbericht zum BFS-OECD Projekt DeSeCo , IHS-Sociological Series No.52 (download: http://www.ihs.ac.at/index.php3?id=550)

Lassnigg Lorenz, Nemeth G. (1999), Personalbedarf im österreichischen Schulwesen, Teil II, Forschungsbericht des IHS, Wien

Lassnigg Lorenz, Paseka A. (Hrsg.) (1997), Schule weiblich - Schule männlich. Zum Geschlechterverhältnis im Bildungswesen, StudienVerlag, Innsbruck-Wien

Lassnigg Lorenz, Pechar Hans (1988), Alternatives to Universities in Higher Education - Country Study: Austria, OECD, Paris

Lassnigg Lorenz, Pollan Wolfgang (1996), Das österreichische Qualifizierungssystem im internationalen Vergleich. In: WIFO-Monatsberichte 12/1996, S.763-780

Lassnigg Lorenz, Stöger E (1999), Evaluation der Bedarfs- und Akzeptanzprüfungsmodelle für FH-Studiengänge. Forschungsbericht des IHS, Wien

Lave Jean, Wenger Etienne (1991), Situated Learning: legitimate perpheral participation, Cambridge University Press, Cambridge

Leitner E. (1992), Spittal an der Drau als Standort einer Fachhochschule. Expertise der Stadt Spittal a.d. Drau., Forschungsbericht, Klagenfurt (im Anhang von Pongratz (1993))

Linhart Markus (1995), Referat „Optimale Größe von Bildungseinrichtungen“ und „Integration von Forschung und Entwicklung“ im Rahmen der Joanneum Research Fachtage „Vision und Realität - Fachhochschulen in Österreich am 25./26. 9. 1995 in Graz"

Löffler Elke (2002), Good Governance als Weiterentwicklung von New Public Management: Verschiedene Ansätze in Europa, in: Tagungsdokumentation der Konferenz „New Public Management - Stand und Entwicklungsperspektiven" in Wien, 19./20. März 2002, KDZ-Zentrum für Verwaltungsforschung ( www.kdz.or.at) 
Lundvall Bengt-Åke, Borrás S. (1999), The Globalising Learning Economy: Implications for Innovation Policy (Report based on contributions from 7 projects under the TSER programme). European Commission (Ed.), Office for Official Publications of the European Communities

Lynch L M. ( Ed.) (1994), Training and the Private Sector. International Comparisons. University of Chicago Press (NBER Comparative Labor Market Series), Chicago

Maresch Helfried (2002), Die Forschung im Fachhochschul-Bereich in der Praxis. In: Prisching Manfred, Lenz Werner, Hauser Werner (Hrsg.) (2002), Die wissenschaftliche Forschung in Österreich. Verlag Österreich, Wien, S. 151-189

Markowitsch Jörg (2001), Praktisches akademisches Wissen - Werte und Bedingungen praxisbezogener Hochschulbildung, WUV-Verlag, Wien

Mayer S., Stampfer M., Sturn D. (2001), Grundkonzeption für ein F\&E-Fachhochschulprogramm. TIG-Gutachten im Auftrag der Fachhochschul-Konferenz, Wien (http://www.cti.ac.at/F\&E_FH/TIG_FH-Konzept.pdf)

Meusberger Peter, Schmidt Andrea (1995), Ausbildungsniveau und regionale Mobilität. Zur Abwanderung von Hochqualifizierten aus Vorarlberg; Abschlußbericht einer Studie im Auftrag der Vorarlberger Landesregierung, hrsg. vom Amt der Vorarlberger Landesregierung

Ministerie van Onderwijs, Cultuur en Wetenschappen (2002), Facts and Figures 2002 (http://www.minocw.nl/english_oud/general/figures/pdf/2.6\%20Higher\%20Professional\%202002.pdf)

MoE (Ministry of Education) Finland (o.J.), Polytechnic Education in Finland - Background Report (http://www.oecd.org/pdf/M00029000/M00029585.pdf.)

MoE (Ministry of Education) Finland (1995), Education, Training and Research in Information Society. A National Strategy, Helsinki (http://www.minedu.fi/infostrategy.html)

Nadornik Wolfgang, Glander Anabel (1997), Kostenanalyse Fachhochschulstudiengänge; Studie im Auftrag des BMWV, Linz

Nadornik Wolfgang, Glander Anabel (1999), Normensystem zur studiengangsbezogenen Rechnungslegung der Fachhochschulerhalter, Leonding

Nadornik Wolfgang, Glander Anabel (1999a), Rechnungslegung der Fachhochschulen - Bericht und Empfehlungen; Studie im Auftrag des BMWV

Nelson R. (1993), National Innovation Systems, Oxford University Press, Oxford

Niederösterreichische Bildungsgesellschaft m.b.H. (o.J.), Homepage www.noe-bildung.at

$\mathrm{OAQ}$ (Organ für Akkreditierung und Qualitätssicherung der Schweizerischen Hochschulen) (2002), Jahresbericht 2001, Bern

Oberösterreichische Industriellenvereinigung (2002), Pressemeldung zur Pressekonferenz „Anforderungen der Industrie an die österreichische Bildungspolitik" vom 7.3.2002

Oberösterreichische Landesregierung (2001), Oberösterreichisches Fachhochschulangebot wächst weiter! Fachhochschulstandort Linz - „Soziales und Gesundheit“ zukunftsweisender Ausbildungsschwerpunkt. Information zur Pressekonferenz von Wirtschafts-Landesrat KommR Josef Fill am 3.12.2001

Oberösterreichische Technologie- und Marketinggesellschaft m.b.H. (1998), Strategisches Programm Oberösterreich 2000+, Linz

OECD (1993), From Higher Education to Employment - Synthesis Report, Paris

OECD (1996), Lifelong Learning for All, Paris

OECD (1998), Redefining Tertiary Education, Paris

OECD (1999), The Response of Higher Education Institutions to Regional Needs, Paris

OECD (2000), Motivating Students for Lifelong Learning, Paris

OECD (2000a), Bildung auf einen Blick. OECD-Indikatoren, Ausgabe 2000, Paris

OECD (2001), Cities and Regions in the New Learning Economy, Paris

OECD (2001a) Lernen für das Leben. Erste Ergebnisse von PISA 2000, Paris

OECD (2002), Review of Education Policy in Finland: the Polytechnic Sector. Examiners Report, DEELSA/ED(2002) vom 23.5.2002, Paris (http://www.oecd.org/pdf/M00030000/M00030262.pdf)

Opetusministeriö (2002), Polytechnic Education in Finland - Background Report, Ministry of Education, Helsinki

ÖROK (Österreichische Raumordnungskonferenz) (2002), Österreichisches Raumentwicklungskonzept 2001. Beschlusstext (http://oerok.gv.at/OEREK2001/) 
Pamer Werner, Heinzelmaier Bernhard (1992), Mehr Bildungschancen durch Fachhochschulen? - Analyse der österreichischen Fachhochschulkonzepte unter besonderer Berücksichtigung ihrer bildungsfördernden Wirkung auf das duale Ausbildungssystem; Forschungsbericht im Auftrag des BMWF, Wien

Pauschenwein Jutta, Jandl Maria, Koubek Anni (Hrsg.) (2001), Telelernen an österreichischen Fachhochschulen. Praxisbeispiele und Möglichkeiten der Weiterentwicklung, Schriftenreihe des Fachhochschulrates 5, WUV-Verlag, Wien

Pechar Hans (1990), Jenseits Alma und Mater. Braucht Österreich Alternativen zu einem Universitätsstudium? in: Die Zukunft 12/1990

Pechar Hans (1996), Finanzierung der Fachhochschulen - Teilprojekt zum Fachhochschulmonitoring, Projektbericht an das BMWFK, Wien

Pechar Hans (1996a), Finanzierung der Fachhochschulen - Teilprojekt zum Fachhochschulmonitoring - interne Arbeitsunterlagen

Pechar Hans (1999), Wien als Fachhochschul-Standort - Empfehlungen zur Fachhochschulentwicklung und finanzierung in Wien, Gutachten im Auftrag des WAFF, Wien

Pechar Hans, Pellert Ada, Pfeffer Tom, Waxenegger Andrea, Ludescher Marcus (1998), Internationalisierung der österreichischen Hochschulen, Endbericht an das BMWV, Wien

Pechar Hans (1999a), Das Zusammenspiel von Bund und Ländern im Fachhochschulbereich - Beitrag zur Studie "Effizienzsteigerung durch Aufgabenbereinigung und Verwaltungsoptimierung im Bundesstaat", Wien

Pechar Hans (2000), Bachelor an Fachhochschulen, Gutachten im Auftrag der Österreichischen FachhochschulKonferenz, Wien

Pechar Hans, Pellert Ada, Pfeffer Tom, Ruis Michael, Unger Martin (1999), HEINE (Higher Education Institutions and the National Economy) - The Institutional Case Studies in Austria, hrsg. vom iff, Wien

Pechar Hans, Wroblewski Angela (1998), Non-Traditional-Students in Österreich. Studienbedingungen bei Nebenerwerbstätigkeit, verspätetem Übertritt und alternativem Hochschulzugang, iff-Forschungsbericht, Wien

Pinkau Klaus et al. (Hrsg.) (1998), Der Universitäts- und Forschungsstandort Deutschland im globalen Markt, Hirzel, Stuttgart/Leipzig

Pongratz Gerhard (1993), Fachhochschule Kärnten - Studienschwerpunkte, Standortfrage; Klagenfurt

Posch Peter, Altrichter Herbert et al. (1992), Schulautonomie in Österreich, BMUK Bildungsforschung 1, Wien

Posch Peter, Altrichter Herbert (1997), Möglichkeiten und Grenzen der Qualitätsevaluation im Schulwesen. Bildungsforschung des BMBWK 12, Studienverlag, Innsbruck-Wien

Pratt John, Hackl Elsa (1999), Breaking the Mould in Austrian Higher Education, in: Higher Education Review, Vol 32, No 1

Pressedienst der Industrie, Pressedienst der Wirtschaftskammer (1998), Pressedienstmeldungen im Rahmen der Präsentation einer Untersuchung des IBW „Berufliche Perspektiven von FachhochschulabsolventInnen“ (Ergebnisse einer Befragung österreichischer Unternehmen, die FachhochschulabsolventInnen beschäftigen) vom 16. März 1998

Pressekonferenz „Aktuelle Ergebnisse aus dem Wiener Wirtschaftsbeirat“, schriftliche Unterlage zur Pressekonferenz am 15.3.2000

Prisching Manfred (2002), Die Regulierung der Fachhochschulen, unveröffentlichter Beitrag zu einer Festschrift für Dr. Peter Schachner-Blazizek

Prisching Manfred, Lenz Werner, Hauser Werner (Hrsg.) (2000), Die Autonomie im Fachhochschulbereich, Schriften zum Bildungsrecht und zur Bildungspolitik Band 2, Verlag Österreich, Wien

Prisching Manfred, Lenz Werner, Hauser Werner (Hrsg.) (2001), Das Verhältnis zwischen Universität und Fachhochschule, Schriften zum Bildungsrecht und zur Bildungspolitik Band 4, Verlag Österreich, Wien

Reinmann-Rothmeier G. \& Mandl H. (1998), Implementation konstruktivistischer Lernumgebungen - revolutionärer Wandel oder evolutionäre Veränderung? (Forschungsbericht Nr. 100), Ludwig-Maximilians-Universität, Lehrstuhl für Empirische Pädagogik und Pädagogische Psychologie, München

Reuke Herrmann (2002), Zwei Seiten einer Medaille: Evaluation und Akkreditierung, in: Reil Thomas, Winter Martin (Hrsg.) (2002), Qualitätssicherung an Hochschulen. Theorie und Praxis, Bertelsmann Verlag GmbH, Bielefeld , $103-110$

Riesman David (1980), On Higher Education. The Academic Enterprise in an Era of Rising Student Consumerism, Jossey-Bass, San Francisco 
Rychen Dominique S., Sagalnik Laura H. (Eds) (2001), Defining and Selecting Key Competences, Hogrefe\&Huber, Seattle

Schedler Klaus (1996), Fachhochschulen in Österreich - ein österreichisches Schicksal?, ibw , Wien

Schedler Kuno, Proeller Isabella (2000), New Public Management, Haupt, Bern-Stuttgart-Wien

Schelling Günther (1999), Evaluation aus Sicht der Auftraggeber, in: Thonhauser Josef, Partry Jean-Luc (Hrsg.) (1999), Evaluation im Bildungsbereich. Wissenschaft und Praxis im Dialog, Studien-Verlag, InnsbruckWien, 67-85

Schenker-Wicki Andrea (2002), Evaluation und Akkreditierung im Schweizer Universitätswesen, in: Reil Thomas, Winter Martin (Hrsg.) (2002), Qualitätssicherung an Hochschulen. Theorie und Praxis, Bertelsmann Verlag $\mathrm{GmbH}$, Bielefeld , 90 - 99

Schinagl Wolfgang (2002), TF2000 - Telelernen an österreichischen Fachhochschulen, WUV-Verlag, Wien

Schindler Götz (2001), Errichtung neuer Fachhochschul-Einrichtungen in Bayern nach 1991 - Standortanalyse und Entscheidungsprozess; in: BMBWK (2001), Regionalisierung im Fachhochschulbereich - Tagungsbericht, Wien

Schmidt Marion (2002), Verschmähter „Master“, in: Die Zeit - Chancen 32/2002

Schneeberger Arthur (2000), Österreichs Bildungsstruktur im internationalen Vergleich. In: ibw (Hrsg.) (2000), Bildung als Schlüssel zur Informationsgesellschaft, Wien, 181-198

Schneeberger Arthur (2001), Lebenslanges Lernen als Schlüssel zur Informationsgesellschaft, ibw-Schriftenreihe Nr.120, Wien

Schwarz Stefanie, Teichler Ulrich (2000), Credit-Systeme an deutschen Hochschulen, in: Schwarz Stefanie, Teichler Ulrich (2000), Credits an deutschen Hochschulen. Kleine Einheiten - große Wirkungen, Luchterhand, Neuwied, 3 - 16

Siemens/ Sitec (1999), Standortentwicklung für die Technische Fachhochschule Wien des Vereins zur Förderung von FHS-Studiengängen - Unterlagen für die Präsentation vom 28.10. 1999

Simmie J. (Ed.) (1997), Innovation, Networks and the Learning Region, Jessica Kingsley, London

SKBF (Schweizerische Koordinationsstelle für Bildungsforschung) (2001), Schweizerische Grundlagendokumentation für die Expertenbesuche. OECD-Examen (http://www.edk.ch/oecd-tertch/d/menu_d.html)

Sohm Kurt (1999), Praxisbezogene Ausbildung auf Hochschulniveau - eine pädagogisch-didaktische Herausforderung, WUV-Verlag, Wien

Sohm Kurt (2002), Referat über den österreichischen Fachhochschulsektor im Rahmen des Workshops "Qualitätssicherung in der Hochschule - Theorie und Praxis" (Wittenberg, 18. - 20. 3. 2002)

Sohm Kurt (2002), Akkreditierung und Evaluierung im österreichischen Fachhochschul-Sektor, in: Reil Thomas, Winter Martin (Hrsg.) (2002), Qualitätssicherung an Hochschulen. Theorie und Praxis. Bertelsmann Verlag $\mathrm{GmbH}$, Bielefeld, 80 - 90

Stadt Wien (2000), Richtlinien für eine Wiener Fachhochschulförderung, Entwurf vom 3.2.2000

Steiner Mario, Herzog-Punzenberger Barbara, Bauer Monika (2000), Bedarfs- und Akzeptanzstudie zur Einrichtung eines Fachhochschul-Studiengangs ,Sozialarbeit und Sozialmanagement', Studie im Auftrag des Vereins zur Förderung von FHS-Studiengängen VFH Wien, Wien, Forschungsbericht des IHS

Steiner Mario, Scheibelhofer Elisabeth, Lassnigg Lorenz, Steger Martin (2001), Adaptation/Anticipation of Higher, Non-University Vocational Education and Training Institutions to Industrial Change: Reflections on and Case Studies of the Austrian Situation; Forschungsbericht im Rahmen des Projekts „European Trends in the Adaptation/ Anticipation of Higher, Non-University Vocational Education and Training Institutions to Industrial Change" der EU-Kommission, Wien , Forschungsbericht des IHS

Steiner Mario, Steger Martin (2001), Fallstudien zur Adaption und Antizipation im österreichischen Fachhochschulsektor, IHS-Sociological Series No. 50, Wien (download: http://www.ihs.ac.at/index.php3?id=550)

Steiner Mario, Steiner Peter, Prenner Peter, Delapina Franz (2001a), Analyse des Beschäftigungs-, Bildungs- und Innovationssystems - Grundlage zur Antizipation von Entwicklungsmöglichkeiten des NÖ. Fachhochschulwesens, Band I; Studie im Auftrag der Niederösterr. Bildungsgesellschaft m.b.H. für Fachhochschul- und Universitätswesen, Forschungsbericht des IHS, Wien

Steiner Mario, Lassnigg Lorenz, Unger Martin (2001b), Antizipation der Entwicklungsmöglichkeiten des NÖ. Fachhochschulwesens - Grundlagen für ein Niederösterreichisches Fachhochschul-Konzept, Band II, Studie im Auftrag der Niederösterr. Bildungsgesellschaft m.b.H. für Fachhochschul- und Universitätswesen, Forschungsbericht des IHS, Wien 
Steiner Mario, Steiner Peter, Lassnigg Lorenz , Prenner Peter (2002), Grundlagen für die Entwicklung eines Systems des Lebenslangen Lernens in Wien. Analysen, Prognosen und strategische Ansätze, Forschungsbericht des Instituts für Höhere Studien, Wien

Steinkellner M. (1998), AbsolventInnenanalyse Jahrgang 1998 des Fachhochschul-Studiengangs Gebäudetechnik Pinkafeld

Storper Michael (1995), The Resurgence of Regional Economics, Ten Years Later: The Region as a Nexus of Untraded Interdependencies; European Journal of Urban and Regional Studies, 2(3), 191-221

Storper Michael (2000), Regional Technology Policies in Europe. Paper Prepared for the Workshop on the Regional Level of Implementation of Innovation and Education \& Training Policies, EU-Commission, DG XII, Brussels, November 23-24, 2000

Sturn D. et al. (1999), Start auf dem Campus: Infrastruktur für Gründungen aus Hochschulen und Forschungseinrichtungen, Forschungsbericht Joanneum-Research, Wien-Graz

Technikum Joanneum (o.J.), Finanzierungsplan 1994 und 1995; Kopie eines Schreibens an die Steiermärkische Landesregierung

Technikum Kärnten (1998), Kärntner Fachhochschul-Konzept 1999-2004 (Unterlage zur Vorlage für eine Regierungssitzung)

Technikum Wien News - Mitteilungsblatt des Vereins zur Förderung von FHS-Studiengängen, Winter 1999/2000

Technologie Impulse Gesellschaft m. b. H. (TIG) (2001a), Ein F\&E-Impulsprogramm für den Fachhochschul-Sektor - Fhplus. Erarbeitung eines Grobkonzepts im Auftrag der FHK, Detailausarbeitung im Auftrag von BMVIT und BMBWK (www.cti.ac.at/F\&E_FH/FHplus_TIG_2001-11-05.pdf)

Technologie Impulse Gesellschaft m. b. H. (TIG) (2001b), Grundkonzeption für ein F\&E Fachhochschulprogramm im Auftrag der FHK (http://www.cti.ac.at/F\&E_FH/TIG_FH-Konzept.pdf)

Technologie Impulse Gesellschaft m. b. H. (TIG) (2002), Präsentation der TiG zum Thema „Fhplus - ein F\&EImpulsprogramm für den Fachhochschul-Sektor" auf der Generalversammlung der Fachhochschulkonferenz 13./14. Mai 2002 in St.Pölten

Techno-Z Salzburg Research (1995), Die digitale Herausforderung. Zur Notwendigkeit von FH-Studiengängen für Telekommunikation und Neue Medien in Österreich, Salzburg

Techno-Z Salzburg Research (o.J.), Studieren am Techno-Z - Fachhochschul-Studiengang Telekommunikationstechnik und -systeme, Salzburg

Tichy Gunther, Darlap P. (1996), Technologie und Bildung, Bericht des Arbeitskreises, in: Handler H. (Hrsg.) (1996), Wirtschaftsstandort Osterreich, BMWA, Wien, 92 - 115

Titscher Stefan et al. (Hrsg.) (2000), Universitäten im Wettbewerb. Zur Neustrukturierung österreichischer Universitäten, Hampp, München

Tödtling-Schönhofer H. et al (1999), Regionale Innovationspolitik 2000, Band 1, ÖIR (Hrsg.), Wien

UNESCO (1996), Manual for statistics on non-formal education, UNESCO Institute for Statistics (UIS), Paris

Urban A. (2001), Das Wissensmanagement von technologie- und innovationspolitischen Aktionen im Netzwerk der Technologiezentren. Studie im Auftrag des BMWA (http://www.bmwa.gv.at/org02/sekvi/vib_fs.htm; aufgrund neuer homepage download nicht mehr möglich)

Voss R. (Hrsg.)(1997), Die Schule neu erfinden. Systemisch-konstruktivistische Annäherungen an Schule und Pädagogik, Luchterhand, Neuwied

Vught F.A. van (1994), Intrinsic and Extrinsic Aspects of Quality Assessment in Higher Education. In: Westerheijden D.F., Brennan J., Maassen P.A.M. (Eds.), Changing Contexts of Quality Assessment: Recent Trends in West European Higher Education (pp. 31-50), Lemma, Utrecht

Vught F.A. van, Wende M.C. van der, Westerheijden D.F. (2002), Globalization and Internationalization: Policy Agendas Compared. In: Fulton O., Enders J. (Eds) (2002), Higher Education in a Globalizing World. International Trends and Mutual Observations, Kluwer, Dordrecht

Wende M.C. van der, Westerheijden D.F. (2001), International Aspects of Quality Assurance With a Special Focus on European Higher Education. In: Quality in Higher Education, 7(3), 233-245.

Westerheijden D.F., Brennan J., Maassen P.A.M. (1994), Changing Contexts of Quality Assessment: Recent Trends in European Higher Education, in: Westerheijden D.F., Brennan J., P.A.M. Maassen (Eds.) (1994), Changing Contexts of Quality Assessment, Lemma, Utrecht

Westerheijden D.F. (2002), Movements Towards a European Dimension in Quality Assurance and Accreditation. Paper presented at the Working Group on the European Dimension of Quality, International Conference on Accreditation and Quality Assurance in Higher Education, 12./13.3.2002, Amsterdam 
Westerheijden D.F. (2003, submitted), Accreditation in the Netherlands: Windmills between Bologna and Seattle, Journal of Studies in International Education

Westerheijden D.F., Leegwater M. (Eds.) (2003, in print), Working on the European Dimension of Quality: Report of the Conference on Quality Assurance in Higher Education as Part of the Bologna Process, Amsterdam, 12-13 March 2002. Ministerie van Onderwijs, Cultuur en Wetenschappen, Zoetermeer

Wilhelmer Peter (2001), Fachhochschulen und Universitäten - einige (empirisch erhärtete) Bemerkungen. In: Prisching Manfred, Lenz Werner, Hauser Werner (Hrsg.) (2001), Das Verhältnis zwischen Universität und Fachhochschule, Schriften zum Bildungsrecht und zur Bildungspolitik Band 4, Verlag Österreich, Wien, 19-39.

Wilson R A (2001), Forecasting Skill requirements at National and Company Levels. In: Training in Europe. Second Report on Vocational Training Research in Europe 2000. Background Report, Vol. 2, OOP-EC, Luxembourg , 561-609 (http://www2.trainingvillage.gr/etv/research/background.asp).

Wimmer Petra (2000), Die ersten Fachhochschul-AbsolventInnen am Arbeitsmarkt. Ergebnisse österreichweiter Erhebungen; Studie im Auftrag der Wirtschaftskammer und der Industriellenvereinigung, ibwSchriftenreihe/ Forschungsbericht Nr. 118, Wien

WR (Wissenschaftsrat) (2002), Empfehlungen zur Entwicklung der Fachhochschulen, Berlin

Zib Christian (1998), Mögliche Rechtsformen für postsekundäre Bildungseinrichtungen; Studie im Auftrag des BMWV, Wien 


\section{Anhang}

\subsection{Ergänzungen zu Kapitel 3}

\subsubsection{Zusammenfassung von Forschungsergebnissen zur Positionierung der FH- AbsolventInnen}

Im folgenden wird eine detaillierte vergleichende Zusammenstellung und Analyse von Forschungsergebnissen zur Positionierung der FH-AbsolventInnen der ersten Jahrgänge im Beschäftigungssystem anhand von drei einschlägigen Studien präsentiert.

Eine Studie (Wimmer 2000) befragte Unternehmen, die FH-AbsolventInnen beschäftigen und gibt Informationen über sechs Unternehmensbereiche (U), für die die AbsolventInnen als geeignet angesehen werden sowie über sieben Kompetenzen $(K)$ und die Karrieremöglichkeiten, die innen zugeschrieben werden. Die FH-AbsolventInnen werden dabei in der Fragestellung direkt mit den UniversitätsabsolventInnen verglichen. Die Einschätzungen zeigen einen hohen Grad an Gleichwertigkeit (der Durchschnittsanteil über alle Dimensionen der Kategorie „beide“ liegt bei $60 \%$, UNI und FH werden im Durchschnitt symmetrisch von je $20 \%$ besser bewertet), indem bei sechs Kompetenzen und drei Unternehmensbereichen sowie bei den Karrierechancen zumindest $50 \%$ der Befragten beide Abschlüsse als gleich gut bewertet haben (vgl. Abbildung 70).

\section{Abbildung 70: Einsatzbereiche und Kompetenzen von FH- und UNI-AbsolventInnen aus der Sicht beschäftigender Betriebe}

\begin{tabular}{|l|lll|l|l|}
\hline & & \\
\hline
\end{tabular}

U: Unternehmensbereich; K: Kompetenzen Quelle: Wimmer 2000. 
Die FH-AbsolventInnen werden überwiegend als für operative Einsatzbereiche als geeignet angesehen, während bei den UniversitätsabsolventInnen wissenschaftliche und innovative Aufgaben dominieren. Trotz der langjährigen und teilweise massiven Kritik an den Universitäten rangieren die heute sehr hoch bewerteten Kompetenzen und Arbeitsbereiche wie betriebliche Entscheidungen (Decision), Unternehmensschnittstellen, Denken in Zusammenhängen (Vernetz) und Kreativität bei Problemlösungen (Kreat-PL) bei den UNIAbsolventInnen auf den oberen Rangplätzen, während sie bei den FH-AbsolventInnen an den unteren Platzen liegen (siehe Tabelle 26).

Tabelle 26: Rangordnung der Einsatzbereiche (U) und Kompetenzen (K) bei FHund UNI-AbsolventInnen in vergleichenden Bewertungen durch Unternehmen

\begin{tabular}{|ll|lr|lr|}
\hline $\begin{array}{l}\text { Rangordnung FH und } \\
\text { UNI geeignet (U) } \\
\text { bzw. kompetent (K) }\end{array}$ & & $\begin{array}{l}\text { Rangordnung FH } \\
\text { besser geeignet }(\mathrm{U}) \\
\text { bzw. kompetent (K) }\end{array}$ & & $\begin{array}{l}\text { Rangordnung UNI } \\
\text { besser geeignet }(\mathrm{U}) \\
\text { bzw. kompetent (K) }\end{array}$ \\
\hline KARRIERE & $\mathbf{7 7 , 9}$ & U operativ & $\mathbf{5 1 , 3}$ & U wiss & $\mathbf{7 5 , 6}$ \\
\hline K Einschul & $\mathbf{7 2 , 5}$ & K praxis & 37,3 & U innovativ & $\mathbf{5 1 , 9}$ \\
\hline K Vernetz & $\mathbf{7 0 , 1}$ & U Kunden & 34,2 & U Decision & 38,5 \\
\hline K Kreat-PL & $\mathbf{6 7 , 5}$ & K team & 29,9 & U Schnittst & 29,1 \\
\hline K Flex-neu & $\mathbf{6 6 , 7}$ & K aktualität & 27,0 & K Vernetz & 16,9 \\
\hline K team & $\mathbf{6 6 , 2}$ & K Einschul & 21,3 & K Kreat-PL & 16,9 \\
\hline U Kunden & $\mathbf{5 9 , 5}$ & K Flex-neu & 19,3 & Karriere & 14,3 \\
\hline K aktualität & $\mathbf{5 9 , 0}$ & U Schnittst & 19,0 & K Flex-neu & 14,0 \\
\hline U Decision & $\mathbf{5 5 , 1}$ & K Kreat-PL & 15,6 & K aktualität & 14,0 \\
\hline U Schnittst & $\mathbf{5 1 , 9}$ & K Vernetz & 13,0 & K praxis & 13,4 \\
\hline K praxis & 49,3 & Karriere & 7,8 & U Kunden & 6,3 \\
\hline U operativ & 48,8 & U innovativ & 7,6 & K Einschul & 6,2 \\
\hline U innovative & 40,5 & U Decision & 6,4 & K team & 3,9 \\
\hline U wiss & 23,1 & U wiss & 1,3 & U operativ & 0,0 \\
\hline
\end{tabular}

U: Unternehmensbereich; K: Kompetenzen

Quelle: Wimmer 2000 
Abbildung 71: Verhältnis der Bewertungen von Einsatzbereichen (U) und Kompetenzen (K) bei FH- und UNI-AbsolventInnen durch Unternehmen

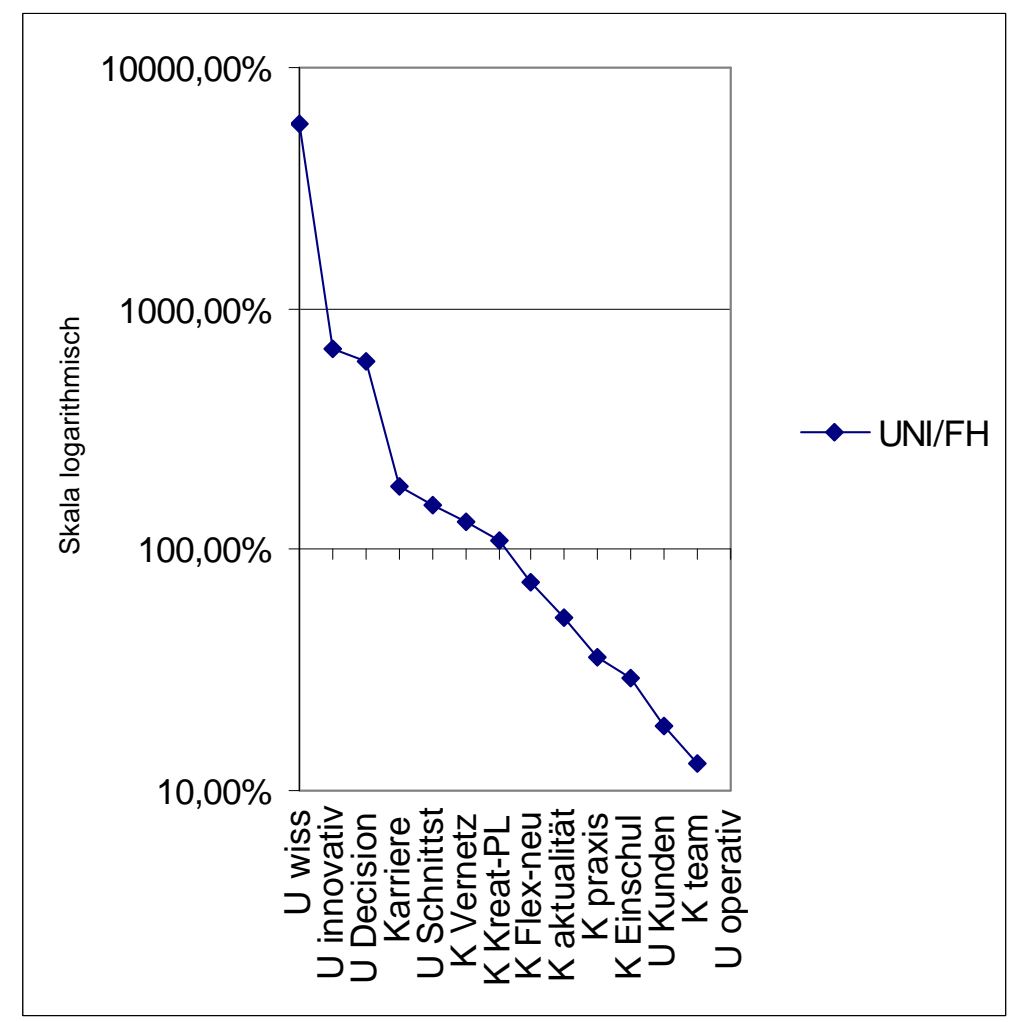

U: Unternehmensbereich; K: Kompetenzen

Quelle: Wimmer 2000

In einer weiteren Studie (Lang/Finder 2000) wurden KMUs, die in Branchen tätig sind, die den größten Teil der FH-AbsolventInnen beschäftigen, nach ihrer Einschätzung von sachlichen Schwächen von UNI- bzw. FH-AbsolventInnen bei fünf allgemeinen (A) und neun spezifischen Kompetenzen befragt. Hier erfolgte die Einschätzung nicht im direkten Vergleich, sondern für die beiden Abschlüsse gesondert. Die Bewertungen wurden nur für jene Unternehmen vorgenommen, die hochqualifizierte Arbeitnehmerlnnen (BHS, FH oder UNI) beschäftigen, darüber hinaus wurden die Antworten für FH-AbsolventInnen auch für jene Unternehmen gesondert ausgewiesen, die tatsächlich FH-AbsolventInnen beschäftigen. 


\section{Abbildung 72: Schwächen von FH- und UNI-AbsolventInnen aus der Sicht von KMUs}

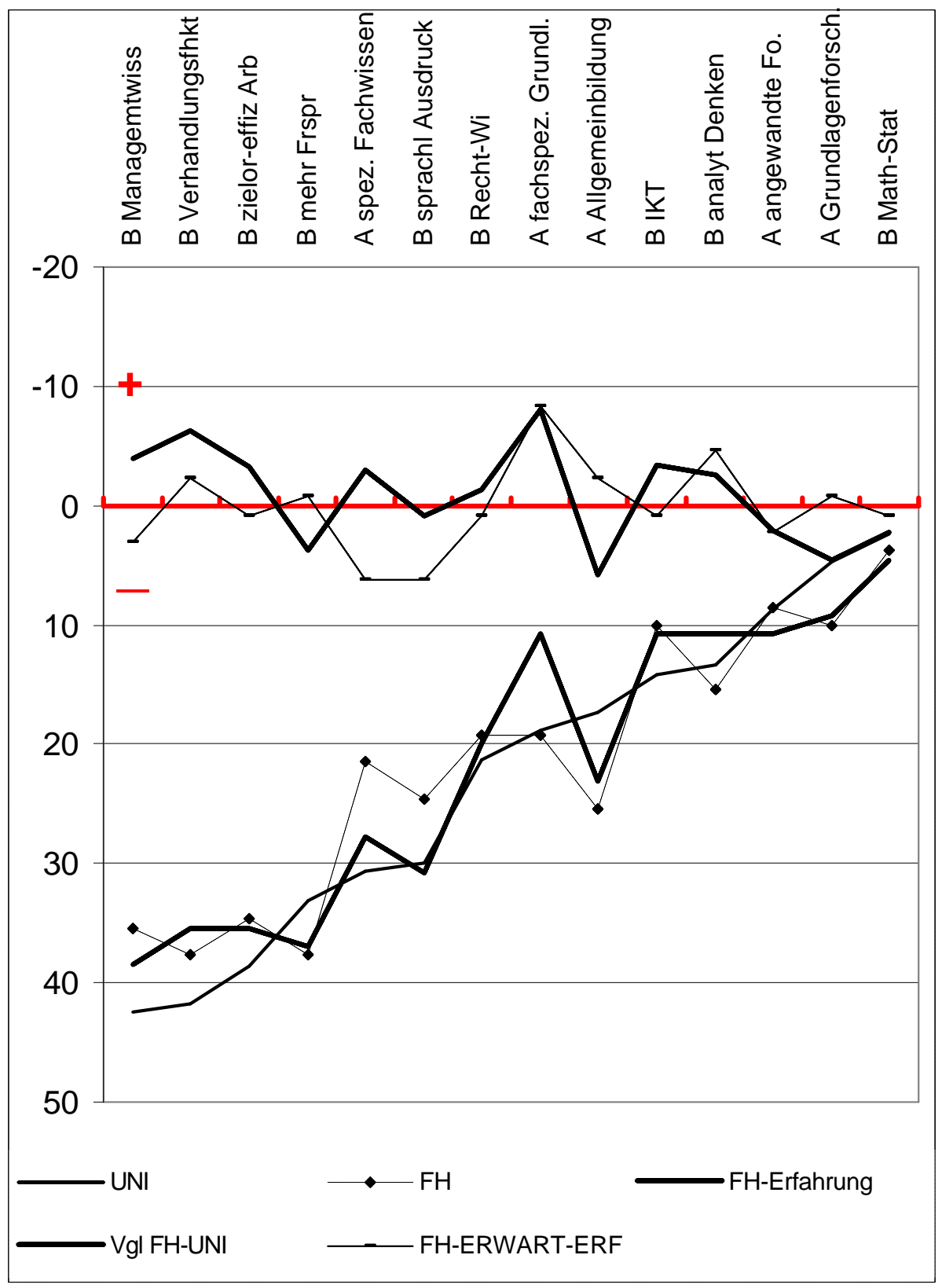

A: allgemeine Kompetenzen; B: spezifische Kompetenzen Quelle: Lang/ Finder 2000

In Abbildung 72 werden die Schwächen in der Rangordnung ausgewiesen, wie sie den UNIAbsolventInnen zugeschrieben werden (die umgekehrte Skala bedeutet niedriger Wert $=$ weniger Schwächen $=$ oben $=$ besser, höherer Wert $=$ mehr Schwächen $=$ unten $=$ schlechter; links sind die häufigsten Schwächen und rechts die geringsten Schwächen der UNI aufgetragen). Der Durchschnittswert der Schwächen ist bei den beiden Abschlüssen gleich, auch zwischen Erwartungen und Erfahrungen bezüglich FH-AbsolventInnen. Die 
wichtigsten Schwächen der UNI-AbsolventInnen sind wirtschaftliche und operative Kompetenzen (Managementwissen, Verhandlungsfähigkeit, effektives Arbeiten), aber auch Fremdsprachenkenntnisse, die geringsten Schwächen werden den UNI-AbsolventInnen bei kognitiven und wissenschaftlichen Kompetenzen (Mathematik/Statistik, Grundlagen- und angewandte Forschung, analytisches Denken) zugeschrieben. Bei den FH-AbsolventInnen ist das Profil sehr ähnlich, zu wenig Fremdsprachenkenntnisse werden stärker als Schwächen gesehen, die Kenntnis fachspezifischer Grundlagen (Spezialisierung) wird im Vergleich zu angewandter Forschung besser bewertet. Im direkten Vergleich zwischen FHund UNI-AbsolventInnen werden den FH-AbsolventInnen größere Schwächen bei den Kompetenzen Grundlagenforschung, Mathematik/Statistik, Allgemeinbildung, angewandte Forschung und Fremdsprachen zugeschrieben. Besser werden sie vor allem in den Bereichen der fachspezifischen Grundlagen, IKT, analytisches Denken und Verhandlungsfähigkeit eingeschätzt.

Für die FH-AbsolventInnen können die Erwartungen und die Erfahrungen gegenübergestellt werden (vgl. Tabelle 27). Besser gegenüber den Erwartungen scheinen sich die FHAbsolventInnen in den Bereichen der fachspezifischen Grundlagen und beim analytischen Denken zu bewähren, mehr Schwächen gegenüber den Erwartungen werden beim spezifischen Fachwissen und beim sprachlichen Ausdruck geortet.

Tabelle 27 Schwächen der UNI- und FH-AbsolventInnen in 14 Kompetenzbereichen

\begin{tabular}{|c|c|c|c|c|c|c|c|}
\hline UNI-Schwächen & $\%$ & $\begin{array}{l}\text { FH-Erfahrung } \\
\text { Schwächen }\end{array}$ & $\%$ & FH/UNI-Vergleich & $\%$ & $\begin{array}{l}\text { FH Erwartung- } \\
\text { Erfahrung }\end{array}$ & $\Delta$ \\
\hline B Math-Stat & 2,4 & B Math-Stat & 4,6 & A fachspez. Grundl. & 57 & A fachspez. Grundl. & $-8,4$ \\
\hline A Grundlagenforsch. & 4,7 & A Grundlagenforsch. & 9,2 & B IKT & 76 & B analyt Denken & $-4,6$ \\
\hline A angewandte Fo. & 8,7 & A fachspez. Grundl. & 10,8 & B analyt Denken & 81 & B Verhandlungsfhkt & $-2,3$ \\
\hline B analyt Denken & 13,4 & B IKT & 10,8 & B Verhandlungsfhkt & 85 & A Allgemeinbildung & $-2,3$ \\
\hline B IKT & 14,2 & B analyt Denken & 10,8 & A spez. Fachwissen & 90 & B mehr Frspr & $-0,8$ \\
\hline A Allgemeinbildung & 17,3 & A angewandte Fo. & 10,8 & B Managemtwiss & 91 & A Grundlagenforsch. & $-0,8$ \\
\hline A fachspez. Grundl. & 18,9 & B Recht-Wi & 20,0 & B zielor-effiz Arb & 92 & B zielor-effiz Arb & 0,8 \\
\hline B Recht-Wi & 21,3 & A Allgemeinbildung & 23,1 & B Recht-Wi & 94 & B Math-Stat & 0,8 \\
\hline B sprachl Ausdruck & 29,9 & A spez. Fachwissen & 27,7 & B sprachl Ausdruck & 103 & B Recht-Wi & 0,8 \\
\hline A spez. Fachwissen & 30,7 & B sprachl Ausdruck & 30,8 & B mehr Frspr & 111 & B IKT & 0,8 \\
\hline B mehr Frspr & 33,1 & B Verhandlungsfhkt & 35,4 & A angewandte Fo. & 124 & A angewandte Fo. & 2,3 \\
\hline B zielor-effiz Arb & 38,6 & B zielor-effiz Arb & 35,4 & A Allgemeinbildung & 134 & B Managemtwiss & 3,1 \\
\hline B Verhandlungsfhkt & 41,7 & B mehr Frspr & 36,9 & B Math-Stat & 192 & B sprachl Ausdruck & 6,2 \\
\hline B Managemtwiss & 42,5 & B Managemtwiss & 38,5 & A Grundlagenforsch. & 196 & A spez. Fachwissen & 6,2 \\
\hline
\end{tabular}

A: allgemeine Kompetenzen; B: spezifische Kompetenzen

Quelle: Lang/Finder 2000

In einer weiteren Studie (Hoyer/Ziegler 2002) wurden die nach Einschätzung der AbsolventInnen im Beruf und im Studium betonten Kompetenzen in einer sehr differenzierten Klassifikation (30 Items) verglichen. Insgesamt werden die abgefragten Items im Beruf um 
einen halben Punkt auf der 5teiligen Skala wichtiger bewertet als im Studium. In der Zusammenfassung nach vier breiten Kompetenzbereichen hat nach den Einschätzungen der AbsolventInnen der Bereich der berufspezifischen Fachkompetenzen den höchsten Stellenwert im Studium, während den persönlichen Handlungskompetenzen im Beruf die größte Bedeutung zugeschrieben wird (die berufliche Bedeutung der Fachkompetenzen wird sogar am geringsten bewertet, jedoch mit sehr kleinen Unterschieden zu den beiden übrigen Kompetenzbereichen: Sozial- und Führungskompetenzen sowie wissenschaftlich-kognitive Kompetenzen). Die grafische Darstellung (Abbildung 73) zeigt die Unterschiede zwischen Beruf und Studium in der Einschätzung der AbsolventInnen. Die größte Diskrepanz besteht im Bereich der persönlichen Handlungskompetenz, die geringste im Bereich der Fachkompetenz, wobei zu betonen ist, dass auch in diesem Bereich die berufliche Bedeutung noch höher eingeschätzt wird als im Studium. Wenn man aus diesen Bewertungen Schlussfolgerungen für das Studium ziehen wollte, heißt das nicht Verringerung der fachlichen Dimension, sondern Stärkung der anderen Kompetenzbereiche.

\section{Abbildung 73: Bewertung von Kompetenzbereichen in Beruf und Studium durch FH- AbsolventInnen}

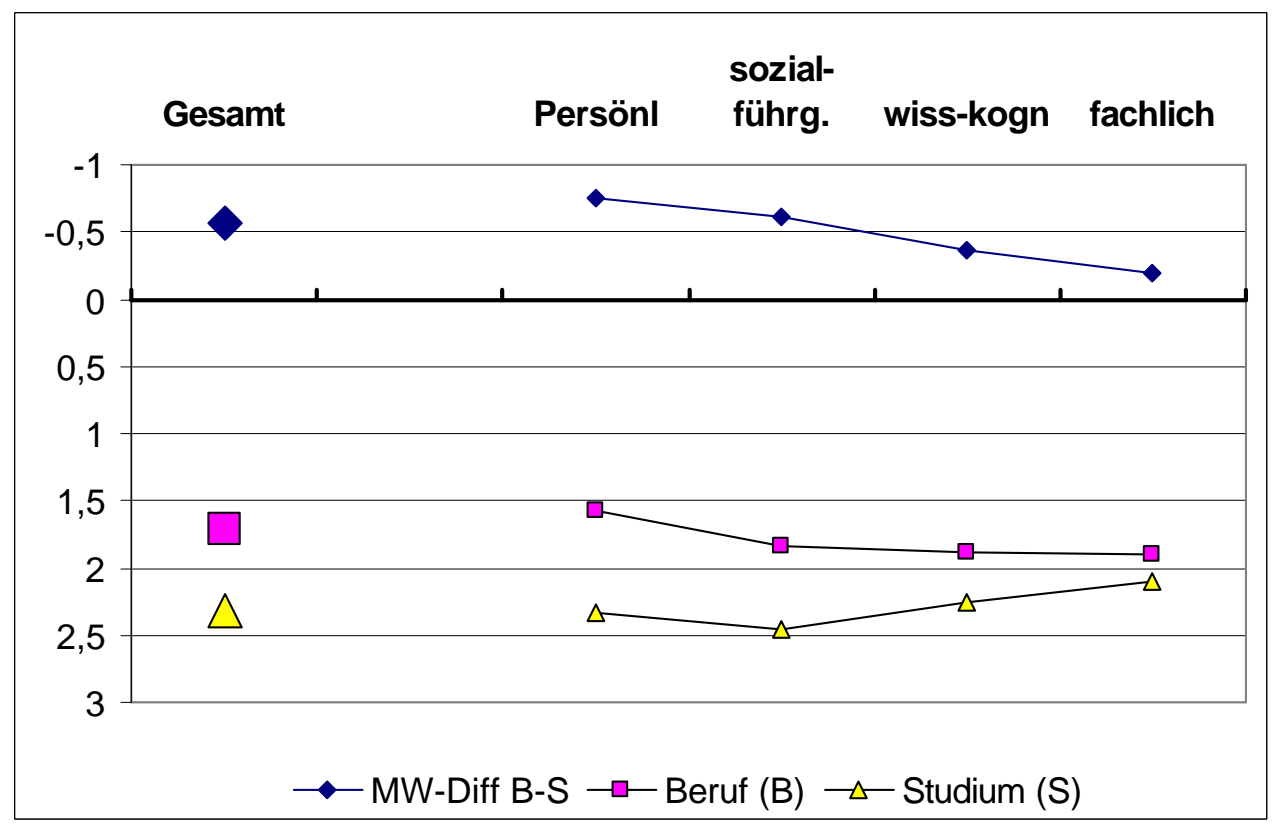

MW-Diff B-S: Mittelwerte der Differenzen zwischen Beruf und Schule.

Quelle: Hoyer/ Ziegler 2002

Teilweise können die Items mit den beiden anderen Studien in bestimmten Ergebnistendenzen verglichen werden. 8 Items sind in der Studie von Lang/Finder (2000) vergleichbar, wobei die Fragestellung nach den Einschätzungen der Schwächen durch KMUs mit FH-Erfahrungen war. Eine ziemlich deutliche Diskrepanz besteht vor allem im Bereich der Fremdsprachenkompetenz, wo die AbsolventInnen einen gewissen Überschuss 
orten, die Betriebe jedoch ein beträchtliches Ausmaß an Schwächen (vgl. Abbildung 74). Weniger deutlich, aber doch tendenziell bemerkenswert ist ein Unterschied in der gegenteiligen Richtung bei den Bereichen analytisches Denken und IKT, wo die AbsolventInnen einen doch deutlichen Mangel angeben, während die Betriebe nur geringe Schwächen sehen. Mit der IBW-Studie (2000) ist der Vergleich nur viel indirekter möglich. Man kann für fünf Items das Ausmaß, in dem die FH-AbsolventInnen besser oder schlechter als die UNI-AbsolventInnen bewertet werden, in Relation zu den Einschätzungen der AbsolventInnen setzen. Im Bereich der Teamfähigkeit erscheint bei diesem Vergleich die Einschätzung der AbsolventInnen vielleicht zu pessimistisch, im Bereich der Entscheidungsfähigkeit korrespondieren die Einschätzungen tendenziell.

\section{Abbildung 74: Kompetenzbereiche von FH- und UNI-AbsolventInnen, Vergleich} zwischen den 3 AbsolventInnenstudien

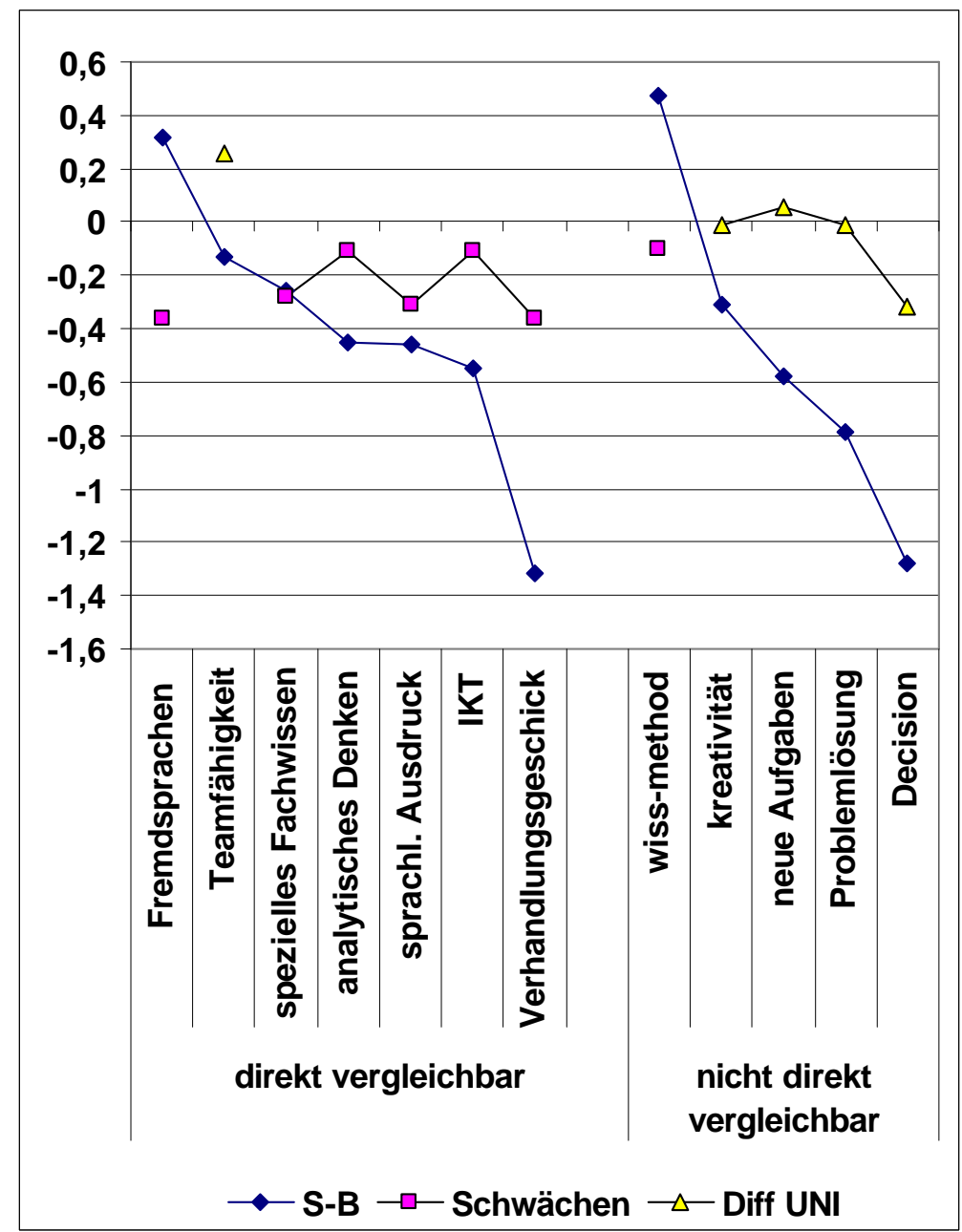

S: Studium

B: Beruf

Quelle: Lang/Finder 2000, Wimmer 2000, Hoyer/Ziegler 2002, IHS-Berechnungen 


\subsubsection{Zur Begrifflichkeit der Berufsfelder bzw. Berufsprofile}

(Auszug aus: Lassnigg / Stöger 1999, 29-32)

Eine weitere Untersuchungsfrage, die bereits in die Berufsfeld- und Qualifikationsforschung hineinreicht, bestand in der Analyse der begrifflichen Fassung der Berufsfelder, die in den Anträgen und in den Bedarfs- und Akzeptanzerhebungen zum Ausdruck kommt. Dabei geht es darum, ansatzweise zu erfassen, in welcher Weise die anwendungsorientierten Profile definiert und umschrieben werden und inwieweit in dieser Hinsicht auch bestimmte Referenzen zur einschlägigen Forschung erkennbar werden.

Im Bereich der Erfassung von Berufsfeldern kann man etwa seit dem Beginn der neunziger Jahre von Tendenzen zu einem Paradigmenwechsel ausgehen, der einerseits zu einer Auflösung der traditionellen Begrifflichkeit und Herangehensweise und andererseits zu neuen Ansatzpunkten und Denkweisen bei der Formulierung von derartigen Feldern, Kategorien oder Begrenzungen führt. Wir befinden uns in einer Übergangszeit, in der die alten Kategorien dabei sind, ihre Bedeutung zu verlieren, aber noch nicht klar ist, wie die neuen aussehen werden.

Die alten Kategorien waren fassbar als Berufe, die auch in statistische Klassifikationen gegossen waren und bis zu einem gewissen Grad ihre Entsprechung in verschiedenen anderen sozialen Kategorisierungen wie Ausbildungen, sozialen Organisationen (Berufsvereinigungen, gewerbliche, gewerkschaftliche Gliederungen etc.) und rechtlichen Kategorien (z.B. Berufsbilder) hatten. Wesentlich an diesen Kategorien war ihre Beständigkeit und (endogene) Anpassung an die vor sich gehenden Veränderungen, die auch bis zu einem gewissen Grad kontrolliert werden konnten. Die Analyse und Beschreibung dieser Veränderungen, die Herausbildung von neuen Berufsgliederungen etc. war ein wichtiger Bestandteil dieses Paradigmas, das in hohem Maße zum Sekundären Sektor bzw. zu den Produktionsberufen assoziiert war. Mit der Tertiärisierung begann sich diese Art der „Beruflichkeit” bereits aufzulösen, was daran erkennbar ist, dass beispielsweise im Bürobereich nie eine ähnlich stark formalisierte berufliche Gliederung entstanden ist wie im Bereich der Produktionsberufe. Dennoch bestand jedoch im Prinzip eine Tendenz und das Bestreben, eine ähnliche Logik der Klassifizierung auch in diesen tertiären Bereichen aufzubauen.

Mit der näheren Analyse von Strukturierungen auf den Arbeitsmärkten und auch mit der Entwicklung der Ansätze der Arbeitsmarktsegmentation und unterschiedlichen Formen der betrieblichen und außerbetrieblichen Qualifizierung und der empirischen Untersuchung der Vorgänge auf den Arbeitsmärkten sind Unterscheidungen zwischen verschiedenen Formen von Qualifikationen und beruflichen Kategorien gemacht worden, die jeweils eine unterschiedliche Bedeutung und auch einen unterschiedlichen Wert auf den Arbeitsmärkten besitzen. Anpassungsprozesse durch externe (numerische) Flexibilität auf beruflichen oder 
unqualifizierten Arbeitsmärkten sind von internen, von informeller Qualifizierung begleiteten Anpassungsprozessen unterschieden worden, die jeweils unterschiedliche Zusammenhänge zu den formalen Ausbildungssystemen aufweisen. Die Diskrepanz zwischen den Systemen beruflicher Gliederung und den Veränderungen der Arbeitsorganisation und Arbeitsteilung ist allenthalben zunehmend beobachtet und festgestellt worden.

In den letzten Jahren, unter den Stichworten der neuen industriellen Revolution, der neuen Produktionskonzepte, der lean production, der flachen Hierarchien und zuletzt der Informationsgesellschaft und der lernenden Ökonomie sind insbesondere zwei neue Richtungen der Konzeptualisierung beruflicher Qualifizierung und beruflicher Qualifikationen entstanden, die sich von den traditionellen Berufen ziemlich deutlich unterscheiden und die insbesondere die traditionellen Berufsfeldanalysen in unterschiedlicher Weise unterlaufen:

- Der Ansatz des Human Resource Development, der die Prozesse des Personaleinsatzes und der begleitenden Qualifizierung in den Unternehmen in den Mittelpunkt stellt und

- der Ansatz der „competence based qualifications”, der eine neue Fassung von Qualifikationen, quasi als Portfolio von nachgewiesenen Kompetenzen vornimmt, wobei die Qualifikationsnachweise stärker additiv, teilweise auch als Ergebnis von sukzessiven Qualifizierungen im Zuge des Erwerbslebens und weniger holistisch, als Ergebnis komplexer formaler Ausbildungen gefasst werden.

Die Entwicklung von Profilen neuer formaler Ausbildungen im Rahmen der österreichischen FH-Studiengänge ist im veränderlichen Umfeld schwer einzuordnen, da diese vorwiegend auf einer pragmatischen Ebene erfolgt. In gewisser Weise geht es um die Definition von neuen Berufs- oder Qualifikationsprofilen, die aus der Vielfältigkeit der Praxis der Berufsausübung vorwiegend in den neuen und dynamischen Bereichen der Wirtschaftstätigkeit „herausgehoben” werden. Referenzen zur einschlägigen Literatur sind im analysierten Material nicht zu erkennen.

Eine nähere Analyse der in den Anträgen beschriebenen Berufsfelder zeigt sehr unterschiedliche „Ereignisräume”, die sehr wenig Systematik und auch einen sehr unterschiedlichen Komplexitätsgrad aufweisen. Berufsfelder werden beispielsweise mit bis zu 70 Stichworten für berufliche Einsatzgebiete gekennzeichnet, was doch eine einigermaßen konkrete Überblickbarkeit in Frage stellt.

Um das Herangehen an die begriffliche Fassung der Berufsfelder etwas zu objektivieren, wurde eine Klassifizierung der verwendeten Begrifflichkeit nach fünf unterschiedlichen Dimensionen vorgenommen, die in der folgenden Abbildung 75 durch die Zusammenstellung der Beispiele veranschaulicht wird. Es zeigt sich, dass die traditionellen Berufsbezeichnungen nur eher selten für die Beschreibung der Profile verwendet werden. Einerseits werden Wirtschafts- bzw. Unternehmensbereiche angeführt, wo die 
Qualifikationen eingesetzt werden sollten, andererseits werden Zusammenstellungen von Kompetenzen (in Begriffen von durch die AbsolventInnen zu erfüllenden Aufgaben und Tätigkeiten) herangezogen.

Im allgemeinen bezeichnen die Beispiele ziemlich komplexe Kompetenzen, deren konkrete Überprüfung im Sinne des „,competence based testing” vermutlich eher schwer fallen würde. 
Abbildung 75: Beschreibung von Berufsfeldern (Übersicht über die verwendeten Begriffe in ausgewählten Anträgen (11 Anträge 1997/981998/99, 17 Anträge 1994/95 - 1996/97)

\begin{tabular}{|c|c|c|c|c|}
\hline $\begin{array}{l}\text { Kompetenzen } \\
\text { (Aufgaben und Tätigkeiten) }\end{array}$ & Position & Berufsbezeichnung & Unternehmensbereich & Branche \\
\hline $\begin{array}{l}\text { Tech 7: Personalbedarfsplanung, Erstellung des } \\
\text { Budgets, } \\
\text { Teambildung/Teamsteuerung, } \\
\text { Ermittlung kostenoptimaler } \\
\text { Fertigungsprogramme } \\
\text { Tech 15: Software selbständig entwickeln., ein } \\
\text { Team von Programmierern leiten } \\
\text { Tech 16: Produktionsplanung- und Kontrolle, } \\
\text { Fertigungsplanung, } \\
\text { Neuproduktentwicklung } \\
\text { Tech 18: das Zusammenwirken zwischen } \\
\text { Automobilindustrie, Zuliefern und } \\
\text { Abnehmern zu verstehen, Projekte zu } \\
\text { leiten, zu organisieren und } \\
\text { auszuführen } \\
\\
\text { Tele 1: Systemanalyse, Koordinierung von } \\
\text { Gruppenarbeit, Rationalisierung } \\
\text { Tele 2: Vertieftes Wissen der Medientechniken, } \\
\text { insbes. der Printtechnologie, der } \\
\text { Kopiertechnik, Bewertung und } \\
\text { Einsatz von Hardwarekomponenten } \\
\text { Tele 3: von der Produktidee zur } \\
\text { Markteinführung, Inhalts- und } \\
\text { Formanalyse } \\
\text { Tele 4: WWW Anwendungen, Gestaltung und } \\
\text { Vorbereitung von Telekonferenzen, } \\
\text { Spracherkennung, Bilderkennung }\end{array}$ & $\begin{array}{l}\text { Tele 2: Führungskraft in KMU, } \\
\text { Mitarbeiter für die diversen } \\
\text { Managementebenen der } \\
\text { Industrie }\end{array}$ & $\begin{array}{l}\text { Tech 7: Betriebs- bzw. } \\
\quad \text { Produktionsmanager } \\
\text { Tech 12: Bauingenieur } \\
\text { Tech 14: Allround-Bauingenieur } \\
\text { Tech 15: Software Engineer } \\
\text { Tech 21: Facility Manager } \\
\text { Tele 3: Multimedia Produzent } \\
\text { Tele 8: Informationsmanager }\end{array}$ & $\begin{array}{l}\text { Tech 7: Produktion, Logistik, } \\
\text { Techn. Planung } \\
\text { Tech 12: Bauablaufsplanung, } \\
\text { Bauleitung, } \\
\text { Bauprojektsteuerung } \\
\text { Tech 16: Marketing, Transport, } \\
\text { Logistik } \\
\text { Tech 18: Entwurf, Fertigung, } \\
\text { Logistik } \\
\text { Technik 22: F\&E, Konstruktion, } \\
\text { Anlageplanung, Vertrieb } \\
\text { Tele 1: Entw., Fertigung, Vertrieb } \\
\text { Tele 2: Produktmanagement, } \\
\text { Systemanalyse, Vertrieb, } \\
\text { Qualitätsmanagement } \\
\\
\text { Tele 4: Systemanalyse, Planung, } \\
\text { Design, Projektleitung } \\
\text { Tele 6: Qualitätssicherung, Produkt } \\
\text { Management, } \\
\text { Vertrieb/Wartung } \\
\text { Tele 15: Marketing-, } \\
\text { Projektleitungs-, } \\
\text { Controllingfunktionen }\end{array}$ & $\begin{array}{l}\text { Tech 16: Sägeindustrie, Holzverarbeitende } \\
\text { Ind., Holztechnik und Holzwirt., } \\
\text { Holzbau } \\
\text { Tech 18: Automobilindustrie, } \\
\text { Mineralölindustrie, Verkehrswirt. } \\
\text { Tele 1: Telekommunikation } \\
\text { Tele 2: indust F\&E, Netzbetreiber, Banken } \\
\quad \text { und Versicherungen, Druck und } \\
\quad \text { Graphik } \\
\text { Tele 3: Multimedia } \\
\text { Tele 4: KMU, Behörden, } \\
\quad \text { Serviceorganisationen } \\
\text { Tele 6: Industrie, Handelsketten, Banken und } \\
\text { Versicherungen }\end{array}$ \\
\hline
\end{tabular}




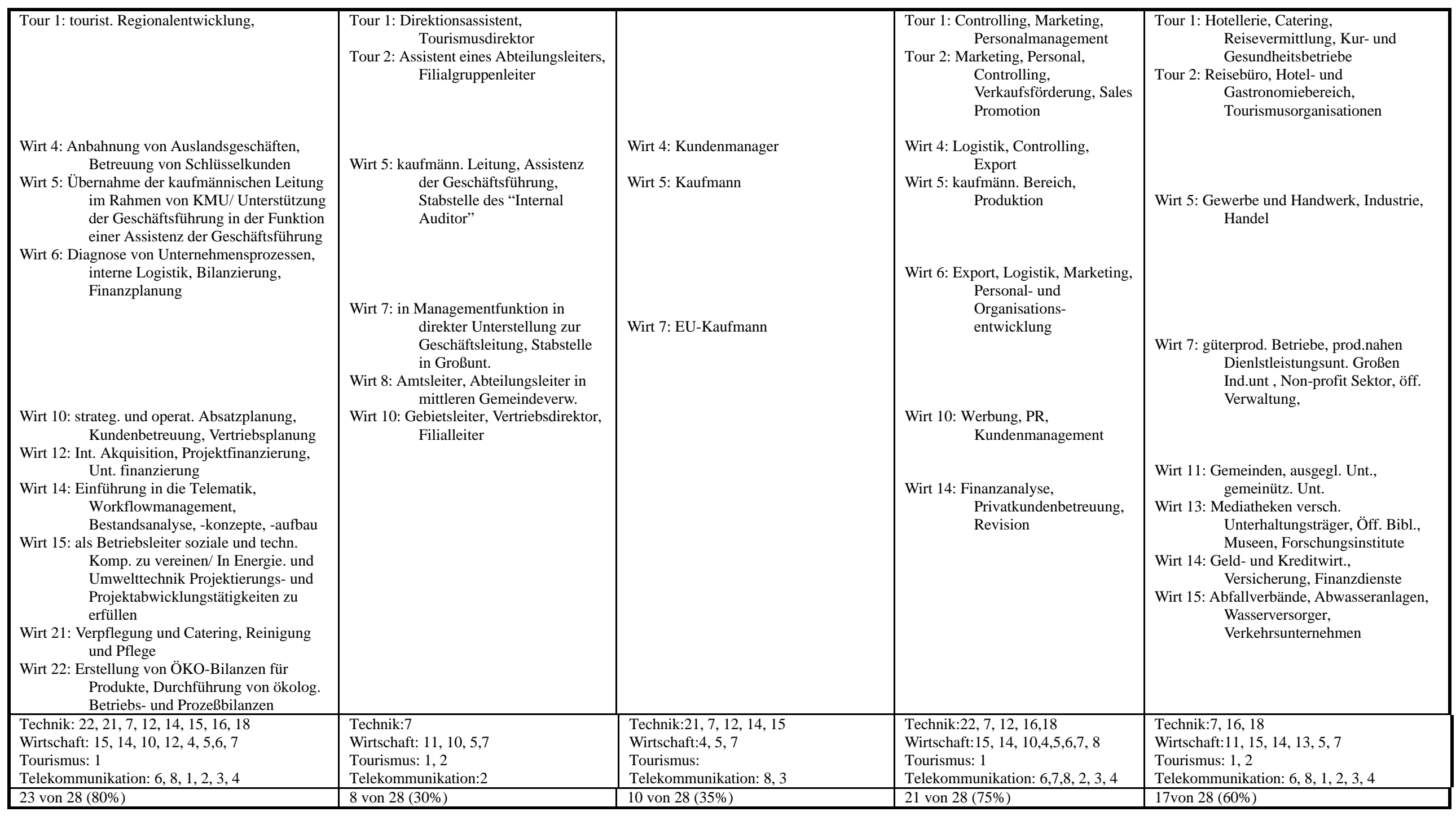




\subsubsection{Synopse der Veränderungen im Umfeld der Bildungspolitik}

\section{Lissabon-Schlussfolgerungen und Innovationspolitik}

Die fortschreitende EU-Integration hat zuletzt vor allem im Zusammenhang mit dem Gipfel von Lissabon neue Zielsetzungen für die Entwicklung der Europäischen Union hervorgebracht, die neue Erfordernisse im Zusammenhang mit wirtschaftlichen und gesellschaftlichen Veränderungen formulieren. Die Fähigkeit zu Innovation und Innovationspolitik hat im Zusammenhang mit der Entwicklung der Wissensgesellschaft und der lernenden Ökonomie ein ganz großes Gewicht bekommen. Verschiedene Politikbereiche und Aktivitäten laufen dabei zusammen: Wissenschafts- und Hochschulpolitik (z.B. Europäischer Forschungsraum, Europäischer Hochschulraum), Wettbewerbs- und Technologiepolitik (z.B. Competitiveness Report, Innovation Observatory), Beschäftigungsund Strukturpolitik (z.B. Joint Employment Report, Europäischer Sozialfonds) und Qualifizierungs- und Bildungspolitik (Memorandum und Mitteilung zum Lebenslangen Lernen, Aktionsprogramm und konkrete Ziele zur offenen Koordinierung im Bereich der Ausbildung), von denen der FH-Sektor in unterschiedlicher Weise betroffen ist. Teilweise werden die Auswirkungen dieser neuen politischen Entwicklungen auf den FH-Sektor davon abhängig sein, inwieweit Österreich eine übergreifende Innovationsstrategie entwickelt und umsetzt. ${ }^{210}$

\section{Bildungspolitik und Standortwettbewerb}

Damit ist ein neuer Stellenwert der Bildungspolitik entstanden, die eine stärker strategische und offensive Bedeutung für "Wettbewerbsfähigkeit“ bzw. "Standortqualität“ bekommen hat. ${ }^{211}$ Beispielsweise sind verschiedene Indikatoren, die sich direkt oder indirekt auf das Bildungswesen beziehen, in den Bereich der Bewertung und des Benchmarking des Beitrages der Mitgliedsstaaten zur globalen Wettbewerbsfähigkeit der Union im Rahmen der Triade einbezogen worden (Bildungsstand der Bevölkerung, hochqualifiziertes Potential, Beteiligung an Weiterbildung etc.). Die Reform der internen Regulation der Bildungssysteme und die Entwicklung von Systemen der Qualitätssicherung, die in Österreich als "latente

210 Die verschiedenen Politikbereiche sind in Österreich bisher nur wenig untereinander koordiniert gewesen, teilweise gibt es in den letzten Jahren Schritte in Richtung verstärkter Kooperation und Koordination, diese führen jedoch auch manchmal zu Regruppierungen, die neuen Koordinationsbedarf erzeugen. Eine systematische Aufarbeitung von strategischen Schritten zur Ausrichtung des Bildungswesens auf die Informationsgesellschaft ist bisher nicht in Sicht. Vgl. dazu z.B. die bereits in der Mitte der 1990er Jahre gesetzten Aktivitäten in Finnland (MoE Finnland 1995).

211 Die Frage, ob man bei Ländern sinnvoll von Wettbewerbsfähigkeit sprechen soll, ist auch unter namhaften Ökonomen durchaus umstritten, vgl. Krugman 1996. 
Zielsetzung" eine wesentliche Neuerung durch den FH-Sektor erfahren hat, ist nur ein Aspekt in diesem Zusammenhang. Die Entwicklung von übergreifenden Zielsetzungen über Strukturen und Dimensionierungen der Ausbildungssysteme ist ebenso wichtig geworden. So wird an der Entwicklung eines flächendeckenden Zielsystems für die Ausbildungssysteme in den Bereichen Qualität, Zugänglichkeit und Offenheit gearbeitet, das die Basis für verschiedene Formen der vergleichenden Beobachtung und des Erfahrungsaustausches der EU-Mitgliedsländer werden soll. Gleichzeitig entwickelt sich die Marktwirtschaft im Bereich des Bildungswesens und manche Länder sehen das Bildungswesen aufgrund seiner volkswirtschaftlichen Größe bereits explizit als „Exportindustrie“212. Hier stellen sich viele offene Fragen, die vor allem auch die Dimensionierung des FH-Sektors betreffen.

\section{Europäische Koordinierung über Ziele, neue Bereitstellungsstrukturen und Monitoring}

Die Initiativen in Richtung der Koordinierung der Bildungspolitik unter den EUMitgliedsstaaten mittels der Entwicklung von konkreten Zielen für die Ausbildungssysteme betreffen drei Ebenen: erstens die Formulierung von Zielen und Indikatoren sowie Verfahren des Erfahrungsaustausches zwischen den Ländern; zweitens die Entwicklung von neuen Bereitstellungsstrukturen im Bereich der Steuerung, Regulierung und Finanzierung, wo unter dem Stichwort „größere Verantwortlichkeit bei den Bildungsinstitutionen“ über neue Verteilungen der Verantwortlichkeiten und Möglichkeiten der Bereitstellung zusätzlicher Mittel diskutiert wird; und drittens die Ebene der Weiterentwicklung der Methoden der Bildungspolitik im Dreieck zwischen der Formulierung von Zielen, der Festlegung von messbaren Ergebnissen und der Entwicklung eines Monitoring-Systems, mit dessen Hilfe die Ziele und Ergebnisse über eine systematische Beobachtung verknüpft werden können. Im FH-Sektor betrifft diese Entwicklung vor allem die Verbindung der dezentralisierten Bereitstellungsstruktur mit der Ebene der bildungspolitischen Steuerung über Ziele, Ergebnisse und Monitoring.

\section{Konkretisierung der bildungspolitischen Ziele für die Bildungssysteme}

Die Koordinierung der Bildungspolitik auf EU-Ebene betrifft erstens die Methode der Zielsteuerung und zweitens die Formulierung von konkreten Zielen und Ergebnissen auf politischer Ebene. Die Übersicht über die Zieldimensionen im EU-Arbeitsprogramm zeigt, dass der FH-Sektor im Prinzip zu den meisten Zielen beitragen kann, einige der Ziele werden auch bereits besonders akzentuiert. Die Formulierung der Ziele macht jedoch klar, dass ein Programm zu ihrer Umsetzung nur sinnvoll für das Bildungswesen insgesamt

212 Vgl. DETYA - Australia 2000. 
formuliert werden kann, wobei sinnvollerweise auch jeweils bestimmte Prioritäten in bestimmten Bereichen gesetzt werden sollten (etwa dort, wo die Abweichung von den Anforderungen besonders groß ist oder wo bestimmte Ziele besonders gut unterstützt werden können). Einige der Ziele werden vermutlich sinnvollerweise als Querschnittsziele zu sehen sein, die in allen Bereichen des Bildungswesens berücksichtigt werden sollten. Für den $\mathrm{FH}$-Sektor ergibt sich bei der Formulierung von Ergebniszielen zumindest in einigen Bereichen ziemlich zwingend eine Abstimmung mit dem Universitätssektor, insbesondere beim Ziel (6) Förderung des Interesses an wissenschaftlichen und technischen Studien und auch beim Ziel (13) Kontakte zur Arbeitswelt, Forschung und Gesellschaft. Der Stellenwert des FH-Sektors im Vergleich zur Universität kann sich je nach der Formulierung von Ergebniszielen und Prioritäten sehr unterschiedlich darstellen. Die Förderung des Zuganges von Frauen zu wissenschaftlichen und technischen Studien sind in diesem Zusammenhang ein wichtiger Aspekt.

\section{Übersicht: Die Ziele im EU-Arbeitsprogramm}

1. Verbesserung der Ausbildung von LehrerInnen und AusbilderInnen

2. Entwicklung der Grundfertigkeiten für die Wissensgesellschaft

3. Entwicklung des Unternehmergeistes

4. Verstärktes Erlernen fremder Sprachen

5. Zugang zu den Informations- und Kommunikationstechnologien für alle

6. Förderung des Interesses an wissenschaftlichen und technischen Studien

7. Bestmögliche Nutzung der Ressourcen

8. Intensivierung von Mobilität und Austausch

9. Stärkung der Europäischen Zusammenarbeit

10. Ein offenes Lernumfeld

11. Unterstützung für aktiven Bürgersinn, gleiche Chancen und gesellschaftlichen Zusammenhalt

12. Lernen muss attraktiver werden

13. Engere Kontakte zur Arbeitswelt und Forschung wie auch zur Gesellschaft im weiteren Sinne 


\section{Lifelong Learning und systemische Koordination}

Mit dem Modell des Lifelong Learning ist ein neues Paradigma für die Gestaltung und Entwicklung der Bildungssysteme in den Vordergrund getreten, das (a) eine Integration von Erstausbildung und Weiterbildung in einem Gesamtsystem anstrebt (in dem die Rolle der Hochschulsysteme erst gefunden werden muss) und (b) eine verbesserte Koordination zwischen den verschiedenen Teilbereichen der Bildungssysteme erfordert (unter Berücksichtigung der formalisierten Systeme und auch der nicht-formalisierten Bildung und des informellen Lernens). Die Schnittstellen im Bildungswesen und die Methoden und Möglichkeiten der Anerkennung nicht-formalisierten Lernens treten dabei in den Vordergrund. Für den FH-Sektor sind damit die Rolle der Weiterbildung, die Zugänglichkeit und die Frage der Anerkennung nicht-formalisierten Lernens direkt betroffen. Die Formulierung einer Strategie zur Förderung des lebensbegleitenden Lernens wird sowohl von der Bildungspolitik als auch von der Beschäftigungs-, Innovations- und Strukturpolitik her angeregt bzw. gefordert. Neben der Entwicklung und Förderung von informellem und nichtformalisiertem Lernen wird der breitere Zugang zu formalen Angeboten als erforderlich angesehen, die Beteiligung an Weiterbildung wird als Erfolgsindikator in den verschiedenen Politikbereichen verwendet. Ein breiter Erfahrungsaustausch auf europäischer Ebene hat zur Definition von wesentlichen Aktionsschwerpunkten zur Förderung des lebensbegleitenden Lernens geführt. ${ }^{213}$ Die Entwicklung von Methoden zur Bewertung von Lernen und die Frage ausreichender Investitionen sind wichtige Aktionsschwerpunkte. Für die FH-Institutionen stellt sich einerseits die Frage der Förderung nicht-traditioneller Zugänge in das reguläre Studium, andererseits aber auch die Frage nach ihrer Beteiligung am Weiterbildungsmarkt im Rahmen ihrer Dienstleistungsfunktion. Welches Gewicht sollen diese beiden Aufgaben haben? Welche Investitionen sollen zu ihrer Erfüllung getätigt werden? Welcher Nutzen ist daraus zu erwarten?

\section{Übersicht: Aktionsschwerpunkte zur Förderung des lebensbegleitenden Lernen}

1. Bewertung des Lernens

2. Information, Beratung, Orientierung

3. Zeit und Geld in Lernen investieren

4. Lernende und Lernangebot zusammenführen

5. Grundqualifikationen

6. Innovative Pädagogik

${ }^{213}$ Siehe EU-Kommission 2001 (http://www.lebenslangeslernen.at/ > aktuelles). 


\section{Neue Kompetenzen als Zentralbegriff inhaltlicher und methodischer Innovation}

An der Schnittstelle zwischen Pädagogik und Bildungspolitik haben sich neue Fragen und Begriffe durchgesetzt, die sich auf die Definition und Auswahl der Inhalte im Wechselspiel mit der Gestaltung der Lernprozesse und der Sicherung der Ergebnisse beziehen. Im Rahmen der Entwicklung konkreter Ziele für die Bildungssysteme auf EU-Ebene und in einem OECD-Projekt214 wird der Entwicklung und Definition von neuen Kompetenzen großes Gewicht gegeben, die aufgrund der gesellschaftlichen und wirtschaftlichen Veränderungen erforderlich werden (Lassnigg/Mayer 2001). An der Schnittstelle von Bildungspolitik und Innovationspolitik wurden neue Begrifflichkeiten über Wissensformen und inre Dynamik entwickelt, die die Ausbildungssysteme vor neue Herausforderungen stellen (vgl. Lundvall/Borras 1999; Lassnigg 1998). Es geht bei diesen Diskussionen darum, dass die Fachkompetenzen als Kernbereiche traditioneller Ausbildungssysteme zunehmend durch verschiedene Formen von weitergehenden Kompetenzen ergänzt werden müssen, die aber noch unzureichend definiert und messbar gemacht werden konnten. Es werden auch bestimmte Zusammenhänge zwischen dem Erlernen der neuen Kompetenzen und den Formen der Lernens und Lehrens hergestellt, die großes Gewicht auf selbstgesteuertes kontextuelles Lernen legen. In der Selbstdarstellung der FH-Studiengänge spielen die neuen Kompetenzen eine große Rolle, die weiter oben referierten Versuche der empirischen Erfassung haben jedoch verschiedene Zweifel hervorgebracht, inwieweit die Vorteile des verschulten Lernens im Hinblick auf zielgerichtete und abschlussorientierte Studien Nachteile im Hinblick auf den Erwerb der geforderten neuen Kompetenzen mit sich bringen. Auf EU-Ebene werden für das Hochschulsystem vor allem die Förderung von Kompetenzen in den Bereichen Mathematik, Naturwissenschaft und Technologie, darunter insbesondere auch Informations- und Kommunikationstechnologien in den nächsten Jahren zunehmend an Relevanz gewinnen. Ziele und Indikatoren im Hinblick auf quantitative und qualitative Verbesserungen in diesen Bereichen sind spezifische Schwerpunkte bei der Entwicklung der konkreten Ziele für die Ausbildungssysteme im Bereich der EU insgesamt. Die Positionierung Österreichs in diesen Bereichen wird sich verstärkt stellen.

\section{Forschungsstrategie als Umsetzung von Innovationspolitik}

Im Rahmen der verstärkten Bedeutung der Innovationspolitik und der Fragen der Entwicklung und Stärkung des Innovationssystems haben die seit Jahrzehnten immer wieder angesprochenen und diskutierten Schwächen in Österreich insbesondere mit der Einrichtung des Rates für Forschung und Technologieentwicklung (FTE-Rat)215 verstärkte operative

\footnotetext{
214 Vgl. dazu die Unterlagen des Projektes "DeSeCo - Definition and Selection of Competencies" (http://www.statistik.admin.ch/stat ch/ber15/deseco/).

215 Siehe: http://www.rat-fte.at/de.php.
} 
Bedeutung erhalten. Die seit langem immer wieder aufgestellte Zielsetzung der Erhöhung der Forschungsquote wurde in Form einer quantitativen Zielsetzung fixiert und verstärkt auf ihre operativen Konsequenzen durchgearbeitet (vgl. Clement et al 2002). Damit sind hinter den globalen quantitativen und finanziellen Zielsetzungen (2,5\% des BIP) abgesehen von der Frage der Aufbringung der Mittel die konkreteren Umsetzungs- und Allokationsprobleme in die Diskussion gekommen: Welche Implikationen hätte die angestrebte erhöhte Forschungsquote für die institutionelle Struktur des Innovationssystems? In welche Bereiche sollen die zusätzlichen Mittel fließen? Welcher Personalbedarf wird dadurch ausgelöst und wie soll dieser gedeckt werden? Es ist klar, dass der FH-Sektor durch diese Fragen unmittelbar betroffen ist, umso mehr, als hier eine zeitliche Koinzidenz zwischen dem beginnenden bzw. sich verstärkenden Aufbau dieser Funktionen im FH-Sektor und der verstärkten Strategie- und Politikentwicklung besteht.

\section{Unternehmen und Regionen im Innovations- und Kompetenzentwicklungssystem}

Im Zusammenhang mit der verstärkten Bedeutung der Innovationstätigkeit für den wirtschaftlichen Erfolg im Zuge der postulierten Veränderungen in Richtung der lernenden Ökonomie und der Wissensgesellschaft sind viele Fragen der Konsequenzen dieser Entwicklungen für die verschiedensten gesellschaftlichen und wirtschaftlichen Akteure auf der Mikro- und Meso-Ebene (Unternehmen, regionale Akteure, Beschäftigte, Sozialpartner, Familien etc.) aufgeworfen worden. Prominente Stellung haben in diesem Zusammenhang Fragen nach der Stellung der Unternehmen im Innovationssystem (Stichworte: Zusammenhang von technologischen und organisatorischen Innovationen, lernende Organisation etc.), Fragen nach der Beziehung zwischen der regionalen und den überregionalen Ebene (Stichworte: Globalisierung und Regionalisierung, lernende Region) und Fragen nach der Beziehung zwischen Innovationssystem und Bildungssystem (Stichworte: lernende Wirtschaft, lernende Gesellschaft, „system of competency building and innovation"). Die Fachhochschuleinrichtungen können aufgrund ihrer Aufgabenstellung und Positionierung gewissermaßen im Schnittpunkt zwischen diesen drei Bereichen (veränderte Unternehmenspraktiken, regionaler Kontext, Beziehung zwischen Ausbildung und Innovation) gesehen werden, wobei von innen erwartet wird, eine aktive Rolle in diesen Beziehungen zu spielen.

\section{Paradigmenwechsel in der Regionalpolitik}

Die Zusammenarbeit der (regionalen) Akteure in (regionalen) Innovationssystemen bzw. die Entwicklung von lernenden Regionen ist ein weiterer Aspekt, der in den letzten Jahren neu akzentuiert wurde. Es wird von einem "Paradigmenwechsel“ in der Regionalpolitik gesprochen, der in der Ablösung des distributiven Fokus durch stärker innovative und impulsgebende Funktionen besteht. Die Zielsetzungen im Zusammenhang mit der 
Regionalisierung und auch die dabei verwendeten Indikatoren und Verfahren der Bewertung können ziemlich klar der distributiven Orientierung zugeordnet werden. Die veränderte regionalpolitische Orientierung bringt vor allem Aspekte des Zusammenwirkens der verschiedenen Akteure im regionalen Raum und die veränderte Positionierung von Hochschulen in den Vordergrund, die im Kapitel 5 über Konzentration näher behandelt werden. Auch das Gewicht und die Bewertung von Mobilität hat sich verändert, sodass die Frage der Positionierung von Hochschulen im Mobilitätsraum, einerseits die Erwartungen an die Mobilitätsbereitschaft der Beteiligten, andererseits aber auch die Effekte von Mobilitätsprozessen neu gestellt werden. 


\subsubsection{Ergänzende Grafiken und Tabellen zu Kapitel 3}

Abbildung 76: Personalpolitik der klein- und mittelbetrieblichen Unternehmen gegenüber BHS, Fachhochschulen, Universitäten

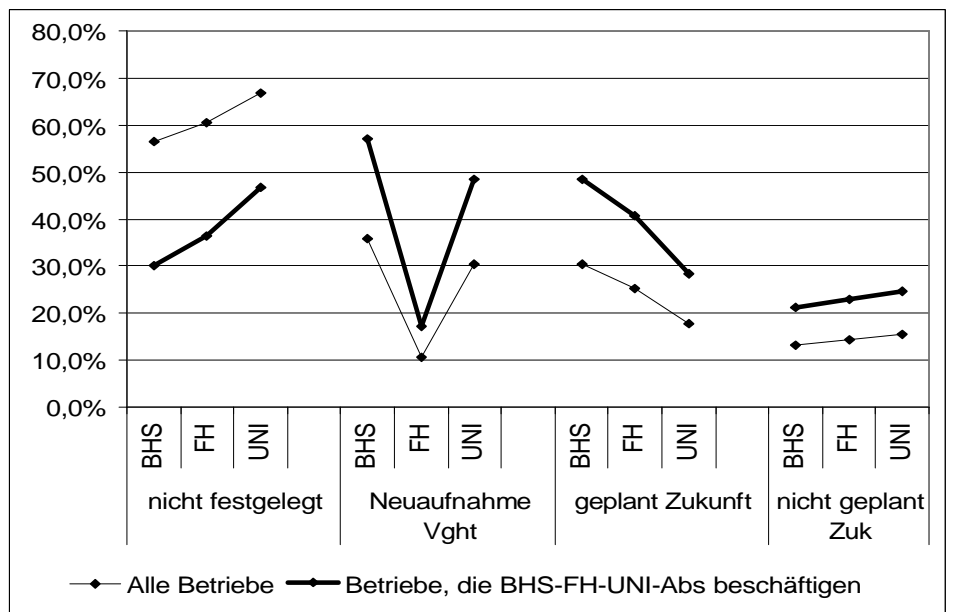

Quelle: Lang/ Finder 2000.

Abbildung 77: Qualifikationsstruktur der Betriebe und das Interesse für Fachhochschulen

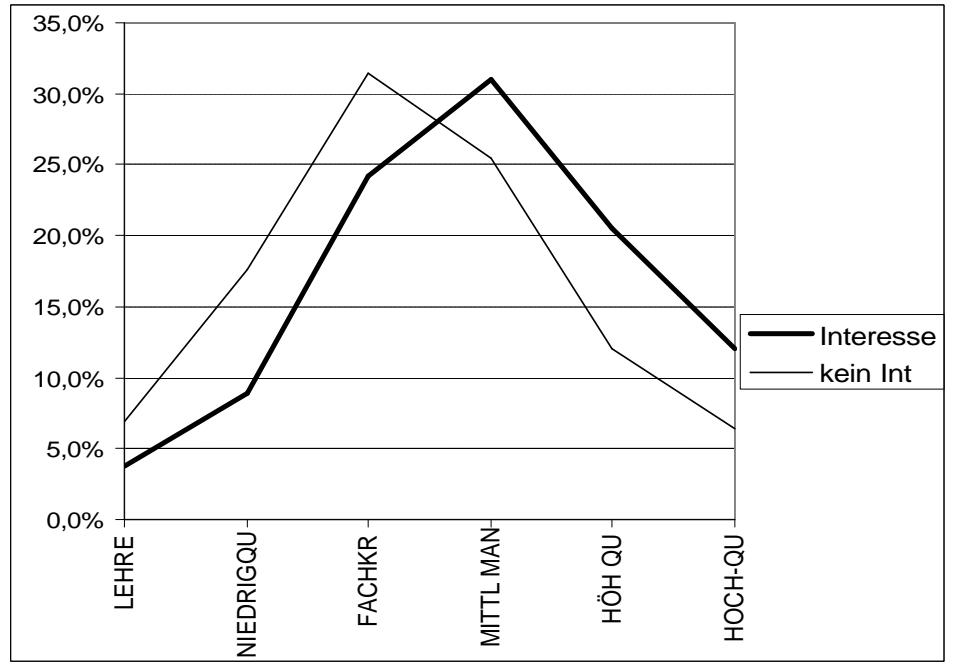

Quelle: Lang/ Finder 2000. 
Tabelle 28: Ausbildungsort der Personen in Weiterbildung 1998/99 (in Prozent)

\begin{tabular}{|c|c|c|c|c|c|c|c|}
\hline Gliederungsmerkmale & 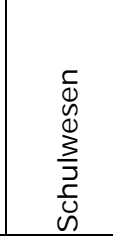 & 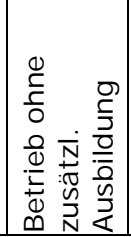 & 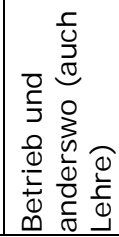 & 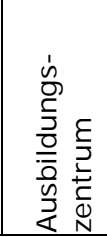 & 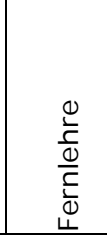 & 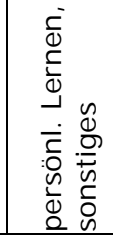 & $\begin{array}{l}\stackrel{\circlearrowright}{E} \\
\stackrel{\xi}{\zeta} \\
\text { जे }\end{array}$ \\
\hline \multicolumn{8}{|l|}{ Geschlecht } \\
\hline Männer & $15,5 \%$ & $10,5 \%$ & $27,8 \%$ & $24,8 \%$ & $10,2 \%$ & $11,2 \%$ & $100 \%$ \\
\hline Frauen & $16,8 \%$ & $8,2 \%$ & $21,8 \%$ & $28,1 \%$ & $8,6 \%$ & $16,6 \%$ & $100 \%$ \\
\hline \multicolumn{8}{|l|}{ Berufsgruppen } \\
\hline Hochqualifizierte Berufe Privatwirtschaft & $16,1 \%$ & $11,9 \%$ & $27,5 \%$ & $24,3 \%$ & $6,6 \%$ & $13,7 \%$ & $100 \%$ \\
\hline Hochqualifiz. Berufe Non-Profit-Sektors & $16,8 \%$ & $9,4 \%$ & $22,1 \%$ & $25,1 \%$ & $7,2 \%$ & $19,3 \%$ & $100 \%$ \\
\hline Qualifizierte Berufe & $14,8 \%$ & $9,4 \%$ & $22,2 \%$ & $30,8 \%$ & $10,8 \%$ & $12,0 \%$ & $100 \%$ \\
\hline Geringqualifizierte/Facharbeiterberufe & $16,4 \%$ & $8,7 \%$ & $29,6 \%$ & $24,2 \%$ & $11,7 \%$ & $9,3 \%$ & $100 \%$ \\
\hline \multicolumn{8}{|l|}{ Bildungsabschluss } \\
\hline PS & $15,4 \%$ & $8,3 \%$ & $31,6 \%$ & $19,6 \%$ & $15,1 \%$ & $10,0 \%$ & $100 \%$ \\
\hline Lehre & $11,6 \%$ & $9,8 \%$ & $29,6 \%$ & $28,3 \%$ & $10,9 \%$ & $9,9 \%$ & $100 \%$ \\
\hline AHS/BMHS & $20,0 \%$ & $10,1 \%$ & $23,2 \%$ & $27,1 \%$ & $6,7 \%$ & $12,9 \%$ & $100 \%$ \\
\hline $\mathrm{FH} / \mathrm{UNI}$ & $16,7 \%$ & $8,1 \%$ & $16,7 \%$ & $24,5 \%$ & $9,6 \%$ & $24,3 \%$ & $100 \%$ \\
\hline \multicolumn{8}{|l|}{ Gemeindengrößenklasse } \\
\hline Gemeinden bis 20.000 Einwohner & $14,4 \%$ & $9,5 \%$ & $28,0 \%$ & $28,5 \%$ & $7,2 \%$ & $12,4 \%$ & $100 \%$ \\
\hline Gemeinden über 20.000 Einwohner & $18,6 \%$ & $9,5 \%$ & $21,1 \%$ & $22,8 \%$ & $13,0 \%$ & $15,0 \%$ & $100 \%$ \\
\hline \multicolumn{8}{|l|}{ Alter } \\
\hline bis 25 Jahre & $29,3 \%$ & $6,2 \%$ & $25,9 \%$ & $25,0 \%$ & $4,6 \%$ & $8,9 \%$ & $100 \%$ \\
\hline 26-45 Jahre & $14,9 \%$ & $10,4 \%$ & $24,0 \%$ & $26,9 \%$ & $9,5 \%$ & $14,3 \%$ & $100 \%$ \\
\hline 46 Jahre und älter & $8,4 \%$ & $9,3 \%$ & $28,6 \%$ & $25,0 \%$ & $13,9 \%$ & $14,9 \%$ & $100 \%$ \\
\hline \multicolumn{8}{|l|}{ Erwerbstätigkeit } \\
\hline Erwerbstätig & $15,7 \%$ & $9,7 \%$ & $25,7 \%$ & $25,9 \%$ & $9,7 \%$ & $13,2 \%$ & $100 \%$ \\
\hline Nicht Erwerbstätige & $25,4 \%$ & $3,6 \%$ & $14,4 \%$ & $32,6 \%$ & $4,4 \%$ & $19,6 \%$ & $100 \%$ \\
\hline Insgesamt & $16,1 \%$ & $9,5 \%$ & $25,2 \%$ & $26,2 \%$ & $9,5 \%$ & $13,5 \%$ & $100 \%$ \\
\hline
\end{tabular}

Anmerkung: Mittel aus den Mikrozensen (Arbeitskräfteerhebung) 1998/1 und 1999/1. Die Lehrlinge sind nicht in den Daten (Labour Force) enthalten, da sich diese ja in Erstausbildung befinden.

Quelle: Statistik Austria, Mikrozensen; Berechnungen: IHS 
Tabelle 29: Ausbildungsort der Personen in Weiterbildung 1998/99 (Absolut)

\begin{tabular}{|c|c|c|c|c|c|c|c|}
\hline Gliederungsmerkmale & 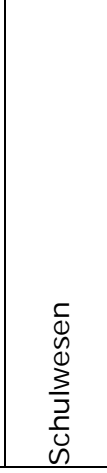 & 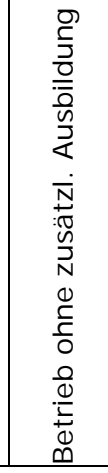 & 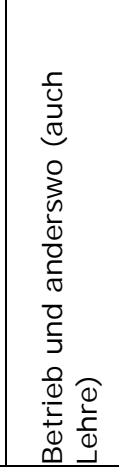 & 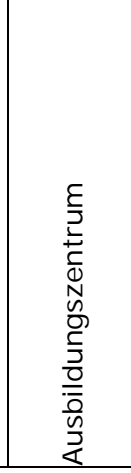 & 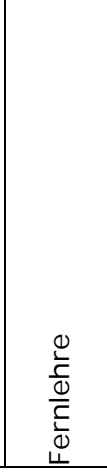 & 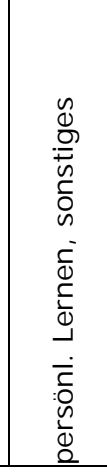 & 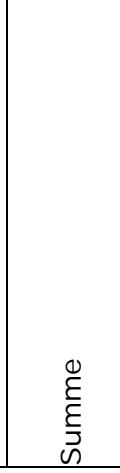 \\
\hline \multicolumn{8}{|l|}{ Geschlecht } \\
\hline Männer & 35822 & 24084 & 63955 & 57183 & 23607 & 25733 & 230384 \\
\hline Frauen & 28732 & 14015 & 37350 & 48078 & 14641 & 28366 & 171184 \\
\hline \multicolumn{8}{|l|}{ Berufsgruppen } \\
\hline Hochqualifizierte Berufe Privatwirtschaft & 7687 & 5673 & 13098 & 11569 & 3122 & 6519 & 47669 \\
\hline Hochqualifiz. Berufe Non-Profit-Sektors & 19629 & 11033 & 25934 & 29394 & 8471 & 22641 & 117103 \\
\hline Qualifizierte Berufe & 15741 & 10031 & 23589 & 32688 & 11442 & 12739 & 106229 \\
\hline Geringqualifizierte/Facharbeiterberufe & 21437 & 11363 & 38684 & 31610 & 15213 & 12200 & 130507 \\
\hline \multicolumn{8}{|l|}{ Bildungsabschluss } \\
\hline PS & 6821 & 3678 & 13990 & 8645 & 6658 & 4417 & 44209 \\
\hline Lehre & 15761 & 13332 & 40265 & 38416 & 14766 & 13437 & 135977 \\
\hline AHS/BMHS & 30732 & 15610 & 35797 & 41686 & 10370 & 19850 & 154045 \\
\hline FH/UNI & 11240 & 5479 & 11254 & 16513 & 6455 & 16395 & 67337 \\
\hline \multicolumn{8}{|l|}{ Gemeindengrößenklasse } \\
\hline Gemeinden bis 20.000 Einwohner & 34423 & 22779 & 67227 & 68288 & 17226 & 29738 & 239681 \\
\hline Gemeinden über 20.000 Einwohner & 30132 & 15320 & 34078 & 36973 & 21022 & 24361 & 161887 \\
\hline \multicolumn{8}{|l|}{ Alter } \\
\hline bis 25 Jahre & 20090 & 4275 & 17764 & 17146 & 3145 & 6064 & 68484 \\
\hline 26-45 Jahre & 37768 & 26382 & 60647 & 68054 & 24008 & 36127 & 252987 \\
\hline 46 Jahre und älter & 6696 & 7442 & 22894 & 20061 & 11095 & 11908 & 80096 \\
\hline \multicolumn{8}{|l|}{ Erwerbstätigkeit } \\
\hline Erwerbstätig & 60362 & 37513 & 98922 & 99872 & 37514 & 50863 & 385045 \\
\hline Nicht Erwerbstätige & 4193 & 587 & 2384 & 5388 & 734 & 3237 & 16522 \\
\hline Insgesamt & 64554 & 38099 & 101305 & 105261 & 38248 & 54099 & 401567 \\
\hline
\end{tabular}

Anmerkung: Mittel aus den Mikrozensen (Arbeitskräfteerhebung) 1998/1 und 1999/1. Die Lehrlinge sind nicht in den Daten (Labour Force) enthalten, da sich diese ja in Erstausbildung befinden.

Quelle: Statistik Austria, Mikrozensen; Berechnungen: IHS 
Tabelle 30: Ausbildungszweck der Personen in Weiterbildung 1998/99 (in Prozent)

\begin{tabular}{|c|c|c|c|c|c|c|c|}
\hline Gliederungsmerkmale & 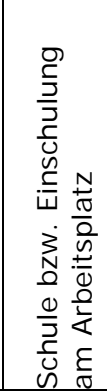 & 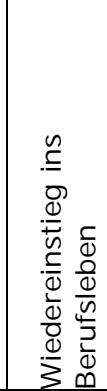 & 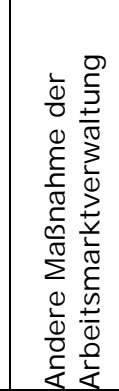 & 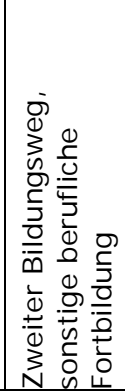 & 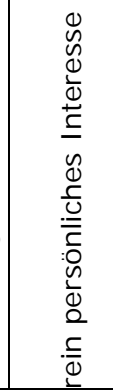 & 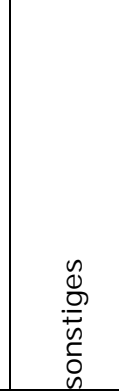 & 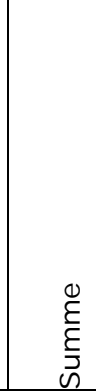 \\
\hline \multicolumn{8}{|l|}{ Geschlecht } \\
\hline Männer & $16,8 \%$ & $0,7 \%$ & $2,6 \%$ & $49,3 \%$ & $18,3 \%$ & $12,3 \%$ & $100 \%$ \\
\hline Frauen & $17,6 \%$ & $2,0 \%$ & $2,3 \%$ & $50,6 \%$ & $18,7 \%$ & $8,9 \%$ & $100 \%$ \\
\hline \multicolumn{8}{|l|}{ Berufsgruppen } \\
\hline Hochqualifizierte Berufe Privatwirtschaft & $17,2 \%$ & $0,7 \%$ & $1,7 \%$ & $50,1 \%$ & $19,4 \%$ & $10,9 \%$ & $100 \%$ \\
\hline Hochqualifiz. Berufe Non-Profit-Sektors & $13,3 \%$ & $0,8 \%$ & $1,7 \%$ & $54,7 \%$ & $17,6 \%$ & $11,9 \%$ & $100 \%$ \\
\hline Qualifizierte Berufe & $17,9 \%$ & $1,7 \%$ & $2,6 \%$ & $48,8 \%$ & $20,0 \%$ & $9,0 \%$ & $100 \%$ \\
\hline Geringqualifizierte/Facharbeiterberufe & $20,1 \%$ & $1,6 \%$ & $3,3 \%$ & $45,9 \%$ & $17,7 \%$ & $11,4 \%$ & $100 \%$ \\
\hline \multicolumn{8}{|l|}{ Bildungsabschluss } \\
\hline PS & $26,5 \%$ & $2,5 \%$ & $4,0 \%$ & $37,5 \%$ & $19,7 \%$ & $9,8 \%$ & $100 \%$ \\
\hline Lehre & $15,4 \%$ & $1,7 \%$ & $2,3 \%$ & $52,0 \%$ & $17,9 \%$ & $10,7 \%$ & $100 \%$ \\
\hline AHS/BMHS & $19,6 \%$ & $0,9 \%$ & $2,2 \%$ & $49,2 \%$ & $18,2 \%$ & $10,0 \%$ & $100 \%$ \\
\hline FH/UNI & $9,0 \%$ & $0,6 \%$ & $2,5 \%$ & $55,0 \%$ & $19,4 \%$ & $13,4 \%$ & $100 \%$ \\
\hline \multicolumn{8}{|l|}{ Gemeindengrößenklasse } \\
\hline Gemeinden bis 20.000 Einwohner & $16,9 \%$ & $1,1 \%$ & $2,6 \%$ & $51,6 \%$ & $17,6 \%$ & $10,3 \%$ & $100 \%$ \\
\hline Gemeinden über 20.000 Einwohner & $17,4 \%$ & $1,5 \%$ & $2,2 \%$ & $47,5 \%$ & $19,7 \%$ & $11,6 \%$ & $100 \%$ \\
\hline \multicolumn{8}{|l|}{ Alter } \\
\hline bis 25 Jahre & $32,7 \%$ & $0,9 \%$ & $2,2 \%$ & $41,9 \%$ & $15,4 \%$ & $6,9 \%$ & $100 \%$ \\
\hline 26-45 Jahre & $13,4 \%$ & $1,5 \%$ & $2,5 \%$ & $53,2 \%$ & $18,3 \%$ & $11,1 \%$ & $100 \%$ \\
\hline 46 Jahre und älter & $15,5 \%$ & $0,6 \%$ & $2,5 \%$ & $46,1 \%$ & $21,8 \%$ & $13,5 \%$ & $100 \%$ \\
\hline \multicolumn{8}{|l|}{ Erwerbstätigkeit } \\
\hline Erwerbstätig & $17,0 \%$ & $0,8 \%$ & $2,3 \%$ & $50,5 \%$ & $18,5 \%$ & $10,9 \%$ & $100 \%$ \\
\hline Nicht Erwerbstätige & $20,5 \%$ & $12,5 \%$ & $5,9 \%$ & $34,3 \%$ & $17,0 \%$ & $9,8 \%$ & $100 \%$ \\
\hline Insgesamt & $17,1 \%$ & $1,2 \%$ & $2,5 \%$ & $49,8 \%$ & $18,5 \%$ & $10,8 \%$ & $100 \%$ \\
\hline
\end{tabular}

Anmerkung: Mittel aus den Mikrozensen (Arbeitskräfteerhebung) 1998/1 und 1999/1. Die Lehrlinge sind nicht in den Daten (Labour Force) enthalten, da sich diese ja in Erstausbildung befinden.

Quelle: Statistik Austria, Mikrozensen; Berechnungen: IHS 
Tabelle 31: Ausbildungszweck der Personen in Weiterbildung 1998/99 (Absolut)

\begin{tabular}{|c|c|c|c|c|c|c|c|}
\hline Gliederungsmerkmale & 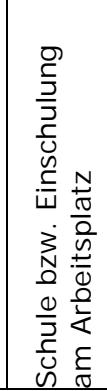 & 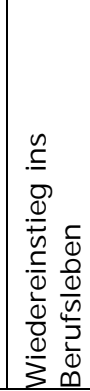 & 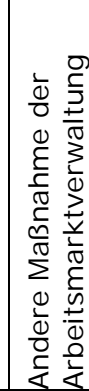 & 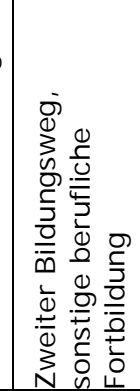 & 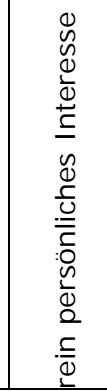 & 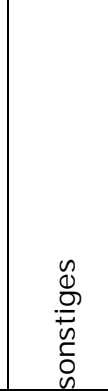 & $\begin{array}{l}\stackrel{d}{E} \\
\stackrel{\xi}{J} \\
\sim\end{array}$ \\
\hline \multicolumn{8}{|l|}{ Geschlecht } \\
\hline Männer & 37569 & 1511 & 5871 & 110234 & 40835 & 27473 & 223492 \\
\hline Frauen & 29266 & 3365 & 3771 & 84289 & 31247 & 14798 & 166736 \\
\hline \multicolumn{8}{|l|}{ Berufsgruppen } \\
\hline Hochqualifizierte Berufe Privatwirtschaft & 8109 & 346 & 817 & 23671 & 9179 & 5133 & 47256 \\
\hline Hochqualifiz. Berufe Non-Profit-Sektors & 15479 & 882 & 2003 & 63751 & 20457 & 13868 & 116440 \\
\hline Qualifizierte Berufe & 18428 & 1704 & 2697 & 50329 & 20636 & 9235 & 103029 \\
\hline Geringqualifizierte/Facharbeiterberufe & 24819 & 1943 & 4125 & 56711 & 21811 & 14036 & 123445 \\
\hline \multicolumn{8}{|l|}{ Bildungsabschluss } \\
\hline PS & 11177 & 1034 & 1683 & 15813 & 8293 & 4138 & 42138 \\
\hline Lehre & 19712 & 2135 & 2958 & 66498 & 22904 & 13755 & 127964 \\
\hline AHS/BMHS & 29866 & 1324 & 3307 & 75076 & 27778 & 15305 & 152656 \\
\hline $\mathrm{FH} / \mathrm{UNI}$ & 6079 & 382 & 1694 & 37135 & 13108 & 9074 & 67471 \\
\hline \multicolumn{8}{|l|}{ Gemeindengrößenklasse } \\
\hline Gemeinden bis 20.000 Einwohner & 38212 & 2441 & 5971 & 116616 & 39714 & 23217 & 226173 \\
\hline Gemeinden über 20.000 Einwohner & 28623 & 2435 & 3671 & 77906 & 32368 & 19054 & 164056 \\
\hline \multicolumn{8}{|l|}{ Alter } \\
\hline bis 25 Jahre & 22091 & 637 & 1455 & 28333 & 10427 & 4672 & 67614 \\
\hline 26-45 Jahre & 32761 & 3761 & 6240 & 130541 & 44824 & 27171 & 245298 \\
\hline $46 \mathrm{~J}$ ahre und älter & 11983 & 478 & 1947 & 35648 & 16831 & 10429 & 77316 \\
\hline \multicolumn{8}{|l|}{ Erwerbstätigkeit } \\
\hline Erwerbstätig & 63569 & 2894 & 8704 & 189071 & 69371 & 40715 & 374323 \\
\hline Nicht Erwerbstätige & 3266 & 1982 & 938 & 5451 & 2711 & 1556 & 15905 \\
\hline Insgesamt & 66835 & 4876 & 9642 & 194522 & 72082 & 42271 & 390229 \\
\hline
\end{tabular}

Anmerkung: Mittel aus den Mikrozensen (Arbeitskräfteerhebung) 1998/1 und 1999/1. Die Lehrlinge sind nicht in den Daten (Labour Force) enthalten, da sich diese ja in Erstausbildung befinden.

Quelle: Statistik Austria, Mikrozensen; Berechnungen: IHS 


\subsection{Ergänzende Grafiken und Tabellen zu Kapitel 5}

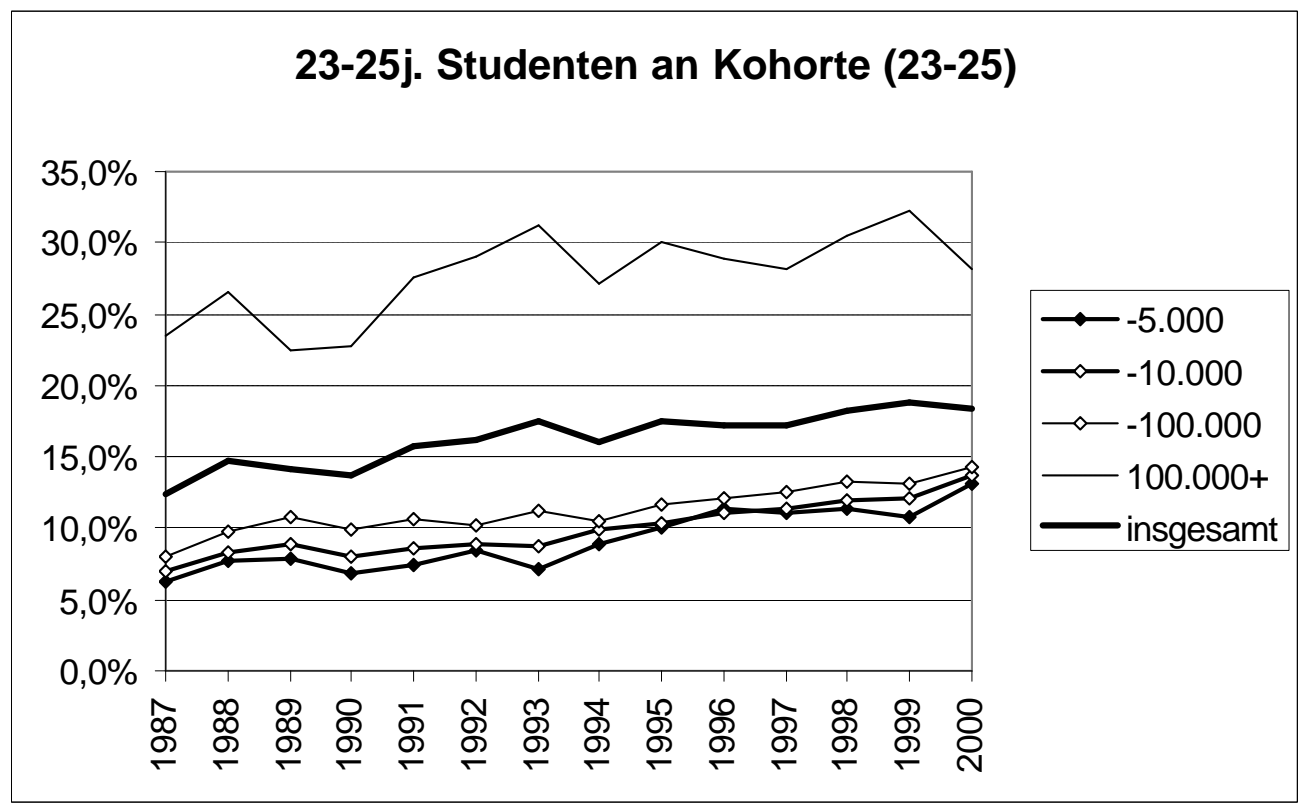

Quelle: Statistik Austria (Mikrozensus), IHS-Berechnungen

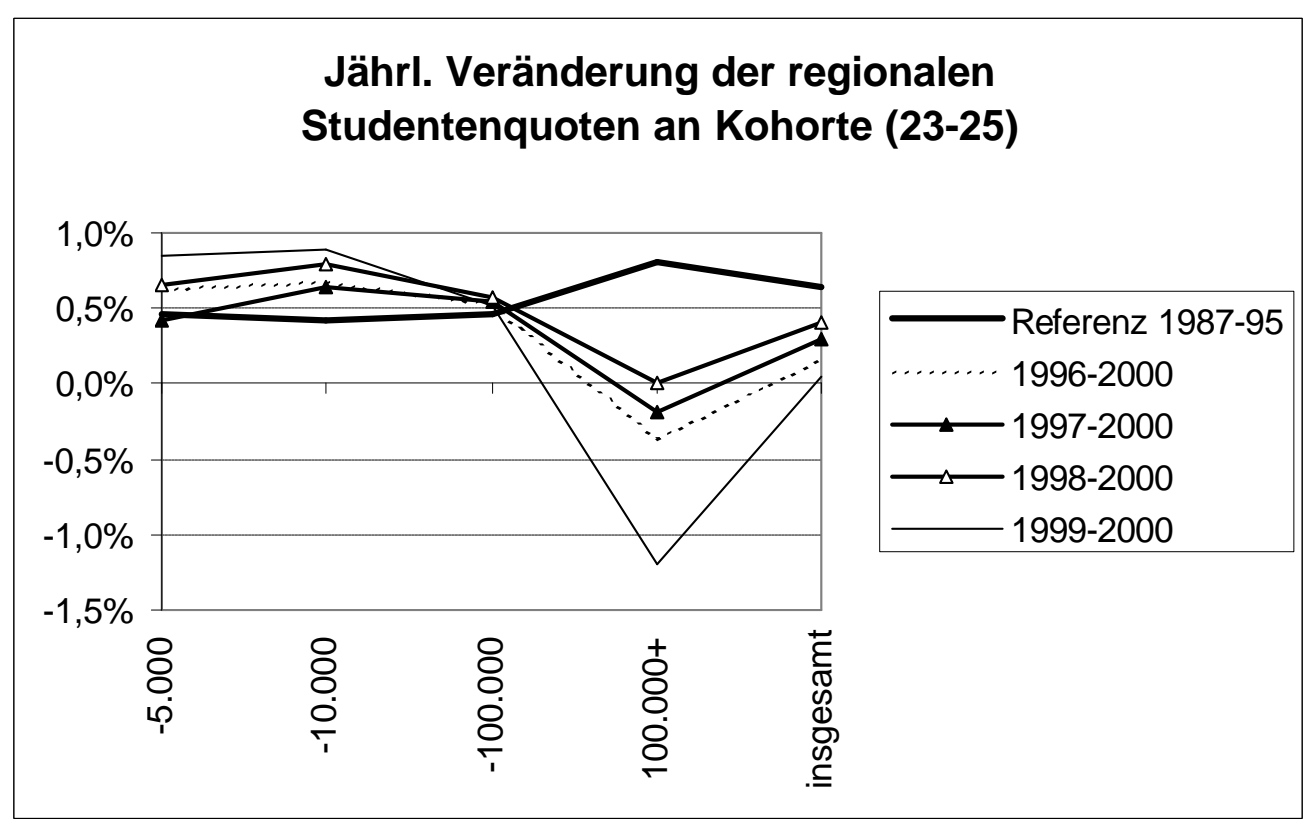

Quelle: Statistik Austria (Mikrozensus), IHS-Berechnungen 


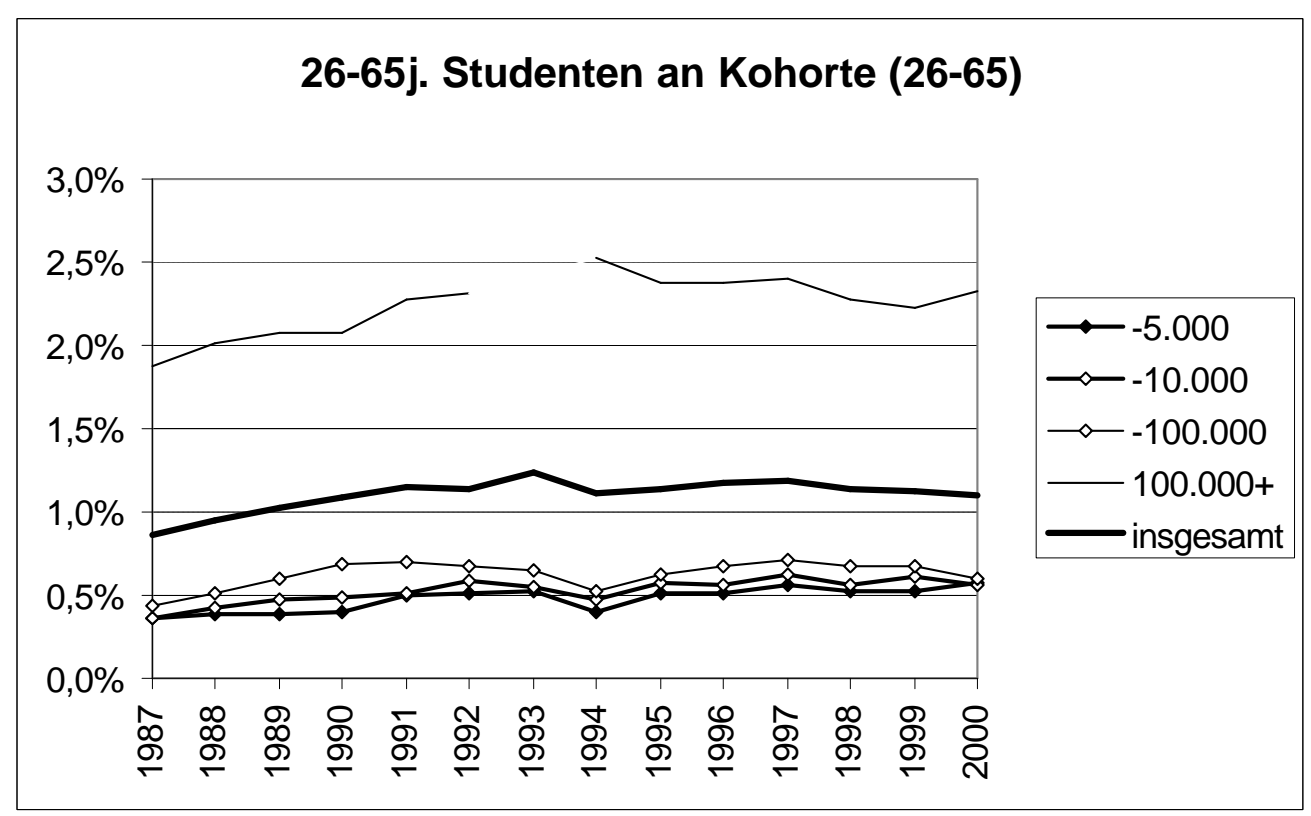

Quelle: Statistik Austria (Mikrozensus), IHS-Berechnungen

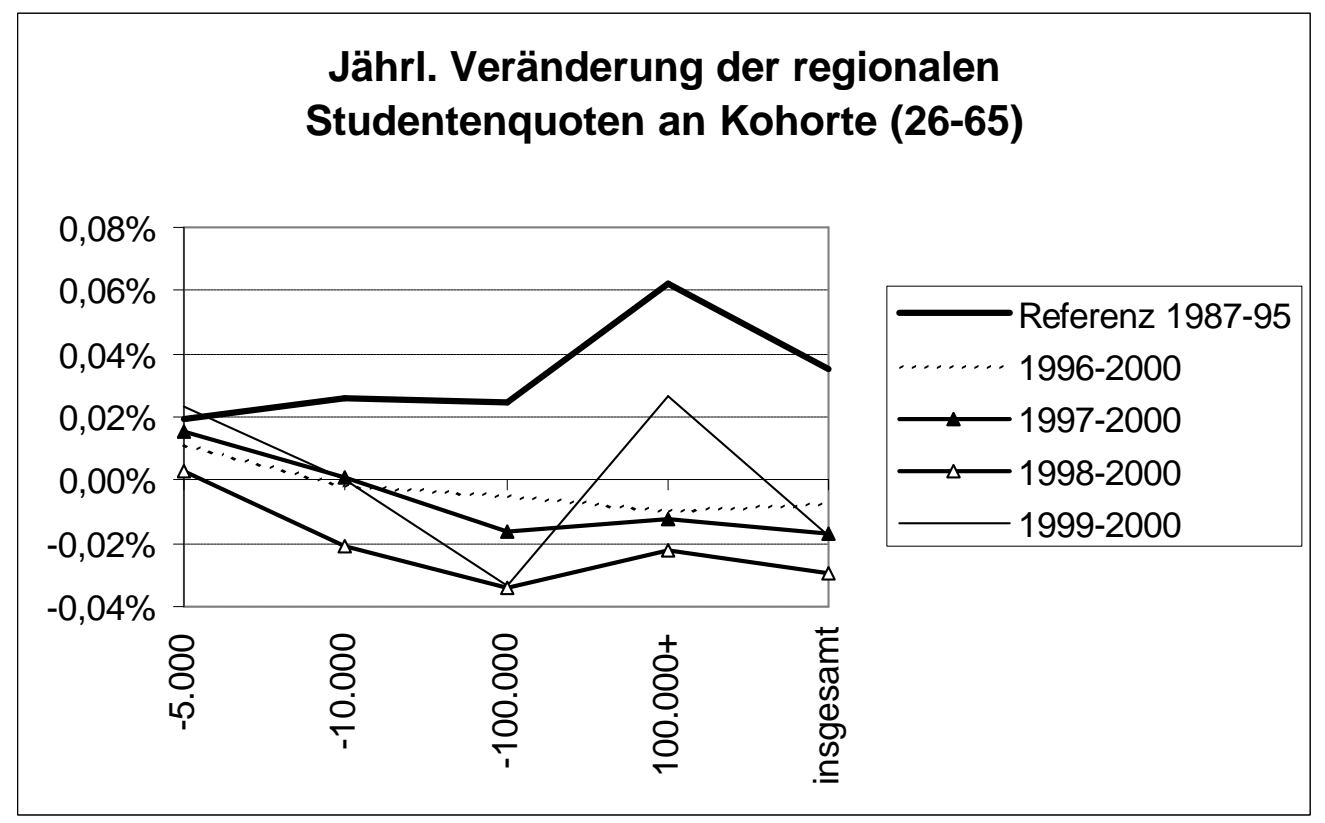

Quelle: Statistik Austria (Mikrozensus), IHS-Berechnungen 


\subsection{Stellungnahmen im Feedback-Prozess}

\subsubsection{Points raised at the feedback meeting with international reviewers}

Vienna, Nov. $11^{\text {th }}, 2002$

Reviewers: Andri Giere (CH), John Pratt (UK), Ulrich Teichler (D). Project team: Lorenz Lassnigg, Martin Unger, Hans Pechar, Ada Pellert (moderator). Elsa Hackl assisted Mr. Pratt in understanding the report which was written in German. First, each of the reviewers made an initial statement. The subsequent discussion, which was based on previous reading and a short introductory presentation, was organised along the chapters of the report. The meeting was concluded by a general discussion.

Some key questions were provided by the project team:

QQUDIS Employment-Qualification-Innovation

\section{Key Questions}

which development pathways (quantitative) ? "critical mass" for FH programmes and institutions ?

regional dispersion - how far? too much diversity?

definition of tasks portfolio sufficient? institutional definition of $\mathrm{FH}$ too weak ?

national policy definition needed? role, structure and tasks of the FH Council ? strengths and weaknesses of the report? 


\section{Summary of the main points discussed at the meeting}

1. Steering mechanisms within this sector. The discussion largely focused on the basic structure of steering mechanisms in the $\mathrm{FH}$ sector, especially on the relationship between political guidance on the one hand and quality assurance on the other hand. The reviewers felt that there was a strong lack of political guidance, and the existing mixture of political steering and quality assurance was also deemed to be rather impractical. According to them, it is a so-called "double accreditation" system, in which responsibilities are shared by the FHR (FH Council) and the ministry. Thus it is not quite clear where political decisions are made, which in turn leads to a certain lack of transparency within the policy formation process, e.g. with regard to the roles of various players or the decision-making process as such. Another consequence would be the problematic role of the FHR, which in some respect is considered to be a "strange creation" with strongly conflicting functions. In addition to its core purpose, i.e. quality assurance, it also used to make various political decisions, e.g. selecting professional areas or localities for FH programmes. Due to the "sketchy nature" of the FHStG (legal regulations) the FHR has adopted a rather broad concept of quality, setting common standards for various key concepts shaping the sector (e.g. the assessment of demand, the concept of an innovative course, a feasible academic environment).

At first, it was recommended to define clear structures for political decisions, giving a say to all the key players, especially the regions and FH institutions. Some sort of buffer organisation (a council), which in every case allows decentralised decision making and at the same time looks at the tertiary education system as a whole, was also considered to be a sensible option. Besides that, the reviewers argued that political decisions should be kept separate from the quality assurance system. While one single comprehensive political decision-making system was deemed to be necessary at a national level, the idea of having a larger number of different agencies for quality assurance was not completely neglected (nevertheless, for practical reasons a comprehensive agency was preferred).

The reviewers also stated that a successful steering system requires a clear relationship between the main actors and a well-functioning coordination system between the actors at different levels. Where should the decisions about national goals be made? How can the balance between national and regional interests be secured? How can the relationship between general, higher-level goals and specific objectives be controlled?

A good relationship between the providers and the academic $\mathrm{FH}$ institutions is also extremely important for the success of the steering system. The latter, which ought to be represented by a strong body, will also be essential for the development of a strong academic identity. The FHK is to some extent understood as a hybrid body - comprising the providers, i.e. the business branch, and the programme directors, i.e. the academic branch 
of the sector - which might in certain cases allow for business considerations to overrule academic concerns.

In any case, however, one should keep in mind that steering at a national level hardly ever works perfectly, and that a well-functioning relationship between the institutions and the political sphere is crucial in this context.

2. Profile of FHs as academic institutions. The profile of FHs as academic institutions should be strengthened as a part of the consolidation process. Visibility at the national and international levels could be improved by presenting entire $\mathrm{FHs}$ rather than single $\mathrm{FH}$ programmes. Strong institutions are particularly important for internationalisation.

The analysis in the report on how a certain standard of resources ("critical mass") could be established was pointed out by the Swiss reviewer as being particularly interesting.

The role of the $\mathrm{FH}$ in strategic management (doing the right thing) and in quality management (doing things right) was stressed as a particularly important aspect in strengthening $\mathrm{FHs}$ as academic institutions. However, the relationship between the providers and the $\mathrm{FH}$ seems to be unclear in this respect. Two key elements that would greatly promote the academic role of the FHs are: (a) developing a strategic plan for the $\mathrm{FH}$, and (b) giving the $\mathrm{FH}$ level a distinct role in managing and controlling the institution.

The issue of multidisciplinarity is not emphasised very much in the report.

3. Aspects of quality assurance. The distinction between the ex-ante dimension of the accreditation process and the post-hoc evaluation was highlighted as a very important issue, especially from the point of view of the British experience. A main point is that the result of the ex-ante evaluation is primarily a positive one, including recommendations of how to make things better, whereas post-hoc evaluations are more strongly geared to find errors, and thus are in danger of primarily getting a "policing" function. A change of this kind was reported to have been executed in the U.K., with the effect that "nothing improved" any more as a result of the post-hoc evaluation procedures. The new predominating post-hoc procedures have led to an overall worsening of the situation, as compared to the old CNAA practices.

The experience of the CNAA could also serve as a good example of how to cope with the problems of the work overload of the FHR: A large number of external reviewers, allocated to a number of subject area committees, were officially listed for the execution of the evaluation procedures. One by-product of this system was a kind of network comprising a relatively high number of renowned experts who were involved in the tasks of quality improvement in the Polytechnics. 
4. Basic trajectories for the FH sector. The mission of becoming a "spearhead of the knowledge economy" was considered to be a proper paradigm for the development of the FH sector. Furthering life-long learning and providing $R \& D$ were taken to be equally essential if this role should be performed successfully. This means that sufficient funding ought to be provided for each of these functions.

More moderate considerations in the report, which tended to give priority to one of these functions over the other, were rejected by the reviewers for not being feasible in the face of various economic and social challenges.

The function of life-long learning needs to be clearly aimed at higher education, which means that FHs should not compete on the general market for further training. This would have to include (a) the provision of appropriate programmes for non-traditional students, (b) development of feasible B. and M. programmes, especially the segment of post-experience master programmes could be an important market for $\mathrm{FHs}$, and (c) programmes offering continuing training for graduates.

5. The funding mechanism. In principle, the suggestion that the funding mechanism should be transferred from the programme level to the institutional level was supported by the reviewers. Yet a more complex formula would be more feasible than the current mechanism which is geared to student numbers alone. Part of the funding could continue to be based on student numbers, but additional funds should be targeted to other basic functions (R\&D, services including life-long learning) as well.

As the British experience shows, a design with too many different specifically targeted funds should be avoided. Instead, a more inclusive overall strategic investment plan could be funded for a certain period of time. The structure and credibility of that plan would be the main subject of the assessment.

6. Demand for qualifications. The reviewers largely agreed with the presented analysis of the aspect of demand. They were to some extent also sceptical of the overall potential of procedures for anticipating a potential demand. The difficulties in assessing demand, especially in dynamic economic sectors, were strongly emphasised. The British example illustrates that business actors do not always know all that much about demand and therefore tend to be somewhat conservative in their assessment. The reviewers also pointed out that the economy tends to make generalist demands rather than asking for specific qualifications.

7. Comparative views. The individual emphasis on the international dimension varies in each chapter. It could also have been somewhat more streamlined.

Important points from the Swiss perspective: 
- FHs can play an important role in the innovation system, improvement of the R\&D element is essential. It seems easier to develop the R\&D function in the technological fields than in other areas (e.g. business)

- Relying on niches is dangerous, especially in view of internationalisation; a unification of the study fields would be important

- A basic difference to the Swiss FH system is that in Austria FHs and UNIs principally compete for the same students. This is not the case in Switzerland, where the relationship to universities is less strained

- Consolidation means improving R\&D, as well as giving the respective institutions a strong identity and visibility.

Important points from the British perspective:

- The CNAA system had important positive aspects, a high number of reviewers were responsible for the improvement of quality in polytechnics

- A change from prospective (ex-ante) evaluation in the accreditation process to ex-post evaluation only may have strong negative effects on the development (negative policing and finding errors vs. positive recommendations for improvement)

- Clear and comprehensive structures for funding seem better than scattered mechanisms due to various items for improvement.

- The funding mechanism should be properly analysed in relation to their consequences, e.g. the funding could follow the logic of consuming (which seems to be the case in Austria where the government buys study places = services) or it could follow the logic of investment (e.g. funding for a strategic plan in analogy to a business plan, which should bring certain returns).

8. Development plan. The reviewers made several summarizing comments about the third development plan. They believe that steering the sector by means of a development plan that includes political priorities is a good practice and should be strengthened in the third period.

The British reviewer recommended some key elements, which should be included in the next development plan for $\mathrm{FHs}$ :

- a brief summary of the success of the existing policy and what it is being built on

- a brief summary of new concerns (e.g. Bologna) and unfinished business 
- consideration of the whole higher education system and the role of the FHs in it (for example, should university numbers be kept constant and expansion take place only in FHs?)

- an indication of the broad policy aims for the FH sector - life-long learning, R\&D, wider access etc..

- the relationship of this to the development of other sectors (health care etc..) and emerging professions not yet in $\mathrm{FH}$ courses

- an indication of future institutional development (e.g. new courses only in existing providers) and of new funding mechanisms to support it

- consideration of the role of the regions (including other provision, e.g. in universities?)

- the role of the FHR in quality and of government in policy and resource decisions

\section{Some more specific comments}

9. Organisation of the report. For readers not so familiar with the Austrian FH sector the report lacks a description of basic elements. The sketch in chapter 1 seems to be a bit too much of a mixture of basic descriptions and evaluative statements.

The quantitative description seems a bit complex, with too much emphasis on selective aspects (especially in chapter 2 ).

The interrelationship between the different themes could have been brought out more strongly.

The evaluation criteria of this report only focus on the original purpose and/or aims of this sector. However, it also seems to be extremely important to take into consideration how the initial goals may change over time.

10. Relationship to the secondary level. The report analyses the FH sector only with regard to how it is related to the higher education sector. But a detailed analysis of the relationship of FHs to schools at the secondary level, especially BHS (which have been explicitly excluded from the study), would also be necessary to understand the role of the $\mathrm{FH}$ sector in the overall education and training system.

11. Teaching staff. The teaching staff was generally considered to be crucial for the evaluation of any institution in higher education. That's why this issue should also have been selected as a special topic in the evaluation. Yet, even though the report included several important analyses about the current state, a comprehensive review of the development of the teaching staff is not given. 
The tensions, resulting from the necessity to develop a strong identity on the one hand and to guarantee continuous improvement on the other hand, are taken to be a core problem in this area.

12. General policy direction. There should be a proper distinction between incremental changes and more substantive changes. This was mainly pointed out in connection with the structure of power relation in this sector, e.g. who has the say about what. The power given to $\mathrm{FH}$ institutions was considered to be a key issue in that respect. The British example, i.e. delegating the accreditation function to certain Polytechnics, was reported to have been rather moderately successful with regard to improvement.

13. Comprehensive consideration of tertiary education. It was mentioned that the $\mathrm{FH}$ sector should increasingly be seen as part of the tertiary sector. Common regulations for the different sectors are thus essential for a proper steering mechanism in higher education.

14. Specification of goals. According to the reviewers the specification of goals and objectives should be extended to all essential functions of FHs (teaching, R\&D, services). Goals and objectives should be aimed at results and formulated in a more concrete fashion. Diversity is important, but a common FH image ought to be built up as well in order to establish "visibility" at an international level.

15. Transparency. It was recommended to make the $\mathrm{FH}$ sector more transparent, especially as to how the institutions are financed and how individual results are produced. In a first step, a common accountancy system (,harmonisierte Kosten- und Leistungsrechnung“) has been implemented in the Swiss FH system. This system has now been extended to include the universities as well; comparable information will be provided soon.

A system of this kind could and should also be used for planning procedures in individual institutions.

16. Concept of internationalisation is too narrow. The reviewers pointed out that an emphasis on the exchange of personnel and cooperation in R\&D activities was as important as the exchange of students. The purpose of this evaluation was thus defined in a somewhat narrow sense.

The linkage between internationalisation and the regional impact of the $\mathrm{FH}$ system is another important point. Internationalisation should therefore be an explicit part of the strategic planning of $\mathrm{FHs}$.

17. Broader programmes. The Swiss reviewer in particular recommended that the titles, i.e. the specific names of individual programmes, should be extended in this connection. Internationalisation could thus be made easier, and a broader definition of the programme 
content could also provide a higher level of flexibility in dealing with employment-related demands. The two-stage programmes in Finland, providing a general initial stage and more specialised "majors" in a second stage were emphasised as good practice in this respect.

18. Full-time and part-time programmes. In the British system these kinds of programmes require different amounts of time: 1 year full-time is equivalent to 2 years part-time.

19 Definition of "non-traditional students". This is a wide concept in research, the use of which must be further specified.

20. New OECD review of higher education. The idea of another OECD review of the development of tertiary education in Austria, which takes into account the experience in the $\mathrm{FH}$ sector as well as the reform of the universities, was brought up.

This exercise ought to provide the ministry with a more comprehensive view of the longerterm development of the system as a whole. 


\subsubsection{Nationale Stellungnahmen}

UNIV. PROF. DR. ELKE GRUBER

Universität Klagenfurt Institut für Erziehungswissenschaft $u$. Bildungsforschung Abteilung für Erwachsenen- und Berufsbildung

Universitätsstrasse 65-67

9020 Klagenfurt

Tel. $++0463 / 2700-1242$

e-mail: elke.gruber@uni-klu.ac.at

Graz, 7.2.2003

\section{Feedback zum Endbericht}

\section{"Evaluierung des Auf- und Ausbaus des Fachhochschulsektors" von Lorenz Lassnigg, Martin Unger, Hans Pechar, Ada Pellert, Don F. Westerheijden}

Der nachfolgende Kommentar ist auf dem Hintergrund einer intensiven Beschäftigung mit dem vorliegenden Bericht sowie meiner dreijährigen Tätigkeit als Mitglied des Österreichischen Fachhochschulrates (2000-2002) entstanden. Im Rahmen dieser Funktion erhielt ich vielfältige Einblicke in den österreichischen Fachhochschulsektor und die Arbeitsweise des österreichischen Fachhochschulrates.

In einem ersten Schritt sollen die Ergebnisse des Berichtes zusammenfassend eingeschätzt werden, in einem zweiten Schritt werde ich auf ausgewählte Ergebnisse in Form von Kommentaren, Anregungen und eigener Meinung näher eingehen. Dabei werden z.T. auch die im Punkt 11.3 des Berichtes (Feedback) angeführten Fragen berücksichtigt.

\section{Zusammenfassende Einschätzung der Ergebnisse des Berichtes}

Das vorliegende Projekt wird in allen Punkten der Ausschreibung gerecht. Es gibt einen fundierten Einblick in die bisherige Entwicklung des FH-Sektors in Österreich und bietet eine gute Grundlage für die Konzeption des dritten Entwicklungs- und Finanzierungsplans für den FH-Sektor. Im originären Sinne einer Evaluationsstudie liefert es keine direkte Anleitung (im Sinne von einfach zu handhabenden „Kochrezepten") sondern bietet ausreichend Stoff und Anknüpfungspunkte für eine weiterführende Diskussion um die künftige Ausgestaltung des FH-Sektors. Diese Diskussion wird jedoch nicht von alle in beginnen, sie muss von verschiedenen Seiten (Bildungspolitik, FH-Rat, FH-Konferenz) aufgenommen und zum „Leben erweckt" werden - was einen Willensbildungsprozess bei den angeführten Akteuren voraussetzt. 
Das methodische Vorgehen der Studie mit seinem Methodenmix an Dokumentenanalyse, Interviews und Review-Verfahren bietet eine solide wissenschaftlich Grundlage zur Erstellung des Berichtes. Als besonders positiv sollen die eingebauten Rückmeldeschleifen hervorgehoben werden, die auch eine Selbstevaluation der Forscherlnnen ermöglichte.

Aus meiner Tätigkeit im FH-Rat sind mir viele der Ergebnisse indirekt bekannt, bei den meisten Akteuren in diesem Bereich dürften diese als implizites Wissen vorhanden sein. Die Hauptleistung des Projektes liegt m.A. nach darin, dieses Wissen nun explizit und gebündelt einer breiteren Öffentlichkeit als Entscheidungsgrundlage zur Weiterentwicklung des $\mathrm{FH}$-Sektors zugänglich gemacht zu haben.

Der Bericht liefert zum einen eine fundierte Bestandsaufnahme des FH-Sektors (global, aber auch in vielen Detailbereichen), gleichzeitig zeigt er kenntnisreich diverse Veränderungspotentiale und -varianten auf (wie z.B. die Frage nach dem Aufgabenprofil künftiger $\mathrm{FH}$ 's, der Funktion des FHR), ohne dass Entscheidungen, in welche Richtung der Sektor entwickelt werden soll, vorweg genommen werden. Die Offenheit des Berichtes ist seine Stärke, in der Folge gilt es nun, die sich aus der Analyse herauskristallisierenden unterschiedlichen Varianten (z.B. mögliche unterschiedliche Entwicklungspfade) breit zu diskutieren. Sonst besteht die Gefahr, dass diese Offenheit zur "Spielwiese" diverser (lokaler politischer) Lobbyisten wird.

Auf einen kleinen Kritikpunkt sei noch kurz hingewiesen, er betrifft die Darstellung der Entwicklungspfade als einem wichtigen Ergebnis des Projektes. Während die stilisierte Darstellung der Entwicklungspfade in ihren Hauptaspekten (Abbildung 68) inhaltlich nachvollziehbar ist, fehlt der Zusammenhang zu den bestimmenden Faktoren in Abbildung 69. Eine eingehende Erläuterung des Zusammenhanges zwischen den beiden Darstellungen würde hier das Gesamtverständnis für die unterschiedlichen Dimensionen der Entwicklungspfade erhöhen.

\section{Kommentare und Anregungen zu ausgewählten Ergebnissen der Studie}

Die folgenden drei Punkte aus der Studie sollten in der zu beginnenden Diskussion um die künftige Entwicklung des FH-Sektors unbedingt Priorität erhalten:

1. Die zunehmende Notwendigkeit einer bildungspolitischen Gesamtkoordination im tertiären Sektor

2. Die Frage nach dem künftigen Entwicklungspfad des FH-Sektors (inklusive der Gestaltung des Aufgabenprofils der FH's und der Standortfrage)

3. Das Überdenken von Funktion und Konstruktion des FH-Rates.

Ad1)

Die zunehmende Notwendigkeit einer bildungspolitischen Gesamtkoordination ergibt sich aus drei Gründen:

1. Die „Pionierphase" des FH-Sektors neigt sich dem Ende zu (manche FH's dürften sich schon in der „Konsolidierungsphase " befinden), die „alten" bildungspolitischen Vorgaben wurden zum Großteil erfüllt. Nun käme es 
darauf an, neue Impulse für diesen Bereich zu setzen und diese auch öffentlich und auf breiter Akteursbasis zu diskutieren.

2. Die neue Universitätsgesetzgebung erfordert einen dringenden Klärungsbedarf über das Verhältnis zu den FH's (siehe vorliegende Studie: Schaffen gleicher Rahmenbedingungen, zunehmende Konkurrenz um Studierende untereinander...).

3. Der Bologna-Prozess wirft ganz neue Fragen für die FH's auf, wie z.b. reicht die ,wissenschaftliche Bedeckbarkeit" von einzelnen FH-Studiengängen aus, um hier auch Masterstudien anzubieten? (In den Niederlanden beispielsweise können Masterstudiengänge an FH's nur in Kooperation mit Universitäten angeboten werden.)

Insgesamt habe ich in den letzten Jahren eine wirklich ernst gemeinte bildungspolitische Diskussion über den $\mathrm{FH}$-Sektor und noch mehr über das Zusammenspiel der Institutionen im tertiären Sektor stark vermisst. Im FH-Rat wurde darüber kaum diskutiert, vielmehr stand das stark expandierende administrative Tagesgeschäft (Akkreditierung von immer mehr Studiengängen etc.) absolut im Vordergrund. Das heißt nicht, dass nicht „Politik" im FH-Sektor gemacht wurde. Diese erfolgte jedoch eher verdeckt, $z$.b. über die Finanzierung bestimmter Studiengänge durch das bm:bwk sowie stark über lokales politisches Lobbying.

Es wird also dringend notwendig sein, einen intensiven öffentlichen Diskussionsprozess auf breiter Akteursbasis zu initiieren und zu führen. Damit könnte der bildungspolitische Gehalt des FH-Sektors wieder ins Bewusstsein der Öffentlichkeit und der Bildungspolitik gerückt werden. Derzeit erfolgt die Diskussion bezüglich FH's - vor allem in den Medien - fast nur mehr unter Marketinggesichtspunkten! (Für die FH's ist diese Entwicklung nicht förderlich, verhindert sie doch Selbstkritik und -reflexion, die für eine Weiterentwicklung dringend notwendig sind.)

Darüber hinaus ist der Vorschlag der AutorInnen der Studie zur Operationalisierung von bildungspolitischen Vorgaben zu unterstützen. Damit könnte einerseits ein Diskussionsprozess über eben diese bildungspolitischen Vorgaben ausgelöst werden, andererseits könnten die erarbeiteten Kriterien als Maßstab künftiger bildungspolitischer Evaluationen dienen. (Bis heute ist z.b. unklar, welche Kriterien an Praxisorientierung, Wissenschaftlichkeit, Internationalität etc. im $\mathrm{FH}$-Bereich angelegt werden sollten, jeder spricht zwar davon, es gibt aber keine Parameter dafür.)

Ad2)

Eine der Hauptfragen im bildungspolitischen Diskurs der nächsten Jahre wird die der weiteren Ausgestaltung des $\mathrm{FH}$-Sektors insgesamt sein. Hier besteht dringender Diskussions- und Handlungsbedarf. Die Ausgestaltung betrifft verschiedene Ebenen, sie werden auf S. 19 der Studie klar angesprochen (a: Entwicklungspfad für den Sektor insgesamt, soll er weiter wachsen, wie ist das Verhältnis zu Unis, welche Ziele werden verfolgt...; b: Standortprofile).

Die Studie hat dankenswerterweise verschiedene Entwicklungspfade formuliert. Meine Präferenz gilt dem Modell der Konsolidierung der Standorte. Das heißt,

- Aufbau von FH's im Sinne einer „kritischen Masse" (wobei die Zahl der 1000 Studierenden aufgrund regional sehr unterschiedlicher Bedingungen nicht 
unbedingt eingehalten werden muss); die sog. „kritische Masse" ist besonders wichtig für die Personalentwicklung, die Entwicklung von F\&E-Aktivitäten, die Internationalisierung etc.;

- Vermeiden neuer Standorte zugunsten eines Ausbaus bestehender (was allerdings nicht bedeuten muss, dass es nicht den einen oder anderen neuen Standort nach genauer Prüfung geben kann);

- keine Fortführung der "Nischenpolitik" (hier halte ich mich an das bildungspolitische Credo von Niklas Luhmann, der den Bedarf künftiger Qualifikationen wie folgt beschreibt: Wir brauchen eine gute Grundbildung bei gleichzeitiger Spezialisierung);

- stattdessen breitere Studiengänge und "gemischte "interdisziplinäre FH's (technische, wirtschaftliche, soziale etc. Studiengänge); auch könnte man hier eigene Weiterbildungsanbieter ansiedeln (siehe Bericht: Stellung von $\mathrm{FH}$ im System des lebensbegleitenden Lernens);

- intensives und nicht extensives Wachstum der Studierendenzahl im FHBereich (wobei hier der Bereich in seiner fachlichen und qualifikatorischen Diversität näher evaluiert werden sollte, d.h. man sollte sich auch nicht scheuen, Studiengänge, wo kein Bedarf mehr besteht, zu schließen).

Interessant fand ich die Überlegungen zur Gestaltung des Aufgabenprofils künftiger FH's. Die Frage nach dem Umfang der Leistungen bzw. des Portfolios der FH's wurde aus meiner Sicht in dieser Direktheit noch nie gestellt. Allerdings gebe ich zu bedenken, dass der FH-Sektor schon jetzt so heterogen und diversifiziert ist, dass eine weitere Differenzierung in eher "forschende" und eher "lehrende" FH's zu einer weiteren Verwirrung in der Landschaft und vor allem bei den Studierenden führen könnte. (Hier sollten wir erst einmal einige Jahre der Umsetzung der BolognaErklärung abwarten, die wahrscheinlich noch genug „Verwirrung" zwischen UniBacc./Masters und FH-Bacc./Masters bringen wird.)

\section{Ad3)}

Die Aufforderung der Autorlnnen der Studie zum Überdenken der Funktion und Konstruktion des FH-Rates ist zu unterstützen. Wie schon angesprochen, haben die administrativen Tätigkeiten die strategischen Entscheidungen im $\mathrm{FH}$-Rat mehr und mehr verdrängt. "Getrieben" vom Altagsgeschäft (vor allem der Akkreditierung neuer Studiengänge) bleibt kaum Zeit für bildungspolitische Überlegungen bzw. Grundsatzdiskussionen (künftige Qualifikationen und damit künftige Studiengänge, pädagogisch-didaktische Ausgestaltung, Fragen der Curriculumentwicklung...). Hinzu kommt, dass dem Rat mit der letzten Novelle des FH-Gesetzes ein wichtiges Entscheidungskriterium - das über die Standortfrage - vom Gesetzgeber aus der Hand genommen wurde (dies sollte übrigens in die Studie noch aufgenommen werden, da es einen entscheidenden Einschnitt in die Entscheidungsmöglichkeit des Rates bildet!). Lokalem politischem Lobbying ist dadurch nur mehr schwer beizukommen.

Die europäische Entwicklung weist auf eine Differenzierung zwischen der bildungspolitischen Beratungsfunktion auf der einen Seite und einer akademischen Qualitätsprüfung (über Akkreditierungs- und Evaluierungsagenturen) auf der anderen Seite hin. Ich plädiere auch dafür, beide Funktionen „sauber" voneinander zu trennen (derzeit liegen die Prioritäten des FH-Rates eher auf der Seite der Akkreditierung und Evaluierung), sie jedoch in unterschiedlichen Gremien bzw. Agenturen unter einem Dach zu belassen. Geeignete Konstruktionen dafür müssten noch entwickelt werden. 
Vorteil dieses Modells wäre, dass es einen Austausch zwischen bildungspolitischen Vorgaben und Qualitätsprüfung gibt (aus meiner Sicht hängen Qualität und Bildungspolitik immer auch zusammen; von einer „abgehobenen“ Qualitätsdefinition halte ich persönlich nicht viel!). Die Funktion der politischen Bildungsberatung in diesem Konstrukt müsste jedoch unbedingt gestärkt werden! Als Nachteil sehe ich, dass der FH-Sektor wieder „unter sich bleibt". Für die dringend anstehende Diskussion über die Positionierung des gesamten tertiären Bildungssektors dürfte dies nicht unbedingt förderlich sein.

Zum Schluss meiner Überlegungen möchte ich noch auf zwei Punkte hinweisen, die relativ schnell angegangen werden sollten:

1. Das Überdenken der Bedarfs- und Akzeptanzanalyse für jeden Studiengang (Stichwort: Makroanalyse statt „Bestätigungsforschung" für jeden einzelnen Studiengang)

2. Entwicklung gezielter Förderprogramme für Frauen sowohl im Bereich der Personalentwicklung als auch im Studierendenbereich (die letzte Absolventlnnenstudie spricht hier eine deutliche Sprache: Unterrepräsentation von Frauen im $\mathrm{FH}$-Bereich; Absolventinnen brauchen deutlich länger für einen Berufseinstieg und verdienen noch dazu wesentlich weniger als ihre männlichen Kollegen)

Ich hoffe, meine Ausführungen finden Ihr Interesse und verbleibe mit den besten Grüßen, elke gruber 
H.-J. Bodenhöfer

Kommentare zu: L. Lassnigg u.a.: Evaluierung des Auf- und Aufbaus des Fachhochschulsektors. IHS, Wien 2002

Trotz des sehr umfangreichen Berichts stellt die Untersuchung keine umfassende Evaluierung der Entwicklung des Fachhochschulsektors in Österreich dar, sondern ist in betonter Weise von den Zielsetzungen des Auftraggebers (bzw. der Spezifikation des Auftrags) bestimmt, die Grundlagen für die Fortschreibung der Entwicklungs- und Finanzierungsplanung des Bundes für den FH-Sektor aufzubereiten.

Nach einem einleitenden Abschnitt zu den Besonderheiten des institutionellen Rahmens für den FH-Sektor, den wichtigsten Entwicklungsdaten für den Zeitraum 1994 - 2002 und einem Überblick zur FH-Entwicklung in vier anderen europäischen Ländern folgt ein zentrales Kapitel zum Stellenwert des FH-Sektors in der österreichischen Bildungs- und Hochschullandschaft, an das sich Abschnitte zu wichtigen Einzelaspekten anschließen: die Umsetzung der Entwicklungs- und Finanzierungsplanungen I und II, die Frage der Standorte im FH-Sektor, die Frage der Internationalisierung (Mobilitätsprogramme, Bologna-Prozess) und das System der Qualitätssicherung im FH-Bereich.

Weitere Aspekte - wie Fragen der Finanzierung und der Kosten von FH-Studiengängen werden nur in allgemeiner und knapper Form behandelt. Das spezifische Finanzierungsmodell für den FH-Bereich - auf Normkosten beruhende Finanzierungszuschüsse des Bundes an die Erhalter als wichtigste Finanzierungsquelle - wird ausführlich dargestellt und kommentiert. Auch aus Gründen der Datenverfügbarkeit beschränkt sich die weitere Analyse jedoch auf die Finanzierungsstruktur des FH-Angebots verschiedener Erhalter bzw. im Vergleich von Bundesländern nach den Finanzierungsanteilen von Bund, Ländern und sonstigen Finanzierungsquellen. Die Kosten einzelner Studiengänge bzw. je Studierende oder je Absolvent, die Streuung der Kosten nach Studiengängen und Erhaltern, Unterschiede der Kostenstruktur oder Vergleiche mit den Kosten vergleichbarer Studiengänge an den Universitäten werden nicht dargestellt. Damit bleibt die Frage der vielfach behaupteten ,Kostengünstigkeit' des FH-Studienangebots, etwa gegenüber den Universitäten, ebenso offen, wie auch mögliche Kostendegressions- und Synergieeffekte, die in der strittigen Standortfrage eine Rolle spielen, oder die Frage von unterschiedlichen Kostenstrukturen und 
,Kostentreibern' (ganz abgesehen von der schwierig zu beantwortenden Frage eines Zusammenhangs von Kosten und Qualität des Leistungsangebots).

Ein anderer Aspekt, der weitgehend ausgeklammert bleibt, betrifft die Zusammenhänge zwischen dem Studienangebot im FH-Bereich und der Entwicklung von Beschäftigungssystem und Arbeitsmarktnachfrage. Zu Recht wird an verschiedener Stelle kritisch angemerkt, dass die ,Bedarfsanalysen' im Zusammenhang von Neuanträgen auf die Einrichtung von Studiengängen notwendig eine enge, quasi Mikro-Perspektive einnehmen und - von methodischen Einwänden im Einzelnen abgesehen - eine Einordnung in (vorzugsweise regionale) Perspektiven der Arbeitsmarktentwicklung nicht zulassen.

Noch stärker problematisiert wird in der Studie auch die ,Nischenpolitik', in die die Interpretation der Zielsetzung innovativer Studienangebote im FH-Bereich geführt hat. Im Ergebnis hat sich ein Angebot von inzwischen 127 unterschiedlich bezeichneten, obwohl teilweise verwandter Studiengänge entwickelt, dessen Vielfalt sowohl die Möglichkeiten der Informationsgewinnung und einer rationalen Studienwahlentscheidung durch Studieninteressenten übersteigt, als auch die Strukturierungsleistung von Zertifikaten für den Arbeitsmarkt überfordert: es ist unmöglich, den einzelnen Studienabschlüssen ein charakteristisches Qualifikationsprofil zuzuordnen und damit auch die Eignung von BewerberInnen bei der Besetzung definierter beruflicher Positionen einzuschätzen.

Die Differenzierung des Angebots bezieht sich dabei auf einen relativ schmalen fachlichen Bereich (,Wirtschaft' und ,Technik') im Unterschied zu dem ebenfalls sehr weit gefächerten Studienangebot der Universitäten; eine größere Zahl von Studiengängen stellt zum Beispiel ein betriebswirtschaftliches Studium mit unterschiedlichen Schwerpunktsetzungen und Spezialisierungen dar, obwohl keiner dieser Studiengänge die Bezeichnung ,Betriebswirtschaftslehre' führt.

Die geforderte Berufsorientierung der Studiengänge schafft noch ein weiteres, grundsätzliches Problem: das der Flexibilität im Zusammenhang von Ausbildung und Beruf an der Schnittstelle des Übergangs in den Beruf und das der beruflichen Mobilität im Berufs- und Karriereverlauf im Sinne der Weiterentwicklung und Anpassung der beruflichen Qualifikation an sich verändernde Tätigkeitsinhalte und -anforderungen, insbesondere in Berufsbereichen, die einem raschen wirtschaftsstrukturellen und technologischen Wandel unterworfen sind. Die Orientierung einer praxisnah und berufsbezogen konzipierten Ausbildung, ausgerichtet auf aktuelle Berufsanforderungen und unmittelbare berufliche Verwertbarkeit sowie auf die unmittelbare Einsatzfähigkeit der AbsolventInnen, unterliegt der Gefahr, dass wechselnde 
Anforderungen im Beschäftigungssystem bereits beim Übergang in den Beruf erhebliche Diskrepanzen zur vermittelten beruflichen Qualifikation auftreten lassen und dass vor allem im Berufsverlauf hohe Anforderungen an die Weiterentwicklung der beruflichen Qualifikation auftreten, für die im Studium unzureichende Grundlagen gelegt wurden. Die entsprechende Frage der Gewichtung fachlich-theoretischer Grundlagen als Fundament eines Prozesses lebenslangen Lernens und unmittelbar verwertbarer berufspraktischer Kenntnisse und Fähigkeiten, die rasch obsolet werden können, ist offensichtlich nicht in einer generellen Weise lösbar, muss jedoch für die Planung eines berufsorientierten, eher speziell ausgerichteten Studienangebots an FH's, wie es die derzeitige Angebotsstruktur dominiert, thematisiert werden.

Einen Hauptpunkt der Analyse und der Empfehlungen der Studie stellt die Notwendigkeit einer fundierten strategischen Planung und Steuerung für die Entwicklung des FH-Sektors dar. Die Entwicklungs- und Finanzierungsplanungen des Bundes stellen im Kern eine Zielprojektion von Studienanfänger- und Studierendenzahlen dar, die sich über die Normkosten-Zuschüsse je Studienplatz in ein Globalbudget für den Finanzierungsbeitrag des Bundes übersetzen. Ergänzt wird diese Planung durch eine Reihe bildungspolitischer Zielvorgaben, die für die Akkreditierungsentscheidungen des FHR wie für die Finanzierungsentscheidung des BMBWK gleichermaßen relevant sind.

Allerdings sind diese Ziele nicht in opernationalisierter Form definiert; ebenso fehlen spezifische Anreize und Programme, die der Zielerreichung dienen könnten (und wie sie in der Studie vorgeschlagen werden). Wie die Studie feststellt, wurde z.B. das Ziel der Strukturbereinigung im postsekundären Sektor, ,nur sehr langsam und in manchen Bereichen bis heute nicht erfolgreich umgesetzt" (die Ausnahme stellt die Aufwertung der Ausbildung an den Sozialakademien zu FH-Studiengängen dar). Auch die Ziele eines erweiterten nichttraditionellen Zugangs zum Hochschulbereich, der erhöhten Chancengleichheit zwischen Frauen und Männern oder die Entwicklung von FH-Standorten zu regionalen Zentren anwendungsorientierter F\&E und Beratung, insbesondere für den KMU-Sektor, sind bislang nur ansatzweise erreicht worden.

Für den Dritten Entwicklungs- und Finanzierungsplan stellen sich damit Fragen einer wirksameren Zielsteuerung, die insbesondere die Struktur von ,Fachhochschulen' (statt gewissermaßen Konglomeraten von Studiengängen), einschließlich deren Stärkung und Autonomisierung gegenüber den Erhaltern, sowie spezifische Anreize und Förderprogramme für Aufgabenbereiche neben der Lehre bzw. für die bildungspolitischen Zielsetzungen 
betreffen. Für die Bestimmung des künftigen Aufgabenprofils der FH's ist jedenfalls das bisherige Steuerungsmodell für den FH-Sektor unzureichend; neben der Frage der Struktur des gesamten post-sekundären und tertiären Bildungssektors, die wesentlich in die Kompetenz des Bundes fällt, kommt hier einer bislang weitgehend fehlenden Entwicklungsplanung für den FH-Bereich auf der Ebene der Bundesländer und unter Einbeziehung der verschiedenen ,stakeholders' besondere Bedeutung zu (Strategische Schwerpunktbildungen, Arbeitsmarktorientierung, Beitrag zum regionalen Innovationssystem, Kooperationsstrukturen im tertiären Bereich, Standortstruktur und Infrastruktur, Strukturen der Selbstorganisation und Selbstverwaltung der FHs bzw. Rolle des Erhalters).

Gegenüber dem bisherigen „bottom-up“-Konzept würde dies die Ergänzung durch eine ,topdown"-Komponente der strategischen Zielorientierung für die Entwicklung des FH-Sektors bedeuten. Unter den in der Studie vorgestellten möglichen „Entwicklungspfaden“ für den FHBereich entspräche diese Strategie dem Szenario „Konsolidierung der Standorte, Aufbau von FHs, breitere Studiengänge (intensives Wachstum)“ unter Einbeziehung der Aspekte des Qualifikationsbedarfs sowie der Mobilisierung des FH-Sektors für die Forschungsstrategie. Die entsprechenden Empfehlungen der Studie für die Fortschreibung der Entwicklungsplanung des Bundes (Entwicklungs- und Finanzierungsplan III) im Sinne erweiteter strategischer bildungspolitischer Zielvorgaben bedeuten eine wesentliche Verbesserung des Steuerungs-, Koordinations- und Kontrollsystems für den FH-Sektor im Rahmen des gesamten tertiären Bildungsbereichs; sie stellen zugleich den Kern des Ertrags der Untersuchung dar.

Der strittigen Standortfrage („,kritische Größe“ vs. Regionalisierung des tertiären Bildungsangebots und Abbau regionaler Disparitäten) widmet die Studie ein eigenes Kapitel, in dem die relevanten Aspekte, die grundlegenden Vorgaben, z.T. auch die internationalen Erfahrungen und Entwicklungen aufgearbeitet werden. Dabei kommt dem Innovationspotential von Hochschulen besondere Bedeutung zu. Anhand der ,Potentialfaktoren': Lehrpersonal, Zahl der Studierenden (über 1.000) und Beteiligung am Impulsprogramm für F\&E versucht die Studie, 3 - 4 Typen von Standorten zu unterscheiden; ebenso wurden die Einschätzungen der Erhalter im Hinblick auf „kritische Größen“ von Standorten und die Einschätzungen von ExpertInnen im Hinblick auf (Größen-)Erfordernisse für das F\&E-Potential von FHs erhoben.

Unabhängig von der Möglichkeit eines Entscheidungsmodells für die Standortwahl, das die relevanten Parameter (Bildungsnachfrage, Beschäftigungsstruktur und Arbeitsnachfrage, 
F\&E-Strukturen und Infrastrukturausstattung etc.) einer optimalen Standortstruktur umfassen müsste, bleibt die Frage der Mindestgröße, von Kostendegressions- und Synergieeffekten, von fordernden Netzwerkstrukturen oder auch der Standortattraktivität für Lehrende und Studierende zunächst offen. Zudem stellt sich diese Frage unterschiedlich für die verschiedenartigen fachlichen Schwerpunkte von FHs (z.B. Betriebswirtschaftslehre oder Technik) sowie für ein verschiedenartiges Aufgabenprofil einzelner Standorte (z.B. Schwerpunkt in der Lehre oder entwickeltes F\&E-Potential).

Weiterführende Schlussfolgerungen können nur aus detaillierteren Kosten-, Kennziffern- und Leistungsindikatoren-Vergleichen für verschiedene Studiengänge, Standorte und FHStrukturen gezogen werden wie sie zumindest in einem Fallstudien-Ansatz durchführbar wären. Die vielfach geäußerte Vermutung, dass die heutige Standortstruktur des FH-Angebots in der Mehrzahl der Fälle durch eine noch ,unterkritische Masse“ charakterisiert ist, die sich mit ineffizienten Strukturen und hohen Kosten der Leistungserstellung verbindet, lässt sich nur in dieser Weise erhärten bzw. auch widerlegen. 


\section{IHs-studie: Entwurf des Endberichts \\ stellungnahme der FHK:}

\section{Vorweg:}

Aus sicht der Erhalter ist es sehr erfreulich, dass zum 10jährigen Jubiläum des FHstG eine so umfangreiche und interessante studie über den Aufbau des Sektors verfasst wird, die wichtige Fragen thematisiert und interessante Zahlen und Durchschnittswerte liefert.

Aufgrund der Komplexität der Fragestellungen ist es einleuchtend, dass im Rahmen der studie nicht alle Probleme einer Lösung zugeführt werden können; in einigen Fällen sind aber die zukünftigen Perspektiven zu wenig herausgearbeitet worden. Für die Entscheidungen und weiteren weichenstellungen in Hinblick auf die Zukunft des sektors wäre es sehr hilfreich, wenn die studie auch die Konsequenzen, dh die zu erwartenden Vor- und Nachteile der vorgestellten Lösungswege skizzieren würde.

Abgesehen davon sollte die studie hinsichtlich zahlreicher Doppelgleisigkeiten, Rechtschreibfehler und strukturierungsmängel überarbeitet werden.

\section{1) Behörden: bestehende struktur und vorgeschlagene zukunfts- Szenarien:}

Den Aussagen zu den Kapazitätsproblemen von FHR und BMBWK ist ebenso beizupflichten wie der Feststellung, dass die Informationspolitik der Behörden in Bezug auf die Erhalter mangelhaft ist. Das spannungsverhältnis innerhalb des FHR aufgrund seiner Funktionen als Behörde und Expertengremium ist deutlich erkennbar und auch nicht zuletzt die Ursache für das unstrukturierte Regelungsverhalten des FHR.

Die Besetzung des FHR mit mehr know-how ist ein guter Vorschlag, es wird aber zu wenig auf die Frage der Zusammensetzung der Mitglieder eingegangen. So ist doch zB der Universitätssektor überdimensional vertreten, dafür fehlen aber VertreterInnen aus anderen wissenschaftlichen und wirtschaftichen Bereichen.

Es ist richtig, dass im FH-Sektor klare Aufgabenabgrenzungen fehlen und dass man sich von öffentlicher seite nun vermehrt strategischen Fragen zuwenden muss; die beiden dargestellten Szenarien zur Behördenstruktur erscheinen allerdings beide nicht zielführend. Gerade die strategischen Fragen werden in den Vorschlägen der AutorInnen Gremien zugeordnet, die nicht nur besonders viele Mitglieder zählen, sondern in ihrer Zusammensetzung auch sehr inhomogen erscheinen (der FHR auf S-FHK_IHS-Studie.doc 
der einen und der vorgestellte Beirat auf der anderen seite). Die Übergabe der wichtigsten strategischen Agenden des BMBWK an überdimensionierte, inhomogene Gremien, ist aus sicht der Erhalter sicherlich nicht der richtige weg. Je größer die Institution, desto schwerfälliger werden gerade die Grundsatzentscheidungen gefällt. Der sektor verdankt seine bisherige Erfolgsgeschichte zu einem großen Teil der hohen Geschwindigkeit und Dynamik der handelnden Personen, sodass es keinen sinn machen würde, diese struktur zu ändern. Die bisherigen Agenden des BMBWK (strategische Aufgaben des Sektors sowie Gesamtkoordination im tertiären sektor) müssen auch künftig unbedingt dort verbleiben (in einem schlanken und effektiven Organ).

Außerdem ist bei dem vorgestellten szenario der Aufwertung des FHR auch der widerspruch innerhalb der studie zu entdecken, dass der Rat von den AutorInnen an anderer stelle bereits als zu differenziert und schwerfällig beurteilt wird und mit seinen Durchführungsaufgaben bereits an Grenzen stößt. Warum sollte man ihm dann auch noch zusätzliche, strategische Fragen übertragen?

Die angedachten wesentlichen Akteure könnten ja in das Beratungsorgan integriert werden, wobei eine Einbeziehung der Länder (und das heist immer gleich x9!) sehr kritisch gesehen wird. Die Länder bringen in die Diskussion eine regionale, nicht aber eine überregionale sichtweise ein und können aufgrund ihrer Eigeninteressen keine objektiven Entscheidungen, den gesamtösterreichischen sektor betreffend, fällen.

Die bereits eingetretene "Verländerung" sowie die faktische, regionale Gliederung des sektors nach den Bundesländergrenzen wird ja in der studie an mehreren stellen auch sehr kritisch gesehen. In einigen Fällen wurden standorte nicht nach bundesländerübergreifenden und regionalen Gesichtspunkten oder nach der Größe des Einzugsgebietes eingerichtet, sondern eher nach Bundesländergrenzen. So kommt es vor, dass FH-Standorte zwar in verschiedenen Bundesländern, tatsächlich aber in sehr großer Nähe zueinander entstanden sind.

Zur Beseitigung der Rechtsunsicherheit bezüglich der zahl reichen Aussagen des FHR (seien es Mitteilungen, Verordnungen, Empfehlungen, Beschlüsse, Rundschreiben) ist eine "Reregulierung " dringend durchuuführen; diese müsste allerdings jedenfalls durch eine andere Institution erfolgen als den FHR selbst

Die FHK hat schon an anderer stelle vehement auf die Gefahren von "Rankings" sowie von Veröffentlichungen von Evaluierungsergebnissen hingewiesen. Diese Art von "Information" ist strikt abzulehnen. Die "vorteile", die in der studie nur sehr rudimentär herausgearbeitet wurden, sind 
gegenüber den Risken einer solchen vorgangsweise zu vernachlässigen.

Es steht außer Zweifel, dass der Sektor in den nächsten Jahren von Politik und Medien am Gelingen der qualitätssicherung gemessen werden wird und das Projekt Fachhochschule - je nach Befund - entweder reüssieren oder scheitern wird. Im Lichte dieser Überlegungen ist es wichtig, zwei Dinge unbedingt auseinanderzuhalten: Die Notwendigkeit der qualitätssicherung und damit auch der Evaluierung im nationalen und internationalen kontext auf der einen seite und die Bewahrung und steigerung der wettbewerbsfähigkeit der FHs im tertiären Bildungssektor auf der anderen seite.

Das ziel der steigerung der Wettbewerbsfähigkeit können die FHs nur erreichen, wenn der sektor nach außen geschlossen auftritt. Eine Veröffentlichung der Ergebnisse würde nicht nur die Konkurrenzsituation unter den FHs unnötig verstärken und nach außen sichtbar machen, sondern auch den anderen Bildungsanbietern im tertiären sektor Tür und Tor öffnen, um Schwächen der FHs - aus dem Zusammenhang gerissen aufzuzeigen und in den Medien zu platzieren. Die Fachhochschulen sind selbstverständlich bereit, sich einer Veröffentlichung der Ergebnisse zu unterziehen, wenn dies nicht nur selektiv für den FH-Bereich sondern für den gesamten sekundären und tertiären Bildungssektor gleichermaßen gelten würde. Damit würde auch eine bessere vergleichbarkeit im Bildungsbereich geschaffen.

\section{2) Zukünftige Entwicklung des Sektors: Felder und Modelle}

Die AutorInnen stellen viele denkmögliche szenarien vor, gehen aber zu wenig auf die vor- und Nachteile der einzelnen Modelle ein bzw geben nur sehr zögerlich Empfehlungen ab.

Aus sicht der FHK ist von den vorgeschlagenen Richtungen vom Grundsatz her nur ein verbessertes Modell "status quo" sinnvoll, das auch eine breite Unterstütuung im sektor selbst hat:

Neben den für dieses Modell angegebenen Kriterien wie: - abgeschwächtes wachstum, - Konsolidierung der standorte, nur begrenzte Eröffnung neuer standorte

müssen aber noch weitere Kriterien Berücksichtigung finden. So ist für das Erreichen eines qualitativen wachstums zB das Abgehen von der Nischenpolitik der letzten Jahre ebenso dringend notwendig wie die verbreiterung des Leistungsangebotes im sektor. Die Diversifikation des Aufgabenportfolios ist für die Beantwortung der Frage der 
Richtung für die Zukunft des FH-Sektors aus unserer sicht sogar als vorfrage zu klären.

Die Wahrnehmung anderer Funktionen (als der Lehre) wie Weiterbildung, Dienstleistung und F+E ist sein sehr wichtiger Ansatz; die Berücksichtigung dieser Bereiche bzw ihre Definition als ziele hängt aber auch sehr stark vom Finanzierungsmodell ab, das dann unbedingt zu überdenken wäre. Zum Beispiel könnten - abgesehen von der studienplatzfinanzierung - Beiträge zu den anderen Funktionen einen Zusatzanreiz für Erhalter darstellen.

Ohne Zusatzfinanzierung ist die verstärkte Konzentration auf andere Funktionen allerdings nicht durchführbar.

In diesem Zusammenhang wäre das Modell einer erhalterbezogenen Finanzierung sicherlich eine sehr gute Lösung.

\section{3) Entwicklungs - und Finanzierungsplanung}

Die Aussage "die steuerung über das steuerungsmittel Geld hat gut funktioniert", kann aus sicht der FHK nicht unterstützt werden.

Durch die Finanzierung von studienplätzen durch andere Körperschaften als den Bund hat der Bund in gewissem Ausmas das "Ruder aus der Hand" gegeben, was die strategische Planung der studienplätze betrifft und die Länder unkoordiniert Einfluss in die Bundespolitik nehmen lässt. Dabei waren nicht Bedarfs- oder standortkriterien vorrangig, sondern das Interesse und der finanzielle Einsatz der Ländervertreter.

Diese Frage muss im neuen Plan unbedingt behandelt und verbessert werden. Es bedarf diesbezüglich klarer Aussagen von seiten der Bundesregierung.

Zur Finanzierung siehe auch das unter Punkt 2 Gesagte.

Die AutorInnen stellen auch die fehlende strukturbereinigung im postsekundären sektor fest. Dieses Defizit kann aus FHsicht nur bekräftigt und die Wichtigkeit der Klarstellung des Verhältnisses der Fachhochschulen zu Universitäten und BHS betont werden.

Auch die Frage, ob Konsolidierung oder weitere Regionalisierung gewünscht ist, ist im nächsten E+F-Plan unbedingt zu behandeln. Der sektor, dh vor allem die Erhalter, benötigt mehr Planungssicherheit als bisher aufgrund der Pläne des Bundes möglich war.

Ebenso wie diese Grundsatzentscheidung ist auch die Lösung der Standortfrage ein Thema, zu dem im kommenden E+F-Plan dringend stellung bezogen werden muss. Es ist notwendig, nachvollziehbare wirtschaftiche und vor allem überregionale Kriterien für die standortentscheidung festzulegen und auf 
Bundesebene eine Klärung herbeizuführen. Auf Ebene der Länder ist das Erstreben einer überregional sinnvollen Lösung sicherlich nicht prioritär.

\section{4) E+F-Plan III}

Die für den E+F-Plan III reklamierten Themen sind alle zu unterstützen.

Die Forderung "Herstellung gleicher Rahmenbedingungen, fairer spielregeln" wird zwar von den AutorInnen aufgestellt, sie wird leider nicht näher konkretisiert.

Es handelt sich hierbei aus sicht der FHK aber um eine zentrale Bedingung für eine Fortschreibung der Erfolgsgeschichte der Fachhochschulen.

Unter anderem bezieht sich diese Forderung auf die Indexierung der Bundesbeiträge, Rechtsbereinigung sowie schaffung von Rechts- und Planungssicherheit für die Zukunft.

Siehe zum E+F-Plan III auch das schon unter Punkt 3 Gesagte. 


\section{Punktation \\ der Ländervertreter zum vorgelegten Entwurf der Studie „Evaluierung des Auf- und Ausbaus des Fachhochschul-Sektors" \\ (Besprechung 28.1.2003)}

$\therefore \quad$ Die Ländervertreter gehen - wie bereits mehrmals darauf hingewiesen - davon aus, dass die vorliegende Studie lediglich einen Teil der Grundlagen für die Erstellung eines neuen Bundesentwicklungs- und Finanzierungsplanes darstellt.

$\therefore \quad$ Die Zielsetzung der beauftragten Studie war unter anderem eine umfassende Bestandsaufnahme sowie eine kritische Analyse des Fachhochschulsektors durchzuführen. Bei genauerer Betrachtung der dargestellten Inhalte der einzelnen Kapitel fällt auf, dass zwischen der gewünschten Analyse einerseits und dem ausgesprochenen Empfehlungen andererseits keine klare Trennung gegeben scheint. Es wird ersucht, die Analysen von den Empfehlungen bzw Schlussfolgerungen klar zu trennen und letztere von verbindlichen Fakten abzuleiten. Bei einigen Kapiteln fehlen die Schlussfolgerungen.

$\therefore \quad$ Hinsichtlich der Analyse des österreichischen Fachhochschul-Sektors sollten die unterschiedlichen Akteure / Institutionen / Organe einer umfassenden Analyse betreffend die Aufgaben und deren tatsächlichen Tätigkeitsfelder unterzogen werden (Stärke- / Schwächeprofil). Für das künftige Rollenbild der Akteure ist diese Analyse besonders wertvoll.

$\therefore \quad$ Die Bundesländervertreter gehen davon aus, dass sie in den erforderlichen Diskussionsprozess für den Entwurf und die Erstellung eines neuen Bundesentwicklungs- und Finanzierungsplanes aktiv eingebunden werden. Die qualitative Bewertung der aus der Studie hervorgehenden Schlussfolgerungen wären mit den Ländervertretern abzustimmen.

× Die wichtigste Aufgabe für den weiteren Prozess einer Entwicklungsplanung für das Fachhochschulwesen ist die Vorgabe von Leitlinien für den gesamten tertiären Bildungsbereich. Die Bundesländervertreter würden es begrüßen, wenn der im Universitätsgesetz 2002 vorgesehene Wissenschaftsrat diese Thematik unter Einbeziehung der Ländervertreter behandelt und dem Fachhochschul-Sektor ein klar abgegrenztes Aufgaben- und Entwicklungsfeld im tertiären Bildungssektor zuordnet. Qualitative Vorgaben für die künftige Entwicklung sollten dabei im Vordergrund stehen.

$\because \quad$ Bei der Gesamtbetrachtung des tertiären Bildungsbereiches sind nicht nur staatliche Bildungseinrichtungen, sondem auch im Zuge der Liberalisierung zuzulassende private Bildungsanbieter zu berücksichtigen 


\subsection{Methodische Hinweise}

\subsubsection{Liste der InterviewpartnerInnen}

Peter Allram, FH-S (Interessenvertretung der Fachhochschul-Studierenden Österreichs Christoph Badelt, Wirtschaftsuniversität Wien

Fritz Bauer, AK OÖ

Irmgard Eisenbach-Stangl, FHR

Friedrich Faulhammer, bm:bwk

Eva Götz, bm:bwk

Wilfried Grätz, Geschäftsstelle des FHR

Elsa Hackl, Universität Wien (vormals bm:bwk)

Maria Hofstätter, AMS

Stefan Humpl, 3s Unternehmensberatung

Monika Hutter, BMF

Werner Jungwirth, FHK

Michael Landertshammer, BWK (vormals FHK)

Christian Mann, NÖ-Bildungsgesellschaft

Jörg Markowitsch, 3s Unternehmensberatung

Andreas Neuhold, Geschäftsstelle des FHR

Herwig Patscheider, Geschäftsstelle des FHR

Peter Piffl-Percevic, Amt der Steiermärkischen Landesregierung

Manfred Prisching, Univ. Graz (vormals FH-Joanneum)

Claus Raidl, Präsident des FHR

Christoph Ramoser, bm:bwk

Günther Schelling, ehemaliger Präsident des FHR

Susanne Schöberl, AK-Wien, FHR

Günther Simonitsch, bm:bwk

Kurt Sohm, Geschäftsstelle des FHR

Monika Vyslouzil, FHR

Gerhard Wagner, VIW (Verband der Informationswirtschaft)

Hanns-Peter Winter, FHR 


\section{Liste der InterviewpartnerInnen für Sekundärauswertung (NÖ-Szenarienprojekt)}

Walter Bauer, Leobersdorfer Maschinenfabrik AG

Heinz Boyer, IMC-Internationales Management Center Krems GmbH

Helmut Detter, Fachhochschule Wiener Neustadt für Wirtschaft und Technik GmbH

Karl Fakler, Arbeitsmarktservice NÖ, Landesgeschäftsstelle

Werner Fröhlich, Donau-Universität Krems

Franz Gritsch, Schoeller-Bleckmann Oilfield Equipment AG

Sigurd Höllinger, Bundesministerium für Bildung, Wissenschaft und Kultur

Wolfgang Horvarth, Amt der NÖ Landesregierung, Geschäftsstelle für Technologie

Anton Hoser, Österreichische Bundesbahnen

Alberich Klinger, NÖ Landesakademie

Raoul Kneucker, Bundesministerium für Bildung, Wissenschaft und Kultur

Josef Kolarz-Lakenbacher, Siemens AG Österreich

Peter Kopacek, Technische Universität Wien

Theodor Krendelsberger, ECO PLUS NÖ Regionale Entwicklungsagentur GmbH

Andreas Kusternig, Amt der NÖ Landesregierung

Christian Mann, NÖ Bildungsgesellschaft mbH für Fachhochschul- und Universitätswesen

Stefan Petters, Electrovac Fabrikation elektrotechnischer Spezialartikel GmbH

Karl Sablik, NÖ Landesakademie

Peter Skalicky, Technische Universität Wien

Matthias Stadler, Gesellschaft zur Durchführung von FH-Studiengängen St. Pölten mbH

Adolf Stricker, NÖ Landesschulrat 


\subsubsection{Datenquellen und -erhebungen}

Neben Sekundärstatistiken der Statistik Austria (v.a. Mikrozensen und Hochschulstatistik) und des BMBWK (v.a. Hörerevidenz, ISCED-Zuordnungen) wurden in erster Linie die Daten aus der BIS-Datenbank (BIS - „Bereitstellung von Informationen über den Studienbetrieb“) des Fachhochschulrates einer erneuten Auswertung unterzogen. ${ }^{216}$ Der Schwerpunkt lag dabei auf der Erstellung von Zeitreihen die insbesondere den Kapiteln 2.2 (Datenüberblick) und 3 (Stellenwert des FH-Sektors) zugrunde liegen.

Zusätzlich wurden mittels einer Befragung der Erhalter und einer Online-Befragung der Studiengangsleitungen im Sommer 2002 eigene Daten erhoben. Beide Fragebögen wurden im Projektbeirat diskutiert und in Zusammenarbeit mit VertreterInnen der FHK ergänzt. Der Versand erfolgte Mitte Juli 2002 per E-Mail ebenfalls über die FHK. Die Erhalter wurden dabei gebeten den ausgefüllten Fragebogen an das IHS retour zu faxen, die Studiengangsleitungen füllten den Fragebogen über die Homepage des IHS aus (ein Musterfragebogen war der E-Mail beigefügt). Aufgrund der Urlaubszeit wurde der Erhebungszeitraum bis Ende August/ Anfang September ausgedehnt.

Der Erhalterfragebogen wurde von 13 Erhaltern (68\%) ausgefüllt, an deren Institutionen rund 80\% der Studierenden des FH-Sektors studieren (lediglich bei den Angaben zu den Finanzen lag die Responserate etwas niedriger). Der Online-Fragebogen der Studiengangsleitungen wurde von 69 StudiengangsleiterInnen (73\%) ausgefüllt, in deren Studiengängen im Studienjahr 2001/02 etwa 75\% der Studierenden inskribiert waren.

Die Themenkomplexe des Erhalterfragebogens waren:

- Stärken/ Schwächen der Studiengänge

- Personal

- Internationalisierung versus Regionalisierung

- Bologna-Prozess (Planungen sowie Vor- und Nachteile von Bakk/Master-Studiengängen)

- Qualitätssicherung

- Zusätzliche Leistungen für Studierende

- Finanzen

- Forschungs-, Beratungs- und Gutachtertätigkeiten

- Rolle der Studiengänge in der Region

- Entwicklungsplanung

216 Die Abfragen der Datenbank erfolgten Anfang Juli 2002. 
Die Themenkomplexe des Studiengangsfragebogens waren:

- Wochenstunden des Studienangebotes

- Konkurrenz zu diversen Universitätsstudienrichtungen

- Lehrpersonal (haupt-/nebenberuflich, Geschlecht, Qualifikation)

- Personalentwicklung

- Aufnahmekriterien für Studierende

- Mobilität der Studierenden und des Lehrpersonals

- Leistungen für Dritte (Vorträge, F\&E)

- Wirtschaftsbereiche an die sich der Studiengang richtet

- Unternehmenskontakte

- Qualitätssicherung 


\subsection{Zeittafel zur Entwicklung des FH-Sektors}

\begin{tabular}{|c|c|c|}
\hline \multicolumn{3}{|l|}{$1990-1992$} \\
\hline $1990 / 12$ & & $\begin{array}{l}\text { Arbeitsübereinkommen zwischen der SPÖ und der ÖVP } \\
\text { über die Bildung einer gemeinsamen Bundesregierung } \\
\text { Koalitionsabkommen: Einrichtung eines } \\
\text { nichtuniversitären postsekundären Bereichs } \\
\text { („,Fachakademien“) in Österreich }\end{array}$ \\
\hline $1991 / 11$ & BMWF, OECD & Seminar „Alternatives to Universities“ in Wien \\
\hline 1992/02 & BMWF & $\begin{array}{l}\text { Erster Entwurf zu einem Bundesgesetz über } \\
\text { Fachhochschul-Studiengänge }\end{array}$ \\
\hline $1992 / 09-1992 / 10$ & OECD & $\begin{array}{l}\text { OECD-Prüfung (Review of Higher Education Policy in } \\
\text { Austria) }\end{array}$ \\
\hline \multicolumn{3}{|l|}{1993} \\
\hline 1993/04 & OECD Paris & $\begin{array}{l}\text { Review of Higher Education Policy in Austria. Examiner`s } \\
\text { Report and Questions }\end{array}$ \\
\hline 1993/05 & Parlament & $\begin{array}{l}\text { Bundesgesetz über Fachhochschul-Studiengänge } \\
\text { (FHStG), BGBI. Nr. 340/1993 }\end{array}$ \\
\hline $1993 / 10$ & & Inkrafttreten des FHStG \\
\hline $1993 / 10$ & FHR & $\begin{array}{l}\text { Ernennung des ersten FHR-Präsidenten (1.10.), } \\
\text { Konstituierung des FHR (21.10.) }\end{array}$ \\
\hline $1993 / 10-1996 / 09$ & $\mathrm{FHR}$ & 1. Funktionsperiode (Schelling) \\
\hline 1994 & & FHR-Präsident: Schelling \\
\hline $1994 / 01$ & FHR & $\begin{array}{l}\text { Information für Antragsteller (IFA 1994) über } \\
\text { Anforderungen an Anträge auf Anerkennung von } \\
\text { Studiengängen als Fachhochschul-Studiengänge und an } \\
\text { Anträge auf Verlängerung der Anerkennung }\end{array}$ \\
\hline 1994/03 & BMWF & $\begin{array}{l}\text { FHS-Entwicklungsplan I: „Entwicklungs- und } \\
\text { Finanzierungsplanung für den Fachhochschulbereich bis } \\
\text { 1999/2000“ }\end{array}$ \\
\hline $1994 / 09$ & & $\begin{array}{l}\text { Die ersten } 10 \text { Fachhochschul-Studiengänge nehmen den } \\
\text { Studienbetrieb auf }\end{array}$ \\
\hline 1995 & & FHR-Präsident: Schelling \\
\hline $1995 / 03$ & FHR & $\begin{array}{l}\text { FHR-Beschluss über Vollmitgliedschaft bei der INQAAHE } \\
\text { (International Network for Quality Assurance Agencies in } \\
\text { Higher Education) }\end{array}$ \\
\hline $1995 / 03$ & FHR & $\begin{array}{l}\text { FHR-Beschluss über „10\%-Regel“: "Der } \\
\text { Fachhochschulrat toleriert, unbeschadet des Nachweises } \\
\text { der erforderlichen Raum-,Ausstattungs-, Personal- und } \\
\text { Finanzressourcen und unbeschadet der vom Bund im } \\
\text { Förderungsvertrag festgesetzten Anzahl geförderter } \\
\text { Studienplätze, die Aufnahme einer um zehn Prozent } \\
\text { höheren Anzahl von Studienbewerbern als Studienplätze } \\
\text { im genehmigten Antrag festgesetzt sind." (FHR-Info Nr. 4) }\end{array}$ \\
\hline $1995 / 06$ & FHR & 1. Informationstag des FHR \\
\hline $1995 / 06$ & FHR & $\begin{array}{l}\text { Verordnung über die Bereitstellung von Informationen } \\
\text { über den Studienbetrieb („Statistikverordnung“ des FHR, } \\
\text { FHR-Info Nr.1) }\end{array}$ \\
\hline $1995 / 07$ & FHR & $\begin{array}{l}\text { Modifikation der „Information für Antragsteller“ (IFA 1995) } \\
\text { (FHR-Info Nr.2) }\end{array}$ \\
\hline
\end{tabular}




\begin{tabular}{|c|c|c|}
\hline $1995 / 11$ & FHK & $\begin{array}{l}\text { Gründung des Vereines "Österreichische } \\
\text { Fachhochschul-Konferenz" }\end{array}$ \\
\hline 1996 & & FHR-Präsident: Schelling \\
\hline $1996 / 04$ & FHR, FHK & $\begin{array}{l}\text { FHR-Beschluss über Beitritt zur EURASHE (European } \\
\text { Association of Institutions in Higher Education) als } \\
\text { Vollmitglied gemeinsam mit der FHK }\end{array}$ \\
\hline 1996/04 & & $\begin{array}{l}\text { Erstmalige Verleihung des akademischen Grads an } \\
\text { Absolventen eines FH-Studiengangs } \\
\text { („Fertigungsautomatisierung“ am Technikum Vorarlberg) }\end{array}$ \\
\hline $1996 / 05$ & FHR & $\begin{array}{l}\text { FHR-Beschluss, der den Erhaltern die Kompensation frei } \\
\text { gewordener Studienplätze (Dropouts) durch eine erhöhte } \\
\text { Aufnahme von Anfängern zu Studienjahresbeginn } \\
\text { ermöglicht (FHR-Info Nr. 6) }\end{array}$ \\
\hline 1996/06 & FHR & Leitfaden für die Lehrevaluation (FHR-Info Nr. 6) \\
\hline $1996 / 07$ & FHR & Berufstitel für Lehrende (FHR-Info Nr. 6) \\
\hline 1996/09 & BMWVK & $\begin{array}{l}\text { Verordnung über das Doktoratsstudium für } \\
\text { Absolventinnen und Absolventen von } 6 \text { Fachhochschul- } \\
\text { Studiengängen technischer Richtung }\end{array}$ \\
\hline 1996/09 & BMWVK & $\begin{array}{l}\text { Verordnung über das Doktoratsstudium für } \\
\text { Absolventinnen und Absolventen von } 4 \text { Fachhochschul- } \\
\text { Studiengängen in den Bereichen Wirtschaft und } \\
\text { Tourismus }\end{array}$ \\
\hline $1996 / 10$ & FHR & $\begin{array}{l}\text { Novelle zur Verordnung über die Bereitstellung von } \\
\text { Informationen über den Studienbetrieb }\end{array}$ \\
\hline 1996/10 -1999/09 & FHR & 2. Funktionsperiode (Schelling) \\
\hline 1997 & & FHR-Präsident: Schelling \\
\hline $1997 / 0$ & BMWV, TIG & $\begin{array}{l}\text { FH-Impulsaktion "Kooperation zwischen FH } \\
\text { Wirtschaft" zur Ingangsetzung bzw. Fortführung des } \\
\text { Technologietransfers; erste Ausschreibungsrunde }\end{array}$ \\
\hline $1997 / 05$ & FHR , FHK & Qualitätssystem FHK/FHR, erste Version \\
\hline $1997 / 05-1997 / 10$ & FHR & $\begin{array}{l}\text { Erste Evaluierung eines FH-Studiengangs im Rahmen } \\
\text { der Verlängerung der Anerkennung }\end{array}$ \\
\hline $1997 / 06$ & FHR , FHK & $\begin{array}{lll}\text { Evaluierungskonzept } \quad \text { und } & \text { Ablauf } & \text { des } \\
\text { Evaluierungsverfahrens (FHR-Info Nr. 8) } & \end{array}$ \\
\hline $1997 / 07$ & FHR & $\begin{array}{l}\text { Änderung im Ablauf des Verfahrens um Anerkennung von } \\
\text { Studiengängen als Fachhochschul-Studiengänge (FHR- } \\
\text { Info Nr. 8) }\end{array}$ \\
\hline $1997 / 09$ & FHR & $\begin{array}{l}\text { Novelle zur Verordnung über die Bereitstellung von } \\
\text { Informationen über den Studienbetrieb (FHR-Info Nr. 9) }\end{array}$ \\
\hline 1998 & & FHR-Präsident: Schelling \\
\hline $1998 / 05$ & Parlament & $\begin{array}{l}\text { Novelle zum FHStG 1993, BGBI. Nr. 72/1998: Schaffung } \\
\text { der Voraussetzungen für zielgruppenspezifische } \\
\text { Weiterqualifizierungsangebote, Beurteilung der } \\
\text { Gleichwertigkeit von ausländischen FH-Studien durch } \\
\text { den FHR, Verlängerung der Frist zur Antragsbearbeitung } \\
\text { durch FHR }\end{array}$ \\
\hline $1998 / 05$ & & $\begin{array}{l}\text { "Sorbonne-Erklärung“ } \\
\text { zuständigen Minister Großbritanniens, Frankreichs, } \\
\text { Deutschlands und Italiens zur Harmonisierung der } \\
\text { Architektur der europäischen Hochschulbildung }\end{array}$ \\
\hline
\end{tabular}




\begin{tabular}{|c|c|c|}
\hline 1998/06 & & $\begin{array}{l}\text { Deklaration über den „Europäischen Raum für } \\
\text { Hochschulbildung“ der zuständigen Minister der } \\
\text { Vertragsstaaten von EU, Europ. Freihandelsorganisation } \\
\text { und assoziierten mittel- und osteurop. Staaten }\end{array}$ \\
\hline 1998/07 & FHR & $\begin{array}{l}\text { Modifikation der „Information für Antragsteller“ (IFA 1998) } \\
\text { (FHR-Info Nr. 11) }\end{array}$ \\
\hline 1998/08 & BMWV & $\begin{array}{l}\text { Verordnung über das Doktoratsstudium für } \\
\text { Absolventinnen und Absolventen von } 7 \text { Fachhochschul- } \\
\text { Studiengängen technischer Richtung }\end{array}$ \\
\hline 1998/08 & BMWV & $\begin{array}{l}\text { Verordnung über das Doktoratsstudium für } \\
\text { Absolventinnen und Absolventen von } 6 \text { Fachhochschul- } \\
\text { Studiengängen wirtschaftlicher Richtung }\end{array}$ \\
\hline 1998/08 & BMWV & $\begin{array}{l}\text { Verordnung über das Doktoratsstudium für } \\
\text { Absolventinnen und Absolventen des Fachhochschul- } \\
\text { Studienganges "Holztechnik und Holzwirtschaft }\end{array}$ \\
\hline 1998/09 & FHR & $\begin{array}{l}\text { Novelle zur Verordnung über die Bereitstellung von } \\
\text { Informationen über den Studienbetrieb (FHR-Info Nr. 12) }\end{array}$ \\
\hline 1998/09 & BMVIT, TIG & Kompetenzzentrenprogramm K plus, Pilotphase \\
\hline 1998/10 & FHR & $\begin{array}{l}\text { Änderung im Ablauf des Anerkennungsverfahrens für } \\
\text { Anträge mit kalkulierter Bundesförderung (FHR-Info Nr. } \\
\text { 13): Abgabe einer Empfehlung des FHR an das BMBWK } \\
\text { über die Förderwürdigkeit von Anträgen }\end{array}$ \\
\hline 1999 & & FHR-Präsident: Schelling \\
\hline 1999/04 & BMWV & $\begin{array}{l}\text { FHS-Entwicklungsplan II: } \\
\text { Finanzierungsplanung für den Fntwicklungs- } \\
\text { 2000/01-2004/05“ }\end{array}$ \\
\hline 1999/04-1999/09 & BMWV, TIG & $\begin{array}{l}\text { FH-Impulsaktion "Kooperation zwischen FH und } \\
\text { Wirtschaft" zur Ingangsetzung bzw. Fortführung des } \\
\text { Technologietransfers; zweite Ausschreibungsrunde }\end{array}$ \\
\hline $1999 / 05$ & FHR & Homepage des FHR (www.fhr.ac.at) \\
\hline 1999/05 & FHR & $\begin{array}{lccc}\text { Beschluss einer } & \text { adaptierten } & \text { Version } & \text { des } \\
\text { "Qualitätssystems } & \text { FHR/FHK" } & \text { (Adaption } & \text { von } \\
\text { Erkenntnissen aus dem Monitoring-Projekt des FHR) } & \end{array}$ \\
\hline 1999/05 & FHR & $\begin{array}{l}\text { Modifikation des „Ablaufverfahrens der Evaluierung und } \\
\text { des Verlängerungsantrages“ }\end{array}$ \\
\hline 1999/08 & Parlament & $\begin{array}{l}\text { Novelle zum Universitäts-Studiengesetz BGBL. Nr. Nr. } \\
\text { 167/1999, mit der an den Universitäten die Möglichkeit } \\
\text { der dreistufigen Studienarchitektur (Bakkalaureats-, } \\
\text { Magister-, Doktoratsstudium) eingeführt wurde }\end{array}$ \\
\hline 1999/08 & BMWV & $\begin{array}{l}\text { Verordnung über die Verleihung der Bezeichnung } \\
\text { "Fachhochschule" an die "Wiener Neustädter Bildungs- } \\
\text { und Forschungs-Gesellschaft m.b.H." }\end{array}$ \\
\hline 1999/09 & BMWV & $\begin{array}{l}\text { Verordnung über das Doktoratsstudium für } \\
\text { Absolventinnen und Absolventen von } 7 \text { Fachhochschul- } \\
\text { Studiengängen technischer Richtung }\end{array}$ \\
\hline 1999/09 & BMWV & $\begin{array}{l}\text { Verordnung über das Doktoratsstudium für } \\
\text { Absolventinnen und Absolventen von } 6 \text { Fachhochschul- } \\
\text { Studiengängen wirtschaftlicher Richtung }\end{array}$ \\
\hline 1999/09 & BMWV & $\begin{array}{l}\text { Verordnung über die Verleihung der Bezeichnung } \\
\text { „Fachhochschule" an die „Fachhochschul-Studiengänge } \\
\text { Vorarlberg-Gesellschaft m.b.H." }\end{array}$ \\
\hline 1999/09 & FHR & $\begin{array}{l}\text { Institutionelle Mitgliedschaft des FHR bei der Deutschen } \\
\text { Gesellschaft für Evaluation }\end{array}$ \\
\hline $1999 / 09$ & FHR & $\begin{array}{l}\text { Modifikation der „Information für Antragsteller“ (IFA 1999) } \\
\text { (FHR-Info Nr. 14) }\end{array}$ \\
\hline
\end{tabular}




\begin{tabular}{|c|c|c|}
\hline $1999 / 10$ & $\mathrm{FHR}$ & $\begin{array}{l}\text { Novelle zur Verordnung über die Bereitstellung von } \\
\text { Informationen über den Studienbetrieb (FHR-Info Nr. 15) }\end{array}$ \\
\hline $1999 / 10-2002 / 9$ & & 3. Funktionsperiode (Rauch, Raidl) \\
\hline 2000 & & FHR-Präsident: Rauch \\
\hline $2000 / 0$ & BMVIT, TIG & $\begin{array}{l}\text { 3. Ausschreibungsrunde der FH-Impulsaktion } \\
\text { "Kooperation zwischen FH und Wirtschaft des BMVIT : } \\
\text { „F\&E-Personal für Fachhochschulen, Strategiekonzepte“ }\end{array}$ \\
\hline $2000 / 03$ & FHR & $\begin{array}{l}\text { Mitgliedschaft des FHR und Teilnahme an der } \\
\text { Gründungskonferenz des ENQA (European Network for } \\
\text { Quality Assurance in Higher Education) }\end{array}$ \\
\hline $2000 / 05$ & FHR & $\begin{array}{l}\text { FHR beschließt Bearbeitung der Anträge aus dem } \\
\text { Bereich „nichtärztliche Gesundheitsberufe“ trotz } \\
\text { ausstehender Klärung der Kostenträgerschaft }\end{array}$ \\
\hline $2000 / 07$ & FHR & $\begin{array}{l}\text { Grundsatzbeschluss des FHR: Konsolidierung der } \\
\text { bestehenden Standorte/Erhalter hat Vorrang vor } \\
\text { Erhöhung der Zahl der Standorte }\end{array}$ \\
\hline $2000 / 09$ & BMBWK & $\begin{array}{l}\text { Verordnung über das Doktoratsstudium für } \\
\text { Absolventinnen und Absolventen der Fachhochschul- } \\
\text { Studiengänge "Industrial Design", "InterMedia" und } \\
\text { "MultiMediaArt }\end{array}$ \\
\hline $2000 / 10$ & FHR & $\begin{array}{l}\text { Novelle zur Verordnung über die Bereitstellung von } \\
\text { Informationen über den Studienbetrieb (FHR-Info Nr. 16): } \\
\text { Reduktion des Datenumfangs, Meldung der Finanzdaten } \\
\text { nur noch an das BMBWK }\end{array}$ \\
\hline $2000 / 10$ & FHR & $\begin{array}{l}\text { Modifikation der „Information für Antragsteller“ (IFA 2000) } \\
\text { (FHR-Info Nr. 17) zukünftig Vorlage von strategischen } \\
\text { Konzepten/Maßnahmen zur Internationalisierung des } \\
\text { beantragten Studiengangs, Vorlage eines } \\
\text { Planungskonzepts für den Ausbau des Bildungsangebots, } \\
\text { Neugestaltung der Richtlinien für die Bedarfs- und } \\
\text { Akzeptanzerhebung }\end{array}$ \\
\hline $2000 / 11$ & BMBWK & $\begin{array}{l}\text { Verordnung über die Verleihung der Bezeichnung } \\
\text { „Fachhochschule" an das „Technikum Kärnten - Verein } \\
\text { zur Errichtung der Fachhochschule Spittal/Drau" }\end{array}$ \\
\hline $2000 / 11$ & BMBWK & $\begin{array}{l}\text { Verordnung: Verleihung der Bezeichnung } \\
\text { "Fachhochschule" an den Verein "Fachhochschul- } \\
\text { Studiengänge Technikum Wien" }\end{array}$ \\
\hline $2000 / 12$ & FHR & $\begin{array}{l}\text { FHR gibt vorbehaltlich der ausstehenden Klärung der } \\
\text { Finanzierungsmodalitäten erstmals } 4 \text { Anträgen aus dem } \\
\text { Bereich „Sozialarbeit“ statt }\end{array}$ \\
\hline 2001 & & FHR-Präsident: Raidl \\
\hline $2001 / 09$ & & $\begin{array}{l}\text { Die ersten Fachhochschul-Studiengänge aus dem } \\
\text { Bereich „Sozialarbeit“ und dem Bereich „nichtärztliche } \\
\text { Gesundheitsberufe“ nehmen den Studienbetrieb auf }\end{array}$ \\
\hline $2001 / 11$ & Parlament & $\begin{array}{l}\text { Novellierung des FHStG } 1993 \text { in Zusammenhang mit } \\
\text { dem Euro-Umstellungsgesetz BGBI. Nr. 136/2001 }\end{array}$ \\
\hline 2002 & & FHR-Präsident: Raidl \\
\hline $2002 / 03$ & BMBWK & $\begin{array}{l}\text { Verordnung: Verleihung der Bezeichnung } \\
\text { "Fachhochschule" an die "bfi Wien Fachhochschul- } \\
\text { Studiengangsbetriebs Gesellschaft m.b.H." }\end{array}$ \\
\hline $2002 / 03$ & BMBWK & $\begin{array}{l}\text { Verordnung: Verleihung der Bezeichnung } \\
\text { "Fachhochschule" an die "International Management } \\
\text { Center Krems-Gesellschaft m.b.H." }\end{array}$ \\
\hline
\end{tabular}




\begin{tabular}{|c|c|c|}
\hline $2002 / 03$ & FHK & $\begin{array}{l}\text { Rahmenübereinkommen zwischen der Österreichischen } \\
\text { Rektorenkonferenz (ÖRK) und der Österreichischen } \\
\text { Fachhochschul-Konferenz (FHK) einerseits und der } \\
\text { Conférence des présidents d'université (CPU) und der } \\
\text { Conférence des directeurs d'écoles et formations } \\
\text { d'ingénieurs (CDEFI) andererseits über die Bewertung } \\
\text { von Studien und die akademische Anerkennung von } \\
\text { Studienabschlüssen , 1. 3. 2002, VOBI.71/2002. } \\
\text { [Frankreich] }\end{array}$ \\
\hline 2002/03 & FHR & $\begin{array}{l}\text { FHR-Beschluss (nach Zustimmung des BMBWK) über } \\
\text { neues Akkreditierungsverfahren ab Mai 2002: } \\
\text { grundsätzliche Trennung der Verfahren zur Klärung der } \\
\text { Finanzierung und der Qualitätsprüfung }\end{array}$ \\
\hline $2002 / 03$ & FHR & $\begin{array}{l}\text { FHR-Beschluss über Änderung des } \\
\text { Evaluierungsverfahrens: zukünftig zwei Arten von } \\
\text { Evaluierungsverfahren „Institutionelle Evaluierung“ und } \\
\text { „Studiengangsbezogene Evaluierung“ }\end{array}$ \\
\hline 2002/05 & Parlament & $\begin{array}{l}\text { Novelle zum FHStG 1993, BGBI. Nr. 58/2002: Einführung } \\
\text { von Bachelor- und Master-Studiengängen an FHS; } \\
\text { generelle Berechtigung zu facheinschlägigen } \\
\text { Doktoratsstudien an Universitäten; FHR wird Kompetenz } \\
\text { zur Standortentscheidung entzogen }\end{array}$ \\
\hline 2002/07 & BMBWK & $\begin{array}{l}\text { Verordnung über das Doktoratsstudium für } \\
\text { Absolventinnen und Absolventen von } 6 \text { Fachhochschul- } \\
\text { Studiengängen technischer Richtung }\end{array}$ \\
\hline $2002 / 07$ & BMBWK & $\begin{array}{l}\text { Änderung der Verordnung über das Doktoratsstudium für } \\
\text { Absolventinnen und Absolventen von Fachhochschul- } \\
\text { Studiengängen wirtschaftlicher Richtung (Entfall } \\
\text { „Infrastrukturwirtschaft") }\end{array}$ \\
\hline 2002/07 & BMBWK & $\begin{array}{l}\text { Verordnung über den Zugang von Absolventinnen und } \\
\text { Absolventen von } 4 \text { Fachhochschul-Studiengängen zum } \\
\text { Doktoratsstudium der Philosophie }\end{array}$ \\
\hline 2002/09 & $\begin{array}{l}\text { BMVIT, } \\
\text { BMBWK }\end{array}$ & $\begin{array}{l}\text { Genehmigung des Förderungsprogramms „FH plus“" zur } \\
\text { Förderung von anwendungsbezogener F\&E im FH-Sektor } \\
(10,9 \text { Mill. } €)\end{array}$ \\
\hline
\end{tabular}




\subsection{Verzeichnis der Abkürzungen}

\begin{tabular}{|c|c|}
\hline AHS & Allgemeinbildende Höhere Schulen \\
\hline BBT & Bundesamt für Berufsbildung und Technologie (Schweiz) \\
\hline BIS & „Bereitstellung von Informationen über den Studienbetrieb“ \\
\hline BHS & Berufsbildende höhere Schulen \\
\hline BMAGS & Bundesministerium für Arbeit, Gesundheit und Soziales \\
\hline BMBF & Bundesministerium für Bildung und Forschung (Deutschland) \\
\hline BMBWK & Bundesministerium für Bildung, Wissenschaft und Kunst \\
\hline BMS & Berufsbildende Mittlere Schulen \\
\hline BMUK & Bundesministerium für Unterricht und Kunst \\
\hline BMUkA & Bundesministerium für Unterricht und kulturelle Angelegenheiten \\
\hline BMVIT & Bundesministerium für Verkehr, Infrastruktur und Technologie \\
\hline BMWF & Bundesministerum für Wissenschaft und Forschung \\
\hline BMWFK & Bundesministerium für Wissenschaft, Forschung und Kunst \\
\hline BMWV & Bundesministerium für Wissenschaft und Verkehr \\
\hline B/M-Studiengänge & Bachelor/Master-Studiengänge \\
\hline $\mathrm{B}-\mathrm{VG}$ & Bundes-Verfassungsgesetz \\
\hline CEDEFOP & European Center for the Development of Vocational Training \\
\hline $\mathrm{CHE}$ & Centrum für Hochschulentwicklung (Deutschland) \\
\hline CHEPS & Center for Higher Education Policy Studies (Niederlande) \\
\hline DVC & Dutch Validation Council \\
\hline ECTS & European Credit Transfer System \\
\hline EFHK & Eidgenössische Fachhochschulkommission (Schweiz) \\
\hline EFP & Entwicklungs- und Finanzierungsplan (für den Fachhochschul-Sektor) \\
\hline ENQA & European Network of Quality Assurance in Higher Education \\
\hline ETH & Eidgenössische Technische Hochschule (Schweiz) \\
\hline $\mathrm{F} \& \mathrm{E}$ & Forschung und Entwicklung \\
\hline FHK & Fachhochschul-Konferenz \\
\hline FHR & Fachhochschulrat \\
\hline FHS & Fachhochschule \\
\hline FHStG & Fachhochschul-Studiengesetz \\
\hline FINHEEC & finnischer Hochschulevaluationsrat \\
\hline FTE & Forschung und Technologieentwicklung \\
\hline HBO-Institutionen & $\begin{array}{l}\text { Einrichtungen der beruflichen Hochschulbildung (hoger } \\
\text { beroepsonderwijs) (Niederlande) }\end{array}$ \\
\hline HBO-Raad & „Fachhochschul“-Rat (Niederlande) \\
\hline $\mathrm{HEI}$ & Higher Education Institution \\
\hline HRG & Hochschul-Rahmengesetz (Deutschland) \\
\hline HRK & Hochschulrektorenkonferenz (Deutschland) \\
\hline Ibw & Institut für Bildungsforschung der Wirtschaft \\
\hline Iff & Institut für interdisziplinäre Forschung und Fortbildung \\
\hline IHS & Institut für Höhere Studien \\
\hline
\end{tabular}




\begin{tabular}{|c|c|}
\hline IKT & Informations- und Kommunikationstechnologien \\
\hline ISCED & $\begin{array}{l}\text { International Standard Classification for Education (Internationale } \\
\text { Standard-Klassifikation des Bildungswesens) }\end{array}$ \\
\hline IWI & Industriewissenschaftliches Institut \\
\hline KMK & Kultusministerkonferenz (Deutschland) \\
\hline KMU & Klein- und mittelbetriebliche Unternehmen \\
\hline LP & Lehrpersonen \\
\hline MBO & Management by Objectives, Ziel-Ergebnis-Steuerung \\
\hline NAO & $\begin{array}{l}\text { Nederlandse Accreditatie Organisatie (Nationale } \\
\text { Akkreditionsorganisation) (Niederlande) }\end{array}$ \\
\hline NACE-Kategorien & $\begin{array}{l}\text { Kategorien der Wirtschaftstätigkeiten-Klassifikation NACE } \\
\text { (Nomenclature générale des Activités économiques dans les } \\
\text { Communautés Européennes) }\end{array}$ \\
\hline NARIC & National Academic Recognition Centre \\
\hline NPM & New Public Management \\
\hline $\mathrm{OAQ}$ & $\begin{array}{l}\text { Organ für Akkreditierung und Qualitätssicherung der Schweizerischen } \\
\text { Hochschulen }\end{array}$ \\
\hline $\mathrm{OE}$ & Organisationsentwicklung \\
\hline OECD & $\begin{array}{l}\text { Organisation for Economic Cooperation and Development } \\
\text { (Organisation für wirtschaftliche Zusammenarbeit und Entwicklung) }\end{array}$ \\
\hline ÖAD & Österreichischer Akademischer Austauschdienst \\
\hline ÖIBF & Österreichisches Institut für Berufsbildungsforschung \\
\hline ÖIR & Österreichisches Institut für Raumplanung \\
\hline ÖROK & Österrreichische Raumordnungskonferenz \\
\hline PE & Personalentwicklung \\
\hline QM & Qualitätsmanagement \\
\hline R\&D & Research and Development \\
\hline SFR & Schweizer Franken \\
\hline STG & Studiengang \\
\hline STJ & Studienjahr \\
\hline SWS & Semesterwochenstunden \\
\hline TIG & Technologie Impulse Gesellschaft m. b. H. \\
\hline UG & Universitätsgesetz 2002 \\
\hline UniStG & Universitäts-Studiengesetz 1997 \\
\hline UOG & Universitäts-Organisationsgesetz \\
\hline VSNU & $\begin{array}{l}\text { Rektorenkonferenz (Vereniging van Samenwerkende Nederlands } \\
\text { Universiteiten) (Niederlande) }\end{array}$ \\
\hline VVI & visitierende und validierende Institute (Niederlande) \\
\hline VZÄ & Vollzeitäquivalente \\
\hline VWGH & Verwaltungsgerichtshof \\
\hline WB & Weiterbildung \\
\hline WIFO & Institut für Wirtschaftsforschung \\
\hline WR & Wissenschaftsrat (Deutschland) \\
\hline
\end{tabular}



Authors: Lorenz Lassnigg, Martin Unger, Hans Pechar, Ada Pellert, Eva Schmutzer-Hollensteiner, Don F. Westerheijden

Title: Review des Auf- und Ausbaus des Fachhochschulsektors

Projektbericht/Research Report

(C) 2003 Institute for Advanced Studies (IHS),

Stumpergasse 56, A-1060 Vienna • ㄲㅛㅛ +43 1 59991-0 • Fax +43 159991-555 • http://www.ihs.ac.at 